ANL-7054

ANL-7054

Argonne Mational Laboratoru

COLLECTED ALGORITHMS 1960-1963

from the

Communications of the Association

for Computing Machinery

by

R. E. Grench and H. C. Thacher, Jr. 


\section{DISCLAIMER}

This report was prepared as an account of work sponsored by an agency of the United States Government. Neither the United States Government nor any agency Thereof, nor any of their employees, makes any warranty, express or implied, or assumes any legal liability or responsibility for the accuracy, completeness, or usefulness of any information, apparatus, product, or process disclosed, or represents that its use would not infringe privately owned rights. Reference herein to any specific commercial product, process, or service by trade name, trademark, manufacturer, or otherwise does not necessarily constitute or imply its endorsement, recommendation, or favoring by the United States Government or any agency thereof. The views and opinions of authors expressed herein do not necessarily state or reflect those of the United States Government or any agency thereof. 


\section{DISCLAIMER}

Portions of this document may be illegible in electronic image products. Images are produced from the best available original document. 


\section{LEG A L NOTICE}

This report was prepared as an account of Government sponsored work. Neither the United States, nor the Commission, nor any person acting on behalf of the Commission:

A. Makes any warranty or representation, expressed or implied, with respect to the accuracy, completeness, or usefulness of the information contained in this report, or that the use of any information, apparatus, method, or process disclosed in this report may not infringe privately owned rights; or

B. Assumes any liabilities with respect to the use of, or for damages resulting from the use of any information, apparatus, method, or process disclosed in this report.

As used in the above, "person acting on behalf of the Commission" includes any employee or contractor of the Commission, or employee of such contractor, to the extent that such employee or contractor of the Commission, or employee of such contractor prepares, disseminates, or provides access to, any Information pursuant to his employment or contract with the Commission, or his employment with such contractor.

Printed in USA. Price $\$ 5.00$. Available from the Clearinghouse for Federal Scientific and Technical Information, National Bureau of Standards.

U. S. Department of Commerce, Springfield, Virginia 


ANL-7054
Mathematics and Computers
(TID-4500, 45th Ed.)
AEC Research and
Development Report

ARGONNE NATIONAL LABORATORY

9700 South Cass Avenue

Argonne, Illinois 60440

COLLECTED ALGORITHMS 1960-1963

from the

Communications of the Association

for Computing Machinery

Compiled and Edited by

R. E. Grench

Reactor Engineering Division

and

H. C. Thacher, Jr.

Reactor Physics Division

RELESASED FIOR ARTROUNCEMMEAT

IN MUCLEAR SCIENICE ABSTRACTS

July 1965

Operated by The University of Chicago

under

Contract W-31-109-eng-38,

with the

U. S. Atomic Energy Commission 


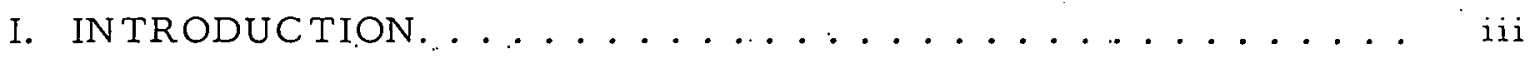

II. SUGGESTIONS TO THE USER. . . . . . . . . . . . v v

III. AN intRoduction to AlgOL .............. vii

- IV. COLLECTED ALGORITHMS (in numerical order) . . . . . . . I

V. SUBJECT INDEX ....................... 140

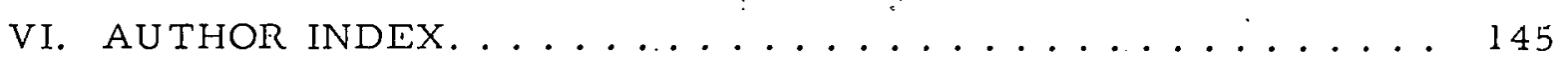




\section{COLLECTED ALGORITHMS 1960-1963 \\ from the \\ Communications of the Association \\ for. Computing Machinery \\ Compiled and Edited by}

R. E. Grench and H. C. Thacher, Jr.

\section{INTRODUCTION}

An algorithm may be defined* as "any method of computation consisting of a comparatively small number of steps; the steps to be taken in a preassigned order and usually involving iteration, which are specifically adapted to the solution of a problem of some particular type." Much of the effort of the casual computer user is devoted to developing and programming algorithms for relatively standard tasks, such as the evaluation of special functions, the solution of systems of linear equations, the estimation of definite integrals, sorting of data, and so forth. Even if he succeeds in finding a description of a suitable procedure, he must often spend considerable effort in translating the rather vague prescriptions of a prose description into a more or less complete computer subroutine.

Normal English prose and conventional mathematical notation are not only unacceptable for input to a computer, but also inconvenient for communicating the dynamic processes of computation from one individual to another. The international ALGOL language can satisfy both these needs. Although its use as a machine input language has grown rather slowly, at least in this country, it is the internationally accepted method of describing numerical calculations in most journals devoted to computation. Many papers describing original research on numerical methods now include an ALGOL program to specify the calculation which was performed. In addition, several journals now include columns of algorithms for common tasks, along with comments on previously published methods.

Among the journals in which algorithms appear more or less regularly are: Communications of the Association for Computing Machinery, BIT (Nordisk Tidskrift for Informations Behandling), Computer Bulletin, and Numerische Mathematik. The algorithms in Numerische Mathematik are outstanding from the standpoints of quality and careful editing. However, they are rather few in number. By far the widest variety of algorithms have been published in the Communications of the Association for Computing Machinery.

\footnotetext{
* The International Dictionary of Applied Mathematics, D. Van Nostrand Co., Inc., Princeton, 1960, p. 32.
} 
In this report, we have collected and reprinted the first 220 algorithms published in this journal, between February 1960 and December 1963, with remarks and certifications pertaining to them which were published through 1964. These algorithms are those which were published while J. H. Wegstein was editor of the Algorithms column. For the benefit of readers who are unfamiliar with the ALGOL language, we have also reprinted a tutorial description of the language by H. R. Schwarz, originally published in the Communications of the Association for Computing Machinery. We wish to express our gratitude to the Association for Computing Machinery and to Dr. Schwarz for permission to reprint this copyrighted material. 


\section{SUGGESTIONS TO THE USER}

The following suggestions are intended to aid the casual computer user who desires a guide in writing a subroutine for a particular task.

This volume includes two indexes: one by general subject, and one by author. In the first, prepared by G. E. Forsythe, the algorithms are arranged according to a modification of the SHARE classification. Having determined the classification which includes the task to be accomplished, a reference to this index will give the numbers and descriptive titles of algorithms in this area. The comments for each algorithm include a statement of what the algorithm accomplishes and a general description of the method used, often with a reference to a more extended discussion. The Certifications (results of tests of the algorithm) and Remarks which follow each algorithm, often contain very helpful information.

If several algorithms appear equally applicable, the user must make a choice. Although there is no substitute for a thorough knowledge of computing methods, or the advice of a qualified consultant, the following criteria may be helpful. (1) More recent algorithms are apt to represent improvements on earlier ones. (2) Wherever possible, use algorithms for which a certification appears. Many of the algorithms in this group were not certified before publication and contain more or less serious errors. The certifications also include numerical results which will be useful in testing your own program. (3) Certain algorithms are written recursively, i.e., call upon themselves. Thïs type of programming is usually inefficient. Many ALGOL translators do not provide for this feature, and hand translation into other languages is usually quite difficult. Thus, recursive algorithms should be avoided if there is any other alternative.

Having selected an algorithm, the task remains of adapting it to the computing system available. If an ALGOL translator is included in your system, this may be fairly trivial. If only a less sophisticated language is provided, the task may be somewhat more tedious, because of the limitations of the target language. Thus, for example, the limitation of Fortran to positive integer subscripts frequently makes the resulting program appear clumsy. The article by Dr. Schwarz will provide more than sufficient instruction in the meaning of the ALGOL, and the ease of translation will depend principally upon the user's familiarity with the language which he is translating.

When the adaptation has been completed, the user should test his program as thoroughly as possible. Tests against the values given in Certifications and problem collections will not only insure against errors in transcription, but will also give an intuitive feeling for the behavior of the method which is often more useful than a rigorous mathematical treatment. 
Testing is, of course, absolutely necessary for uncertified algorithms; where the user may have to contend with minor or major errors in the original program. If errors are discovered, their prompt dissemination by publication in the Communications of the Association for Computing Machinery is highly recommended. 


\title{
An Introduction to ALGOL
}

\author{
H. R. Schwarz \\ Swiss Federal Institute of Technology, Zurich, Switzerland
}

\section{Programming Languages in General}

To solve a numerical problem with the use of a computer; the solution must be described in terms of the computer, i.e.. in computer notation, before it can be given to the machine. Unfortunately, however, the computer notations are not only much different from classical mathematical notation but also different among themselves, and many of the difficulties of programming may be attributed to these variations.

Frequent attempts have been made to bridge the gap between computer and classical mathematical notation by devising notations somewhere between the two extremes in order to combine the advantages of both:

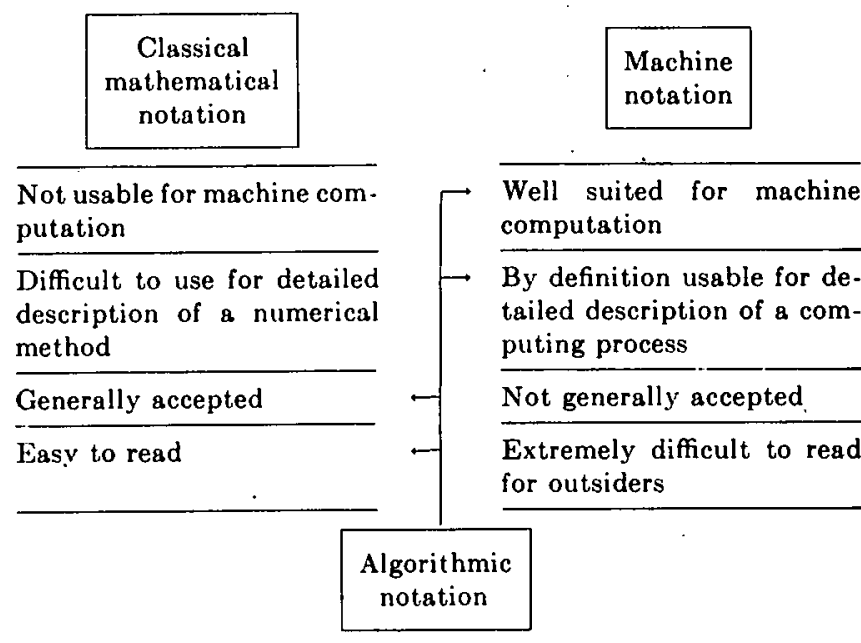

Such algorithmic notations, as we shall call them, have the appearance of classical mathematical notation but include certain dynamic elements which remind one of ordinary programming. Before explaining the technical details, however, let us review the historical facts. (More information may be found in $[1,2,3]$.)

In 1951 Rutishauser worked out a notation for numerical processes which could have been translated into machine notation by the machine itself. Unfortunately this translation could not be done by the small relay computer $\mathrm{Z} 4$. which was then in operation in the Swiss Federal Institute. Moreover, the notation was somewhat clumsy and not very efficient, and therefore it was never used in practise. On the other hand, it did prove for the first time that programming could be made fully automatic.

*Comm. ACM 5 (Feb 1962), 82-95.
However, this early work [1] on automatic programming did not receive much attention until the Fortran system was announced. Shortly after that (more exactly, after the Darmstadt meeting on computing machines in 1955), a European group for automatic programming was formed that subsequently worked out an algorithmic notation which was ready for publication at the end of 1957. However,-it-was-decided-to ${ }^{-}$hold ${ }^{-}$back-the-publication and to $^{-1}$ try to discuss the subject with the ACM. In a joint conference of the ACM group with the GAMM group (GAMM was the official name of the European group mentioned above) which took place in May 1958 at Zurich, the two groups combined their proposals for algorithmic notations into one and thus Algol was born [4].

After the preliminary publication, much interest in the subject developed and the final result of further discussions and publications was the January 1960 Conference in Paris. At this conference a completely new version of Algol was created and its concept was published in [5].

Prior to the Paris Conference, Rutishauser held some lectures on the first version of ALGOL at the Summer Conference in Chapel Hill, N.C. from August 17 to 28, 1959. These lectures have been published [6], and the present paper represents an adaptation of them to Algol 60 in accordance with a suggestion at the Matrix Symposium held by SIAM in Gatlinburg, April 24-29, 1961.

I wish to acknowledge my indebtedness to Prof. H. Rutishauser for his generous support at that time, thus enabling me to write the present paper.

\section{A First Look at ALGOL}

By definition, Algol is a means of describing numerical processess in an easily readable form that is ready for automatic translation into a machine program. The description of a numerical method in terms of ALGOL is called the Algol program for the method. Such an Algol program consists of statements like the following:

$$
x:=2 \times p \times q ; \quad y:=2 \times q \times q-p \times p
$$

which mean that the variables on the left side of the symbol $:=$ should be given the values which result from the expressions on the right side. Further features allow one to describe repeated execution of a statement for several values of a subscript (subscripts are always written in square brackets):

$$
\begin{aligned}
& h:=0 ; \\
& \text { for } k:=1 \text { step } 1 \text { until } n \text { do } h:=h+a[k] \times b[k]
\end{aligned}
$$


Here $h$ obtains the value zero at the beginning; then for every $k$ the product $a[k] \times b[k]$ is added to $h$; thus at the end $h$ is the inner product of the two vectors $a$ and $b$. This example shows already that the for statement corresponds to a loop in ordinary programming. The treatment of nested loops is described later.

In computations, frequently operations must be carried out only if a certain condition is fulfilled; an example may illustrate the treatment of such cases in AlgoI:

$$
\text { if } \mathrm{z}<0 \text { then } \mathrm{z}:=0
$$

Here $\mathrm{z}$ is unchanged if $\mathrm{z} \geq 0$, because then the statement $\mathrm{z}:=0$ is skipped. But if $z$ is negative, $z$ is replaced by zero; thus in the whole, the statement replaces $z$ by $\operatorname{Max}(0, \mathrm{z})$.

An additional feature is the label, which allows one to mark any statement of an ALGOL program. This may be used for referring to statements in explanations, but more important is the possibility to jump to such labeled statements. Example:

$$
\begin{aligned}
& \text { a33: } x:=p \times x+q \times y \\
& \vdots \\
& \text { go to } \text { a33 } \\
& \vdots
\end{aligned}
$$

Here a33 is a label and go to a.33 means an unconditional jump to the statement carrying that label; i.e. go to corresponds to the unconditional jump order in ordinary programming. Conditional jumps can be treated by combination of if and go to statements:

$$
\text { if } x \neq 0 \text { then go to a } 33
$$

Except for such go to statements (and conditional statements, which are explained later), the statements of an ALGol program are carried out in sequence.

\section{Variables and Expressions}

In $\$ 2$ we have given a preliminary description of some features of ALGoL. In order to enable the reader to make full use of AlgoL, exact definitions are given in the AlgoL report [5]. It is the purpose of this paper to give further explanations and examples in order to make the Algol report more familiar.

(a) The basic symbols from which Algol is built up are listed in (AR2). ${ }^{1}$ The meanings of the operators need no further explanations, except $\neg$ and $\equiv$, which are the symbols for logical negation and equivalence.

(b) For another class of delimiters no symbols were available; therefore words had to be used in place of them. These words, which in handwriting and typewriting are always underlined and in printing are always set in boldface type, are called word delimiters; they have, like $\times$ and + , fixed meanings and should not be translated into other languages.

${ }^{1}$ In this way we shall cite sections of the ALGOL Report [5]: (AR2) refers to section 2. (c) An important notion is the identifier I. An identifier is a string of letters and digits that begins with a letter. Thus xe7c, p, a, p123 are identifiers, but 17 and 7 ae are not.

In Algol, identifiers are used as names of variables and other entities occurring in computations. They may be chosen quite freely. Thus a variable may be given a name like vcrit or z2asymm, and greek letters may be transcribed into "alpha" or "omega". However, no delimiters may be used within an identifier, e.g. v-crit is not allowed as an identifier, because it could not be distinguished from the difference of the variables $\mathrm{v}$ and crit.

Moreover, it should be kept in mind that some few combinations (see AR3.2.4) are already reserved as names of the elementary functions of analysis and should therefore not be used. But besides these exceptions, identifiers have no fixed meanings like the word delimiters, from which they are distinguished by the boldface type used for the latter.

(d) In (AR3 to 5), the rules are given for building up expressions, statements and declarations from the basic symbols. To understand these rules, let us consider an example:

〈subscripted variable $\rangle::=\langle$ array identifier $\rangle$ [(subscript list $\rangle]$

(See AR3.1.1.) By applying the recursive syntactic definition, this example has the following meaning: A subscripted variable (collective abbreviation $\mathrm{V}$ ) consists of an array identifier I, followed by a sequence of arithmetic expressions $\mathrm{E}$ in square brackets and separated by commas. Examples:

$$
\mathrm{a}[\mathrm{k}+1, \mathrm{k}-\mathrm{i}] ; \mathrm{b}[0.5 \times(\mathrm{i}+\mathrm{k})+0.5 \times \mathrm{abs}(\mathrm{i}-\mathrm{k})] ; \mathrm{c}[\mathrm{i}, 3, \mathrm{k}]
$$

As the definition of $\mathrm{V}$ occurs in (AR3.1.1), expressions (which are admitted as subscripts) are not yet defined, but it is to be understood that anything is admitted as $\mathrm{E}$ that fits the definition of arithmetic expressions given later in (AR3.3) [see also below under (e)]. Since according to this definition arithmetic expressions may themselves contain subscripted variables, the following construction is allowed:

$$
\mathbf{a}[\mathrm{u}[\mathbf{k}], \mathbf{v}[\mathbf{k}], \mathbf{w}[\mathrm{u}[\mathrm{j}], \mathbf{v}[\mathbf{j}]]]
$$

In most cases, however, the subscript positions of subscripted variables will be occupied just by simple variables and integers (these are special cases of expressions).

(e) (AR3.3) shows the rules for the construction of arithmetic expressions, which are the backbone of ALGoL:

First, the syntactic rules in (AR3.3.1) define numbers, variables (simple or subscripted), and functions (to be defined later) as expressions. Thus, 17 and a[k] are expressions.

Second, any connection of expressions according to the rules of arithmetic (including exponentiation) is again an expression. Finally, an arbitrary expression, if enclosed in parentheses, is again an expression, i.e. one may write additional parentheses at will.

Third, in Algol even conditional arithmetic expressions are admitted, which means that according to a condition 
one of two alternative expressions is chosen. An example may illustrate this feature:

$$
\text { (if } m<n \text { then } m \text { else } n \text { ) }
$$

Here the resulting value of that conditional expression is taken as $m$ if $m$ is less than $n$ and as $n$ in the opposite case. Thus the expression has the meaning of $\operatorname{Min}(n, m)$.

The operational meaning of an expression is partially given by the composition rules; it is always the number which results if the expression is evaluated with the actual values of the occurring variables along the rules of ordinary arithmetic. There are, however, a few cases like $a / b / c$ where ${ }^{2}$ the operational meaning must be defined by additional rules (so-called precedence rules). The precedence rules of ALgol are given in (AR3.3.5.1).

(f) The definition of Boolean expressions (AR3.4) is quite analogous to the definition of arithmetic expressions, and the operational meaning can also be defined analogously. But, in addition, Boolean expressions are generated by arithmetic relations (equations and inequalities) of the general form: $E_{1} \rho E_{2}$, where $E_{1}$ and $E_{2}$ are arithmetic expressions and $\rho$ is any of the six relational operators. Thus,

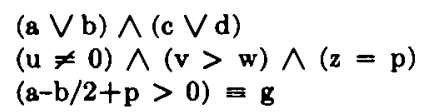

(the parentheses may even be omitted)

are Boolean expressions. The operational meaning of $E_{1} \rho E_{2}$ is

true, if the relation holds with the actual values of the variables occurring in $\mathrm{E}_{1}$ and $\mathrm{E}_{2}$;

false, if the relation does not hold.

A Boolean variable or expression cannot take values other than true or false.

(g) Since the general form of a function already uses the notion of parameter, general functions are therefore explained under procedures. Functions have the form $I_{f}(E, E, \ldots, E)$, i.e. a function name $I_{f}$ (an identifier) followed by a list of expressions in parentheses. The operational meaning is a single number (the value of the function). How this value is computed from the actual values of the expressions $E$ must be defined by a corresponding procedure declaration (see $§ 8 . a$ ).

(h) A label must be either an identifier or an integer; thus 17 and $A b 7$ are labels. Labels may be used to mark certain statements in an Algol program.

A simple designational expression may be either a label as defined above, or (formally) a variable with one single subscript position (a switch variable), i.e. $\mathrm{L}$ or $\mathrm{I}[\mathrm{E}]$. The operational meaning is in either case a label, namely $\mathrm{L}$ if the expression is already a label $\mathrm{L}$, but if it is a switch

\footnotetext{
${ }^{2}$ Such situations should be avoided by writing a sufficient number of parentheses, but notwithstanding an operational meaning must be defined also for such expressions as long as they obey the composition rules formally.
}

variable $I[E]$ the operational meaning must be defined by a switch declaration for the identifier I (see \$5.c).

As in the case of arithmetic expressions, conditional designational expressions are also admitted. Example:

$$
\text { if } k>n \text { then } 130 \text { else } s[k]
$$

\section{Statements}

Expressions may be interpreted as orders to compute something according to given rules, but otherwise they have no independent dynamic meaning. Only the statements of Algol are essentially dynamic; they are the sentences of the language.

For statements we introduce the collective abbreviation $S$, which means that in all following composition rules any statement is admissible for an $\mathrm{S}$, provided this is not explicitly forbidden.

\section{(a) Compound Statements and Blocks (AR4.1)}

Before defining the various kinds of statements, we refer to the possibility that any sequence of statements can be grouped together into one statement (which is then called a compound statement) just by enclosing them between the word delimiters begin and end:

$$
\text { begin } \mathrm{S} ; \mathrm{S} ; \ldots ; \mathrm{S} \text { end }
$$

Such a compound statement ${ }^{3} S_{c}$ may be used in place of any $S$ occurring in definitions of elements of Algol. It may be used also in the above definition; therefore some of the statements in a compound statement may already be compound, e.g.:

begin $\mathrm{S} ; \mathrm{S}$; begin $\mathrm{S} ; \mathrm{S} ; \mathrm{S} ; \mathrm{S} ; \mathrm{S}$ end end

On the other hand, there is one restriction: Only statements and declarations (see below) may be enclosed between begin and end; therefore it is not permissible to group a sequence like

$$
\mathbf{S} ; \mathbf{S} ; \text { begin } \mathbf{S} ; \mathbf{S} ; \mathbf{S}
$$

together into one compound statement, since begin is not a statement or declaration. In other words, if a statement $S$ is at the same time part of two different statements $S_{c}$ and $S_{c c}$, then either $S_{c} \subset S_{c c}$ or $S_{c c} \subset \dot{S}_{c}$.

If a sequence of statements is preceded by some declarations D (see \$5) and enclosed between begin and end, this combination is called a block. It is of the general form

$$
\text { begin } \mathrm{D} ; \mathrm{D} ; \ldots ; \mathrm{D} ; \mathrm{S} ; \mathrm{S} ; \ldots ; \mathrm{S} ; \mathrm{S} \text { end }
$$

Such a block introduces a new level of nomenclature, which means that all declarations in the head of the block are only valid inside the block. Outside the block the declared quantities have no existence; thus they are said to be local quantities to the block.

On the other hand, each of the statements S above may again be a compound statement or a block. Thus the

\footnotetext{
${ }^{3}$ Note that the statements are separated from each other by semicolons, but no semicolon is needed after begin or before end.
} 
notion of nonlocal entities is introduced: An entity occurring in a block but not being declared inside this block is called nonlocal and represents the same quantity inside the block and at least in the level immediately outside it.

(b) Assignment Statement (AR4.2)

An assignment statement, $\mathrm{V}:=\mathrm{E}$ for example, assigns a new value, namely the value of the expression $E$ at the moment, to the variable $V$ on the left side of the assignment symbol $:=$. It must be kept in mind that in contrast to classical mathematical notation an assignment like $\mathbf{x}:=\mathbf{x}+\mathbf{E}$ is meaningful in Algol, since this simply means that the value of $x$ is increased by the actual value of the expression $E$.

The Boolean assignment statement is defined by analogy as $\mathrm{V}:=\mathrm{B}$ where $\mathrm{B}$ is a Boolean expression and $\mathrm{V}$ is a Boolean variable. Example:

$$
\text { decide }:=(a=0) \equiv(b=0)
$$

This gives the variable "decide" the following value:

$$
\begin{aligned}
& \text { true, if } a=b=0 \text { or } a \times b \neq 0 \\
& \text { false, otherwise. }
\end{aligned}
$$

The assignment statement may be used also in an extended form: the same value of an expression may be assigned to several variables of the same declared type (see \$5, type declarations). Example:

$$
\mathrm{i}:=\mathrm{k}:=\mathrm{p}:=1
$$

This assignment statement gives the variables $i, k$ and $p$ (of type real or integer) the value 1 .

(c) Labeled Statements

Any statement can be furnished with a label, which is written in front of the statement and separated from it by a colon. Thus

$$
\mathrm{L}: \mathrm{S}
$$

is a labeled statement and may be used in place of any $S$ in the Algol definitions. Example:

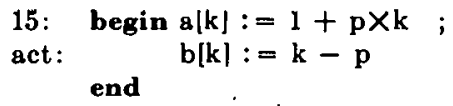

(d) Go To Statements (AR4.3).

Go to statements interrupt the normal sequence of execution of statements. The statement

$$
\text { go to } \mathrm{D}^{\mathbf{L}}
$$

where $D^{L}$ is a designational expression, has the operational meaning of a jump to the statement whose label $L^{\prime}$ is defined by $\mathrm{D}^{\mathrm{L}}$. If $\mathrm{D}^{\mathrm{L}}$ is already a label, then this is $\mathrm{L}^{\prime}$. But if $D^{L}$ is a switch variable I[E], then the label $L^{\prime}$ is the label in the $\mathbf{k}^{\text {th }}$ position of the switch declaration for the identifier $\mathrm{I}$, where $\mathrm{k}$ is the integer nearest to the actual value of the expression $E$ (see also the explanations for the switch declarations, \$5.c, where an example also is given). If $\mathrm{D}^{\mathrm{L}}$ is a conditional designational expression, the label $L^{\prime}$ is defined accordingly.

It must be kept in mind that after a jump to a statement L : S has occurred, then, beginning with the statement
$\mathrm{L}: \mathrm{S}$, the statements are again carried out in sequence until a new jump takes place; but no automatic return to the origin of the jump will occur.

An exception has to be added: If the designational expression $D^{L}$ is not defined, e.g. if the subscript expression of the switch variable lies outside the range from 1 to $n$, where $n$ is the length of the switch variable, then the go to statement has no effect and the next following statement is carried out.

(e) If Statements and Conditional Statements (AR4.5)

The general form of an if statement is

if $B$ then $S_{\text {uneoad }}$

where $B$ is a Boolean variable or expression and $S_{\text {uncond }}$ is an unconditional statement. It has the following operational meaning: The statement immediately following if $B$ then is executed if and only if the actual value of $B$ is true. Thus the if statement occurring in an Algol program changes the normal sequence of execution of statements only if the value of $B$ is false; in that case the unconditional statement $\mathrm{S}_{\text {uncond }}$ is skipped, but no other change occurs. Example:

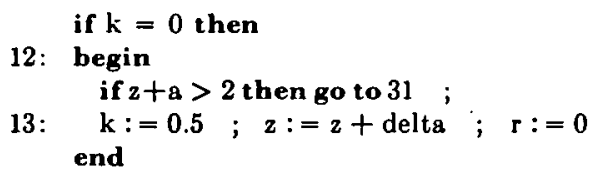

Here the statement immediately following if $\mathrm{k}=0$ then is the compound statement with the label 12. Therefore in case $\mathrm{k} \neq 0$ this compound statement is skipped as a whole and the computation is resumed after end. If $\mathbf{k}=0$, then the compound statement, i.e. the single statements contained in it, are executed: ${ }^{4}$ If $\mathrm{z}+\mathrm{a}>2$, then a jump to 31 occurs; otherwise, the three statements following label 13 are executed, after which the computation proceeds with the statement after end.

An extended form of an if statement is the conditional statement, which is of the general form:

\section{if $B$ then $S_{\text {uncond }}$ else $S$}

Here $B$ is a Boolean variable or expression, $\mathbf{S}_{\text {uncond }}$ an unconditional statement, and $\mathrm{S}$ an arbitrary statement, i.e. it may be itself a conditional statement. A conditional statement has the following operational meaning: According to the actual value of $\mathrm{B}$ one of the two alternative statements $S_{\text {uncond }}$ and $S$ is selected. If $B$ has the value true, then $S_{\text {uncond }}$ is executed and $S$ is skipped; if $B$ has the value false, then $S_{\text {uncond }}$ is skipped and $S$ is executed. Thus the normal sequence of statements is changed in any case by a conditional statement. Example:

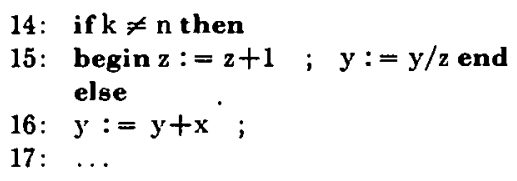

A compound statement is unconditional regardless of the statements contained in it. 
The same operational effect may be achieved by the following explanatory program:

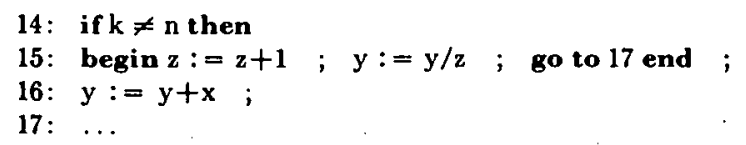

(f) For Statements (AR4.6)

The general form of a for statement is:

$$
\text { for } \mathrm{V}:=\ell \text { do } \mathrm{S}
$$

where $\ell$ represents a list of elements as defined below and $S$ is any statement. The for statement causes the variable $\mathrm{V}$ (the running subscript) to run through the actual values of all expressions in the list in succession, and for every value of $\mathrm{V}$ the statement $\mathrm{S}$ will be executed exactly once. After exhaustion of the list, the normal sequence of execution is resumed, heginning with the statement-after $S$.

In most cases an element of the for list $\ell$ will be given in the form

$$
E_{1} \text { step } E_{2} \text { until } E_{3}
$$

which is an abbreviation for $E_{1}, E_{1}+E_{2}, E_{1}+2 E_{2}, \ldots$, $\mathrm{E}_{1}+n \mathrm{E}_{2}$, where $\mathrm{n}$ is defined as the largest integer such that (for the actual values of the $E^{\prime} s$ ): $n \leq\left(E_{3}-E_{1}\right) / E_{2}$. If the resulting $\mathrm{n}$ is negative, then the list element is defined as empty; in this case the statement $S$ is not carried out at all for that element.

However, this explanation does not cover the full generality of such an element and is only valid in the case where the arithmetic expressions $E_{2}$ and $E_{3}$ do not depend on variables changed during the execution of the statement S. A concise description of the operational meaning of the general form of such a for list element is given in (AR 4.6.4.2). Example:

$$
\text { for } i:=1 \text { step } 1 \text { until } n \text { do } v[i]:=0
$$

The components of a vector $\mathrm{v}$ are set to zero for the indices running from 1 to $\mathrm{n}$.

In other cases a for list element may consist of a single arithmetic expression and a for statement may look like

$$
\text { for } V:=E_{1}, E_{2}, \ldots, E_{0} \text { do } S
$$

where the $E_{k}$ stand for arithmetic expressions. The statement $\mathrm{S}$ is executed $\mathrm{n}$ times while $\mathrm{V}$ has the values of $E_{1}, E_{2}, \ldots, E_{n}$ consecutively. The values of the arithmetic expressions are computed and assigned to $\mathrm{V}$ just before the execution of the statement $S$. This fact may be important if one or several of the expressions $E_{k}$, except the first, depend on variables calculated inside the statement S. Examples:

for $\mathrm{p}:=0.1,0.2,0.5,0.8,0.9,0.95$ do $\mathrm{S}$;

for $k:=a, b, a+b$ do $S$

In the first example, the expressions in the list are only numbers. In the second example, $\mathrm{S}$ is executed for $\mathrm{k}$ having the values of $a, b$, and their sum.

A third kind of an element of the for list is the so-called while element. It has the general form

$$
\text { E while B }
$$

where $\mathrm{E}$ is an arithmetic and $\mathrm{B}$ a Boolean expression. The statement $\mathbf{S}$ which stands under control of such a while element is executed for the actual value of $\mathrm{E}$ assigned to the running variable $\mathrm{V}$ as many times as the Boolean expression B has the value true. Example:

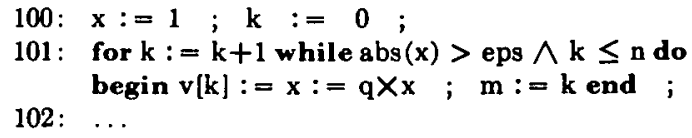

The two statements labeled by 100 and 101 together have the operational effect that the components of a vector $\mathrm{v}$ are assigned the values of a geometric progression with quotient $q$ and starting with $v[1]:=q$. This is done as long as the elements of the geometric progression are greater than a tolerance eps and $\mathbf{k}$ does not exceed the value of $n$. In statement 102 (or following) the number $m$ of assigned components is available.

It must be mentioned that the running variable $\mathrm{V}$ of a for statement is undefined when the loop is left, because of exhaustion of the for list. Thus no value of the variable $\mathrm{k}$ in the last example is available in statement 102 (or following); for this reason the running value of $k$ is assigned to another variable $\mathrm{m}$.

On the other hand, the actual value of the running subscript is defined outside the for statement if and only if the statement is left by a go to statement.

Example 1: A vector $\mathrm{v}$ is supposed to have strictly positive components. A program is established to check this assumption, and if it is not true, the index of the first negative component should be available:

50: for $\mathrm{i}:=1$ step 1 until $\mathrm{n}$ do

51: if $v[i] \leq 0$ then go to nonpos ;

52: $\ldots$

If all components $v[i]$ are positive, then the computation goes on with statement 52 . Otherwise, the for statement is left by the go to statement leading to the statement labeled nonpos. There $i$ is defined and this information may be used.

The most general form of a for list is a concatenation of the three kinds of elements, separated by commas. Thus in the example,

for $k:=1$ step 1 until $i-1, i+1$ step 1 until $n$ do $S$

the variable $k$ runs from 1 to $n$ in steps of 1 with the exception of $i \quad(1 \leq i \leq n)$. If $i=1$, then the first element is considered to be empty and the same holds for the second element for $\mathrm{i}=\mathrm{n}$.

Example 2: Simultaneous computation of $\mathrm{f}(\mathrm{x})=\mathrm{f}$ and $f^{\prime}(x)=f 1$ for a polynomial $f(x)=\sum_{k=0}^{n} a[k] x^{k}$ with Horner's rule.

$\mathrm{f}:=\mathrm{f} 1:=0$;

for $k:=n$ step -1 until 0 do

begin $f 1:=f 1 \times x+f ; f:=f \times x+a[k]$ end

In this example, after setting the values $f=f 1=0$, the compound statement consisting of the two statements $\mathrm{f} 1:=\ldots$ and $\mathrm{f}:=\ldots$ is executed $\mathrm{n}+1$ times in succession,-after which we have the required results. 
This example shows that if several statements must be put under control of the same for clause (or the same if clause), they must be enclosed by begin and end.

(g) Dummy Statements (AR4.4)

A dummy statement has no operational effect and acts like an empty order in ordinary programming. It is introduced by putting an additional semicolon. A dummy statement may serve to place a label at a place where it would not be allowed otherwise. Example:

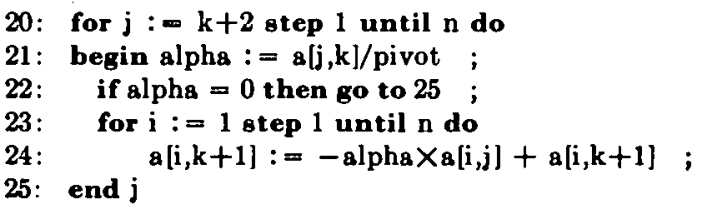

If alpha $=0$, then a go to statement refers to the end of the j-loop. The label " 25 " labels the dummy statement preceding end $j$ and introduced by the semicolon at the end of statement 24 .

\section{Declarations}

As explained in (AR5), declarations are interspersed in an Algol program to state certain facts. They are needed to make the program self-contained, so that no additional information is required and the computer may understand it. (On the other hand, the human reader of such a program might understand it in most cases without the declarations.) As already stated in \$4.a, the declarations are only valid for the block in the head of which they stand.

\section{(a) Type Declarations (AR5.1)}

For any simple variable occurring in an ALgol program a declaration concerning its type must be given. Three different types are possible: real, integer, Boolean. Examples of type declarations are:

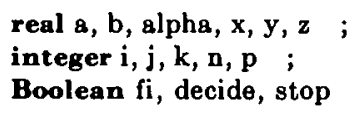

If a quantity is declared to be of type real, this ineans that it may be given positive and negative real values including zero. If a quantity occurring in an ALgol program is essentially integer, it should be declared so by an integer declaration. This has the following operational meaning: If for some reason a noninteger value is assigned to any of the variables listed in the declaration, this is immediately rounded to the nearest integer. But it should be understood that this rounding has only the purpose to round the value of an expression which is theoretically integer-valued to an exact integer, and not to replace the entier function.

Example: In the assignment statement

$$
k:=n \times(n+1) \times(n+2) / 6
$$

the expression on the right side is integer-valued for all integers $n$, but the compúted value may turn out to differ by a small amount from an integer. If $\mathrm{k}$ is declared to be an integer, the value of the expression is in all cases corrected to the nearest integer.

It must be noted, however, that the actual values of expressions occurring in subscript positions are automatically (without integer declaration) rounded to the next integer, but this rounding concerns only the values of the expressions and has no effect whatsoever on the variables in these expressions.

A Boolean variable (taking only the values true and false) occurring in an Algol program must be declared so by a Boolean declaration. On the other hand, Boolean expressions, especially those which are generated by arithmetic relations $\mathrm{E}_{1} \rho \mathrm{E}_{2}$, must not be declared as Boolean.

(b) Array-Declarations (AR5.2)

For any subscripted variable occurring in an ALGOL program, exact bounds for the values of the subscripts must be given. This is done by array declarations, which must appear in the block for which they are valid. General form:

$$
\text { array } I, I, \ldots, I[\ell], I, I, \ldots, I[\ell], I, \ldots
$$

In the list we have several groups of identifiers I, with each group followed by a list of subscript pairs in square brackets. The expressions of a subscript pair are separated by a colon and the pairs in the list are separated by commas.

Each group $I, I, \ldots, I[\ell]$ indicates that the variables I,I, . .,I are subscripted variables with as many subscripts as there are pairs in the list $\ell$. Moreover, the first expression of a pair gives the lower and the second expression the upper bound for the subscripts in the corresponding positions. Furthermore, these bounds are common for the group. It must be kept in mind that the expressions in the subscript pairs may depend only on variables which are non-local to the block in which the array declaration stands Thus in the outermost block of a program the subscript bounds in array declarations must be constant.

All subscripted variables as declared by an array declaration above are understood to be of type real. If, on the other hand, the declared variables are of type integer or Boolean, the array declaration has to be preceded by the corresponding type declarator integer or Boolean. Examples:

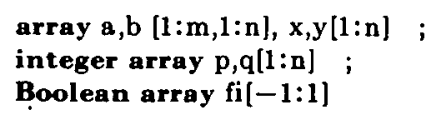

Here $a$ and $b$ are real variables with two subscripts each, the first running from 1 to $\mathrm{m}$, the second from 1 to $\mathrm{n}$ (rectangular matrices), and $x$ and $y$ are real variables with one single subscript running from 1 to $n$. $p$ and $q$ are both integer-valued variables whose single subscript runs from 1 to $\mathrm{n}$ and $\mathrm{fi}$ is declared to be a Boolean variable.

Remark Concerning own: Variables defined by type or array declarations may also be declared to preserve their values when the block is left by preceding the cor- 
responding declarations by the declarator own. For further details, see (AR5).

(c) Switch Declaration (AR5.3)

General form:

$$
\text { switch } I:=D_{1}^{L}, D_{2}^{L}, \ldots, D_{n}^{L}
$$

where $I$ is an identifier (the name of the switch) and the $\mathrm{D}_{\mathbf{k}}^{\mathbf{L}}$ are designational expressions (see $\S 3 . \mathrm{h}$ ). In most cases, the $D_{k}{ }^{k}$ will simply be labels. With this definition, go to $I[E]$ has the operational meaning go to $D_{k}{ }^{L}$, where $\mathrm{k}$ is the integer nearest to the actual value of $\mathrm{E}$. But if the resulting $\mathrm{k}$ lies outside the interval $\mathbf{l} \leq \mathrm{k} \leq \mathrm{n}$ ( $n$ is the number of switch entries), the go to statement is skipped and the normal sequence of operation is resumed with the following statement.

Some of the $D_{k}{ }^{2}$ occurring in the switch declaration may again be of the form $I[E]$, in which case we have multiple switches.

As an example, we lengthen the program for computing $f(x)$ and $f^{\prime}(x)$ by Horner's rule (given as an example in \$4.f) for $n \leq 3$ :

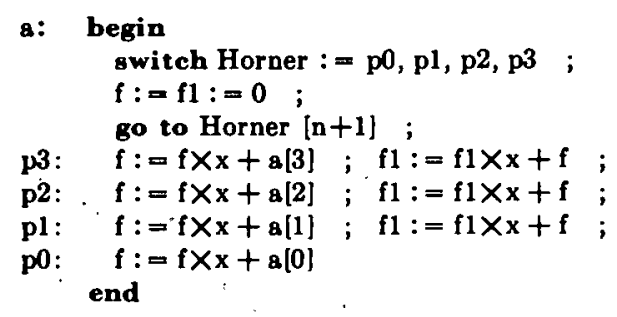

If one tries to use this program for $\mathrm{n}>3$, then go to Horner $[n+1]$ will not be executed, since Horner [n+1] is then outside the range of the switch declaration. Therefore the program should check whether $\mathrm{n}>3$ and should give an alarm, or a comment (see AR2.3) should be added to the example above in the form:

comment:- This program should not be used for $n>3$

\section{Examples}

Though the possibilities of writing programs are somewhat limited because we have not yet treated procedures, we shall give some examples here.

Example 1: The first example concerns the numerical integration of a differential equation of second order with given initial values. We choose the method of Adams' extrapolation, which consists of the following formulae:

$$
\begin{gathered}
y(x+h)=y(x)+h y^{\prime}(x)+h^{2}\left[\frac{1}{2} y^{\prime \prime}(x)+\frac{1}{6} \nabla y^{\prime \prime}(x)\right. \\
\left.+\frac{1}{8} \nabla^{2} y^{\prime \prime}(x)+\ldots\right] \\
y^{\prime}(x+h)=y^{\prime}(x)+h\left[y^{\prime \prime}(x)+\frac{1}{2} \nabla y^{\prime \prime}(x)\right. \\
\left.+\frac{5}{12} \nabla^{2} y^{\prime \prime}(x)+\ldots\right]
\end{gathered}
$$

where the $\nabla^{\mathrm{k}} \mathrm{y}^{\prime \prime}(\mathrm{x})$ are the backward differences of $\mathrm{y}^{\prime \prime}$ at the point $x$ and for the interval $h$. In contrast to other proposals, we start the integration by an iterative process (statements 2 to 8 ) which uses the same formulae as the forward integration (statements 10 onwards).
The quantities $\mathrm{x} 0, \mathrm{y} 0, \mathrm{z} 0, \mathrm{~m}, \mathrm{xE}, \mathrm{h}$ are supposed to be nonlocal to the block (which is thought of as a part of a larger program) and are therefore considered to be given at the moment of entering this block. Equally the variables $x, y, z$ must be nonlocal quantities to the block, so that they are available outside the block as the final result of the computation.

begin

comment: $x 0, y 0, z 0$ are the initial values for $x, y, y^{\prime} . m$ is the order of the method, it must be chosen $\leq 6$. $x E$ is the end of the integration, $h$ is the integration step. $w[0]$ is the second derivative, $w[k]$ its $k^{\text {th }}$ backward difference. The values of $x, y$, $z, w[k]$ for different values of $x$ are not distinguished by a subscript ;

real we, $p, q$; integer $v, r, j ;$ Boolean beta, decide ; array $b, c, w[0: 6]$;

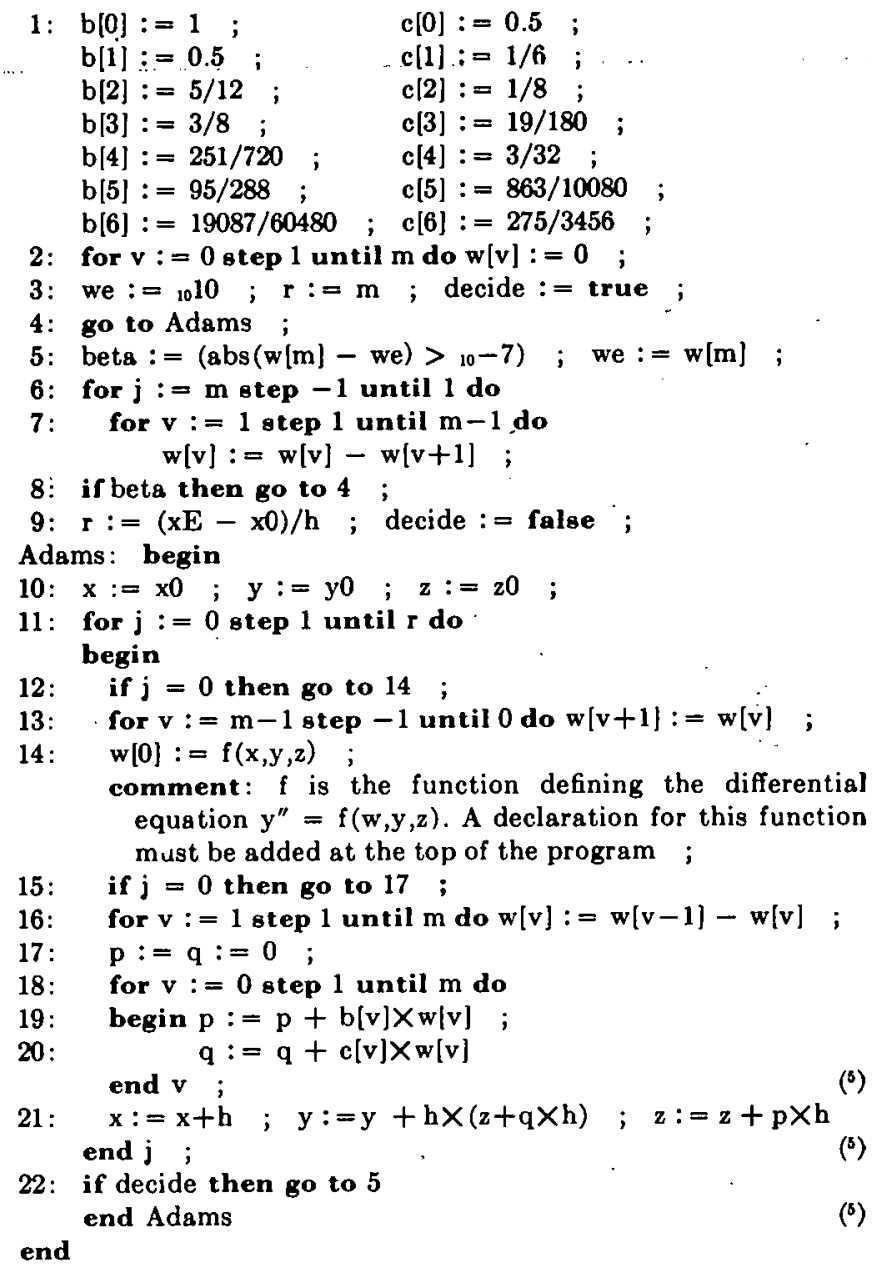

This method is based on the following principle:

The leading row of backward differences (which are unknown at the beginning) is first filled out with zeros

It should be noted that between end and the next semicolon or next end or next else, any string of symbols may be written (but not containing end, else or a semicolon) without any effect on the computation. This serves solely to show the reader which compound statement is closed by the end in question, but the machine does not need this additional information. 
(statement 2). With this leading row we integrate $\mathrm{m}$ steps ahead with the formulae of Adams (statement 4), since r in the compound statement Adams means the number of steps to be integrated. After this we may build up a new difference table from the $m^{\text {th }}$ row backwards by keeping the $\mathrm{m}^{\text {th }}$ difference constant (statements 6 and 7 ).

In this way we obtain a new leading row of backward differences, with which we again integrate $m$ steps forward. This is repeated until the $\mathrm{m}^{\text {th }}$ differences of two successive runs are nearly equal (statements 5 and 8 ; note that "we" is the $\mathrm{m}^{\text {th }}$ difference of the foregoing run). As soon as beta is false, we start integrating ahead a sufficient number of steps to reach $\mathrm{xE}$ (statements 10 and following).

Example 2: Computation of $\tan (\mathrm{x})$ with Lambert's continued fraction (1770).

Starting with $a[0]=0, b[0]=b[1]=1, a[1]=x$, the recursion formulae (not in ALgol!)

$$
\begin{aligned}
& \mathrm{a}[\mathrm{k}+1]=(2 \mathrm{k}+1) \mathrm{a}[\mathrm{k}]-\mathrm{x}^{2} \mathrm{a}[\mathrm{k}-1] \\
& \mathrm{b}[\mathrm{k}+1]=(2 \mathrm{k}+1) \mathrm{b}[\mathrm{k}]-\mathrm{x}^{2} \mathrm{~b}[\mathrm{k}-1]
\end{aligned}
$$

generate two sequences of numbers such that

$$
\lim _{\mathbf{k} \rightarrow \infty} \mathrm{a}[\mathrm{k}] / \mathrm{b}[\mathbf{k}]=\tan (\mathrm{x})
$$

exists for any (also complex) $\mathrm{x}$ which is not an odd multiple of $\frac{1}{2} \pi$, but even for the poles the sequence behaves reasonably, since then the reciprocal values converge to zero. The continued fraction converges quite fast-even for $x=5$ (with two poles between $x$ and the origin!) we obtain

$$
\mathrm{a}[12] / \mathrm{b}[12]=-3.380515027 \ldots
$$

in place of

$$
\tan (5) \quad=-3.380515006 \ldots
$$

There is, however, one difficulty which arises frequently in setting up the program for a problem of that kind: we do not know how many steps it will take in general for $\mathrm{x}$ to reach a certain accuracy. ${ }^{6}$ On the other hand, numbers $a[k], b[k]$ are generated by the process, for which subscript ranges must be declared in an array declaration, and for this the number of steps should be known.

However, in examples like this, where $a[k+1]$ and $b[k+1]$ depend only on a limited number of predecessors (general case: $a[k+1]=f(a[k], a[k-1], \ldots, a[k-m])$ with fixed $\mathrm{m}$ ), we may help ourselves by limiting the range of $\mathrm{k}$ to a finite interval $\mathrm{J}$, and then, as soon as $\mathrm{k}$ reaches one end of $J$, begin again at the other end, so that $k+1$ and $\mathrm{k}-\mathrm{m}$ are always in $\mathrm{J}$.

In this problem we would restrict $k$ to the interval from 0 to 21 , say, and then, as soon as $\mathrm{k}$ reaches 20 , redefine $a[0]:=a[20], a[1]:=a[21], \quad$ and the same for $b$. If needed, we may combine this with a division of the four

\footnotetext{
${ }^{6}$ In this example we can restrict $x$ to a finite interval because of the periodicity of the function. This would also give a limit for $k$ for a given accuracy. We do not make use of this possibility, since it might not exist in other cases.
}

numbers by the same constant in order to keep them in range.

The question remains of when to stop the computation. Without proof, I say that

$$
\operatorname{abs}\left(x^{2 k+1} /(a[k+1] \times a[k]+b[k+1] \times b[k])\right)<10^{-10}
$$

is a useful criterion and will lead to about 10-digit accuracy. With this, the following Algov program results:

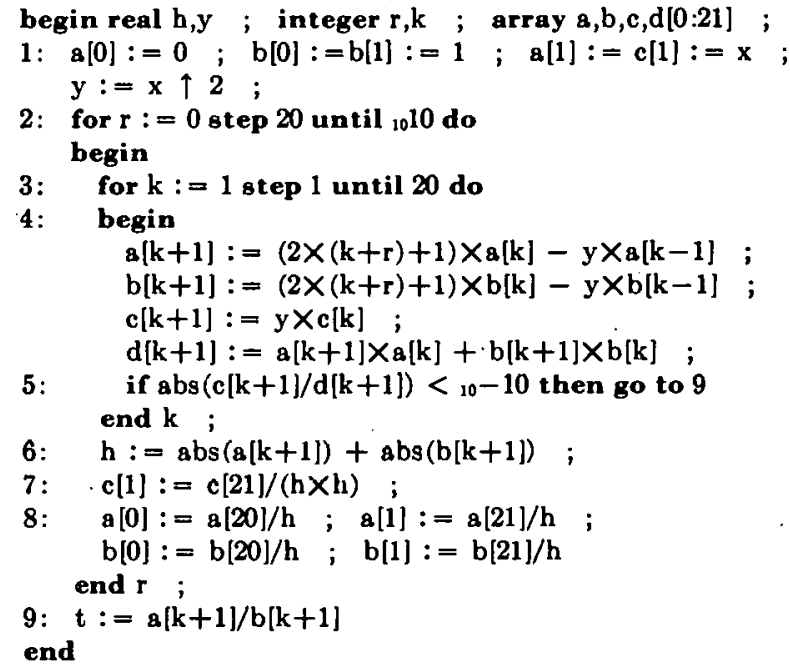

REmark: The block is understood to be a part of a larger program. It uses the nonlocal quantities $x$ (the argument) and $t$ (the result $\tan (x))$.

Example 3: This example includes one of the permunent functions: Let $y[0], y[1], \ldots, y[100]$ be 100 values of a function $y[k]=f(x 0+k h)$; then for a given $x$ in the interval $x 0 \leq x \leq x 0+100 h, f(x)$ can be computed approximately by linear interpolation:

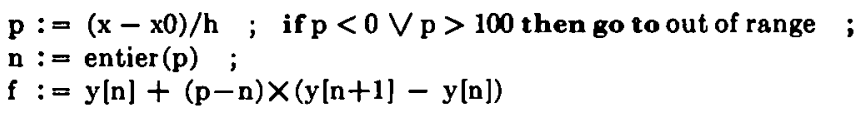

In this example no declarations are given for any of the variables. $x 0, x$ and $h$ must be given. If the argument $x$ lies outside the given range, the conditional go to statement leads to the statement labeled "out of range", where provision must be made for such a case.

\section{Errors in an ALGOL Program}

As in ordinary programming, one may make errors in writing an Algol program. These errors are more dangerous than in ordinary programming, since the whole translating process must be repeated if the generated machine program turns out to be wrong. Still worse is the effect of errors if such an Algol program is published. We distinguish several kinds of errors.

\section{(a) Syntactical Errors}

If an ALGOL program actually violates the composition rules, then it is called syntactically wrong. Such errors are in general already detected by the routine which should translate it into machine program. They may also 
be found very easily by proofreading, since they are in most cases quite obvious. Examples:-

Omission of one of a pair of brackets.

Omission of a begin or an end.

Two operation symbols in succession like $+x$.

Omission of a semicolon or other separating symbol.

An error happening sometimes is the omission of the multiplicstion symbol, since this is usual in mathematical notation, but not allowed in Algor.

Underlining of a word other than the word delimiters (see the list of those in AR2).

\section{(b) Operational Errors}

By operational errors we mean errors which destroy the operational meaning of parts of a program. Such errors are much harder to detect; in most cases they will pass the translation routine, but the generated machine program will be defective. On the other hand, a careful reader will be able to detect these errors, even if he is not familiar.with the problem to be solved.

Examples: (i) A variable occurs on the right side of an assignment statement dynamically before a value has been assigned to it, or the assignment for such a variable is bypassed by a jump:

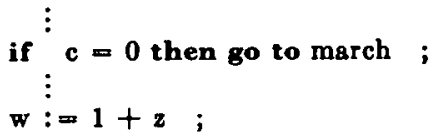

This is operationally meaningless for $c=0$, if $w:=1+z$ is the first statement involving $w$ in that program.

(In the examples treated so far there are many cases where a variable occurs the first time [or exclusively] on the right side of an assignment statement, but those are input variables for which-in an actually used programvalues will be assigned by input operations. These input operations have not been treated so far.)

(ii) A subscript exceeds the range which is prescribed by the array declaration.

(iii) A variable declared as Boolean occurs in an arithmetic expression or vice versa.

\section{(c) Programming Errors}

It is of course still possible that a program is syntactically and operationally correct but all the same--because of an oversight - does not describe the numerical method which it should. No rules can be given to prevent. errors of this kind. One can only check the Algol program carefully, and sometimes only a test run will detect an error. Quite tricky errors, which even a test computation will hardly detect, are those which only slow down the convergence of iteration methods without leading to wrong results.

\section{Procedures}

Procedures are the subroutines of Algou. Their purpose is the same as that of library subroutines of ordinary programming: they serve to simplify ALGoL programs.
As in ordinary programming, we distinguish between the description of a subroutine (procedure declaration) and the call or application of a subroutine (procedure statement or function call).

\section{(a) Procedure Declaration (see AR5.4)}

A procedure declaration consists of a heading and the statement describing the procedure. The general form of the heading of a procedure declaration is:

procedure $I(p, p, \ldots, p)$; value $\ell$; 〈specification part 〉;

Here $I$ is an identifier (the name of the procedure), the p's are the formal parameters of the procedure (in the form of identifiers). In general, these parameters are separated by commas, but one may also replace a comma by a parameter delimiter of the form $) \lambda \lambda \ldots \lambda$ ( where $\lambda$ stands for any letter. By inserting such parameter de-limiters-the-meaning of the-formal-parameters-may-be explained. They do not have any operational effect.

In the value part, value $\ell ;, \ell$ represents a list of those formal parameters which have to be treated as local quantities in the procedure body. Thus the operational effect of such a value part is the transfer of the values of all these listed parameters to storage places local in respect to the procedure body. If these formal parameters are assigned new values during execution of the procedure body, these values will not be available outside the procedure. As a consequence, formal parameters the values of which are wanted as results of the procedure must not be listed in the value part. Of course, the value part may be empty.

The specification part gives some information about the type and kind of the formal parameters. In most cases this additional information is not needed and the reader of the program could understand it without additional help, and thus these specifications are quite optional. However, there exist Algol translators (Alcor hardware group) for which all formal parameters must be specified, and anyhow a procedure is easier to read if all specifications are given.

After the heading, the statement which follows is needed to describe the procedure. In generul, the statement is a compound statement or even a block if local (temporary) quantities are declared.

Example 1: Computation of the inner product of two vectors $\mathrm{a}$ and $\mathrm{b}$ with $\mathrm{n}$ components each:

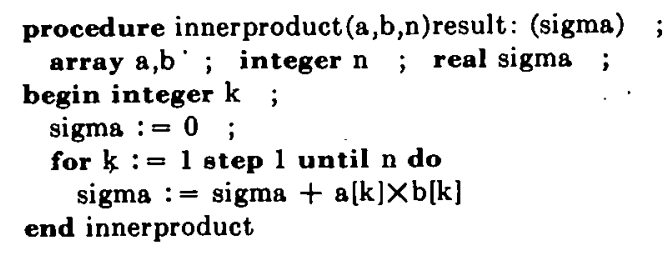

The procedure innerproduct has the property that it has only one output parameter which is a simple variable, i.e. it produces one single number. In such a case the procedure might be defined as a function by omitting the result parameter sigma. But then the output parameter 
has to be given the name of the procedure and the procedure declaration must be preceded by a corresponding type declarator (see AR5.4.4):

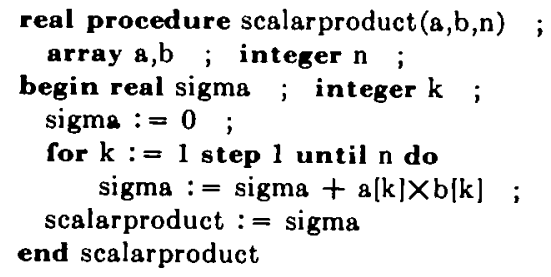

Remarks: Inside the procedure body at least one assignment statement for scalarproduct has to occur. But on the other hand, the variable scalarproduct must not appear on the right side of an assignment statement, because this would mean for its part an activation of the procedure scalarproduct. Therefore the auxiliary variable sigma has to be introduced.

In both examples the formal parameters $a, b$ and $n$ could be listed in the value part. But this is not necessary, because no new values are assigned to these parameters. In the first example, however, sigma must not appear in the value part.

Example 2: Gauss-elimination (without search for pivots) for the solution of $\sum_{k=1}^{n} a[i, k] \times x[k]+b[i]=0$ $(\mathrm{i}=1,2, \ldots, \mathrm{n})$. The input parameters are: the matrix $a[i, k]$, the constant terms $b[i]$, the number of equations $n$. The output parameter is: the solution $x[k]$.

If we denote the coefficients of the reduced equations of Gauss' method by the same identifiers a and b as the originally given elements, we obtain the following procedure: ${ }^{7}$

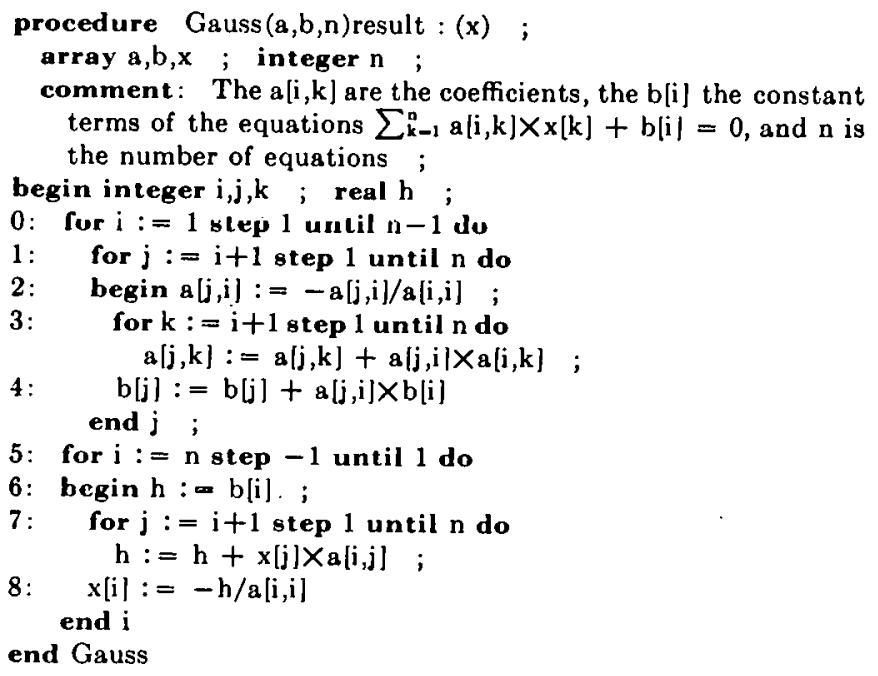

We distinguish the two parts: elimination proper (statements 0 to 4), and back substitution (statements 5 to 8). The procedure shows clearly the three typical loops of the elimination: The i-loop, beginning with 0 (counting

\footnotetext{
' If the elements $a[i, k]$ and $b[i]$ should not be changed by the execution of the procedure, they should be listed in the value part.
}

the reduction steps), the j-loop, beginning with statement 1 (counting the rows in every reduction step) and the k-loop, beginning with statement 3 (counting the elements in one row of the matrix $a[i, k])$.

It must be noted that this program is not foolproof insofar as some of the a $[\mathrm{i}, \mathrm{i}]$ which appear as denominators in statements 2 and 8 may vanish (for positive definite symmetric matrices this cannot happen). Similar situations may arise also in other examples treated here.

\section{(b) Procedure Statements}

General form:

$I(p, p, \ldots, p)$

$I$ is the name of the procedure and must coincide with the name in the corresponding procedure declaration; the p's are now the actual parameters. The types of the parameters-but not their names-must coincide with those in the corresponding procedure declaration (see the explana-. tions and restrictions in (AR4.7.3 to 4.7.8)).

An example may show the application of the procedure statements:

Let $A=(a[i, k])$ be a matrix with $n$ rows and $p$ columns; we want to orthonormalize the columns, i.e. to decompose $A$ as follows: $A=A_{N} R$, where $A_{N}$ is a $(n \times p)$-matrix with orthonormal columns and $R$ a triangular $(p \times p)$-matrix. Since $A^{T} A=R^{T} A_{N}{ }^{T} A_{N} R=R^{T} R$, we could obtain $R$ by triangular (Gauss-Cholesky) decomposition of the matrix $\mathbf{A}^{\mathbf{T}} \mathbf{A}$. The following method is numerically safer, since it avoids the generally ill-conditioned matrix $\mathrm{A}^{\mathrm{T}} \mathrm{A}$ : (the elements of the resulting matrix $A_{N}$ are again denoted by $\mathrm{a}[\mathrm{i}, \mathrm{k}])$ :

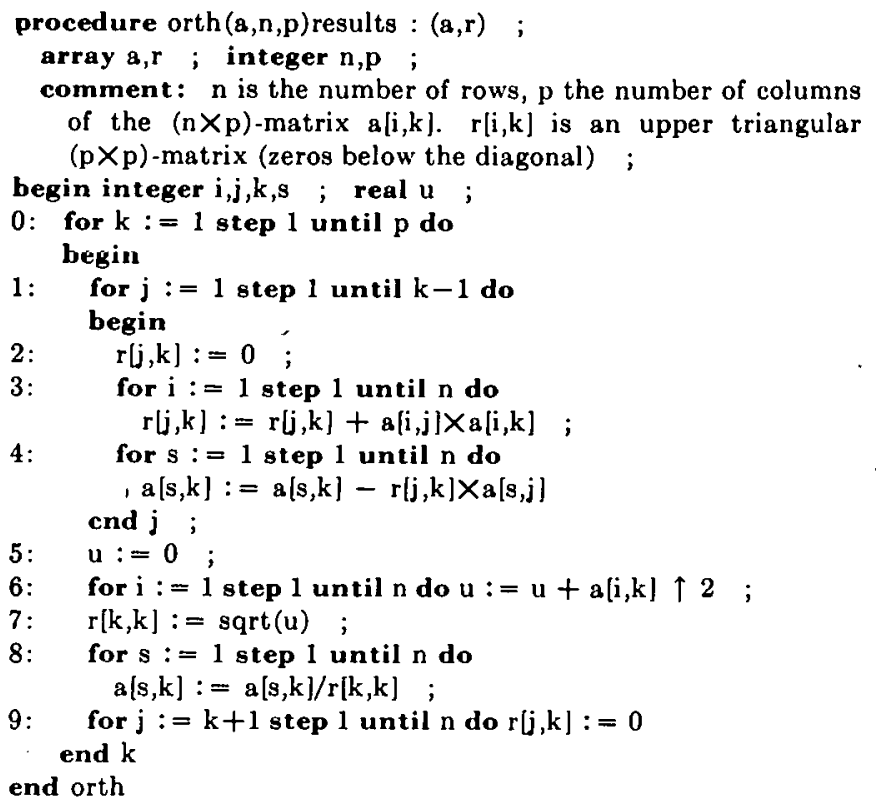

$k$ counts the number of reduction steps; in the $k^{\text {th }}$ step already the $\mathrm{k}-1$ first columns of $\mathrm{a}[\mathrm{j}, \mathrm{k}]$ are orthonormal. Then for $j=1,2, \ldots, k-1$ the inner product of the $k^{\text {th }}$ with the $\mathrm{j}^{\text {th }}$ column of $\mathrm{a}[\mathrm{j}, \mathrm{k}]$ is computed (statements 2 and 3 ) and then the $\mathrm{k}^{\text {th }}$ column made orthogonal to the 
$\mathrm{j}^{\text {th }}$ (statement 4). In statements 5 to 8 , the $\mathrm{k}^{\text {th }}$ column is normalized and finally in statement 9 , the subdiagonal zeros of the $\mathrm{k}^{\text {th }}$ column of the matrix $\mathrm{r}[\mathrm{j}, \mathrm{k}]$ are filled in.

One sees that this program is itself written as a procedure. This should be done whenever a calculation is selfcontained and general enough that it has a chance to be used later as a subroutine. Procedure orth certainly fits into this frame, because we can easily solve least square problems with it. The equations

$\sum_{1}^{p} \mathrm{a}[\mathrm{i}, \mathrm{k}] \times \mathrm{x}[\mathrm{k}]+\mathrm{v}[\mathrm{i}]=0 \quad(\mathrm{i}=1,2, \ldots, \mathrm{n} \quad ; \mathrm{n} \geq \mathrm{p})$

are solved by

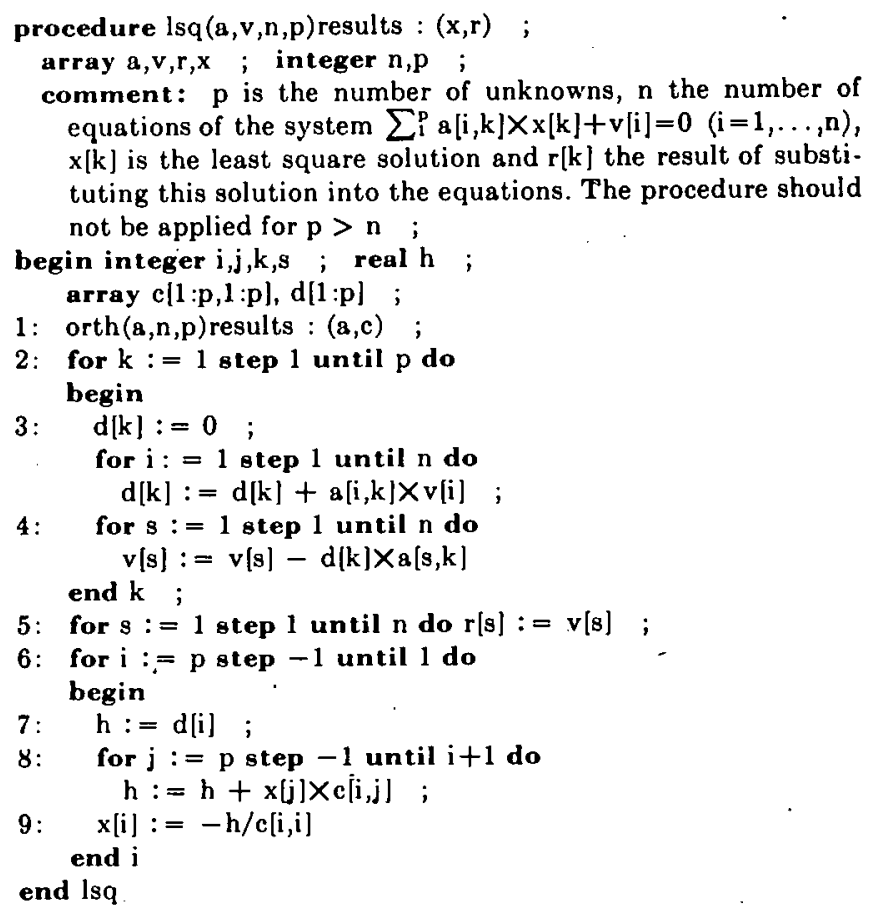

In this example we have in addition to the array specification in the heading (for the formal parameters) an array declaration at the beginning of the procedure body which concerns variables occurring only inside the procedure. It must be remembered that these variables are not available outside the procedure.

The example shows further that the names of parameters and variables in the procedure declaration are not determinative for the names to be used for the same quantities in the main program. In lsq, for instance, $r$ denotes the residual vector of the least square solution which has nothing to do with the $r$ occurring in the procedure declaration of orth and which in lsq is denoted by $\mathrm{c}$.

In (AR5.4.6) the possibility of adding procedures expressed in machine language (machine oriented procedures) to ALGOL is indicated. In order to show what this may mean, we treat here the development of a function (given by an arbitrary rule for computing its values) into a series of Chebyshev polynomials for a given interval [7]:

$$
f(x)=\frac{1}{2} c_{0}+\sum_{k=1}^{\infty} c_{k} T_{k}(x, a, b),
$$

where

$$
T_{k}(x, a, b)=\cos \left[n \times \arccos \frac{2 x-a-b}{b-a}\right] .
$$

The $T_{k}(x,-1,1)$ are generally denoted by $T_{k}(x)$, the $\mathrm{T}_{\mathrm{k}}(\mathrm{x}, 0,1)$ by $\mathrm{T}_{\mathrm{k}}^{*}(\mathrm{x})$.

For $f(x)=1 /(1+3 x)$ we have

$$
f(x)=\frac{1}{2}+\sum_{1}^{\infty} 3^{-k} T_{k}(x, 0,1)
$$

which converges quite well in $(0,1)$, whereas the power series diverges for $x \geq 1 / 3$.

The method of computing the coefficients $c_{k}$ is given in many textbooks. Numerically we can proceed as follows: For an increasing number of $n$ we compute the exact Newtonian interpolation polynomial for the following $\mathrm{n}+1$ points (Chebyshev abscissae):

$$
t_{k}=\frac{1}{2}(a+b)+\frac{1}{2}(b-a) x_{k},
$$

where

$$
\mathrm{x}_{\mathrm{k}}=\cos (\mathrm{k} \pi / \mathrm{n}) \quad \mathrm{k}=0,1,2, \ldots, \mathrm{n} .
$$

For these polynomials, the coefficients of the (finite) Chebyshev development are given explicitly as follows:

$$
c_{k}=\frac{2}{n}\left[\frac{1}{2} f\left(t_{0}\right)+\sum_{s=1}^{n-1} f\left(t_{\theta}\right) x_{s \times k}+\frac{1}{2}(-1)^{k} f\left(t_{n}\right)\right] .
$$

Then we increase $\mathrm{n}$ until either an upper limits for $\mathrm{n}$ is reached, or two successive n's yield approximately the same coefficients $c_{k}$. To avoid the latter happening accidentally when the computed c's are still far from the true values, we compute the values of the function and the Chebyshev series at a random point in the interval $(a, b)$.

Of course, $n$ need not necessarily run through all integers, but only through a selection (approximately a geometrical sequence of quotient 1.25).

In order to obtain the Chebyshev abscissae for a given $\mathrm{n}$ in full machine accuracy, we presume that a machineoriented procedure,

procedure $\operatorname{cs}(n)$ results : (x) ; integer $n \quad ; \operatorname{array} x \quad$;

is available, generating the values $x[k]=\cos (k \pi / n)$ for $\mathrm{k}=0,1, \ldots, 2 \mathrm{n}$. To obtain full machine accuracy special programming techniques are necessary, and thus it is impossible to express the computation in terms of ALGOL.

The same remark holds for the function random which generates a' random or pseudorandom number lying in the open interval $-1<$ random $<1$. This procedure

$$
\text { real procedure random ; }
$$

must also be available in machine notation. It should be noted that this is at the same time an example of a pro- . cedure which has no parameters. 
Thus we obtain:

procedure Chebyshev(a,b,s,eps,phi,f)results : (c,n)exit : (nonconvergence) ;

real a,b,eps ; integer s,n ; Boolean phi ; real procedure $f$; array c ; label nonconvergence ; comment : $a$ and $b>a$ are the bounds for the interval. $s$ is an upper bound for the order up to which the coefficients must be computed at most. eps is a tolerance for leaving the procedure. phi decides whether the interpolation polynomial of exact order $\mathrm{s}$ is wanted (phi $=$ true) or the infinite Chebyshev series ( $p h i=$ false). $f$ is the function to be developed. $c[k]$ are the coefficients wanted. $n$ is the order of the approximating polynomial used to meet the requirements. If the upper bound s has proved insufficient to fulfill the tolerance, then the procedure is left through the emergency exit nonconvergence ;

begin real $d, g, h, z, y$, urandom ; integer $k, a l p h a, v$; array $x[0: 2 \times s], u[0: s], w[0: s+1] ;$

1: for $\mathrm{k}:=1$ step 1 until $\mathrm{s}$ do $\mathrm{c}[\mathrm{k}]:=0$;

2: if phi then $n:=s$ else $n:=1$;

3: $\operatorname{cs}(n)$ results: $(x)$;

4: for $\mathrm{k}:=0$ step 1 until $\mathrm{n}$ do $\mathrm{u} \mid \mathrm{k}]:=\mathrm{f}((\mathrm{a}+\mathrm{b}+(\mathrm{b}-\mathrm{a}) \times \mathrm{x}[\mathrm{k}]) / 2) ;$

5: for $k:=0, n$ do $u[k]:=u[k] / 2$

6: $d:=\mathrm{g}:=0$;

7: for $k:=0$ step 1 until $n$ do begin

8: $\quad \mathrm{g}:=\mathrm{g}+\mathrm{abs}(\mathrm{c}[\mathrm{k}])$

9: $\quad \mathrm{h}:=0 ;$ alpha $:=0$;

10: for $\mathrm{v}:=0$ step 1 until $\mathrm{n}$ do begin

11: $\quad \mathrm{h}:=\mathrm{h}+\mathrm{x}[\mathrm{alpha}] \times \mathrm{u}[\mathrm{v}] ;$ alpha $:=$ alpha $+\mathrm{k}$;

12: $\quad$ if alpha $\geq 2 \times n$ then alpha $:=$ alpha $-2 \times n$

$$
\text { end } v \text {; }
$$

13: $\mathrm{z}:=2 \times \mathrm{h} / \mathrm{n} ; \mathrm{d}:=\mathrm{d}+\mathrm{abs}(\mathrm{z}-\mathrm{c}[\mathrm{k}]) ; \mathrm{c}[\mathrm{k}]:=\mathrm{z}$ end $k$;

14: if phi then go to 25 ;

15: $y:=$ random ;

16: urandom $:=\mathrm{f}((\mathrm{a}+\mathrm{b}+(\mathrm{b}-\mathrm{a}) \times \mathrm{y}) / 2)$;

17: $w[n]:=w[n+1]:=0$;

18: for $k:=n-1$ step -1 until 0 do $w[k]:=2 \times y \times w[k+1]-w[k+2]+c[k]$

19: $\mathrm{d}:=\mathrm{d}+\mathrm{sbs}(\mathrm{w}[0]-\mathrm{y} \times \mathrm{w}[1]-0.5 \times \mathrm{c}[0]-$ urandom $) ;$

20: $\mathrm{g}:=\mathrm{g}+$ abs(urandom) ;

21: if $\mathrm{d} / \mathrm{g}<$ eps then go to 25 .

22: if $\mathbf{n}=\mathbf{s}$ then go to nonconvergence ;

23: $\mathrm{n}:=\operatorname{entier}(1.25 \times \mathrm{n}+2) \quad ; \quad$ if $\mathrm{n}>\mathrm{s}$ then $\mathrm{n}:=\mathrm{s}$;

24: go to 3 ;

25: end Chebyshev

Explanations referring to the statement labels:

1: At the beginning all $s+1$ coefficients $c[k]$ are flled in as zeros (in order that the comparison of two successive sets of coefficients be meaningful at the very beginning.

2: $\mathbf{n}$ is given its starting value according to the Boolesn variable phi. If phi is true, then the interpolation polynomial of order $\mathrm{s}$ is wanted. In the opposite case the development is required and thus we start with $\mathrm{n}=1$ and increase later in statement 23.

3: Procedure statement for calculating the values $\cos (k \pi / n)$ as accurately as possible.

4: The values of the function $f$ for the $n+1$ Chebyshev abscissae are computed; these values are called $u[k]$.

5: The boundary values of the function are halved in order to simplify the summation in statement 10 . This is an example where the running of the subscript is given by a list.

6: Initial values for the summations in statements $8,13,19$ and 20.
7: The $k$-loop begins, $k$ counting the coefficients $c[k]$

8: Summation of the values $|c[k]|$, where $c[k]$ are the coefficients corresponding to the foregoing value of $n$. This summation - is always meaningful, since the $c[k]$ are defined up to $k=8$ by virtue of statement 1 .

9: Initial values for the computation of $c[k]$. Note that the two sssignment statements cannot be combined into one because the two variables are of different type.

10-12: Summation in order to obtain the $c[k]$. Alpha is the quantity $\mathrm{k} \times \mathrm{v}$ reduced modulo $2 \times \mathrm{n}$ to the interval $0 \leq$ alpha $<$ $2 \times n$.

13: An auxiliary variable $z$ is assigned the new value of $c[k]$. Then the summation of the values $|z-c[k]|$ takes place, where the $c[k]$ is still the coefficient corresponding to the foregoing value of $n$. At last $c[k]$ gets its computed value.

14: If the interpolation polynomial of exact order $s$ was wanted (phi has the value true), the computation is finished and the procedure is left by the go to statement leading to the very end of the procedure.

15, 16: A random number denoted by $y$ is generated by invoking the function random, and the function $f$ is computed for a random point of the interval $(a, b)$.

17, 18: Clenshaw's recursion formula for the evaluation of the Chebyshev series at the randorn point.

19: The absolute discrepancy of the function and the series at the random point is added to $\mathrm{d}$.

20: Furthermore $g$ is increased by the absolute value of the function at the random point.

21: If $d / g$, which is something like the relative error of the coefficients, fulfills the tolerance, then the procedure is left.

22: If, on the other hand, $n$ has already reached its maximum value $\mathrm{s}$ (with $\mathrm{phi}=$ false), then the given tolerance is too small for the upper bound 8 , the process did not converge, and thus the procedure is left by the exit label nonconvergence.

23: If $\mathbf{n}<\mathrm{s}$ and $\mathrm{d} / \mathrm{g}>\mathrm{eps}$, then $\mathrm{n}$ is increased. Although $\mathrm{n}$ is declared to be an integer (by the specification in the procedure heading) the entier function should be used for rounding the expression. If the increased $n$ exceeds $s$, then it is reduced.

24: After this we start anew with statement 3 .

25: A dummy statement is introduced by the semicolon after the last statement in order to be able to put a label to which a go to statement (statements 14 and 21) can refer.

There remain a few words to be said about the application of such a procedure, because of the free function $f$ and the exit label, nonconvergence, occurring in the declaration.

If in an ALGOL program the procedure is called by the statement

Chebyshev $(-1,1,100,10-5$, false)proc:(rho)result:(c,eta)exit:

(color)

then this means that the Chebyshev coefficients of the function rho must be computed for the interval $(-1,1)$ with a tolerance of $10^{-5}$ and with an $n$ equal at most to 100 , and that the computation in case of nonconvergence has to proceed with the statement labeled with color (and not with the statement following the procedure statement). This requires that in the same Algol program there must be a statement with the label color and a function declaration for the function rho. The function must be defined by a type procedure declaration

real procedure $\operatorname{rho}(x) ; \operatorname{real} x$; 
Without much explanation we give here the ALgol program for the numerical inversion of matrices with the method of Gauss-Jordan as proposed by $\mathrm{H}$. Rutishauser [8]. The program described uses pivotal search and exchange of the pivotal row and column with the $k^{\text {th }}$.row and column. Thus in the $\mathrm{k}^{\text {th }}$ Jordan step the $\mathrm{k}^{\text {th }}$ diagonal element becomes the pivot. This makes the determination of the pivot in the remaining matrix simpler but makes a final reordering of the matrix necessary.

procedure gjr(a,n,eps)result : (a)exit : (singular) ;

array $a$; integer $n$; real eps ; label singular ;

comment: $n$ is the order of the matrix $a[i, k]$ to be inverted. eps is a tolerance for acceptance of the singularity of the given matrix and singular is the emergency exit in case of a singular matrix ;

begin integer $i, j, k$; real pivot, $z$;

array $b, c[1: n] ;$ integer array $p, q[1: n]$;

1: for $k:=1$ step 1 until $n$ do.

begin

comment: determination of the pivot element;

pivot: $=0$;

for $i:=k$ step 1 until $n$ do

for $\mathrm{j}:=\mathrm{k}$ step 1 until $\mathrm{n}$ do

if $\operatorname{abs}(\mathrm{a}(\mathrm{i}, \mathrm{j}])>\mathrm{abs}$ (pivot) then

begin pivot $:=a[i, j] ; p[k]:=\mathrm{i} ; q[k]:=\mathrm{j}$ end ;

7: $\quad$ if abs(pivot) $\leq$ eps then go to singular ;

comment: exchange of the pivotal row with the $k^{\text {th }}$ row ;

8: $\quad$ if $\mathrm{p}[\mathrm{k}] \neq \mathrm{k}$ then

for $\mathrm{j}:=1$ step 1 until $\mathrm{n}$ do

begin $z:=a[p[k], j] ; a[p[k], j]:=a[k, j] ;$ $a[k, j]:=z$

end $j$;

comment: exchange of the pivotal column with the $k^{\text {th }}$ column ;

11: if $q[k] \neq k$ then

12: for $\mathrm{i}:=1$ step 1 until $\mathrm{n}$ do

13: $\quad \operatorname{begin} z:=\mathrm{s}[\mathrm{i}, \mathrm{q}[\mathrm{k}]] ; \mathrm{a}[\mathrm{i}, \mathrm{q}[\mathrm{k}]]:=\mathrm{a}[\mathrm{i}, \mathrm{k}]$; $a[i, k]:=2$

end $i$;

comment: Jordan step ;

for $j:=1$ step 1 until $n$ do

begin

if $\mathrm{j}=\mathrm{k}$ then

begin $b[j]:=1 /$ pivot $;, c[j]:=1$ end

else

begin $b[j]:=-a[k, j] /$ pivot $; c[j]:=a[j, k]$ end ;

$a[k, j]:=a[j, k]:=0$

end $j$;

for $i:=1$ step 1 until $n$ do

for $j:=1$ step 1 until $n$ do

end $k$;

$a[i, j]:=a[i, j]+c[i] \times b[j]$

comment: reordering of the matrix ;

23: for $k:=n-1$ step -1 until 1 do

begin

24: if $\mathrm{p}[\mathrm{k}] \neq \mathrm{k}$ then

25: for $i:=1$ step 1 until $n$ do

26: $\quad$ begin $z:=a[i, p[k]] ; a[i, p[k]]:=a[i, k] \quad$;

$$
\mathrm{a}[\mathrm{i}, \mathrm{k}]:=\mathrm{z}
$$

end $\mathrm{i}$;

27: if $q[k] \neq k$ then

28: for $\mathrm{j}:=1$ step 1 until $n$ do

29: $\quad$ begin $z:=a[q[k], j] ; a[q[k], j]:=a[k, j] ;$

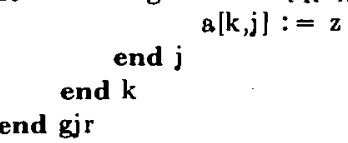

\section{APPENDIX}

\section{Input-Output Operations}

In the ALGoL report nothing is said about input-output operations. But each computation has to start with some initial values which depend on the specific problem to be treated and so have to be given through some input medium into the machine. On the other hand, the desired results of the computation have to be transmitted to the world outside of the machine by means of output installations. Therefore each Algol program which actually has to be run on a computer will necessarily contain statements concerning input and output operations. Such operations have not been treated so far in this paper, but they must be defined by procedures which of course have to be written in machine code (see AR5.4.6). Examples of such machine oriented procedures are:

(a) Suppose that there exists a standard input medium (paper-tape,-magnetic tape, cards)-and that -all-input data needed by the program should be read in by this means. Thus the principal result of the procedure

$$
\text { real.procedure read ; }
$$

will be the next following number standing on the input medium. Normally the procedure is applied in assignment statements like

$$
\mathrm{n}:=\mathrm{read} ; \mathrm{m}:=\text { read } ;
$$

where the variables $\mathrm{n}$ and $\mathrm{m}$ are given their actual values.

(b) Suppose that there exists a typewriter for printing the results. Then a procedure for printing one value is for instance:

$$
\text { procedure print }(V)
$$

The procedure statement

$$
\operatorname{print}(\mathbf{a}[\mathbf{i}, \mathbf{k}])
$$

will cause the printing of the actual value of a[i,k].

Apart from the output procedure print $(V)$ a. procedure controlling the line feed of the typewriter might be desirable for listing the results. Thus we need, e.g.

$$
\text { procedure newline ; }
$$

An example may show the use of these three procedures. A mtarix $\mathbf{A}=\left(a_{\mathbf{i k}}\right)$ of order $\mathbf{n}$ should be inverted by applying the procedure gjr (see the end of $\S 8$ ):

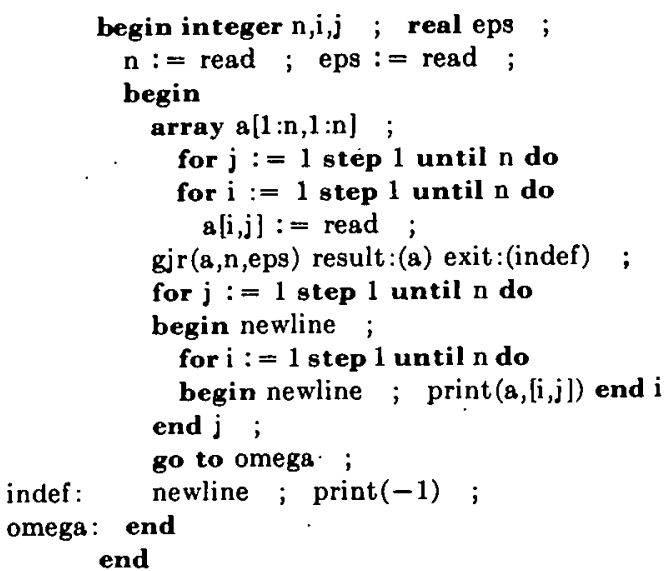


The program assumes that the actual values of the order $n$, the tolerance eps and the elements of the matrix (column by column) are prepared on the input medium. In case of nonsingularity the inverted matrix is printed column by column, each column separated from the other by an additional empty space. Otherwise only the number -1 is printed to indicate the singularity of the given matrix.

Furthermore, the example uses four procedures as nonlocal quantities. How these procedures are entered in relation to the program depends very much on the internal organization of a computer and how the specific translator for it is built. But this compiler oriented question lies beyond the scope of the present paper.

Further and more complicated examples of inputoutput procedures can be invented according to the special equipment of the computer and the purpose which they have to fulfill. Even such input and output facilities which do not exist at the present time, but will be available in the future, can be handled by introducing appropriate procedures. Therefore it must be mentioned here that there is no need to introduce new elements to ALGol for such purposes, which might become obsolete as soon as new peripheral equipment is introduced.

\section{REFERENCES}

1. Rutishatser, H. Automatische Rechenplanfertigung bei programmgesteuerten Rechenmaschinen. Mitt. Inst. angew. Math. ETH. Verlag Birkhäuser, Basel (1952).

2. Symposium on Automatir. Programming, 13-14 May 1954. Office of Naval Research, Washington.

3. Rutishauser, H. Massnahmen zur Vereinfachung des Programmierens. Nachr.-tech. Fachber. Bd. 4. Verlag Vieweg (1956).

4. Perlis, A. J. and Samelson, K. Preliminary report.-international algebraic language ALGOL. Comm. ACM 1 (Dec. 1958), or Numer. Math. 1 (1959), 41-60.

5. NAUR, P. (Ed.), ET AL. Report on the algorithmic language ALGOL 60. Comm. ACM 3 (1960), 299-314, or Numer. Math. 2 (1960), 106-136.

6. Rutishauser, H. Notes on application of ALGOL to numerical analysis. Summer Conference on Frontier Research in Digital Computers, 1959, Chapel Hill, N. C.

7. Nat. Bur. Standards. Tables of the ('hebyshev Polynomials. Appl. Math. Ser. Vol. 9. U. S. Gov't. Printing Office, Washington, D. C. (1952).

8. Rutishauser, H. Zur Matrizeninversion nach Gauss-Jordan. Z.AMP 10 (1959), 281-291. 
COLLECTED ALGORITHMS

(in numerical order) 
1. QuadI

R. J. Herbold

National Bureau of Standards, Washington 25, D. C.

comment

QuadI is useful when integration of several functions of same limits at same time using same point rule is desired. The interval $(a, b)$ is divided into $m$ equal subintervals for an $n$-point quadrature integration. $p$ is the number of functions to be integrated. $w_{k}$ and $u_{k}$ are normalized weights and abscissas respectively, where $k=1,2,3, \cdots, n$. $u_{k}$ must be in ascending order. $P(B, j)=:(c)$ is a procedure which must be supplied by the programmer. It evaluates (c) the function (as indicated by $j$ ) for $B . I_{j}$ is the result of integration for function $j$.;

procedure QuadI $\left(a, b, m, n, p, w_{k}, u_{k}, P(B, j)=:(c)\right)=:(I j)$ begin

Quad I... $\quad \therefore \quad$ h $:=(b-a) / m$

for $\quad j:=1(1) p ; I_{j}:=0$

$$
\text { A }:=a-h / 2
$$

for $\quad i:=1(1) \mathrm{m}$

L1

L2

$$
\begin{array}{ll}
\text { begin } & A:=A+h \\
\text { for } & k:=1(1) n \\
\text { begin } & B:=A+(h / 2) \times u_{k}
\end{array}
$$$$
\text { for } \quad j:=1(1) p
$$

L3:

$\begin{array}{ll}\text { begin } & P(B, j)=:(c) \\ & I_{i}:=I_{j}+w_{k} \times c \\ & \text { end } L 1 \\ \text { for } & j:=1(1) p \\ & I_{j}:=(h / 2) \times I_{j} \\ \text { return } & \\ \text { integer } & (j, k, i) \\ \text { end } & \text { QuadI }\end{array}$

\section{ROOTFINDER}

\section{J. Wegstein}

National Bureau of Standards, Washington 25, D. C.

comment

This procedure computes a value of $g=x$ satisfying the equation $x=f(x)$. The procedure calling statement gives the function, an initial approximation $a \neq 0$ to the root, and a tolerance paramater $\epsilon$ for determining the number of significant figures in the solution. This accelerated iteration or secant method is described by the author in Communications, June, 1958.

procedure $\operatorname{Root}(f(), a, \epsilon)=:(g)$

begin

Root

$\mathrm{b}:=\mathrm{a} \quad ; \mathrm{c}:=\mathrm{f}(\mathrm{b}) \quad ; \mathrm{g}:=\mathrm{c}$

if $(c=a) ;$ return

$\mathrm{d}:=\mathrm{a} ; \mathrm{b}:=\mathrm{c} \quad ; \quad \mathrm{e}:=\mathrm{c}$.

$c:=f(b)$

$g:=(d \times c-b \times e) /(c-e-b+d)$

if $(a b s((g-b) / g) \leqq t) ;$ return

$\mathrm{e}:=\mathrm{c} ; \mathrm{d}:=\mathrm{b} \quad ; \mathrm{b}:=\mathrm{g} ;$ go to Hob

\section{CERTIFICATION}

2. Rootfinder, J. Wegstein, Communications $A C M$, February, 1960

Henry C. Thacher, Jr., ${ }^{*}$ Argome National Laboratory, Argonne, Illinois

Rootfinder was coded for the Roval-Precision LGP-30 Computer, using an interpretive floating point system with 28 bits of significance. The translation from ALGOL was made by hand. Provision was made to terminate the iteration after ten cycles if convergence had not been secured.

The program was tested against the following functions:

(1) $f(x)=(x+1)^{1 / 3}$

$($ Root $=1.3247180)$

(2) $f(x)=\tan x$

(3. $\alpha) \quad f(x)=2 \pi \alpha+\tan ^{-1} x \quad(\alpha=1,2,3,4)$

(4. $\alpha) \quad f(x)=\sinh \alpha x$

$(\alpha=-1.2,-0.5,0.5,1.2)$

Selected results were as follows:

\begin{tabular}{cclccl}
$\mathrm{f}(\mathrm{x})$ & $\alpha$ & \multicolumn{1}{c}{$\mathrm{x}_{\mathrm{k}-1}$} & $\mathrm{x}_{\mathrm{k}}$ & \\
1 & 1.3 & $10^{-7}, 10^{-6}$ & $1.32+7233$ & 1.3258637 & $(1)$ \\
1 & 1.3 & $10^{-5}$ & & $1.32 \overline{47165}$ & $(1)$ \\
2 & 5 & $10^{-3}$ & -.4674691 & $-.36021 \overline{288}$ & $(1,2)$ \\
2 & 4 & $10^{-3}$ & +.84880381 &.$+ \overline{69496143}$ & $(1,2)$ \\
3.1 & 1 & $10^{-5}$ & & $7 . \overline{7252531}$ & \\
3.2 & 2 & $10^{-5}$ & & $14.06615 \overline{5}$ & \\
3.3 & 3 & $10^{-5}$ & & 20.371026 \\
3.4 & 4 & $10^{-5}$ & & 26.665767
\end{tabular}

(1) No convergence after 10 iterations. Underlined figures are incorrect.

(2) For this function, $f^{\prime}(0)=1$; so convergence is not to be expected at this root. However, the algorithm did not find any other root.

It should be noted that the convergence criterion used fails for a zero root. The provision to terminate after a given number of cycles is therefore essential. Also, double precision is desirable.

REMARK ON ALGORITHM 2

ROOTFINDER (J. Wegstein, Communications $A C M$, February, 1960)

Henry C. Thacher, Jr., ${ }^{*}$ Argonne National Laboratory, Argonne, Illinois

$$
\frac{y_{k}-Y}{y_{k-1}-Y}=\frac{\left(y_{k-2}-Y\right) f^{\prime \prime}}{2\left(f^{\prime}-1\right)+\left(y_{k-1}-y_{k-2}\right) f^{\prime \prime}}+O\left(y_{k-1}-Y\right)^{3}
$$

where $Y$ is the desired root, and the derivatives $f^{\prime}$ and $f^{\prime \prime}$ are evaluated there. Convergence is thus second order, provided that $\left|\mathrm{f}^{\prime \prime}\right|\left|\mathrm{y}_{\mathrm{k}-1}-\mathrm{Y}\right|<2\left|\mathrm{f}^{\prime}-1\right|$.

The algorithm is, however, somewhat unstable numerically because of the factor $f\left(y_{k-1}\right)-f\left(y_{k-2}\right)-y_{k-1}+y_{k-2}$ in the de nominator.

Experience has shown that the minimum for $\epsilon$ is about one half the precision being used. Provision to indicate when roundoff errors are causing random oscillations of $g$ would be a desirable addition.

The criterion used for terminating the iteration renders the algorithm unsuitable for a zero root. A preliminary test for a zero root would be desirable. In addition, the algorithm should include provision for exit after a stated number of iterations.

Algorithm 15 appears to offer advantages along these lines.

* Work supported by the U. S. Atomic Energy Commission.

This algorithm has the convergence factor 
REMARKS ON ALGORITHMS 2 AND 3 (Comm. $A C M$, February 1960), ALGORITHM 15 (Comm. $A C M$, August 1960) AND ALGORITHMS 25 AND 26 (Comm. ACM, November 1960)

J. H. WiLKinson

National Physical Laboratory, Teddington.

Algorithms 2, 15, 25 and 26 were all concerned with the cal. culation of zeros of arbitrary functions by successive linear or quadratic interpolation. The main limiting factor on the accuracy attainable with such procedures is the condition of the method of evaluating the function in the neighbourhood of the zeros. It is this condition which should determine the tolerance which is allowed for the relative error. With a well-conditioned method of evaluation quite a strict convergence criterion will be met, even when the function has multiple roots.

For example, a real quadratic root solver (of a type similar to Algorithm 25) has been used on ACE to find the zeros of triple. diagonal matrices $T$ having $t_{i i}=a_{i}, t_{i+1, i}=b_{i+1}, t_{i, i+1}=$ $c_{i+1}$. As an extreme case $I$ took $a_{1}=a_{2}=\cdots=a_{6}=0, a_{6}=$ $a_{7}=\cdots=a_{10}=1, a_{11}=2, b_{i}=1, c_{i}=0$ so that the function which was being evaluated was $x^{5}(x-1)^{6}(x-2)$. In spite of the multiplicity of the roots, the answers obtained using floating-point arithmetic with a 46-bit mantissa had errors no greater than $2^{-4}$. Results of similar accuracy have been obtained for the same problem using linear interpolation in place of the quadratic. This is because the method of evaluation which was used, the twoterm recurrence relation for the leading principal minors, is a very well-conditioned method of evaluation. Knowing this, I was able to set a tolerance of $2^{-6}$ with confidence. If the same function had been evaluated from its explicit polynomial expansion, then a tolerance of about $2^{-7}$ would have been necessary and the multiple roots would have obtained with very low accuracy.

To find the zero roots it is necessary to have an absolute tolerance for $\left|x_{r+1}-x_{r}\right|$ as well as the relative tolerance condition. It is undesirable that the preliminary detection of a zero root should be necessary. The great power of rootfinders of this type is that, since we are not saddled with the problem of calculating the derivative, we have great freedom of choice in evaluating the function itself. This freedom is encroached upon if we frame the rootfinder so that it finds the zeros of $x=f(x)$ since the true function $x-f(x)$ is arbitrarily separated into two parts. The formal advantage of using this formulation is very slight. Thus, in Certification 2 (June 1960), the calculation of the zeros of $x=\tan x$ was attempted. If the function $(-x+\tan x)$ were used with a general zero finder then, provided the method of evaluation was, for example

$$
\begin{gathered}
x=n \pi+y \\
\tan x-x=-n \pi+\frac{\frac{y^{2}}{3}-\frac{y^{5}}{30}-\cdots}{\cos y},
\end{gathered}
$$

the multiple zeros at $x=0$ could be found as accurately as any of the others. With a slight modification of common sine and cosine routines, this could be evaluated as

$$
-n \pi+\frac{(\sin y-y)-y(\cos y-1)}{1+(\cos y-1)}
$$

and the evaluation is then well-conditioned in the neighbourhood of $x=0$. As regards the number of iterations needed, the restriction to 10 (Certification 2) is rather unreasonably small. For example, the direct evaluation of $x^{60}-1$ is well conditioned, but starting with the values $x=2$ and $x=1.5$ a considerable number of iterations are needed to find the root $x=1$. Similarly a very large number are needed for Newton's method, starting with $x=2$. If the time for evaluating the derivative is about the same as that for evaluating the function (often it is much longer), then linear interpolation is usually faster, and quadratic interpolation much faster, than Newton.

In all of the algorithms, including that for Bairstow, it is use ful to have some criterion which limits the permissible change from one value of the independent variable to the next [1]. This condition is met to some extent in Algorithm 25 by the condition S4, that $\operatorname{abs}(\mathrm{fprt})<\operatorname{abs}(\mathrm{x} 2 \times 10)$, but here the limitation is placed on the permissible increase in the value of the function from one step to the next. Algorithms 3 and 25 have tolerances on the size of the function and on the size of the remainders $r 1$ and r0 respectively. They arc very difficult tolcrances to aseign since these quantities may take very small values without our wishing to accept the value of $x$ as a root. In Algorithm 3 (Comm. ACM June 1960) it is useful to return to the original polynomial and to iterate with each of the computed factors. This eliminates the loss of accuracy which may occur if the factors are not found in increasing order. This presumably was the case in Certification 3 when the roots of $x^{6}+7 x^{4}+5 x^{3}+6 x^{2}+3 x+2=0$ were attempted. On ACE, however, all roots of this polynomial were found very accurately and convergence was very fast using singleprecision, but the roots emerged in increasing order. The reference to slow convergence is puzzling. On $\mathrm{ACE}$, convergence was fast for all the initial approximations to $p$ and $q$ which were tried. When the initial approximations used were such that the real root $x=-6.3509936103$ and the spurious zero were found first, the remaining two quadratic factors were of lower accuracy, though this was, of course, rectified by iteration in the original polynomial. When either of the other two factors was found first, then all factors were fully accurate even without iteration in the original polynomial [1].

\section{REFERENCE}

[1] J.H. WiLkinson. The evaluation of the zeros of ill-conditioned polynomials Parts I and II. Num. Math. 1 (1959), 150-180.

\section{Solution of Polynomial Equation by Bairstow- HitchCock Method}

A. A. Grau

Oak Ridge National Laboratory, Oak Ridge, Tenn. -

procedure

BAIRSTOW (n, a [ ], eps0, eps1, eps2, eps3, K) = :

(m, x[l, y[ ], nat [ ], ex[ ]);

comment The Bairstow-Hitchcock iteration is used to find successively pairs of roots of a polynomial equation of degree $n$ with coefficients $a_{i}$ ( $i=0,1, \cdots, n$ ) where $a_{n}$ is the constant term. On exit from the procedure, $m$ is the number of pairs of roots found, $x[i]$ and $y[i] \quad(i=1, \cdots, m)$ are a pair of real roots if nat $[i]=1$, the real and imaginary parts of a complex pair if nat[i] $=-1$, and ex[i] indicates which of the following conditions was met to exit from the iteration loop in finding this pair:

1. Remainders, rl, r0, become absolutely less than eps1

2. Corrections, incrp, incrq, become absolutely less than eps2. 
3. The ratios, incrp/p, incrq/q, become $a b$ solutely less than eps3.

4. The number of iterations becomes $K$.

In the last case, the pair of roots found is not reliable and no further effort to find additional roots is made. The quantity eps 0 is used as a lower bound for the denominator in the expressions from which incrp and incrq are found.;

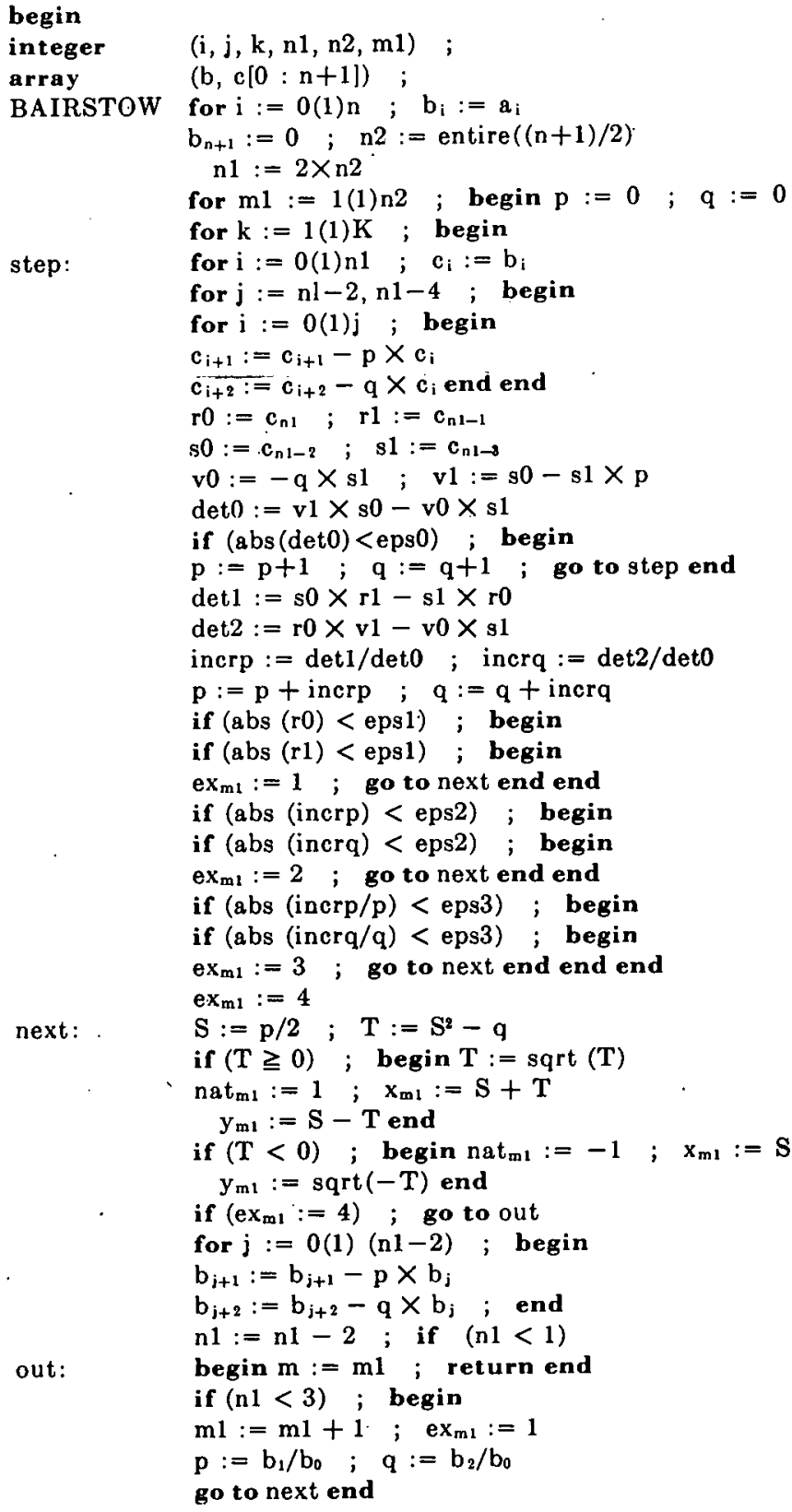

end end

\section{CERTIFICATION}

3. Solution of Polynomial Equation by BairstowHitcheock Method, A. A. Grau, Communications ACM, February, 1960 .

Henry C. Thacher, Jr., ${ }^{*}$ Argonne National Laboratory, Argonne, Illinois.

-Bairstow was coded for the Royal-Precision LGP-30 computer, using an interpretive floating point system (24.2) with 28 bits of significance. The translation from ALGOL was made by hand.

The following minor corrections were found necessary.

a. $\operatorname{det} 2:=\mathrm{r} 0 \times \mathrm{v} 1-\mathrm{v} 0 \times \mathrm{s} 1$ should be $\operatorname{det} 2:=\mathrm{r} 0 \times \mathrm{v} 1$ $-\mathrm{v} 0 \times \mathrm{r} 1$

b. $S:=p / 2$ should be $S:=-p / 2$.

After these were made, the program ran smoothly for the following equations:

$$
\begin{aligned}
x^{4}-3 x^{3}+20 x^{2}+44 x+43=0 \quad x & =-.97063897 \pm 1.0058076 i \\
x & =-2.4706390 \pm 4.6405330 i \\
x^{6}-2 x^{5}+2 x^{4}+x^{3}+6 x^{2}-6 x+8 & =0 \\
x & =0.50000000 \pm 0.86602539 i \\
x & =1.0000000 \pm 1.0000000 i \\
x & =1.5000000 \pm 1.3228756 i \\
x^{5}+x^{4}-8 x^{3}-16 x^{2}+7 x+15= & 0 \\
x & =.000000005,{ }^{* *}-0.99999999 \\
x & =3.0000000,0.999999999 \\
x & =-2.0000000 \pm 1.0000000 \mathrm{i}
\end{aligned}
$$

With the equation $x^{5}+7 x^{4}+5 x^{3}+6 x^{2}+3 x+2=0$ convergence was slow, and full accuracy was not obtained. However, the equation with reciprocal roots, $2 \mathrm{x}^{5}+3 \mathrm{x}^{4}+6 \mathrm{x}^{3}+5 \mathrm{x}^{2}+7 \mathrm{x}+$ $1=0$, converged rapidly.

* Work supported by the U. S. Atomic Energy Commission.

** Spurious zero real roots are introduced for equations of odd order.

\section{CERTIFICATION OF ALGORITHM 3}

SOLUTION OF POIYNOMIAL, EQUATION BY

BARSTOW-HITCHCOCK (A. A. Grau, Comm. ACM

Feb. 1960)

John Herndox

Stanford Research Institute, Menlo Park, California

Bairstow was transliterated into BALGOL and tested on the Burroughs 220. The corrections supplied by Thatcher, Comm. ACM, June 1960, were incorporated. Results were correct for equations for which the method is suitable. $x^{4}-16=0$ is one of those which gave nonsensical results. Seven-digit results were obtained for 12 test equations, one of which was $x^{6}-2 x^{5}+2 x^{4}+$ $x^{3}+6 x^{2}-6 x+8=0$. 
CERTIFICATION OF ALGORITHM 3

SOLUTION OF POLYNOMIAL EQUATIONS BY

BAIRSTOW HITCHCOCK METHOD (A. A. Grau,

Comm. ACM, February, 1960)

JAMES S. VAXDERGRAFT

Stanford Lniversity, Stanford, California

Bairstow was coded for the Burroughs 220 computer using the Burroughs Algol. Conversion from Algol, 60 was made by hand on a statement-for-statement basis. The integer declaration had to be extended to include $n, k, n, N A T, E X$, and the corrections noted in the certification by Henry C. Thacher, Jr., Communications ACM, June, 1960, were incorporated.

By selecting the input parameters carefully, all branches of the routine were tested and the program ran smoothly. The following polynomials equations were solved:

$$
\begin{aligned}
x^{6}-14 x^{4}+49 x^{2}-36=0, & x= \pm 1.0000000 \\
x & = \pm 1.9999998 \\
x & = \pm 3.0000001
\end{aligned}
$$

$$
\begin{aligned}
x^{8}-30 x^{6}+273 x^{4}-820 x^{2}+576=0, & x= \pm 1.0000000 \\
x & = \pm 2.0000000 \\
& x= \pm 2.9999999 \\
& x= \pm 4.0000001
\end{aligned}
$$

Several minor errors were found in the certification by $\mathrm{Mr}$. Thacher. The constant term in the first polynomial should be 54 instead of 43, the second pair of roots for that polynomial should be $+2.4706339+4.6405330 \mathrm{i}$, and the second pair of roots for the second polynomial should be $-1.0 \pm i$.

\section{SEE REMARKS ALGORITHM 2}

\section{Bisection Routine}

S. Gorn

University of Pennsylvania Computer Center Philadelphia, $\mathrm{Pa}$.

comment This procedure evaluates a function at the end-points of a real interval, switching to an error exit (fools exit) FLSXT if there is no change of sign. Otherwise it finds a root by iterated bisection and evaluation at the midpoint, halting if either the value of the function is less than the free variable $c$ or two successive approximations of the root differ by less than 1 . e should be chosen of the order of error in evaluating the function (otherwise time would be wasted), and $e l$ of the order of desired accuracy. el must not be less than two units in the last place carried by the machine or else indefinite cycling will occur due to round-off on bisection. Although this. method is of 0 order, and therefore among the slowest, it is applicable to any continuous function. The fact that no differentiability conditions have to be checked makes it, therefore, an 'old work-horse' among routines for finding real roots which have already been isolated. The free variables y1 and y2 are (presumably) the end-points of an interval within which there is an odd number of roots of the real function $F, \alpha$ is the temporary exit for the evaluation of $\mathbf{F}$;

procedure Bisec(y1, y2; $\epsilon, \epsilon 1, F()$, FLSXT) $=.:(x)$

begin

Bisec: $\quad \mathrm{i}:=1 ; \mathrm{j}:=1 ; \mathrm{k}:=1 ; \mathrm{x}:=\mathrm{y} 2$

$\alpha: \quad f:=F(x) ;$ if $(\operatorname{abs}(f) \leqq \epsilon) ;$ return

go to $\gamma_{1}$
First val: $i:=2 ; f 1:=f ; x:=y 1 ;$ go to $\alpha$

Succ val : if $(\operatorname{sign}(f)=\operatorname{sign}(f 1)) ;$ go to $\delta_{j} ;$ go to $7 k$

Sec val: $j:=2 ; k:=2$

Midpoint: $\quad x:=y 1 / 2+y 2 / 2 ;$ go to $\alpha$.

Reg 8: $\quad$ y2 :=x

Precision: if $(\operatorname{abs}(y 1-y 2) \geqq \epsilon 1) ;$ go to Midpoint return

$\operatorname{Reg} \eta: \quad \dot{y} 1:=x$; go to Precision

integer $(i, j, k)$

switch $\gamma:=$ (First val, Succ val)

owitch $\delta:=$ (FLSXT, Reg $\delta$ )

owitch $\eta:=$ (Sec val, Reg $\eta$ )

end Bisec

\section{CERTIFICATION OF ALGORITHM 4}

BISECTION ROUTINE (S. Gorn, Comm. ACM, March 1960)

Patty Jane Rader, * Argonne National Laboratory, Argonne, Illinois

Brsec was coded for the Royal-Precision LGP-30 computer, using an interpretive floating point system (24.2) with 28 bits of significance.

The following minor correction was found necessary.

a: go to $\gamma_{1}$ should be go to $\gamma_{1}$

After this correction was made, the program ran smoothly for $F(x)=\cos x$, using the following parameters:

$\begin{array}{lllll}n & \text { y2 } & . & a & \text { Results } \\ 0 & 1 & .001 & .001 & \text { FLSXT } \\ 0 & 2 & .001 & .001 & 1.5703 \\ 1.5 & 2 & .001 & .001 & 1.5703 \\ 1.55 & 2 & .1 & .1 & 1.5500 \\ 1.5 & 2 & .001 & .1 & 1.5625\end{array}$

These combinations test all loops of the program.

5. Bessel Function I, Series Expansion

Dorothea S. Clarke

General Electric Co., FPLD, Cincinnati 15, Ohio

comment Compute the Bessel function $I_{n}(X)$ when $n$ and $X$ are within the bounds of the series expansion. The procedure calling statement gives $n, X$ and an absolute tolerance $\delta$ for determining the point at which the terms of the summation become insig. nificant. Special case : $I_{0}(0)=1$;

procedure $I(n, X, \delta)=:(I s)$

begin

I:

if

$8:-0 ;$ sum $:=0$

$(\mathrm{n} \neq 0) ;$ go to STRT

if $\quad(X=0) ;$ begin $I s:=1$; return end

STRT: $\quad$ summ $:=1$; go to SURE

sfac $:=1$

if $(s=0)$; go to HRE

for $t:=1(1) s$

sfac $:=\operatorname{sfac} \times t$

HRE: $\quad$ snfac : $=$ sfac

for $\quad t:=s+1(1) s+n$

snfac : $=$ snfac $\times t$

summ $:=\operatorname{sum}+(X / 2)^{a+2 \times a n} /(\operatorname{sfac} X$ snfac $)$

SURE: if $(\delta<$ abs (summ - sum))

begin $\quad s:=8+1 ;$ sum $:=$ summ ; go to STRT end

Is := summ ; return

end 
6. Bessel Function I, Astuptotic Expansion Dorothea S. Clarke

General Electric Co., FPLD, Cincinnati 15, Ohio

comment Compute the Bessel Function $I_{u}(X)$ when $n$ and $X$ are within the bounds of the asymptotic expansion. The procedure calling statement gives $n, X$ and an absolute tolerance $\delta$ for determining the point at which the terms of the summation become in. significant;

procedure $\mathbf{I}(\mathbf{n}, \mathbf{X}, \boldsymbol{\delta})$ : (IA)

begin

I:

$r:=1 ;$ pe $:=\left(4 \times n^{2}-1\right) /(8 \times X)$

sum $:=-$ pe

Repeat:

$r: \mathbf{r}+1$

pe $:=$ pe $X .\left((2 \times n)^{2}-(2 \times r-1)^{2}\right) /(r \times 8 \times X)$

if

( $\delta<$ abs (pe))

begin

sum, : = sum $+(-1)^{r} \times$ pe ; go to Repeat end IA : $=(1+$ sum $) \times(\exp (X) /$ sqrt $(2 \times \pi \times X))$ return

end

\section{Euclidian Algorithm}

Robert Claussen

General Electric Co., Cincinnati 15, Ohio

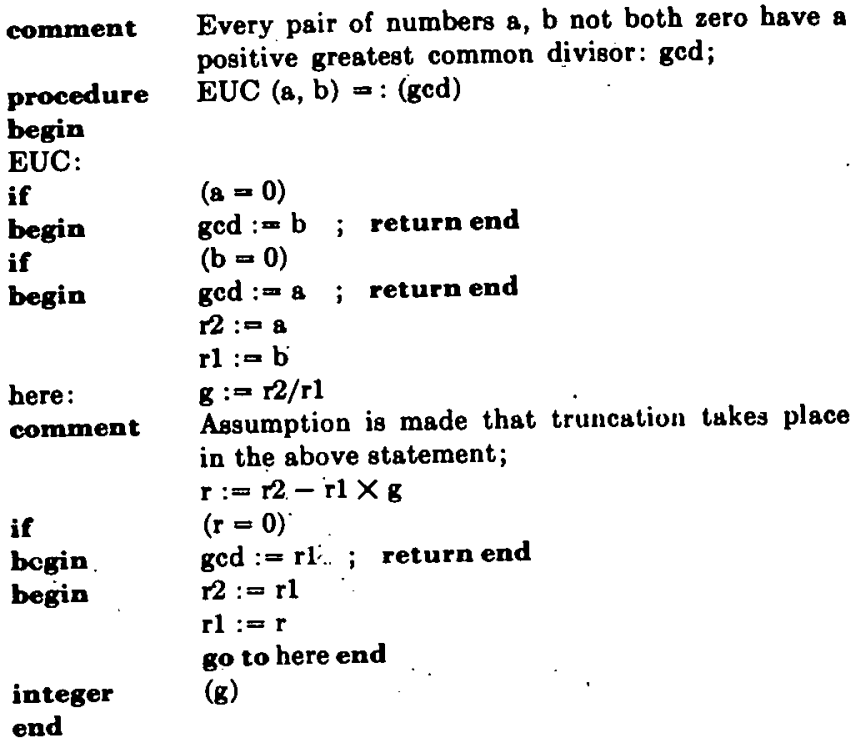

\section{ALGORITHM 8}

EULER SUMMATION

3 (May 1960), 318 P. NAUR

procedure euler (fct, sum, eps, tim) ; value eps, tim ; integer tim ; real procedure fet ; real sum, eps ; comment euler computes the sum of fet(i) for $i$ from zero up to infinity by means of a suitably refined euler transformation. The summation is stopped as soon as tim times in succession the absolute value of the terms of the transformed series are found to be less thsn eps. Hence, one should provide a function fet with one integer argument, an upper bound eps, and an integer tim. The output is the sum sum. euler is particularly efficient in the case of a slowly convergent or divergent alternating series ; begin integer $\mathrm{i}, \mathrm{k}, \mathrm{n}, \mathrm{t} \quad$; $\operatorname{array} \mathrm{m}[0: 15] \quad ; \quad$ real $\mathrm{mn}, \mathrm{mp}, \mathrm{ds}$; $i:=n:=t:=0 ; m[0]:=\operatorname{fct}(0) ; \operatorname{sum}:=m[0] / 2 ;$ nextterm: $i:=i+1 ; m n:=f \operatorname{ct}(i)$;

for $k:=0$ step 1 until $n$ do begin $\mathrm{mp}:=(\mathrm{mn}+\mathrm{m}[\mathrm{k}]) / 2 ; m[k]:=\mathrm{mn}$; $\mathrm{mn}:=\mathrm{mp}$ end means ;

if $(a b s(m n)<a b s(m[n])) \wedge(n<15)$ then

begin ds $:=\mathrm{mn} / 2 ; \mathrm{n}:=\mathrm{n}+1 ; \mathrm{m}[\mathrm{n}]:=$ mn end sccept

else ds : $=\mathrm{mn}$;

sum $:=$ sum $+d s$;

if $a b s(d s)<e p$ then $t:=t+1$ else $t:=0$;

end euler

if $t<$ tim then go to nextterm

\section{CERTIFICATION OF ALGORITHM 8}

EULER SUMMATION [P. Naur et al. Comm. ACM 3, May 1960]

Henry C.. Thacher, Jr.*

Argonne National Laboratory, Argonne, Ill.

- Work supported by the U. S. Atomic Energy Commission

The body of euler was tested on the LGP-30 computer using the Dartmouth SCALP translator. No errors were detected.

The program gave excellent results when used to derive the coefficients for the expansion of $\ln (1+x)$ in shifted Chebyshev polynomials from the first ten terms of the power series. For $n=0,1$, $2,3,4$, the coefficient of $x^{i}$ in the power. series was multiplied by the coefficient of $T_{n}^{*}(x)$ in the expression of $x^{i}$ in terms of the $T_{n}^{*}(x)$. The product, for $i=1,2, \cdots, 10$ was used as $f c t(i)$ in the program. Results for $n=0$ were as follows:

$\begin{array}{cccc}i & f c t(i) & d s & s u m \\ 1 & +0.50000000 & - & - \\ 2 & -0.18750000 & +0.07812500 & +0.3281250 \\ 3 & +0.10416667 & +0.05729166 & +0.3854167 \\ 4 & -0.068359375 & -0.005940758 & +0.3794759 \\ 5 & +0.049218750 & -0.001928713 & +0.3775471 \\ 6 & -0.037597656 & -0.001357019 & +0.3761900 \\ 7 & +0.029924665 & +0.0001742393 & +0.3763642 \\ 8 & -0.024547577 & +0.0000571311 & +0.3764212 \\ 9 & +0.020607842 & +0.0006395427 & +0.3764607 \\ 10 & -0.017619705 & -0.0000055069 & +0.3764551 \\ \text { True Value } & & +0.3764528129 \ldots\end{array}$

Errors less than $0.2 \times 10^{-6}$ were also found for $n=1,2,3,4,5$, $6,7,8$ and 9

This technique appears to be a useful supplement to direct telescoping (Algorithms 37 and 38) and to the methods recommended by Clenshaw', for slowly convergent power series.

1 Clenshaw, C. W., Chebyshev Series for Mathematical Functions. National Physical Laboratory Math Tables, Vol. 5, London, H.M.S.O. (1962).

\section{AIGORITEM 9 RUNGE-KUTTA INTEGRATION}

3 (May 1960), 318 P. NAUR

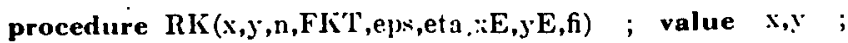
integer $n$; Boolean $f$; real $x, e p s, e t a, x E$; array Y,YE ; procedure FKT ;

comment : RK integrates the system $y_{k}{ }^{\prime}=f_{k}\left(x, y_{1}, y_{2}, \ldots, y_{n}\right)$ $(k=1,2, \ldots, n)$ of differential equations with the method of RungeFuttat with automatic search for appropriate length of integration step. Parameters are: The initial values $x$ and $y[k]$ for $x$ and the unknown functions $y_{k}(x)$. The order $n$ of the system. The procedure FKT $(x, y, n, z)$ which represents the system to be integrated, i.e. the set of functions $f_{k}$. The tolerance values eps and eta which 
govern the accuracy of the numerical integration. The end the integration interval $x E$. The output parameter $y E$ which represents the solution at $x=x E$. The Boolean variable $f$, which must always be given the value true for an isolated or first entry into RIi. If however the functions y must be available at several meshpoints $x_{0}, x_{1}, \ldots, x_{n}$, then the procedure must be called repeatedly (with $x=x_{k}, x E=x_{k+1}$, for $k=0,1, \ldots, n-1$ ) and then the later calls may occur with $f=$ false which saves computing time. The input parameters of FKT must be $x, y, n$, the output parameter $z$ represents the set of derivatives $z[k]=f_{k}(x, y[1], y[2], \ldots, y[n])$ for $x$ and the actual $y$ 's. A procedure comp enters as a non-local identifier ;

begin

array $2, y 1, y 2, y 3[1: n]$; real $x 1, x 2, x 3, H$; Boolean out ; integer $k, j$; own real $s, H_{s}$;

procedure RKIST $(x, y, h, x e, y e)$; real $x, h, x e$; array y,ye ;

comment : RKIST integrates one single RUNGE-KUTTA with initial values $x, y[k]$ which yields the output parameters $x e=x+h$ and $y e[k]$, the latter being the solution at xe. Important: the parameters $n, F K T, z$

begin enter RK1ST as nonlocal entities ;

array $w[1: n], a[1: 5]$; integer $k, j$;

$\mathrm{a}[1]:=\mathrm{a}[2]:=\mathrm{a}[5]:=\mathrm{h} / 2 \quad ; \mathrm{a}[3]:=\mathrm{a}[4]:=\mathrm{h} ;$

xe $:=\mathrm{x}$;

for $k:=1$ step 1 until $n$ do $y e[k]:=w[k]:=y[k]$;

for $j:=1$ step 1 until 4 do

begin

FKT $(x e, w, n, z)$;

$\mathrm{xe}:=\mathrm{x}+\mathrm{a}[\mathrm{j}]$;

for $k:=1$ step 1 until $n$ do

begin

$w[k]:=y[k]+a[j] \times z[k]$

$y e[k]:=y e[k]+a[j+1] \times z[k] / 3$

end $k$

end $j$

end RK1ST ;

Begin of program:

if fi then begin $\mathrm{H}:=x E-x \quad ; s:=0$ end else $H:=H_{s} ;$ out $:=$ fulse ;

$A$ A : if $(x+2.01 \times H-x E>0) \equiv(H>0)$ then

begin $\mathrm{Hs}:=\mathrm{H} ;$ out $:=$ true ; H := $(x E-x) / 2$ end if ;

RK1ST $(x, y, 2 \times H, x 1, y 1)$;

BB: RK1ST $(x, y, H, x 2, y 2) ; \operatorname{RK} 1 S T(x 2, y 2, H, x 3, y 3)$;

for $k:=1$ otep 1 until $n$ do

if $\operatorname{comp}(\mathrm{y} l[k], y 3[k], e t a)>$ eps then go to $\mathrm{CC}$;

comment : $\operatorname{comp}(a, b, c)$ is a function designator, the value of which is the absolute value of the difference of the mantissae of $a$ and $b$, after the exponents of these quantities have been made equal to the largest of the exponents of the originally given parameters a,b,c ;

$\mathrm{x}:=\mathrm{x} 3$; if out then go to DD;

for $k:=1$ step 1 until $n$ do $y[k]:=y 3[k]$;

if $s=5$ then begin $s:=0 ; H:=2 \times H$ end if ; $\mathrm{s}:=\mathrm{s}+1 ;$ go to $\mathrm{AA}$;

$\mathrm{CC}: \mathrm{H}:=0.5 \times \mathrm{H} ;$ out $:=$ fulse $; \mathrm{x1}:=\mathrm{x} 2 ;$

for $k:=1$ step 1 until $n$ do $y 1[k]:=y 2[k]$;

go to $\mathrm{BB}$;

DD: for $k:=1$ step 1 until $n$ do $y E[k]:=y 3[k]$

end $\mathrm{RH}^{*}$

${ }^{8}$ This RK-program contuins some new ideas which are related to ideas of S. GILL, A process for the step-by-step integration of differential equations in an automatic computing machine, Proc. Camb. Phil. Soc. Vol. 47 (1951) p. 96; and E. Fröberg, On the solution of ordinary differential equations. with digital computing machines, Fysiograf. Sällsk. Lund, Förhd. $20 \mathrm{Nr}$. 11 (1950) p. 136-152. It must be clear, however, that with respect to computing time and round-off errors it may not be optimal, nor has it actually been tested on a computer.
10. Evaluation of the Chebyshev Polynomial $T_{n}(X)$ BY Recursion

G. M. Galler

National Bureau of Standards, Washington 25, D. C.

comment This procedure computes the Chebysher polynomial $T_{n}(X)=\cos \left(n \times \cos ^{-1}(X)\right)$ for any given real argument, $X$, and any order, $n$, by the recursion formula below;

real procedure $\operatorname{Ch}(n, X)$;

real

$X$; integer $n$;

begin real

$\mathrm{a}, \mathrm{b}, \mathrm{c} ;$ integer $\mathrm{i}$;

$\mathrm{a}:=1 ; \mathrm{b}:=\mathrm{X}$;

if $n=0$ then $c:=a$ else if $n=1$ then

$c:=b$ else for $i:=2$ step 1 until $n$ do

begin $c:=2 \times X \times b-a ;$ $a:=b ; b:=c$

end

$\mathrm{Ch}:=\mathrm{c}$

end

CERTIFICATION OF ALGORITHM 10

CHEBYSCHEV POLYNOMIAL $\mathrm{T}_{\mathrm{n}}(\mathrm{x}$ ) (Galler, Comm. $A C M$, June, 1960)

JoHN HERNDON

Stanford Research Institute, Menlo Park, California

When transliterated into BALGOL and tested on the Burroughs 220, $\mathrm{Ch}(\mathbf{n}, \mathrm{x})$ gave better than 7-digit accuracy for $\mathbf{n}=\mathbf{0}$, $1,4,8$ and $x=.01, .2, .7$. It gave answers when $x>1$ which corresponded to the value of the series with $x$ substituted.

11. Evaluation of the Hermite Polynomial $H_{n}(X)$ BY RECuRsion

G. M. Galler

National Bureau of Standards, Washington 25, D. C.

comment

This procedure computes the Hermite polynomial

$H_{a}(X)=(-1)^{n} \times e^{X^{2}} \times\left(d^{n} / d X^{2}\left(e^{-x^{2}}\right)\right)$ for any given real argument, $X$, and any order, $n$, by the recursion formula below;

real procedure $\mathrm{He}(\mathrm{n}, \mathrm{X})$;

integer

n ; real $\mathbf{X}$;

begin real

$a, b, c$; integer $i$;

$a:=1 ; b:=2 X$

if $n=0$ then $c: m a$ else if $n=1$ then

$c:=$ b else for $i:=1$ step 1 until $n-1$ do

begin $c:=2 \times x \times b-2 \times i \times a$;

a := b ; b :=c

end

$\mathrm{He}:=\mathrm{c}$

end 
12. Evaluation of the Laguerre Polynomial $\mathrm{L}_{n}(\mathrm{X})$ by REcursion

G. M. Galler

- National Bureau of Standards, Washington 25 D. C.

comment

This procedure computes the Laguerre polynomial

$L_{n}(X)=e^{X} \times\left(d^{n} / d X^{n}\left(X^{n} \times e^{-X}\right)\right)$ for any given real argument, $X$, and any order, $n$, by the recursion formula below;

real procedure $\mathrm{La}(\mathrm{n}, \mathrm{X})$;

integer $n$; real $X$;

begin real $\quad a, b, c$; integer $i$;

$\mathrm{a}:=1 ; \mathrm{b}:=1-\mathrm{X}$;

if $n=0$ then $c:=a$ else if $n=1$ then

$c:=b$ else for $i=1$ step 1 until $n-1$ do

begin $c:=(1+2 \times i-X) \times b-(i \uparrow 2) \times 8$;

$a:=b ; b:=c$

end

$\mathrm{La}:=\mathrm{c}$

end

13. Evaluation of the Legendre Polynomial $P_{n}(X)$ BY Recursion

G. M. Galler

National Bureau of Standards, Washington 25 D. C.

comment

This procedure computes the Legendre polynomial

$P_{n}(X)=\left(1 /\left(2^{n} X n !\right)\right) \times d^{n} / d X^{n}\left(X^{2}-1\right)^{n}$ for

any given real argument, $X$, and any order, $n$, by the recursion formula below;

real procedure

integer

$\operatorname{Le}(n, X)$;

begin real

$\mathrm{n}$; real $\mathrm{X}$;

$a, b, c$; integer $i$;

$\mathrm{a}:=1 ; \mathrm{b}:=\mathrm{X}$;

if $n=0$ then $c:=a$ else if $n=1$ then

c $:=b$ else for $i:=1$ step 1 until $n-1$ do

begin $c:=b \times X+(i /(i+1)) \times(X \times b-a)$;

- $a:=b ; b:=c$

end

Le $:=\mathrm{c}$

end

CERTIFICATION OF ALGORITHM 13

LEGENDRE POLYNOMIAL $\mathrm{P}_{\mathrm{n}}(\mathrm{x})$ (Galler, Comm. ACM, June 1960)

Joh: Herndon

Stanford Research Institute, Menlo Park, California

When transliterated into BALGOL and tested on the Burroughs $220, \operatorname{Le}(\mathrm{n}, \mathrm{x})$ gave 7 -digit accuracy for $\mathrm{n}=0,1,4,9$ and $\mathrm{X}=.01$, $2,7,1.9,5.0$.
ALGORITHM 14. COMPLEX EXPONENTIAL INTEGRAL

\section{A. Beam}

National Bureau of Standards, Washington 25, D. C.

procedure $\operatorname{EKZ}(\mathrm{x}, \mathrm{y}, \mathrm{k}, \epsilon, \mathrm{u}, \mathrm{v}, \mathrm{n}) ; \operatorname{real} \mathrm{x}, \mathrm{y}, \mathrm{k}, \epsilon, \mathrm{u}, \mathrm{v}$ :

integer $n$;

comment

EKZ computes $w(z, k)=u+i v=z^{k} e^{z} \int_{z}^{\infty} e^{-t} d t / t^{k}$

from the continued fraction representation found in H. S. Wall, Continued Fractions, Chap. 18 (D. Van Nostrand, New York, 1948). Input parameters are $x, y, k$, and $\epsilon$ where $z=x+i y$. Successive convergents are computed as follows: For $n=2,3,4$, $\cdots, D_{n}=z /\left(z+M \times D_{n-1}\right), R_{n}=$ $\left(D_{n}-1\right) R_{n-1}, C_{n}=C_{n-1}+R_{n}$, where $M$ is $k+(n-2) / 2$ or $(n-1) / 2$ according to whether $n$ is even or odd, and $D_{1}=R_{1}=C_{1}=1$. Computation is stopped when $C_{n}$ and $C_{n-1}$ agree to the significance specified by $\epsilon$. The corresponding index $\mathbf{n}$ is available after use of the procedure. This method is valid in the entire complex plane exrept. for the origin and the negative real axis. Convergence is too slow to be practical for $|\mathrm{z}|<.05$. Also for some range within the half-strip $|y|<2$, $\mathrm{x}<0$ (this range depends on $\mathrm{k}$ ). The method is valid for complex $k$, but only real $k$ is considered in this procedure;

begin real $t 1, t 2, t 3, M, K, c, a, d, b, g, h, c 1$;

integer $m$;

comment $\mathrm{R}=\mathrm{a}+\mathrm{ib}, \mathrm{D}=\mathrm{c}+\mathrm{id}, \mathrm{C}=\mathrm{g}+\mathrm{ih}$; $\mathrm{el}:=\epsilon \uparrow 2$

$\mathrm{u}:=\mathrm{c}:=\mathrm{a}:=1 ; \mathrm{v}:=\mathrm{d}:=\mathrm{b}:=0$

$\mathrm{n}:=1 ; \mathrm{K}:=\mathrm{k}-1 ;$

BACK: $\quad \mathrm{g}:=\mathrm{u} ; \mathrm{h}:=\mathrm{v} ; \mathrm{n}:=\mathrm{n}+\mathrm{l}$;

$\mathrm{m}:=\mathrm{n} \div 2$;

if $2 \times m=n$ then $M:=m+K$ else $M:=m$;

$\mathrm{t} 1:=\mathrm{x}+\mathrm{M} \times \mathbf{c} ; \mathrm{t} 2:=\mathrm{y}+\mathrm{M} \times \mathrm{d} ;$

$\mathrm{t} 3:=\mathrm{t} 1 \uparrow 2+\mathrm{t} 2 \uparrow 2$;

c : $=(\mathrm{x} \times \mathrm{tl}+\mathrm{y} \times \mathrm{t} 2) / \mathrm{t} 3$

$\mathrm{d}:=(\mathrm{y} \times \mathrm{t} 1-\mathrm{x} \times \mathrm{t} 2) / \mathrm{t} 3$;

$\mathrm{t} 1:=\mathrm{c}-1 ; \mathrm{t} 2:=\mathrm{a} ;$

$\mathrm{a}:=\mathrm{a} \times \mathrm{tl}-\mathrm{d} \times \mathrm{b} ; \mathrm{b}:=\mathrm{d}^{\prime} \times \mathbf{t} 2+\mathrm{t} 1 \times \mathrm{b} ;$ $\mathrm{u}:=\mathrm{g}+\mathrm{a} ; \mathrm{v}:=\mathrm{h}+\mathrm{b} ;$

if $(a \uparrow 2+b \uparrow 2) /(u \uparrow 2+v \uparrow 2)>$ cl then go to BACK ;

end

\section{CERTIFICATION OF ALGORITHAI 14}

COMILLX EXPONENTIAL INTEGRAL (A. Beam,

Comm. AC:II, July, 1960)

P. J. Raber axi Hexry C. Thacher; Jk.*

Argonne National Laboratory, Argonne, Illinois

Ek' was programmed by hand for the Roval-Precision LGP-30 computer, using a $28-b i t$ matutissal foating-point interpretive system (24.2 modified). To farcilitate comparison with existing tables (National Bureau of Statudirds Applied Mathematies Series 51 and 37 ), the real and imaginary parts of $E_{k}(z)$ were computed from $"$ and $v$. Results are shown in the following table. In all ratses, the values angeed with tabulated vallues within the tolerance specificd.

$\begin{array}{ccccc}\times \times & y & k & \epsilon & n \\ 1 \times 10^{-8} & 1.0 & 1 & 10^{-1} & 7 \\ 1 \times 10^{-8} & 1.0 & 1 & 10^{-2} & 1+ \\ 1 \times 10^{-8} & 1.0 & 1 & 10^{-3} & 24 \\ 1 \times 10^{-8} & 1.0 & 1 & 10^{-1} & 37\end{array}$




$\begin{array}{ccccr}1 \times 10^{-8} & 1.0 & 1 & 10^{-5} & 52 \\ 1 \times 10^{-8} & 1.0 & 1 & 10^{-6} & 70 \\ 1 \times 10^{-8} & 1.0 & 1 & 10^{-7} & 90 \\ 1 \times 10^{-8} & 1.0 & 1 & 10^{-8} & 114 \\ 1 \times 10^{-8} & 2.0 & 1 & 10^{-6} & 37 \\ 1 \times 10^{-8} & 3.0 & 1 & 10^{-6} & 26 \\ 1 \times 10^{-8} & 4.0 & 1 & 10^{-6} & 21 \\ 1.0 & 1 \times 10^{-8} & 1 & 10^{-6} & 40 \\ 1.0 & 1.0 & 1 & 10^{-6} & 34 \\ 1.0 & 2.0 & 1 & 10^{-6} & 26 \\ 1.0 & 3.0 & 1 & 10^{-6} & 21 \\ 2.0 & 1 \times 10^{-8} & 1 & 10^{-6} & 23 \\ 2.0 & 1.0 & 1 & 10^{-6} & 22 \\ 2.0 & 2.0 & 1 & 10^{-6} & 20 \\ 2.0 & 3.0 & 1 & 10^{-6} & 17 \\ 3.0 & 1 \times 10^{-8} & 1 & 10^{-6} & 17 \\ 3.0 & 1.0 & 1 & 10^{-6} & 17 \\ 3.0 & 2.0 & 1 & 10^{-6} & 16 \\ 3.0 & 3.0 & 1 & 10^{-6} & 15 \\ 4.0 & 0.0 & 0 & 10^{-6} & 20 \\ 4.0 & 0.0 & 1 & 10^{-6} & 15 \\ 4.0 & 0.0 & 2 & 10^{-6} & 16 \\ 4.0 & 0.0 & 3(1) 14 & 10^{-6} & 17 \\ 4.0 & 0.0 & 15,16 & 10^{-6} & 16\end{array}$

It thus appears that the algorithm gives satisfactory accuracy, but that in certain ranges of the variables, the time required mav be excessive for extensive use.

\section{ALGORITHM 15}

ROOTFINDER II (Modification of Algorithm 2. RooTFINDER, J. Wegstein, Communications ACM, February, 1960)

Henry C. Thacher, Jr., ${ }^{*}$ Argonne National Laboratory, Argonne, Illinois

procedure ROOT II (f, a, eps, $n, g, c, m$ ); integer $n, m$; real procedure f; real a, eps, $g, c$;

comment ROOT II computes a value of $g=y$ satisfying the equation $y=f(y)$. The iteration will converge to $Y$ providing that at some time in the iteration $\mathrm{a} g$ is reached such that $\mathrm{abs}(\mathrm{g}-\mathrm{Y}) \times$ $\operatorname{abs}(\mathrm{d}(\mathrm{df} / \mathrm{dy}) / \mathrm{dy})<2 \times \operatorname{abs}((\mathrm{df} / \mathrm{dy})-1)$, where the derivatives are evaluated at $Y$. Input includes (1) $f$, a procedure for computing $f(y), \quad(2) a$, an initial approximation to the root, (3) eps, a tolerance for the relative error in $\mathrm{g}$, and (4) $\mathrm{n}$, a maximum number of iterations to be performed. Output includes: (1) $\mathrm{g}$, the required root, (2) $c=f(g)-g$, (3) $\mathrm{m}, \mathrm{a}$ parameter indicating the success of the procedure. If the tolerance was not met, $m<0$. $|\mathrm{m}-1|$ gives the number of times that the correction to $\mathrm{g}$ exceeded the preceding one, an indication of instability. ;

begin integer $\mathrm{j}$; real $b, d, h$;

$\mathrm{m}:=1 ;$ if $\mathrm{f}(0)=0$ then begin $\mathrm{g}:=0$; go to return end

else $g:=f(a) ; \quad b:=d:=c:=a-g ;$

if $c=0$ then go to return else

for $j:=1$ step 1 until $n$ do begin $c:=f(g)-g$; if $(\mathrm{abs}(\mathrm{c} / \mathrm{g})<$ eps then go to return else $\mathrm{h}:=$ b/c;

if $\mathrm{h}<0 \vee \mathrm{h}>2$ then $\mathrm{m}:=\mathrm{m}+1$ else

$\mathrm{d}:=\mathrm{d} /(\mathrm{h}-1) ; \mathrm{b}:=\mathrm{c} ; \mathrm{g}:=\mathrm{g}+\mathrm{d}$ end iteration

comment if the system is known to be stable, the if clause of the last statement can be omitted;

$\mathrm{m}:=-\mathrm{m}$ return end

CERTIFICATION OF ALGORITHM 15

ROOTFINDER II (Revision by Henry C. Thacher, Jr., Communications ACM, August, 1960)

Henry C. Thacher, Jr., * Argonne National Laboratory, Argonne, Illinois

The revision of ROOTFINDER suggested in the preceding remark was programmed by hand for the Royal Precision LGP-30 computer, using a 28-bit mantissa floating point interpretive system (24.2).

The program was tested for the following equations:

(1.k) $\mathrm{f}(\mathrm{y})=\arctan \mathrm{y}+\mathrm{k} \pi \quad(\mathrm{k}=0,1,2,3,4,0,8)$

(2) $f(y)=\left(y^{3}+1\right)^{1 / 3}$

(3) $f(y)=y^{3}-1$

These both have the root 1.3247180428

$\left.\begin{array}{l}\text { (4.k) } f(y)-\sinh \alpha_{k} y \\ (5 . k) f(y)=\cosh \alpha_{k} y\end{array}\right\}\left(\alpha_{1}=-1.2, \alpha_{2}=-0.5, \alpha_{8}=0.5, \alpha_{4}=1.2\right)$

Typical results of these tests were as follows:

\begin{tabular}{|c|c|c|c|c|c|}
\hline$f(y)$ & e & a & $\mathbf{g}$ & {$[f(g-1)-g-1] \times 1$} & Remarks \\
\hline 1.0 & $10^{-8}$ & 1.0000 & 0.0000000 & 0.00 & \\
\hline $1.1^{\circ}$ & $10^{-3}$ & 3.1415 & 4.4934094 & 0.15 & \\
\hline 1.2 & $10^{-*}$ & 6.2832 & 7.7252518 & 0.60 & \\
\hline 1.3 & $10^{-8}$ & 9.4248 & 10.904122 & 0.00 & \\
\hline 1.4 & $10^{-8}$ & 12.5664 & 14.066194 & 0.00 & \\
\hline 1.6 & $10^{-3}$ & 18.8496 & 20.371303 & 0.60 & \\
\hline 1.8 & $10^{-3}$ & 25.1327 & 26.666054 & 0.60 & \\
\hline 1.2 & $10^{-\mathrm{s}}$ & 1.3 & 1.3247179 & 0.00 & \\
\hline 1.2 & $10^{-8}$ & 0.5 & 1.3247179 & 0.00 & . \\
\hline 3 & $10^{-\infty}$ & 9.0 & 4.4804900 & $197.74 \times 10^{7}$ & Diverged 2 times, not con- \\
\hline 3 & $10^{-0}$ & 5.0 & 1.3482797 & $\left..51 \times 10^{7}\right\}$ & verged after 20 iter. \\
\hline 3 & $10^{\rightarrow}$ & 3.0 & 1.3247180 & 0.0 & Converged in less than 20 iter. \\
\hline 3 & $10^{\rightarrow} \rightarrow$ & 2.0 & & & $\begin{array}{l}\text { Diverged } 2 \text { times. Term. with } \\
h=1\end{array}$ \\
\hline 3 & $10^{-}$ & 1.1 & 1.3247180 & 1384.24 & $\begin{array}{l}\text { Diverged } \theta \text { times. Converged } \\
\text { after } 20 \text { iter. }\end{array}$ \\
\hline 3 & $10^{-}$ & 1.0 & & & Terminated when $\mathrm{g}$ became 0 . \\
\hline 3 & $10 \rightarrow$ & 0.8 & 1.3247180 & 0.00 & $\begin{array}{l}\text { Diverged } 4 \text { times. Conv. in } \\
\text { less than } 20 \text {. }\end{array}$ \\
\hline 3 & $10^{-0}$ & 0.6 & 1.6161598 & $4.39 \times 10^{7}$ & $\begin{array}{l}\text { Diverged } 2 \text { times. No conv. } \\
\text { after } 20 \text {. }\end{array}$ \\
\hline 4. $\mathrm{k}$ & $10^{-0}$ & 1.0 & 0.00000000 & $0 \quad 0.00000000$ & For all k. \\
\hline 5.1. 8.4 & $10^{-8}$ & 1.0 & 0.09179585 & $50.793 \times 10^{7}$ & $\begin{array}{l}\text { Diverged } 7 \text { times. No conv. } \\
\text { dfter } 20 \text { iter. }\end{array}$ \\
\hline $6.2,8.3$ & $10^{-8}$ & 1.0 & 1.11787755 & 50.037 & \\
\hline
\end{tabular}

Function (3) is of particular interest, since it does not converge for most algorithms. With the Wegstein iteration, convergence was obtained, or would have been obtained with a few more iterations for a wide range of initial guesses.

\section{REMARK ON ALGORITHM 15}

ROOTFINDER II (Henry C. Thacher, Jr., Comm. $A C M$, August 1960)

George E. Forsythe and John G. Herriot, Stanford University, Stanford, California

As pointed out by Lieberstein (Comm. ACM, January 1959, p. 5), this algorithm is precisely the Newton method of chords or the scant method applied to $g(x)=f(x)-x=0$. Thus convergence is not of second order but rather (for simple roots) of order $\frac{1}{2}(\sqrt{ } \overline{5}-1)=1.618$, as shown by Jeeves (Comm. ACM, August 1958 , pp. 9-10). In the first portion of the algorithm b, c, d, should be set equal to $g-a$ instead of $a-g$ in order to be consistent with 
the iteration portion. Doing this will usually cut down the number of iterations. Not only is a preliminary test for a zero root desirable but the possibility that $\mathrm{g}$ may be zero at any stage of the iteration should be considered in writing the return criterion. The possibility that $h=1$ should also be checked and appropriate action taken. Algorithm 26 takes care of these matters and also corrects some minor errors in Algorithm 15. This method is certainly not the best rootfinder that could be written.

SEE ALGORITHM 2

\section{ALGORITHM 16 \\ CROUT WITH PIVOTING \\ George E. Forsythe \\ Stanford University, Stanford, California \\ real procedure INNERPRODUCT $(u, v)$ index : (k) start : (s) finish : (f); \\ value $s, f$; integer $k, s, f ;$ real $u, v$; \\ comment INNERPRODUCT forms the sum of $u(k) \times$ $v(k)$ for $k=8, s+1, \ldots, f$. If $s>f$, the value of INNERPRODUCT is zero. The substitution of a very accurate inner product procedure would make CROUT more accurate; \\ begin \\ real $\mathrm{h}$; \\ $\mathrm{h}:=0 ;$ for $\mathrm{k}:=\mathrm{s}$ step 1 until $\mathrm{f}$ do $\mathrm{h}:=\mathrm{h}+\mathrm{u} \times \mathrm{v}$; \\ INNERPRODUCT $:=\mathrm{h}$ \\ end INNERPRODUCT; \\ procedure CROUT (A, b, $n, y$, pivot, INNERPRODUCT); value $n$; array $A, b, y$, pivot; integer $n$, pivot; real procedure INNERPRODUCT; \\ comment This is Crout's method with row interchanges, as formulated in reference [1], for solving $A y=b$ and transforming the augmented matrix $[A \cdot b]$ into its triangular decomposition $\mathrm{LU}$ with all $L[k, k]=1$. If $A$ is singular we exit to 'singular,' a non-local label. pivot[k] becomes the current row index of the pivot element in the $k$-th column. Thus enough information is preserved for the procedure SOLVE to process a new right-hand side without repeating CROUT. The accuracy obtainable from CROUT would be much increased by calling CROUT with a more accurate inner product procedure than INNERPRODUCT;}

begin

integer $k, i, j$, imax, $p$; real TEMP, quot;

for $k:=1$ step 1 until $n$ do

1: begin

TEMP : $=0$;

for $\mathrm{i}:=\mathrm{k}$ step 1 until $\mathrm{n}$ do

2: begin

$A[i, k]:=A[i, k]-\operatorname{INNERPRODUCT}(\mathbf{A}[\mathbf{i}, \mathbf{p}], \mathbf{A}[\mathbf{p}, \mathbf{k}]$, $\mathrm{p}, 1, \mathbf{k}-1)$

if $\operatorname{abs}(\mathrm{A}[\mathrm{i}, \mathrm{k}])>$ TEMP then

3: begin

TEMP $:=\operatorname{abs}(A[i, k]) ; \quad \operatorname{imax}:=i$

end 3

end 2;

pivot $[k]:=$ imax;

comment We have found that $A[i m a x, k]$ is the largest

pivot in column $k$. Now we interchange rows $k$ and imax;

if $\operatorname{imax} \neq k$ then
4: begin for $j:=1$ step 1 until $n$ do

5: begin

TEMP $:=A[k, j] ; A[k, j]:=A[i \max , j] ;$

$A[\operatorname{imax}, j]:=$ TEMP

end 5;

TEMP $:=\mathrm{b}[\mathrm{k}] ; \mathrm{b}[\mathrm{k}]:=\mathrm{b}[\mathrm{imax}] ; \mathrm{b}[\mathrm{imax}]:=$ TEMl

end 4;

comment The row interchange is done. We proceed to the , elimination;

if $\mathrm{A}[\mathrm{k}, \mathrm{k}]=0$ then go to singular;

for $i:=k+1$ step 1 until $n$ do

begin quot $:=1.0 / \mathrm{A}[\mathrm{k}, \mathrm{k}] ; \mathrm{A}[\mathrm{i}, \mathrm{k}]:=$ quot $\times \mathrm{A}[\mathrm{i}, \mathrm{k}]$ end;

for $\mathrm{j}:=\mathrm{k}+1$ step 1 until $\mathrm{n}$ do

$A[k, j]:=\mathbf{A}[\mathbf{k}, \mathbf{j}]-\operatorname{INNERPRODUCT}(\mathbf{A}[\mathbf{k}, \mathbf{p}]$, $A[p, j], p, 1, k-1)$

$b[k]:=b[k]-\operatorname{INNERPRODUCT}(A[k, p], b[p], p$, $1, k-1)$

end 1;

comment The triangular decomposition is now finished, and we do the back sulustilution;

for $k:=n$ step -1 until 1 do

$y[k]:=(b[k]-\operatorname{INNERPRODUCT}(A[k, p], y[p], p$, $\mathrm{k}+1, \mathrm{n}) / \mathrm{A}[\mathrm{k}, \mathrm{k}]$

end CROUT;

procedure SOLVE (B, c, n, z, pivot, INNERPRODUCT);

value $n$; array $B, c, z$, pivot; integer $n$, pivot;

real procedure INNERPRODUCT;

comment SOLVE assumes that a matrix $A$ has already been transformed into B by CROUT, but that a new column $c$ has not been processed. SOLVE solves the system $\mathrm{Az}=c$, and the output $\mathrm{z}$ of SOLVE is precisely the same as the output $y$ of the procedure statement CROUT (A, c, n, y, pivot, INNERPRODUCT). However, SOLVE is faster, because it does not repeat the triangularization of $A_{\text {; }}$

begin

integer $\mathrm{k}$; real TEMP.

for $k:=1$ step 1 until $n$ do

begin

TEMP := c[pivot[k]]; $c[\operatorname{pivot}[k]]:=c[k] ; c[k],:=$ TEMP; $c[k]:=c[k]-$ INNERPRODUCT $(B \mid k, p]$, $c[p], p 1, k-1)$

end;

for $k:=n$ step -1 until 1 do

$\mathrm{z}[\mathrm{k}]:=(\mathrm{c}[\mathrm{k}]-\mathrm{INNERPRODUCT}(\mathrm{B}[\mathrm{k}, \mathrm{p}], \mathrm{z}[\mathrm{p}], \mathrm{p}$, $\mathbf{k}+1, \mathbf{n}) / \mathrm{B}(\mathbf{k}, \mathbf{k}]$

end SOLVE

\section{REFERENCE}

[1] J. H. Wilkinson, theory and practice in linear systems, pp. 43-100 of JoHn W. CARR III (editor), Application of Advanced Numerical Analysis to Digital Computers, (Lectures given at the University of Michigan, Summer 1958, College of Engineering, Engineering Summer Conferences, Ann Arbor, Michigan [1959]). 
REMARK ON ALGORITHM 16

CROUT WITH PIVOTING (G. E. Forsythe, Comm. $A C M, 3$ (Sept. 1960), 507-8.)

Henry C. Thacher, Jr., ${ }^{*}$ Argonne National Laboratory, Argonne, Illinois

This procedure contains the following errors:

a. In SOLVE, the expression

$c[k]:=c[k]-$ INNERPRODUCT

$(B[k, p), c\{p], p \quad 1, k-1)$

should read:

$c[k]:=c[k]-$ INNERPRODUCT

(B $[k, p], c[p], p, 1, k-1)$

b. In CROUT, the specification part should read:

array $A, b, y$; integer $n$; integer array pivot ;

c. In SOLVE, the specification part should read:

array $B, c, z$; integer $n$; integer array pivot ;

The efficiency of the algorithm will be improved by the following changes:

a. In the elimination phase of CROUT, replace

for $\mathrm{i}:=k+1$ step 1 until $n$ do by

begin quote $:=1.0 / \mathrm{A}[\mathrm{k}, \mathrm{k}] ; \mathrm{A}[\mathrm{i}, \mathrm{k}]:=$ quot $\mathrm{XA}[\mathrm{i}, \mathrm{k}]$ end ;

quot $:=1.0 / \mathrm{A}[\mathrm{k}, \mathrm{k}]$; for $\mathrm{i}:=\mathrm{k}+1$ step 1 until $\mathrm{n}$ do $A[i, k]:=$ quot $X A[i, k]$;

b. Omit INNERPRODUCT from the formal parameter list in both CROUT and SOLVE, and declare INNERPRODUCT either locally, or globally. This avoids any reference to INNERPRODUCT in the calling sequence produced by a compiler.

It is also to be noted that a minor modification of CROUT allows it to be used to evaluate the determinant of $A$.

All of these suggestions are included in a later algorithm.

\section{REMARK ON ALGORITHM 16}

CROUT WITH PIVOTING (G. Forsythe, Communications ACM, September, 1960)

George E. Forsithe

Stanford University, Stanford, California

\section{QUERY}

Perhaps the most basic procedure for an ALGOL library of matrix programs is an inner product procedure. The procedure Innerproduct given on page 311 of [1] is fairly difficult to comprehend, and probably poses great difficulties for most translating routines. I merely copied its form in writing a modifed inner product routine for [2].

My query is: How should one write an inner product procedure in ALGOL?

\section{REFERENCES}

1. Peter Naur (editor), J. W. Backus, et al., Report on the algorithmic language ALGOL 60, Comm. Assoc. Comp. Mach. $\$$ (1960), 299-314.

2. Georae E. Forsythe, Crout with pivoting in AlGOL 60, Comm. Assoc. Comp. Mach. 3 (1960), 507-508.

\section{ALGORITHM 17}

TRDIAG

C. F. Sprague III

General Atomic Division of General Dynamics Corp., San Diego, California

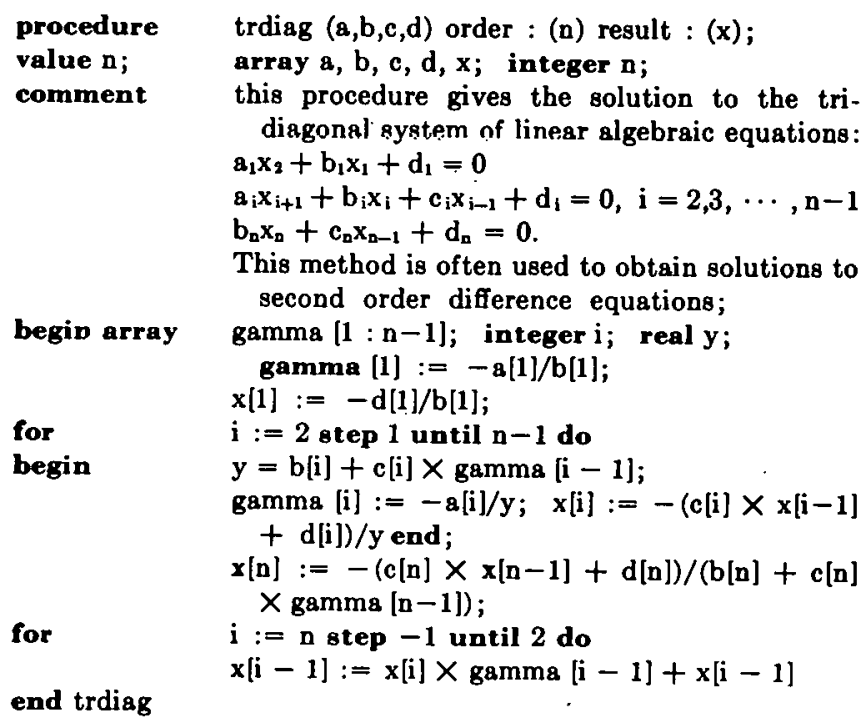

\section{ALGORITHM 18 \\ RATIONAL INTERPOLATION BY CONTINUED FRACTIONS}

R. W. FLOYD

Armour Research Foundation, Chicago, Illinois

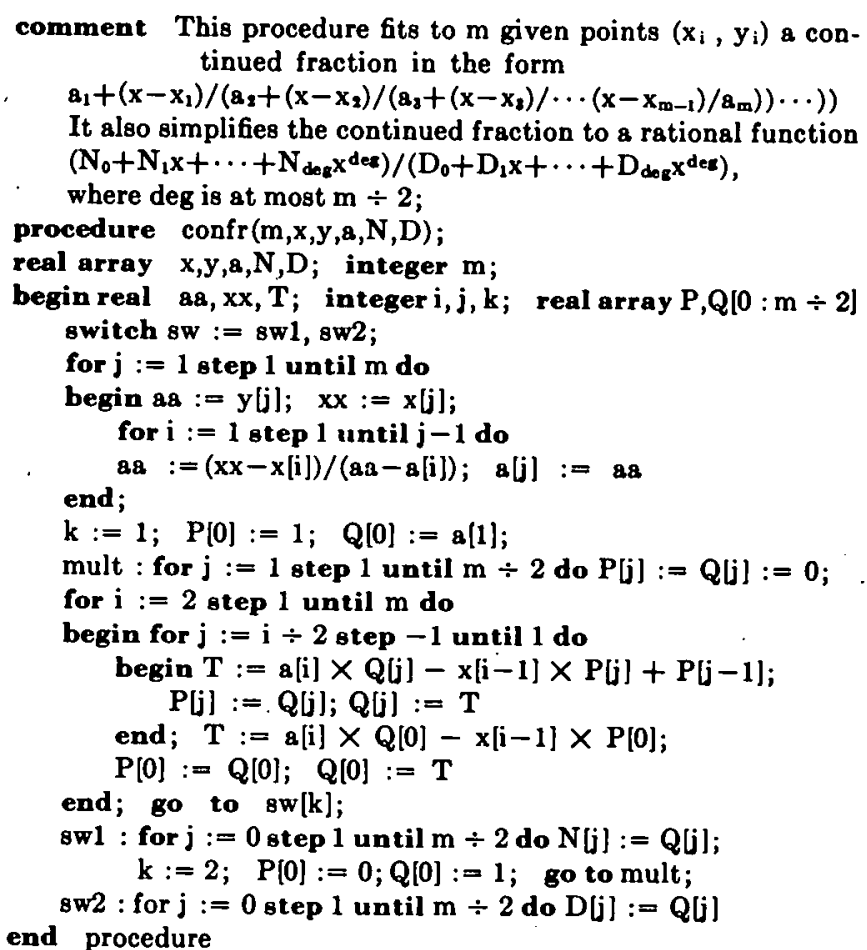




\section{CERTIFICATION OF ALGORITHM 18 \\ RATIONAL INTERPOLATION BY CONTINUED FRACTIONS}

[R. W. Floyd, Comm. ACM., Sept. 1960]

Henry C. Thacher, JR.*

Reactor Engineering Div., Argonne National Lab.,

Argonne, Ill.

* Work supported by the U. S. Atomic Energy Commission

The body of procedure confr was tested with the Algol translator system written for the LGP-30 computer by the Dartmouth College Computer Center. No syntactical errors were found in the procedure body, except for a missing semicolon after the array delcaration. The translated algorithm gave satisfactory results when tested on values of $(4 x+1) /(x+4)$ at any three of the points $x=1,2,3,4$. When all four points were used, a division overflow occurred in the statement for $i:=1$ step 1 until $j-1$ do $a a:=$ $(x x-x[i]) /(a a-a[i])$; which forms the reciprocal differences. An overflow of this type will occur whenever $y[j]$ is approximated to high accuracy by one of the continued fractions based only on the points $x[i], i=1,2, \cdots, k$ with $k$ less than $j$. Unless $i=j-1$, the difficulty may be overcome by setting $a g$ equal to the largest real representable in the computer whenever division overflow would occur. When $i=j-1$, the difficulty is irretrievable, and the data points must be reordered.

\section{ALGORITHM 19 \\ BINOMIAL COEFFICIENTS \\ Richard R. KEnYon \\ Computing Laboratory, Purdue University, Lafayette, Indiana}

comment This procedure computes binomial coefficients $\mathbf{C}_{\mathbf{m}}{ }^{n}=\mathbf{n} ! / \mathbf{m} !(n-m) !$ by the recursion formula $C_{i+1}^{2}=(n-1) C_{i}{ }^{n} /(i+1)$ starting from $\mathrm{C}_{0}{ }^{\mathrm{a}}=1$;

integer procedure $\mathbf{C}(\mathbf{m}, \mathbf{n})$;

integer $\quad m, n$;

begin integer $i, a, b$;

$\mathrm{a}:=1$;

if $2 \times m>n$ then $b:=n-m$ else

$\mathbf{b}:=\mathbf{m}$;

for $i:=0$ step 1 until $b$ do

begin $a:=(n-i) \times a \div(i+1)$ end

$\mathrm{C}:=8$

end

Binomial Coefficients

\section{CERTIFICATION OF ALGORITHM 19}

BINOMIAL COEFFICIENTS [Richard R. Kenyon, Comm. ACM Oct., 1960]

Richard George*

Particle Accelerator Div., Argonne National Lab., Argonne, Ill.

- Work supported by the U. S. Atomic Energy Commission.

This procedure was tested on the LGP-30, using the compiler Algol-30 from Dartmouth College Computation Center. The following changes were found necessary:

(1) Within the comment, the line

$$
C_{i+1}^{n}=(n-1) C_{i}^{n} /(i+1)
$$

should be

$$
C_{i+1}^{n}=(n-i) C_{i^{n}} /(i+1)
$$

(2) The line defining the iteration loop

for $i:=0$ step 1 until $b$ do

should be

(3) The sequence

$$
\text { for } i:=0 \text { step } 1 \text { until } b-1 \text { do }
$$

should be.

$$
\begin{array}{ll}
\text { end } \quad C:=a & \text { end } \\
\text { end } \quad C:=a & \text { end }
\end{array}
$$

\section{REMARK ON ALGORITHM̆ 19}

RINOMIAL COEFFICIENTS (Richard R. Kenyon,

Comm. ACM, Oct. 1960)

Bichard Steck

Armour Research Foundation, Chicago 16, Ill.

The for clause of Algorithm 19 should read:

$$
\text { for } i:=0 \text { step } 1 \text { until } b-1 \text { do }
$$

With this correction the algorithm was certified on the Armour Research Foundation UNIVAC 1105.

The recursion formula stated in the comment should read:

$$
\mathrm{C}_{i+1}^{n}=(n-i) \mathrm{C}_{1}{ }^{\prime \prime} /(i \mid 1) \text {. }
$$

\section{ALGORITHM 20 \\ REAL EXPONENTIAL INTEGRAL}

S. Peavy

National Bureau of Standards, Washington 25, D.C.

real procedure

comment

begin

\section{CERTİICATION OF ALGORITHM 20}

REAL EXPONENTIAL INTEGRAL (S. Peavy, Comm. $A C M$, Oct. 1960)

William J. Alexander* and Henry C. Thacher, Jr. * Argonne National Laboratory, Argonne, Illinois

Expint ( $x$ ) was programmed for the LGP-30 computer, using both a $7 \mathrm{~S}$ floating-point compiler (ACT III) and an $8 \mathrm{~S}$ floatingpoint interpretive code (24.2). Constants given to more than $7 \mathrm{~S}$ (or to $8 \mathrm{~S}$ for the 24.2 program) were rounded to $7 \mathrm{~S}$ (or $8 \mathrm{~S}$ ). 
After changing the constant .005519968 to .05519968 , both programs gave acceptable accuracy over the range tested.

The $8 \mathrm{~S}$ (24.2) program was compared with the $9 \mathrm{D}$ values given for $-\mathrm{E}_{\mathrm{i}}(-\mathrm{x})$ in Mathematical Tables Project, Tables of Sine, Cosine, and Exponential Integrals, Volume II (1940) for the set of values $x=0.1(0.1) 1.0(1.0) 10.0$. The largest discrepancy found was $-16 \times 10^{-8}$ for $x=0.1$. For $x$ greater than 1 , all values tested were good to $8 \mathrm{~S}$.

For computing real values of the exponential integral, this algorithm is much faster than EKZ (Algorithm 13). For $x<1$, the ratio of speeds was of the order of 20 .

\section{REMARK ON ALGORITHM 20}

REAL EXPONENTIAL INTEGRAI، (S. l'eavy, Comm: $A C M$, October 1960)

S. Peavy

National Bureau of Standards, Washington, D. C.

A printing error has been called to our attention by J. A. Beutler of E. I. duPont de Nemours and Co. I.ines 15 through 17 of Algorithm 20 should read

$z:=((((.00107857 \times \times-.00976004) \times x$

$+.05519968) \times x-.24991055) \times x$

$+.99999193) \times x-.577215606-\ln (x)$

\section{ALGORITHM 21}

\section{BESSEL FUNCTION FOR A SET OF INTEGER ORDERS}

\section{W. Börsch-Supan}

National Bureau of Standards, Washington 25, D. C.

procedure BESSELSETINT $(x, n, \epsilon, J) ;$ value $x, n, \epsilon$; real $x, \epsilon$; integer $n$; real array $J$;

comment: This procedure computes the values of the Bessel functions $J_{p}(x)$ for real argument $x$ and the set of all integer orders from 0 up to $n$ and stores these values into the array $J$, whose subscript bounds should include the integers from 0 up to $\mathrm{n}$. $\mathrm{n}$ must be nonnegative.

The computation is done by applying the recursion formula backward from $\mathrm{p}=\mathrm{k}$ down to $\mathrm{p}=0$ as described in $M T A C 11$ (1957), 255-257. $k$ is chosen to yield errors less than $10^{-5}$ approximately after the first application of the recursion. The recursion is repeated with a larger $k$ until the difference between the results of the two last recursions doesn't exceed the given bound $\epsilon>0$. The steps in increasing $\mathrm{k}$ are chosen in such a way that the errors decrease at least by a factor of approximately $10^{-5}$. There is no protection against overflow. ;

begin real dist, rec0, rec1, rec2, sum, max, err ; integer $k, p$; Boolean $s$; real array Jbar $[0: n]$; if $x=0$ then

begin $J[0]:=1 \quad ;$ for $p:=1$ step 1 until $n$ do $J[p]:=0$; go to Exit

end ;

dist : $=$ if $\operatorname{abs}(x) \geqq 8$ then $5 \times$ abs $(x) \uparrow(1 / 3)$ else 10 ;

$k:=\operatorname{entier}(($ if $\operatorname{abs}(x) \geqq n$ then abs $(x)$ else $n)+$ dist $)+1$; $s:=$ false ;

Rec: $\operatorname{rec} 0:=0$; rec1 $:=1$; sum $:=0$;

for $p:=k$ step -1 until 1 do

begin J[if $p>n+1$ then $n$ else $p-1]:=\operatorname{rec} 2:=$ $2 \times \mathrm{p} / \mathrm{x} \times \operatorname{rec} 1-\operatorname{rec} 0$; if $p=1$ then sum $:=$ sum + rec2

else if $\mathrm{p} \div 2 \times 2 \neq \mathrm{p}$ then sum :=

sum $+2 \times$ rec2

rec0 $:=$ rec1 ; rec1 $:=\operatorname{rec} 2$

end recursion;

Norm: for $p:=0$ step 1 until $n$ do $J[p]:=J[p] /$ sum ;

if 8 then

begin $\max :=0$;

for $p:=0$ step 1 until $n$ do

begin err $:=$ abs $(J[p]-J b a r[p])$;

if err $>\max$ then $\max :=$ err

end maximum error ;

end then

if $\max \leqq \epsilon$ then go to Exit

else $8:=$ true ;

for $p:=0$ step 1 until $n$ do $J b a r[p]:=J[p]$;

$k:=$ entier $(k+$ dist $)$;

go to Rec ;

Exit: end BESSELSETINT

\section{ALGORITHM 22 \\ RICCATI-BESSEL FUNCTIONS OF FIRST AND SECOND KIND}

H. OsER

National Bureau of Standards, Washington 25, D. C.

procedure RICCATIBESSEL ( $x, n, e p s, S, C)$;

value $x, n$, eps ;

real $x$, eps; integer $n$; real array $S, C$;

comment: RICCATIBESSEL computes $S_{k}(x)=(\pi x / 2)+J_{k+1}(x)$ and $C_{k}(x)=-(\pi x / 2)+Y_{k+k}(x)$ for real $x \neq 0$ and all integer values of $k$ from 0 through $n$ with a prescribed (absolute) accuracy eps. The computation is done by using the recursion relations of the cylinder functions. For abs $(x)>n$ both $S_{k}(x)$ and $C_{k}(x)$ are computed by using the recursions for ascending orders. For $n>\operatorname{abs}(x)$ the functions $S_{k}(x)$ are obtained by using the recursion in descending orders. (See STEGUNAbramowitz, $M T A C 11,1957,255-257$ ). Reaching out two different intervals beyond the order $n$, the two vectors $S_{k}{ }^{1}(x)$ and $S_{k}{ }^{2}(x)$ are checked if the maximum component of their difference meets the tolerance eps. If this is not the case a maximum of 10 iterations is set up to achieve the required absolute accuracy. Initial values $S_{k \max }$ and $S_{k \max -1}$ for the backward iteration are computed from the corresponding values $C_{k \max -1}$ and $C_{k \max }$. No check of accuracy is done in case $n<\operatorname{abs}(x)$. Both $C_{k}(x)$ and $S_{k}(x)$ are affected in this case by errors of the same order of magnitude as the sub. routines for $\sin (\mathrm{x})$ and $\cos (\mathrm{x})$;

begin real $r 1, r 2, r 3, r 4, r 5, r 6$, step, acc, $\max , a, b, d 1, d 2$; integer $i, k, l$, imax ;

real array $W[0: n]$;

switch $\mathrm{P}:=$ initial, improve ;

acc $:=106$;

step: $={ }_{10} 3$;

imax : $=10$

comment: These constants may be chosen differently, but caution has to be taken because of overflow. acc sets an initial iteration to give roughly a 6-place accuracy. Subsequent iterations should improve the result to 3 more places each ; 


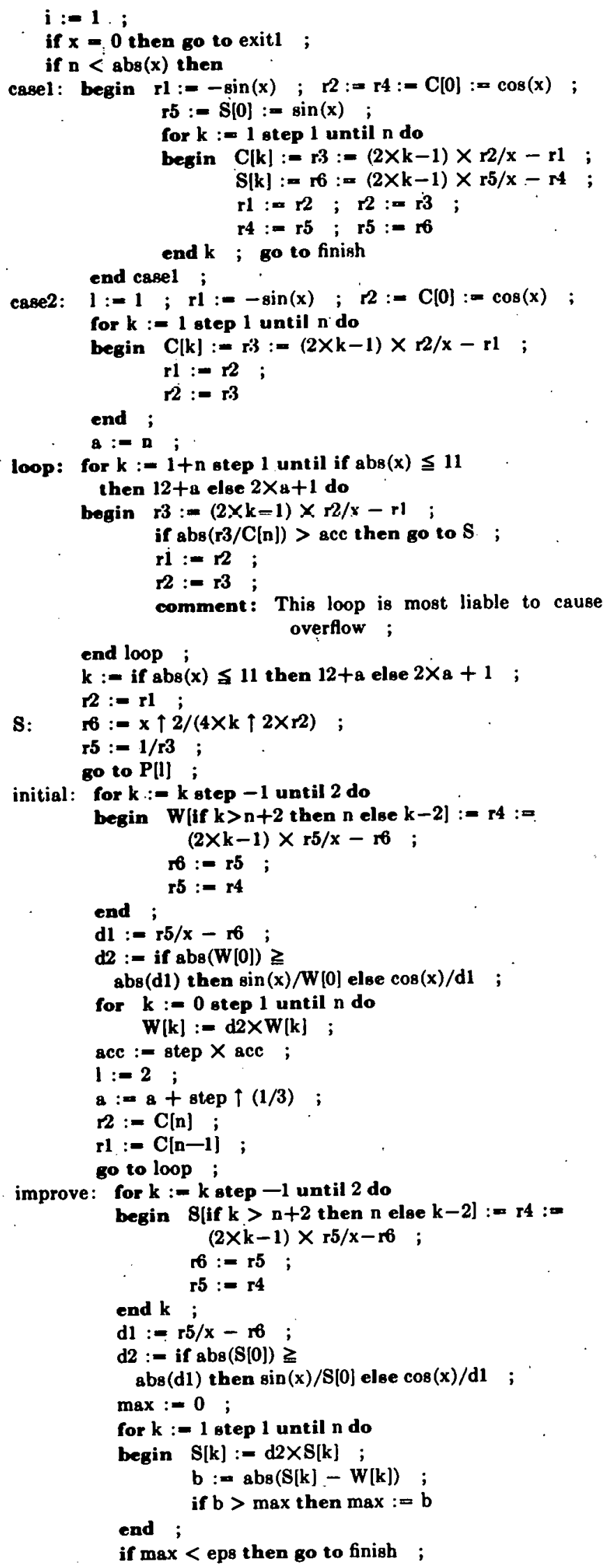

for $k:=0$ step 1 until $n$ do $W[k]:=\mathbf{S}[\mathbf{k}]$;

acc : $=$ step $X$ acc ;

if $\mathrm{i} \geqq$ imax then go to exit2 ;

$\mathrm{i}=\mathrm{i}+1 ; \mathrm{a}:=\mathrm{a}+\operatorname{step} \uparrow(1 / 3) ;$

$\mathrm{r} 2:=\mathrm{C}[\mathrm{n}] ; \mathrm{rl}:=\mathrm{C}[\mathrm{n}-1] ;$ go to loop ;

exitl : go to finish.; comment : $x=0$;

exit2: go to finish ;

comment: maximum number of iterations reached ;

Gnish: end RICCATIBESSEL

\section{ALGORITHM 23 \\ MATH SORT \\ Wallace Feurzeig}

Laboratories for Applied Science, University of Chicago, Chicago, Ill.

procedure MATHSORT (INVEC, OUTVEC, TOTEVEC, $n, k$, SETFUNC) ; value $n, k$;

array INVEC, OUTVEC ;

integer array TOTEVEC ;

integer procedure SETFUNC ;

integer $n, k$;

begin comment MATHSORT is a fast sorting algorithm which produces a monotone rearrangement of an arbitrarily ordered set of $n$ numbers (represented by the vector INVEC) by a surprising though familiar device. The resultant sorted set is represented by the vector OUTVEC. The key field, i.e. the ordered set of bits (or bytes) on which the sort is to be done, is obtained by some extraction-justification function denoted SETFUNC. The key field allows the representation of $k$ possible values denoted $0,1, \ldots, k-1$.

The procedure determines first of all the exact frequency distribution of the set with respect to the key, i.e. the number of elements of INVEC with key field value precisely equal to $j$ for all $j$ between 0 and $k-1$. The cumulative frequency distribution TOTEVEC [i] $=\sum_{j-0}^{i}$ (Number of elements of INVEC with key value $=j$ ) is then computed for $0 \leqq i \leqq k-1$. This induces the direct assignment (storage mapping function) of each element of INVEC to a unique cell in OUTVEC. This assignment (like the determination of the frequency distribution) requires just one inspection of each element of INVEC. Thus the algorithm requires only $2 n$ "look and do" operations plus $k-1$ additions (to get the cumulative frequency distribution).

The algorithm can be easily and efficiently extended to handle alphabetic sorts or multiple key sorts. To sort on another key the same algorithm is applied to each new key field with the new INVEC designated as the last induced ordering (i.e. the current OUTVEC). The algorithm has been used extensively at LAS on binary as well as decimal machines both for internal memory sorts and (with trivial modification) for large tape sorts ;

for $i:=1$ step 1 until $n$ do TOTEVEC[SETFUNC (INVEC[i])] := TOTEVEC [SFTFUUNC(INVEC[i])] + 1 ;

for $i:=1$ step 1 until $k-1$ do TOTEVEC $[i]:=$ TOTEVEC $[i]+$ TOTEVEC $[i-1]$;

for $i:=1$ step 1 until $n$ do begin OUTVEC[TOTEVEC[SETFUNC(INVEC[i])]] $:=$ INVEC[i] ; TOTEVEC[SETFUNC(INVEC[i])] := TOTEVEC[SETFUNC(INVEC[i])] - 1 ; end

end MATHSORT. 
CERTIFICATION OF ALGORITHM 23

MATHSORT (Wallace Feurzeig, Comm. ACM, Nov., 1960)

Russell W. Ranshaw

University of Pittsburgh, Pittsburgh, Pa.

The MATHSORT procedure as published was coded for the IBM 7070 in Fortran. Two deficiencies were discovered:

1. The TOTVEC array was not zeroed within the procedure. This led to some difficulties in repeated use of the procedure.

2. Input vectors already in sort on nonsort fields were unsorted. That is, given the sequence

$$
31,21,32,22,33 \text {, }
$$

Mathsort would produce, for a sort on the 10's digit:

$$
22,21,33,32,31 \text {, }
$$

which is definitely out of sequence.

The following modified form of the procedure corrects these difficulties. Note the transformation of symbols.

procedure MATHSORT (I, O, T, n, k, S); value $n, k$; array $I, O$; integer array $T$; integer procedure $S$; integer $n, k$;

begin for $\mathrm{i}:=0$ step 1 until $k-1$ do $T[i]:=0$; for $i:=1$ step 1 until $n$ do $T[S(I[i])]:=T[S(I[i])]+1$; for $\mathrm{i}:=\mathrm{k}-2$ step -1 until 0 do $\mathrm{T}[\mathrm{i}]:=\mathrm{T}[\mathrm{i}]+$ $\mathrm{I} \mid \mathrm{i}+1 \mathrm{i}$

for $i:=1$ step 1 until $n$ do

begin $O[\mathrm{n}+1-\mathrm{T}[\mathrm{S}(\mathrm{I}[\mathrm{i}])]]:=\mathrm{I}[\mathrm{i}]$;

$\mathrm{T}[\mathrm{S}(\mathrm{I}[\mathrm{i}])]:=\mathrm{T}[\mathrm{S}(\mathrm{I}[\mathrm{i}])]-1$;

end MATHSORT.

Using the MATHSORT procedure ten times and having the procedure $S$ supply each digit in order, 1000 random numbers of 10 digits each were sorted into sequence in 31 seconds. The method of locating the lowest element, interchanging with the first element, and continuing until the entire list has been so examined yielded a complete sort on the same 1000 random numbers in 227 seconds. Using the Table-Lookup-Lowest command in the 7070 yielded 56 seconds for the same set of random numbers.

\section{AIGOIRITHM 24}

\section{SOLLTION OF TRI-DIAGONAL LINEAR EQUA- TIONS}

\section{B. LEAVENWORTH}

American Machine \& Foundry Co., Greenwich, Conn.

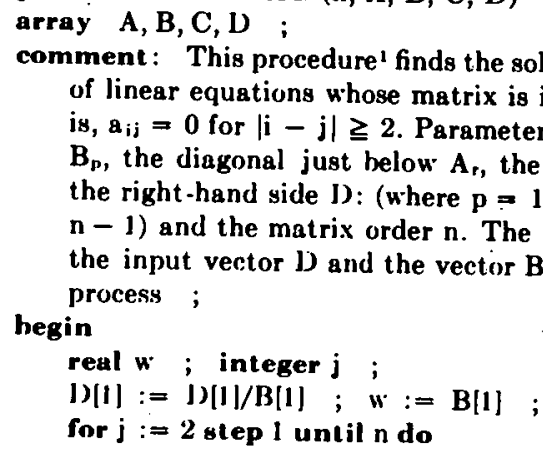
process ; of linear equations whose matrix is in tridiagonal form, that is, $a_{i j}=0$ for $|i-j| \geqq 2$. Parameters are: the main diagonal $B_{p}$, the diagonal just below $A_{r}$, the diagonal just above $C_{r}$, the right -hand side $\mathrm{J}$ ): (where $\mathrm{p}=1, \ldots, \mathrm{n}$ and $\mathrm{r}=1, \ldots$; $n-1)$ and the matrix order $n$. The solution vector replaces the input vector $\mathrm{J}$ and the vector $\mathrm{B}$ is also destroyed in the

$$
\begin{aligned}
& \text { begin } B \mid j-1]:=C[j-1] / w ; w:=B[j]-A[j-1] \\
& \times \mathrm{B} \mid \mathrm{j}-1] \text {; } \\
& \mid)(j|:=(I)| j \mid-A[j-1] \times 1)[j-1]) / w \text { end ; } \\
& \text { for } j:=1 \text { step } 1 \text { until } n-1 \text { do } \\
& \text { end ThII)A(i } \\
& \text { I) }[n-j \mid:=1)[n-j]-B(n-j \mid \times 1)(n-j+1]
\end{aligned}
$$

'D. W. Peaceman and H. H. Rachford, Jr., The Numerical Solution of Puraholic and Elliptic Jifferential Equations, Journal of the Suc. for Ind. and Applied Math. Vol. 3 March 1955.

\section{AI,GOIRITHM 25}

REAI, ZEROS OF AN AIRBITRARY FUNCTION

B. LEAVENWORTH

American Machine and Foundary Co., Greenwich, Conn.

procedure ZEROS(n, C, FUNCTION, m, ep1, ep2, ep3, eta) ; integer $n, m$; real epl, ep2, ep3, eta ; array $C$; procedure FUNCTION :

comment: This procedure finds the real zeros of an arbitrary function using Muller's method ${ }^{1,2}$ and is adapted from FORTRAN code by Frank. ${ }^{8}$ Each iteration determines a zero of the quadratic passing through the last three function values. Parameters include the number of roots desired $n$. If $\mathrm{C}_{\mathrm{i}}$ is zero, starting values are $-1,1,0$ respectively. If $\mathrm{C}_{\mathrm{i}}=\beta$ then the starting values are $.9 \beta, 1.1 \beta, \beta$. The procedure F.UNCTION (rt, frt) must be supplied to evaluate the function value $\mathrm{frt}$, given the argument $\mathrm{rt} . \mathrm{m}$ is the maximum number of iterations permitted. epl is the relative convergence criterion on successive iterates. ep2 is the absolute convergence criterion on the function value. eta is the spread for multiple roots, that is, if $\left|r t-C_{i}\right|<$ ep? where $C_{i}$ is a previously found root, then $\mathrm{rt}$ is replaced by $\mathrm{rt}+$ eta ;

begin integer $L, j k, i, m m ;$ real $p, p 1, p 2, x 0, x 1, x 2, r t$, frt, fprt, d, dd, di, 'h, bi, den, dn, dm, tem ;

switch S : Si, S2, S3, S4 ;

for $L:=1$ step 1 until $m$ do

begin $\mathrm{jk}:=0 ;$ if $\mathrm{C}[\mathrm{L}]=\mathbf{0}$ then go to initial else go to assign ;

initial: $\mathrm{p}:=-1, \mathrm{p} 1:=1 ; \mathrm{p} 2:=0 ;$ go to start ;

assign: $p:=.9 \times \mathrm{C}[\mathrm{L}] ; \mathrm{p} 1:=1.1 \times \mathrm{C}[\mathrm{L}] ; \mathrm{p} 2:=\mathrm{C}[\mathrm{L}] ;$

start: rt : $=p$; go to fn;

enter: go to $S[$ if $j k<4$ thew $j k$ else 4$]$;

Sl: rt := pl ; $x 0:=$ fprt ; go to fn ;

S2: rt := p2; $x 1:=$ fprt ; go to fn ;

S3: $\quad x 2:=$ fprt $; h:=$ if $C[L]=0$ then -1

else $-.1 \times \mathrm{C}[\mathrm{L}] ; \mathrm{d}:=-.5$;

loop: dd : $=1+d ;$ bi : = x $0 \times d \uparrow 2-x 1 \times d d \uparrow 2 \times \times 2 \times$ $(d d+d)$

den : $=$ bi $\uparrow 2-4 \times \times 2 \times d \times d d \times(x 0 \times d-(x 1 \times d d)+\times 2)$; if den $\leqq 0$ then den $:=0$ else den $:=$ sqrt.(den) ;

dn $:=b i+$ den ; dm $:=b i-d e n ;$

if abs(dn) $\leqq \mathrm{abs}(\mathrm{dm})$ then den $:=\mathrm{dm}$ else den $:=\mathrm{dn}$;

if den $=0$ then den $:=1$;

di : $=-2 \times \times 2 \times$ dd $/$ den ; h := di $\times h \quad ; \quad r t:=r t+h ;$ go to if $\mathrm{abs}(\mathrm{h} / \mathrm{rt})<\mathrm{epl}$ then call else fn ;

S4: if abs(fprt) $<$ abs $(x 2 \times 10)$ then begin $x 0:=x 1 ; x 1:=x 2 ; x 2:=$ fprt ; $d:=d i$; go to loop end else begin di $:=$ di $\times .5 ; h:=h \times .5$; $\mathrm{rt}:=\mathrm{rt}-\mathrm{h} ;$ go to $\mathrm{fn}$ end ;

fn: $j k:=j k+1 ;$ if $j k<m$ then $m m:=1$ else $m m:=0$; call: $\operatorname{FUNCTION}(\mathrm{rt}, \mathrm{frt}) ;$ if $\mathrm{mm}=1$ then go to compute else go to root ; 
compute: fprt := frt ;

for $i:=2$ step 1 until $L$ do

begin tem := rt $-\mathrm{C}[i-1] ;$ if abs $($ tem $)<e p 3$ then go to change else fprt : $=f \mathrm{prt} / \mathrm{tem}$ end

test: if $\operatorname{abs}(\mathrm{frt})<\mathrm{ep} 2 \wedge$ abs $(\mathrm{fprt})<$ ep2 then go to root

else go to enter ;

change: rt := $\mathrm{t}+\mathrm{etu} ; \mathrm{jk}:=\mathrm{jk}-1$; go to fn ;

root: $C[L]:=$ rt end $L$

end ZEROS

1). E. MLller, A Method for Solving Algebraic Equations Using an Automatic Computer, MTAC 10 (1956).

2 W. L. Frank, Finding Zeros of Arbitrary Functions, J. ACM 6 (1958)

${ }^{3}$ W. L. Frank, RWGRT, General Root Finder 704 Fortran Source Language Subroutine Share Distribution * 635. Parameters used by Frank are: epl $=10^{-6}$, ep2 $=10^{-20}$, ep3 $=10^{-20}$, eta $=10^{-3}$.

\section{REMARK ON ALGORITHM 25}

REAL ZEROS OF AN ARBITRARY FUNCTION

(B. Leavenworth, Comm. ACM, November 1960)

Robert M. Collinge

Burroughs Corporation, Pasadena, California

On attempting to use this algorithm, I discovered the two following errors:

(1) The line following the SWITCH statement should read: for $L:=1$ step 1 until $n$ do

(2) The line starting with the label loop: should read: loop: dd := $1+d ; \quad b i=x 0 \times d \uparrow 2-x 1 \times d d \uparrow 2$ $+\times 2 \times(d d+d) ;$

With these two modifications incorporated the algorithm was translated into the language of the Burroughs Algebraic Compiler and has been used successfully on the Burroughs $220 \mathrm{Com}$ puter.

SEE ALGORIT:IM 2

\section{ALGORITHM 26}

ROOTFINDER III (Modification of Algorithm 15. Rootfinder II, Henry C. Thacher, Jr., Comm. ACM, August. 1960)

Jонх G. Herriot, Stanford University, Stanford, California

procedure ROOTIII (f, a, eps, $n, g, c, m$ ) ; integer $n, m$; real procedure $f$; real $a$, eps, $\mathrm{g}, \mathrm{c}$;

comment ROOTIII computes a value of $\mathrm{g}=\mathrm{y}$ satisfying the equation $y=f(y)$. The iteration will converge to $Y$ providing that at some time in the iteration $\mathrm{a} g$ is reached such that $\operatorname{abs}(\mathrm{g}-\mathrm{Y}) \times \operatorname{abs}(\mathrm{d}(\mathrm{df} / \mathrm{dy}) / \mathrm{dy})<2 \times \operatorname{abs}\left(\left(\mathrm{df} / \mathrm{d} y^{y}\right)-1\right)$, where the derivatives are evaluated at $Y$. Input includes: (1) $f$, a procedure for computing $f(y),(2)$ a, an initial approximation to the root, (3) eps, a tolerance for the relative error in $g$, and (4) $n$, a maximum number of iterations to be performed. Output includes: (1) g, the required root, (2) $c=f(g)-g,(3) \mathrm{m}$, a parameter indicating the success of the procedure. If the tolerance was not met $m<0$. The number $|\mathrm{m}|-1$ gives the number of times that the correction to $\mathrm{g}$ exceeded the preceding one. If $f(y)-y$ has the same value for two successive approximations to $\mathrm{g}$, then $\mathrm{h}=1$, and we exit to "alarm", a nonlocal label. Alarm should provide a means of deciding whether $\mathbf{g}$ is an acceptable root or not. ; begin integer $\mathbf{j}$; real $b, d, h$;

$\mathrm{m}:=1 ; \mathrm{j}:=0 ; \mathrm{c}:=\mathbf{0}$;

if $f(0)=0$ then begin $g:=0$;

go to return end ;

$\mathrm{g}:=\mathrm{f}(\mathrm{a}) . ; \mathrm{b}:=\mathrm{d}:=\mathrm{c}:=\mathrm{g}-\mathrm{a} ;$

if $\mathrm{c}=0$ then go to return ;

for $j:=1$ step 1 until $n$ do.

begin $c:=f(g)-g$;

if $\operatorname{abs}(\mathrm{c}) \leqq \mathrm{abs}(\mathrm{g}) \times$ eps then go to return ;

h $:=b / c$;

if $\mathrm{h}=1$ then go to alarm;

if $h>0 \wedge h<2$ then $m:=m+1$;

$\mathrm{d}:=\mathrm{d} /(\mathbf{h}-1) \quad ; \quad b:=c ; g:=g+d$

end iteration ;

$\mathbf{m}:=-\mathbf{m}$; return : end

SEE ALGORITHM 2

ALGORITHM 27

ASSIGNMMENT

Roland SiLver

MIT Lincoln Laboratory, ${ }^{*}$ Lexington, Massachusetts

procedure Assignment(d, $n, x)$; value $n$; integer $n$; array $d$; integer array $x$;

comment: Assignment determines that permutation $x$ of the integers $[1: n]$ for which the sum $(i:=1(1) n)$ of the elements $d[i, x[i]]$ of the $n \times n$ matrix $d$ is a minimum. $n \geqq 2$. For more complete information see: An Algorithm for the Assignment Problem, Roland Silver, Comm. $A C M$, Nov. 1960, p. 605 ;

begin

switeh Switch := NEXT, L1, NEXT1, MARK ;

array $a[1: n, 1: n]$;

integer array $c[1: n], c b[1: n]$, lambda[1:n], mu[1:n], $r[1: n], y[1: n]$;

integer cbl, $q 1, \mathrm{cl} 0, \mathrm{i}, \mathrm{j}, \mathrm{k}, \mathrm{l}, \mathrm{rl}, \mathrm{rs}, \mathrm{sw}$;

comment:

INITIALIZE ;

for $\mathrm{i}:=1$ step 1 until $\mathrm{n}$ do

begin $\min :=\mathrm{d}[\mathrm{i}, 1]$;

for $j:=2$ step $l^{\prime}$ until $n$ do if $d[i, j]<\min$ then min $:=\mathrm{d}[\mathrm{i}, \mathrm{j}]$;

for $j:=1$ step 1 until $n$ do $a[i, j]:=d[i, j]-\min$

end $\mathrm{i}$;

for $j:=1$ step 1 until $n$ do

begin $\min :=\mathrm{a}[1, \mathrm{j}]$;

for $i:=2$ step 1 until $n$ do if $a \mid i, j]<\min$ then $\min$ $:=\mathbf{a}|\mathbf{i}, \mathrm{j}|$;

for $i:=1$ step 1 until $n$ do $a[i, j]:=a[i, j]-\min$

end $j$;

for $i:=1$ step 1 until $n$ do $x[i]:=y[i]:=0$;

for $i:=1$ step 1 until $n$ do

begin

for $j:=1$ step 1 until $n$ do

begin

if $\mathrm{a}[\mathrm{i}, \mathrm{j}] \neq 0 \vee \mathrm{x}[\mathrm{i}] \neq 0 \vee \mathrm{y}[\mathrm{j}] \neq 0$ then go to $\mathrm{J} \mathrm{I}$;

$x[i]:=j ; y[j]:=i$

J1: $\quad$ end $j$;

end $i$;

START: comment: Start labeling

$\mathrm{rl}:=\mathrm{cl}:=0 ; \mathrm{rs}:=1$;

for $i:=1$ step 1 until ndo 


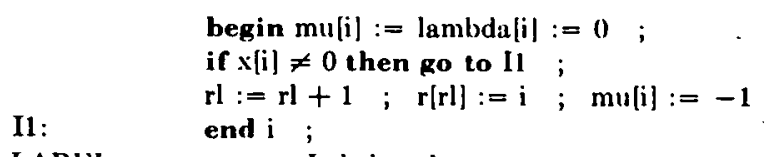

LABBEL: comment: Label and scan ;

$\mathrm{i}:=\mathrm{r}[\mathrm{rs}] ; \mathrm{rs}:=\mathrm{rs}+1 ;$

for $j:=1$ step 1 until $n$ do

begin if $a[i, j] \neq 0$ or lambda $[j \mid \neq 0$ then go to $\mathrm{J} 2$;

lambda $[\mathrm{j}]:=\mathrm{i} ; \quad \mathrm{cl}:=\mathrm{cl}+1 ; \operatorname{clcl}]:=\mathrm{j}$.;

if $\mathrm{y}[\mathrm{j}]=0$ then go to MARI ;

2. $\quad \mathrm{rl}:=\mathrm{rl}+1 ; \mathrm{r}[\mathrm{rl}]:=\mathrm{y}[\mathrm{j}]^{\prime} ; \operatorname{mu}[\mathrm{y}[\mathrm{j}] \mathrm{l}:=\mathrm{i}$ end $\mathrm{j}$;

if $\mathrm{rs} \leqq \mathrm{rl}$ then go to LABEL ;

comment :

RENORMALIZE ;

sw $:=1 ; \quad$ clo $:=\mathrm{cl} ; \quad$ cbl $:=0 ;$

for $j:=1$ step 1 until $n$ do

begin if lambda[j] $\neq 0$ then go to $\mathrm{J} 3$;

J3:

cbl $:=\operatorname{cbl}+1 ; \operatorname{cb}[\mathrm{cbl}]:=\mathrm{j}$

$\min :=\mathrm{a}[\mathbf{r}[\mathrm{i}], \mathbf{c b}[\mathbf{i}]] \quad ;$

for $k:=1$ atep 1 until rl do

begin

for $l:=1$ step 1 until cbl do if $a[r[k], \operatorname{cb}[1]\} \leqq \min$ end $k$;

then $\min :=a[r[k], c b[1]]$

for $i:=1$ step 1 until $n$ do

begin if $\mathrm{mu}[\mathrm{i}] \neq 0$ then go to $\mathrm{I} 2$;

for $l:=1$ step 1 until cl0 do $a[i, c[1]]:=a[i, c[1]]+\min ;$ go to 13 ;

12: for $1:=1$ step 1 until cbl do

begin $a[i, c b[1]]:=a[i, c b[1]]-\min ;$

go to Switch[sw] ;

NEXT: if $a[i, c b[1] \neq 0 \vee$ lambda $[$ cb $[1]] \neq 0$ then go to $\mathrm{L} 1$; lambda[cb[l]] := i ;

if $y[c b[I]]=0$ then

begin $\mathrm{j}:=\mathrm{cb}[\mathrm{l}] ;$ sw $:=2 ;$ go to $\mathrm{Ll}$ end ;

$\mathrm{cl}:=\mathrm{cl}+\mathbf{1} ; \mathbf{c}[\mathbf{c l}]:=\mathrm{cb}[\mathrm{l}] ;$

$\mathrm{rl}:=\mathrm{rl}+1 ; \mathrm{r}[\mathrm{rl}]:=\mathrm{y}[\mathrm{cb}[\mathrm{l}]] ;$

L1: end 1 ;

I3: end $i$;

go to Switch $[\mathrm{sw}+2]$;

NEXT1: if cl0 $=$ cl then go to LABEL ;

for $\mathrm{i}:=\operatorname{cl} 0+1$ step 1 until cl do mu[y[c[i]]] := c[i] ;

go to LABEL ;

MARK: comment : mark new column and permute ;

$y[j]:=i:=$ lambda $[j]$;

if $x[i]=0$ then begin $x[i]:=j ;$ go to

START end ;

$k:=j ; j:=x[i] ; x[i]:=k \quad$;

go to MARK

end Assignment

* Operated with support from the U. S. Army, Navy and Air Force.

[Note: The reader should distinguish between the letter 1 and the figure 1 , both of which appear in the above algorithm.-Ed.]

\section{CERTIFICATION OF ALGORITHM 27}

ASSIGNMENT [Roland Silvers, Comm. ACM 3 , Nov. 1960].

ROBERT D. WitTY

Burroughs Corp., Detroï, Mich.

Assignment was successfully run on the Burroughs B5000 using Burroughs extended Algol 60.

\begin{tabular}{rrrrrr}
\multicolumn{8}{c}{ Input } & Array \\
60 & 0 & 0 & 76 & 0 & 0 \\
0 & 40 & 18 & 0 & 60 & 24 \\
60 & 16 & 2 & 4 & 0 & 40 \\
0 & 27 & 18 & 3 & 55 & 75 \\
0 & 40 & 62 & 16 & 11 & 53 \\
28 & 4 & 10 & 84 & 0 & 16
\end{tabular}

Solution Vector: $X(6,4,3,1,5,2)$

The following changes were made in the algorithm prior to its successful run:

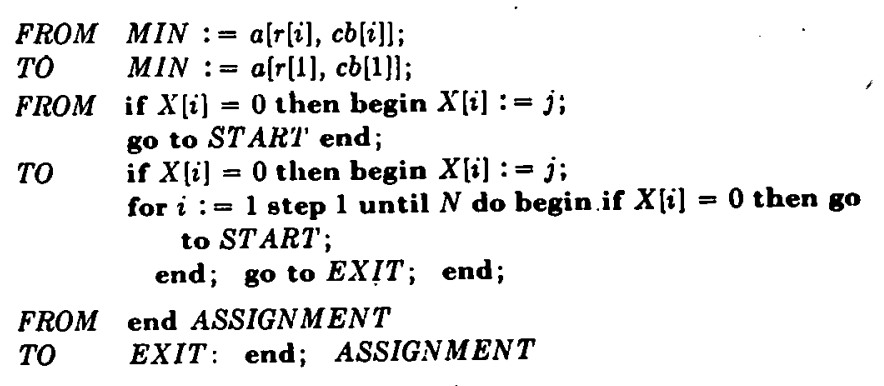

\section{CERTIFICATION OF ALGORITHM 27}

ASSIGNMENT [Roland Silver, Comm. ACM, Nov. 1960]

Albert Newhouse

University of Houston, Houston, Texas

The ASSIGNMENT algorithm was translated into MAD and successfully run on the IBM $709 / 7094$ after the following corrections were made:

All references to array $a$ and $d$ refer to the same array, i.e. change all $a[i, j]$ to $d[i, j]$. Furthermore:

(a) 3rd line after $L A B E L$ : comment: Label and scan; should read

begin if $d[i, j] \neq 0 \vee$ lambda $[j] \neq 0$ then go

(b) first line after $J 3$ : end $j$; should read $\min :=d[r[1], c b[1]]$

(c) line I2: should read

12: for $l:=1$ step 1 until $c b l$ do

Since there is no provision made for this algorithm to end the following additions were made:

(1) in the integer declaration add the variable: flag

(2) first line after $S T A R T$ : comment: ... add the line flag : $=n$

(3) first line before $I 1$ : end $i$; change to read $r l:=r l+1 ; \quad r[r l]:=i ; \quad m u[i]:=-1 ;$ flag $:=$ flag -1

(4) add a line after $I 1$ : end $i$; if $f$ lag $=n$ then go to FINI;

(5) change the last line of the algorithm to read: FINI: end Assignment

In order to obtain the minimum value of the $\sum_{i=1}^{n} a_{i x i}$ (in the following called total) the following additions may be made: 
Add a real variable tolal and

(A) new line after INITIALIZE; total : $=0$;

(B) new line after the first end $i$; total $:=$ total $+\min$;

(C) new line after the first end $j$; total $:=$ lotal $+\min$;

(1)) after the line end $k$; after $J 3$ : end $j$; add the line

tolal $:=$ total $+(r l+c b l-n) \times \min ;$

\section{ALGORITHM 28 \\ LEAST SQUARES FIT BY ORTHOGONAL POLY: NOMIALS \\ JohN G. MaCKINNEY}

General Kinetics Incorporated, Arlington 6, Virginia

procedure LSFIT ( $f, x l, x m, m, k$, alpha, beta, sigma, s, p) ; value $x 1, x m, m, k$; real $x 1 ; x m$; integer $\mathbf{m}, \mathbf{k}$;

real array $f$, alphs, beta, sigma, s, p. ;

comment LSFIT accepts $m$ values of the function $f$ at equal intervals of the abscissa from $x 1$ through $x m$, and obtains in $p[0]$ through $p[k]$ the coefficients of the best polynomial approximation of degree $k$ or less (least squares) as programmed by George E. Forsythe, Journal SIAM 5, no. 2, June 1957, with only minor variations. The output values alpha $[1: k]$, beta $[0: k]$, and $s[0: k]$ enable the user to make final adjustments to the results, according to the statistic sigma $[0: k]$. LSFIT uses the procedure POLYX (a, b, c, d, n) to transform its results from the interval $(-2,2)$ to the interval (x1, xm) ;

begin integer i, j ; real dummy, x, xone, deltax, delsq, omega, lastw, thisw ;

real array cthisp, cpoly $[0: k]$, clastp $[-1: k]$, lastp, thisp $[1: \mathrm{m}]$;

Boolean swx ;

comment Initialization ;

swx := true ; beta $[0]:=$ clastp $[0]:=$ clastp $[-1]:=$

delsq $:=$ omega $:=0$;

cthisp [0] := 1 ; thisw := m ;

for $i:=1$ step 1 until $m$ do

begin delsq $:=$ delsq $+\mathrm{f}[\mathrm{i}] \uparrow 2$;

thisp [i] $:=1 ;$ lastp [i] $:=0$;

omega $:=$ omega $+\mathrm{f}[\mathrm{i}]$ end ;

$\mathrm{s}[0]:=$ cpoly $[0]:=$ omega/thisw ;

delsq : $=$ delsq $-\mathrm{s}[0] \times$ omega ;

sigma $[0]:=\operatorname{delsq} /(\mathbf{m}-1)$;

comment Transformation of abscissa ; $\mathrm{i}:=\mathbf{m}+\mathbf{2}$;

if $2 \times i=m$ then deltax $:=4 /(m-1)$ else deltax $:=$

$4 / \mathrm{m}$; xone := -2 ;

comment Main Computation loop ;

for $i:=0$ step 1 until $k-1$ do

begin dummy $:=0 ; x:=$ xone ;

1: for $j:=1$ step 1 until $m$ do

begin dummy $:=\operatorname{dummy}+x \times$ thisp $[j] \uparrow 2$;

$x:=x+$ deltax end ;

2: alpha $[i+1]:=$ dummy/thisw ;

lastw := thisw ;

thisw $:=$ omega $:=0$;

$x:=$ xone ;

3: for $\mathrm{j}:=1$ step 1 until $\mathrm{m}$ do

begin dummy $:=$ beta [i] $\times$ lastp [i] ;

lastp $[j]:=$ thisp $[j]$; thisp $[j]:=(x-\operatorname{alpha}[i+1]) \times$ thisp [j]

- dummy

thisw := thisw + thisp $[j] \uparrow 2$;

omega $:=$ omega $+f[j] \times$ thisp $[j]$;

$\mathrm{x}:=\mathrm{x}+$ deltax end ;

4: beta $[i+1]:=$ thisw / lastw ;

$\mathrm{s}[\mathrm{i}+\mathrm{l}]:=$ omega / thisw ;

delsq $:=$ delsq $-\mathrm{s}[\mathrm{i}+1] \times$ omega ;

sigma $[\mathrm{i}+1]:=\operatorname{delsq} /(\mathrm{m}-\mathrm{i}-1)$;

if swx then go to 6 ;

5: cpoly $[i+1]:=0 ;$ go to 9 ;

comment Termination of main loop when higher power will not improve fit ;

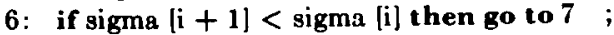
swx := false ; go to 5 ;

comment Recursion for polynomial coefficients ;

7: for $\mathrm{j}:=0$ step 1 until $\mathrm{i}$ do

begin dummy := clastp [j] $\times$ beta [i] ; clastp $[j]:=$ cthisp [j] ;

cthisp $[\mathrm{j}]:=$ clastp $[\mathrm{j}-1]-$ alpha $[\mathrm{i}+1] \times$ cthisp $[\mathrm{j}]-$ dummy ;

cpoly $[j]:=$ cpoly $[j]+s[i+1] \times$ cthisp $[j]$ end ;

8: cpoly $[\mathrm{i}+1]:=\mathrm{s}[\mathrm{i}+1]$

cthisp $[i+1]:-1$;

9: clastp $[i+1]:=0$ end of main

computation loop, transformation of polynomial follows ;

begin real $a, b$;

a $:=\operatorname{deltax} \times(\mathrm{m}-1) /(\mathrm{xm}-\mathrm{x} 1)$

$\mathrm{b}:=$ xone $-\mathrm{a} \times \mathrm{xl}$;

POLYX (a, b, cpoly, p, k) end

end of LSFIT

REMARK ON ALGORITHM 28

LEAST-SQUARES FIT BY ORTHOGONAL POLYNOMIALS (John G. MacKinney, Comm. ACM 8 (Nov. 1960))

D. B. MAcMillaN

Knolls Atomic Power Laboratory, General Electric Co., Schenectady, N. Y.

The algorithm obtains the coefficients of the fitted polynomial of lowest degree such that an increase in the degree would cause an increase in the statistic sigma (sigma squared in Forsythe's notation). A significant decrease in sigma, as one goes from a fitted polynomial to one of higher degree, indicates that the increase in degree causes an improvement in the fit to the function underlying the data, rather than merely following more closely the random variations about that function introduced by the physical measurement process.

If one of the orthogonal polynomials, say the one of $i$ th degree, is missing from the underlying function, and some of the orthogonal polynomials of higher degree are present, then the fitted polynomial of $i$ th degree will not be a real improvement over that of $(i-1)$-th degree, but higher order fitted polynomials will be a real improvement. For example, in one of our recent routine problems the coefficient of the second degree orthogonal polynomial was quite small, and the first few values of sigma, starting with sigma (1), were $.255, .264, .062, .046, .048$. The algorithm would have chosen the first degree fitted polynomial as "best", but the third and fourth degree fitted polynomials were clearly better than it.

This loophole may be plugged by modifying the algorithm so it computes the coefficients of the polynomial of lowest degree $i$ for which it is true that

$$
\text { sigma }(i+1) \geq \operatorname{sigma~(i)~}
$$

and that

$$
\operatorname{sigma}(j) \geq .6 \operatorname{sigma}(i) \quad j=i+2, i+3, \cdots, k \text {, }
$$

(.6 was chosen arbitrarily). 


\section{ALGORITHM 29}

\section{POLYNOMIAL TRANSFORMER}

JoHN G. MACKINNEY

General Kinetics Inc., Arlington 6, Virginia

procedure POLYX (a, b, c, d, n) ; value $a, b, n$; integer $\mathrm{n} ; \operatorname{real} \mathrm{a}, \mathrm{b}$; real array $c, d$;

comment POLYX computes coefficients $\mathrm{d} 0, \mathrm{dl}, \ldots$, dn of the transformed polynomial $p(t)$ given $c 0, c l, \ldots$. cn of $p(x)$ where $x=a t+b$;

begin integer $i, j, k ; \operatorname{real} \operatorname{array} z, w[0: n]$

$w[0]:=z[0]:=1 ; d[0]:=c[0] ;$

for $i$ :- 1 step 1 until $n$ do

begin $w[i]:=1 ; z[i]:=b \times z[i-1] ;$ $d[0]:=d[0]+c[i] \times z[i]$

end of initialization ;

for $j:=1$ step 1 until $n$ do

begin $w[0]:=w[0] \times a ; d[j]:=c[j] \times w[0] ;$ $\mathbf{k}:=1$;

for $\mathrm{i}:=\mathrm{j}+1$ step 1 until $n$ do

begin $w[k]:=a \times w[k]+w[k-1] ;$ $d[j]:=d[j]+c[i] \times w[k] \times z[k] ;$

end $k:=k+1$ end

end of POLYX polynomial transformer

\section{ALGORITHM 30}

NUMERICAL SOLUTION OF THE POLYNOMIAL EQUATION

K. W. Ellenderger

Missile Division, North American Aviation, Downey, California

procedure ROOTPOL (n, a, L, F, u, v, CONV) ;

value $n, a, L, F$; integer $L, F, n$;

array $a, u, v, C O N V$;

comment The Bairstow and Newton correction formulae are used for a simultaneous linear and quadratic iterated synthetic division. The coefficients of a polynomial of degree $n$ are given as $a_{1}(i=0, i, \ldots, n)$ where $a_{n}$ is the constant term. The coefficients are scaled by dividing them by their geometric mean: The Bairstow or Newton iteration method will nearly always converge to the number of figures carried, F, either to root values or to their reciprocals. If the simultaneous Newton and Bairstow iteration fails to converge on root values or their reciprocals in $\mathbf{L}$ iterations, the convergence requirement will be successively reduced by one decimal figure. This program anticipates and protects against loss of significance in the quadratic synthetic division. (Refer to "On Programming the Numerical Solution of Polynomial Equations," by $\mathbf{K}$. W. Ellenberger, Commun. ACM $\$$ (Dec. 1960), 644-647.) The real and imaginary part of each root is stated as $u[i]$ and $v[i]$, respectively, together with the corresponding constant, CONV $i$, used in the convergence test. This program has been used successfully for over a year on the Bendix G15-D (Intercard System) and has recently been coded for the IBM 709 (Fortran System);

begin integer $i, j, m$; $\operatorname{array} h, b, c, d, e[-2: n]$; real $t, K, p s, q s, p t, q t, s, r e v, r$;

ROOTPOL: $\quad b_{-1}:=b_{-2}:=c_{-1}:=c_{-2}:=d_{-1}:=d_{-2}:=e_{-1}:=$ $\mathbf{e}_{-2}:=0$;

for $j:=0$ step 1 until $n$ do $h_{j}:=a_{j} ; t:=1$; $\mathrm{K}:=10^{\mathrm{p}}$;
ZROTEST: if $\mathrm{h}_{\mathrm{n}}=0$ then

begin $u_{n}:=0 ; v_{a}:=0 ; \operatorname{CONV}_{a}:=K$; $\mathbf{n}:=\mathbf{n}-\mathbf{1} ;$ go to ZROTEST

end ;

INIT: if $\mathrm{n}=0$ then go to RETURN ;

ps $:=$ qs $:=$ pt $:=$ qt $:=\mathrm{s}:=0$;

rev $:=1 ; \mathrm{K}:=10^{\mathrm{F}}$;

if $n=1$ then

begin $r:=-h_{1} / h_{0} ;$ go to LINEAR

end ;

for $j:=0$ otep 1 until $n$ do

begin

if $h_{j}=0$ then $s:=s$ elses $s:=s+\log (a b s(h j))$

end $; \mathrm{s}:=\mathrm{s}^{10}$;

for $j:=0$ step 1 until $n$ do $h_{j}:=h_{j} / 8$;

if abs $\left(h_{1} / h_{0}\right)<a b s\left(h_{n-1} / h_{n}\right)$ then

REVERSE: begin $t:=-t ; m:=$ entier $((n+1) / 2)$;

for $j:=0$ step 1 until $m$ do

begin $8:=h_{\mathfrak{l}} ; h_{\jmath}:=h_{n-j} ; j_{n-j}:=8$

end

end ;

if $q \mathrm{qs} \neq 0$ then

begin $p:=p s ; q:=q s ;$ go to ITERATE

end ;

if $h_{n-2}=0$ then

begin $q:=1 ; p:=-2$

end else

begin $q:=h / h_{n-2} ; p:=\left(h_{n-1}-q \times h_{n-1}\right) / h_{n-1}$ end ;

if $\mathbf{n}=\mathbf{2}$ then go to QADRTIC ; $\mathbf{r}:=0$;

ITERATE: for $i:=1$ step 1 until $L$ do begin

BAIRSTOW: for $\mathrm{j}:=0$ step 1 until $n$ do begin $b_{j}:=h_{j}-p \times b_{j-1}-q \times b_{j-2} ;$

$c_{j}:=b_{j}-p \times c_{j}-1-q \times c_{j-2}$

end ;

if $n_{n-1}=0$ then go to BNTEST

if $b_{n-1}=0$ then go to BNTEST;

if abs $\left(h_{n-1} / b_{n-1}\right)<K$ then go NEWTON ;

$b_{a}:=h_{0}-q \times b_{n-2} ;$

BNTEST:

if $b_{n}=0$ then go to QADRTIC ;

if $K<$ abs $\left(h_{n} / b_{n}\right)$ then go to QADRTIC ;

NEWTON: for $j:=0$ step 1 until $n$ do

begin $d_{j}:=h_{j}+r \times d_{j-1} ; e_{j}:=d_{j}+r \times e_{j-1}$ end ;

if $d_{n}=0$ then go to LINEAR ;

if $K<$ abs $\left(h_{b} / d_{n}\right)$ then go to LINEAR ;

$c_{n-1}:=-p \times c_{n-2}-q \times c_{n-2}$;

$s:=c_{n-2}^{2}-c_{n-1} \times c_{n-8}$;

if $s=0$ then

begin $p:=1 p-2 ; q:=q \times(q+1)$

end else

begin $p:=p+\left(b_{n-1} \times c_{n-2}-b_{n} \times c_{n-2}\right) / s ;$

$q:=q+\left(-b_{n-1} \times c_{n-1}+b_{n} \times c_{n-2}\right) / 8$

end ;

if $e_{n-1}=0$ then $r:=r-1$ else $r:=r-d_{n} / e_{n-1}$

end $;$ ps $:=$ pt ; qs $:=$ qt $;$ pt $:=$ p ;

qt $:=q$;

if rev $<0$ then $K:=K / 10 ; r e v=-r e v ;$

go to REVERSE ;

LINEAR: if $\mathrm{t}<0$ then $\mathrm{r}:=1 / \mathrm{r} ; \mathrm{u}_{\mathrm{n}}:=\mathrm{r} ; \mathrm{v}_{\mathrm{a}}:=0$;

$\operatorname{CONV}_{\mathrm{a}}:=\mathrm{K} ; \mathrm{n}:=\mathrm{n}-1$;

for $j:=0$ step 1 until $n$ do $h_{j}:=d_{\mathfrak{l}}$;

if $n=0$ then go to RETURN ;

go to BAIRSTOW ;

QADRTIC: if $\mathrm{t}<0$ then

begin $p:=p / q ; q:=1 / q$

end ;

if $0<\left(q-(p / 2)^{2}\right)$ then 
RETURN: ond

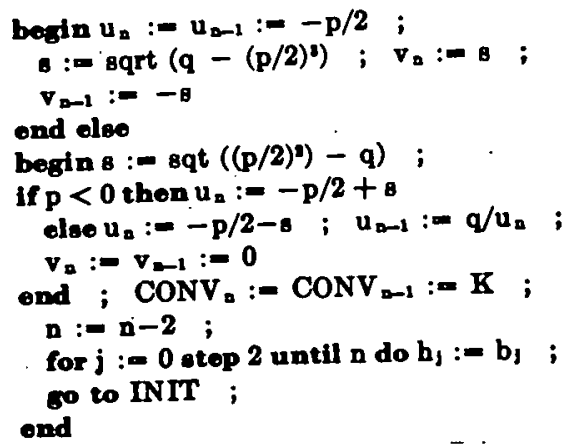

CERTIFICATION OF ALGORITHM 30

ILMERICAL SOLUTION OF THE POLYNOMIAL EQUATION (K. W. Ellenberger, Comm. ACM, Dec. 1960)

William J. Alexander

Argonne National Laboratory, Argonne, Ill.

ROOTPOL was coded by hand for the LGP 30 using the ACT-III Compiler with 24 bits of significance. The following corrections were found necessary.

(a) $b_{-1}:-b_{-2}:=c_{-1}:=c_{-2}:=d_{-1}:=d_{-2}:=e_{-1}:=\theta_{-1}:=0$ should be

$b_{-1}:=b_{-2}:=c_{-1}:=c_{-1}:=d_{-1}:=e_{-1}:=h_{-1}:=0$

(b) $\mathrm{m}:=$ entier $((\mathrm{n}+1) / 2)$ should be

$m:=$ entier $((n-1) / 2)$

(c) $j_{0-1}:=8$ should be $h_{n-1}:=s$

(d) $q:=h / h_{a-2}$ should be $h_{a} / h_{\mathrm{a}-s}$

(c) $c j:=b_{j}-p \times c_{j}-1-q \times c_{j-2}$ should be

$c_{j}:=b_{j}-p \times c_{j-1}-q \times c_{j-1}$

(f) If $n_{n-1}=0$ then go to BNTEST should be

if $h_{a-1}=0$ then go to BNTEBT

(g) $8:=$ sqrt $\left(\mathrm{q}-(\mathrm{p} / 2)^{2}\right)$ should be

B : $=$ eqrt $\left(q-(p / 2)^{2}\right)$

(h) for $j:=0$ step 2 until $n$ do $b_{j}:=b_{j}$ should be for $j:=0$ step 1 until $n$ do $h_{j}:-b_{j}$

(i) go to BAIRSTOW should be go to ITERATE

The following correction was found necessary in the given example (Refer to "On Programming the Numerical Solution of Polynomial Equations," by K. W. Ellenberger, Comm. ACM s, Dec., 1830) :

$f(x)=(.10088) 10^{8} x^{4}-(.08913) 10^{6} x^{2}+(.10000) 10^{4} x+$ $(.10000) 10^{1}=0$ should be

$f(x)=(.10098) 10^{2} x^{4}-(.98913) 10^{6} x^{2}-(.10990) 10^{4} x^{2}+$ $(.10000) 10^{8} x+(.10000) 10^{1}=0$

With these corrections the results obtained agree with those given in the example.

For equations of higher order it was found necessary to avoid repeated scaling of the reduced equation in order to prevent floating point overflow. The range on the exponent in the ACT III system in $-32 \leqq e \leqq 31$.

Further floating point overflow difficulties were experienced when certain coefficients in the reduced equation became small but not zero. The following additions were made to avoid this fault:

(a) for $\mathrm{j}:=0$ step 1 until $\mathrm{n}$ do $\mathrm{h}_{\mathrm{j}}:=\mathrm{d}_{\mathrm{j}}$ was replaced by for $j:=0$ step 1 until $n$ do begin if sbs $\left(h_{j} / d_{j}\right)<K$ then $h_{j}:=d_{j}$ else $h_{j}:=0$ end

(b) for $j:=0$ step 1 until $n$ do $h_{j}:=b_{j}$ was replaced by for $j:=0$ step 1 until $n$ do begin if abs $\left(h_{j} / h_{j}\right)<K$ then $h_{j}:=b_{j}$ else $h_{j}:=0$ end
With the above changes the following results were obtained:

$$
\begin{gathered}
x^{4}-3 x^{3}+20 x^{2}+44 x+54=0 \\
x=-.9706390 \pm 1.005808 i \\
x=2.470639 \pm 4.640533 i \\
x^{6}-2 x^{5}+2 x^{4}+x^{2}+6 x^{2}-6 x+8=0 \\
x=-.9999999 \pm .9999999 i \\
x=1.500000 \pm 1.322876 i \\
x=.5000002 \pm .8660251 i \\
x^{5}+x^{4}-8 x^{8}-16 x^{2}+7 x+15=0 \\
x=3.000001 \\
x=-2.000000 \pm 1.000001 i \\
x=-.9999997 \\
x=.9999998
\end{gathered}
$$

\section{CERTIFICATION OF ALGORITHM 30}

NUMERICAL SOLUTION OF THE POLYNOMIAL

EQUATION [K. W. Ellenberger, Comm. ACM 3

(Dec. 1960), as corrected in the previous Certification

by William J. Alexander, Comm. ACM \& (May 1961)]

Kalman J. Cohen

-Graduate-School-of--Industrial-Administration;-Carnegie-

Institute of Technology, Pittsburgh, Pa.

The ROOTPOL procedure originally published by Ellenberger as corrected and modified by Alexander was coded for the Bendix G20 in 20-GATE. Some serious errors were found in the third and fourth lines above the statement labelled "REVERSE" in Ellenberger's Algorithm which were not mentioned in Alexander's Certification. First, the function " $\log ^{\prime}$ " is not a standard function in Algol 60; it is clear from the context, however, that Ellenberger intends this to be the logarithm function to the base 10. Second, Ellenberger's Algorithm failed to divide the accumulated sum of the logarithms by $n+1$ before taking the antilogarithm.

The correct, and slightly simplified, manner in which the third and fourth lines above the statement labelled "REVERSE" should read is:

if $h_{j} \not 0$ then $s:=\ln \left(\operatorname{abs}\left(h_{j}\right)\right)$

end; $s:=s /(n+1) ; s:=\exp (s)$

With these corrections, the numerical results obtained essentially agree with those reported by Alexander.

\section{ALGORITHM 31}

\section{GAMMA FUNCTION}

Robert M. Collinge

Burroughs Corporation, Pasadena, California

real procedure Gamma $(x)$; real $x$;

comment For $\mathrm{x}$ in the range $2 \leq \mathrm{x} \leq 3$ an approximating poly. nomial is used. In this range the maximum absolute error $\epsilon(x)$ is $|c(x)|<0.25 \times 10^{-7}$. For $x>3$ we write $\Gamma(x)=(x-1)(x-2)$ $\ldots(x-n) \Gamma(x-n)$ where $2 \leq(x-n) \leq 3$, and for $x<2$ we write $\Gamma(x)=\frac{\Gamma(x+n)}{x(x+1) \ldots(x+n-1)}$ where $2 \leq(x-n) \leq 3$. For $x=0$ or a negative integer $\Gamma(x)$ is set eaual io a large value $10^{50}$

begin

real $h, y$;

$\mathrm{h}:=1.0 ; \mathrm{y}:=\mathrm{x}$

Al: if $y=0$ then $h:=10^{50}$

else if $\mathrm{y}=2.0$ then go to $\mathrm{A} 2$

else if $\mathrm{y}<2.0$ then begin

$\mathrm{h}:=\mathrm{h} / \mathrm{y} ; \mathrm{y}:=\mathrm{y}+1.0 ;$ go to $\mathrm{Al}$ end 
else if $y \geq 3.0$ then begin

$\mathrm{y}:=\mathrm{y}-1.0 ; \mathrm{h}:=\mathrm{h} \times \mathrm{y} ;$ go to $\mathrm{Al}$ end

else begin $y:=y-2.0$

$\mathrm{h}:=((((((.0016063118 \times \mathrm{y}+.0051589951) \times \mathrm{y}$

$+.0044511400) \times y+.0721101567) \times y$

$+.0821117404) \times y+.4117741955) \times y$

$+.4227874605) \times y+.9999999758) \times h$ end;

A2: Gamma : $=\mathrm{h}$ end Gamma.

CERTIFICATION OF ALGORI'THM 31

GAMMA FUNCTION [R. M. Collinge, Comm. ACM,

Feb. 61]

Peter G. Behrenz

Mathematikmạkinnämnden, Stockholm, Sweden

GAMMA was successfully run on FACIT EDB using FAcTTAlool 1, which is a realization of Alool 60 for FACIT EDB. No changes in the program were necessary. The relative error was as stated in the comment of GAMMA about $10^{\circ}$

\section{ALGORITHM 32}

MULTINT

R. Don Freeman JR.

Michigan State University, East Lansing, Michigan

real procedure MULTINT (n, Low, Upp, Funev, s, P, u, w); value $n$;

real procedure Low, Upp, Funev; array s, u, $w$; integer $n$;

comment MULTINT will perform a single, double, triple,...,

$T$-order integration depending on whether $n=1,2, \ldots, T$. The result is:

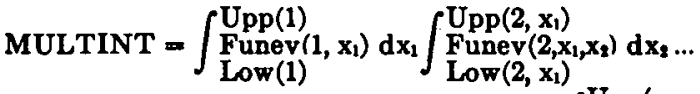

$$
\begin{aligned}
& \text { Upp }\left(n, x_{1}, \ldots, x_{n-1}\right) \\
& \int \underset{\operatorname{Low}\left(n, x_{1}, \ldots, x_{n-1}\right)}{\operatorname{Fun}\left(x_{1}, \ldots, x_{0}\right)} d x_{n}
\end{aligned}
$$

The variable of integration is $x[j] . \quad j=1$ refers to the outermost integral, $j=n$, the innermost integral. The code divides each interval equally into $s[j]$ subintervals and performs a $P$-point Gaussian integration on each subinterval with weight functions $w[k[j]]$ and abscissas $u[k[j]] . P$ is the size of the arrays of weight functions and abscissas and must be provided by the main code along with these arrays.

Since the values $x[1], x[2], \ldots, x[n]$, are stored in an array, sa are $a, b, c, d, r$, it is necessary to substitute an integer for the upper bound $T$ of these arrays before the program is executed. This means, for example, if 3 is substituted for $T$, then the procedure will not do a 4th order integral unless it is retranslated with $T \geq 4$.

The values of the lower and upper bounds and functions must of course be specified at the time of use. If each of these constituted a separate procedure, it would require writing and translating $3 \mathrm{n}$ different procedures. This is eliminated by grouping them into Low, Upp, and Funev which compute the lower and upper bounds and value of the functions respectively in each of the jth integrals. Since these are each essentially a collection of "subprocedures," the first statement of each should be a switch directing the code to the "subprocedure" which is used in the jth integral. Note that, for example, $\operatorname{Low}(3, x)$ is formally a function of $x[1], x[2], \ldots, x[T]$; this is done merely because it is more convenient to make $\operatorname{Low}(j, x)$ formally a function of the whole array $x$ for all $j$. Actually of course $\operatorname{Low}(3, x)$ would be a function of $x[1]$ and $x[2]$ only; begin real array $a, b, c, d, r, x[1: T]$;

integer array $k, h[1: T]$; real $f$; integer $j, m$;

for $j:=1$ step 1 until $T$ do

$\mathrm{m}:=1$ $\mathrm{x}[\mathrm{j}]:=\mathbf{0 . 0}$

$\mathrm{r}[\mathrm{n}+1]:=\mathrm{d}[\mathrm{n}+1]:=1.0$;

setup: for $j:=m$ step 1 until $n$ do

begin

$$
\mathbf{a}[\mathrm{j}]:=\operatorname{Low}(j, x) \text {; }
$$

$\mathrm{b}[\mathrm{j}]:=\operatorname{Upp}(\mathbf{j}, \mathbf{x})$;

$d[j]:=(b[j]-a(j]) / s[j] ;$

$\mathrm{c}[\mathrm{j}]:=\mathrm{a}[\mathrm{j}]+0.5 \times \mathrm{d}[\mathrm{j}]$

$x[j]:=c[j]+0.5 \times d[j] \times u[1]$;

$r[j]:=0.0$;

$h[j]:=k[j]:=1$; end;

$$
\mathbf{j}:=\mathbf{n} \text {; }
$$

sum: $\quad f:=$ Funev $(j, x)$;

$r[j]:=r[j]+r[j+1] \times d[j+1] \times f \times w[k[j] \mid ;$

if $(k[j]<P)$ then go to labk;

if $(h[j]<s[j])$ then go to labh;

$j:=j-1$;

if $(j=0)$ then go to exit;

go to sum:

labb: $\quad h[j]:=h[j]+1$;

$c[j]:=a[j]+(b[j]-0.5) \times d[j] ;$

$k[j]:=1$

go to initalx;

labk: $\quad k[j]:=k[j]+1$;

initalx: $\quad x[j]:=c[j]+0.5 \times d[j] \times u[k[j]]$;

if $(j=n)$ then go to sum;

$\mathrm{m}:=\mathrm{j}+\mathbf{1}$;

go to setup;

exit: MLLTINT : $=r[1] \times \mathrm{d}[1] \times 0.5 \uparrow \mathrm{n} ;$ end

\section{CERTIFICATION OF ALGORITHM 32}

MULTINT [R. Don Freeman, Comm. ACM, Feb. 1961]

Henry C. Thacher, JR.*

Reactor Engineering Div., Argonne National Laboratory, Argonne, Ill.

- Work supported by the U. S. Atomic Energy Commission.

The procedure was transcribed into the ACT-III language for the LGP-30 computer, and was tested on the integrals:

(1) $\int_{0}^{1} \int_{0}^{1} \int_{0}^{1} \int_{0}^{1} k[\cos u-7 u \sin u$

$$
\left.-6 u^{2} \cos u+u^{2} \sin u\right] d w d x d y d z=\sin k
$$

where $u=k v 0 x y z$, and

(2)

$$
\begin{aligned}
\int_{0}^{1} \int_{0}^{\sqrt{1-x^{2}}} \int_{0}^{\sqrt{1-x^{2}-y^{2}}} & \frac{d z d y d x}{x^{2}+y^{2}+(z-k)^{2}} \\
& =\pi\left(2+\frac{1}{2}\left(\frac{1}{k}-k\right) \log \left|\frac{1+k}{1-k}\right|\right)
\end{aligned}
$$

The Alool procedures for the second integral are:

real procedure Low $(j, x)$;

Low := 0;

real procedure $U p p(j, x) ;$ comment $z \equiv x[3], \quad y \equiv x[2], \quad x \equiv$ $x(1)$;

begin

integer $i$; real lemp;

Lemp $:=1.0$;

for $i:=j-1$ step -1 until 1 do

temp $:=\operatorname{temp}-x[j] \times x[j]$

$U p p:=\operatorname{sqrt}(t e m p)$

end; 
real procedure Funev $(j, x)$;

comment The real parameter $k$ is global;

Punev : $=$ if $j<3$ then 1.0 else $1 /(x[1] \times x[1]+x[2] \times x[2]+(x[3]-k)$ $\uparrow 2)$;

The first integral was tested only with $8[j]=1$, and with various Gaussian formulas for integrals over the interval $(-1,+1)$. Hesults were as follows:

\begin{tabular}{clrlr} 
true & \multicolumn{1}{c}{$-\mathbf{1 . 0 0 0 0 0 0 0}$} & 0.0000000 & -1.0000000 & 0.0000000 \\
$p=2$ & 0.993704 & -0.0333603 & +0.020166 & 6.881490 \\
$p=3$ & 1.000032 & 0.0000848 & -1.061651 & -0.597419 \\
$p=4$ & 0.999999 & 0.0000001 & -0.998407 & +0.0027035 \\
$p=5$ & 1.000000 & -0.0000002 & -1.000028 & -0.0007857
\end{tabular}

For the second integral, two values of $s=8[1]=8[2]=8[3]$ were used, and two values of $p$. Results were as follows:

\begin{tabular}{ccccc}
$c$ & \multicolumn{2}{c}{$1 / 2$} & \multicolumn{2}{c}{2} \\
true & \multicolumn{1}{c}{11.46027376} & \multicolumn{2}{c}{1.10609687} \\
8 & 1 & 2 & 1 & 2 \\
$p=2$ & 5.454460 & 11.838651 & 1.0368770 & 1.1184305 \\
$p=3$ & 9.361666 & 12.408844 & 1.1343551 & 1.1094278
\end{tabular}

The effect of the pole at $(0,0, k)$ is obvious.

For the algorithm to run in any compiler, the semicolon following $x[T]$; in the fourth line above the end of the comment must be deleted. The array bounds on the arrays $r$ and $d$ must be increased to $[1: T+1]$.

For 8 system which permits variable array bound, the introduction of the integer $T$ appears superfluous. For such a syatem, $T$ may be replaced by $n$ throughout with a probable gain in efficiency. For most translators, the presence of undefined elements in an array will not cause difficulties, provided these elements do not appear in an expression before they are assigned a value. The statement "for $j:=1$ step 1 until $T$ do $x|j|:=0.0$;" is thus superfluous. The semicolon before the end which precedes the label "sum" also appesrs unnecessary.

In spite of these minor corrections, the algorithm appears to be extremely convenient for multiple quadratures over arbitrary regions using the Cartesian product of any explicit one-dimensional formula (and not merely a Gaussian formula) for integrating over the range $[-1,1]$. If endpoints are used in the formula, it will, of course, repeat the calculation for each section of the range.

\section{ALGORITHII 33}

\section{FACTORIAL}

\section{F. LIPP}

RCA Digital Computation and Simulation Group, Moorestown, New Jersey

\section{real procedure Factorial (n);} value $n$; integer $n$;

comment This procedure makes use of the implicitly defined recursive property of Algol to compute $n !$;

begin Factorial $:=$ if $n=0$ then 1 . else $n \times$ Factorial $(n-1)$ end
ALGORITHM 34

\section{GAMMA FUNCTION}

M. F. LIPP

RCA Digital Computation and Simulation Group, Moorestown, New Jersey

real procedure - Gamma $(x)$; real $x$;

comment This procedure generalizes the recursive factorial routine, finding $\Gamma(1+x)$ for reasonable values of $x$. Accuracy vanishes for large $x(|x|>10)$ and for negative $x$ with small fractional parts. For $x$ being a negative integer the impossible value zero is given;

begin test: if $x<0$ then go to minus else if $x<1$ then begin integer $i$; real $y$; array a $[1: 8]$

$\mathrm{a}[1]:=-.57719165$;

a $[2]:=.98820589 ;$ \& $[3]:=-.89705694$;

a $[4]:=.91820686$;

a $[5]:=-.75670408 ;$ a $[6]:=.48219939$;

a $[7]:=-.19352782$;

a $[8]:=.03586834 ; y:=$ a $[1]$;

for $i:=2$ step 1 until 8 do $y:=y \times x+a$ [i] ;

Gamma : $=y$ end hastings

else Gamma : $=x \times$ Gamma $(x-1)$; go to endgam; minus: if $x=-1$ then Gamma $:=0$ else

Gamma := Gamma $(x+1) / x$;

endgam : end gam

REMARK ON ALGORITHM 34

GAMMA FUNCTION [M. F. Lipp, Comm. ACM 4

(Feb. 1961)]

Margaret L. Johnson and Ward Sangren

Computer Applications, Inc., San Diego, Calif.

The coefficients used in the calculation of the Hasting's polynomial are used in reverse order. The algorithm should have

$\mathrm{a}[1]=-.19352782 ; \quad \mathrm{a}[2]=.48219939 ; \mathrm{a}[3]=-.75670408 ;$

$\mathrm{a}[4]=.91820686 ; \quad \mathrm{a}[5]=-.89705694 ; \quad \mathrm{a}[6]=.98820589 ;$

$\mathrm{a}[7]=-.57719165 ; \mathrm{a}[8]=1.0$;

$y=.03586834$

for $i:=1$ step 1 until 8 do $y:=y \times x+z[i]$;

Further, since Gamma $(x)=\Gamma(1+x)$, the divisor $x$ in the statement labeled minus should be $x+1$.

\section{ALGORITHM 35}

\section{SIEVE}

T. C. Woop

RCA Digital Computation and Simulation Group, Moorestown, New Jersey

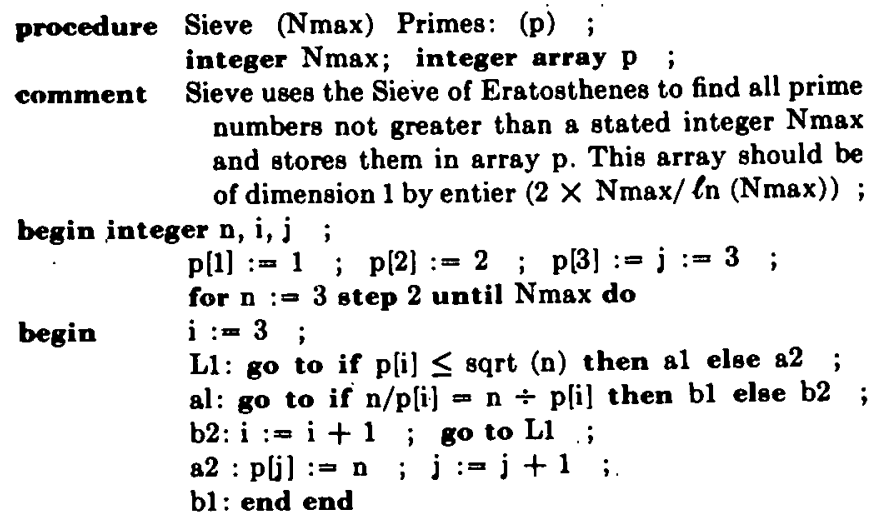
numbers not greater than a stated integer $\mathrm{Nmax}$ and stores them in array $p$. This array should be of dimension 1 by entier $(2 \times \mathrm{Nmax} / \ln (\mathrm{Nmax}))$;

begin integer $n, i, j$; $p[1]:=1 ; p[2]:=2 ; p[3]:=j:=3$; for $n:=3$ step 2 until Nmax do

begin $\quad i:=3$;

L1: go to if $p[i] \leq$ sqrt (n) then 81 else 82 ;

al: go to if $n / p[i]=n \div p[i]$ then b1 else b2 ; b2: $\mathrm{i}:=\mathrm{i}+1 ;$ go to $\mathrm{L} 1$; a2 $: p[j]:=n ; j:=j+1$;

b1 : end end 
$\therefore$ CERTIFICATION OF ALGORITHM 35

SIEVE (T. C. Wood, Comm. ACM, March 1961)

P. J. Brown

University of North Carolina, Chapel Hill, N. C.

SIEVE was transliterated into GAT for the UNIVAC 1105

and successfully run for a number of cases.

The statement:

go to if $n / p[i]=n \div p[i]$ then b1 else b2;

was changed to the statement:

go to if $n / p[i]-n \div p[i]<.5 / N \max$ then b1 else b2;

loundoff error might lead to the former giving undesired results.

\section{CERTIFICATION OF ALGORITHM 35}

SIEVE [T. C. Wood, Comm. ACM. Mar. 1961]

J. S. Hillmore

Elliott Bros. (London) Ltd., Borehamwood, Herts., England

The stutement:

go to if $n / p[i]=n \div p[i]$ then $b 1$ else $b 2$

was changed to the statement:

go to if $(n \div p[i]) \times p[i]=n$ then $b 1$ else $b 2$;

This avoids any inaccuracy that might result from introducing real arithmetic into the evaluation of the relation.

The modified algorithm was successfully run using the Elliott A LGol translator on the National-Elliot.t 803.

\section{ALGORITHM 36}

'TCHEBYCHEFF

\section{A. J. GIanNI}

IRCA Digital Computation and Simulation Group, Moorestown, New Jersey

procedure tchebycheff $(t, x, m, \ell)$;

real array $t, x$; integer $\ell, \mathrm{m}$;

comment given a set of $m+1$ values of $x$ stored in a onedimensional array whose subscripts run from 0 thru $m$ at least, construct $a$ table of $t_{n}(x), n=$ $0,1, \cdots, l$ and store it in the two-dimensional array $t$, where you find $t_{n}(x[m])$ as $t[n, m]$;

begin integer $\vec{i}, k, n$;

for $k:=0$ step 1 until $m$ do begin $t[0, k]:=1$; $t[1, k]:=x[k]$ end ;

for $n:=2$ step 1 until $\ell$ do for $i=0$ step 1 until $m$ do

end tcheby
ALGORITHM 37

TELESCOPE 1

K. A. Brons

RCA Advanced Programming Group, Pennsauken, N. J.

procedure Telescope $1(\mathrm{~N}, \mathrm{~L}$, eps, limit, c) ; value limit, $\mathrm{L}$; integer $N$; real $L$, eps, limit ; array $c$;

comment: Telescope 1 takes an $\mathrm{N}$ th degree polynomial approximation $\sum_{k=0}^{N} c_{k} x^{k}$ to a function which was valid to within eps $\geq 0$ over an interval $(0, L)$ and reduces it, if possible, to a polynomial of lower degree, valid to within limit $>0$. The initial coefficients $c_{k}$ are replaced by the final coefficients, and the deleted coefficients are replaced by zero. The initial eps is replaced by the final bound on the error. $\mathrm{N}$ is replaced by the degree of the reduced polynomial. $\mathbf{N}$ and eps must be variables.

This procedure computes the coefficients given in the Techniques Department of the ACM Communications, Vol. 1, No. 9, from the recursion formula

$$
8_{k-1}=-8_{k} \cdot \frac{k \cdot L \cdot(2 k-1)}{2(N+k-1) \cdot(N-k+1)} ;
$$

start:

begin integer $k$; array $d[0: N]$;

if $N<1$ then go to exit ; $d[N]:=-c[N]$; for $k:=N$ step -1 until 1 do

$\mathrm{d}[\mathbf{k}-1]:=-\mathrm{d}[\mathrm{k}] \times \mathbf{L} \times \mathbf{k} \times(\mathbf{k}-0.5) /$

$((\mathbf{N}+\mathbf{k}-1) \times(\mathbf{N}-\mathbf{k}+1))$;

if eps + abs $(d[0])<$ limit then

begin eps := eps + abs $(d[0])$;

for $k:=N$ step -1 until 0 doc $[k]:=c[k]+d[k]$; $N:=N-1$; go to start end ;

exit: end

\section{CERTIFICATION OH ALGORITHM, 37}

TELESCOPE 1 [K. A. Brons, Comm. ACM, Mar., 1961]

Henky C. Thacher, Jr.*

Reactor Engineering Div., Argonne National Lab., Argonne, Ill.

* Work supported by the U.S. Atomic Energy Commission.

The hody of Telescope 1 was compiled and tested on the LGP-30 using the ALGOL 60 translator system developed by the Dartmouth College Computer Center. No syntactical errors were found, and the program ran satisfactorily. The 10th degree polynomial obtained by truncating the exponential series was telescoped using $\lim =.1_{10}-2$ and $L=1.0$. The result was $N=3$, eps $=$ $.2103005_{10}-3$, and coefficients $+.9997892,-.9930727,+.4636493$, - .1026781. The error curve for the telescoped polynomial was computed for $x=0(.02) 1.0$. The error extrema were bounded by eps to within $0.5 \%$. The discrepancy is within the range of input conversion and round-off error.

\section{CERTIIICATION OF ALGORITHMI 37}

TELFSCOPE 1 [K. A. Brons, Comm. $A C M$, Mar. 1961] J.AMES F. BRIDGES

Ni Iichigan State University, East Lansing, Mich.

This procedure was tested on the CICC $160 \mathrm{~A}$, using $160 \mathrm{~A}$ ForTran. The 10th degree polynomial obtained by truncating the series $\exp (-x)$ was telescoped using $L=1$ and $\lim =0.001$. The result was $N=3$, eps $=0.21061862_{10}-3$ and coefficients $+0.99978965,-0.99307236,+0.46364955,-0.10267767$. The error curve was computed for $x=0(0.02) 1.0$ and no error exceeded eps, the worst error being $2 \%$ of eps less than eps. 
This result is in close agreement with that of Henry $C$. Thatcher, Jr. in his Certification (Comm. $A(C M$, Aug. 1962). Mr. Thatcher has pointed out that he inadvertantly referred to the series for $\exp (-x)$ as the "exponential series" thereby inferring the positive series $\exp (+x)$. There is also a typographical error in his eps. It should be $+0.2103505_{10}-3$.

\section{ALGORITHM 38 TELESCOPE 2}

\section{K. A. Brons}

RCA Advanced Programming, Pennsauken, N. J.

procedure Telescope 2 (N, I, eps, limit, c) ; value limit, L : integer $\mathbf{N}$; real $L$, eps, limit ; array c ; comment Telescope 2 takes an $\mathrm{N}$ th degree polynomial approximation $\sum_{k=0}^{N} c_{k} x^{k}$ to a function which was valid to within eps $\geq 0$ over an interval $(-L, L)$ and reduces it, if possible; to a polynomial of lower degree, valid to within limit $>0$. The initial coefficients $c_{k}$ are replaced by the final coefficients, and deleted coefficients are replaced by zero. The initial eps is replaced by the final bound on the error, and $N$ is replaced by the degree of the reduced polynomial. $\mathbf{N}$ and eps must be variables.

This procedure computes the coefficients given in the Techniques Department of the ACM Communicstions, Vol. 1; No. 9, from the recursion formula

$$
a_{k}=-a_{k} \frac{k \cdot L^{2}(k-1)}{(N+k-2) \cdot(N-k+2)} ;
$$

begin integer $k$; real 8 ; array $d[0:-N]$ : stsrt: if $N<2$ then go to exit; $d[N]:=-c[N]$; , for $k$ : $=\mathbf{N}$ step -2 until 2 do $\mathrm{d}[\mathrm{k}-2]:=-\mathrm{d}[\mathrm{k}] \times \mathrm{L} \uparrow 2 \times \mathrm{k} \times(\mathrm{k}-1) /$ $((\mathbf{N}+k-2) \times(N-k+2))$;

if $(N / 2)-$ entier $(N / 2)=0$ then $s:=d[0]$ else $\mathrm{s}:=\mathrm{d}[\mathbf{1}] / \mathrm{N}$;

If eps + abs(s) $<$ limit then begin eps : = eps + abs(s) ;

for $k$ :- $N$ step -2 until 0 do $c[k]:=c[k]+d[k]$;

exit:
ALGORITHM 39

CORRELATION COEFFICIENTS WITH MATRIX MULTIPLICATION

\section{Papken Sassouni}

Burroughs Corporation, Pasadena, California

procedure NÖRM (x) number of rows: (m) number of columns: (n) normalized output: (y) standard deviations: (8) ;

value $\mathrm{m}, \mathrm{n} ;$ integer $\mathrm{m}, \mathrm{n}$; $\operatorname{array} \mathrm{x}, \mathrm{y}, \mathrm{s}$;

comment Given an observation matrix [x] consisting of observations $x_{i j}$ on a population, NORM will calculate

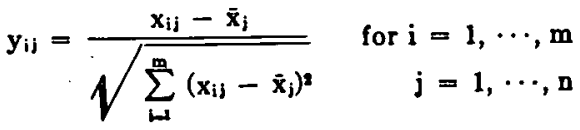

and the standard deviations

$$
s_{i}=\sqrt{\frac{\sum_{i=1}^{m}\left(x_{i i}-\ldots \bar{x}_{j}\right)^{2}}{m}}
$$

where $\bar{x}_{j}$ is the mean of observations on the $j$-th factor ;

begin integer $i, j$; real $r, b, c, b$; $r:=$ sqrt $(m)$; for $j:=1$ step 1 until $n$ do

1 : begin $\quad h:=0$;

for $i:=1$ step 1 until $m$ do

$\mathrm{h}:=\mathrm{h}+\mathrm{x}[\mathrm{i}, \mathrm{j}] ; \mathrm{h}:=\mathrm{h} / \mathrm{m} ; \mathrm{b}:=0$;

for $i^{i}:=1$ step 1 until $\mathrm{m}$ do

2: begin $\quad c:=\dot{x}[i, j]-b ; b:=b+c \uparrow 2 ; y[i, j]:=c$ end 2 . ;

b : = sqrt (b)

for $i:=1$ step 1 until $m$ do

$y[i, j]:=y[i, j] / b ; s[j]:=b / r$

end 1

end NORM ;

comment The normalization is now completed, and we are ready to compute the correlation matrix ;

procedure TRANSMULT. (y) number of rows: (m) number of columns: (n) symmetrical square matrix result: (z) ;

value $\mathrm{m}, \mathrm{n}$; integer $\mathbf{m}, \mathbf{n}$; array $\mathbf{y}, \mathbf{z}$;

comment This procedure multiplies two matrices, the first being the transpose of the second. The result is a symmetrical matrix with respect to the main diag. onal, therefore only the lower part of it, including the main diagonal, is computed. The upper half is obtained by equating corresponding elements;

begin integer $i, j, k$; real $h$;

for $j:=1$ step 1 until $n$ do

for $i:=j$ step 1 until $n$ do

hegin $\quad h:=0$;

for $k:=1$ step 1 until $\mathrm{m}$ do

$h:=h+y[k, i] \times y[k, j] ; z[i, j]:=h$;

if $i \neq j$ then $z[j, i]:=b$

end $i$

end TRANSMULT. [ $z]$ is the square matrix of the correlation coefficients of the initial observation matrix $[\mathbf{x}]$ 


\section{ALGORITHM 40}

\section{CRITICAL PATH SCHEDULING}

B. LEAVENWORTH

American Machine \& Foundry Co., Greenwich, Conn.

procedure CRITICALPATH (n, I, J, DIJ, ES, LS, EF, LF, TF FF) ;

integer $n$; integer array I, J, DIJ, ES, LS, EF, LF, TF, FF ;

comment: Given the total number of jobs $n$ of a project, the vector pair $I_{k}, J_{k}$ representing the $k$ th job, which is thought of as an arrow connecting event $I_{k}$ to event $J_{k}\left(I_{k}<J_{k}\right.$, $k=1 \cdots, n$ ), and a duration vector $(D I J)_{k}$, CRITICALPATH determines the earliest starting time (ES) $k$, latest starting time $(\mathrm{LS})_{k}$, earliest completion time $(\mathrm{LF})_{k}$, Iatest completion time $(L F)_{k}$, the total float $(T F)_{k}$, and the free float $(F F)_{k}$. $I_{1}$ must be 1 and the $I_{k}, J_{k}$ must be in ascending order. For example, if the first three jobs are labelled $(1,2)$, $(1,3),(3,4)$, then the $I, J$ vectors are $(1,1,3)$ and $(2,3,4)$ respectively. The critical path is given by each arrow whose total float is zero. The following non-local labels are used for exits: out1 $-I_{k}$ not less than $J_{k} ;$ out2 $-I_{k}$ out of sebegin quence ; out $3-I_{k}$ missing;

integer $k$, index, max, min ; integer array $t i$, te $[1: n]$; index $:=1$;

for $k:=1$ step 1 until $n$ do

begin

if $I[k] \geqq J[k]$ then go to outl ;

if $I[k]<$ index then go to out2;

if $I[k]>$ index $\wedge I[k] \neq$ index +1 then go to out3 ;

C: end ;

if $I[k]=$ index +1 then index $:=I[k]$

for $k:=1$ step 1 until $n$ do $\operatorname{ti}[k]:=t e[k]:=0$

for $k:=1$ step 1 until $n$ do

begin

$\max :=\mathrm{ti}[\mathbf{I}[\mathbf{k}]]+\mathrm{DIJ}[\mathbf{k}]$

if $\mathrm{ti}[\mathrm{J}[\mathrm{k}]]=0 \vee \mathrm{ti}[\mathrm{J}[\mathrm{k}]]<\max$ then

$\operatorname{ti}[\mathbf{J}[\mathbf{k}]]:=\max$

A : end ti ;

$\operatorname{te}[J[\mathbf{n}]]:=\operatorname{ti}[\mathrm{J}[\mathbf{n}]] ;$

begin

for $k:=n$ step -1 until 1 do

$\min :=\operatorname{te}[J[k]]-D I J[k]$

if $\operatorname{te}[\mathrm{I}[\mathrm{k}]]=0 \mathrm{~V} \operatorname{te}[\mathrm{I}[\mathrm{k}]]>\min$ then

B: end te ;

$\operatorname{te}[\mathbf{I}[\mathrm{k}]]:=\min$

for $k:=1$ step 1 until $n$ do

begin

ES[k]:= ti[I[k]] ;

$\mathbf{L S}[\mathbf{k}]:=\operatorname{te}[\mathbf{J}[\mathbf{k}]]-\mathrm{DIJ}[\mathbf{k}]$

$E F[k]:=\operatorname{ti}[\mathbf{I}[\mathbf{k}]]+$ DIJ[k] ;

$\mathbf{L F}[\mathbf{k}]:=$ te $[\mathbf{J}[\mathbf{k}]]$;

$\operatorname{TF}[k]:=\operatorname{te}[J[k]]-\operatorname{ti}[\mathbf{I}[\mathbf{k}]]-\mathrm{DIJ}[\mathbf{k}]$

end

$F F[k]:=t i[J[k]]-t i[I[k]]-D I J[k]$

end CRITICALPATH

\section{REFERENCES}

(1) James E. Kellet, Jr. and Morgan R. WAlker, "CriticalPath Planning and Scheduling," 1959 Proceedings of the Eastern Joint Computer Conference.

(2) M. C. Frishberg, "Least Cost Estimating and Scheduling - Scheduling Phase Only," IBM 650 Program Library File No. 10.3.005.
CERTIFICATION OF ALGORITHM 40

CRITICAL PATH SCHEDULING (B. Leavenworth, Comm. ACM, Mar. 1961)

Neal P. Alexander

Union Carbide Olefins Company, South Charleston, West Virginia

The Critical Path Scheduling algorithm was coded in Fortran for the IBM 7070. The following changes were made:

(a) ti $[\mathrm{k}]:=$ te $[\mathrm{k}]:=0$;

should be

ti $[\mathbf{k}]:=0$;

te $[k]:=9999$

(b) if te $[\mathrm{I}[\mathrm{k}]]=0 \mathrm{~V}$ te $[\mathrm{I}[\mathrm{k}]]>$ min then

should be if te $[I[k]]>\min$ then

This change permits a value of 0 to be calculated for te $[I[k]]$ and remain as the minimum value.

In the statement if $\mathrm{ti}[\mathrm{J}[\mathrm{k}]]=0 \mathrm{Vti}[\mathrm{J}[\mathrm{k}]]<\max$ then

the part of the statement "ti $[J[k]]=0$ " is redundant and can be omitted.

\section{CERTIFICATION OF ALGORITHM 40}

CRI'TICAL PATH SCHEDULING [B. Leavenworth, Comm. ACM 4 (Mar. 1961), 152; 4 (Sep. 1961), 392; 5 (Oct. 1962), 513]

Irvin A. Hoffman (Reed 7 Feb. 1964)

Woodward Governor Co., Rockford, Ill.

The Critical Path Scheduling algorithm was coded in FAST for the NCR315. The modifications suggested by Alexander [Comm. $A C M 4$ (Sept. 1961)] were included. Results were correct in all tested cases. However, the example of the $I, J$ vectors given in the comment is incorrect, as it would cause the exit out $3-I_{k}$ missing.

[Eviton's Note. There are also two semicolons which should be removed from the comment of Algorithm 40.-G.E.F.]

\section{CERTIFICATION OF ALGORITHM 40}

CRITICAL PATH SCHEDULING [B. Leavenworth,

Comm. ACM (Mar. 1961)]

Lars Hellberg

Facit Electronics AB, Solna, Sweden.

The Critical Path Scheduling algorithm was transliterated into Facit-Alool-1 and tested on the FAcIT EDB. The modifications suggested by Alexander [Comm. ACM (Sept. 1961)] were included. Results were correct in all tested schedules.

\section{ALGORITHM 41}

EVALUATION OF DETERMINANT

JOSEF G. Solomon

RCA Digital Computation and Simulation Group, Moorestown, New Jersey

real procedure Determinant $(A, n)$;

real array $A$; integer $n$;

comment This procedure evaluates a determinant by triangularization; 


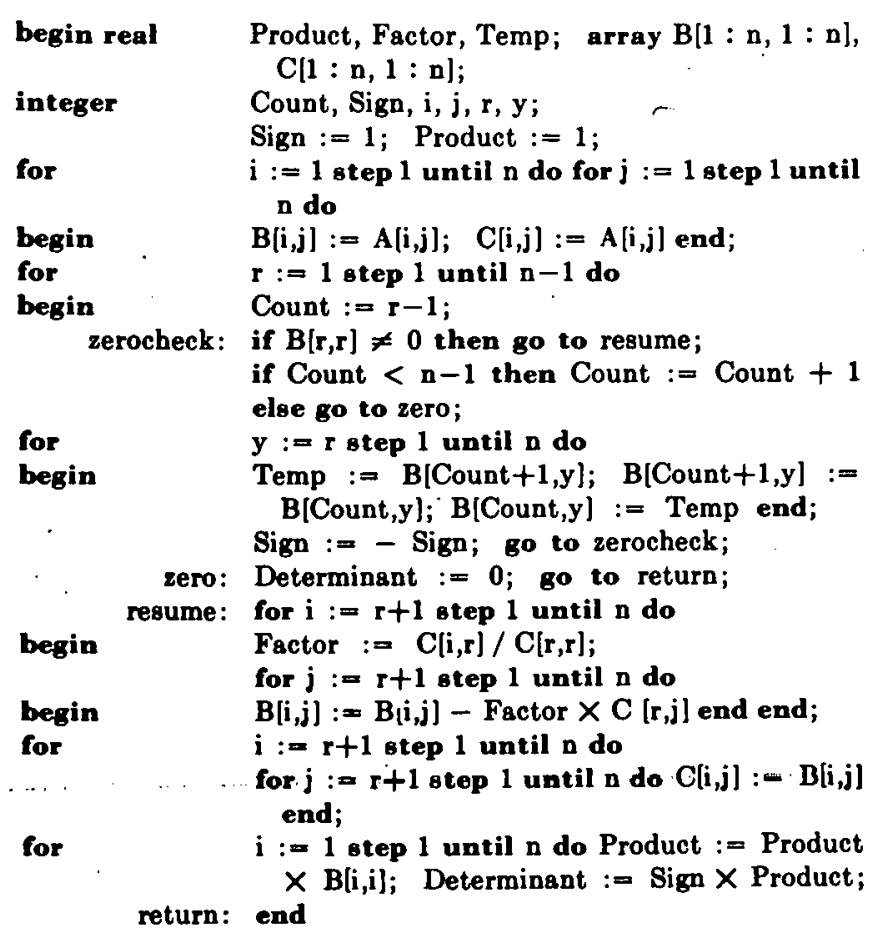

\section{CERTIFICATION OF ALGORITHM 41}

EVALUATION OF DETERMINANT [Josef G. Solomon, RCA Digital Computation and Simulation Group, Moorestown, N. J.]

Bruce H. Freed

Dartmouth College, Hanover, N. H.

When Algorithm 41 was translated into Scalp for running on the LGP-30, the following corrections were found necessary:

1. In the " $y$ " loop after " $B[$ Count, $y]:=T e m p$ " and before the

"end" insert

"Temp := C[Count+1,y];

$C[$ Count $+1, y]:=C[$ Count, $y]$;

$C[$ Count,y $]:=$ Temp"

2. "Sign" is an Alaol word when uncapitalized. However, many systems (if not all) do not recognize the difference between small and capital letters. For this reason "Sign" was changed to "ssign" for the LGP-30 run (and in the revision which follows later).

The following addition might be made in the specification as a concession to efficiency: "value $A, n$;".

The following changes might be made to make the Algorithm less wordy:

1. for "Ssign $:=1$; Product $:=1 ;$ "

put "Ssign $:=$ Product $:=1 ;$;"

2. for "begin $B[i, j]:=A[i, j] ; \quad C[i, j]:=A[i, j]$ end;" put " $B[i, j]:=C[i, j]:=A[i, j] ; "$

3. for "begin $B[i, j]:=B[i, j]-$ Factor $\times C[r, j]$ end end;" put " $B[i, j]:=B[i, j]-F a c t o r \times C[r, j]$ end;"

The above corrections and changes were made and the program was run with the correct results, as follows:

$$
\begin{gathered}
A=\left(\begin{array}{rrr}
10.96597 & 35.10765 & 96.72356 \\
2.35765 & -84.11256 & .87932 \\
18.24689 & 22.13579 & 1.11123
\end{array}\right) \\
\text { Determinant }=.15273131006
\end{gathered}
$$

Hand calculation on a desk calculator gives the value of the determinant for the above matrix as 152,731.3600.
$A=\left(\begin{array}{rrrr}1.0 & 3.0 & 3.0 & 1.0 \\ 1.0 & 4.0 & 6.0 & 4.0 \\ 1.0 & 5.0 & 10.0 & 10.0 \\ 1.0 & 6.0 & 15.0 & 20.0\end{array}\right) \quad$ Determinant $=.9999999_{10}+00$

The above matrix, being a finite segment of Pascal's triangle, has determinant equal to 1.00000000 .

$$
A=\left(\begin{array}{lll}
0.0 & 0.0 & 0.0 \\
5.0 & 9.0 & 2.0 \\
7.0 & 5.0 & 4.0
\end{array}\right) \quad \text { Determinant }=.000000010+00
$$

This is, of course, exactly correct.

Finally, one major change can be made which does away with several instructions and reduces variable storage requirements by $n^{2}$. This change is the complete removal of matrix $C$ from the program. It is extraneous.

The revised Algorithm was translated into SCALP and run on the LGP-30 with exactly the same results as above.

The revised Algorithm 41 follows.

\section{ALGORITHM 41, REVISION}

EVALUATION OF DETERMINANT [Josef G. Solomon, RCA Digital Computation and Simulation Group, Moorestown, N. J.]

Bruce H. Freed

Dartmouth College, Hanover, N. H.

real procedure determinant $(a, n)$;

real array $a$; integer $n$; value $a, n$;

comment This procedure evaluates a determinant by triangularization;

begin real product, factor, temp;

array $b[1: n, 1: n]$;

integer count, ssign, $i, j, r, y$;

ssign $:=$ product $:=1$;

for $i:=1$ step 1 until $n$ do

for $j:=1$ step 1 until $n$ do

$b[i, j]:=a[i, j]$

for $r:=1$ step 1 until $n-1$ do

begin count $:=r-1$;

zerocheck: if $b[r, r] \neq 0$ then go to resume;

if count $<n-1$ then count := count +1 else go to zero;

for $y:=r$ step 1 until $n$ do

begin temp $:=b[$ count $+1, y]$;

$b[$ count $+1, y]:=b[$ count,$y]$;

$b[$ count, $y]:=$ temp end;

ssign := -ssign;

go to zerocheck;

zero: determinant $:=0$; go to return;

resume: for $i:=r+1$ step 1 until $n$ do

begin factor $:=b[i, r] / b[r, r]$;

for $j:=r+1$ step 1 until $n$ do

$b[i, j]:=b[i, j]-$ factor $\times b[r, j]$ end end

for $i:=1$ step 1 until $n$ do

product $:=$ product $\times b[i, i]$;

determinant $:=$ ssign $\times$ product;

return: end

\section{REMARK ON REVISION OF ALGORITHM 4}

EVALLATION OF DETERMINANT [Josef G. Solomon, Comm. A(M 4 (Apr. 1961), 176; Bruce H. Freed,

Comm. ACM 6 (Sept. 1963); 520]

Leo J. Rotexberg (Recd 7 Oct. 63)

Box 2400, 362 Memorial Dr., Cambridge, Mass.

While desk-checking the program an error was found. For $\mathrm{ex}$ ample, the algorithm as published would have calculated the valuc zero as the deterninant of the matrix 


$\left[\begin{array}{llll}0 & 0 & 0 & 1 \\ 0 & 0 & 1 & 0 \\ 0 & 1 & 0 & 0 \\ 1 & 0 & 0 & 0\end{array}\right]$

The error lies in the search for a nonzeru element in the rth column uf the matrix $b$.

Editor's . Note. Appirently the best general deterninant evaluators in this section are imbedded in the linear equation solvers Algorithm 43 [C'oum. A (:II \& (Apr. 1961), 176, 182; and 6 (Aug. 19(33), 445] and Algorithm 135 [Comm. ACM 5 (Nov. 1962), 553, 557]. They search each column for the largest pivot in absolute value. Algorithm 41 searches only for a nonzero pivot in each column, and will therefore fail for the natrix

$$
\left[\begin{array}{lll}
2^{-1} & 1 & 1 \\
1 & 1 & 2 \\
1 & 1 & 1
\end{array}\right]
$$

if $\iota \gg s$, for a machine with s-bit floating point.

It is hoped that soon a good determinant evaluator will be published to take the place of Algorithm +1.- (i. E. F.

\section{ALGORITHM 42 \\ INVERT}

T. C. Wood

RCA Digital Computation and Simulation Group, Moorestown, New Jersey

procedure Invert (A) order: (n) Singular: (s) Inverse: (A1); array $A, A l$; integer $n, s$, value $n$;

comment This procedure inverts the square matrix $A$ of order $\mathrm{n}$ by applying a series of elementary row operation to the matrix to reduce it to the identity matrix. These operations when applied to the identity matrix yield the inverse A1. The case of a singular matrix is indicated by the value $s:=1$;

begin comment augment matrix $A$ with identity matrix; array $a[1: n, 1: 2 \times n]$; integer $i, j$;

for $i^{*}:=1$ step 1 until $n$ do

for $j:=1$ step 1 until $2 \times n$ do

if $j \leqq n$ then $a[i, j]:=A[i, j]$ else

if $j=n+1$ then $a[i, j]:=1.0$ else $a[i, j]:=0.0$; comment begin inversion;

for $i:=1$ step 1 until $n$ do

begin integer $k, \ell$, ind; $j:=\ell:=i$; ind $:=\mathbf{s}:=0$;

L1: if $\mathrm{a}[\ell, \mathrm{j}]=0$ then

begin ind $:=1 ;$ if $\ell<\mathrm{n}$ then begin $\ell:=\ell+1$;

go to $L 1$ end

else begin $s:=1$; go to $\mathrm{L} 2$ end end;

if ind $=1$ then for $\mathrm{k}:=1$ step 1 until $2 \times \mathrm{n}$ do

begin real temp;

temp $:=\mathrm{a}[\ell, \mathrm{k}]$

$\mathrm{a}[\ell, \mathrm{k}]:=\mathrm{a}[\mathrm{i}, \mathrm{k}]$

$a[i, k]:=$ temp end $k$ loop;

for $k:=j$ step 1 until $2 \times n$ do $a[i, k]:=a[i, k] / a[i, j] ;$

for $\ell:=1$ step 1 until $n$ do

if $\ell \neq \mathrm{i}$ then for $\mathrm{k}:=1$ step 1 until $2 \times \mathrm{n}$ do $\mathrm{a}[\ell, \mathrm{k}]:=\mathrm{a}[\ell, \mathrm{k}]-\mathrm{a}[\mathrm{i}, \mathrm{k}] \times \mathrm{a}[\ell, \mathrm{j}]$

end $i$ loop;

for $i:=1$ step 1 until $n$ do

for $j:=1$ step 1 until $n$ do

L2: end of procedure
CERTIFICATION OF ALGORITHM 42

INVERT (T. C. Wood, Comm. ACM, Apr., 1961)

Anthony W. Knapp and Paul Shaman

Dartmouth College, Hanover, N. H.

INVERT was hand-coded for the LGP-30 using machine language and the 24.0 floating-point interpretive system, which carries 24 bits of significance for the fractional part of a number and five bits for the exponent. The following changes were found necessary:

(a) if $\mathrm{j}=\mathrm{n}+1$ then $\mathrm{a}[\mathrm{i}, \mathrm{j}]:=1.0$ else $\mathrm{a}[\mathrm{i}, \mathrm{j}]:=0.0$; should be

if $j=n+i$ then $a[i, j]:=1.0$ else $a[i, j]:=0.0$

(b) for $\mathrm{k}:=\mathrm{j}$ step 1 until $2 \times \mathrm{n}$ do

$\mathbf{u}[\mathrm{i}, \mathrm{k}]:=\mathbf{a}[\mathbf{i}, \mathbf{k}] / \mathbf{a}[\mathrm{i}, \mathrm{j}]$;

should be

for $k:=2 \times n$ step -1 until i do

$a[i, k]:=a[i, k] / a[i, i]$

(c) if $1 \neq \mathrm{i}$ then for $\mathrm{k}:=1$ step 1 until $2 \times \mathrm{n}$ do $a[l, k]:=a[l, k]-a[i, k] \times a[l, j]$

should be

if $l \neq i$ then for $k:=2 \times n$ step -1 until $i$ do $a[l, k]:=n[l, k]-a[i, k] \times a[l, i] ;$

Given these changes, $j$ becomes superfuous in the second $i$ loop, and the other references to $j$ may be changed to references to $i$.

INVERT obtained the following results:

The computer inverted a 17-by-17 matrix whose elements were integers less than ten in absolute value. When the matrix and its inverse were multiplied together, the largest nondiagonal element in the product was -.00003 . Most nondiagonal elements were less than .00001 in absolute value.

INVERT was tested using finite segments of the Hilbert matrix. The following results were obtained in the $4 \times 4$ case:

$\begin{array}{rrrr}16.005 & -120.052 & 240.125 & -140.082 \\ -120.052 & 1200.584 & -2701.407 & 1680.917 \\ 240.126 & -2701.411 & 6483.401 & -4202.217 \\ -140.082 & 1680.920 & -4202.219 & 2801.446\end{array}$

The exact inverse is:

$$
\begin{array}{rrrr}
16 & -120 & 240 & -140 \\
-120 & 1200 & -2700 & 1680 \\
240 & -2700 & 6480 & -4200 \\
-140 & 1680 & -4200 & 2800
\end{array}
$$

INVERT was also coded for the LGP-30 in machine language and the 24.1 extended range interpretive system. This system, which uses 30 significant bits for the fraction, obtained the following as the inverse of the $4 \times 4$ Hilbert matrix:

$\begin{array}{rrrr}16.000 & -120.001 & 240.001 & -140.001 \\ -120.001 & 1200.006 & -2700.015 & 1680.010 \\ 240.001 & -2700.016 & 6480.037 & -4200.024 \\ -140.001 & 1680.010 & -4200.024 & 2800.016\end{array}$

The program coded in the 24.0 interpretive system successfully inverted a matrix consisting of ones on the minor diagonal and zeros everywhere else.

\section{REMARKS ON ALGORITHM 42}

INVERT [T. C. Wood, Comm. ACM, Apr. 1961]

P. NAUR

Regnecentralen, Copenhagen, Denmark

INVERT cannot be recommended since it does not search for pivot and therefore will give poor accuracy. This is contirmed by the figures quoted by Knapp and Shaman in their certification [Comm. ACM \& (Nov. 1961), 498]. The results obtained by them 
using 30 significant bits for the fraction may be compared directly with those obtained using INVERSION II (Algorithm 120) and gjr with the Gier Alaol system (see certification below). Inverting the $4 \times 4$ segment of the Hilbert matrix, the largest error in any element is found to be:

INVERT (Knapp and Shaman) INVERSION II) (see certification of gjr $\quad$ Alg. 120)

$\begin{array}{cl}\text { Subscripts } & \text { Error } \\ 3,3 & \mathbf{0 . 0 3 7} \\ 3,3 & \mathbf{0 . 0 3 0 6} \\ 4,3 & 0.00010\end{array}$

In view of this basic shortcoming of Algorithm 42, it is unnecessary to report on other features of it.

CORRECTION TO EARLIER REMARKS ON ALGORITHM 42 INVERT, ALG. 107 GALSS'S IIETHOD, ALG. 120 INVERSION II, AND gjr [P. Naur, Comm. $A C M$, Jan. 1963, 38-40.]

P. Naur

Regnecentralen, Copenhagen, Denmark

George. Forsy the, Stanford University, in a private communi. cation has informed me of two major weaknesses in my remarks on the above algorithms:

1) The computed inverses of rounded Hilbert matrices are compared with the exact inverses of unrounded Hilbert matrices, instead of with very accurate inverses of the rounded Hilbert matrices.

2) In criticizing matrix'inversion procedures for not searching for pivot, the errors in inverting positive definite matrices cannot be used since pivot searching seems to make little difference with such matrices.

It is therefore clear that although the figures quoted in the earlier certification are correct as they stand, they do not substantiate the claims I have made for them.

To obtain a more valid criterion, without going into the considerable trouble of obtaining the very accurate inverses of the rounded Hilbert matrices, I have multiplied the calculated inverses by the original rounded matrices and compared the results with the unit matrix. The largest deviation was found as follows:

\begin{tabular}{cccc} 
& \multicolumn{3}{c}{ Maximim deviation from elements of the tnit matrix } \\
\cline { 2 - 4 } Order & INVERSION $I I$ & $g j$ & Ratic \\
2 & $-1.49_{10}-8$ & $-1.49_{10}-8$ & 1.0 \\
3 & $-4.77_{10}-7$ & $-8.34_{10}-7$ & 0.57 \\
4 & $-9.54_{10}-6$ & $-3.43_{10}-5$ & 0.28 \\
5 & $-7.32_{10}-4$ & $-4.58_{10}-4$ & 1.6 \\
6 & $-1.61_{10}-2$ & $-1.42_{10}-2$ & 1.1 \\
7 & $-5.78_{10}-1$ & $-5.47_{10}-1$ & 1.1 \\
8 & $-1.20_{10}-2$ & $-1.38_{10} 1$ & 8.7 \\
9 & $-4.91_{10} 1$ & $-2.22_{10} 1$ & 2.2
\end{tabular}

This criterion supports Forsythe's criticism. In fact, on the basis of this criterion no preference of INVERSION II or $g$ jr can be made.

The calculations were made in the Gier Algol system, which has floating numbers of 29 significant bits.
ALGORITHM 43

\section{CROUT WITH PIVOTING II}

Henry C. Thacher, JR.*

Argonne National Laboratory, Argonne, Illinois

real procedure INNERPRODUCT $(u, v)$ index : $(k)$ start : (s) finish : (f);

value $s, f$; integer $k, s, f$; real $u, v$;

comment INNERPRODUCT forms the sum of $u(k) \times v(k)$ for $k=s, s+1, \ldots$ f. If $s>f$, the value of INNERPRODUCT is zero. The substitution of a very accurate inner product procedure would make CROUT more accurate;

comment INNERPRODUCT may be declared in the head of any block which includes the block in which CROUT is de. clared. It may be used independently for forming the inner product of vectors;

begin

real $h$;

$\mathrm{h}:=0 ;$ for $\mathrm{k}:=\mathrm{s}$ step 1 until f do $\mathrm{h}:=\mathrm{h}+\mathrm{u} \times \mathrm{v}$; INNERPROIJUCT $:=\mathrm{h}$

end INNERPRODUCT;

procedure CROUT II (A, b, n, y, pivot, det, repeat)

comment This procedure is a revision of Algorithm 16, Crout With Pivoting by George E. Forsythe, Comm. ACM 3, (1960) 507-8. In addition to modifications to improve the running of the program, and to conform to proper usage, it provides for the computation of the determinant, det, of the matrix $A$. The solution is obtained by Crout's method with row interchanges, as formulated in reference [1], for solving $\mathrm{Ay}=\mathrm{b}$ and transforming the augmented matrix $[\mathrm{A}$ b] into its triangular decomposition $\mathrm{LU}$ with all $\mathrm{L}(\mathrm{k}, \mathrm{k})=1$. If $\mathrm{A}$ is singular we exit to 'singular,' a nonlocal label. pivot $(k)$ becomes the current row index of the pivot element in the $k$-th column. Thus enough information is preserved for the procedure to process a new right-hand side without repeating the triangularization, if the boolean parameter repeat is true. The accuracy obtainable from CROUT would be much increased by calling CROUT with a more accurate inner product procedure than INNERPRODUCT.

The contributions of Michael F. Lipp and George E. Forsythe by prepublication review and pointing out several errors are gratefully acknowledged;

comment Nonlocal identifiers appearing in this procedure are: (1) The nonlocal label 'singular', to which the procedure exits if $\operatorname{det} A=0$, and (2) the real procedure 'INNERPRODUCT' given above;

value $n$; array $A, b, y$; integer $n$; integer array pivot; real det; Boolean repeat;

begin

integer $k, i, j$, imax, $p$; real TEMP, quot;

$\operatorname{det}:=1$; if repeat then go to 6 ;

for $k:=1$ step 1 until $n$ do

1: begin

TEMP : = 0;

for $\mathrm{i}:=\mathrm{k}$ step 1 until $\mathrm{n}$ do

2: begin

$A[i, k]:=A[i, k]-$ INNERPROI)UCT $(A[i, p], A[p, k]$, $\mathrm{p}, \mathbf{1}, \mathbf{k}-\mathbf{1})$; if $\operatorname{abs}(\mathrm{A}[\mathrm{i}, \mathrm{k} \mid)>\mathrm{TEMP}$ then

3: begin

$\operatorname{TEMP}:=\operatorname{abs}(\mathrm{A}[\mathrm{i}, \mathrm{k}]) ; \quad \operatorname{imax}:=\mathrm{i}$

end 3

end 2;

pivot $[k]:=i \max$

comment We have found that $\mathrm{A}[\mathrm{imax}, \mathrm{k}]$ is the largest pivot in column k. Now we interchange rows $k$ and imax; 
begin

TEMP $:=A[k, j] ; A[k, j]:=A[i m a x, j] ; A[i \max , j]$ $:=\mathrm{TEMP}$

end 5 ;

TEMP $:=b[k] ; b[k]:=b[i m a x] ; b[i m a x]:=$ TEMP

end 4;

comment The row interchange is done. We proceed

to the elimination;

if $\mathrm{A}[\mathrm{k}, \mathrm{k}]=0$ then go to singular;

quot $:=1.0 / \mathrm{A}[\mathrm{k}, \mathrm{k}]$;

for $\mathrm{i}:=k+1$ step 1 until $n$ do

$\mathrm{A}[\mathrm{i}, \mathrm{k}]:=$ quot $\times \mathbf{A}[\mathbf{i}, \mathbf{k}]$;

for $\mathrm{j}:=\mathrm{k}+1$ step 1 until $\mathrm{n}$ do

$\mathrm{A}[\mathrm{k}, \mathrm{j}]:=\mathrm{A}[\mathrm{k}, \mathrm{j}]$ - INNERPRODUCT $(\mathrm{A}[\mathrm{k}, \mathrm{p}]$, $A[p, j], p, 1, k-1)$;

$b[k]:=b[k]^{*}-$ INNERPRODUCT $(A[k, p], b[p]$, p; i, k-1)

end 1 ; go to 7 ;

comment The triangular decomposition is now finished, and we skip to the back substitution;

6: begin comment This section is used when the formal parameter repeat is true, indicating that the matrix $\mathbf{A}$ has previously been decomposed into triangular form by CROUT II, with row interchanges specified by pivot, and that it is desired to solve the linear system with a new vector $b$, without repeating the triangularization; for $k:=1$ step 1 until $n$ do

begin

TEMP : = b $[$ pivot $[k]] ; \quad b[\operatorname{pivot}[k]]:=b|k| ; b[k]:=$ TEMP; $b[k]:=b[k]-$ INNERPRODUCT end 6 ; $(A[k, p], b[p], p, 1, k-1)$ end;

7: for $k:=n$ step -1 until 1 do

8: $\quad$ begin if $\neg$ repeat then $\operatorname{det}:=A[k, k] \times \operatorname{det}$;

$y[k]:=(b[k]$ - INNERPRODUCT $(A[k, p], y[p], p$, $\mathrm{k}+1, \mathrm{n}) / \mathrm{A}[\mathrm{k}, \mathrm{k}]$

end 8 ;

end CROUT II;

REFERENCE:

(1) J. H. Wilkinson, Theory and practice in linear systems. In John W. Carr III (editor), Application of Advanced Numerical Analysis to Digital Computers, pp. 43-100 (Lectures given at the University of Michigan, Summer 1958, College of Engineering, Engineering Summer Conferences, Ann Arbor, Michigan [1959]).

\section{CERTIFICATION OF ALGORITHM 43}

CROUT II (Henry C. Thacher, Jr., Comm. ACM, 1960)

Hentry C. Thacher, Jr.*

Argonne National Laboratory, Argonne, Illinois

CIROUT II was coded by hand for the Royal Precision LGP-30 computer, using a 28-bit mantisa floating point interpretive system ( 24.2 modified).

'The program was tested against the linear system:

$\mathrm{A}=\left(\begin{array}{rrrr}12.1719 & 27.3941 & 1.9827 & 7.3757 \\ 8.1163 & 23.3385 & 9.8397 & 4.9474 \\ 3.0706 & 13.54344 & 15.5973 & 7.5172 \\ 3.0581 & 3.1510 & 6.9841 & 13.1984\end{array}\right) \quad \mathrm{b}=\left(\begin{array}{l}6.6355 \\ 6.1304 \\ 4.6921 \\ 2.5393\end{array}\right)$

with the following results:
$\mathrm{A}^{\prime}=\left(\begin{array}{cccc}12.171900 & 27.394100 & 1.9827000 & 7.3756999 \\ 0.25226957 & 6.6327021 & 15.097125 & 5.6565352 \\ 0.25124262 & -0.56260107 & 14.979620 & 14.527683 \\ 0.66680633 & 0.76468695 & -0.20207132 & -1.3606142\end{array}\right)$

$b^{\prime}=\left(\begin{array}{c}6.6354999 \\ 3.0181653 \\ 2.5702026 \\ -0.082780734\end{array}\right) \quad$ pivot $=\left(\begin{array}{l}1 \\ 3 \\ 4 \\ 4\end{array}\right) \quad y=\left(\begin{array}{l}0.15929120 \\ 0.14691771 \\ 0.11257482 \\ 0.060840712\end{array}\right)$

$\operatorname{det}=-1645.4499$. All elements of $\mathrm{Ab}-\mathrm{y}$ were less than $10^{-7}$ in magnitude. Identical results were obtained with the same $b$, and repeat true. With the same $b$ and the last row vector of $A$ replaced by $(19.1927,33.4409,25.1298,5.2811)$, i.e. A $4, \mathrm{j}=\mathrm{A} 1 \mathrm{j}$, $+2 \mathrm{~A} 2, \mathrm{j}-3 \mathrm{~A} 3, \mathrm{j}$, the results were:

$\operatorname{det}=0.10924352 \times 10^{-3}$,

$\mathrm{y}=\left(0.29214425 \times 10^{8},-0.12131172 \times 10^{8}, 0.72411923 \times 10^{7}\right.$, $\left.-0.51018392 \times 10^{7}\right)$.

Failure to recognize this singular matrix is due to roundoff, either in the data input or in the calculation.

\section{CERTIFICATION OF ALGORITHM 43}

CROUT II [Henry Thacher, Jr., Comm. ACM (1960), 176]

C. Domingo and F. Rodriguez-GiL

Universidad Central, Caracas, Venezuela

CIROUT II was coded in PUC-R2 and tested in the IBM-1620. Two types of INNERPROIDUCT subruutines were used. The first one finds the scalar product in fixed-point arithmetic to increase accuracy, using an accumulator of 32 digits. The second one uses ordinary floting-point with eight significative figures.

Using a unit matrix as right-hand side, a $6 \times 6$ segment of $\mathrm{Hil}$ bert matrix was inverted. The inverse was inverted again.

The maximum difference between this result and the original segment of Hilbert matrix was:

I'sing fixed-point INNERPROIUUC. . . . . 8.2420 $\times 10^{-4}$

$\left(1\right.$ alue of determinant............... $\left.4.7737088 \times 10^{-18}\right)$ Using foating-point INNERPROI)UC .... 3.014016 $\times 10^{-2}$

( ialue of determinant . . . . . . . . . . $4.4950721 \times 10^{-18}$ )

Two typographical errors were observed in the algorithm:

The statement:

$$
b[k]:=g[k]-\text { INNERPRODUCT }(A[k, p], b[p], p, i, k-1)
$$

should be :

$$
b[k]:=b[k] \text { - INNERPRODỤCT }(A[k, p], b[p], 1, k-1)
$$

The statement:

$y[k]:=(b[k]-$ INNERPRODUCT $(A|k, p|, y[p], p, k+1, n) / A[k, k]$ should be:

$y[k]:=(b \mid k]-$ INNERPROI)UCT $(A[k, p], y[p], p, k+1, n)) / A[k, k]$

Storage may be saved eliminating the array $y$ and using instead the array $b$, in which the solution is formed.

A previous certification of this algorithm [Comm. ACM 4, + (Apr. 1961), 182] was tested again with the same results. Two errors were detected in the certification: The row that must replace the last row of $A$ in order to obtain a singular matrix must he : $\begin{array}{llll}19,1927 & 33.4409 & -251298 & -5.2811\end{array}$ 
ALGORITHM 44

BESSEL FUNCTIONS COMPUTED RECURSIVELY

Maria E. WoJcickI

RCA Digital Computation and Simulation Group, Moorestown, New Jersey

procedure Bessfr(N, FX, LX, Z) Result: (J, Y);

value $\mathbf{L X}, \mathbf{F X}, \mathrm{N}$;

real $F X, L X, Z$; real array $J, Y$; integer $N$;

comment Bessel Functions of the first and second kind, $J_{P}(X)$ and $Y_{P}(X)$, integral order $P$, are computed by recursion for volues of $X, F X \leqq X \leqq L X$, in steps of $Z$. The functions are computed for values of $P, \quad 0 \leqq P \leqq N$. M[SUB], the initial value of $P$ being chosen according to formulae in Erdelyi's Asymptotic Expansions. The computed values of $J_{P}(X)$ and $Y_{P}(X)$ are stored as column vectors for constant argument in matrices $J, Y$ of dimension $(N+1)$ by entier $((\mathrm{LX}-\mathrm{FX}) / \mathrm{Z}+1)$;

begin real PI, X, GAMMA, PAR, LAMDA, SUM, SUM1; integer $P, S U B$, MAXSUB;

\section{$P I:=3.14159265$}

GAMMA $:=.57721566$,

PAR. $: 63.0=1.5 \times \ln (2 \times P I)$;

MAXSUB := entier $((L X-F X) / Z)$;

begin real array JHAT $[0: N, 0:$ MAXSUB];

integer array $M[0$ :MAXSUB $]$;

SUB : $=0$

for $X:=F X$ step $Z$ until LX do

begin if $(X>0) \wedge(X<10)$ then $M[S U B]:=2 \times$ entier $(X)+9$ else

begin real $A L O G$;

ALOG : = $($ PAR $-1.5 \times \ln (\mathrm{X})) / \mathrm{X}$

$M[S U B]:=$ entier $(X \times(\exp (A L O G)+\exp$ $(-A L O G)) / 2$ ) end;

if $\mathrm{N}>\mathrm{M}$ [SUB] then

begin for $P:=M$ [SUB] +1 step 1 until $N$ do

$J[P, S U B]:=0$ end;

JHAT [M [SUB], SUB] := $10 \uparrow(-9)$;

comment Having set the uppermost $\mathrm{J}_{P}(X)$ to a very small number we are now going to compute all the $J_{P}(X)$ down to $\mathbf{P}=\mathbf{0}$;

i) for $P:=M$ [SUB] step -1 until 1 do

JHAT $[\mathrm{P}-1$, SUB] : $=2 \times \mathrm{P} / \mathrm{X} \times \mathrm{JHAT}[\mathrm{P}, \mathrm{SUB}]-\mathrm{JHAT}$

$[\mathrm{P}+1, \mathrm{SUB}]$;

SUM := SUM1 :=0;

for $P:=2$ step 2 until $(M[S U B] \div 2)$ do

SUM $:=$ SUM + JHAT $[P$, SUB $]$;

LAMDA : $=$ JHAT $[0$, SUB $]+2 \times$ SUM;

for $P:=0$ step 1 until $N$ do

$\mathrm{J}[\mathrm{P}, \mathrm{SUB}]:=$ JHAT $[\mathrm{P}, \mathrm{SUB}] /$ LAMDA;

comment $J_{P}(X)$ have been computed by use of $\hat{J}_{P}(X)$;

for $P:=2$ step 2 until (M [SUB] $\div 2$ ) do

SUM1 $:=$ SUM1 $+(-1) \times(-1) \uparrow \mathbf{P} \div \mathbf{J}[2 \times \mathbf{P}$, SUB $]$ $/ 2 / \mathrm{P}$

$\mathrm{Y}[0, \mathrm{SUB}]:=2 / \mathrm{PI} \times(\mathrm{J}[0, \mathrm{SUB}] \times(\mathrm{GAMMA}+\ln (\mathrm{X} / 2))$ $+4 \times$ SUM1)

for $P:=0$ step 1 until (M/SUB]-1) do

$\mathbf{Y}[\mathrm{P}+1, \mathrm{SUB}]:=(-2 / \mathrm{PI} / \mathrm{P}+\mathrm{J}[\mathrm{P}+1, \mathrm{SUB}] \times \mathbf{Y}[\mathrm{P}$, SUB])/J [P, SUB];

SUB $:=$ SUB +1 end end end

\section{ALGORITHM 45}

INTEREST

Peter Z. Ingerman

University of Pennsylvania, Philadelphia, Pa.

procedure monpay (i, B, L, t, $k, m$, tol, goof)

comment This procedure calculates the periorlic payment necessary to retire a loan when the interest rate on the loan varies (possibly from period to period) as a function of the asyet-unpaid principal.

The formal parameters are: $i$, array identifier for the vector of interest rates; $-B$, array identifier for the minimum amounts at which the corresponding $i$ applies; $-L$, the amount to be borrowed; $-t$, the number of periods for which the loan is to be taken out; $-k$, the number of different interest rates (and upper limit for vectors $i$ and $B$ ); $-m$, the desired periodic payment; -tol, the allowable deviation of $m$ from some ideal; and goof, the error exit to use if convergence fails. The only output parameter is $\mathrm{m}$. For further discussion, see Comm. ACM 3 (Oct. 1960), 542;

begin array $h, S[1: k, 1: t], M, X[1: k]$;

integer array $T, a, b[1: 1 k]$;

integer $p, q, r, 8 a, s b, I, i b, m b, n b$;

comment This section sets up the procedure;

for $p:=1$ step 1 until $k$ do

begin for $q:=1$ step 1 until $t$ do

begin $h_{p, q}:=i_{p} q^{q}$ $S_{p . q}:=\left(h_{p, q}-1\right) /\left(i_{p}-1\right)$ end;

if $p=1$ then $X_{p}:=0$ else $X_{p}:=B_{p} \times\left(i_{p-1}-i_{p}\right)$;

$M_{p}:=L \times\left(h_{p, t} / S_{p, t}\right)$ end;

sa $:=\mathrm{sb}:=\mathrm{ib}:=\mathrm{mb}:=0 ; \mathrm{nb}:=\mathrm{t}$;

for $p:=1$ step 1 until $k$ do

begin $a_{p}:=$ entier $\left(B_{p+1} / M_{p+1}+0.5\right)-8 a$;

$\mathrm{sa}:=\mathrm{sq}+\mathrm{a}_{\mathrm{p}}$

$T_{p}:=b_{p}:=$ entier $\left(B_{p+1} / M_{p}-0.5\right)-s b ;$

$s b:=s b+b_{p}$;

if $b_{p}>m b$ then

begin ib $:=p ; n b:=n b-m b ; \quad m b:=b p$ end

else $n b:=n b-b_{p}$ end;

$\mathrm{T}_{\mathrm{ib}}:=\mathrm{nb}$

$\mathrm{I}:=1$;

for $p:=1$ step 1 until $k$ do

$I:=I \times\left(a_{p}-b_{p}+1\right)$;

comment Having counted the number of possible iterations and established a set of trial values for the $T_{a}{ }^{\prime} B, a$ trial $m$ is found;

$\mathrm{D}:=1 ; \mathrm{E}:=\mathrm{F}:=0$.

newm: for $p:=1$ step 1 until $k$ do

begin $D:=D \times h_{p} . T_{p}$;

$\mathrm{u}:=1$;

if $\dot{p} \neq 1$ then for $q:=1$ step 1 until $p-1$

do $u:=u \times h_{q}, T_{a}$;

$E:=E+S_{p}, T_{p} \times u$

$\mathbf{v}:=0$

if $p \neq 1$ then for $r:=1$ step 1 until $p$

do $v:=v+X_{r}$

$F:=F+u \times v$ end;

$\mathrm{m}:=(\mathrm{L} \times \mathrm{D}+\mathrm{F}) / \mathrm{E}$;

comment Now find out whether $m$ is good enough;

$q:=1 ; \quad F:=D:=0$;

for $p:=1$ step 1 until $t$ do

begin get $F: F:=(D+m-E) /\left(1+i_{q}\right)$; if $B_{q+1} \geqq F$ then $D:=F$ else $q:=q+1$;

if $D \neq F$ go to get $F$ end;

if abs $(D-L) \leqq$ tol then go to exit;

comment If not within tolerance, adjust $T_{n}$ 's and try again; 


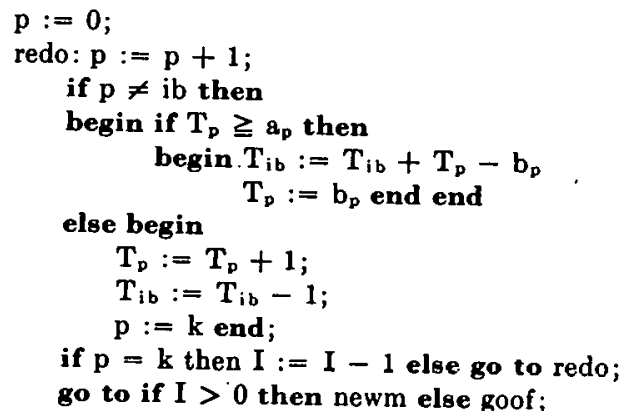

if $p=$ k then $I:=1-1$ else go to redo

exit: end monpay;

CERTIFICATION OF ALGORITHM 45

INTEREST [Peter Z. Ingerman, Comm. ACM Apr. 1961 and Oct. 1960]

CarL B. Wright

Dartmouth College, Hanover, N. H.

INTEREST was translated into Dartmouth College Computation Center's "Self Contained ALGOL Processor" for the RoyalMcBee LGP-30. When using SCALP, memory capacity is severely limited and thus it was necessary to run this program in two blocks. Block I ended with the computation of I, and Block II started with the "newm" loop. After making the changes listed below, test problems using up to three interest rates and up to 18 time periods were used with the following results:

\begin{tabular}{|c|c|c|c|c|c|}
\hline Loan & Periods & Interest Rales & Payments & $\underset{\text { Balance }}{\text { Final }}$ & Tolerance \\
\hline$\$ 100.00$ & 1 & 0.05 & $\$ 105.00$ & $\$ 0.00$ & $\$ 0.25$ \\
\hline 1800.00 & 10 & 0.03 & 211.01 & 0.05 & 4.50 \\
\hline 875.65 & 8 & 0.08 to 500.00 & & & \\
\hline 750.00 & 18 & $\begin{array}{c}0.05 \text { over } 500.00 \\
0.06 \text { to } 5000.00 \\
0.05 \text { to } 10,000.00 \\
0.04 \text { over } 10,000.00\end{array}$ & 139.78 & -1.49 & 2.19 \\
\hline
\end{tabular}

* Hand calculation.

It is noted that in each case the final balance is within the prescribed tolerance $(0.0025$ of the loan).

In the following corrections bracketed subscripts replace ordinary subscripts and exponentiation is represented by $\uparrow$ rather than superscript.

The following corrections should be made in the Note on Interest in the October, 1960, issue of Comm. ACM:

1. Definition of $B[n]$ : Replace "minimum" by "maximum". Replace " $j[n]$ " by " $j[n-1]$ ".

2. Define $B[k+1] \equiv L$.

3. Definition of $K[n]$ : Replace " $B[n]$ " by " $B[n+1]$ ". dure:

The following corrections were found necessary in the proce-

1. The upper limit of the vector $B$ is $k+1$, not $k$. It is not neces. sary to change the upper limit of the $I$-vector. (See correction 4
below.)

2. $D, E, F, u, v$ were not declared and must be declared as real.

3. In the array declaration replace " $M[1: k]$ " by " $M[1: k+1]$ ". 4. As $j$ approaches $0, i$ approaches 1 and $\lim (h / S)=1 / t$. Thus
for $j[k+1]=0, i(k+1]$ for $j[k+1]=0, i[k+1]=1$, and $M[k+1]=L / t$. Thus after insert

$M[k+1]:=L / t ; \quad B[k+1]:=L ;$

5. In the conditional statement following computation of $b[r]$ replace " $>$ " by " $\geqq$ ". 6. In same conditional statement, next line, " $m b:=b p$ " should
read " $m b:=b[p]$ ".
7. $D:=1 ; E:=F:=0$;

newm: for $p:=1$ step 1 until $k$ do

should be changed to

newm: $D:=1 ; \quad E:=F:=0$;

for $p:=1$ step 1 until $k$ do

8. begin get $F: F:=(D+m-E) /(1+i[q])$; if $B[q+1] \geqq F$ then $D:=F$ else $q:=q+1$; if $D \neq F$ go to get $F$ end;

should be changed to read as follows:

begin get $F: \quad F:=(D+m) / i[q]$;

if $B[q+1] \geqq F$ then $D:=F$ else

begin if $q<k$ then $q:=q+1$ else $D:=F$ end; if $D \neq F$ then go to get $F$ end;

Note that the "then" in the last line was omitted from the original procedure.

9. In the "redo" loop insert a semicolon after the statement $T[i b]:=T[i b]+T[p]-b[p]$;

10. In the "redo" loop, next line, omit the second "end".

11. In the "redo" loop,

should be changed to

$$
p:=k \text { end }
$$

$$
p:=k \text { end end }
$$

\section{ALGORITHM 46 EXPONENTIAL OF A COMPLEX NUMBER}

JoHN R. HERNDON

Stanford Research Institute, Menlo Park, California

procedure $\operatorname{EXPC}(a, b, c, d)$; value $a, b$; real $a, b, c, d$;

comment This procedure computes the number, $c+d i$, which is equal to $\mathrm{e}^{(\mathrm{s}+\mathrm{bi})}$;

begin $c:=\exp (\mathrm{a})$; d : $=\mathrm{c} \times \sin (\mathrm{b})$;

end EXPC; c $:=c \times \cos (b)$

CERTIFICATION OF ALGORITHM 46

EXPONENTIAL OF A COMPLEX NUMBER (J. R. Herndon, Comm. ACM \& (Apr., 1961), 178)

A. P. RELPH

Atomic Power Div., The English Electric Co., Whetstone, England

Algorithm 46 was translated using the Deuce Algol compiler, no corrections being required, and gave satisfactory results.

\section{ALGORITHM 47 ASSOCIATED LEGENDRE FUNCTIONS OF THE FIRST KIND FOR REAL OR IMAGINARY ARGUMENTS}

JoHN R. HERNDON

Stanford Research Institute, Menlo Park, California

procedure LEGENDREA $(m, n, x, r)$; value $m, n, x, r$; integer $m, n$; real $x, r$; 
comment This procedure computes any $P_{a}{ }^{m}(x)$ or $P_{n}{ }^{m}$ (ix) for $n$ an integer less than 20 and $m$ an integer no larger than $n$. The upper limit of 20 was taken because (42)! is larger than $10^{\circ}$. Using a modification of this procedure values up to $n=35$ have been calculated. If $P_{n} m(x)$ is desired, $r$ is set to zero. If $r$ is nonzero, $P_{n}{ }^{m}$ (ix) is computed;

begin

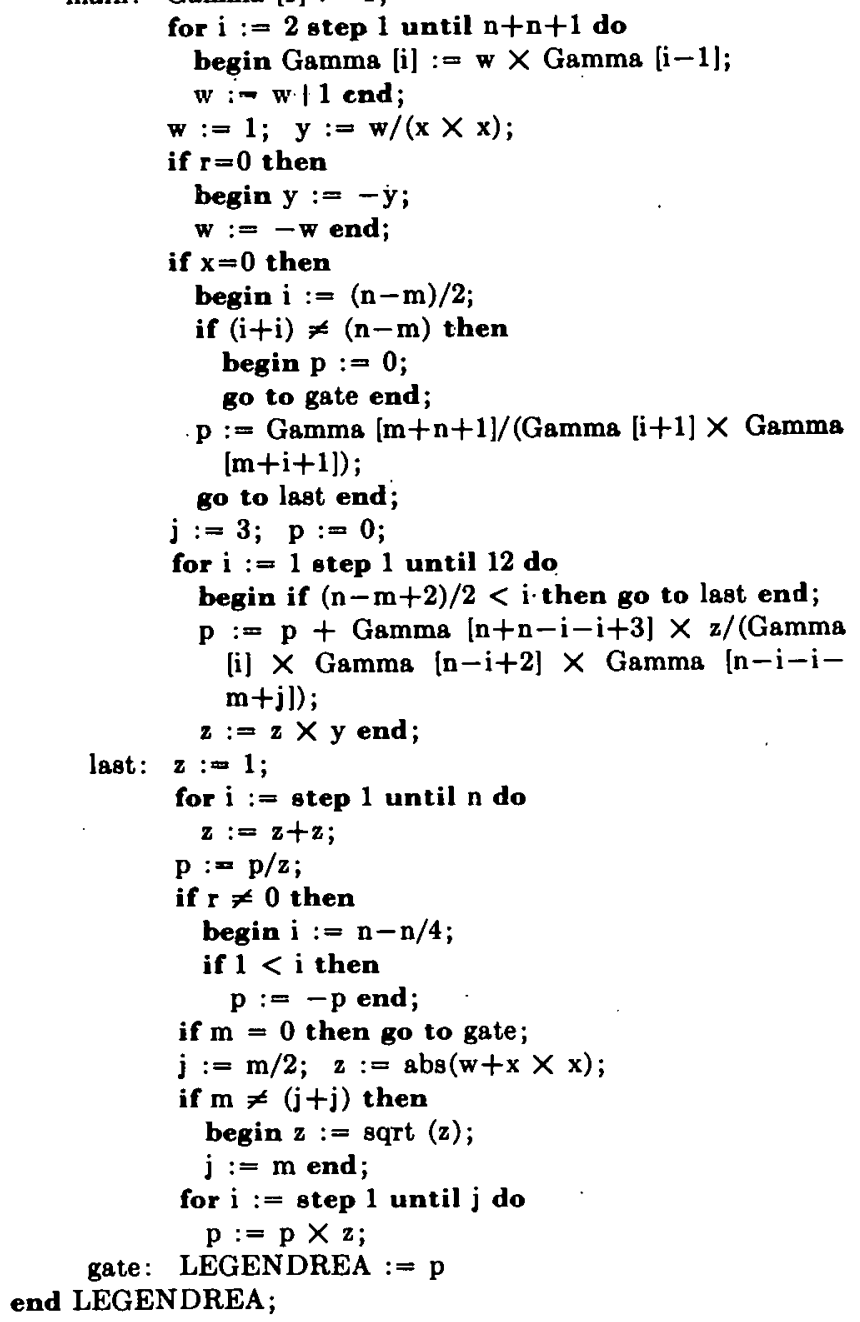

\section{CERTIFICATION OF ALGORITHM 47}

ASSOCIATED LEGENDRE lUNCTIONS OF THE FIRST KIND IOOR REAL OR IMIAGINARY ARGUMENTS [John R. Herndon, Comm. ACM, Apr. 1961] Richard George*.

Argonne National Laboratory, Argonne, III.

- Work supported by United States Atomic Energy Commission.

This procedure was programmed in ForTram for the IBM 1620 and was tested with a number of real arguments. A few errors were detected:

1. In the following sequence the end must be removed:

begin if $(n-m+2) / 2<i$ then go to last end;

2. In these, the lower bound of 1 is needed:

$$
\begin{aligned}
& \text { for } i:=\text { step } 1 \text { until } n \text { do } \\
& \text { for } i:=\text { step } 1 \text { until } j \text { do }
\end{aligned}
$$

3. There are four places where integer arithmetic is clearly intended and we must substitute the symbol $\div$ for the symbol $/$.

In addition, it might be mentioned that the statement.

$$
\text { if } n=m \text { then go to main; }
$$

could be omitted from the ALGOL program without harm, though the ForTran version requires it. Here, and elsewhere in the procedure, one might make an equivalent but more succinct statement. With change in style, the variable $j$ could be eliminated.

\section{ALGORITHM 48 LOGARITHM OF A COMPLEX NUMBER} John R. Herndon Stanford Research Institute, Menlo Park, California

procedure $\operatorname{LOGC}(a, b, c, d)$; value $a, b$; real $a, b, c, d$; comment This procedure computes the number, $c+d i$, which is equal to $\log _{e}(a+b i)$;

begin $c:=$ sqrt $(a \times a+b \times b)$; $\mathrm{d}:=\arctan (\mathrm{b} / \mathrm{a})$; c : $=\log (c)$; if a $<0$ then $d:=d+3.1415927$

end LOGC;

\section{CERTIFICATION OF ALGORITHM 48}

LOGARITHM OF A COMPLEX NUMBER (J. R. Herndon, Comm. ACM 4 (Apr., 1961), 179) A. P. RELPH

Atomic Power Div., The English Electric Co., Whetstone, England

Algorithm 48 was translated using the DEUCE Algol compiler, after certain modifications had been incorporated, and then gave satisfactory results.

The original version will fail if $a=0$ when the procedure for arctan is entered. It also assumes that $-\pi / 2<d<3 \pi / 2$, whereas the principal value for logarithm of a complex number assumes $-\pi<d \leqq \pi$.

Incidentally, the ALgol 60 identifier for natural logarithm is In, not log.

The modified procedure is as follows: 
procedure LOGC ( $a, b, c, d)$; value $a, b$; real $a, b, c, d$;

comment This procedure computes the number $c+d i$ which is equal to the principal value of $\log _{e}(a+b i)$. If $a=0$ then $c$ is put equal to $-{ }_{1047}$ which is used to represent "- infinity";

begin integer $m, n$

$$
\begin{aligned}
& \mathrm{m}:=\operatorname{sign}(\mathrm{a}) ; \mathrm{n}:= \operatorname{sign}(\mathrm{b}) ; \\
& \text { if } \mathbf{a}=\mathbf{0} \text { then begin } \mathrm{c}:=-1047 ; \\
& \mathrm{d}:=1.5707963 \times \mathrm{n} ; \\
& \text { go to } \mathrm{k}
\end{aligned}
$$

end;

$$
\begin{aligned}
& c:=\operatorname{sq} r t(a \times a+b \times b) \\
& c:=\ln (c) \\
& d:=1.5707963 \times(1-m) \times(1+n-n \times n)+\arctan (b / a) ;
\end{aligned}
$$

k: end LOGC;

\section{REMARK ON ALGORITHM 48}

LOGARITHM OF A COMPLEX NUMBER [John R.

Herndon, Comm. ACM 4 (Apr. 1961)]

Margaret L. Johnson and Ward Sangren

Computer Applications, Inc., San Diego, Calif.

Considerable care must be taken in using the arctan function. In Algorithm 48 two such difficulties are ignored. First, it is necessary, because of a resulting division by zero, to deal separately with the case where the real part of the complex number is zero. Second, if the real part of the complex number is negative and the argument of the logarithm is to have a value between $-\pi$ and $\pi$ then the action depends upon the sign of the imaginary part of the complex number. For clarity the following procedure exhibits in sequence the alternatives:

procedure I,OGC (a, b, c, d); value $a, b$; real $a, b, c, d$; comment This procedure computes the number $c+d i$ which is equal to $\log _{e}(a+t i)$. It is assumed that the arctan has a value between $-\pi / 2$ and $\pi / 2$.

begin if a $>0$ then begin THETA $:=0$; go to SOL end; if $a<0 \wedge b \geqq 0$ then begin THETA $:=3.1415927$; go to $S O L$ end

if $\mathrm{a}<0 \wedge \mathrm{b}<0$ then begin THETA $:=-3.1415927$; go to $S O L$ end;

if $a=0 \wedge b=0$ then begin $c:=d:=0$; go to RETURN end;

if $a=0 \wedge h>0$ then begin $c:=\ln (b) ; d:=1.570963$; go to RETURN end;

if $\mathrm{a}=0 \wedge \mathrm{b}<0$ then begin $\mathrm{c}:=\ln (\mathrm{abs}(\mathrm{b}))$; $d:=1.570963$; go to RETURN end;

SOL: $d:=\arctan (b / a)+$ THETA;

$c:=\operatorname{sqrt}(a \times a+b \times b)$

c: $:=\ln (\mathrm{c})$;

RETURN : end I.OGC

\section{REMARK ON REMARKS ON ALGORITHM 48 [B3]} LOGARITHM OF A COMPLEX NUMBER [John R. Herndon, Comm. ACM 4 (Apr. 1961), 179; 5 (Jun. 62), $347 ; 5$ (Jul. 62), 391]

David S. Collens (Recd. 24 Jan. 1964 and 1 Jun. 1964)

Computer Laboratory, The University, Liverpool 3, England

This procedure was designed to compute $\log _{e}(a+b i)$, namely $c+d i$, and although some very necessary precautions about its use have already been stated, some points seem to have escaped notice. In particular, A. P. Relph [Comm. ACM, June 1962, 347] remarked that if $a=0$, then $c$ becomes '-infinity', but this is only the case if $b=0$ also. Margaret L. Johnson and Ward Sangren [Comm. ACM, July 1962,391] conceded that $a=b=0$ was a special case, but wrongly gave zero as the result. The only reasonable way of dealing with this case is to exit to some nonlocal label and to let the user decide whether to terminate his program or to assign particular values to $c$ and $d$. The obvious values to use here are, for $c$, a negative number, larger than the largest which would be given by the procedure, and possibly zero for $d$. (In an implementation where $2^{-129}$ is the smallest representable nonzero number, the largest negative value of $c$ possible is -89.416.) Finally, in the Johnson-Sangren version of the procedure, the last conditional statement should read

$$
\text { if } \begin{aligned}
a & =0 \wedge b<0 \text { then begin } c:=\ln (a b s(b)) ; \\
d & :=-1.570963 ; \text { go to } R E T U K N \text { end; }
\end{aligned}
$$

the omission of the minus sign in the original being probably typographical in origin.

\section{ALGORITHM 49 \\ SPHERICAL NEUMANN FUNCTION}

JoHN R. HERNDON

Stanford Research Institute, Menlo Park, California

real procedure SPHBEN $(r, x)$; value $r, x$; real $r, x$; comment This procedure computes the spherical Neumann function $(\pi / 2 x) N_{t+1 / 2}(x)$. Infinity is represented by $10^{47}$;

begin real $z, g, t$; if $x=0$ then

begin $8:=10 \uparrow 47$;

go to gate

end;

$s:=-\cos (x) / x$;

if $r=0$ then

go to gate;

$\mathrm{t}:=\sin (\mathrm{x}) / \mathrm{x}$;

for $g:=1$ step 1 until $r$ do

begin $\mathrm{z}:=\mathrm{s}$;

$\mathrm{s}:=\mathrm{s} \times(\mathrm{g}+\mathrm{g}-1) /(\mathrm{x}-\mathrm{t})$ :

$\mathbf{t}:=\mathbf{z}$

end;

gate: SPHBEN := s

end SPHBEN;

ALGORITHM 50

INVERSE OF A FINITE SEGMENT OF THE HILBERT MATRIX

JöHN R. HERNDON

Stanford Research Institute, Mcnlo Park, California

procedure INVHILBERT $(\mathrm{n}, \mathrm{S})$; value $\mathrm{n} ;$ real $\mathrm{n}$; real array $S$;

comment This procedure computes the elements of the inverse of an $\mathrm{n} \times \mathrm{n}$ finite segment of the Hilbert matrix and stores them in the array $S$;

begin real $i, j, k$;

$\mathrm{S}[1,1]=\mathrm{n} \times \mathrm{n}$;

for $i:=2$ step 1 until $n$ do

begin

$S[i, i]:=(n+i-1) \times(n-i+1) /((i-1) \times(i-1)) ;$

$S[i, i]:=S[i-1, i-1] \times S[i, i] \times S[i, i]$ end; 


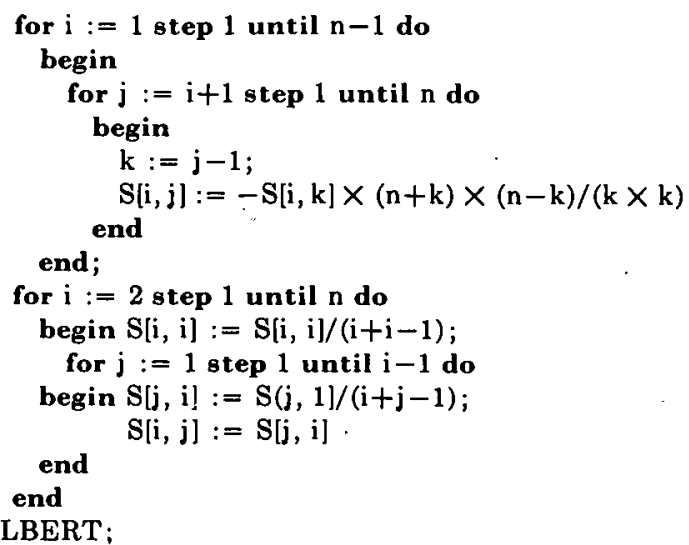

CERTIFICATION OF ALGORITHM 50

INVERSE OF A FINITE SEGMENT OF THE HILBERT MATRIX [J. R. Herndon, Comm. ACM \& (Apr. 1961)]

B. RANDELL

Atomic Power Division, The English Electric Co., Whetstone, England

INVHILBERT was translated using the Deuce Algol compiler and the following corrections being needed.

1. $\mathrm{S}[1,1]=\mathrm{n} \times \mathrm{n}$, replaced by $\mathrm{S}[1,1]:=\mathrm{n} \times \mathrm{n}$;

2. $\quad S[j, i]:=S(j, 1] /(i+j-1)$

replaced by $S[j, i]:=S[j, i] /(i+j-1)$

The compiled program, which used a 20 bit mantissa floating point notation then produced the following $4 \times 4$ segment

$\begin{array}{rccc}16.0 & -120.0 & 240.0002 & -140.0 \\ -120.0 & 1200.0 & -2700.0 & 1680.0019 \\ 240.0 & -2700.0 & 6480.0 & -4200.0 \\ -140.0 & 1680.0019 & -4200.0 & 2800.0039\end{array}$

\section{REMARKS ON AND CERTIFICATION OF A.L-} GORTHM 50

INVERSE OF A FINITE SEGMENT OF THE HILBERT MATRIX [J. R. Herndon, Comm. ACM, Apr. 1961]

P. NAUR

Regnecentralen, Copenhagen, Denmark

In addition to inserting the corrections indicated by $B$. Randcll [Comm. ACM 5 (Jan. 1962), 50], we have modified and simplified the algorithm as follows:

1. The types of $n, i, j$ and $k$ have been changed to integer. This saves roundoff operstions in subscripts.

2. Explicit multiplications have been replaced by squaring. This saves code length and execution time, at least in a compiler like ours for the GiEn.

3. Repeated references to subscripted variables have been eliminated, partly with the aid of an additional simple working variable, $w$, partly by using simultaneous assignments.

4. An unnecessary begin end pair has been removed.

In total, these changes, in addition to reducing the code length, have increased speed by a factor of 1.6 .

The resulting algorithm is as follows:

procedure INVHILBERT $(n, S)$;

value $n$; integer $n$; real array $S$;

comment ALG. 50: This procedure computes the elements of the inverse of an $n \times n$ finite segment of the Hilbert matrix and stores them in the array $S$. The Hilbert matrix has the elements HILBERT $[i, j]=1 /(i+j-1)$. The segments of this are known to be increasingly ill-conditioned with increasing size; begin integer $i, j, k ;$ real $w$

$w:=S[1,1]:=n \uparrow 2$

for $i:=2$ step 1 until $n$ do $w:=S[i, i]:=w \times((n+i-1) \times$ $(n-i+1) /(i-1) \uparrow 2) \uparrow 2$;

for $i:=1$ step 1 until $n-1$ do for $j:=i+1$ step 1 until $n$ do begin

$k:=j-1$;

$S[i, j]:=-S[i, k] \times(n+k) \times(n-k) / k \uparrow 2$

end;

for $i:=2$ step 1 until $n$ do for $j:=1$ step 1 until $i$ do

$S[i, j]:=S[j, i]:=S[j, i] /(i+j-1)$

end INVHILBERT;

Both the original version and the above improved one have been run successfully on the Gier ALGOL system (30-bit mantissa). The test program included:

(a) Output of the $4 \times 4$ matrix, to be compared with the results of Randell [loc. cit.]. Results:

$\begin{array}{rrrr}16.000000 & -120.000000 & 240.000000 & -140.000000 \\ -120.000000 & 1200.000000 & -2700.000000 & 1680.000000 \\ 240.000000 & -2700.000000 & 6480.000000 & -4200.000000 \\ -140.000000 & 1680.000000 & -4200.000000 & 2799.999977\end{array}$

(b) For $n:=1$ step 1 until-15, the inverse of the segment was calculated by INVHILBERT and multiplied by the segment of the Hilbert matrix, and the result was compared with the unit matrix. The maximum error was divided by the largest element of the inverse to form a relative error. Some of the results, which were entirely satisfactory throughout, are given below:

$\begin{array}{clccc}\text { Order } & \begin{array}{c}\text { Element of } \\ \text { max error }\end{array} & \text { abs (max error) } & \begin{array}{c}\text { Iargest element of } \\ \text { INVHILBERT }\end{array} & \text { Relative error } \\ 3 & S[3,3] & 2.38_{10}-7 & 1.92_{10} 2 & 1.24_{10}-9 \\ 6 & S[2,4] & 4.39_{10}-3 & 4.41_{10} 6 & 9.96_{10}-10 \\ 9 & S[2,8] & 1.24_{10} 2 & 1.22_{10} 11 & 1.01_{10}-9 \\ 12 & S ! 5,9] & 1.54_{10} 6 & 3.66_{10} 15 & 4.21_{10}-10 \\ 15 & S[1,12] & 1.06_{10} 11 & 1.15_{10} 20 & 9.22_{10}-10\end{array}$

(c) The time for a call of the revised INVHILBERT was found as follows:

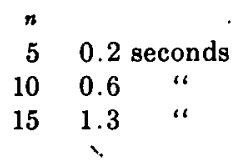

ALGORITHM 51

\section{ADJUST INVERSE OF A MATRIX WHEN AN ELEMENT IS PERTURBED}

JoHn R. HERndon

Stanford-Research Institute, Menlo Park, California

procedure ADJUST (n, d, i, j, A, B); value $i, j, n, d$; integer $i, j, n$; real $d$; real array $A, B$;

comment If the $n \times n$ matrix $A=M^{-1}$ and a change, $d$, is made in the $i, j$-th element of $M$ this procedure will calculate the corrected matrix for $\mathrm{M}^{-1}$ by adjusting matrix $\mathrm{A}$. The adjusted matrix is stored in $B$;

begin integer $r, s$;

real $t$;

$\mathrm{t}:=\mathrm{d} /(\mathrm{A} \mid \mathrm{j}, \mathrm{i}] \times \mathrm{d}+1)$;

for $r:=1$ step 1 until $n$ do

begin for $s:=1$ step 1 until $n$ do $B[r, s]=A[r, s]-t \times A[r, i] \times A j, s]$ end

end ADJUST 
CERTIFICATION OI ALGORITHM 5̈I

ADJUST INVERSE Ol' A MATRIX WHEN AN

FILEMENT IS PERTURBED [John R. Herndon,

Comm. ACM 4 (Apr. 1961)]

Richard George*

Argonne National Laboratory, Argonne, IIl.

This procedure was programmed in Fortran and reduced to machine code mechanically. It was run on the Argonne-built computing machine, George. A floating-point routine was used which allows maximum accuracy to 31 bits.

The procedure was tested for matrices with $n$ ranging from 2 to 10 . For each value of $n$, there were 20 successive trials; each trial consisted of a random perturbation of a randomly selected element of the matrix $M$, followed by a use of ADJUST, followed by the matrix multiplication $N:=B \cdot M$. For each trial, the adjustment was evaluated by computing

$$
\operatorname{sum}:=\left\{\sum_{i=1}^{n} \sum_{j=1}^{n} N[i, j]\right\}-n \text {. }
$$

For random perturbations between -1.0 and +1.0 , the value of sum never exceeded $2.0_{10}-8$.

There are two typographical errors present:

$$
B[r, s]=\Lambda[r, s]-t \times A[r, i] \times A j, s] \text { end }
$$

should be

$$
B[r, s]:=A[r, s]-t \times A[r, i] \times A[j, s] \text { end }
$$

\section{ALGORITHM 52}

\section{A SET OF TEST MATRICES}

JOHN . R. HERndon

Stanford Research Institute, Menlo Park, California

procedure TESTMATRIX $(n, A)$; value $n$; integer $n$; real array $A$

comment This procedure places in $A$ an $n \times n$ matrix whose inverse and eigenvalues are known. The $n$-th row and the $n$-th column of the inverse are the set: $1,2,3, \ldots, n$. The matrix formed by deleting the $n$-th row and the $n$-th column of the inverse is the identity matrix of order $n-1$;

begin

$$
\begin{aligned}
& \text { integer } i, j ; \\
& \text { real } t, c, d, f ; \\
& c:=t \times(t+1) \times(t+t-5) / 6 ; \\
& d:=1 / c ; \\
& A[n, n]:=-d ; \\
& \text { for } i:=1 \text { step } 1 \text { until } n-1 \text { do } \\
& \quad \text { begin } f:=i ; \\
& \quad A[i, n]:=d \times f ; \\
& A|n, i|:=A[i, n] ; \\
& A[i, i]:=d \times(c-f \times f) ; \\
& \quad \text { or } j:=1 \text { step } 1 \text { until } i-1 \text { do } \\
& \quad \text { begin } t:=j ; \\
& \quad A[i, j]:=-d \times f \times t ; \\
& \quad A[j, i]:=A[i, j] \\
& \quad \text { end }
\end{aligned}
$$

end TESTMATRIX;
CERTIFICATION OF ALGORITHMI i2

A SET OF TEST MATRICES [J. R. Herndon, Comm. ACAI, Apr. 1961]

J. S. Hillmore

Elliott Bros. (London) Ltd., Borehamwood, Herts., England

The algorithm was corrected as recommended by $\mathrm{H}$. E. Gillert in his certification [Comm. ACM, Aug. 1961] and then successfully. run using the Elliot t Algou translator on the National-Elliot t. 803. The matrices so generated were used to test the matrix inversion procedure GJR given by $H$. $R$. Schwarz in his article "An Introduction to Al.Gol." [Comm. ACM, Feb). 1962]

\section{CERTIFICATION OF ALGORITHM 52}

A SET OF TEST MATRICES (J. R. Herndon, Comm. $A C M$, Apr. 1961)

H. E. Gilbert

University of California at San Diego, La Jolla, Calif.

The statement

$$
c:=t \times(t+1) \times(t+t-5) / 6 ;
$$

was changed to

$$
\text { c }:=n \times(n+1) \times(n+n-5) / 6 ;
$$

to make the inverse have the form described in the algorithm. The algorithm was translated to Forman and tested with a matrix eigenvalue program on the CDC 1604 computer at UCSD.

The eigenvalues for the $20 \times 20$ test matrix are:

$$
\begin{array}{cl}
1 . & 1.000000 \\
2 . & 1.000000 \\
\vdots & \vdots \\
19 . & .01636693 \\
20 . & -.02493833
\end{array}
$$

\section{REMARKS ON AND CERTIFICATION OF} ALGORITHM 52

A SET OF TEST MATRICES [J. R. Herndon, Comm. ACM, Apr. 1961]

P. NAUR

Regnecentralen, Copenhagen, Denmark

In addition to inserting the correction indicated by $\mathrm{H}$. $\mathrm{E}$. Gilbert [Comm. ACM (Aug. 1961), 339] the algorithm was simplified by using the simultaneous assignment and by eliminating the local variables $t$ and $f$. The resulting algorithm is as follows:

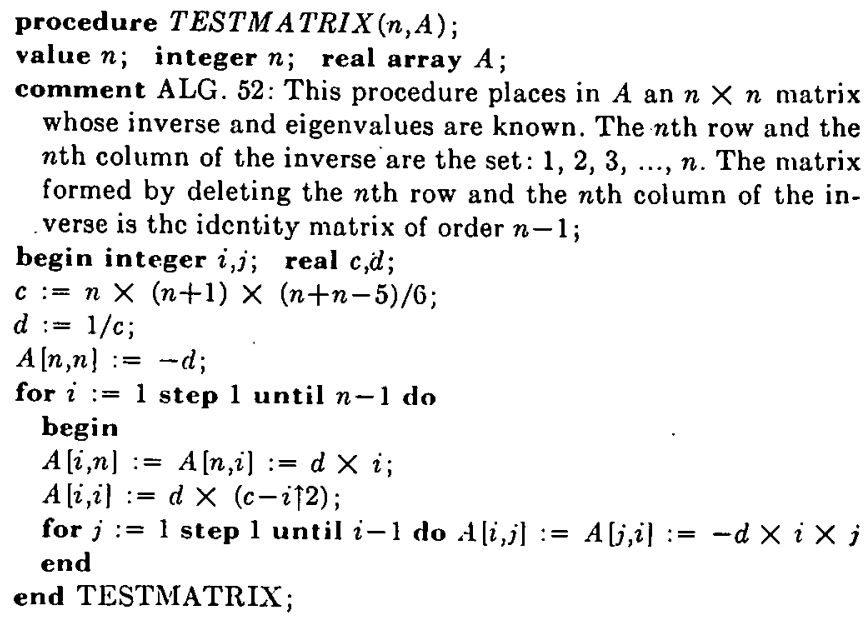


This version of the algorithm was successfully run in the GIER Algol system together with the inversion procedures INVERSION II and gjr (see Certification of Algorithm 120 below). From the figures produced by INVERSION II it looks as if the determinant of these matrices is given by $6 /(n(n+1)(5-2 n))$, which is also the value of the element $A[n, n]$. For $n>3$ the absolutely greatest element is $A[1,1]=1+A[n, n]$.

\section{REMARK ON ALGORITHM 52}

A SET OF TEST MATRICES (John R. Herndon, Comm. $A C M$, Apr. 1961)

G. H. DUBAY

University of St. Thomas, Houston, Tex.

In the assignment statement

$$
c:=t \times(t+1) \times(t+t-5) / 6 ; \quad(a)
$$

the $t$ is undefined. A suitable definition would be provided by preceding (a) with $\mathbf{t}:=\mathbf{n}$;

ADDITIONAL REMARKS ON ALGORITHM 52 A SET OF TEST MATRICES [J. R. Herndon, Comm. $A C M$ (Apr. 1961), 180]

P. NAUR

Regnecentralen, Copenhagen; Denmark

From an inspection of the results of eigenvalue-finding algorithms I conclude that all but two of the eigenvalues of TESTMATRIX are unity while the two remaining are given by the expressions $6 /(p \times(n+1))$ and $p /(n \times(5-2 \times n))$ where

$$
p=3+\text { sqrt }((4 \times n-3) \times(n-1) \times 3 /(n+1)) .
$$

These expressions have been used for the determination of absolute errors of the eigenvalues calculated by JACOBI, Algorithm 85, and Householder Tridiagonalisation, etc. as reported below. They were also used to calculate the following table (using GIER ALGOL, with 29 significant bits):

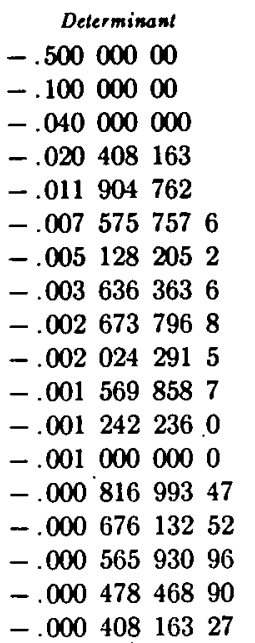

Eigenralues Differing from unity

$\begin{array}{llllll}.224 & 744 & 87 & -2.224 & 744 & 9 \\ .153 & 112 & 89 & -.653 & 112 & 89 \\ .113 & 238 & 08 & -.353 & 238 & 08 \\ .088 & 290 & 570 & -.231 & 147 & 71 \\ .071 & 428 & 571 & -.166 & 666 & 67 \\ .059 & 386 & 081 & -.127 & 567 & 90 \\ .050 & 422 & 549 & -.101 & 704 & 60 \\ .043 & 532 & 383 & -.083 & 532 & 383 \\ .038 & 097 & 478 & -.070 & 183 & 039 \\ .033 & 718 & 770 & -.060 & 034 & 559 \\ .030 & 128 & 103 & -.052 & 106 & 125 \\ .027 & 139 & 206 & -.045 & 772 & 747 \\ .024 & 619 & 013 & -.040 & 619 & 013 \\ .022 & 470 & 157 & -.036 & 359 & 046 \\ .020 & 619 & 902 & -.032 & 790 & 288 \\ .019 & 012 & 916 & -.029 & 765 & 605 \\ .017 & 606 & 429 & -.027 & 175 & 807 \\ .016 & 366 & 903 & -.024 & 938 & 332\end{array}$

The figures for $n=20$ agree very well with the results quoted by H. E. Gilbert in his certification [Comm. ACM 4 (Aug. 1961), 339].
ALGORITHM 53

NTH ROOTS OF A COMPLEX NUMBER

John R. Herndon

Stanford Research Institute, Menlo Park, California

procedure NTHROOT ( $n, r, u$, REAL, UNREAL); value $n, r, u$; integer $n$; real $r, u$; real array REAL, UNREAL;

comment This procedure computes the $n$ roots of the equation $x^{n}=r+u i$. The real parts of the roots are stored in the vector REAL [ ]. The imaginary parts are stored in the corresponding locations in the vector UNREAL [ ];

hegin integer $\mathrm{nl}, \mathrm{n} 2 ;$ real en, th, $\mathrm{s}$, th 1 ; REAL $\{\mathrm{n}\}:=0$; en $:=1 / n$;

if $u=0$ then begin $s:=(\operatorname{abs}(r)) \uparrow \mathrm{en}$; th $:=0$; go to main end;

if $r=0$ then

begin $s:=(\operatorname{abs}(u)) \uparrow$ en;

th $:=1.5707963$;

if $u<0$ then

th $:=-$ th

go to main end;

$\mathrm{s}:=(\mathrm{r} \times \mathrm{r}+\mathrm{u} \times \mathrm{u}) \uparrow(\mathrm{en} / 2)$;

th $:=\arctan (u / r)$;

main: if $\mathrm{r}<0$ then

th $:=$ th +3.1415926

th: $:=$ en $\times$ th;

th1 := $6.2831853 \times \mathrm{en}$;

for $\mathrm{n} 2:=1$ step 1 until $\mathrm{n}$ do begin REAL [n2] := $8 \times \cos (\mathrm{th})$; UNREAL $[\mathrm{n} 2]:=\mathrm{s} \times \sin (\mathrm{th})$ th $=$ th + th 1 end

end NTHROOT;

\section{REMARK ON ALGORITHM 53}

Nth ROOTS OF A COMPLEX NUMBER (John R.

Herndon, Comm. ACM 4, Apr. 1961)

C. W. Nestor, Jr.

Oak Ridge National Laboratory, Oak Ridge, Tennessee

A considerable saving of machine time for $N \geqq 3$ would result from the use of the recursion formulas for the sine and cosine in place of an entry into a sine-cosine subroutine in.the do loop associated with the $\mathrm{N}$ th roots of a complex number. That is, one could use

$$
\begin{aligned}
& \sin (n+1) \theta=\sin n \theta \cos \theta+\cos n \theta \sin \theta \\
& \cos (n+1) \theta=\cos n \theta \cos \theta-\sin n \theta \sin \theta,
\end{aligned}
$$

ut the cost of some additional storage.

We have found this procedure to be very efficient in problems dealing with Fourier analysis, as suggested by $G$. Goerzel in chapter 24 of Mathematical Methods for Digital Computers.

\section{ALGORITHM 54}

GAMMA FUNCTION FOR RANGE 1 TO 2

JOHN R. HERNDON

Stanford Research Institute, Menlo Park, California

real procedure $Q(x)$; value $x$; real $x$,

comment This procedure computes $\Gamma(x)$ for $1 \leqq x \leqq 2$. This is a reference procedure for the more general gamma function procedure. $\Gamma(x)=Q(x-1)$; 
begin

end

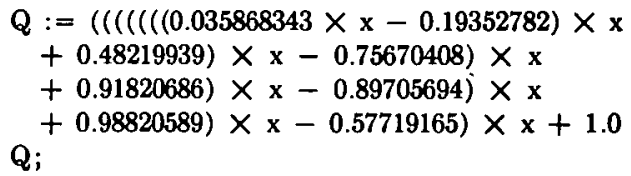

Q;

\section{ALGORITHM 55 \\ COMPLETE ELLIPTIC INTEGRAL OF THE FIRS'T KIND}

JoHn R. HERNDON

Stanford Research Institute, Menlo Park, California

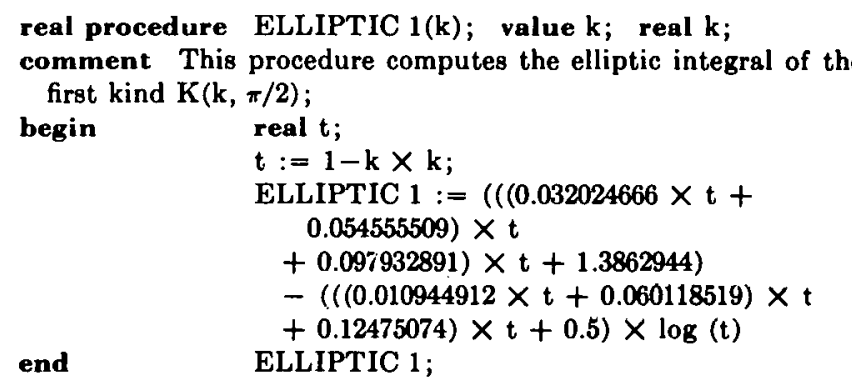

\section{CERTIFICATION OF ALGORITHM 55}

COMPIETE ELIII'TIC INTEGRAL OF THE l'IRST

KIXD [John R. Herndon, ('omm. ACM, Apr. 196i1]

and

CLRTIFICATION OF AI,GORITHM 149

COMPLETE ELIIPTIC INTEGRAL [J. N. Merner, Comm. ACM, Dec. 1962]

Heniry C. Thacher, Jk.

Reactor Eng. Div., Argonne National Laboratory,

Argonne, Ill.

*Work supported by the U.S. Atmmic Energy Commission.

The bodies of Algorithm 55 and of the second procedure of Algorithm 149 were tested on the I, (il'-30 computer using ScAl.l', the 1)artmouth "LOA1)-AN1-(io" translator for a substantial sul)set of Aldow 60. The floating-point arithmetic for this translator carries $7+$ significant digits.

In addition to modifications required because of the limitations of the ACAL' subset, the following need correction:

In Algorithm 55:

1. The constant $0.05+55.509$ should be 0.054544409 .

2. The function log should be ln.

In procedure EIIP 2 of Algorithm 149, the statement $a:=c$ should be $a:=C$.

The parameters of Algorithm 1.19 are related to the complete elliptic integral of the first kind by: $K=a \times \operatorname{ELIP}(a, b)$ where the parameter $m=k^{2}=1-b / a$.

The maximum approximation error in Algorithm 55 is given by Hastings as about $0.610-6$. In addition there is the possibility of serious cancellation error in forming the complementary palame.ter $t=1-k \times k$. For $k$ ne:ur 1 , errors as great as 4 significint digits were sustained. In these regions, the complementary parameter itself is a fal more satisfactory parameter.

The accuracy obtainable with Algorithm 149 is limited only by the arithmetic accuracy and the amount of effort which it is desired to expend. Six-figure accuracy was obtained with 5 applications of the arithmetic-geometric mean for $a=1000, b=2$, and with one application for $a=500, b=500$.
Neither algorithm is sutisfactory for $k=1$. The behavior for Algorithm 55 will be governed by the error exit from the logarithn! procedure. Under these circumstances, Algorithm 149 goes into an endless loop. Algorithm 149 may also go into an endless loop of the terminating constant $(10-8$ in the published algorithm) is tor small for the arithmetic being used. For the Scalp arithmetic it. was found necessary to increase this tolerance to $5.0_{10}-7$. The resulting values of the elliptic integrals were, however, accurate to within 2 in the 7 th significant digit (6th decimal).

The relative efficiency of the two algorithms will depend st.rongly on the efficiency of the square-root and logarithm sub). routines. With most systems, Algorithm 55 will provide sufficient accuracy, and will be more efficient. If a square-root operation or a highly efficient square-root subroutine is available. Algurithm 149 may well be the better niethod.

\author{
ALGORITHM 56 \\ COMPLETE ELLIPTIC INTEGRAL OF THE \\ SECOND KIND \\ JoHN R. HERndoN \\ Stanford Research Institute, Menlo Park, California \\ real procedure ELLIPTIC 2(k); value $k$; real $k$ : \\ comment This procedure computes the elliptic integral of the \\ second kind $E(k, \pi / 2)$; \\ begin

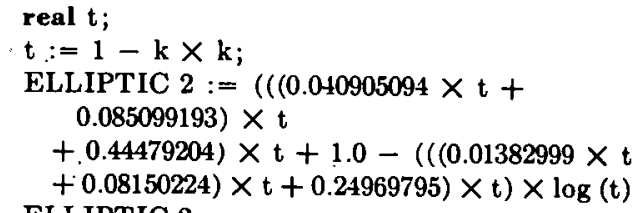

\section{ALGORITHM 57 \\ BER OR BEI FUNCTION}

JoHn R. HERndon

Stanford Research Institute, Menlo Park, California

real procedure $\operatorname{BERBEI}(r, z)$; value $r, z$; real $r, z$;

comment This procedure computes $\operatorname{ber}(z)$ if $r$ is set equal to zero. bei( $\mathrm{z})$ is produced if $\mathrm{r}$ equals 1.0 ; begin

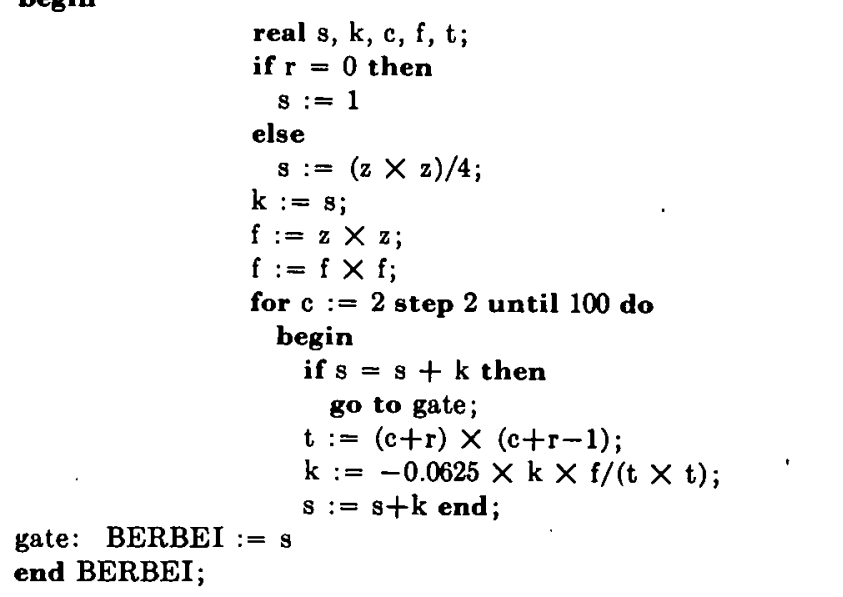


CERTIFICATION OF ALGORITHM

BER OR BEI FUNCTION [J. R. Herndon, Comm. ACM

4 (Apr. 1961)]

A. P. RELPH

The English Electric Co. Whetstone, England

Algorithm 57 was translated using the Detce Algol compiler. No corrections were required, and the results were satisfactory

\section{CERTIFICATION OF AIGORITHN i7}

BER OR BEI l:LNCTION [John R. Herndon, Comm. $A C . M$, Apr. 1961]

IIENRY C. THACHER, JR.*

Reactor Engineering Div., Argonne National Lab., Argonne, Ill.

* Work supported by the U.S. Atomic Energy Commission.

The body of Algorithm 57 was. tested on the LCil'-30 using the Algol 60 translator developed by the Dartmouth College Computer Center. No synt actical errors were found. For $z=0.1(0.1) 1.0$, with a $7+$ significant decimal arithmetic routine, the program gave results with errors less than 5 (and for $z=1(1) 5$ less than 12) $i^{n}$ the seventh digit. For large values of $z$, serious cancellation rrors maly occur. For example, for $z=20$, more than 2 decimals of significance cain be lost in this way.

\section{AI,GORITHM 58 MATRIX INVERSION \\ Donali) Cohen \\ Burroughs Corporation, Pasadena, Calif.}

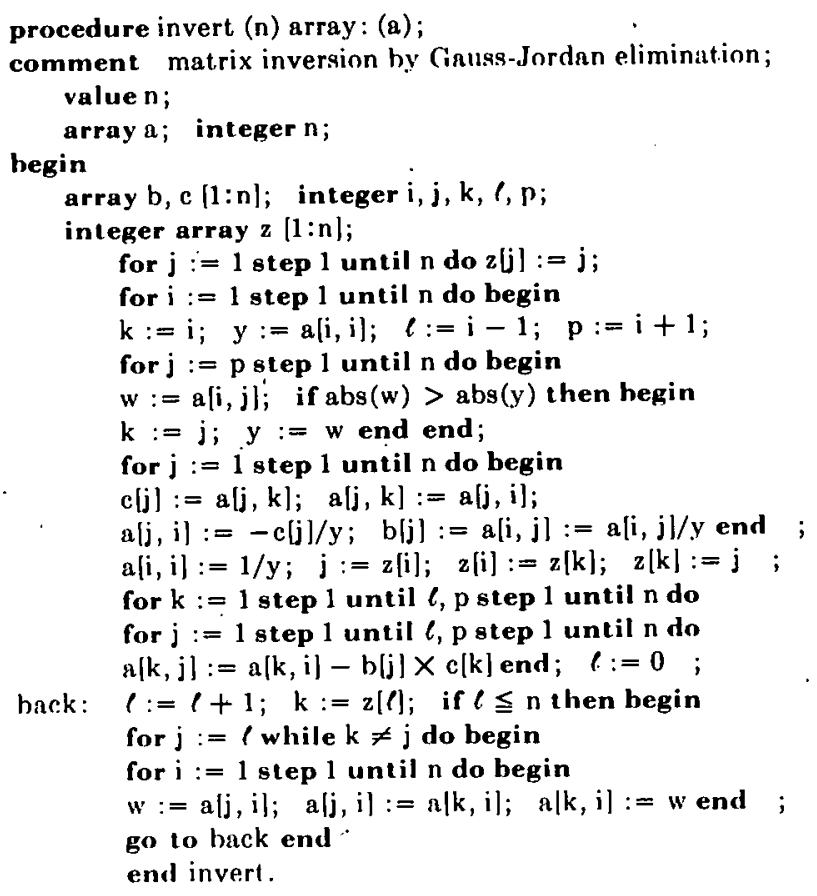

CEIRTIPICATION ()F AICIORITHM 58

MATRIX INVERSION (I)onald Cohen, Comm. $A C: M / 4$, May 1961)

Richard) A. Congels

Yalem Computer Center, St. Louis University, St. Iouis, Mo.

Invert was hand-coded in FurTran for the IBM 1620. The following corrections were found necessary:

The statement $a_{k, j}:=a_{k, i}-b_{j} \times c_{k}$ should be

$$
a_{k, j}:=a_{k, j}-b_{j} \times c_{k}
$$

The statement go to back should be changed to

$$
\mathrm{i}:=\mathrm{z}_{\mathrm{k}} ; \mathrm{z}_{\mathrm{k}}:=\mathrm{z}_{\mathrm{j}} ; \mathrm{z}_{\mathbf{j}}:=\mathrm{i} \text {; go to back }
$$

After these corrections were made, the program was checked by inverting a $6 \times 6$ matrix and then inverting the result. The second result was equal to the original matrix within round-off.

\section{CERTIFICATION OF ALGORITHM is}

MATIIX INVERSION-[Donăld Cohen, Comm. ACM, May, 1961]

RICharl) George*

Particle Accelerator Div., Argonne / National Lab., Argonne, Il!.

This procedure was programmed in Furtrax and reduced to machine code mechanically. It was run on the Argonne-built computing machine, GEORGE. A floating-point routine was used which allows maximum accuracy to 31 bits.

There are a number of errors of various types:

(1) There are eight begin's and only seven end's.

(2) The line

$$
a[k, j]:=a[k, i]-b[j] \times c[k] \text { end; }
$$

should be

$$
a[k, j]:=a[k, j]-b[j] \times c[k] \text { end; }
$$

(3) The permutation of rows of the inverted matrix and permutation of elements of the integer array $z$ must be carried out simultaneously. This algorithm fails to do this, and consequently the matrix at the time of exit from the procedure is left in a permuted condition.

(4) The algorithm permits the statement

$$
k:=z[l]
$$

to be executed even though the declarations place an upper limit of $n$ on the integer array $z$, and the test for $l \leqq n$ has not yet been made. Obviously, Mr. Cohen's compiling system would allow an out-of-bounds array look-up. One could easily incorporate into an ALGOL compiler a guard against such illicit array references, and therefore the published algorithm might be considered machine dependent.

(5) This algorithm requires $3 n^{2}$ divisions, most of which are unnecessary. By inserting the statement

$$
y:=1.0 / y
$$

at the proper place, one may accomplish the obvious economy of reducing this to only $n$ divisions plus $2 n^{2}$ multiplications.

(6) If a matrix should be singular (or nearly so), some pivot element will be zero (or very small), and a test should be made, with provision for a jump to $A L A R M$, a non-local label.

(7) The identifiers $w$ and $y$ should be declared within this procedure to avoid trouble.

(8) This algorithm omits calculation of the determinant of the matrix. This could be computed with very little extra effort. 
The revised algorithm was then tested on the LGP-30 computer, using AlgoL-30, a small subset of Algol. Within the restrictions of this subset, the program worked satisfactorily on test matrices.

\section{REMARK ON ALGORITHM 58 \\ MATRIX INVERSION [D. Cohen, Comm. ACM, May 61]}

Peter G. Behrenz

Matematikmaskinnmänden, Box 6131, Stockholm 6, Sweden

invert was run on Facit EDB using Facit-Algol 1. Some changes in the procedure had to be made:

1. $y$ and $w$ had to be declared in the procedure-body as real $y, w$

2. The last part of the procedure starting with $l:=0$; which should interchange the matrix rows did not work correctly, even with the corrections proposed by R. A. Conger [Comm. ACM, June 62 ]. We propose the following code:

for $l:=1$ step 1 until $n$ do begin

$k:=z[l] ;$ for $j:=l$ while $k \neq j$ do begin

for $i:=1$ step 1 until $n$ do begin

$w:=a[j, i] ; \quad a[j, i]:=a[k, i] ; a[k, i]:=w$ end;

$i:=z[k] ; \quad z[k]:=z[j] ; \quad k:=z[j]:=i$ end end end invert

If the matrix $a$ is singular, the value of the pivot element $y$ will once be zero or very nearly zero and division by zero would occur in the course of the calculation. It would therefore be advantageous to introduce an empirical tolerance parameter epsilon into the procedure.

To calculate the determinant of the matrix $a$ it is only necessary to put three more statements into the code. With these augmentations invert should read:

procedure invert ( $n, a$, epsilon, determinant);

value $n$, epsilon; real epsilon, determinant;

array $a$; integer $n$;

begin real $y, w$; integer $i, j, k, l, p$;

array $b, c[1: n]$; integer array $z[1: n]$;

delerminant $:=1$.

followed by the same code as before until

$y:=w$ end end

delerminant $:=y \times$ delerminant;

if $k \neq i$ then determinant $:=-$ determinant;

if $a b s(y)<$ epsilon then go to singular;

followed by the same code as before with the changes mentioned in the certification by R. A. Conger [Comm. $A C M$, June 62] and the changes given above. singular should be a nonlocal label in the main program.

\section{REMARK ON ALGORITHM is}

MATRIX INVERSION [Donald Cohen, Comm. $A C M$, May, 1961]

George Struble

\section{University of Oregon, Eugene, Oregon}

For the last seven lines, beginning with $a \mid k, j]:=a[k, i]$, substi tute:

$a[k, j]:=a[k, j]-b[j] \times c[k \mid$ end;

$l:=0$;

back: $\quad l:=l+1$;

again: $k:=z[l]$

if $k \neq l$ then

begin for $i:=1$ step 1 until $n$ do

hegin $w:=a \mid l, i]$

$a[l, i]:=a \mid k, i]$

$a[k, i]:=w$ end;

$z[l]:=z[k]$

$z|k|:=k$;

go to again end

else if $l \not \neq n$ go to back

end invert

ALGORITHM 59

ZEROS OF' A REAL POLI'NOMIAL BY RESULTANT JROCEDURE

F. H. Bareiss and M. A. Fisherkelder

Argome National Laboratory, Argonne, Ill.

procedure RES (n, c, alpha, mu, re, im, rt, gc) ; value $n$, $c$, alpha ; integer $n$, alpha ; integer array mu ; array c, re, im, rt, gc ;

comment RES finds simultaneously all zeros of a polynomial of degree $n$ with real coefficients, $c_{j}(j=0, \ldots n)$, where $c_{n}$ is the constant term. The real part, $r_{i}$, and imaginary part, $\mathrm{im}_{\mathrm{i}}$, of each zero, with corresponding multiplicity, mu $\mathrm{u}_{1}$, and remainder term, $\mathrm{rt}_{\mathrm{i}},(\mathrm{i}=1, \ldots, \mathrm{n})$, are found and a polynomial with coefficients $\mathrm{gc}_{\mathbf{j}}(\mathrm{j}=0, \ldots, n)$, is generated from these zeros. Alpha provides an option for local or nonlocal selection of $M$, the number of root-squaring iterations, and delta and epsilon, acceptance criteria. If alpha $=1$, these parameters are assigned locally. If alpha $=2, \mathrm{M}$, delta and epsilon are set equal to the global parameters $\mathrm{Mp}$, deltap, and epsilonp, respectively. In cases where zeros may he found more than once, the superfluous ones are eliminated by factorization. The method has been described by $\mathrm{E}$. H. Bareiss (J. ACM 7, Oct. 1960, pp. 346-386).

begin integer $M$; real delta, epsilon ; switch $U:=$ U1, U2 ;

go to $\mathbf{U}$ [alpha];

U1:

U2:

START:

SYNTHETIC DIV:

SQUARING OPERATION
$\mathrm{M}:=10 ;$ delta $:=0.2 ;$ epsilon $:=10^{-8}$; go to START ;

$M:=M p$; delta $:=$ deltap ; epsilon := epsilonp ;

begin integer $C T$, nu, nuc, beta, $m, j, j c, k$, $\mathrm{i}, \mathrm{p}$; Boolean ROOT ;

real $X, Y, G X, r p$; array $a$, ac $[0: n, 0: M]$, $R, R c, t[0: n]$,

$\mathrm{s}[-1: \mathrm{n}], \mathrm{ag}[-2: \mathrm{n}], \mathrm{rh}, \mathrm{q}, \mathrm{G}, \mathrm{F}[1: 2 \times \mathrm{n}]$; switch $\mathrm{S}:=\mathrm{S} 1, \mathrm{~S} 2 ;$ switch $\mathrm{T}:=\mathrm{T} 1, \mathrm{~T} 2$;

gwitch $\mathrm{V}:=\mathrm{V} 1, \mathrm{~V} 2$;

real procedure min $(u, v) ; \operatorname{real} u, v$; $\min :=$ if $\mathrm{u} \leqq \mathrm{v}$ then $\mathrm{u}$ else $\mathrm{v}$;

real procedure SYND ( $W, Q, I, T)$;

integer $\mathrm{I} ;$ real $\mathrm{W}, \mathrm{Q}$; array $\mathrm{T}$;

begin $s[-1]:=0 ; s[0]:=T[0] ;$ for $\mathrm{m}:=1$ step 1 until $I$ do $\mathrm{s}[\mathrm{m}]:=\mathrm{T}[\mathrm{m}]-\mathrm{W} * \mathrm{~s}[\mathrm{~m}-1]-\mathrm{Q} \times \mathrm{s}$ $[\mathrm{m}-2]$;

if $\mathrm{Q}=0$ then SYND $:=$ abs (s[I]) else SYND $:=a b s(W / 2 \times s[I-1]+s(I])$ end SYND ;

CT $:=$ beta $:=1 \quad ;$ for $j:=0$ step 1 until n do a $[j, 0]:=c[j]$;

begin integer $e 1$; real $h$; for $m:=$ 1 step 1 until $M$ do begin for $j:=1$ step 1 until $n$ do 
RII): begin $h:=0$; for el $:=1$ step 1 until $\min (n-j, j)$ do $h:=h+(-1) \uparrow$ el $\times a[j-e l, m-1] \times a$ $(\mathrm{j}+\mathrm{el}, \mathrm{m}-1)$;

$\mathrm{a}[\mathrm{j}, \mathrm{m}]:=(-1) \uparrow \mathrm{j} \times(\mathrm{a}[\mathrm{j}, \mathrm{m}-1] \uparrow$ $2+2 \times h)$ end end end ;

for $\mathrm{j}:=0$ step 1 until $\mathrm{n}$ do $\mathrm{R}[\mathrm{j}]:=(-1) \uparrow$

$j \times \mathbf{a}[\mathrm{j}, \mathrm{M}-1] \uparrow 2 / \mathbf{a}[\mathrm{j}, \mathrm{M}]$;

$$
\mathrm{j}:=0 ; \mathrm{nu}:=1 \text {; }
$$

if $(1-\operatorname{delta} \leqq R[j]) \wedge(R[j] \leqq 1+$ delta $)$ then

begin $\mathrm{rp}:=(\mathrm{a}[\mathrm{j}, \mathrm{M}] / \mathrm{a}[\mathrm{j}-\mathrm{nu}, \mathrm{M}]) \uparrow(1 /(2 \uparrow$

MXnu)) ;

go to $\mathrm{T}$ [beta] end ;

$\mathrm{nu}:=\mathrm{nu}+\mathbf{1}$

$\mathrm{j}:=\mathrm{j}+1 ;$ if $\mathrm{j}=\mathrm{n}$ then go to $\mathrm{S}$ [heta] else go to RI) ;

nu :=1; goto 2 ;

$\mathrm{rh}[\mathrm{CT}]:=\mathrm{rp} ; \mathrm{X}:=\mathrm{rp}+$ epsilon $X \mathrm{rp} ;$ $Y:=X+$ epsilon $X$ rp ;

for $\mathrm{k}:=0$ step 1 until $\mathrm{n}$ do $\mathrm{t}[\mathrm{k}]:=$ abs (c[k]) ; $\mathrm{F}^{\prime}\left[\mathrm{CLI}^{\prime}\right]:=$ SYNI $(Y, 0, \mathrm{n}, \mathrm{t})-$ SYND $(\mathrm{X}, 0, \mathrm{n}, \mathrm{t})$;

$\mathrm{G}[\mathrm{CT}]:=\mathrm{SYND}(\mathrm{rh}[\mathrm{CT}], 0, \mathrm{n}, \mathrm{c}) ;$ if $\mathrm{F}[\mathrm{CT}]>\mathrm{G}[\mathrm{CT}]$ then

begin ROOT $:=$ true ; $q[\mathrm{CT}]:=0$; $\mathrm{CT}:=\mathrm{CT}+1 \quad ; \mathrm{F}[\mathrm{CT}]:=\mathrm{F}[\mathrm{CT}-1]$ end ; rh $[\mathrm{CT}]:=-\mathrm{rp} ; \mathrm{G}[\mathrm{CT}]:=\mathrm{SYNI}$ (rh $[\mathrm{CT}], 0, \mathrm{n}, \mathrm{c})$;

if $\mathrm{F}[\mathrm{CT}]>\mathrm{G}[\mathrm{CT}]$ then begin ROOT := true ; $\mathrm{q}[\mathrm{CT}]:=0 ; \mathrm{CT}:=\mathrm{CT}+1$; $\mathrm{F}[\mathrm{CT}]:=\mathrm{F}[\mathrm{CT}-1]$ end ; if $\mathrm{nu}=1$ then go to 2 ;

$q[\mathrm{CT}]:=\mathrm{rp} \uparrow 2 ;$ nuc $:=\mathrm{nu} ; \mathrm{jc}:=\mathrm{j}$;

for $j:=0$ step 1 until $n$ do

begin $R c[j]:=\mathbb{R}[j] ; \operatorname{ac}[j, M]:=a[j, M]$ end ;

begin real $h$; array $b[-1: n+1$, $-1: n+1], A[1: n]$, r $[0: n, 0: n], C B[-1: n+1]$;

b $[-1,0]:=\mathrm{CB}[-1]:=\mathrm{CB}[\mathbf{n}+\mathbf{1}]:=0$; for $\mathrm{j}:=0$ step 1 until $\mathrm{n}$ do

CB $[j]:=c[j] ; b[0,0]:=1 ;$ for $k:=$ 1 step 1 until $n$ do

begin $b[k,-1]:=0 ;$ for $j:=0$ step 1 until $k$ do

$b[k+1, j]:=b[k, j-1]-q[C T] \times b$ $[\mathbf{k}-1, \mathrm{j}]$

b $[k+1, k+1]:=h:=0$; for $j:=$ $\mathrm{n}-\mathrm{k}$ step -1 until 0 do

$h:=h+(C B[j] \times C B[k+j]-C B(j-1]$ $\times \mathrm{CB}[\mathrm{k}+\mathrm{j}+\mathbf{1}]) \times \mathrm{q}[\mathrm{CT}] \uparrow(\mathrm{n}-\mathrm{k}-\mathrm{j})$; $A[k]:=(-1 \uparrow k \times h \quad ;$ for $j:=0$ step 1 until $k-1$ do

begin $r[0, j]:=0 ; r[k, j]:=r[k-1, j]+$ $A[k] \times b[k, j]$ end ;

$\mathbf{r}[\mathrm{k}, \mathrm{k}]:=\mathrm{A}[\mathrm{k}]$ end ; beta $:=2$; for $\mathrm{j}:=0$ step 1 until $\mathrm{n}$ do

a $[j, 0]:=I[n, j]$ end ; go to SQUARING OPERATION ;

if $(\mathrm{rp} / 2) \uparrow 2 \geqq q[\mathrm{CT}]$ then go to $3 ; \mathrm{rh}$ $[\mathrm{CT}]:=\mathrm{rp}$;

G [CT] := SYND (rh [CT], q [CT], n,c) ; if $\mathrm{F}[\mathrm{CT}]>\mathrm{G}[\mathrm{CT}]$ then

begin $\mathrm{CT}:=\mathrm{CT}+1 ; \mathrm{F}[\mathrm{CT}]:=\mathrm{F}$. $[\mathrm{CT}-1] ; \mathrm{q}[\mathrm{CT}]:=\mathrm{q}[\mathrm{CT}-1]$ end ; rh $[\mathrm{CT}]:=-\mathrm{rp} ; \mathrm{G}[\mathrm{CT}]:=\mathrm{SYN} \mathrm{C}$ [rh [CT], $\mathrm{q}[\mathrm{CT}], \mathrm{n}, \mathrm{c})$;

if $\mathrm{F}[\mathrm{CT}]>\mathrm{G}[\mathrm{CT}]$ then begin $\mathrm{CT}:=\mathrm{CT}$ $+1 ; F[C T]:=F[C T-1] ;$

$q[C T]:=q[C T-1 \mid$ end ; go to 3 ;

for $j:=0$ step 1 until $n$ do begin $a[j, M]:=$ ac $[\mathrm{j}, \mathrm{M}]$

$R[j]:=\operatorname{Rc}[j \mid$ end $; j:=j c \quad ; \quad$ beta $:=1$; if ROOT then go to 3 else

nu := nuc ; go to 1 ;

ag $[-2]:=$ ag $[-1]:=0 ;$ ag $[0]:=1$; for $\mathrm{j}:=1$ step 1 until $\mathrm{n}$ do

$\operatorname{ag}[\mathbf{j}]:=0 ; \mathrm{k}:=\mathbf{1} \quad ; \mathrm{i}:=\mathbf{n} \quad ; \mathrm{m}:=\mathbf{1}$;

for $j:=0$ step 1 until $n$ do

$\mathrm{t}[\mathrm{j}]:=\mathrm{c}[\mathrm{j}] ;$

MUIT:

$\mathrm{mu}[\mathrm{m}]:=0 ; \mathrm{p}:=$ if $\mathrm{q}[\mathrm{k}]=0$ then 1 else 2 ;

IT:

V1:

V2:

E:

$\mathrm{GX}:=\mathrm{SYNl})(\mathrm{rh}[\mathrm{k}], \mathrm{q}[\mathrm{k}], \mathrm{i}, \mathrm{t}) ;$ if $\mathrm{F}[\mathrm{k}]$ $>\mathrm{GX}$ then

begin for $\mathrm{j}:=1$ step 1 until $\mathrm{n}$ do

ag $[j]:=a g[j]-\operatorname{rh}[k] \times$ ag $[j-1]+q$

$[\mathrm{k}] \times \mathrm{ag}[\mathrm{j}-2]$;

$\mathrm{mu}[\mathrm{m}]:=\mathrm{mu}[\mathrm{m}]+\mathrm{p} ; \mathrm{i}:=\mathbf{i}-\mathbf{p} ;$

for $\mathrm{j}:=0$ step 1 until $i$ do

$t[j]:=s[j] ;$ go to IT end else if $\mathrm{mu}[\mathrm{m}] \neq 0$ then begin

rt $[\mathrm{m}]:=\mathrm{G}[\mathrm{k}]$; go to $\mathrm{V}[\mathrm{p}]$ end else go to $\mathrm{D}$;

$\operatorname{re}[\mathrm{m}]:=\operatorname{rh}[\mathrm{k}] ; \operatorname{im}[\mathrm{m}]:=0 ;$ go to $\mathrm{E} ;$ re $[\mathrm{m}]:=\operatorname{rh}[\mathrm{k}] / 2 ; \operatorname{im}[\mathrm{m}]:=\operatorname{sqrt}(\mathrm{q}[\mathrm{k}]-$ re $[\mathrm{m}] \uparrow 2)$;

$\mathrm{m}:=\mathrm{m}+1$;

D: $\quad \mathrm{k}:=\mathrm{k}+1 ;$ if $\mathrm{k} \leqq \mathrm{CT} \wedge \mathrm{m} \leqq \mathrm{n}$ then go to MULT ;

for $j:=0$ step 1 until $n$ do gc $[j]:=\operatorname{ag}[j]$ end end RES

\section{ALGORITHM 60 \\ ROMBERG INTEGRATION}

F. L. BAUER

Gutenberg University, Mainz; Germany

real procedure rombergintegr (fct, $\ell_{\mathrm{gr}}, \mathrm{rgr}$, ord) ;

value $\ell_{\mathrm{gr}}, \mathrm{rgr}$, ord ;

real $\ell_{\mathrm{gr}}, \mathrm{rgr}$; integer ord ; real procedure fct ; comment rombergintegr is the value of the integral of the function fet between the limits $\ell_{\mathrm{gr}}$ and $\mathrm{rgr}$, calculated by the algorithm of Romberg with an error term of the order $2 \times$ ord +2 , ord $\geqq 0$ Computation time will roughly be doubled when ord is increased by 1 ;

begin

real array $\mathrm{t}[1$ : ord +1$]$;

real $\ell, u, m$;

integer $f, b, j, n$;

$\ell:=\mathrm{rgr}-\ell \mathrm{gr}$

$\mathrm{t}[1]:=(\mathrm{fct}(\operatorname{lgr})+\mathrm{fct}(\mathrm{rgr})) / 2$;

$\mathbf{n}:=1$;

for $h:=1$ step 1 until ord do

begin $u:=0$;

$m:=\ell /(2 \times n)$; 


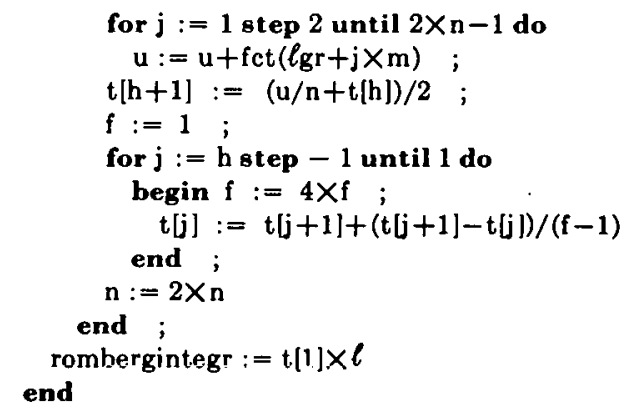

CERTIFICATION OF ALGORITHM 60 ROMBERG INTEGRATION (F. L. Bauer, Comm. $A C A$, June 1961)

Karl Heinz Buchner

Lurgi Gesellschaft fur Mineraloltechnik m.b.H., Frankfurt, Germany

Since August 1961, the Rombert Integration has been successfully applied in Forran language to various problems on an IBM 1620. Due to its elegant method and the memory saving features, the Romberg Integration has succeeded other methods in our program library, e.g., the Newton-Cotes integration of order 10.

Reference is made to Stiefel, Numerische Mathermatik (Teubner Verlag. Stuttgart). Stiefel discusses in his book various methods of numerical integration including the Romberg algorithm.

\section{CERTIIICATION OF ALGORITHM 60}

ROMBERG INTEGRATION (F. L. Bauer, Comm. $A C M$, June, 1961)

Henry C. ThaCher, JR.*

Argonne National Laboratory, Argonne, Ill.

* Work supported by the U. S. Atomic Energy Commission.

This procedure was translated to the ACT III compiler language for the Royal Precision LGP-30 computer. This system provides $7+$ significant decimal digits. The program was used to integrate $x^{n}$ between the limits 0.01 and 1.1 , and between the limits 1.1 and 0.01 . The results in Table I were obtained. The pole at 0 for negative $n$ affords a test of the reliability of the method when the higher derivatives of the integrand are large. The agreement between integrations in the forward and backward directions is an indication of the effects of round-off error.

It is apparent that the procedure gives results well within the noise level for the positive powers, and that even the effect of a closely adjacent singularity for the negative powers can be overcome.

The flexibility of the algorithm would be improved by adding to the formal parameters a procedure, check, to decide if sufficient accuracy had been obtained without carrying through the entire iteration. A possible form for this procedure would be:

procedure check $(t 1, t 2, f$, exit);

real $\mathrm{t} 1, \mathrm{t} 2$;

label exit;

integer $\mathrm{f}$;

begin if abs $((\mathrm{t} 2-\mathrm{t} 1) \times \mathrm{f}) / \mathrm{t} 1<$ tolerance $\wedge \mathrm{f}>$ minimum order then go to exit end.

The global variables tolerance, which is the maximum relative difference between approximations of increasing order, and the minimum acceptable order should be selected by the programmer for the exigencies of the problem. A check of this sort is clearly not as sound as an a priori estimate of the necessary order, but is frequently an acceptable expedient.
The Romberg quadrature algorithm is analyzed in the following references:

Romberg, W. Vereinfachte -numerische Integration. Det Kongelinge Norske Videnskaber Selskab Forhandlinger 28, (1955), 30-36.

Stiefel, E., and Rutishauser, H. Remarques concernant l'integration numerique. Comptes Rendus Acad. Scil (Paris) 252, (1961), 1899-1900.

TABLE I. InTEgration of $\int_{0.01}^{1.1} x^{n} d x$ AND $\int_{1.1}^{0.01} x^{n} d x$

\begin{tabular}{lcccc}
\hline \multicolumn{1}{c}{$n$} & 0 & +12 & +12 & -1 \\
\hline True Value & 1.0900000 & $.2(5555932$ & -.26555932 & 4.7004831 \\
Order 1 & 1.0899997 & $.5707(8812$ & $-.570768+2$ & 19.641113 \\
Order 2 & 1.0899997 & .30614608 & -.30614626 & 10.656923 \\
Order 5 & 1.0899991 & .26555693 & -.26555818 & 4.9017590 \\
Order 10 & & & & 4.7002345 \\
\hline
\end{tabular}

\begin{tabular}{lccc}
\hline \multicolumn{1}{c}{$n$} & -1 & -5 & -5 \\
\hline True lalue & -4.7004831 & $.25000000 \times 10^{8}$ & $-18.1666667 \times 10^{8}$ \\
Order.1 & $-19.6+1125$ & $18.1660555 \times 10^{8}$ & $-.25000000 \times 10^{8}$ \\
Order 2 & -10.6569029 & $8.4777719 \times 10^{8}$ & $-8.4777766 \times 10^{8}$ \\
Order 5 & -4.9017805 & $1.0408634 \times 10^{8}$ & $-1.04086+40 \times 10^{8}$ \\
Order 10 & -4.7004402 & $.25000715 \times 10^{8}$ & $-.25000727 \times 10^{8}$ \\
Order 12 & & $.24999291 \times 10^{8}$ & $-.25001311 \times 10^{8}$
\end{tabular}

REMARK ON ALGORITHM 60 [D1]

ROMBERG INTEGRATION [F. L. Bauer, Comm. ACM 4 (June 1961) 255; 5 (Mar. 1962), 168; 5 (Nay 1962), 281]

Henry C. Thacher, Jr.* (Recd. 20 Feb. 1964 and 23 Mar.. 1964)

Argonne National Laboratory, Argonne, Ill.

- Work supported by the U. S. Atomic Energy Commission.

The Romberg integration algorithm has been used with great success by many groups $[1,2]$, and appears to be among the most generally reliable quadrature methods available. It is, therefore, worth pointing out that it is not entirely foolproof, and that a significant class of in tegrands exists for which the extrapolated values are poorer estimates of the integral than the corresponding trapezoidal sums.

The validity of the Romberg procedure depends upon the possibility of expanding the error of the trapezoidal rule in powers of $h^{2}$, where $h$ is the stepsize. One expansion of this type is the EulerMaclaurin sum formula. An alternative expression may be obtained from the Fourier series expansion. The coefficients of $h^{2 r}$ in the Euler Maclaurin formula are proportional to the difference of the values of the $(2 r+1)$-th derivative at the two ends of the range. Thus, any integral for which the odd derivatives of the integrand either vanish or are equal at the limits will not be improved by Romberg extrapolation. Among the common examples of such integrals are integrals of periodic functions over a period and integrals for which the derivatives vanish at both limits. An example of the last type is the integral approximation to the modified Hankel function [3], $e^{x} K_{p}(x)=\int_{0}^{L} e^{x(1-\cosh t)} \cosh (p t) d t$, where $L$ is taken so large that the contribution of the integral from $L$ to $\infty$ may be neglected. Several other examples are given under the heading "Exceptional cases" by Bauer, Rutishauser and Stiefele [7]. This paper is among the most extensive discussions of the Romberg method in English.

The algorithm also fails when the expansion of the error term contains other powers of $h$ along with the even ones. Rutishauser [4] discusses estimating integrals of the form $\int_{0}^{a} f(x) d x=$ $\int_{0}^{a}(\varphi(x) / \sqrt{ } x) d x$. If such integrals are estimated by the trapezoidal 
rule, assigning the value 0 to $f(0)$, the error may be expressed in the form $\sum c_{k} h^{2 t}+\sqrt{ } h \sum d_{k} h^{k}$. Although the standard Romberg extrapolation fails when applied to this sequence of estimates, Rutishauser presents a modified procedure which is effective.

The extrapolation is also invalid when the integrand is discontinuous, although this exception is trivial from the computational standpoint.

It has also been pointed out $[5,6]$ that the Romberg procedure may amplify round-off errors. The losses, while significant, do not appear prohibitive for most applications.

REFERENCES:

1. Thacher, H. C., JR. Certification of algorithm 60. Comm. ACM 5 (Mar. 1962), 168.

2. Buchner, K. H. Certification of algorithm 60. Comm. ACM 5 (May, 1962), 281.

3. Fetris, H. E. Algorithm 163, modified Hankel function. Comm. ACM 6 (Apr. 1963), 161-2; 6 (Sep. 1963), 522.

4. Rutishauser, $H$. Ausdehnung des Rombergschen Prinzips. Numer. Math. 6 (1963), 48-54.

5. McKequan, W. M. Personal communication, Sept. 1963.

6. Engeli, M. Personal communication, Jan. 1964.

7. Bauer, F. L., Rutighausfir, H., and Stiefele, E. New aspects in numerical quadrature. Proc. Symp. Appl. Math 15, $1863,192-218$.

\section{ALGORITHM 61 \\ PROCEDURES FOR RANGE ARITHMETIC \\ Allan GibB* \\ University of Alberta, Calgary, Alberta, Canada \\ begin \\ procedure RANGESUM (a, b, c, d, e, f); \\ real $a, b, c, d, e, f$;}

comment The term "range number" was used by P.S. Dwyer, Linear Computations (Wiley, 1951). Machine procedures for range arithmetic were developed about 1958 by Ramon Moore, "Automatic Error Anslysis in Digital Computation," LMSD Report 48421, 28 Jan. 1959, Lockheed Missiles and Space Division, Palo Alto, California, 59 pp. If a $\leqq x \leqq b$ and $c \leqq y \leqq d$, then RANGESUM yields an interval $[e, f]$ such that $e \leqq(x+y)$ $\leqq f$. Because of machine operation (truncation or rounding) the machine sums $a+c$ and $b+d$ may not provide safe end-points of the output interval. Thus RANGESUM requires a non-local real procedure ADJUSTSUM which will compensate for the machine arithmetic. The body of ADJUSTSUM will be dependent upon the type of machine for which it is written and so is not given here. (An example, however, appears below.) It is assumed that ADJUSTSUM has as parameters real $v$ and $w$, and integer $i$, and is accompanied by a non-local real procedure CORRECTION which gives an upper bound to the magnitude of the error involved in the machine representation of a number. The output ADJUSTSUM provides the left end-point of the output interval of RANGESUM when ADJUSTSUM is called with $\mathrm{i}=-1$, and the right end-point when called with $\mathrm{i}=1$. The procedures RANGESUB, RANGEMPY, and RANGEDVD provide for the remaining fundamental operations in range arithmetic. RANGESQR gives an interval within which the square of a range number must lie. RNGSUMC, RNGSUBC, RNGMPYC and RNGDVDC provide for range arithmetic with complex range arguments, i.e. the real and imaginary parts are range numbers;

begin

e : = ADJUSTSUM $(a, c,-1)$;

$f:=\operatorname{ADJUSTSUM}(b, d, 1)$

end RANGESUM. procedure RANGESUB ( $a, b, c, d, e, f)$;

real $a, b, c, d, e, f$;

comment RANGESUM is a non-local procedure;

begin

RANGESUM $(a, b,-d,-c, e, f)$

end RANGESUB;

procedure RANGEMPY (a, b, c, d, e, f);

real $a, b, c, d, e, f$;

comment ADJUSTPROD, which appears at the end of this procedure, is analogous to ADJUSTSUM above and is a nonlocal real procedure. MAX and. MIN find the maximum and minimum of a set of real numbers and are non-local;

begin

real $v, w$

if $a<0 \wedge c \geqq 0$ then

1: begin

$$
\mathrm{v}:=
$$

end 1 ;

if $a \geqq 0$ then

2: begin if $\mathrm{c} \geqq 0$ then

3:begin

$e:=a \times c ; f:=b \ddot{x} d ;$ goto 8

end 3;

$\mathrm{e}:=\mathrm{b} \times \mathrm{c}$;

if $d \geqq 0$ then

4: begin

$f:=b \times d ; g \circ$ to 8

end 4;

$f:=\mathrm{a} \times \mathrm{d} ;$ goto 8

5: end 2;

if $b>0$ then

6: begin

if $d>0$ then

begin

$e:=\operatorname{MIN}(a \times d, b \times c)$;

$f:=\operatorname{MAX}(a \times c, b \times d) ;$ go to 8

end 6 ;

$e:=b \times c ; f:=a \times c ;$ go to 8

end 5 ;

$f:=a \times c$;

if $\mathrm{d} \leqq 0$ then

7: begin

e : $=b \times d ;$ go to 8

end 7 ;

e $:=a \times d$

8: $\mathrm{e}:=$ ADJUSTPROD $(\mathrm{e},-1)$

$f:=\operatorname{ADJUSTPROD}(f, 1)$

end RANGEMPY;

procedure RANGEDVD (a, b, c, d, e, f);

real $a, b, c, d, e, f$;

comment If the range divisor includes zero the program exists to a non-local label "zerodvsr". RANGEDVD assumes a non-local real procedure ADJUSTQUOT which is analogous (possibly identical) to ADJUSTPROD;

begin

if $\mathrm{c} \leqq 0 \wedge \mathrm{d} \geqq 0$ then go to zerodvsr;

if $\mathrm{c}<0$ then

1: begin

if $b>0$ then

2: begin

e : $=\mathrm{b} / \mathrm{d} ;$ go to 3

end 2;

$\mathrm{e}:=\mathrm{b} / \mathrm{c}$;

3: if $a \geqq 0$ then

4: begin

$f:=a / c ;$ go 108

end 4 ;

$f:=a / d ;$ go io 8

end 1; 


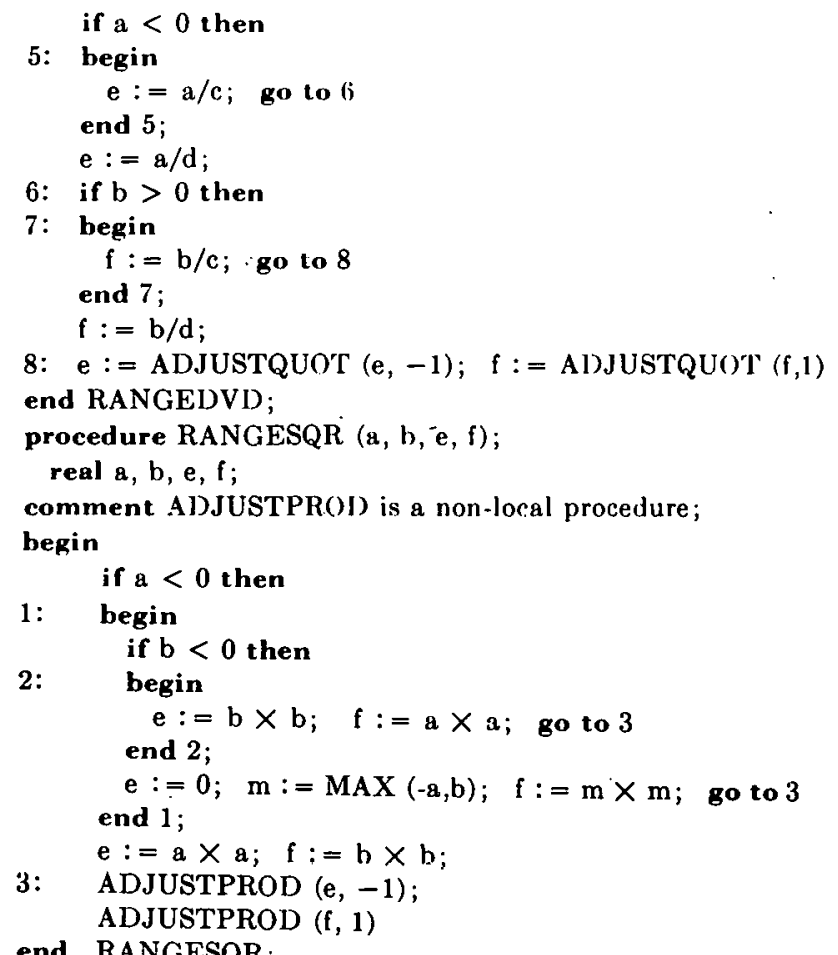

end RANGESQR;

procedure RNGSUMC (aL, aR, bL, bU; cL, cR, dL, dU, eL, eR, $f L, f U)$

real aL, aR, bL, bU, cL, cR, dL, dU, eL, eR, fL, fU;

comment Rangesum is a non-lacal procedure;

begin

RANGESUM (aL, aR, cL, cR, eL, eR);

RANGESUM (bL, bU, dL, dU, fL, fU)

end RNGSUMC;

procedure RNGSUBC (aL, aR, bL, bU, cL, cR, dL, dU, eL, eR, $f \mathrm{~L}, \mathrm{fU}$ ) :

real aL, aR, bL, bU, cL, cR, dL, dU, eL, eR, fL, fU;

comment RNGSUMC is a non-local procedure;

begin

RNGSUMC (aL, aR, bL, bR, $-c R,-c L,-d U,-d L, e L, e R$, $\mathrm{fL}, \mathrm{fU}$ )

end RNGSUBC;

procedure RNGMPYC (aL, aR, bL, bU, cL, cR, dL, dU, eL, $e R, f L, f U$ );

real aL, aR, bL, bU, cL, cR, dL, dU, eL, eR, fL, fU;

comment RANGEMPY, RANGESUB, and RANGESUM are

non-local procedures;

begin

real $\mathrm{L} 1, \mathrm{R} 1, \mathrm{~L} 2, \mathrm{R} 2, \mathrm{~L} 3, \mathrm{R} 3, \mathrm{~L} 4, \mathrm{R} 4$;

RANGEMPY (aL, aR, cL, cR, L1, R1);

RANGEMPY (bL, bU, dL, dU, L2, R2);

RANGESUB (L1, R1, L2, R2, eL, eR);

RANGEMPY (aL, aR, dL, dU, L3, R3);

RANGEMPY (bL, bU, cL, cR, L4, R4);

RANGESUM (L3, R3, L4, R4, fL, fU);

end RNGMPYC;

procedure RNGDVDC (aL, aR, bL, bU, cL, cR, dL, dU, eL, $\mathrm{eR}, \mathrm{fL}, \mathrm{fU}$ );

real $a L, a R, b L, b U, c L, c R, d L, d U, e L, e R, f L, f U$;

comment RNGMPYC, RANGESQR, RANGESUM, and

RANGEDVD are non-local procedures;

begin

real L1, R1, L2, R2, L3, R3, L4, R4, L5, R5;

RNGMPYC (aL, aR, bL, bU, cL, cR, -dU, -dL, L1, R1, L2, R2);

RANGESQR (cL, cR, L3, R3);

RANGESQR (dL, dU, L4, R4);

RANGESUM (L3, R3, L4, R4, L5, R5);

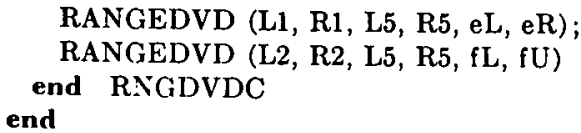

\section{EXAMPLE}

real procedure CORRECTION (p); real p;

comment CORRECTION and the procedures below are intended for use with single-precision normalized floating-point arithmetic for machines in which the mantissa of a floating-point number is expressible to s significant figures, base b. Limitations of the machine or requirements of the user will limit the range of $p+o b^{m} \leqq|p|<b^{n+1}$ for some integers $m$ and $n$. Appropriate integers must replace $\mathrm{s}, \mathrm{b}, \mathrm{m}$ and $\mathrm{n}$ below. Signal is a non-local label. The procedures of the example would be included in the same block as the range procedures above;

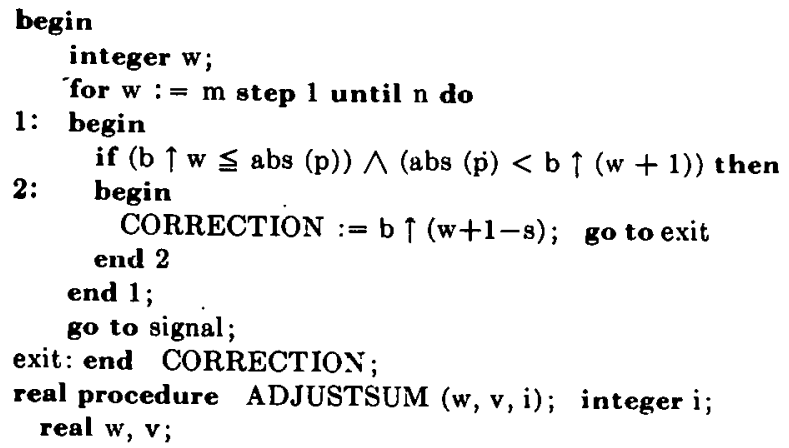
with machine which, when operating in floating point addition, simply shift out any lower order digits that may not be used. No attempt is made here to examine the possibility that every digit that is dropped is zero. CÓRRECTION is a non-local real procedure which gives an upper bound to the magnitude of the error involved in the machine representation of a number;

begin

real $\mathrm{r}, \mathrm{cw}, \mathrm{cv}, \mathrm{cr}$;

$\mathbf{r}:=\mathbf{w}+\mathbf{v}$

if $w=0 \vee v=0$ then go to 1

cw : = CORRECTION $(w)$;

cv $:=$ CORRECTION $(v)$;

cr $:=$ CORRECTION (r);

if $\mathrm{cw}=\mathrm{cv} \wedge \mathrm{cr} \leqq \mathrm{cw}$ then go to 1 ;

if sign ( $\times$ sign $(w) \times \operatorname{sign}(v) \times \operatorname{sign}(r))=-1$ then go to 1 ;

ADJUSTSUM : $=r+i \times \operatorname{MAX}(\mathrm{cw}, \mathrm{cv}, \mathrm{cr})$; go to exit;

1: ADJUSTSUM : $=r$;

exit: end ADJUSTSUM;

real procedure ADJUSTPROD $(p, i)$; real $p$; integer $i$;

comment ADJUSTPROD is for machines which truncate when lower order digits are dropped. CORRECTION is a non-local real procedure;

begin

if $p \times i \leqq 0$ then

1: begin

ADJUSTPROD $:=p ;$ go to out

end 1;

ADJUSTPROD : $=p+i \times$ CORRECTION $(p)$

out: end ADJUSTPROD;

comment Although ordinarily rounded arithmetic is preferable to truncated (chopped) arithmetic, for these range procedures truncated arithmetic leads to closer bounds than rounding does.

* These procedures were written and tested in the Burroughs 220 . version of the ALGOL language in the summer of 1960 at Stanford University. The typing and editorial work were done under Office of Naval Research Contract Nonr-225(37). The author wishes to thank Professor George E. Forsythe for encouraging this work and for assistance with the syntax of ALGOL 60 . 


\section{ALGORITHM 62}

A SET OF ASSOCIATE LEGENDRE POLYNOMIALS

OF THE SECOND KIND*

JohN R. HERndon

Stanford Research Institute, Menlo Park, California

comment This procedure places a set of values of $\mathrm{Q}_{\mathbf{n}}{ }^{\mathrm{m}}(\mathrm{x})$ in the array $Q[$ ] for values of $n$ from 0 to $n$ max for a particular value of $m$ and a value of $x$ which is real if $r i$ is 0 and is purely imaginary, ix, ortherwise. $R[$ ] will contain the set of ratios of successive values of $Q$. These ratios may be especially valuable when the $\mathrm{Q}_{\mathrm{u}}{ }^{\mathrm{m}}(\mathrm{x})$ of the smallest size is so small as to underflow the machine representation (e.g. $10^{-65}$ if $10^{-51}$ were the smallest representable number). $9.9 \times 10^{45}$ is used to represent infinity. Imaginary values of $x$ may not be negative and real values of $x$ may not be smaller than 1.

Values of $\mathrm{Q}_{\mathrm{n}}{ }^{\mathrm{m}}(\mathrm{x})$ may be calculated easily by hypergeometric series if $x$ is not too small nor $(n-m)$ too large. $Q_{n} m(x)$ can be computed from an appropriate set of values of $\mathrm{P}_{\mathrm{n}}{ }^{\mathrm{m}}(\mathrm{x})$ if $\mathrm{x}$ is near 1.0 or ix is near 0 . Loss of significant digits occurs for $x$ as small as 1.1 if $\mathrm{n}$ is larger than 10. Loss of significant digits is a major difficulty in using finite polynomial representations also if $n$ is larger than $m$. However, QLEG has been tested in regions of $x$ and $n$ both large and small;

procedure $Q L E G(m, n \max , x, r i, R, Q)$; value $m, n \max , x, r i$;

begin real $t, i, n, q 0, s$; real $m, n \max , x, r i$; real array $R, Q$;

$\mathbf{n}:=20$;

if $n \operatorname{nmax}>13$ then

$\mathrm{n}:=\mathrm{nmax}+7$

if ri $=0$ then

begin if $m=0$ then

$\mathrm{Q}[0]:=0.5 \times \log ((x+1) /(x-1))$ else

begin $t:=-1.0 / \operatorname{sqrt}(\mathrm{x} \times \mathrm{x}-1)$;

$\mathrm{q} 0:=0$

$\mathrm{Q}[0]:=\mathrm{t}$;

for $\mathrm{i}:=1$ step 1 until $m$ do

begin $s:=(x+x) \times(i-1) \times t$

$\times \mathrm{Q}[0]+(3 \mathrm{i}-\mathrm{i} \times \mathrm{i}-2) \times \mathrm{q} 0$;

$\mathrm{q} 0:=\mathrm{Q}[0]$

if $\quad \mathrm{x}=1$ then

$\mathrm{Q}[0]:=\mathrm{s}$ end end;

$\mathrm{Q}[0]:=9.9 \uparrow 45$;

$\mathbf{R}[\mathbf{n}+1]:=\mathbf{x}-\operatorname{sqrt}(\mathbf{x} \times \mathbf{x}-1)$;

for $i-:=n$ step -1 until 1 do

$R[i]:=(i+m) /((i+i+1) \times x$

$+(\mathrm{m}-\mathrm{i}-1) \times \mathrm{R}[\mathrm{i}+\mathbf{1}])$;

go to the end;

if $\mathrm{m}=0$ then

begin if $x<0.5$ then

$Q[0]:=\arctan (x)-1.5707963$ else

$\mathrm{Q}[0]:=-\arctan (1 / \mathrm{x})$ end else

begin $t:=1 / \operatorname{sqrt}(x \times x+1)$;.

q0 $:=0$;

$\mathrm{Q}[0]:=\mathrm{t}$;

for $\quad i:=2$ step 1 until $m$ do

begin $8:=(x+x) \times(i-1) \times t \times Q[0]$

$+(3 \mathrm{i}+\mathrm{i} \times \mathrm{i}-2) \times \mathrm{q} 0$;

$\mathrm{q} 0:=\mathrm{Q}[0]$

$\mathrm{Q}[0]:=\mathrm{s}$ end end;

$R[n+1]:=x-\operatorname{sqrt}(x \times x+1)$;

for $i:=n$ step -1 until 1 do

$R[i]:=(i+m) /((i-m+1) \times R[i+1] i$

$-(\mathrm{i}+\mathrm{i}+1) \times \mathrm{x})$;

for $i:=1$ step 2 until nmax do

$\mathrm{R}[\mathrm{i}]:=-\mathrm{R}[\mathrm{i}]$; the: for $\mathrm{i}:=1$ step 1 until nmax do

end QLEG;

$$
\mathrm{Q}[\mathrm{i}]:=\mathrm{Q}[\mathrm{i}-1] \times \mathrm{R}[\mathrm{i}]
$$

* This procedure was developed in part under the sponsorship of the Air Force Cambridge Research Center.

\section{REMARK ON ALGORITHM 62}

A SET OF ASSOCIATE LEGENDRE POLYNOMIALS

OF THE SECOND KIND (John R. Herndon, Comm.

$A C M$ \& (July, 1961))

JoHn R. HeRndon

Stanford Research Institute, Menlo Park, California

In regard to Algorithm 62 in Communications of the $A C M$, two errors were found:

The 14th line of the procedure

for $i:=1$ step 1 until $m$ do

should read

for $i:=2$ step 1 until $m$ do

The 35 th line

$$
+(3 \mathrm{i}-\mathrm{iXi}-2) \mathrm{Xq0}
$$

should read

$$
+(3 \mathrm{i}-\mathrm{i} \times \mathrm{i}-2) \times \mathrm{q} 0
$$

The procedure $Q L E C$ was developed from the standard recurrence formula

$$
(n+m-1) Q_{n-2}^{m}=(2 n-1) \cdot x \cdot Q_{n-1}^{m}-(n-m) Q_{n}{ }^{m} .
$$

Invert and multiply by $(n+m-1) Q_{n-1}^{m}$.

$$
\frac{Q_{n-1}^{m}}{Q_{n-2}^{m}}=\frac{(n+m-1)}{(2 n-1) \cdot x-(n-m) Q_{n}^{m} / Q_{n-1}^{m}},
$$

or

$$
R_{n-1}^{m}=\frac{(n+m-1)}{(2 n-1) \cdot x-(n-m) R_{n}{ }^{m}} .
$$

Analysis (and testing) shows that, for $n$ large, this infinite continued fraction need only be carried to about eight terms for eightdigit accuracy if the final term is evaluated with the asymptotic value derived by setting

$$
R_{n-1}^{m}=R_{n}{ }^{m}, \lim _{n \rightarrow \infty} R_{n}{ }^{m}=x \pm \sqrt{x^{2}-1},
$$

the minus sign being chosen since in general $Q_{n}{ }^{m}<Q_{n-1}^{m}$. The formulas pertaining to purely imaginary parameters follow readily. The value of

$$
Q_{0}{ }^{0}(x)=\frac{1}{2} \log _{e} \frac{x+1}{x-1}
$$

while

$$
Q_{1}{ }^{0}(x)=x \cdot Q_{0}{ }^{0}(x)-1
$$

and

$$
Q_{0}^{\prime}(x)=\frac{-1}{\sqrt{x^{2}-1}} .
$$

Other values are derived using the ratios $R_{n}{ }^{m}(x)$ and/or the recurrence formula

$$
{Q_{n}{ }^{m}}=-\frac{2(m-1) x}{\sqrt{x^{2}-1}} Q_{n}^{m-1}+(n-m+2)(n+m-2) Q_{n}^{m-2} .
$$

The derivation of the expression for $Q_{0}{ }^{\circ}(i x)$ is not trivial and proceeds as follows: 


$$
\begin{aligned}
i \cdot Q_{0}{ }^{\circ}(i x) & =\frac{1}{2} \log _{e} \frac{i x+1}{i x-1}=\frac{1}{2} \log _{e}\left[-\frac{x^{2}-1}{x^{2}+1}+\frac{2 x}{x^{2}+1}\right] \\
e^{a+i b} & =e^{a} \cdot e^{i b}=e^{a} \cos b+i \sin b .
\end{aligned}
$$

Thus

$$
\tan b=\frac{-2 x}{1-x^{2}}
$$

and

$$
Q_{0}{ }^{0}(i x)=(\arctan x-\pi / 2) i .
$$

\section{ALGORITHM 63}

\section{PARTITION}

\section{A. R. Hoare}

Elliott Brothers Ltd., Borehamwood, Hertfordshire, Eng.

procedure partition $(A, M, N, I, J)$; value $M, N$;

array $A$; integer $M, N, I, J$;

comment $I$ and $J$ are output variables, and $A$ is the array (with subscript bounds $M: N$ ) which is operated upon by this procedure. Partition takes the value $X$ of a random element of the array $A$, and rearranges the values of the elements of the array in such a way that there exist integers $I$ and $J$ with the following properties:

$\mathrm{M} \leqq \mathrm{J}<\mathrm{I} \leqq \mathrm{N}$ provided $\mathrm{M}<\mathrm{N}$

$A[R] \leqq X$ for $M \leqq R \leqq J$

$A[R]=X$ for $J<R<I$

$A[R] \geqq X$ for $I \leqq R \leqq N$

The procedure uses an integer procedure random $(M, N)$ which chooses equiprobably a random integer $F$ between $M$ and $N$, and also a procedure exchange, which exchanges the values of its two parameters;

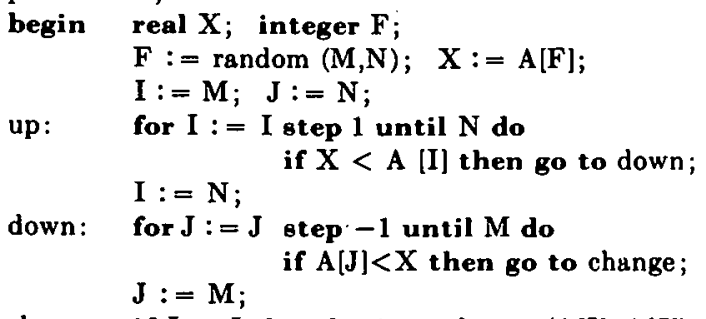

change: if $I<J$ then begin exchange $(A[I], A[J])$; $\mathbf{I}:=\mathbf{I}+\mathbf{1} ; \mathbf{J}:=\mathbf{J}-\mathbf{1}$ go to up

end

else if $I<F$ then begin exchange (A[I], $A[F]$ );

$$
I:=I+1
$$

end

else if $\mathrm{F}<\mathrm{J}$ then begin exchange $(\mathrm{A}[\mathrm{F}], \mathrm{A}[\mathrm{J}])$

$$
\mathrm{J}:=\mathrm{J}-1
$$

end ;

end partition
CERTIFICATION OF ALGORITHMS 63, 64, 65

PARTITION, QUICKSORT, FIND [C. A. R. Hoare, Comm. ACM, July 1961]

J. S. Hillmore

Elliott Bros. (I.ondon) Ltd., Borehamwood, Herts., England

The body of the procedure find was corrected to read:

begin integer $I, J$;

if $M<N$ then begin partition $(A, M, N, I, J)$;

if $K \leqq I$ then find $(A, M, J, K)$

else if $J \leqq K$ then find $(A, I, N, K)$

end find end

and the trio of procedures was then successfully run using the Elliott A LGol translator on the National-Elliott 803.

The author's estimate of $\frac{1}{3}(N-M) 1 n(N-M)$ for the number of exchanges required to sort a random set was found to be correct. However, the number of comparisons was generally less than $2(N-M) 1 n(N-M)$ even without the modification mentioned below.

The efficiency of the procedure quicksort was increased by changing its body to read:

begin integer $I, J$;

if $M<N-1$ then begin partition $(A, M, N, I, J)$; quicksort $(\mathcal{A}, M, J)$; quicksort $(A, I, N)$

end

else if $\dot{N}-M=1$ then begin if $A[N]<A[M]$ then

end exchange $(A[M], A[N])$

end quicksort

This alteration reduced the number of comparisons involved in sorting a set of random numbers by $\$-5$ percent, and the number of entries to the procedure partition by $25-30$ percent.

CERTIFICATION OF ALGORITHMS 63, 64 AND 65, PARTITION, QUICKSORT, A.ND FIND, [Comm. $A C M$, July 1961]

B. Randell and L. J. Russell

The English Electric Company Ltd., Whetstone, England

Algorithms 63, 64, and 65 have been tested using the Pegasus Algol 60 Compiler developed at the De Havilland Aircraft Company Ltd., Hatfield, England.

No changes were necessary to Algorithms 63 and 64 (Partition and Quicksort) which worked satisfactorily. However, the comment that Quicksort will sort an array without the need for any extra storage space is incorrect, as space is needed for the organization of the sequence of recursive procedure activations, or, if implemented without using recursive procedures, for storing information which records the progress of the partitioning and sorting.

A misprint ('if' for 'if' on the line starting 'else if $J \leqq K$ then ..') was corrected in Algorithm 65 (Find), but it was found that in certain cases the sequence of recursive activations of Find would not terminate successfully. Since Parlition produces as output two integers $J$ and $I$ such that elements of the array $A[M: N]$ which lie between $A[J]$ and $A[I]$ are in the positions that they will occupy when the sorting of the array is completed, Find should cease to make further recursive activations of itself if $K$ fulfills the condition $J<K<I$.

Therefore the conditional statement in the body of Find was changed to read

if $K \leqq J$ then find $(A, M, J, K)$

else if $I \leqq K$ then find $(A, I, N, K)$

With this change the procedure worked satisfactorily. 


\section{ALGORITHM 64 \\ QUICKSORT}

C. A. R. HoAre

Elliott Brothers Ltd., Borehamwood, Hertfordshire, Eng.

procedure quicksort $(A, M, N)$; value $M, N$; array $A$; integer $M, N$;

comment Quicksort is a very fast and convenient method of sorting an array in the random-access store of a computer. The entire contents of the store may be sorted, since no extra space is required. The average number of comparisons made is $2(M-N)$ In $(N-M)$, and the average number of exchanges is one sixth this amount. Suitable refinements of this method will be desirable for its implementation on any actual computer;

begin integer $I, J$;

if $\mathrm{M}<\mathrm{N}$ then begin partition $(A, M, N, I, J)$; quicksort $(A, M, J)$; quicksort $(A, I, N)$

end

end quicksort

SEE ALGORITHM 63 .

\section{ALGORITHM 65}

FIND

C. A. R. Hoare

Elliott Brothers Ltd., Borehamwood, Hertfordshire, Eng.

procedure find $(A, M, N, K)$; value $M, N, K$;

array $A$; integer $M, N, K$;

comment Find will assign to $A[K]$ the value which it would have if the array $A[M: N]$ had been sorted. The array $A$ will be partly sorted, and subsequent entries will be faster than the first; begin

integer $\mathrm{I}, \mathrm{J}$;

if $\mathrm{M}<\mathrm{N}$ then begin portition $(A, M, N, I, J)$;

if $K \leqq I$ then find $(A, M, I, K)$

else if $\mathrm{J} \leqq K$ then find $(A, J, N, K)$

end find

end

SEE AIGORITHM 63

\section{ALGORITHM 66}

INVRS

JoHN CAFFReY

Director of Research, Palo Alto Unified School District, Palo Alto, California

procedure Invrs $(t)$ size $:(n)$; value $n$; real array $t$; integer $\dot{n}$;

comment Inverts a positive definite symmetric matrix $t$, of order $n$, by a simplified variant of the square root method. Replaces the $n(n+1) / 2$ diagonal and superdiagonal elements of $t$ with elements of $t^{-1}$, leaving subdiagonal elements unchanged. Advantages: only $n$ temporary storage registers are required, no identity matrix is used, no square roots are computed, only $n$ divisions are performed, and, as $\mathbf{n}$ becomes large, the number of multiplications approaches $\mathrm{n}^{3} / 2$;

begin integer $i, j, s$; real array $v \mid l: n-1)$; real $y$, pivot;

for $s:=0$ step 1 until $n-1$ do

hegin pivot $:=1.0 / t[1,1]$;

begin pivot $:=1.0 / t[1,1]$;

comment If $t[1,1] \leqq 0, t$ is not positive definite;

for $i:=2$ step 1 until $n$ do $v[i-1]:=t[1, i]$;

for $i:=1$ step 1 until $n-1$ do

begin $t[i, n]:=y:=-v[i] \times$ pivot;

for $j:=i$ step 1 until $n-1$ do

$\mathrm{t}[\mathrm{i}, \mathrm{j}]:=\mathrm{t}[\mathrm{i}+1, \mathrm{j}+1]+\mathrm{v}[\mathrm{j}] \times \mathrm{y}$

end;

$\mathrm{t}[\mathrm{n}, \mathrm{n}]:=-$ pivot

end;

comment At this point, elements of $\mathrm{t}^{-1}$ occupy the original array space but with signs reversed, and the following statements effect a simple reflection;

for $i:=1$ step 1 until $n$ do

for $j:=i$ step 1 until $n$ do $t[i, j]:=-t[i, j]$

end Invrs

\section{CERTIFICATION OF ALGORITHM 66}

INVRS (J. Caffrey, Comm. ACM. July 1961)

B. Randell, C. G. Broyden.

Atomic Power Division, The English Electric Company, Whetstone, England.

INVRS was translated using the Deuce Algol Compiler, and needed the following correction.

The repeat of the line,

$$
\text { begin pivot := } 1.0 / \mathrm{t}[1,1] \text {; }
$$

was deleted.

The compiled program, which used a 20 bit mantissa floating point notation, was tested using Wilson's matrix

$\begin{array}{rrrr}5 & 7 & 6 & 5 \\ 7 & 10 & 8 & 7 \\ 6 & 8 & 10 & 9 \\ 5 & 7 & 9 & 10\end{array}$

and gave results

$\begin{array}{rrrr}67.9982 & -40.9991 & -16.9995 & 9.9997 \\ -40.9991 & 24.9995 & 9.9997 & -5.9998 \\ -16.9995 & 9.9997 & 4.9998 & -2.9999 \\ 9.9997 & -5.9998 & -2.9999 & 1.9999\end{array}$

(The output routine completed the symmetric matrix)

INVRS will in fact invert non-positive symmetric matrices, the only restriction appearing to be that the leading minors of the matrix must be non-zero. The variable $T[1,1]$ takes as its successive values ratios of the $(r+1)$ th to the $r$ th leadng minors of the matrix, and if it becomes zero the variable 'pivot' cannot be computed.

The following matrix, for which the successive values of $T[1,1]$ were $+2,-2,-1,-0.6,+5$ gave results correct to one unit in the fifth significant figure.

$\begin{array}{rrrrr}2 & -3 & 1 & -1 & 4 \\ -3 & 2 & -4 & 3 & -2 \\ 1 & -4 & -3 & 2 & 4 \\ -1 & 3 & 2 & -2 & -3 \\ 4 & -2 & 4 & -3 & 2\end{array}$


CERTIFICATION OF ALGORITHM 66

INVRS (J. Caffrey, Comm. ACM, July 1961)

JohN CAFFrey

Palo Alto Unified School District, Palo Alto, California

INVRS was translated using the Burroughs 220 Algebraic Computer (BALCOM) at Stanford University, using 8-digit floatingpoint arithmetic. The misprint noted by Randell and Broyden (Comm. ACM, Jan. 1962, p. 50) was corrected, and the same example (Wilson's $4 \times 4$ matrix) was used as a test case. The resulting inverse was:

$$
\begin{array}{rrrr}
68.0000 & -41.0000 & -17.0000 & 10.0000 \\
& 25.0000 & -10.0000 & -6.0000 \\
& & 5.0000 & -3.0000 \\
& & & 2.0000
\end{array}
$$

It may also be useful to note that the determinant of the matrix may be obtained as the successive product of the pivots. That is, if $t_{i}(=T(1,1))$ is the $i$ th pivot of a matrix of order $n$,

For the above input example,

$$
\text { determinant }=\prod_{i}^{n} t_{i} \text {. }
$$

$$
\text { determinant }=1.0
$$

Randell and Broyden's observation concerning the apparent limitation of INVRS to positive definite cases is correct: That is, any nonsingular real symmetric matrix (positive, indefinite, or negative) may be inverted using this algorithm. The original INVRS should therefore be modified as follows:

$$
\text { if pivot }=0 \text { then go to singular; }
$$

Randell and Broyden's second example (of order 5) was also used as a test case, with the resulting inverse:

$$
\begin{array}{rrrrr}
-.0000 & .9999 & .0000 & .0000 & .9999 \\
& 1.5333 & -.7333 & -.1333 & .7999 \\
& & -.8666 & -1.0666 & -.5999 \\
& & . & -1.4666 & -.1999
\end{array}
$$

.2000

determinant $=-14.999999$

An attempt to invert the inverse of the $4 \times 4$ segment of the Hilbert matrix, as presented by Randell (Comm. ACM, Jan. 1962 , p. 50), yielded the following results:

$\begin{array}{llll}.9999 & .4999 & .3333 & .2499 \\ & .3333 & .2499 & .1999 \\ & & .1999 & .1666 \\ & & & .1428\end{array}$

determinant $=6048020.6$

\footnotetext{
ALGORITHM 67

CRAM

JoHN CAFFrey

Director of Research, Palo Alto Unified School District, Palo Alto, California

procedure $\operatorname{CRAM}(n, r, a)$ Result: (f); value $n, r$; integer $n, r$; real array $a, f$;

comment CRAM stores, via an unspecified input procedure READ, the diagonal and superdiagonal elements of a square symmetric matrix e, of order $n$, as a pseudo-array of dimension $1: n(n+1) / 2$. READ $(u)$ puts one number into $u$. Elements $e[i, j]$ are add ressable as $a[c+j]$, where $c=(2 n-i)(i-1) / 2$ and $c[i+1]$ may be found as $c[i]+n-i$. Since $c[1]=0$, it is simpler to develop a table of the $c[i]$ by recursion, as shown in the sequence labelled "table". Further manipulation of the elements so stored is illustrated by premultiplying a rectangular matrix $f$, of order $n, r$, by the matrix e, replacing the elements of $f$ with the new values, requiring a temporary storage array $v$ of dimension $1: n$;
}

begin integer $i, j, k, m$; real array $v[1: n] ;$ real $s$;

integer array $c[1: n]$;

table: $\mathrm{j}:=-\mathrm{n} ; \mathrm{k}:=\mathrm{n}+1 ;$ for $\mathrm{i}:=1$ step 1 until $\mathrm{n}$ do begin

$\mathrm{j}:=\mathrm{j}+\mathrm{k}-\mathrm{i} ; \quad \mathrm{c}[\mathrm{i}]:=\mathrm{j}$ end;

load: for $i:=1$ step 1 until $n$ do

begin for $\mathrm{j}:=\mathrm{i}$ step 1 until $\mathrm{n}$ do $\operatorname{READ}(\mathrm{v}[\mathrm{j}]) ; \mathrm{m}:=$ c[i];

for $\mathrm{k}:=\mathrm{i}$ step $\mathrm{l}$ until $\mathrm{n}$ do $\mathrm{a}[\mathrm{m}+\mathrm{k}]:=\mathrm{v}[\mathrm{k}]$ end;

premult: for $\mathrm{j}:=1$ step 1 until $\mathrm{r}$ do

begin for $i:=1$ step 1 until $n$ do

begin $s:=0.0$;

for $k:=1$ step 1 until $i$ do

hegin $\mathrm{m}:=\mathrm{c}[\mathrm{k}] ; \mathrm{s}:=\mathrm{s}+\mathrm{a}[\mathrm{m}+\mathrm{i}]$

$X f[k, j]$ end;

for $k:=i+1$ step 1 until $n$ do end;

$$
s:=s+a[m+k] \times f[k, j] ; \quad v[i]=s
$$

for $k:=1$ step 1 until $n$ do $f[k, j]=v[k]$

end CRAM

end

\section{CERTIFICATION OF ALGORITHM 67}

CRAM (J. Caffrey, Comm. ACM 4 (July 1961), 322)

A. P. RELPH

Atomic Power Divi, The Finglish Electric Co., Whetstone, England

CRAM was translated using the Devce Algol compiler with the following corrections:

$$
\mathrm{V}[\mathrm{i}]=\mathrm{S} \text { was changed to } \mathrm{V}[\mathrm{i}]:=\mathrm{S}
$$

$\mathrm{f}[\mathrm{k}, \mathrm{j}]=\mathrm{V}[\mathrm{k}]$ was changed to $\mathrm{f}[\mathrm{k}, \mathrm{j}]:=\mathrm{V}[\mathrm{k}]$

It is quicker not to use the table of the Cli] in the "load" sequence and instead use the following sequence:

load: $m:=n \times(n+1) / 2$;

for $\mathrm{i}:=1$ step 1 until $m$ do $\operatorname{READ}(\mathrm{a}[\mathrm{i}])$;

\section{ALGORITHM 68 \\ AUGMENTATION}

H. G. RICE

Computer Sciences Corp., Palos Verdes, Calif.

real procedure $\operatorname{Aug}(x, y)$; value $x, y$; integer $x, y$;

comment This algorithm makes use of the implicitly defined recursive properties of AlgOL procedures to compute the augment of $x$ by $y$, using the basic technique of incrementation by unit step size;

begin Aug : $=$ if $\mathrm{x}=0$ then (if $\mathrm{y}>\mathrm{x}$ then (Aug $(\mathrm{y}-1, \mathrm{x})+1$ ) else $y$ )

else Aug $(x-1, y+1)$ end Aug

\section{CERTIFICATION OF ALGORITHM 68}

AUGMENTATION (H. G. Rice, Comm. $A C M$, Aug. 1961)

L. M. Breed

Stanford University, Stanford, Calif.

AUGMENTATION was transliterated into BALGOL for the Burroughs 220, and proved successful in a number of test cases. However, the following algorithm has exactly the same effect and is considerably simpler:

real procedure $\operatorname{Aug}(x, y)$; value $x, y$; integer $x, y$;

begin if $x<0$ then $L$ : go to L else Aug : $=x+y$ end Aug 
ALGORITHM 69

CHAIN TRACING

Brian H. Mayoh

Regnecentralen, Gl. Carlsbergvet. 2, Copenhagen.

procedure CHAIN tracing (iteration counter, number of identifiers, number of identifier links, final-linkage matrix, couples);

Boolean array final linkage matrix;

integer array couples;

integer iteration counter, number of identifiers, number of identifier links;

begin comment This procedure is given a list of pairs of integers, the second being related to the first in some way. It finds those pairs of integers which are related to esch other if the relation is transitive. It is supplied with,

couples a matrix whose bound pairlist is $[1: 2,1:$ number of identifier links] where couples $[2, \mathrm{i}]$ is related to couples $[1, i]$ in some way.

final linkage matrix a matrix whose bound pair list in [1:number of identifiers, 1 :number of identifiers] and into which the proceduro puts true if the second subscript expression is an integer which is related to the integer corresponding to the first subscript expression, if the relation is irreflexive then the diagonal entries of this matrix are false.

iteration counter a place for the procedure to put the length of the longest chain it finds. CHAIN tracing can be applied to any system which can be represented by a Turing machine by letting the integers in couples correspond to the Turing machine states. Two integers $j, k$ are related if there is an input symbol which causes state $j$ to change to state $k$. If the Turing machine always stops whatever the sequence of input symbols, then its final linkage matrix will have false for all leading diagonal entries;

integer $i, j$;

Boolean array working linksge matrix [1: number of identifiers: 1 :number of identifiers];

Boolean procedure PROGRESS;

begin PROGRESS := false;

for $i:=1$ step 1 until number of identifiers

do for $j:=1$ step 1 until number of identifiers

do begin if Working linkage matrix $[i, j]=\neg$ Final

linkage matrix $[i, j]$ then PROGRE\&S :- true;

Final linkage matrix [i, j] :- Working linkage matrix $[i, j]$

end of comparison

end of PROGRESS;

BEGIN OF PROGRAM:

for iteration counter : $=-1,0$, iteration counter +1 while PROGRESS

do for $i:=1$ step 1 until number of identifier links

do for $j:=1$ step 1 until number of identifiers

do begin if iteration number $=-1$

then Final linkage Matrix [couples $[1, i], j]$

$:=$ Working linkage Matrix [couples $[1, \mathrm{i}], \mathrm{j}]$

$:=$ couples $[2, i]=j$

else Working linkage Matrix [couples $[1, i], j]$ $:=$ Working linkage Matrix [couples $[1, \mathrm{i}], \mathrm{j}]$ $\checkmark$ Working linkage Matrix [couples $[2, \mathrm{i}], \mathrm{j}]$;

end of CHAIN tracing;
ALGORITHM 70

INTERPOLATION BY AITKEN

Charles J. Mifsud

General Electric Co., Bethesda, Md.

procedure $\operatorname{AITKEN}(x, f, n, X, F)$; real array $x, f$; integer $n$; real $X, F$;

comment If given $x_{0}, x_{1}, \ldots x_{n}, n+1$ abscissas and also given $f\left(x_{0}\right), f\left(x_{1}\right), \ldots f\left(x_{n}\right), n+1$ functional values, this procedure generates a Lagrange polynomial, $F(X)$ of the $n$th degree so that $F\left(x_{i}\right)=f\left(x_{i}\right)$. Hence, for any given value $X$, a functional value $F(X)$ is generated. The procedure is good for either equal or unequal intervals of the $x_{\mathfrak{i}}$. Aitken's interative scheme is used in the generation of $F(X)$. Since the $f$ array is used for temporary storage, as the calculation proceeds its original values are destroyed;

begin integer $i, j, t$;

for $j:=0$ step 1 until $n-1$ do

begin $t:=j+1$

for $i:=t$ step 1 until $n$ do

$f[i]:=((X-x[j]) \times f[i]-(X-x[i]) \times f[j]) /$ $(x[i]-x[j])$ end

$F:=f[n]$

end

CERTIFICATION OF ALGORITHM 70

INTERPOLATION BY AITKEN (C. J. Mifsud, Commi. $A C M 4$ (Nov. 1961)]

A. P. RELPH

The English Electric Co., Whetstone, England

Algorithm 70 was translated using the DeUce ALGoL compiler and gave satisfactory results after semicolons had been added to

and

$$
\mathrm{t}:=\mathrm{j}+1 \text { to make it } \mathrm{t}:=\mathrm{j}+1 \text {; }
$$$$
(\mathrm{x}[\mathrm{i}]-\mathrm{x}[\mathrm{j}]) \text { end to make it }(\mathrm{x}[\mathrm{i}]-\mathrm{x}[\mathrm{j}]) \text { end; }
$$

The identifier $t$ can be eliminated and the algorithm shortened by the following changes:

Replace begin integer $\mathrm{i}, \mathrm{j}, \mathrm{t}$; by begin integer $\mathrm{i}, \mathrm{j}$;

Replace $\mathrm{t}:=\mathrm{j}+1$;

for $i:=t$ step 1 until

by for $\mathrm{i}:=\mathrm{j}+1$ step 1 until n do

\section{ALGORITHM 71}

PERMUTATION

R. R. Coveyou and J. G. Sullivan

Oak Ridge National Laboratory, Oak Ridge, Tenn.

procedure PERMUTATION (I, P, N);

value $I, N$; integer $N$; integer array $P$; boolean $I$;

comment This procedure produces all permutations of the integers from 0 thru $N$. Upon entry with $I=$ false the procedure initializes itself producing no permutation. Upon each successive entry into the procedure with $I=$ true a new permutation is stored in $\mathrm{P}[0]$ thru $\mathrm{P}[\mathrm{N}]$. When the process has been exhausted a sentinel is set: 


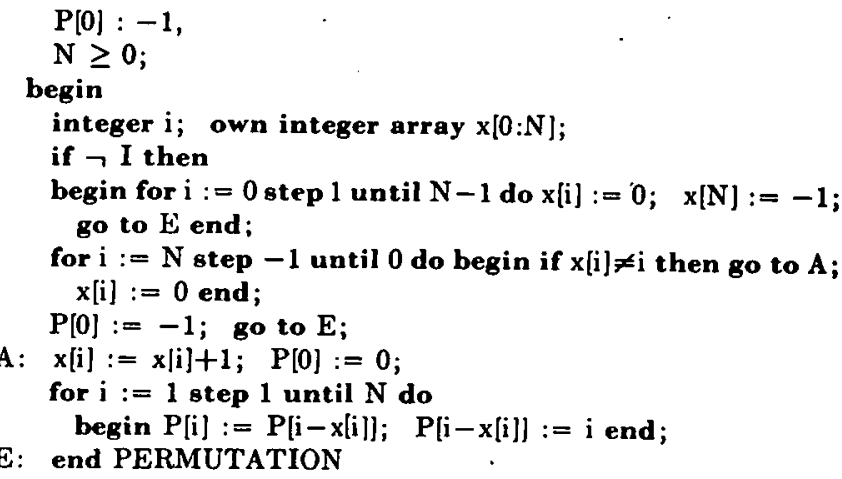

\section{CERTIFICATION OF ALGORITHM 71}

PERMUTATION (R. R. Coveyou and J. G. Sullivan, Comm. ACM, Nov. 1961)

P. J. Brown

University of North Carolina, Chapel Hill, N. C.

PERMUTATION was transliterated into GAT for the UNIvaC 1105 and successfully run for a number of cases.

\section{CERTIFICATION OF ALGORITHM 71}

PERMUTATION [R. R. Coveyou and J. G. Sullivan, Comm. $A C M$, Nov. 1961]

\section{J. S. Hillmore}

Elliott Bros. (London) Ltd., Borehamwood, Herts., England

The algorithm was successfully run using the Elliott AlgoL translator on the National-Elliott 803 . The integer array $x$ was made a parameter of the procedure in order to avoid having an own array with variable bounds.

\section{CERTIFICATION OF ALGORITHM 71}

PERMUTATION (R. R. Coveyou and J. G. Sullivan, Comm. ACM, Nov. 1961)

J. E. L. Peck and G. F. Schrack

University of Alberta, Calgary, Alberta, Canada

PERMUTATION was translated into Fortran for the IBM 1620 and it performed satisfactorily. The own integer array $x[0: n]$ may be shortened to $x[1: n]$, provided corresponding corrections are made in the first two for statements.

However, PERMUTE (Algorithm 86) is superior to PERMU. TATION in two respects

(1) PERMUTATION, using storage of order $2 \mathrm{n}$, is designed to permute the specific vector $0,1,2, \cdots, n-1$ rather than an arbitrary vector. Thus storage of order $3 n$ is required to permute an arbitrary vector. PERMUTE, in contrast, only needs storage of order $2 \mathrm{n}$ to permute an arbitrary vector.

(2) PERMUTE is built up from cyclic permutations. The number of permutations actually executed internally (the redundant ones are suppressed) by PERMUTE is asymptotic to $(e-1) n$ ! rather than $n$ !. In spite of this, PERMUTE is distinctly faster (1316 against 2823 seconds for $n=8$ ) than PERMUTATION. If $t_{n}$ is the time taken for all permutations of a vector with $n$ components, and if $r_{n}=t_{n} / n t_{n-1}$, then one would expect $r_{n}$ to be close to 1. Experiment with small values of $n$ gave the following results for $r_{n}$.

$\begin{array}{rccc}\text { n } & 6 & 7 & 8 \\ \text { PERMUTE } & 0.96 & 0.99 & 1.00 \\ \text { PERMUTATION } & 1.10 . & 1.13 & 1.12\end{array}$

Is there yet a faster way to do it?

See also: C. Tompkins, "Machine Attacks on Problems whose Variables are Permutations", Proceedings of Symposia in Applied Mathematics, Vol. VI: Numerical Analysis (N. Y., McGraw-Hill, 1956).
ALGORITHM 72

COMPOSITION GENERATOR

L. Hellerman and S. Ogden

IBM-Product Development Laboratory, Poughkeepsie, N. Y.

procedure comp (c, $k)$; value $k$; integer array $c$; integer $k$;

comment Given a $k$-part composition $\mathrm{c}$ of the positive integer $\mathrm{n}$, comp generates a consequent composition if there is one. If comp operates on each consequent composition after it is found, all compositions will be generated, provided that $1,1, \ldots, 1$, $n-k+1$ is the initial $c$. If $c$ is of the form $n-k+1,1,1, \ldots, 1$, there is no consequent, and $c$ will be replaced by a $k$ vector of 0's. Reference: John Riordan, An Introduction to Combinatorial Analysis, John Wiley and Sons, Inc., New York, 1958, Chapter 6;

begin integer $j$; integer array $d[1: k]$;

if $\mathrm{k}=1$ then go to last;

for $j:=1$ step 1 until $k$ do $d[j]:=c[j]-1$;

test: if $\mathrm{d}[\mathrm{j}]>0$ then go to set;

$\mathrm{j}:=\mathrm{j}-1$;

go to if $j=1$ then last else test;

Bet: $d[j]:=0$;

$d[j-1]:=d[j-1]+1$;

$\mathrm{d}[\mathrm{k}]:=\mathrm{e}[\mathrm{j}]-2$;

for $j:=1$ step 1 until $k$ do $c[j]:=d[j]+1$;

go to exit;

last: for $j:=1$ step 1 until $k$ do $c[j]:=0$;

exit: end comp

\section{CERTIFICATION OF ALGORITHM 72}

COMPOSITION GENERATOR [L. Hellerman and $\mathbf{S}$.

Ogden, Comm. ACM, Nov. 1961]

D. M. Collison

Elliott Bros. (London) Ltd., Borehamwood, Herts., England

After

the statement

$$
\text { for } j:=1 \text { step } 1 \text { until } k \text { do } d[j]:=c[j]-1 \text {; }
$$

$$
j:=k \text {; }
$$

should be inserted (see ALGOL 60 report, para 4.6.5). With this alteration, the algorithm was successfully run using the Elliott Algol translator on the National-Elliott 803.

\section{ALGORITHM 73}

\section{INCOMPLETE ELLIPTIC INTEGRALS}

David K. JeFreHSON

U. S. Naval Weapons Laboratory, Dahlgren, Virginia procedure ellint (k, phi, E, F);

value $k$, phi

real phi, F, $k, E$;

comment ellint computes the value of the incomplete elliptic integrals of the first and second kinds, $F(p h i, k)$ and $E$ (phi, $k$ ), where phi is in radians. If $|\mathrm{k}|>1$ or $|\mathrm{phi}|>\pi / 2, \mathrm{E}$ and $\mathrm{F}$ will be set equal to $100,000,000$, otherwise they will contain the computed integrals. For the formulation of this procedure, see DiDonato, A. R., and Hershey, A. V., "New Formulae for Computing Incomplete Elliptic Integrals of the First and Second Kind", J. ACM 6, 4 (Oct. 1959); 
begin real $\mathrm{kp}$, sinphi, $\mathrm{n}$, cosphi;

real array $H[1: 2], A[1: 2]$, sigma $[1: 4], L[1: 2], M[1: 2]$, $\mathrm{N}[1: 2], \mathrm{T}[1: 2]$, del $[1: 4]$;

sigma $[1]:=$ sigma $[2]:=$ sigma $[3]:=$ sigma $[4]:=0$;

H $[1]:=1$;

n : $=0$;

$\operatorname{sinphi}:=\sin (\mathrm{phi})$

if abs $(k \times$ sinphi) $\leq \tanh (1)$ then go to small else if abs $(k) \leq$ $1 \wedge \operatorname{abs}(\mathrm{phi}) \leq \pi / 2$ then go to large;

$\mathrm{E}:=\mathrm{F}:=100000000$;

go to stop;

small: $A[1]:=$ phi;

step 1: $\mathrm{n}:=\mathrm{n}+\mathbf{1}$;

$\operatorname{cosphi}:=\cos (\mathrm{phi})$;

$\mathrm{E}:=(2 \times \mathrm{n}-1) /(2 \times \mathrm{N})$;

$\mathrm{H}[2]:=\mathrm{E} \times \mathrm{k} \uparrow 2 \times \mathrm{H}[1]$;

$A \cdot[2]:=E \times A[1]-\operatorname{sinphi} \uparrow(2 \times n-1) \times$ cosphi $/(2 \times n)$; del [1] := H [2] $\times \mathrm{A}[2]$;

del $[2]:=-\mathrm{k} \uparrow 2 \times \mathrm{H}[1] \times \mathrm{A}[2] /(2 \times \mathrm{n})$;

. sigma [1] := sigma [1] $+\operatorname{del}[1]$;

sigma $[2]:=$ sigma $[2]+\operatorname{del}[2]$;

$\mathbf{H}[1]:=. \mathbf{H}[2]$;

$A[1]:=A[2] ;$

if abs ((sigma [1] $+\operatorname{del}[1])-$ sigma [1]) $>0 \wedge$ phi $\times$ sinphi $\uparrow(2 \times n) \geq A$ [2] then to step 1 ;

$F:=$ phi + sigms $[1]$

E := phi + sigma [2];

go to stop;

large: $k p:=$ sqrt $(1-k \uparrow 2)$

A $[1]:=1$

$L[1]:=M[1]:=N[1]:=0 ;$

step 2: $\mathbf{n}:=\mathrm{n}+1$

$\mathrm{E}:=(2 \times \mathrm{n}-1) /(2 \times \mathrm{n})$

F := abs $(k) \times$ sqrt $(1-\operatorname{sinphi} \uparrow 2) \times(1-k \uparrow 2 \times$ sinphi

$\uparrow 2) \uparrow((2 \times n-1) /(2 \times n))$

$\mathbf{H}[2]:=\mathbf{E} \times \mathbf{H}[1]$;

$A[2]:=E \uparrow 2 \times \mathrm{kp} \uparrow 2 \times A[1]$;

$\mathrm{L}[2]:=\mathrm{L}[1]+1 /(\mathrm{n} \times 2 \times \mathrm{n}-1))$;

$M[2]:=(M[1]-F \times H[2]) \times((2 \times n+1) /(2 \times n+2)) \uparrow 2 \times$ $\mathrm{kp} \uparrow 2$;

$\mathrm{N}[2]:=(\mathrm{N}[1]-\mathrm{F} \times \mathrm{H}[1]) \times \mathrm{E} \times(2 \times \mathrm{n}+1) \times \mathrm{kp} \uparrow 2 /(2 \times$ $\mathrm{n}+2)$;

$\operatorname{del}[1]:=M[2]-A[2] \times L[2] ;$

del [2] :=N [2] $-\mathbf{E} \times \mathbf{k p} \uparrow 2 \times \mathbf{A}[1] \times \mathrm{L}[2]+\mathrm{kp} \uparrow 2 \times A$ [1] $/((2 \times n) \uparrow 2)$;

$\operatorname{del}[3]:=A[2] ;$

$\operatorname{del}[4]:=(2 \times n+1) \times A[2] /(2 \times n+2)$;

sigma $[1]:=$ sigma $[1]+\operatorname{del}[1]$;

sigma [2] := sigma [2] + del [2];

sigma $[3]:=$ sigma $[3]+\operatorname{del}[3]$;

sigma $[4]:=$ sigma $[4]+\operatorname{del}[4]$;

$H[1]:=H[2]$;

A [1] := A [2];

L [1] := L [2];

$M[1]:=M[2]$

$N[1]:=N[2]$;

if abs ((sigma [1] $+\operatorname{del}[1])-\operatorname{sigma}[1])>0$ then go to step 2;

$\mathrm{T}[1]:=\ln (4 /(\mathrm{sqrt}(1-\mathrm{k} \uparrow 2 \times \operatorname{sinphi} \uparrow 2)+\operatorname{abs}(\mathrm{k}) \times \operatorname{sqrt}(1-$ sinphi $\uparrow 2))$;

$\mathrm{T}[2]:=$ abs $(\mathrm{k}) \times \operatorname{sqrt}((1-\operatorname{sinphi} \uparrow 2) /(1-\mathrm{k} \uparrow 2 \times \operatorname{sinphi} \uparrow 2))$;

$\mathrm{F}:=\mathrm{T}[1] \times(1+\operatorname{sigma}[3])+\mathrm{T}[2] \times \ln (.5+.5 \times$ abs $(\mathrm{k} \times$ sinphi)) + sigma $[1]$;

$\mathrm{E}:=(.5+\operatorname{sigma}[4]) \times \mathrm{kp} \uparrow 2 \times \mathrm{T}[1]+1-\mathrm{T}[2] \times(1-\mathrm{abs}$ $(\mathrm{k} \times$ sinphi)) + sigma [2];

stop: end
CERTIFICATION OF ALGORITHM 73

INCOMPLETE ELLIPTIC INTEGRALS (David K.

Jefferson, Comm. ACM, Dec. 1961)

Dean C. Kriebel

U. S. Naval Weapons Laboratory, Dahlgren, Virginia

This algorithm was originally coded in Nonc machine language and K. Pearson's incomplete elliptic integral tables of the first and second kind generated. (See DiDonato, A. R., and Hershey, A. V., "New Formulae for Computing Incomplete Elliptic Integrals of the First and Second Kind",.J.ACM 6, 4 (Oct. 1959)).

The algorithm was coded for the MAD Compiler exactly as written in ALGoL and run on an IBM 7090. Forty cases were computed with $\mathrm{K}$ ranging from $0^{\circ}$ to $90^{\circ}$ and $\mathrm{PHI}$ ranging from $0^{\circ}$ to $90^{\circ}$. The results contained eight significant digits which agreed with the DiDonato and Hershey tables to within 0 to 2 units in the 8 th digit. (This may be attributed to the decimal to binary, binary to decimal input-output conversion used with a binary computer as compared to straight decimal computation on the Nonc.)

\section{CERTIFICATION OF ALGORITHAI 73}

INCONIPLETE ELLIPTIC INTEGRAIS [David Ki

Jefferson, Comm. AC./ Dec. 1961]

R. P. VAN DE RIET

Mathematical Centre, Amsterdam

The algorithm contained three misprints:

The 26th line of the procedure

should read

$$
E:=(2 \times n-1) /\left(2 \times N^{\circ}\right)
$$

The 4(ith line of the procedure

$$
E:=(2 \times n-1) /(2 \times n) ;
$$

should read

$$
\uparrow 2) \uparrow((2 \times n-1) /(2 \times n)) \text {; }
$$

$\uparrow 2) \uparrow((2 \times n-1) / 2) /(2 \times n)$

The 4Gth line of the procedure

$$
L[2]:=L[4]+1 /(n \times 2 \times n-1)) \text {; }
$$

should read

$$
L[2]:=L[1]+1 /(n \times(2 \times n-1)) \text {; }
$$

The program was run on the $X 1$ computer of the Mathematical Centre. For $p h i=45^{\circ}, k=\sin \left(10^{\circ}\left(10^{\circ}\right) 180^{\circ}\right), E$ and $F^{\prime}$ were calculated. The result contained 12 significant digits.

Comparison with a 12-decimal table of Legendre-Limde (1931) showed that the 12th digit was affected with an error, at most 4 units large. After about 10 minutes of calculation (i.e. more than 100 cycles) no results were obtained for $k:=\sin 89^{\circ}$, phi $=1^{\circ}$ and the calculation was discontinued.

REMARKS. As phi is unchanged during the calculation, we placed the statement $\cos p h i:=\cos (p h i)$ in the beginning of the program, to be certain that the cosine was not calculated 30 or more times. Moreover, in the expression for $T[1]$ and $T[2]$, sqr (1-sin phi $\uparrow 2)$ was replaced by cos.phi, so that loss of significant figures does not occur.

The expression. $2 \times n$ was changed in a new variable, to obtain a more rapid program.

\section{CERTIFICATION OF ALGORITHM 73}

INCOMPLETE ELLIPTIC INTEGRALS [David ' $K$.

Jefferson, Comm. ACM 4, Dec. 1961]

\section{Noelle A. Meyer}

E. I. du Pont de Nemours \& Co., Wilmington, Del.

Ellint was hand-coded in Fortran for the IBM 7070. The following corrections were made:

The statement

should be

$$
E:=(2 \times n-1) /(2 \times N) ;
$$

$$
E:=(2 \times n-1) /(2 \times n) ;
$$


The statement

$F:=a b s(k) \times \operatorname{sqr} t(1-\sin p h i \uparrow 2) \times(1-k \uparrow 2 \times \sin p h i \uparrow 2) \uparrow$

$((2 \times n-1) /(2 \times n))$;

should be

$F:=(a b s(h) \times \operatorname{sqr} l(1-\sin p h i \uparrow 2) \times$

The statement

$$
(1-k \uparrow 2 \times \sin p h i \uparrow 2) \uparrow(n-.5)) /(2 \times n)
$$

should be

$$
L[2]:=L[1]+1 /(n \times 2 \times n-1)) ;
$$

$$
L[2]:=L[1]+(1 /(n \times(2 \times n-1)) ;
$$

In order to accommodate negative $\phi$ the following changes were made :

The statement

if $a b s((\operatorname{sigma}[1]+\operatorname{del}[1])-\operatorname{sigma}[1])>0 \wedge p h i \times \operatorname{sinphi} \uparrow$

$(2 \times n) \geq A[2]$ then go to step 1 ;

was changed to

if $a b s((\operatorname{sigma}[1]+\operatorname{del}[1])-\operatorname{sigma}[1])>0 \wedge a b s(p h i \times \sin p h i \uparrow(2 \times n))$ $\geq a b s(A[2])$ then go to step 1 ;

Also the following was inserted before the last statement (stop: end)

if $p h i<0$ then go to wait else go to stop;

wait: $F:=-F$;

$$
E:=-E
$$

The revised algorithm yielded satisfactory answers when compared with the DiDonato and Hershey tables. Differences occurred in the eighth significant digit as shown in the following difference tables.

\section{DIFFERENCE TABLES}

\section{F-TABLE}

$\theta$ (in degrees)

\begin{tabular}{ccccc} 
(in degrees) & 0 & 30 & 60 & 90 \\
0 & 0. & 0. & 0. & 0. \\
30 & $-1 \times 10^{-8}$ & $-1 \times 10^{-8}$ & $-1 \times 10^{-8}$ & $-3 \times 10^{-8}$ \\
60 & $1 \times 10^{-8}$ & $1 \times 10^{-8}$ & $2 \times 10^{-8}$ & $-3 \times 10^{-8}$ \\
90 & 0. & $2 \times 10^{-8}$ & $6 \times 10^{-8}$ & 0. \\
\multicolumn{5}{c}{} \\
0 & 0. & $E-\mathrm{TABLE}$ \\
30 & $-1 \times 10^{-8}$ & $-1 \times 10^{-8}$ & $-1 \times 10^{-8}$ & $-1 \times 10^{-8}$ \\
60 & $1 \times 10^{-8}$ & $1 \times 10^{-8}$ & $-7 \times 10^{-8}$ & $3 \times 10^{-8}$ \\
90 & 0. & 0. & $1 \times 10^{-8}$ & 0.
\end{tabular}

REMARK ON ALGORITHM 73

INCOMPLETE ELLIPTIC INTEGRALS [David K.

Jefferson, Comm. ACM (Dec. 1961)]

DAvid K. JefFERSON

U. S. Naval Weapons Laboratory, Dahlgren, Virginia

In regard to Algorithm 73 , two errors were found:

The 34th line of the procedure

$$
\begin{aligned}
F:= & a b s(k) \times \text { sgrt }(1-\operatorname{sinphi} \uparrow 2) \\
& \times(1-k \uparrow 2 \times \operatorname{sinphi} \uparrow 2) \uparrow((2 \times n-1) /(2 \times n)) ;
\end{aligned}
$$

should read

$$
\begin{aligned}
F & :=a b s(k) \times \operatorname{sqrt}(1-\sin p h i \uparrow 2) \\
& \times(1-\mathrm{k} \uparrow 2 \times \operatorname{sinphi} \uparrow 2) \uparrow((2 \times n-1) / 2) /(2 \times n) ;
\end{aligned}
$$

The 37 th line

$$
L[2]:=L[1]+1 /(n \times 2 \times n-1)) ;
$$

should real

$$
L[2]:=L[1]+1 /(n \times(2 \times n-1)) ;
$$

In addition, efficiency is improved by interchanging lines 13 and 14 :

$$
\text { Step 1: } \begin{array}{ll}
n:=n+1 ; \\
& \operatorname{cosphi}:=\cos (p h i) ;
\end{array}
$$

can be replaced by

$$
\operatorname{cosphi}:=\cos (p h i) \text {; }
$$

Slep 1: $n:=n+1$;

\section{ALGORITHM 74}

\section{CURVE FITTING WITH CONSTRAINTS}

J. E. L. Реск,

University of Alberta, Calgary, Alberta, Canada

procedure Curve fitting ( $k, a, b, m, x, y, w, n, a l p h a, b e t a, s, s g m s q, x 0$, gamma, $\mathbf{c}, \mathbf{z}, \mathbf{r}$ );

comment This procedure finds, by the method of least squares, the polynomial of degree $n, k \leq n<k+m$, whose graph contains $\left(a_{1}, b_{1}\right), \cdots,\left(a_{k} b_{k}\right)$ and approximates $\left(x_{1}, y_{1}\right), \cdots$, $\left(x_{m}, y_{m}\right)$, where $w_{i}$ is the weight attached to the point $\left(x_{i}, y_{i}\right)$. The details will be found in the reference cited below, where a similar notation is used. A nonlocal label "error" is assumed;

value $a, x, y$, w; integer $k, m, n, r$; real $x 0$, gamma; array a, b, x, y, w, alpha, beta, s, sgmsq; c, z;

begin integer $i, j$; array $w 1[1: k]$; real $p, f$, lambda ;

comment We shall first define several procedures to be used in the main program, which begins at the label START;

procedure Evalue (x, nu);

comment This procedure evaluates $f=s_{0} p_{0}+s_{1} p_{1}+\cdots+$ $\mathrm{s}_{\nu} \mathrm{p}_{\nu}$, where $\mathrm{p}_{-1}(\mathrm{x})=0, \mathrm{p}_{0}(\mathrm{x})=1, \beta_{0}=0$ and $\mathrm{p}_{\mathrm{i}+1}(\mathrm{x})$ $=\left(x-\alpha_{i}\right) p_{i}(x)-\beta_{i} p_{i-1}(x)^{\prime}, i=0,1, \cdots, \nu-1$. The value of $\mathrm{p}_{\nu}(\mathrm{x})$ remains in $\mathrm{p}$;

real $x$; integer $n u$;

begin real $p 0$, temp; integer $i ; \quad p 0:=0 ; p:=1 ; f:=s[0]$;

for $i:=0$ step 1 until nu-1 do

begin temp :=

$p:=(x-$ alpha $[i]) \times p-$ beta $[i] \times p 0$

$p 0:=$ temp; $f:=f+p \times s[i+1]$ end $i$

end Evalue;

procedure Coda $(n, c)$;

comment This procedure finds the $c$ 's when $c_{0}+c_{1} x+\cdots+$ $c_{n} x^{n}=s_{0} p_{0}(x)+\cdots+s_{n} p_{n}(x)$;

integer $n$; array $c$;

begin integer $\mathrm{i}, \mathrm{r}$; real $\mathrm{tl}, \mathrm{t} 2$; array $\mathrm{pm}, \mathrm{p}[0: \mathrm{n}]$;

for $r:=1$ step 1 until $n$ do

$\mathrm{c}[\mathrm{r}]:=\operatorname{pm}[\mathrm{r}]:=\mathrm{p}[\mathrm{r}]:=0$

$\operatorname{pm}[0]:=0 ; \quad p[0]:=1 ; \quad c[0]:=s[0] ;$

for $i:=0$ step 1 until $n-1$ do

begin $t 2:=0$

for $r:=0$ step 1 until $i+1$ do

begin $t 1:=(t 2-$ alpha[i] $\times \mathrm{p}[\mathrm{r}]-$ beta[i] $\times \mathrm{pm}[\mathrm{r}]) / \mathrm{lambda}$

$\mathrm{t} 2:=\mathrm{pm}[\mathrm{r}]:=\mathrm{p}[\mathbf{r}] ; \quad \mathrm{p}[\mathrm{r}]:=\mathrm{t} \mathbf{1}$;

$c[r]:=c[r]+t l \times s[i+1]$ end $r$

end $i$

end Coda;

procedure GEFYT ( $\mathrm{n}, \mathrm{n} 0, \mathrm{x}, \mathrm{y}, \mathrm{w}, \mathrm{m})$

comment This is the heart of the main program. It computes the $\alpha_{i}, \beta_{i}, s_{i}, \sigma_{i}^{2}$, using the method of orthogonal polynomials, as described in the reference;

integer $\mathrm{n}, \mathrm{n} 0, \mathrm{~m}$; array $\mathrm{x}, \mathrm{y}, \mathrm{w}$;

begin real dsq,wpp, wpp0,wxpp,wyp,temp;

integer $i, j$, freedom; array $p, p 0[1: m]$; boolean exact;

if $n-n 0>m \vee n<n 0$ then go to error;

beta $\mid n 0]:=\operatorname{dsq}:=\operatorname{wpp}:=0 ;$ exact $:=n-n 0 \geq m-1$;

for $\mathrm{j}:=1$ step 1 until $\mathrm{m}$ do

begin $p[j]:=1 ; \quad p 0[j]:=0 ; \quad w p p:=w p p+w[j] ;$

if $\neg$ exact then $d s q:=d s q+w[j] \times y[j] \times y[j]$ end initialise;

for $i:=n 0$ step 1 until $n$ do

begin freedom $:=m-1-(i-n 0) ; \quad$ yyp $:=$ wxpp $:=0$

for $\mathrm{j}:=1$ step 1 until $m$ do

begin temp $:=w[j] \times p[j] ;$

if $\mathrm{i}<\mathrm{n}$ then wxpp $:=w \times w p p+\operatorname{temp} \times \mathrm{x}[\mathrm{j}] \times \mathrm{p}[\mathrm{j}]$;

if freedom $\geq 0$ then wyp $:=$ wyp $+\operatorname{temp} \times y[j]$ end $j$;

if freedom $\geq 0$ then $s[i]:=$ wyp/wpp;

if $\neg$ exact then begin dsq $:=d s q-s[i] \times s[i] \times w p p$

sgmsq[i] : $=\mathrm{dsq} /$ freedom end if ; 
if $\mathrm{i}<\mathrm{n}$ then begin alpha[i] $:=\operatorname{wxp} / \mathrm{wpp} ; \quad$ wpp0 $:=$ wpp;

$\operatorname{wpp}:=0$;

for $j:=1$ step 1 until $m$ do

begin temp $:=(x[j]-$ alpha[i] $) \times p[j]-$ beta[i] $\times$ p0[j];

$w p p:=w p p+w[j] \times$ temp $\times$ temp;

$p 0[j]:=p[j] ; p[j]:=$ temp end $j ;$

beta[i+1] $:=$ wpp $/$ wpp 0 end if

end $\mathrm{i}$

end GEFYT;

START: for $j:=1$ step 1 until $k$ do

begin $w 1[j]:=1 ; \quad a[j]=(a[j]-x 0) /$ gamma end $j$;

GEFYT (k,0,a,b,wl,k);

comment This finds the polynomial of degree $k-1$ whose graph contains $\left(a_{1}, b_{1}\right), \cdots,\left(a_{k}, b_{k}\right)$ supplying the $\alpha_{i}, \beta_{i}, s_{i}, \quad 0 \leq \mathrm{i} \leq k$;

begin real rho; rho $:=0$;

for $j:=1$ step 1 until $m$ do

begin $r h o:=$ rho $+w[j]$;

$x[j]:=(x[j]-x 0) /$ gamma end $j ;$ rho $:=m /$ rho;

comment The factor $\rho$ is used to normalize the weights. We shall now put $s_{k}=0$ in order to evaluate $p_{k}(x)$ and the polynomial of degree $k-1$ simultaneously;

$\mathbf{s}[\mathrm{k}]:=0$;

for $\mathrm{j}:=1$ step 1 until $\mathrm{m}$ do

begin Evalue $(x[j], k)$;

if $\mathbf{p}=0$ then go to error;

$y[j]:=(y[j]-f) / p$

$w[j]:=w[j] \times p \times p \times$ rho end $j$

end rho;

comment We have now normalized the weights and adjusted the weights and ordinates ready for the least squares approximation;

GEFYT (n,k,x,y,w,m);

comment The coefficients $\alpha_{i}, \beta_{i}, \quad 0 \leq i<n$, and $s_{i}, \quad 0 \leq i \leq n$ are now ready. The polynomial may be evaluated for $x=z_{1}, z_{2}$, $\cdots, z_{r}$, but the variable must be adjusted first. Note that we may evaluate the best polynomial of lower degree by deoreasing $\mathbf{n}$;

begin real $x$;

for $j:=1$ step 1 until $r$ do

begin $x:=(z[j]-x 0) /$ gamma;

Evalue $(x, n)$; comment the values of $z_{j}$ and $f$ should now be printed; end $j$;

comment We may now adjust the coefficients for scale and then find the coefficients of the power series $c_{0}+c_{1} x+\cdots+c_{n} x^{n}=$ $\mathrm{s}_{0} \mathrm{p}_{0}(\mathrm{x})+\cdots+\mathrm{s}_{\mathrm{n}} \mathrm{p}_{\mathrm{n}}(\mathrm{x})$;

for $i:=0$ step 1 until $n-1$ do

begin alpha[i] := alpha[i] $\times$ gamma $+\mathrm{x} 0$;

beta[i] $:=$ beta[i] $\times$ gamma end $i$; lambda::= gamma;

Coda (n,c);

comment We may now re-evaluate the polynomial from the power series;

for $j:=1$ step 1 until $r$ do

begin $\mathrm{x}:=\mathrm{z}[\mathrm{j}] ; \mathrm{f}:=\mathrm{c}[\mathrm{n}]$;

for $i:=n-1$ step -1 until 0 do

$f:=f \times x+c[i]$

comment the values of $x$ and $f$ should now be printed; end $j$ end $x$

end Curve fitting

Reference: Peck, J. E. L. Polynomial curve fitting with constraint, Soc. Indust. Appl. Math. Rev. (1961).

CERTIFICATION OF ALGORITHM 74

CURVE FITTING WITH CONSTRAINTS .J. E.

Peck, Comm. ACM, Jan. 62]

KAzuo Isoda

Japan Atomic Energy Research Institute, Tokai, Ibaraki, Japan

Algorithm 74 was hand-compiled into SOAP IIa for the IBM 650 and run successfully with no corrections except the case in which the origin $(0,0)$ are given as both a constraint and a sample.

\section{ALGORITHM 75}

\section{FACTORS}

J. E. L. PECK,

University of Alberta, Calgary, Alberta, Canada

procedure factors $(\mathrm{n}, \mathrm{a}, \mathrm{u}, \mathrm{v}, \mathrm{r}, \mathrm{c})$;

comment This procedure finds all the rational linear factors of the polynomial $\dot{a}_{0} x^{n}+a_{1} x^{n-1}+\cdots+a_{n-1} x+a_{n}$, with integral coefficients. An absolute value procedure abs is assumed;

value $n, a$; integer $r, n, c$; integer array $a, u, v$;

begin comment $W e$ find whether pdivides $a_{0}, 1 \leq p \leq\left|a_{0}\right|$ and $q$ divides $a_{n}, \quad 0 \leq q \leq\left|a_{n}\right|$. If this is the case we try $(p x \pm q)$; integer $p, q, a 0$, an ;

$\mathbf{r}:=0 ; \quad \mathbf{c}:=1 ;$ comment $r$ will be the number of linear factors and $c$ the common constant factor;

TRY AGAIN : a0 :=a[0]; an $:=a[n]$;

for $p:=1$ step 1 until abs $(a 0)$ do

begin if $(\mathrm{a} 0 \div \mathrm{p}) \times \mathrm{p}=\mathrm{a} 0$ then

begin comment $p$ divides $a_{0}$;

for $q:=0$ step 1 until abs(an) do

begin if $q=0 \vee(a n \div q) \times q=$ an then

begin comment $q$ divides $a_{n}$ (or $q=0$ ). If $p=q$ we may have a common constant factor, therefore; if $q$ $>1 \wedge \mathrm{p}=1$ then

begin integer $\mathrm{j}$ :

for $\mathrm{j}:=1$ step 1 until $\mathrm{n}-1$ do

if $(a[j] \div q) \times q \neq a[j]$ then go to NO CONSTANT;

for $j:=0$ step 1 until $n$ do

$\mathrm{a}[\mathrm{j}]:=\mathbf{a}[\mathrm{j}] / \mathrm{q}$;

$c:=c \times q ;$ go to TRY AGAIN

end the search for a common constant factor.;

NO CONSTANT:

begin comment try $(\mathrm{px}-\mathrm{q})$ as a factor;

integer $f, g, i ; f:=a 0 ; g:=1$;

comment we try $x=q / p$;

for $i:=1$ step 1 until $n$ do

begin $g:=g \times p ; f:=f \times q+a[i] \times g$

end evaluation;

if $f=0$ then

begin comment we have found the factor $(p x-q)$; $\mathbf{r}:=\mathrm{r}+\mathbf{1} ; \mathrm{u}[\mathrm{r}]:=\mathrm{p} ; \mathrm{v}[\mathrm{r}]:=\mathrm{q}$;

comment there are now $r$ linear factors;

begin comment we divide by $(p x-q)$;

integer $i, t ; t:=0$;

for $i:=0$ step 1 until $n$ do

begin $a[i]:=t:=(a[i]+t) / p ; t:=t \times q$

end $i$;

$\mathrm{n}:=\mathbf{n}-\mathbf{1}$

end reduction of polynomial. Therefore;

go to if $\mathrm{n}=0$ then REDUCED else TRY AGAIN

end discovery of $p x-q$ as a factor. But

if we got this far it was not a factor so try $p x+q$;

$q:=-q ;$ if $q<0$ then go to NO CONSTANT

end trial of $p x \pm q$,

end $q$ divides $a_{n}$ and

end of $q$ loop.

end $p$ divides $a_{0}$, also

end $p$ loop, which means;

REDUCED: if $\mathrm{n}=\mathbf{0}$ then

be $\quad \therefore \mathrm{a} 0 ; \mathrm{a} 0:=1$

end it ..

end factors procedure. There are now $r(r>0)$, rational linear factors $\left(u_{i} x-v_{i}\right), \quad 1<i<r$, and the reduced polynomial of reduced degree $n$ replaces the original. The common constant factor is c. Acknowledgments to Clay Perry. 


\section{CERTIFICATION OF -ALGORITHM 75}

FACTORS [J. E. L. Peck, Comm. ACM, Jan. 1962]

J. S. Hillmore

Elliott Bros. (London) Ltd., Borehamwood, Herts., England

The following changes had to be made to the algorithm:

(1) For. if $g>1 \wedge p=1$ then

put if $q>1 \wedge p=q$ then

(2) For begin $c:=c \times a 0 ; \quad a 0:=1$ end

put begin $c:=c \times a[0] ; \quad a[0]:=1$ end

(3) For if $q=0 \vee(a n \div q) \times q=$ an then

put if (if $q=0$ then true else $($ an $\div q) \times q=a n$ ) then

This change is necessary to ensure that the term $(a n \div q)$ is not evaluated when $q=0$.

The algorithm, thus modified, was successfully run using the Elliott Algol translator on the National-Elliott 803.

\section{CERTIFICATION OF ALGORITHM 75}

FACTORS [J. E. L. Peck, Comm. ACM 5 (Jan. 1962)]

A. P. RELPH

The English Electric Co., Whetstone, England

Algorithm 75 was translated using the DeUCE ALgol compiler and gave satisfactory results after the following corrections had been made:

begin if $q=0 \vee($ an $\div q) \times q=a n$ then

begin if $q>1 \wedge p=1$ then

was changed to

begin if $q \leqq 1$ then go to NO CONSTANT;

if $($ an $\div q) \times q=$ an then

begin if $p=q$ then

begin $c:=c \times a 0 ; \cdot a 0:=1$

end

was changed to

begin $c:=c \times a[0] ; \quad a[0]:=1$

end

There are now $r(r>0)$ rational linear factors $\left(u_{i} x-v_{i}\right)$, $1<\mathrm{i}<r$,

was changed to

If $r>0$ there are now $r$ rational linear factors $\left(u_{i} x-v_{i}\right), 1 \leqq i \leqq r$,

To return to the state $(p=1, q=0)$ after every factor or constant is found is inefficient. This can be avoided by substituting a $[0]$ and $a[n]$ for the identifiers $a 0$ and an respectively. The procedure then becomes:

procedure factors $(n, a, u, v, r, c)$; value $n, a$;

integer array $a, u, v$;

integer $r, n, c$;

begin integer $p, q$;

$\mathbf{r}:=0 ; \quad \mathrm{c}:=1$;

ZERO: if $a[n]=0$ then

begin $r:=r+1 ; \quad u[r]:=1 ; v[r]:=0 ; \quad n:=n-1 ;$ go to ZERO

end;

for $p:=1$ step 1 until abs (a[0]) do

begin if $(a[0] \div p) \times p=a[0]$ then

begin for $q:=1$ step 1 until abs $(a[n])$ do

begin if $q=1$ then go to NO CONSTANT;

TRY AGAIN: if $(a[n] \div q) \times q=a[n]$ then

begin integer $j$;

for $j:=0$ step 1 until $n-1$ do if $(a[j] \div q) \times q \neq a[j]$ then go to NO CONSTANT; for $\mathrm{j}:=\mathbf{0}$ step 1 until $\mathbf{n}$ do

$\mathrm{a}[\mathrm{j}]:=\mathrm{a}[\mathrm{j}] / \mathrm{q}$;

c : $=\mathrm{c} \times \mathrm{q} ;$ go to TRY AGAIN end;

NO CONSTAN $\mathrm{T}$ begin integer $f, g, i ; f:=a[0]$; $\mathrm{g}:=1$

for $\mathrm{i}:=1$ step 1 until $\mathrm{n}$ do

begin $g:=g \times p$;

$\mathrm{f}:=\mathrm{f} \times \mathrm{q}+\mathrm{a}[\mathrm{i}] \times \mathrm{g}$

end;

if $[=0$ then

begin $r:=r+1 ; \quad u[r]:=p$;

$\mathrm{v}[\mathrm{r}]:=\mathrm{q}$

begin integer $i, t ; t:=0$;

for $i:=0$ step 1 until $n$ do

begin $a[i]:=t:=(a[i]+t) / p$;

$\mathrm{t}:=\mathrm{t} \times \mathrm{q}$

end;

$\mathrm{n}:=\mathrm{n}-1$

end

go to if $\mathrm{n}=0$ then REDUCED

else NO CONSTANT.

end ;

$\mathrm{q}:=-\mathrm{q} ;$ if $\mathrm{q}<0$ then go to $\mathrm{NO}$

CONSTANT end

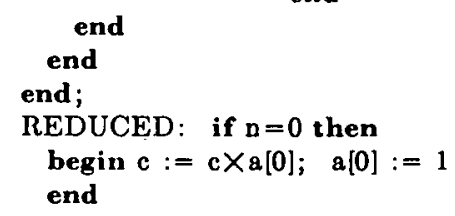

end

\author{
ALGORITHM 76 \\ SORTING PROCEDURES \\ IVAN Flores \\ Private Consultant, Norwalk, Connecticut
}

comment The following Algov 60 algorithms are procedures for the sorting of records stored within the memory of the computer. These procedures are described in detail, flow-charted, compared, and contrasted in "Analysis of Internal Computer Sorting" by Ivan Flores $[J . A C M 8$ (Jan. 1961)]. Although sorting is usually a business computer application, it can be described completely in ALGOL if we stretch our imagination a little. Sorting is ordering with respect to a key contained within the record. If the key is the active record, the sorting is trivial. A means is required to extract the key from the record. This is essentially string manipulation, for which no provision, as yet, has been made in ALGOL. We circumambulate this difficulty by defining an integer procedure $K(I)$ which "creates" a key from the record, I. Algol does provide for machine language code substitutions, which is one way to think of $K(I)$. This could be more accurately represented by using the string notation proposed by Julien Green ("Remarks on ALGOL and Symbol Manipulation," Comm. ACM 2 (Sept. 1959), 25-27]. The function sub $(\$, \mathrm{i}, \mathrm{g})$ represents the procedure, $\mathbf{K}(\mathrm{I})$. \$ corresponds to the record I, i corresponds to the starting position of the key and $g$ corresponds to the length of the key. Both $i$ and $g$ are values which must be specified when the sort procedure is called for as a statement instead of a declaration. 
Another factor, which might vex some, is that the key might be alphabetic instead of numeric. Then, of course, $K(I)$ would not be integer. It would, however, be string when such is defined eventually. Note, also, that keys are frequently compared. This is done using the ordering relations " $>$ " for "greater than," etc. These are not really defined in the ALgol statement [NAUR, Peter, et Al. "Report on the Algorithmic Language Algol 60". Comm. ACM 3 (May 1960), 294-314]. They can simply be defined so that $Z>Y>\cdots>A>9>\cdots>1>0$. Also the assignment $\mathrm{X}[\mathrm{i}]:=\mathrm{z}$ should be interpreted as "Assign the key ' $z$ ' which is larger than any other key." For any sort procedure $(I, N, S)$, " $I$ " is the set of unsorted records, " $N$ " is their number, and " $\mathrm{S}$ " the sorted set of records.

Caution, these algorithms were developed purely for the love of it: No one was available with the combined knowledge of sorting and ALGOL to check this work. Hence each algorithm should be carefully checked before use. I will be glad to answer any questions which may arise;

Sort insert $(I, N, S)$; value $N$; array $I[1: N], S[1: N]$; integer procedure $\mathrm{K}(\mathrm{I})$; integer $\mathrm{N}$;

begin integer $i, j, k$;

$\mathrm{S}[1]:=\mathrm{I}[1]$;

for $i:=2$ step 1 until $N$ do begin

for $\mathrm{j}:=\mathrm{i}-1, \mathrm{j}-\mathrm{l}$ while $\mathrm{K}(\mathrm{I}[\mathrm{i}])>\mathrm{K}(\mathrm{S}[\mathrm{j}])$ do

for $k:=i$ step -1 until $j+1$ do

$\mathrm{S}[\mathrm{k}]:=\mathrm{S}[\mathrm{K}-1]$

$\mathrm{S}[\mathrm{j}+1]:=\mathrm{I}[\mathrm{i}]$ end end

Sort count $(I, N, S)$; value $N$; array $I[1: N], S[1: N]$;

integer procedure $K(I)$; integer $N$;

begin integer array $C[1: N]$; integer $i, j$;

for $i:=1$ step 1 until $N$ do $C[i]:=0$;

for $\mathrm{i}:=2$ step 1 until $\mathrm{N}$ do

for $j:=1$ step 1 until $i-1$ do

if $\mathrm{K}(\mathrm{I}[\mathrm{i}])>\mathrm{K}(\mathrm{I}[\mathrm{j}])$ then $\mathrm{C}[\mathrm{i}]:=\mathrm{C}[\mathrm{i}]+1$

else $\mathrm{C}[\mathrm{j}]:=\mathrm{C}[\mathrm{j}]+1$;

for $i:=1$ step 1 until $N$ do

$\mathrm{S}[\mathrm{C}[\mathrm{i}]]:=\mathrm{I}[\mathrm{i}]$ end

Sort select $(I, N, S)$; value $N$; array $I[1: N], S[1: N]$;

integer procedure $K(I)$; integer $N$;

begin integer $i, j, A, h$;

for $i:=1$ step 1 until $N$ do begin

$\mathbf{h}:=\mathbf{K}(\mathbf{I}[\mathbf{1}])$;

for $\mathrm{j}:=2$ step 1 until $\mathrm{N}$ do

if $h>K(I[j])$ then begin $h:=K(I[j]) ; A:=j$ end;

$\mathrm{S}[\mathrm{i}]:=\mathrm{I}[\mathrm{A}]$

$\mathrm{I}[\mathrm{A}]:=\mathrm{z}$ end end

Sort select exchange $(I, N)$; value $N$; array $I[1: N]$;

integer procedure $K(I)$; integer $N$;

begin integer $h, i, j, H$; real $T$;

for $i:=1$-step 1 until $N$ do begin

$\mathrm{H}:=\mathrm{K}(\mathrm{I}[\mathbf{i}]) ; \mathrm{h}:=\mathbf{i}$;

for $\mathrm{j}:=\mathrm{i}+1$ step 1 until $\mathrm{N}$ do

if $K(I[j])<H$ then begin

$\mathrm{H}:=\mathrm{K}(\mathrm{I}[\mathrm{j}]) ; \mathrm{h}:=\mathrm{j}$ end

$\mathrm{T}:=\mathrm{I}[\mathrm{i}] ; \mathrm{I}[\mathrm{i}]:=\mathrm{I}[\mathrm{h}] ; \mathrm{I}[\mathrm{A}]:=\mathrm{T}$ end end

Sort binary insert $(I, N, S)$; value $N$; array $I[1: N], S[1: N]$;

integer procedure $\mathrm{K}(\mathrm{I})$; integer $\mathrm{N}$;

begin integer $i, k, j, l$;

if $K(I[1])<K(I[2])$ then begin

$S[1]:=I[1] ; S[2]:=I[2]$ end

else begin $S[1]:=I[2] ; \quad S[2]:=I[1]$ end;

start: for $i:=3$ step 1 until $N$ do begin

$\mathrm{j}:=(\mathrm{i}+1) \div 2$;

find spot: $\quad$ for $k:=(i+1) \div 2,(k+1) \div 2$ while $k>1$ do if $K(I[i])<K(S[j])$ then $j:=j-k$

else $j:=j+k$;

if $K(I[i]) \geq K(S[j])$ then $j:=j-l$

move items: for $1:=\mathrm{i}$ step -1 until $\mathrm{j}$ do

$\mathrm{S}[1+1]:=\mathrm{S}[1]$

enter this

one: $\quad \mathrm{S}[\mathrm{j}]:=\mathrm{I}[\mathrm{i}]$ end end

Sort address calculation (I,N,S,F); value $\mathrm{N}$;

array $S[1: M], I[1: N]$; integer procedure $F(K), K(I)$;

integer $\mathrm{N}, \mathrm{M}$;

begin integer $\mathrm{i}, \mathrm{j}, \mathrm{G}, \mathrm{H}, \mathrm{F}, \mathrm{M}$;

$\mathrm{M}:=\operatorname{entier}(2.5 \times \mathrm{N})$

for $\mathrm{i}:=1$ step 1 until $M$ do $S[i]=0$;

Address: for $\mathrm{i}:=1$ step 1 until $\mathrm{N}$ do begin

$F:=\mathbf{F}(\mathbf{K}(\mathrm{I}[\mathrm{i}]))$;

if $S[F]=0$ then begin $S[F]:=I[i]$;

go to NEXT end

else if $\mathrm{K}(\mathrm{S}[\mathrm{F}])>\mathrm{K}(\mathrm{I}[\mathrm{i}])$ then go to SMALLER;

LARGER: for $\mathrm{H}:=\mathrm{F}, \mathrm{H}+1$ while $\mathrm{K}(\mathrm{S}[\mathrm{H}])<\mathrm{K}(\mathrm{I}[\mathrm{i}])$ do

for $G:=H, G+1$ while $K(S[G]) \neq 0$ do

for $j:=G$ step -1 until $H+1$ do

$\mathrm{S}[\mathrm{j}]:=\mathrm{S}[\mathrm{j}-1]$;

$\mathrm{S}[\mathrm{H}]:=\mathrm{I}[\mathrm{i}]$; go to NEXT;

SMALLER: for $\mathrm{H}:=\mathrm{F}, \mathrm{H}-1$ while $\mathrm{K}(\mathrm{S}[\mathrm{H}])>\mathrm{K}(\mathrm{I}[\mathrm{i}])$ do

for $G:=H, G-1$ while $K(S[G]) \neq 0$ do

for $j:=G$ step 1 until $H-1$ do

$S[j]=S[j+1]$

$\mathrm{S}[\mathrm{H}]:=\mathrm{I}[\mathrm{i}]$

NEXT: end end

Sort quadratic select $(I, N, S)$; value $N$; array $I[1: N], S[1: N]$; integer procedure $K(I)$; integer $N$; begin integer $i, j, k, C, D, J, M$; integer array $C[1: M], D[1: M]$; array $\mathrm{I}[1: \mathrm{M}, 1: \mathrm{M}]$

Divide inputs: $\mathrm{M}:=$ entier $(\operatorname{sqrt}(\mathrm{N}))+1 ; \mathrm{j}:=\mathrm{k}:=1$; for $\mathrm{i}:=1$ step 1 until $\mathrm{N}$ do begin

$\mathrm{I}[\mathrm{j}, \mathrm{k}]:=\mathrm{I}[\mathrm{i}] ; \mathrm{k}:=\mathrm{k}+1$;

if $k>M$ then begin $k:=1$;

$j:=j+1$ end end

Fill up inputs: $\quad \mathrm{I}[\mathrm{j}, \mathrm{k}]:=\mathrm{z} ; \mathrm{k}:=\mathrm{k}+1$;

if $k>M$ then begin $k:=1 ; j:=j+1$ end

if $j \leq M$ then go to Fill up inputs;

Set controls: $\quad$ for $j:=1$ step 1 until $M$ do begin

$\mathrm{C}[\mathrm{j}]:=\mathrm{K}(\mathbf{I}[\mathbf{j}, \mathbf{1}]) ; \mathrm{D}[\mathrm{j}]:=1$;

for $\mathrm{k}=2$ step 1 until $\mathrm{M}$ do

if $\mathrm{C}[\mathrm{j}]>\mathrm{K}(\mathrm{I}[\mathrm{j}, \mathrm{k}])$ then begin

$\mathrm{C}[\mathrm{j}]:=\mathrm{K}(\mathrm{I}[\mathrm{j}, \mathrm{k}]) ; \mathrm{D}[\mathrm{j}]:=\mathrm{k}$ end end;

$\mathrm{i}:=1$;

Find least: $\quad \mathrm{C}:=\mathrm{C}[1] ; \quad \mathrm{D}:=\mathrm{D}[1] ; \quad \mathrm{J}:=1$;

for $\mathrm{j}:=2$ step 1 until $M$ do

if $\mathrm{C}>\mathrm{C}[\mathrm{j}]$ then begin $\mathrm{C}:=\mathrm{C}[\mathrm{j}]$;

$\mathrm{D}:=\mathrm{D}[\mathrm{j}] ; \quad \mathrm{J}:=\mathrm{j}$ end;

Fill file: $\quad \mathrm{S}[\mathrm{i}]:=\mathrm{I}[\mathrm{J}, \mathrm{D}] ; \mathrm{i}:=\mathrm{i}+\mathrm{I} ; \mathrm{I}[\mathrm{J}, \mathrm{D}]:=\mathrm{z}$;

if $\mathrm{i}=\mathrm{N}+1$ go to STOP;

Reset controls: for $\mathrm{j}:=\mathrm{J}$ do begin

$\mathrm{C}[\mathrm{j}]:=\mathrm{K}(\mathbf{l}[\mathrm{j}, 1]) ; \quad \mathrm{D}[\mathrm{j}]:=1$;

for $k:=2$ step 1 until $M$ do

if $\mathrm{C}[\mathrm{j}]>\mathrm{K}(\mathrm{I}[\mathrm{j}, \mathrm{k}])$ then begin $\mathrm{C}[\mathrm{j}]:=$

$\mathrm{K}(\mathrm{I}[\mathrm{j}, \mathrm{k}] ; \mathrm{D}[\mathrm{j}] \mathrm{:}:=\mathbf{k}$ end end;

go to Find least;

STOP: $\quad$ end

Presort quadratic selection $(\mathrm{I}, \mathrm{N}, \mathrm{S})$; value $\mathrm{N}$; array $I[1: N], S[1: N]$; integer procedure $K(I)$; integer $N$;

begin integer $i, j, k, C, J, M$; integer array $C[1: M], D[1: M]$ array $\mathrm{I}[1: \mathrm{M}, 1: \mathrm{M}]$ 
Divide inputs: $\quad \mathrm{M}:=$ entier $(\operatorname{sqrt}(\mathrm{N}))+1 ; j:=\mathrm{k}:=1$;

for $\mathrm{i}:=1$ step 1 until $\mathrm{N}$ do begin

$\mathrm{I}[\mathrm{j}, \mathrm{k}]:=\mathrm{I}[\mathrm{i}] ; \quad \mathbf{k}:=\mathbf{k}+1$;

if $k>M$ then begin $k:=1$;

$\mathrm{j}:=\mathrm{j}+1$ end end

Fill up inputs: $I[j, k]:=z ; k:=k+1$;

if $k>M$ then begin $k:=1 ; j=j+1$ end

if $\mathrm{j} \leq \mathrm{M}$ then go to Fill up inputs;

First sort: $\quad$ for $j:=1$ step 1 until $M$ do

sort select exchange $(\mathrm{I}[\mathrm{j}, \mathrm{k}], \mathrm{M})$;

Set controls: for $\mathrm{j}:=1$ step 1 until $\mathrm{M}$ do begin

$C[j]:=K(I[j, 1]) ; D[j]:=1$ end

$\mathrm{i}:=1$;

Find least: $\quad \mathrm{C}:=\mathrm{C}[1] ; \mathrm{J}:=1$;

for $\mathrm{j}:=1$ step 1 until $M$ do

if $\mathrm{C}>\mathrm{C}[\mathrm{j}]$ then begin $\mathrm{C}:=\mathrm{C}[\mathrm{j}]$;

$\mathrm{J}:=\mathrm{j}$ end;

Fill file:

$\mathrm{S}[\mathrm{i}]:=\mathrm{I}[\mathrm{J}, \mathrm{D}[\mathrm{J}]] ; \quad \mathrm{i}:=\mathrm{i}+1$;

if $\mathrm{i}=\mathrm{N}+1$ go to STOP

Reset control: for $\mathrm{j}:=\mathrm{J}$ do begin

$\mathrm{I}[\mathrm{j}]:=\mathrm{I}[\mathrm{j}]+1$;

if $\mathrm{D}[\mathrm{j}]>\mathrm{M}$ then $\mathrm{C}[\mathrm{j}]:=\mathrm{z}$ else $\mathrm{C}[\mathrm{j}]:=$ $\mathbf{K}(\mathrm{I}[\mathrm{j}, \mathrm{D})[\mathrm{j}]])$ end

STOP: go to Find least;

end

Sort binary merge $(I, N, S)$; value $N$; array $I[1: N]$;

integer procedure $\mathrm{K}(\mathrm{I})$; integer $\mathrm{N}$;

begin real array $S[1: N]$;

integer array $A[0: 1,0: J[a]], B[0: 1,0: K[b]], A l o c[0: 1,0: J[a]]$,

Bloc $[0: 1,0: K[b]], J[0: 1], K[0: 1], j[0: 1], k[0: 1]$;

integer $a, b, i, j, k$;

distribute: $\quad a:=b:=j[0]:=j[1]:=1$;

for $\mathrm{i}:=1$ step 1 until $\mathrm{N}$ do begin

if $\mathrm{K}(\mathrm{I}[\mathrm{i}])<\mathrm{K}(\mathrm{I}[\mathrm{i}-1]$ then

if $\mathrm{a}=1$ then $\mathrm{a}:=0$ else $\mathrm{a}:=1$

$A[a, j[a]]:=\mathbf{K}(I[i]) ; \quad A l o c[a, j[a]]:=i$; $\mathrm{j}[\mathrm{a}]:=\mathrm{j}[\mathrm{a}]+1$ end

$\begin{array}{ll} & \mathrm{J}[0]:=\mathrm{j}[0] ; \mathrm{J}[1]:=\mathrm{j}[1] ; \\ \text { next sort: } & \text { begin } \mathrm{a}:=\mathrm{b}:=\mathrm{j}[0]:=\mathrm{j}[1]:=\mathrm{k}[0]:=\end{array}$ $\mathrm{k}[1]:=1$;

two inputs: $\quad$ if $A[1, j[1]] \leq A[0, j[0]\}$ then a $:=1$ else $a:=0$;

$\mathrm{B}[\mathrm{b}, \mathrm{k}[\mathrm{b}]]:=\mathrm{A}[\mathrm{a}, \mathrm{j}[\mathrm{a}]]$;

$\operatorname{Bloc}[\mathrm{b}, \mathrm{k}[\mathrm{b}]]:=\operatorname{Aloc}[\mathrm{a}, \mathrm{j}[\mathrm{a}]]$;

$\mathrm{j}[\mathrm{a}]:=\mathrm{j}[\mathrm{a}]+1 ; \quad \mathrm{k}[\mathrm{b}]:=\mathrm{k}[\mathrm{b}]+1 ;$

if $A[a, j[a]] \geq A[a, j[a]-1]$ then go to two inputs else

if $\mathrm{a}=1$ then $\mathrm{a}:=0$ else $\mathrm{a}:=1$;

single step: $\quad \mathrm{B}[\mathrm{b}, \mathrm{k}[\mathrm{b}]]:=\mathrm{A}[\mathrm{a}, \mathrm{j}[\mathrm{a}]]$;

Bloc $[\mathrm{b}, \mathrm{k}[\mathrm{b}]]:=$ Aloc $[\mathrm{a}, \mathrm{j}[\mathrm{a}]]$;

$\mathrm{j}[\mathrm{a}]:=\mathrm{j}[\mathrm{a}]+1 ; \quad \mathrm{k}[\mathrm{b}]:=\mathrm{k}[\mathrm{b}]+1$;

if $A[a, j[a]] \geq A[a, j[a]-1]$ then go to single step;

switch file:

check rollout:

if $b=1$ then $b:=0$ else $b:=1$;

for $a:=0,1$ do

if $\mathrm{j}[\mathrm{a}]=\mathrm{J}[\mathrm{a}]$ then go to rollout;

go to two inputs;

rollout:

$\mathbf{B}[\mathbf{b}, \mathbf{k}[\mathbf{b}]]:=\mathbf{A}[\mathbf{a}, \mathrm{j}[\mathrm{a}]]$;

Bloc $[b, k[b]]:=$ Aloc $[a, j[a]]$;

$\mathrm{k}[\mathrm{b}]:=\mathrm{k}[\mathrm{b}]+1 ; \quad \mathrm{j}[\mathrm{a}]:=\mathrm{j}[\mathrm{a}]+1$;

if $\mathrm{j}[\mathrm{a}]=\mathrm{J}[\mathrm{a}]$ then go to interchange files;

if $A[a, j[a]]<A[a, j[a]-1]$ then

if $b=1$ then $b:=0$ else $b:=1$;

go to rollout;

interchange files: $\mathbf{K}[0]:=\mathrm{k}[0] ; \mathrm{K}[1]:=\mathrm{k}[1]$;

if $K[0]=1$ then go to output end

for $b:=1,0$ do begin

for $k[b]:=1$ step 1 until $K[b]$ do begin

$A[b, k[b]]:=B[b, k[b]] ;$

Aloc $[\mathrm{b}, \mathrm{k}[\mathrm{b}]]:=\mathrm{Bloc}[\mathrm{b}, \mathrm{k}[\mathrm{b}]]$;

$J[b]:=K[b]$ end end

output:

go to next sort;

for $\mathrm{i}:=1$ step 1 until $\mathrm{N}$ do

end 
The routine was originally programmed as an open subroutine for the IBM 704 in Fortran II and occupied 323 memory locations. It is based upon a Lagrange interpolation scheme specialized for averaged second order parabolas. The technique finds the slope of a function numerically defined at points $1,2,3$ and 4 by fitting a parabola through the points $1,2,3$, and another parabola through the points 2,3 , and 4 . $\therefore$ The slope then, at point 2 , is the average analytical derivative of the two parabolas, i.e. the coefficients of the parabola through points 1,2 and $3\left(a_{1} x_{2}{ }^{2}+b_{1} x_{2}+c_{1}\right)$ and the coefficients of the parabola through points 2,3 , and $4 \quad\left(a_{2} x_{2}{ }^{2}+b_{2} x_{2}+c_{2}\right)$ are determined by applying Lagrange's equations as shown below. The arithmetic mean of these coefficients $a=\left(a_{1}+a_{2}\right) / 2$, $\mathrm{b}=\left(\mathrm{b}_{1}+\mathrm{b}_{2}\right) / 2, \quad \mathrm{c}=\left(\mathrm{c}_{1}+\mathrm{c}_{2}\right) / 2$ are used to supply the slope in the interval from 2 to 3 , namely $(2 a x+b)$.

The interpolation is calculated in similar fashion, except the final formula is that a parabola $\left(a x^{2}+b x+c\right)$.

The integration is performed likewise by a curve fitting process, e.g. the integral between any two points say 2 and 3 is the average integral of the two parabolas between the independent coordinate limits for points 2 and 3 . The averaging process is done for each interval along the abscissa as the results obtained are accumulated to evaluate the definite integral.

Applying Lagrange's equations, the coefficients $a, b$, and $c$ may be found by defining: $\mathrm{T}_{\mathbf{j}}=y / \prod_{\mathbf{i}-1, i \not j}^{\mathrm{j}}\left(\mathbf{X}_{\mathbf{j}}-\mathbf{X}_{\mathbf{i}}\right)$ where $y=f(x), \quad n=3, j=1,2, \cdots, n$, then $a=\sum_{i=1}^{n} T_{i}$, $b=\sum_{i-1}^{a} T_{i} \sum_{j-1, j \neq i}^{a} X_{i}, \quad c==\sum_{i=1}^{n} T_{i} \prod_{j-1, j}^{n} \not X_{j} ;$

begin real $c a, c b, c c, a, b, c, s y l, \because u$, terml, term2, term3, da, dif, sum;

integer $\mathrm{jm}, \mathrm{js}, \mathrm{jul}, \mathrm{ia}, \mathrm{ib}$;

start: $\quad$ witch alpha $:=\mathrm{L} 1, \mathrm{~L} 1, \mathrm{~L} 12$; switch bets := L9, L5, L6;

switch gamma $:=\mathrm{L} 10, \mathrm{~L} 11$; switch delta $:=\mathrm{L} 8$, L8, L13;

comment For interpolation, differentiation or integration set $\mathrm{jt}=1,2$, or 3 respectively;

go to alpha $[j t]$;

L1: if $x a r g \geq x a[n o p]$ then go to L2;

if $x a r g \geq x a[n o p-1]$ then go to L2;

if $x a r g \leq x a[1]$ then go to $L 3$;

if $x a r g \leq x a[2]$ then go to $\mathrm{L} 3$; go to $\mathrm{LA}$;

L2: $\quad$ jm $:=$ nop -1 ; js $:=1$; go to term;

L3: $\quad j m:=2 ;$ js $:=1$; go to term;

comment Locate argument;

L4: for ia $:=2$ step 1 until nop do begin

if $x a$ [ia] > xarg then go to L7; jm := ia end;

comment Before loop is complete xarg $\leq \mathrm{xa}$ [ia];

L5: $\quad$ ca $:=a$; cb $:=b$; cc $:=\mathrm{c}$; js $:=3$; jm := $\mathrm{jm}+1$; go to term;

I6: $\quad a:=(c a+a) / 2 ; \quad b:=(c b+b) / 2 ; \quad c:=(c c+c) / 2$; go to L9;

L7: js:=2; go to term;

L8: go to beta [js];

L9: go to gamma $[j t]$;

comment Interpolation, $\mathrm{jt}=1$;

L10: $\quad \mathrm{da}:=\mathrm{a} \times \times \times \operatorname{xarg} \uparrow 2+\mathrm{b} \times \mathrm{xarg}+\mathrm{c} ;$ go to exit1;

comment Differentiation, $\mathrm{jt}=2$;

L11: dif : $=2 \times x a r g+b$; go to exit2;

comment Integration, $\mathrm{jt}=3$;

L12: $\quad$ sum $:=0 ;$ syl $:=$ xlo; jul $:=$ nop -1 ; $\mathrm{ib}:=2$;

L16: for jm := ib step 1 until jul do begin;

comment Lagrange formulae;

term1 := ya $[j m-1] /((x a[j m-1]-x a[j m]) \times$ $(\mathrm{xa}[\mathrm{jm}-1]-\mathrm{xa}[\mathrm{jm}+1]))$;

term2 := ya $[j m] /((x a \cdot[j m]-x a[j m-1]) \times$ $(x a[j m]-x a[j m+1]))$; term3 : = ya $[j m+1] /((x a[j m+1]-x a[j m-1]) \times$

$(x a[j m+1]-x a[j m]))$;

$\mathrm{a}:=$ term $1+$ term $2+$ term 3 ;

$b:=-(x a[j m]+x a[j m+1]) \times \operatorname{term} 1-(x a$

$\left.\left.[j m-1]+x a_{u} m+1\right]\right) \times$ term $2-(x a[j m-1]+$ $x a$ ljm]) $\times$ term 3 ;

$c:=x a[j m] \times x a[j m+1] \times$ terml $+x a[j m-1] \times$

$x a[j m+1] \times$ term $2+x a[j m-1] \times x a[j m] \times$

term3; go to delta [jt];

L13: if jm $\not 2$ then go to $\mathrm{Ll4}$;

ca : = a; cb := b; cc := c; go to L15;

L14: $\quad$ ca $:=(a+c a) / 2 ; \quad c b:=(b+c b) / 2 ; \quad c c:=$

$(\mathrm{c}+\mathrm{cc}) / 2$;

L15: " syu : = xa $[\mathrm{jm}]$;

sum $:=\operatorname{sum}+\mathrm{ca} \times($ syu $\uparrow 3-\operatorname{syl} \uparrow 3) / 3+\mathrm{cb} \times$

(syu $\uparrow 2-$ syl $\uparrow 2) / 2+$ cc $\times$ (syu - syl);

ca $:=\mathrm{a} ;$ cb $:=\mathrm{b} ;$ cc $:=\mathrm{c} ;$ syl $:=$ syu end;

comment End of loop on [jm] index;

sum $:=\operatorname{sum}+\mathrm{ca} \times(\operatorname{xup} \uparrow 3$-syl $\uparrow 3) / 3+\mathrm{cb} X$

(xup $\uparrow 2$-syl $\uparrow 2$ ) $/ 2+$ cc $\times($ xup - sy1); go

to exit3;

term: $\quad$ ib $:=\mathrm{jm}$; jul $:=\mathrm{ib}$; go to Ll6;

comment The results for interpolation, differentiation, and

integration are $\mathrm{da}$, dif, and sum respectively;

exit1: AVINT : = da; go to exit;

exit2: AVINT : = dif; go to exit;

exit3: AVINT : $=$ sum;

exit: end

\section{CERTIFICATION OF ALGORITHM 77}

AVINT (Paul E. Hennion, Comm. ACM 5, Feb., 1962)

Victor E. WhitTier

Computations Res. Lab., The Dow Chemical Co., Midland, Mich.

AVINT was transliterated into BAC-220 (a dialect, of ALGOL-58) and was tested on the Burroughs 220 computer. The following minor errors were found:

1. The first statement following label Lil should read:

dif $:=2 \times a \times x a r g+b$;

2. The semicolon (;) at the end of the line beginning with the label L16 should be deleted.

3. There appears to be a confusion between " 1 " (numeric) and "l" (alphabetic) following label L12. This portion of the program should read:

L12: sum $:=0 ;$ syl $:=$ xlo; jul $:=$ nop $-1 ;$ ib $:=2$;

After making the above corrections the procedure was tested for interpolation, differentiation, and integration using $e^{x}, \log X$, and $\sin X$ in the range $(1.0 \leqq X \leqq 5.0)$. Twenty-one values of each of these functions, evenly spaced with respect to $\dot{X}$ and accurate to at least 7 significant digits, were tabulated in the above range. Then the procedure was tested. The following table indicates approximately the accuracy obtained:

\begin{tabular}{|c|c|c|c|}
\hline \multicolumn{4}{|c|}{ Number of Significant.Digits } \\
\hline $\begin{array}{l}\text { Funciron } \\
e^{x}\end{array}$ & $\geqq 4^{*}$ & $\geqq 2$ & $\geqq 4$ \\
\hline $\log X$ & $\geqq 4^{*}$ & $\geqq 2$ & $\geqq 3$ \\
\hline $\sin X$ & $\geq 4^{*}$ & $\geqq 2$ & $\geqq 4$ \\
\hline
\end{tabular}

* Except for interpolation between the first two points in the table.

The above results are quite reasonable in view of the relatively large increment in $X$. Tests using smaller increments in $X$ and uneven spacing of $X$ were also satisfactory.

It was also discovered that for integration the following restrictions must be observed:

1. $x l o \leqq x a(1)$.

2. $x u p \geqq x a$ (nop). 
REMARK ON ALGORITHM 77

INTERPOLATION, DIFFERENTIATION, AND INTEGRATION [P. E. Hennion, Comm. ACM 5, Feb. 1962]

P. E. Hennion

Giannini Controls Corp., Berwyn, Penn.

It was brought to my attention through the CERTIFICATION OF ALGORITHM 77 AVINT (V. E. Whittier, Comm. ACM, June, 1962) that restrictions on the upper and lower limits of integration existed, i.e., (1) $x 10 \leqq x a(1)$. (2) $x u p \geqq x a($ nop). To remove these restrictions the following two changes should be made.

1. Replace the two lines starting at line $L 12$ : and ending after the statement $i b:=2$; with the following code:

L12: $\operatorname{sum}:=0 ;$ syl $:=x 10 ; i b:=2 ; j u 1:=$ nop;

for $i a:=1$ step 1 until nop do begin

if $x a[i a] \geqq x 1 o$ then go $t o L 17 ; \quad i b:=i b+1$; end;

L17: for $i a:=1$ step 1 until nop do begin

if $x u p \geqq x a[j u 1]$ then go to $L 18 ; j u 1:=j u 1-1$; end;

L18: $j u 1:=j u 1-1$

2. Change line $L 13$ : to read

$L 13$ : if $j \neq i b$ then go to $L 14$;

\section{ALGORITHMI 78}

RATIONAL ROOTS OF' POLYNOMIALS WITH INTEGER COEFICIENTS

C. Perry

University of California at San Diego, La Jolla, California

comment This Algol procedure, named ratfact, for finding rational roots of polynomials with integer coefficients is a pedagogical example illustrating the use of the for statement described in section 4.6.3. Also, an extension suggested by J. Peck of the well-known polynomial evaluation by nesting, i.e. Horner's method, is used. The polynomial $f(x)=a_{0}+a_{1} x+$ $\cdots+a_{n} x$ " with integer coefficients and with $a_{0} a_{n} \neq 0$ has a lowest term rational root $p / q$ if and only if $a_{0} q^{n}+a_{1} q^{n-1} p+$ $\cdots+a_{n-1} q p^{n-1}+a_{n} p^{n}=0$, also $q$ must be a factor of $a_{n}$ and $p$ a factor of $a_{0}$. Procedure Ratfact outputs the nonzero rational roots $\mathrm{p} / \mathrm{q}$ by execution of the procedure whose formal name is print. The output procedure uses the string whose formal name is format for control of the output format

procedure ratfact (a, $n$, print, format);

integer array a $[0: n]$; integer $n$; procedure print; string format;

begin integer $i, p, q, r, t, f, g$;

p loop: for $p:=1$ step 1 until abs $(a[0])$ do

begin comment if $p$ is not a factor of a [0] or $q$ is not a factor of $a \mid n]$ then skip to the end of the loop for advance in the respective for list;

if $\mathrm{a}[0] \neq(\mathrm{a} \mid 0] \div \mathrm{p}) \times \mathrm{p}$ then go to 1

else $q$ loop: for $q:=1$ step 1 until abs $(a[n])$ do

begin if $a[n] \neq(a[n] \div q) \times q$ then go to 2

else

begin comment root test and print; comment start polynomial evaluation;

$\mathrm{f}:=\mathrm{g}:=\mathrm{a}[0] ; \mathrm{t}:=\mathrm{p}$

for $i:=1$ step 1 until $n$ do

begin $r:=a \mid i] \times t$;

$f:=f \times q+r$

$\mathrm{g}:=-\mathrm{g} \times \mathrm{q}+\mathrm{r}$

$\mathrm{t}:=\mathrm{t} \times \mathrm{p}$

end polynomial evaluation; comment computing $r$ saves one subscript evaluation;

if $f=0$ then print (format, $p, q$ );

if $\mathrm{g}=0$ then print (format, $-\mathrm{p}, \mathrm{q}$ );

comment print is the formal name of the procedure

to be used to output the variables in the format

specified by the string whose formal name is format; end root test and print;

2: end q loop;

1: end $p$ loop;

end ratfact, without overflow test.

CERTIFICATION OF ALGORITHM 78

RATFACT (C. Perry, Comm. ACM 5, Teb. 1962)

M. H. Halstead

Navy Electronics Laboratory, San Diego, Calif.

RATFACT was copied in the Navy Electronics Laboratory International Algol Compiler, Neliac, and tested on the UNivac M-490 Countess and the CDC 1604. Polynomials of order 2 through 6 were tested. No corrections were found necessary. It was noted that a polynomial whose coefficients included $a$, common factor would producc superfluous values of $p / q$, in whish this fraction was indecd $a$ root, but one in which $p$ and $q$ contained a common factor.

REMIAR ON ALGORITHAI 78

RATIONAL ROOTS OF POLYNOMIALS WITH INTEGER COEFFICIENTS [C. Perry, Comm. $A C M$, Feb. 1962]

D. MI. Collisox

Elliott Bros. (London) Ltd., Borehamwood, Herts., England

The algorithm was successfully run using the Elliot Algor, translator on the National-Elliott 803 . It was noticed that a multiple rational root will only be printed once by the procedure.

\section{ALGORITHM 79}

\section{DIFFERENCE EXPRESSION COEHFICIENTS}

Thomas P. Giammo

Space Technology Lahoratories, Inc., Los Angeles, California

procedure dicol ( $k, n, x p, x t a b$, coef);

value $k, n$; integer $k, n$; real $x p$;

array $x$ tab, coef;

comment dicol produces the coefficients for the $\mathrm{n}$ ordinates (corresponding to the abscissae, $x$ tab) in the $n$-point finite difference expression for the $\mathrm{k}$-th derivative evaluated at $\mathrm{xp}$. The method used is to determine the analytic expression for the $k$-th derivative of each coefficient in the $n$-point Lagrangian interpolation formula and evaluate it at $x p$. Note that $\mathrm{k}=0$ will produce the Lagrangian interpolation coefficients them. selves;

begin integer array xuse $[1: n-1]$; real factk, sum, denom, part;

integer $i$, terms, $j$, $m$, high;

factk $:=1.0 ;$ for $\mathrm{i}:=2$ step 1 until $\mathrm{k}$ do factk $:=\mathrm{i} \times$ factk; terms : $=n-k-1$; if terms $<0$ then go to $\mathrm{Z}$;

for $\mathrm{j}:=1$ step 1 until $\mathrm{n}$ do 
loop: begin sum $:=0$; denom $:=1.0 ;$ part $:=1.0$;

for $i:=1$ step 1 until $n$ do

if $\mathrm{i} \neq \mathrm{j}$ then denom $:=\operatorname{denom} \times(x \operatorname{xab}[j]-\operatorname{xtab}[\mathrm{i}])$; if terms $=0$ then go to $Y$; $\mathrm{m}:=1 ;$ high $:=1$;

$A: \quad$ if $($ high $=j) \vee(x$ tab $[$ high] $=x p)$ then A1: begin high := high +1 ; go to $A$ end $A 1$; if high $>n$ then $A 2$ : begin $m:=m-1$; if $m>0$ then

A3: begin high := xuse $[\mathrm{m}]+1$; go to $\mathrm{A}$ end $\mathrm{A3}$; go to $X$ end $A 2$;

xuse $[\mathrm{m}]:=$ high; $m:=m+1$;

if $m \leqq$ terms then begin high $:=$ high +1 ; go to $A$ end;

for $i:=1$ step 1 until terms do

part := part $\times(x p-x$ tab $[x u s e[i]])$;

sum $:=$ sum + part; $m:=$ terms; part $:=1.0$;

high $:=$ xuse [terms] +1 ; go to $\mathrm{A}$;

$Y:$ sum $:=1.0$;

$X:$ coef $[j]:=$ sum $\times$ factk/denom end loop;

go to EXIT;

$\mathrm{Z}:$ for $\mathrm{i}:=1$ step 1 until $\mathrm{n}$ do coef $[\mathrm{i}]:=0$;

EXIT: end dicol

\section{CERTIFICATION OF ALGORITHM 79}

DIFFERENCE EXPRESSION COEFFICIENTS

[Thomas Giamo, Comm. ACM, Feb. 1962]

\section{Eva S. Clark}

University of California at San Diego, La Jolla, California

The procedure was translated into Fortran and run on the CDC 1604. Reasonable accuracy was obtained for $k=0,4 \leqq n \leqq 12$. For increasing $n$ and increasing $k$, the accuracy diminished. It was found that the execution time increased rapidly as $n$ was increased. For $k=0$, the following results were obtained:

$\begin{array}{rc}n & \text { Approximale Number of Machine:Operations } \\ 4 & 1.3 \times 10^{3} \\ 6 & 6.9 \times 10^{3} \\ 8 & 3.8 \times 10^{4} \\ 10 & 1.8 \times 10^{5} \\ 12 & 8.6 \times 10^{5}\end{array}$

The author indicated in a letter that the procedure was developed for use with small $n$ and small $k$.

\section{ALGORITHM 80 \\ RECIPROCAL GAMMA FUNCTION OF REAL ARGUMENT}

William Holsten

University of California at San Diego, La Jolla, California

real procedure $R G R(x)$; real $x$; real procedure $R G A M$; comment Procedure RGAM computes the real reciprocal Gamma function of real $x$ for $-1<x<1$, utilizing Horner's method for polynomial evaluation of the approximation polynomial. RGR extends the range of RGAM by use of the formulae

(1) $1 / \operatorname{Gamma}(x-1)=(x-1) / \operatorname{Gamma}(x)$ for $x<-1$,

(2) $1 / \operatorname{Gamma}(x+1)=1 / x \times \operatorname{Gamma}(x)$ for $x<1$;

begin real $y$;

if $x=0$ then begin $R G R:=0$; go to EXIT end

if $x=1$ then begin RGR := ; go to EXIT end

if $\mathrm{x}<1$ then go to $\mathrm{BB}$; $y:=1$

$\mathrm{x}:=\mathrm{x}-1 ; \mathrm{y}:=\mathrm{y} \times \mathrm{x} ;$ if $\mathrm{x}>1$ then go to $\mathrm{AA}$;

if $x=1$ then begin $R G R:=1 / y$; go to EXIT end

RGR := RGAM(x)/y; go to EXIT;

BB: if $x=-1$ then begin RGR :=0; go to EXIT end

if $\dot{x}>-1$ then begin RGR := RGAM(x);

go to EXIT end

$\mathbf{y}:=\mathbf{x}$;

CC: $\quad x:=x+1 ;$ if $x<-1$ then begin $y:=y \times x$; go to $\mathrm{CC}$ end

RGR := RGAM $(x) \times y$;

EXIT: end RGR;

real procedure $\operatorname{RGAM}(x)$; real $x$; integer $i$;

real array $B[0: 13]$;

comment The algorithm for this routine was adapted from

"University of Illinois Digital Computer, Auxiliary Library

Routine B-17-328", by John Ehrman. Reference may also be made to Algorithm 34, dated February, 1961. Approximation accuracy is $\pm 2^{-35}$;

begin real $z$;

$\mathrm{B}[0]:=1.000000000000 ; \mathrm{B}[1]:=-.422784335092$;

$\mathrm{B}[2]:=-.233093736365 ; \mathrm{B}[3]:=+.191091101162$;

$\mathrm{B}[4]:=-.024552490887 ; \mathrm{B}[5]:=-.017645242118$

$\mathrm{B}[6]:=+.008023278113 ; \mathrm{B}[7]:=-.000804341335$;

$\mathrm{B}[8]:=-.000360851496 ; \quad \mathrm{B}[9]:=+.000145624324$

$\mathrm{B}[10]:=-.000017527917 ; \quad \mathrm{B}[11]:=-.000002625721 ;$

$\mathrm{B}[12]:=+.000001328554 ; \mathrm{B}[13]:=-.000000181220$;

$\mathrm{z:}=\mathrm{B}[13]$;

for $\mathrm{i}:=12$ step -1 until 0 do $z:=\mathrm{z} \times \mathrm{x}+\mathrm{B}[\mathrm{i}]$;

RGAM : $=\mathrm{z} \times \mathrm{x} \times(\mathrm{x}+1)$

end RGAM;

\section{ALGORITHM 81}

\section{ECONOMISING A SEQUENCE 1}

Brian H. MaYOH

Digital Computer Laboratory, University of Illinois, Urbana, Ill.

procedure ECONOMISER 1 (desired property, costs, n, C);

array costs; integer $n$;

Boolean procedure desired property;

Boolean array $\mathrm{C}$

begin comment Given a finite, monotonely increasing sequence of positive numbers, looked upon as prices, ECONOMISER 1 selects the cheapest subsequence with a given property. The formal parameters are: Desired property, a function designator to answer the question: Does the subsequence held in array $C$ possess the required property? $n$ is (number of elements in the sequence) +1 . Costs is an array of size $[1: n]$. Costs[1] to costs[n-1] hold the numbers of the sequence and $\operatorname{costs}[n]$ is any arbitrary number greater than the sum of all other elements of costs. $C$ is an array of the same size and indicates a subsequence by the rule: $C[i] \equiv$ element $i$ of the original sequence is in the subsequence. At exit from ECONOMISER 1, $C$ indicates the cheapest subsequence. It is supposed that the original sequence has the desired property.;

integer $\mathrm{d}, \mathrm{j}, \mathrm{k}, \ell$; real $\mathrm{i}$;

for $\mathrm{j}:=1$ step 1 until $n$ do $C[j]:=j=1 ; d:=0$; reenter: $\mathrm{d}:=\mathrm{d}+1$;

INSIDE: begin own real array prices $[1: d]$; own Boolean array alternatives $[1: d, 1: n]$; procedure ENTER SUCCESSORS; 


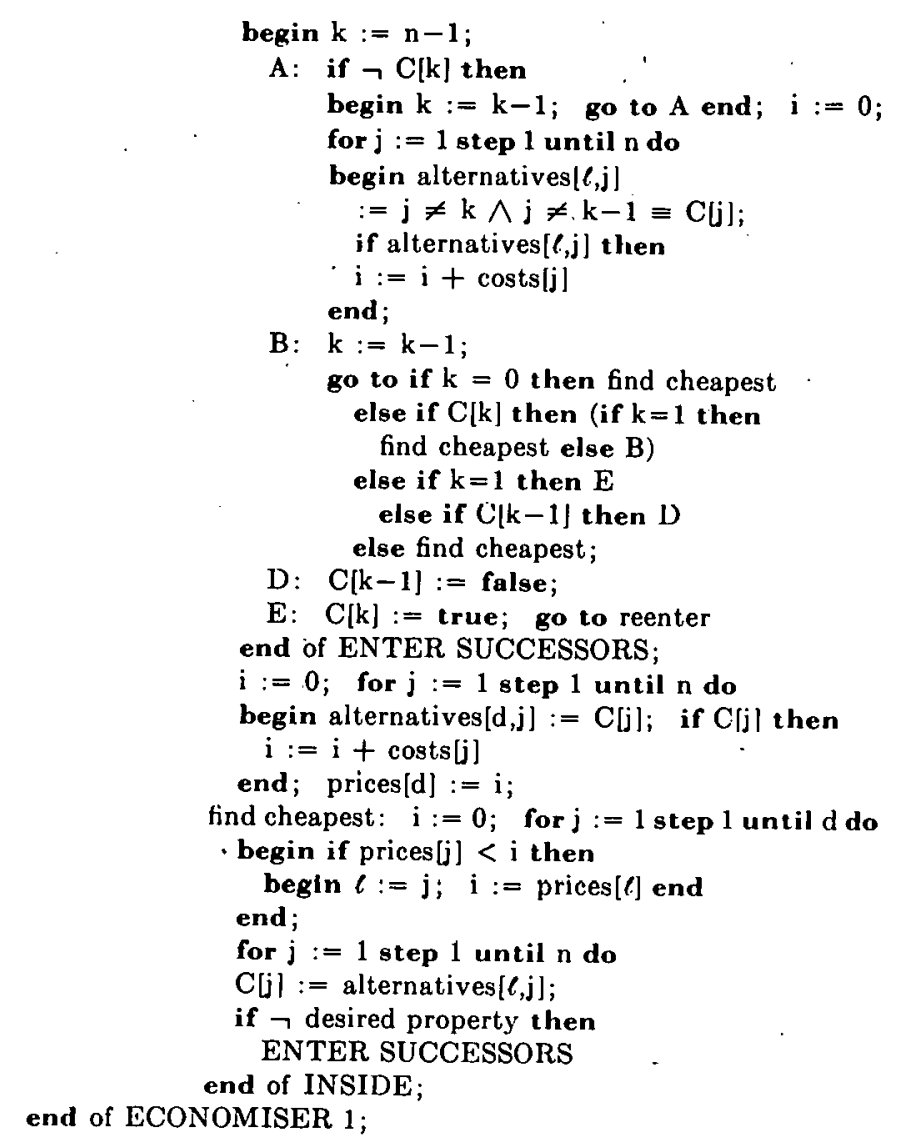

\section{ALGORITHM 82}

\section{ECONOMISING A SEQUENCE 2}

BRIAN H. MAYOH

Digital Computer Laboratory, University of Illinois, Urbana, Ill.

procedure ECONOMISER 2 (desired property, costs, n, C, r, Reject list); Boolean procedure desired property;

integer $n, r$; array costs; Boolean array Reject list;

begin comment In some applications of ECONOMISER 1, it is simple to establish that some subsequences are redundant in the sense that any sequence containing them is certainly not the cheapest subsequence with the desired property. For such applications ECONOMISER 2 avoids all unnecessary calls of desired property. The new formal parameters are: $r$ a variable whose value is initially 0 and is increased by 1 every time that desired property discovers a new redundant subsequence. Reject list an array of size $[1: \mathrm{r}, 1: \mathrm{n}]$. Reject list $[\mathrm{a}, \mathrm{b}]$ carries the answer to: Is element $b$ of the original sequence in the $a^{\text {th }}$ redundant subsequence found by desired property?;

real $i$; integer $d, j, k, \ell$; Boolean gapfilled, first time; procedure INSIDE (entrymaker); Boolean entrymaker:

begin own real array prices $[1: d]$;

own Boolean array alternatives $[1: d, 1: n]$; procedure ENTER SUCCESSORS;

hegin integer $c$; Boolean array ssq[1:n]; for $j:=1$ step 1 until $n$ do ssq[j] := $C[j]$; $\mathrm{c}:=\mathrm{n}-1$
A: if $\neg \mathrm{ssq}[\mathrm{c}]$ then begin $\mathrm{c}:=\mathrm{c}-1$; go to $\mathrm{A}$ end : $\mathrm{C}[\mathrm{c}]:=$ false; $\mathrm{C}[\mathrm{c}+1]:=$ true;

INSIDE (true); gapfilled $:=$ true;

B: $\quad c:=c-1$; go to if $c=0$ then $F$ else if ssq[c] then

(if $c=1$ then $F$ else $B$ ) else if $c=1$ then

$E$ else if ssqlc-1) then $D$ else $F$;

$D: \operatorname{ssq}[c-1]:=$ false;

$\mathrm{E}:$ for $\mathrm{j}:=1$ step 1 until $n$ do $C[j]:=\operatorname{ssq}[j] \equiv j \neq c$; INSIDE (true);

F: end of ENTER SUCCESSORS;

if entrymaker then

begin for $j:=1$ step 1 until $r$ do

begin for $k:=1$ step 1 until $n$ do

begin if $\neg \mathrm{C}[\mathrm{k}] \wedge$ Reject list $[j, \mathrm{k}]$ then

go to $\mathrm{G}$ end;

$\mathrm{G}$ : end;

ENTER SUCCESSORS; go to $\mathrm{H}$;

$\mathrm{i}:=0$; if gapfilled then $\mathrm{d}:=\mathrm{d}+1$;

for $\mathrm{j}:=1$ step 1 until $\mathrm{n}$ do

begin alternatives[if gapfilled then

d else $\ell, \mathrm{j}]:=\mathrm{C}[\mathrm{j}]$;

if $\mathrm{C}[\mathrm{j}]$ then $\mathrm{i}:=\mathrm{i}+\operatorname{costs}[\mathrm{j}]$

end; prices[if gaptilled then d else $\ell]:=\mathrm{i}$

end; if first time $V \neg$ entrymaker then

begin $i:=0$; gapfilled $:=$ first time $:=$ false;

for $j:=1$ step 1 until d do

begin if prices $[j]<i$ then

begin $\ell:=\mathrm{j} ; \quad \mathrm{i}:=\operatorname{prices}[\ell]$ end

end;

for $\mathrm{j}:=1$ step 1 until $\mathrm{n}$ do

$\mathrm{C}[\mathrm{j}]:=$ alternatives $[\ell, \mathrm{j}]$;

if desired property then go to found;

ENTER SUCCESSORS; go to reenter

end;

$H$ : end of INSIDE;

for $\mathrm{j}:=1$ step 1 until $n$ do $C[j]:=j=1$;

$\mathrm{d}:=0 ;$ first time $:=$ gapfilled $:=$ true;

reenter: INSIDE (first time);

found:

end of ECONOMISER 2;

\section{ALGORITHM 83}

\section{OPTIMAL CLASSIFICATION OF OBJECTS}

BRIAN H. MAYOH

Digital Computer Laboratory, University of Illinois, Urbana, Ill.

procedure OPTIMUM COVERING FINDER (Pattern, population, set number, set prices, chosen sets, bounds, overflow); Boolean array. Pattern, chosen sets; integer population, set number, bounds; array set prices; label overflow;

begin comment The number of objects in some given set is given by population. The procedure is given a classification of these objects by a collection of overlapping subsets. A cost is assigned to each subset. Then OPTIMUM COVERING FINDER selects the cheapest subcollection such that every object is contained in at least one of the subsets of the subcollection. set prices[i] carries the cost of subset $i$. Pattern is an array of size [1:set number, 1 :population] such that Pattern $[a, b] \equiv$ does subset $a$ include object $b$. chosen sets $[i]$ finally 
carries the answer to the question: Is set $i$ in the cheapest subcollection? The programmer must restrict the amount of space available to the procedure by setting bounds. From experience bounds $=$ set number $\uparrow 2$ suffices to avoid most alarm exits to overflow.;

Boolean array $\mathrm{C}[1$ :population], D[1:bounds, 1:population], $R, S[1$ :bounds, 1 : set number $]$;

integer $a, b, d, r, s$;

Boolean procedure HAVE WE A COVERING;

begin procedure $A D D$ to $(Q, q, f)$; integer $q$; real $f$; Boolean array $Q$;

begin if $q=$ bounds then go to overflow else $q:=q+1$; for $a:=1$ step 1 until set number do $Q[q, a]:=f$

end; for a := 1 step 1 until population do $\mathrm{C}[\mathrm{a}]:=$ false;

for a := 1 step 1 until set number do

begin if chosen sets[a] then

for $b:=1$ step 1 until population do

$\mathrm{C}[\mathrm{b}]:=\mathrm{C}[\mathrm{b}] \vee$ Pattern[a,b]

end; for $a:=1$ step 1 until population do

begin if $\neg \mathrm{C}[\mathrm{a}]$ then go to $\mathrm{E}$ end;

go to found;

$E$ : for $d:=1$ step 1 until s do

begin for $b:=1$ step 1 until population do

begin if $C[b] \wedge \neg D[d, b]$ then go to try another end; ADD to ( $R, r$, chosen sets[a]);

for $b:=1$ step 1 until set number do

begin if chosen sets[b] $\wedge \neg \mathrm{S}[\mathrm{d}, \mathrm{b}]$ then

ADD to $(R, r, S[d, a] \vee a=b)$

end; go to $\mathrm{F}$;

try another:

end of for statement labelled $\mathrm{E}$;

AlJ) to (S, s, chosen sets[a]);

for $\mathrm{a}:=1$ step 1 until population do $\mathrm{D}[\mathrm{s}, \mathrm{a}]:=\mathrm{C}[\mathrm{a}]$;

F: HAVE WE A COVERING $:=$ false

end; $r:=s:=0$;

ECONOMISER 2 (HAVE WE A COVERING, set prices,

set number, $r, R$, chosen sets);

found: end

\section{ALGORITHM 84}

\section{SIMPSON'S INTEGRATION}

PaUl E. Hennion

Giannini Controls Corporation

Astromechanics Research Division, Berwyn, Penn.

real procedure $\operatorname{SIM}(n ; a, b, y)$;

value $n, a, b$; real $a, b$; integer $n$; array $y$;

comment This is a method for obtaining the approximate value of the definite integral of a continuous function when the integral cannot be evaluated in elementary functions. Given $y=f(x)$ and the $\int_{a}^{b} y d x$ to be evaluated. Plot the curve $f(x)$, and divide $[a, b]$ evenly into $n$ equal parts, erecting the ordinates $y_{0}, y_{1}, \cdots, y_{n}$. Then the approximate value of the definite integral by Simpson's rule states that:

$$
\int_{a}^{b} f(x) d x=\frac{b-a}{3 n}\left(y_{0}+4 y_{1}+2 y_{2}+\cdots+4 y_{n-1}+y_{n}\right)
$$

begin real $s$; integer $i$;

$\mathrm{s}:=(\mathrm{y}[0]-\mathrm{y}[\mathrm{n}]) / 2$;

for $\mathrm{i}:=1$ step 2 until $\mathrm{n}-1$ do $\mathrm{s}:=\mathrm{s}+2 \times \mathrm{y}[\mathrm{i}]+\mathrm{y}[\mathrm{i}+1]$;

$\operatorname{SIM}:=2 \times(b-a) \times s /(3 \times n)$

end
CERTIFICATION OF ALGORITHM 84

SIMPSON'S INTEGRATION [P. E. Hennion, Comm.

ACM, Apr. 62]

Peter G. Behrenz

Matematikmaskinnïmnden, Stockholm, Sweden

SIM was successfully run on FACIT EDB using F ACIT-Algor. 1 , which is a realization of ALGOL 60 for FACIT EDB. No changes in the program were necessary. To test SIM some polynomials were integrated.

\section{CERTIFICATION OF ALGORITHM 84}

SIMPSON'S INTEGRATION [P. E. Hennion, Comm. $A C M 5$ (Apr. 1962)]

A. P. RELPH

The English Electric Co., Whetstone, England

Simpson's Integration was translated using the Deuce Algol compiler and, with no corrections, gave satisfactory results.

It is not stated in the comment that. integer $n$ needs to be even.

\section{REMARK ON ALGORITHM 84}

SIMPSON'S INTEGRATION [Paul E. Hennion. Comm. ACMI, Apr. 1962]

Richard George*

Particle Accelerator Div., Argonne National Lab., Argonne, III.

* Work supported by the U. S. Atomic Energy Commission.

In performing integration by the use of Simpson's rule, it is well known that the interval $[a, b]$ must be divided evenly into $n$ equal parts, and that it is essential for $n$ to be an even number.

In the published algorithm, there is neither a comment on this important restriction, nor a programmed test for the parity of $n$. It is therefore a potential trap for the unwary programmer

\section{ALGORITHM 85 \\ JACOBI}

Thomas G. Evans

Bolt, Beranek, and Newman*, Cambridge, Mass.

* This work has been sponsored by the Air Force Cambridge Research Laboratories, OAR (USAF), Detection Physics Lab. oratory, under contract AF 19(628)-227.

procedure JACOBI (A, S, n, rho);

value $n$, rho; integer $n$; real rho; real array $A, S$; comment This procedure finds all eigenvalues and eigenvectors of a given square symmetric matrix by a modified Jacobi (iterative) method (cf. J. Greenstadt, "The determination of the characteristic roots of a matrix by the Jacobi method," in Mathematical Methods for Digital Compulers, A. Ralston and H. S. Wilf, eds.). JACOBI is given a square symmetric matrix of order $n$ stored in the array $A$. The initial contents of the array $S$ are immaterial, as $S$ is initialized by the procedure. At exit the $k^{\text {th }}$ column of the array $S$ contains the $k^{\text {th }}$ of the $n$ eigenvectors of the given matrix, and the diagonal element $A[k, k]$ of the array $A$ is the corresponing $k^{\text {th }}$ eigenvalue. The parameter rho is the "accuracy" requirement" introduced in the above reference, where a detailed flow chart of the method is given. The significance of rho is that the iteration terminates when, for every off-diagonal ele- 
ment $A[i, j]$, abs $(A[i, j])<(r h o / n) \times$ norml, where norm1 is a function only of the off-diagonal elements of the original matrix; begin real norm1, norm2, thr, mu, omega, sint, cost, int1, v1, v2, v3;

integer $i, j, p, q$, ind;

comment Set array $S=n \times n$ identity matrix;

for $\mathrm{i}:=1$ step 1 until $\mathrm{n}$ do

for $j:=1$ step 1 until $i$ do

if $i=j$ then $S[i, j]:=1.0$

else $S[i, j]:=S[j, i]:=0.0$

comment Calculate initial norm (norm1), final norm (norm2), and threshold (thr);

int1 $:=0.0$;

for $i:=2$ step 1 until $n$ do

for $\mathrm{j}:=$ step 1 until $\mathrm{i}-1$ do intl $:=$ int $1+2.0 \times \mathrm{A}[\mathrm{i}, \mathrm{j}] \uparrow 2$

norm1 $:=$ sqrt (int); norm2 $:=($ rho $/ \mathrm{n}) \times$ norm1;

thr $:=$ norm 1 ; ind $:=0$;

main: thr $:=\operatorname{thr} / \mathrm{n}$;

comment The sweep through the off-diagonal elements begins here;

main 1: for $q:=2$ step 1 until $n$ do

for $p:=1$ step 1 until $q-1$ do

if abs $(A[p, q]) \geqq$ thr then

begin ind $:=1 ; \mathrm{v} 1: \doteq A[\mathrm{p}, \mathrm{p}] ; \mathrm{v} 2:=\mathrm{A}[\mathrm{p}, \mathrm{q}]$; v3 := A[q, q]; mu : $-0.5 \times(\mathrm{v} 1-\mathrm{v} 3)$;

omega := (if $\mathrm{mu}=0.0$ then 1 else sign $(\mathrm{mu})$ ) $\times$ $(-\mathrm{v} 2) / \mathrm{sqrt}(\mathrm{v} 2 \uparrow 2+\mathrm{mu} \uparrow 2)$

sint $:=$ omega/sqrt $(2.0 \times(1.0+\operatorname{sqrt}(1.0-$ omega $\uparrow 2))$ );

cost $:=\operatorname{sqrt}(1.0-\sin t \uparrow 2)$

for $i:=1$ step 1 until $n$ do

begin intl $:=A[i, p] \times \operatorname{cost}-A[i, q] \times$ sint;

$A[i, q]:=A[i, p] \times \sin t+A[i, q] \times$ cost;

$A[i, p]:=$ int1;

int1 $:=\mathrm{S}[\mathrm{i}, \mathrm{p}] \times$ cost $-\mathrm{S}[\mathrm{i}, \mathrm{q}] \times$ sint;

$\mathrm{S}[\mathrm{i}, \mathrm{q}]:=\mathrm{S}[\mathrm{i}, \mathrm{p}] \times \sin \mathrm{t}+\mathrm{S}[\mathrm{i}, \mathrm{q}] \times \cos \mathrm{t}$ $\mathrm{S}[\mathrm{i}, \mathrm{p}]:=$ inti

end :

for $\mathrm{i}:=$ step 1 until $\mathrm{n}$ do

begin $A[p, i]:=A[i, p] ; A[q, i]:=A[i q]$ end;

$\mathrm{A}[\mathrm{p}, \mathrm{p}]:=\mathrm{v} 1 \times \operatorname{cost} \uparrow 2+\mathrm{v} 3 \times \operatorname{sint} \uparrow 2-2.0 \times$

v2 $\times \sin t \times$ cost

$\mathrm{A}[\mathrm{q}, \mathrm{q}]:=\mathrm{v} 1 \times \sin \mathrm{t} \uparrow 2+\mathrm{v} 3 \times \operatorname{cost} \uparrow 2+2.0 \times$ v2 $\times$ sint $\times$ cost;

$\mathrm{A}[\mathrm{p}, \mathrm{q}]:=\mathrm{A}[\mathrm{q}, \mathrm{p}]:=(\mathrm{v} 1-\mathrm{v} 3) \times \sin \mathrm{t} \times \cos \mathrm{t}+$ end; $\mathrm{v} 2 \times(\operatorname{cost} \uparrow 2-\operatorname{sint} \uparrow 2)$

comment Now test to see if current tolerance exceeded and

if not, whether tinal tolerance reached;

if ind $=1$ then begin ind : $=0$; go to main 1 end

else if thr $>$ norm 2 then go to main

end JACOBI

CERTIFICATION OF ALGORITHM 85

JACOBI [T. G. Evans, Comm. ACAI, Apr. 1962]

J. S. Hillmore

Elliott Bros. (London) Ltd., Borehamwood, Herts., England

The statement

omega := (if $m u=0.0$ then 1 else $\operatorname{sign}(m u))$

$\times(-V 2) / \operatorname{sqrl}(V 2 \uparrow 2+m u \uparrow 2)$

was changed to

omega $:=$ if $m u=0.0$ then -1.0 else $-\operatorname{sign}(m u)$

X. $V 2 / \operatorname{sqr} l(V 2 \uparrow 2+m u \uparrow 2)$

When $m u=0$, the original statement reduces to

omega $:=-V 2 /$ squt $(V 2 \uparrow 2)$;

and a truncation error in the evaluation of the square root can make the magnitude of omega slightly. greater than unity. As a result, an error stop occurs during execution of the next statement when an at tempt is made to evaluate sqrl $(1-$ omega $\uparrow 2)$.

In its modified form the algorithm has been successfully rum using the Elliott ALgol translator on the National-Elliot. 803 . Matrices of order up to fifteen have been solved, yielding eigenvalues and eigenvectors with an overall accuracy of seven decimal places.

\section{CERTIFICATION OF ALGORITHA 8;}

JACOBI [Thomas G. Evans, Comm. ACM (Apr. 1962), 208]

P. NaUR

Regnecentralen, Copenhagen, Demmark

We have first run this algorithm in the (iIER Algol system with the following corrections included:

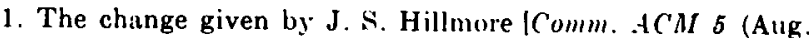
1962), 440] with capital $V$ changed to $r$.

2. The 4th for clause corrected to read:

$$
\text { for } j:=1 \text { step } 1 \text { until } i-1 \text { do }
$$

3. The last for clause corrected to read

$$
\text { for } i:=1 \text { step } 1 \text { until } n \text { do }
$$

On closer examination we have found, however, that a significant number of superfluous operations could be eliminated in the innermost loop by rewriting the two for statements at the center of the algorithm as a single for statement, to read as follows:

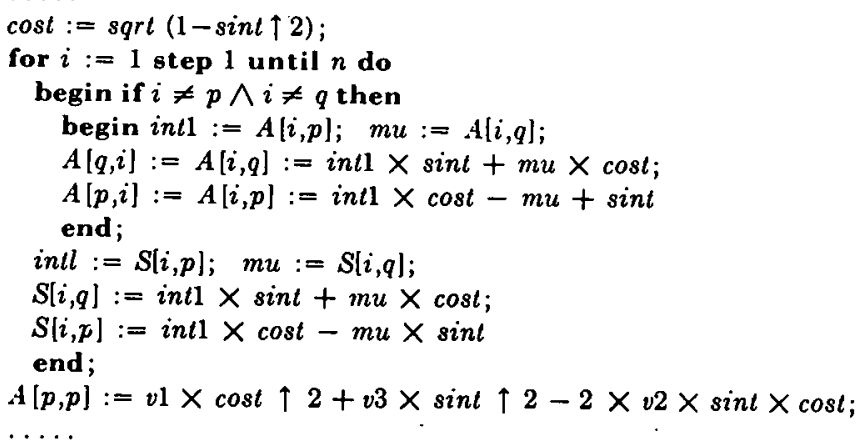

This revision is particularly advantageous in systems having a comparatively slow subscript mechanism, such as Gier Algol, because it eliminates more than 3 out of 8 references to subscripted variables.

JACOBI has been tried with two different sets of matrices having known eigenvalues. In both cases a test program was set up to find the range of errors of the eigenvalues computed by JACOBI. In addition, the relations $A v-\lambda v=0$ ( $A$ is the given matrix, $v$ an eigenvector, and $\lambda$ the corresponding eigenvalue) and $A-(S T)$ LAMBDA $S=0$ ( $S$ is the matrix having the eigenvectors as columns and $S T$ its transpose, and LAMBDA is the diagonal matrix of the eigenvalues) were used as checks. The test matrices were TESTMATRIX calculated by the revised algorithm 52 given in Comm. ACM 6 (Jan. 1963), 39, and the following matrix suggested by Mr. H. B. Hansen :

\section{HBH TESTMATRIX $[j, i]=$ HBH TESTMATRIX $[i, j]$

$$
=n+1-j \quad j \geqq i
$$

having the eigenvalues $0.5 /(1-\cos ((2 \times i-1) \times p i /(2 \times n+1)))$. 
The results were as shown in Table 1 (Gier Algol, works with floating numbers of 29 significant bits)

The compile time for the program which produced one of these tables was about 40 seconds. Run times were as follows:

\begin{tabular}{|c|c|c|c|c|}
\hline \multirow{4}{*}{$\begin{array}{c}\text { Kho } \\
10-3\end{array}$} & $n$ & \multicolumn{2}{|c|}{$\begin{array}{c}\text { Origina! algorithm } \\
\text { TESTMATRLX ALG. 52 HBH } \\
\text { TF.STMATRIX } \\
\text { (seconds) }\end{array}$} & \multirow{2}{*}{$\begin{array}{c}\text { Revised algo- } \\
\text { rilhm HBH } \\
\text { TESTMATRIX } \\
\text { (seconds) } \\
3\end{array}$} \\
\hline & 5 & & & \\
\hline & 10 & & & 22 \\
\hline & 15 & & & 70 \\
\hline $10-5$ & 5 & 3 & & 5 \\
\hline & 10 & 5 & 41 & 29 \\
\hline & 15 & 13 & 148 & 99 \\
\hline $10-8$ & 5 & 4 & 7 & 6 \\
\hline & 6 & 5 & 12 & \\
\hline & 7 & 5 & 18 & \\
\hline & 8 & 5 & 25 & $\cdot$ \\
\hline & 10 & 13 & & 38 \\
\hline & 15 & 22 & & 116 \\
\hline
\end{tabular}

From these figures it looks as if TESTMATRIX, Algorithm 52, is atypical as far as solution by means of JACOBI is concerned. The much higher accuracy obtained for this matrix as compared with the $\mathrm{HBH}$ matrix points in the same direction.

For further comparison it may be mentioned that the algorithms published by J. H. Wilkinson [Num. Math. 4 (1962), 35t376 also have been tested successfully with Gier Algol. Wilkinson's algorithms reduce the matrix to tridiagonal form by means of Householder's method and use Sturm sequences to find the eigenvalues and inverse iteration to find the eigenvectors. In Gier ALGoL this method is about 1.3 times as fast as JACOBI for the range of matrices considered here. JACOBI has the advantage that the eigenvectors are properly orthogonal, even in the case of multiple eigenvalues, and also has a much simpler logic. On the other hand if only some of the eigenvalues and/or eigenvectors are sought Wilkinson's algorithms will of ten offer much higher speed than JACOBI, which always finds them all.

SEE NEXT PAGE FOR TABLES

\section{ALGORITHM 86 \\ PERMUTE}

J. E. L. Peck and G. F. Schrack

University of Alberta, Calgary, Alberta, Canada

procedure PERMUTE $(\mathrm{x}, \mathrm{n})$;

array $x$; integer $n$;

comment Each call of PERMUTE executes a permutation of the first $n$ components of $x$. It assumes a nonlocal Boolean variable 'first', which when true causes the procedure to initialise the signature vector $p$. Thereafter 'first' remains false until after $n$ ! calls;

begin own integer array $p[2: n]$; integer $i, k$;

if first then

begin for $\mathrm{i}:=2$ step 1 until $\mathrm{n}$ do $\mathrm{p}[\mathrm{i}]:=\mathrm{i} ;$ first $:=$ false

end initialise;

for $k:=2$ step 1 until $n$ do begin integer $\mathrm{km}$; real $\mathrm{t}$;

$\mathrm{t}:=\mathrm{x}[1] ; \mathrm{km}:=\mathrm{k}-1$

for $i:=1$ step 1 until $\mathrm{km}$ do

$\mathrm{x}[\mathrm{i}]:=\mathrm{x}[\mathrm{i}+\mathbf{1}$;

$\mathrm{x}[\mathrm{k}]:=\mathrm{t} ; \mathrm{p}[\mathrm{k}]:=\mathrm{p}[\mathrm{k}]-1 ;$

if $p[k]: \neq 0$ then go to EXIT;

$\mathrm{p}[\mathrm{k}]:=\mathrm{k}$

end $\mathrm{k}$;

first := true;

EXIT: end PERMUTE

CERTIFICATION OF ALGORITHM 86

PERMUTE [J. E. L. Peck and G. F. Schrock, Comm. ACII, Apr. 1962]

D. M. Collison

Elliott Bros. (London) Ltd., Borehamwood, Herts., England

The algorithm was successfully run using the Elliott Algol translatior on the National-Elliott 803. Values of $n$ used were 0,1 , $2,3,4$.

\section{ALGORITHM 87 \\ PERMUTATION GENERATOR}

JoHN R. HowelL

Orlando Aerospace Division, Martin Marietta Corp., Orlando, Florida

procedure PERMUTATION (N, K);

value $K, N$; integer $K$; integer array $N$;

comment This procedure generates the next permutation in lexicographic order from a given permutation of the $K$ marks $0,1, \cdots,(K-1)$ by the repeated addition of $(K-1)$ radix $K$. The radix $K$ arithmetic is simulated by the addition of 9 radix 10 and a test to determine if the sum consists of only the original $K$ digits. Before each entry into the procedure the $K$ marks are assumed to have been previously specified either by input data or as the result of a previous entry. Upon each such entry a new permutation is stored in $N[1]$ through $N[K]$. In case the given permutation is $(K-1),(K-2), \cdots, 1,0$, then the next permutation is taken to be $0,1, \cdots,(K-1)$. A Fortran subroutine for the IBM 7090 has been written and tested for several examples;

begin integer $i, j$, carry;

for $\mathrm{i}:=1$ step 1 until $\mathrm{K}$ do

if $\mathrm{N}[\mathrm{i}]-\mathrm{K}+\mathrm{i} \neq 0$ then go to add;

for $\mathrm{i}:=1$ step 1 until $\mathrm{K}$ do $\mathrm{N}[\mathrm{i}]:=\mathrm{i}-1$;

go to exit;

add: $\quad \mathrm{N}[\mathrm{K}]:=\mathrm{N}[\mathrm{K}]+9$;

for $\mathrm{i}:=1$ step 1 until $K-1$ do

begin if $K>10$ then go to $B$;

carry $:=N[K-i+1] \div 10$; go to $\mathrm{C}$;

B: $\quad$ carry := N $[\mathrm{K}-\mathrm{i}+1] \div \mathrm{K}$;

C: if carry $=0$ then go to test

$\mathrm{N}[\mathrm{K}-\mathrm{i}]:=\mathrm{N}[\mathrm{K}-\mathrm{i}]+$ carry;

$\mathrm{N}[\mathrm{K}-\mathrm{i}+1]:=\mathrm{N}[\mathrm{K}-\mathrm{i}+1]-10 \times$ carry

end $i$;

test: for $\mathrm{i}:=1$ step 1 until $K$ do if $\mathrm{N}[\mathrm{i}]-(\mathrm{K}-1)>0$

then go to add:

for $\mathrm{i}:=1$ step 1 until $\mathrm{K}-1$ do

for $\mathrm{j}:=\mathrm{i}+1$ step 1 until $\mathrm{K}$ do

if $N[i]-N[j]=0$ then go to add;

exit: end PERMUTATION GENERATOR 
CERTIFICATION OF ALGORITHM 87

PERMUTATION GENERATOR [John R. Howell,

Comm. ACM (Apr. 1962)]

G. F. Schrack and M. Shimrat

University of Alberta, Calgary, Alb., Canada

PERMUTATION GENERATOR was translated into ForTRAN for the IBM 1620 and it performed satisfactorily. The algorithm was timed for several small values of $n$. For purposes of comparison we include the times (in seconds) for PERMULEX (Algorithm 102).

\begin{tabular}{r|rrrrr}
$n$ & 3 & 4 & 5 & 6 & 7 \\
\hline PERMUTATION Ginerator & 3 & 41 & 558 & - & - \\
PERMLLEA & - & 3 & 6 & 37 & 278
\end{tabular}

As can be seen from this table, PERMUTATION GENERATOR is considerably slower. It is probable that one could speed up PERMUTATION GENERATOR to a great extent by rearranging the algorithm in such a manner that the digits of a number to a certain base are permuted rather than the elements of a sequence.

\section{CERTIFICATION OF ALGORITHM 85}

TABLE 1

HBH TESTMATRIX

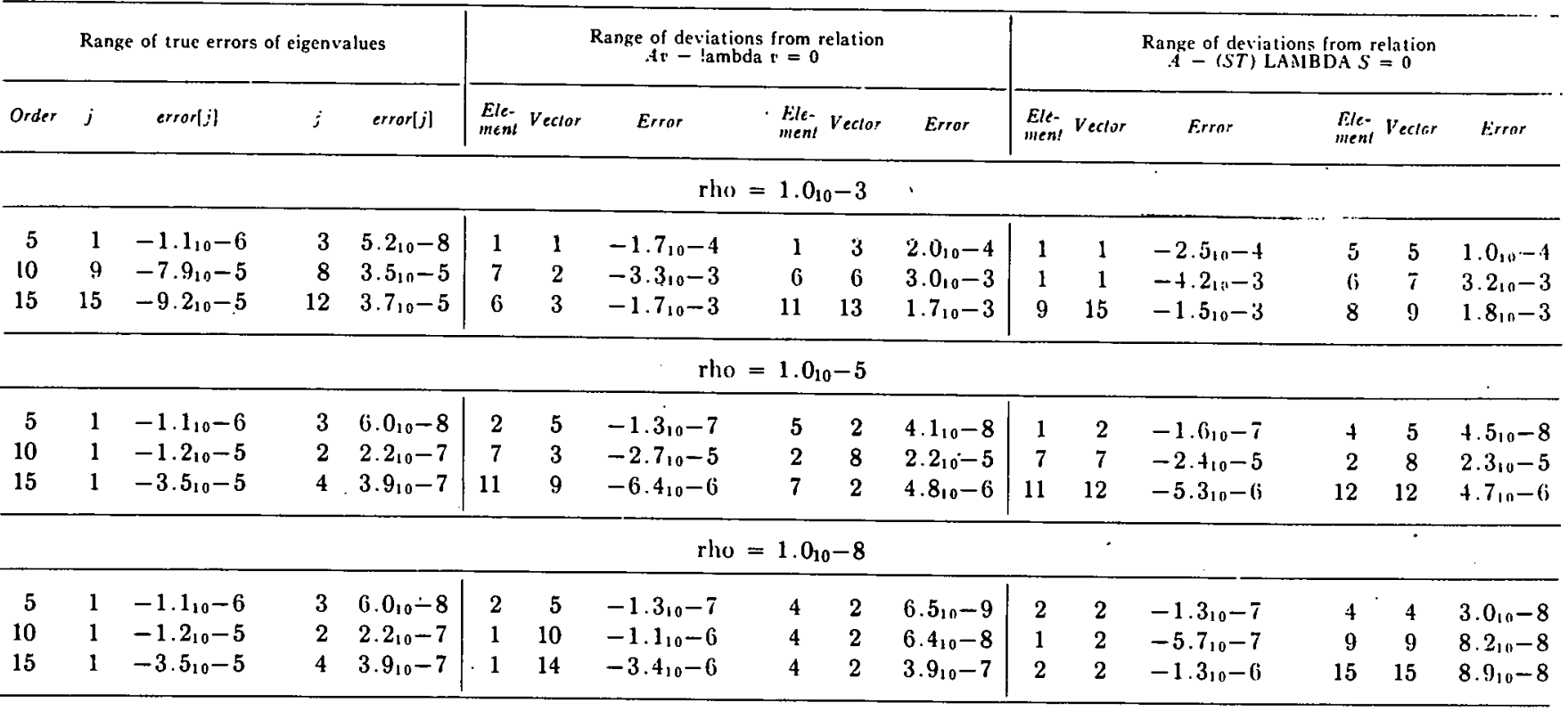

TESTMATRIX, Algorithm 52

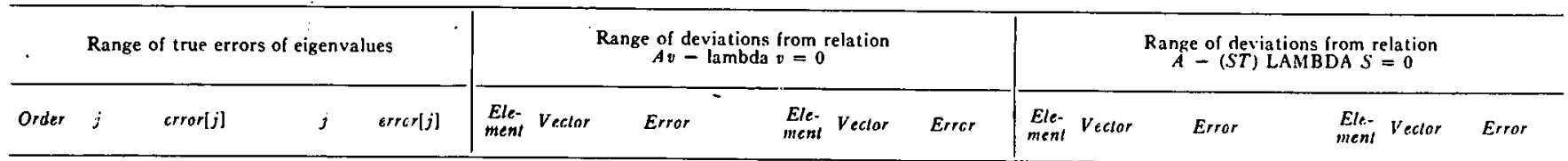

rho $=1.0_{10}-5$

\begin{tabular}{rrrrl|rrrrrrrrrrrrr}
5 & 4 & $-1.0_{10}-8$ & 1 & .0 & 5 & 5 & $-3.3_{10}-8$ & 5 & 4 & $4.3_{10}-8$ & 5 & 5 & $-5.1_{10}-8$ & 4 & 4 & $3.9_{10}-8$ \\
10 & 8 & $-1.1_{10}-8$ & 4 & .0 & 7 & 7 & $-1.210-8$ & $\therefore$ & 9 & 6 & $1.3_{10}-8$ & 7 & 8 & $-5.1_{10}-9$ & 6 & 6 & $2.0_{10}-8$ \\
15 & 13 & $-1.1_{10}-8$ & 6 & .0 & 14 & 14 & $-9.3_{10}-9$ & 10 & 10 & $9.4_{10}-9$ & 8 & 9 & $-1.9_{10}-9$ & 10 & 10 & $1.3_{10}-8$ \\
\hline
\end{tabular}

rho $=1.0_{10}-8$

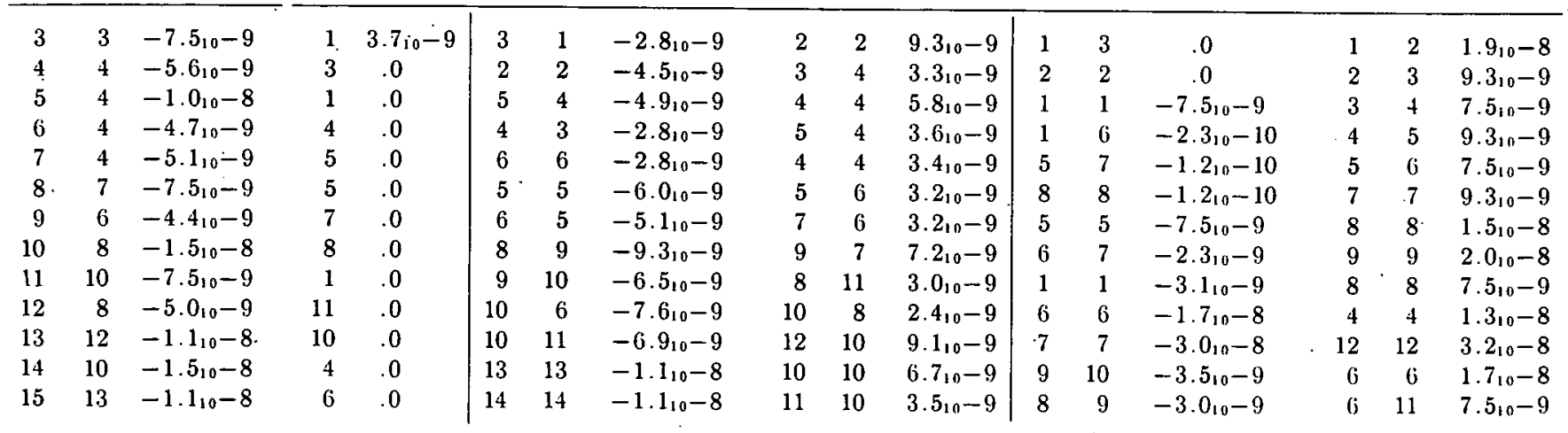


CERTIFICATION OF ALGORITHM 87

PERMUTATION GENERATOR. [John R. Howell,

Comm: $A C M I$, Apr. 1962]

D. M. Collisor

Elliott Bros. (London) Ltd., Borehnmwood, Herts., England

The array $N$ was removed from the value list in order that the permutations might be available outside the procedure. The algorithm was then run successfully with the Elliot t. Alcool translator on the National-Elliott 803. It was rather slower than Algorithm 86.

\section{ALGORITHM 88}

EVALUATION OF ASYMPTOTIC EXPRESSION FOR THE FRESNEL SINE AND COSINE INTEGRALS

JoHN L. CUNDIFF

Engineering Experiment Station, Georgia Institute of Technology, Atlanta, Ga.

real procedure FRESNEL (u) Result: (frcos, frsin); value (u);

comment This procedure evaluates the Fresnel sine and cosine integrals for large $u$ by expanding the anymptotic series given by

$$
\begin{aligned}
S(u)=\frac{1}{2}-\frac{\cos (x)}{\sqrt{2 \pi x}} & {\left[1-\frac{1 \cdot 3}{(2 x)^{2}}+\frac{1 \cdot 3 \cdot 5 \cdot 7}{(2 x)^{4}}-\cdots\right] } \\
& -\frac{\sin (x)}{\sqrt{2 \pi x}}\left[\frac{1}{2 x}-\frac{1 \cdot 3 \cdot 5}{(2 x)^{3}}+\frac{1 \cdot 3 \cdot 5 \cdot 7 \cdot 9}{(2 x)^{6}}-\cdots\right]
\end{aligned}
$$

and

$$
\begin{aligned}
C(u)=\frac{1}{2}-\frac{\sin (x)}{\sqrt{2 \pi x}} & {\left[1-\frac{1 \cdot 3}{(2 x)^{2}}+\frac{1 \cdot 3 \cdot 5 \cdot 7}{(2 x)^{4}}-\cdots\right] . } \\
- & -\frac{\cos (x)}{\sqrt{2 \pi x}} \cdot\left[\frac{1}{2 x}-\frac{1 \cdot 3 \cdot 5}{(2 x)^{3}}+\frac{1 \cdot 3 \cdot 5 \cdot 7 \cdot 9}{(2 x)^{5}}-\cdots\right]
\end{aligned}
$$

in which $x=\pi u^{2} / 2$. Reference: Pearcey, T. Table of the Fresnel Integral to Six Decimal Places. The Syndics of the Cambridge University Press, Melbourne, Australia (1956).;

begin pi $:=3.14159265 ;$ arg $:=$ pi $\times(u \uparrow 2) / 2$; temp $:=1$; argsq $:=1 /(4 \times(\arg \uparrow 2)) ;$ term $:=-3 \times$ argsq; series := $1+$ term; $N:=3$;

first: $\quad$ if temp $=$ series then go to second; temp := series; termi := term;

term := $=-$ termi $\times(4 \times N-7) \times(4 \times N-5) \times($ argsq); if abs(term) $>$ abs(termi) then go to second; series := temp + term; $\mathrm{N}:=\mathrm{N}+1 ;$ go to first;

second: series $2:=\frac{1}{2} \times \arg ;$ temp $:=0$; term $:=$ series 2 ; $\mathrm{N}:=2$;

loop: if series2 = temp then go to exit; termi := term; term : $=-$ termi $\times$ argsq $\times(4 \times N-5) \times(4 \times N-3)$; if $\operatorname{abs}($ term) $>$ abs(tcrmi) then @o to exit; temp $:=$ series2; series2 $:=$ temp + $1 \mathrm{crm}$; $\mathrm{N}:=\mathrm{N}+1$; go to lool?;

exit: if $\mathrm{u}<0$ then half $:=-\frac{1}{2}$ else half $:=\frac{1}{1}$;

frcos $:=$ half $+(\sin (\arg ) \times$ series $-\cos (\arg )+\operatorname{series} 2) /$ $($ pi $\times$ 11):

frsin $:=$ half $-(\cos (\arg ) \times \operatorname{series} 2+\sin (\arg ) \times$ series $) /$ (pi $\times u)$

end FRESNEL;
REMARK ON ALGORITHMS 88, 89 AND 90

EVALUATION OF THE FRESNEL INTEGRALS

[J. L. Cundiff, Comm. ACM, May 1962]

Malcolm D. Gray

The Boeing Co., Seattle, Wash.

While coding these algorithms in FORTRAN for the IBM 7094, modifications were required (both in the formulation and in the language) before execution with any degree of speed and accuracy could be obtained. In the process it was found that the reference, Pearcy, contains an error in the formula for $C(u)$. This error is contained in Algorithm 88 in the formula

$$
C^{\prime}(u)=\frac{1}{2}-\frac{\sin (x)}{\sqrt{2 \pi x}} \mid \quad l-\cdots .
$$

The first minus sign above should be a plus sign.

After the necessary modifications were made, the three algorithms were found to be too large and uneconomical for our usige. A single algorithm, incorporating these three procedures, was written and is in current usage in a computer program which refpuires several thousand evaluations of each Fresnel integral.

\section{ALGORITHM 89}

EVALUATION OF THE FRESNEL SINE INTEGRAL JoHN L. CUNDIFF

Engineering Experiment Station, Georgia Institute of Technology, Atlanta, Ga.

real procedure FRESNELSIN (u) Result: (frsin); value u; comment This algorithm computes the Fresnel sine integral defined by,

$$
S(u)=\int_{0}^{u} \sin \pi t^{2} / 2 d t,
$$

by evaluating the series expansion

$$
S(x)=\sqrt{\frac{2 x}{\pi}}\left[\frac{x}{3}-\frac{x^{3}}{7 \cdot 3 !}+\frac{x^{5}}{11 \cdot 5 !}-\frac{x^{7}}{15 \cdot 7 !}+\cdots\right]
$$

where $x=\pi u^{2} / 2$. Reference: PEARCEY, T. Table of the Fresnel Integral to Six Decimal Places. The Syndics of the Cambridge University Press, Melbourne, Australia (1956).;

begin $\mathrm{Pi} 2:=1.5707963 ; \mathrm{x}:=\mathrm{Pi} 2 \mathrm{x}(\mathrm{u} \uparrow 2) ;$ frsin $:=\mathrm{x} / 3$; frsqr $:=x \uparrow 2 ; N:=3$; term $:=(-x \times$ frsqr $) / 6$; frsini $:=$ frsin + term $/ 7$;

Loop: if $\mathrm{frsin}=$ frsini then go to exit; frsin := frsini; term : $=-$ term $\times$ frsqr $/((2 \times N-1) \times(2 \times N-2))$; frsini $:=$ frsin + term $/(4 \times N-1) ; N:=N+1$; go to Loop;

exit: frsin $:=$ frsini $\times u$

end FRESNELSIN;

\section{SEE ALGORITHM 88}




\section{ALGORITHM 90 \\ EVALUATION OF THE FRESNEL COSINE INTE- GRAL}

JoHn L. CUndifF

Engineering Experiment Station, Georgia Institute of Technology, Atlanta, Ga.

real procedure FRESNELCOS $(u)$ result: (frcos); value $(u)$; comment This algorithm computes the Fresnel cosine integral defined by

$$
C(u)=\int_{0}^{u} \cos \frac{\pi t^{2}}{2} d t,
$$

by evaluating the series expansion

$$
C(u)=\sqrt{\frac{2 x}{\pi}}\left[1-\frac{x^{2}}{5 \cdot 2 !}+\frac{x^{1}}{9 \cdot 4 !}-\frac{x^{6}}{13 \cdot 6 !}+\cdots\right],
$$

where $x=\pi u^{2} / 2$. Reference: P'Earcey, T., Table of the Fresnel Integral to Six Decimal Places. The Syndics of the Cambridge University Press, Melbourne, Australia (1956).;

begin pi2 $:=1.5707963 ; \quad x:=$ pi2 $\times(u \uparrow 2) ;$ frcos $:=1$; xsqr $:=x \uparrow 2 ; \quad N:=3$; term $:=-x s q r / 2$; frcoi $:=1+($ term $/ 5)$;

loop: if frcoi $=$ frcos then go to exit; term := -term $\times$ $x \operatorname{sqr} /((2 \times \mathrm{N}-2 \times(2 \times \mathrm{N}-3)) ;$ frcos $:=$ frcoi; frcoi $:=$ frcos + term $/(4 \times N-3) ; N:=N+1 ;$ go to loop: exits: freos := u $\times$ freos

end FRESNELCOS;

SEE ALGORITHM 88

\section{ALGORITHM 91}

\section{CHEBYSHEV CURVE-FIT}

\section{Albert Newhouse}

University of Houston, Houston, Texas

procedure CHEBFIT $(m, n, X, Y)$; integer $m, n$; array $X, Y$; comment This procedure fits the tabular function $Y(X)$ (given as $m$ points $(X, Y)$ ) by a polynomial $P=\sum_{i=0}^{n} A_{i} X^{i}$. This polynomial is the best polynomial approximation of $Y(X)$ in the Chebyshev sense. Reference: Stiffer, E. Numerical Methods of Tchebycheff Approximation, U. of Wisc. Press (1959), 217-232;

begin array $X[1: m], Y[1: m], T[1: m], A[0: n], A X[1: n+2]$,

$A Y[1: n+2], A H[1: n+2], B Y[1: n+2], B H[1: n+2]$

integer array IN $[1: n+2]$; real TMAX, $H$; integer $i$, j, k, imax;

comment Initialize;

$\mathrm{k}:=(\mathrm{m}-1) /(\mathrm{n}+1)$;

for $1:=1$ step 1 until $n+1$ do IN $[i]:=(i-1) \times k+1$; $\mathrm{IN}[\mathrm{n}+2]:=\mathrm{m}$;

START: comment Iteration begins;

for $i:=1$ step 1 until $n+2$ do

begin $A X[i]:=X[I N[i]]$;

$A Y[i]:=Y[I N[i]]$

end $i$;

$$
\mathrm{AH}[\mathrm{i}]:=(-1) \uparrow(\mathrm{i}-1)
$$

DIFFERENCE: comment divided differences;

for $i:=2$ step 1 until $n+2$ do

begin

for $j:=i-1$ step 1 until $n+2$ do

begin $B Y[j]:=A Y[j]$;

end $\mathrm{j}$; for $j:=i$ step 1 until $n+2$ do

begin $A Y[j]:=(B Y[j]-B Y[j-1]) /$

$(A X[j]-A X[j-i+1])$;

$A H[j]:=(B H[j]-B H[j-1]) /$

end $j$;

end $i$;

$(A X[j]-A X[j-i+1])$

$\mathrm{H}:=-\mathrm{AY}[\mathrm{n}+2] / \mathrm{AH}[\mathrm{n}+2]$;

POLY: comment polynomial coefficients;

for $i:=0$ step 1 until $n$ do

$\operatorname{bcgin} A[\mathrm{i}]:=\mathrm{AY}[\mathrm{i}]+\mathrm{AH}[\mathrm{i}] \times \mathrm{H}_{\text {; }}$

end $i$;

$$
B Y[i]:=0
$$

$\mathrm{BY}[1]:=1$; TMAX $:=\operatorname{abs}(\mathrm{H}) ; \quad i \max :=\mathrm{IN}[1]$;

for $i:=1$ step 1 until $n$ do

begin

for $\mathrm{j}:=0$ step 1 until $\mathrm{i}-1$ do

begin

$\mathrm{BY}^{\prime}[\mathrm{i}+1-\mathrm{j}]:=\mathrm{BY}[\mathrm{i}+\mathrm{l}-\mathrm{j}]-\mathrm{BY}[\mathrm{i}-\mathrm{j}] \times \mathrm{X}[\mathrm{IN}[\mathrm{i}]] ;$

$A[j]:=A[j]+A[i] \times B Y^{\prime}[i+1-j]$

end $j$;

end $i$;

ERROR: comment compute deviations;

for $i:=1$ step 1 until $m$ do

begin $T[i]:=A[n]$;

for $j:=0$ step 1 until $n$ do $T[i]:=T[i] X[i]+A[n-j]$;

$\mathrm{T}[\mathrm{i}]:=\mathrm{T}[\mathrm{i}]-\mathrm{Y}[\mathrm{i}]$;

if alss $(\mathrm{T}[\mathrm{i}])<$ TMAX then go to $\mathrm{Ll}$;

TMAX := abs(T[i]);

$\operatorname{imax}:=i$

L1: $\quad$ end $i$;

for $i:=1$ step 1 until $n+2$ do

begin

if imax $<$ IN [i] then go to L2;

if $\operatorname{inax}=\mathrm{IN}[\mathrm{i}]$ then go to FIT end end $i$;

L2: if $\mathrm{T}[\mathrm{imax}] \times \mathrm{T}[\mathrm{IN}[\mathrm{i}]]<0$ then go to $\mathrm{L} 3$;

IN[i] := imax;

go to START;

L3: if $\operatorname{IN}[1]<$ imax then go to L4;

for $i:=1$ step 1 until $n+1$ do $\operatorname{IN}[n+3-i]:=\operatorname{IN}[n+2-i]$.

IN $[i]:=$ imax;

go to START;

L4: if IN $[n+2] \leqslant$ imax then go to L5;

IN $[i-2]:=$ imax;

go to START;

L5: for $i:=1$ step 1 until $n+1$ do $\operatorname{IN}[i]:=\operatorname{IN}[i+1]$;

IN $[n+2]:=$ imax

go to START;

FIT: end CHEBFIT

\section{CERTILICATION OI' ALGORITHM 91}

CHEBYSHEV CURVEFIT [A. Newhouse, Comm. $A C M$, May 1962]

ROBERT P. HALE

University of Adelaide, Adelaide, South Australia

The CHEBFIT algorithm was translated into ForTran and successfully run on an IBM 1620 when the following alterations: were made :

(a) 2nd line after

should read

comment Initialize;

for $i:=1$ step 1 until $n+1$ do $I N[i]:=(i-1) \times k+1$;

(b) 2nd and 3rd lines after

Poly: comment polynomial coefficients;

should read

begin $A[i]:=A Y[i+1]+A H[i+1] \times H ; B Y[i+1]:=0$ 
(c) 3 rd line af ter

ERROR: comment compute deviations;

should read

for $j:=1$ step 1 until $"$ do $T[i]:=T[i] \times \times[i]+A[n-j]$

(d) Immediately before st:utement $I .2$ insert $i:=n+2$; (:1s for list may be exhausted and $i$ no longer defined).

(e) 2 nd line after statement $I .3$ shomid read $I N[1]:=$ imar;

(f) lst line after statement $l . t$ shomld read $I .1[i-1]:=i m a r$;

\section{REMARKS ON ALGORITHM 91}

CHEBYSHEV CURVE TIT [A. Newhouse, Comm.

ACM 5 (May 1962), 281; 6 (April 1963), 167]

Peter NaUr (Recd. 27 Sept. 1963)

Regnecentralen, Copenhagen, Denmark

In addition to the corrections noted by R. P. Hale [op. cit., April 1963) the following are necessary: the procedure body.

2. The identifier $A$ must be included as a formal parameter. sequence.

4. comment cannot follow the colon following a label. This occurs in four places.

5. The end following go to FIT must be removed.

In addition, a large number of details can be made more concise and unnecessary operations can be eliminated. Also, it seems
1. The arrays $X, Y$, and $A$ cannot be declured to be local within

3 . It should be noted that the $X[i]$ must form a monotonic desirable to produce the maximum deviation as a result.

S1: for $\mathrm{j}:=1$ step 1 until $\mathrm{n}$ do

for $i:=1$ step 1 until $n$ do $C[i, j]:=0$;

for $j:=1$ step 1 until $n$ do

begin for $\mathrm{k}:=1$ step 1 until $\mathrm{n}$ do

begin $C[j, j]:=C[j, j]+W[j, k] \times U[k, j]$ end;

if $j=1$ then $Z:=B[j] / C[j, j]$ else $Z:=1 / C[j, j]$;

for $k:=1$ step 1 until $n$ do

begin $X[k]:=Z \times W[j, k]$;

$\mathbf{W}[\mathbf{j}, \mathbf{k}]:=\mathrm{X}[\mathbf{k}]$

end $k$;

for $k:=1$ step 1 until $n$ do

begin if $k=j$ then go to $S 2$ else

for $p:=1$ step 1 until $n$ do

$\mathrm{C}[\mathrm{k}, \mathrm{j}]:=\mathrm{C}[\mathrm{k}, \mathrm{j}]+\mathrm{U}[\mathrm{p}, \mathrm{j}] \times \mathrm{W}[\mathrm{k}, \mathrm{p}]$

if $\mathrm{j}=1$ then $b h:=B[j]$ else $b h:=1$;

if $k=1$ then $b 1:=B[j]$ else $b 1:=0$;

for $p:=1$ step 1 until $n$ do

begin $X[p]:=b h \times W[k, p]+(b 1-C[k, j]) \times$

$\mathrm{W}[\mathrm{j}, \mathrm{p}]$;

$\mathrm{W}[\mathrm{k}, \mathrm{p}]:=\mathrm{X}[\mathrm{p}]$

$$
\text { end } p \text {; }
$$

S2: $\quad$ if $k=j \wedge j=n$ then go to $S 3$

end $j$;

end $k$;

S3: for $j:=$ step 1 until $n$ do

if $\operatorname{absf}(\operatorname{absf}(\mathrm{C}[\mathrm{j}, \mathrm{jl})-\operatorname{absf}(\mathrm{B}[1]))>$ eps then go to $\mathrm{S4}$;

go to $\mathrm{S} 6$;

S4: if kount $>0$ then go to $\mathrm{S} 5$ else go to $\mathrm{S} 6$;

S5: kount := kount -1 ;

go to $\mathrm{S} 1$;

S6: for $\mathrm{j}:=$ step 1 until $\mathrm{n}$ do

$\mathrm{X}[\mathrm{j}]:=\mathrm{W}[1, \mathrm{j}]$;

S7: end SIMULTANEOUS

\section{ALGORITHM 93 \\ GENERAL ORDER ARITHMETIC \\ Millard H. Perstein \\ Control Data Corp., Palo Alto, Calif.}

procedure SIMULTANEOUS ( $U, W, C, X, B, n$, kount, eps, absf) ;

array $U, W, C, X, B$; integer $n$, kount ;

real eps; real procedure absf;

comment This procedure solves the problem $\mathrm{Ux}:=\mathrm{b}$ for the vector $x$. It assumes the problem written in the form $x^{\prime} U^{\prime}:=b^{\prime}$, where ' denotes transpose. The procedure is completed in n cycles and may be iterated kount times (kount $\leqq 6$ ). The transpose of $U$ is in $U[$,$] and the row vector b^{\prime}$ is in $B$. The integer $n$ is the dimension of $\mathrm{U}$, and the solution row vector $\mathrm{x}^{\prime}$ is in $\mathrm{X}$. The matrix $\mathrm{C}$ is a check of accuracy. It should have $\mathrm{b}^{\prime}$ in its first row, the first element $b_{1}$ of $b^{\prime}$ along its main diagonal, and zeros elsewhere. The real number eps checks to see how close the actual result is to this theoretical one. Also if we let $b^{\prime}:=$ $(1,0, \cdots, 0)$, then this procedure finds the inverse $W[$,$] of U$. The function absf finds the absolute value of its argument. Tlie procedure chooses the column vectors of $U$ as the row vectors of $W$ in the $0^{\text {tb }}$ cycle of the first iteration. For all subsequent iterations, the row vectors of $W$, computed at the $n^{\text {th }}$ cycle of the last iteration, are the row vectors of $W$. in the $0^{\text {th }}$ cycle ;

begin integer $i, j, k, p$; real $b h, b 1 ; Z$;

for $j:=1$ step 1 until $n$ do

for $i:=1$ step 1 until $n$ do $W[j, i]:=U[i, j]$ procedure arithmetic (a, b, c, op);

comment This procedure will perform different order arithmetic operations with $b$ and $c$, putting the result in $a$. The order of the operation is given by op. For op $=1$ addition is performed. For $o p=2$ multiplication, repeated addition, is done. Beyond these the operations are non-commutative. For $o p=3$ exponentiation, repeated multiplication, is done, raising $b$ to the power $c$. Beyond these the question of grouping is important. The innermost implied parentheses are at the right. The hyper-exponent is always c. For $o p=4$ tetration, repeated exponentiation, is done. For op $=5,6,7$, etc., the procedure performs pentation, hexation, heptation, etc., respectively.

The routine was originally programmed in ForTran for the Control Data 160 desk-size computer. The original program was limited to tetration because subroutine recursiveness in Sontrol Data 160 Fortras has been held down to four levels in the interests of economy.

The input parameter, $b, c$, and $o p$, must be positive integers, not zœro;

begir. own integer $d, e, f$, drop;

if op $=1$ then

begin $a:=b+c ;$ go to 1

end if op $=2$ then $\mathrm{d}:=0$; integer a, b, c, op; 
else $d:=1 ;$ e $:=c ;$ drop $:=$ op -1 ;

for $f:=1$ step 1 until e do

begin arithmetic $(a, b, d$, drop);

$$
\mathrm{d}:=\mathbf{a}
$$

end:

1: end arithmetic

\section{CERTIFICATION OF ALGORITHM 93 .}

GENERAL ORDER ARITHMETIC [Millard H. Perslein, Comm. ACM (June 1962)]

Richard George

Particle Accelerator Div. Argonne National Laboratory, Argonne, Ill.

Algorithm 93 was programmed for the IBM 1620, using "Fortran-recursion" (i.e., generous use of the copy rule). The program ran without any modifications and was tested through tetration. Further levels were available, but were too timeconsuming to reach.

\section{ALGORITHM 94 \\ COMBINATION}

JEROME KuRTZBERG

Burroughs Corp., Burroughs Laboratories, Paoli, Pa.

procedure COMBINATION $(J, N, K)$; value $N, K$; integer array $\mathbf{J}$; integer $\mathbf{N}, \mathbf{K}$;

comment This procedure generates the next combination of $N$ integers taken $K$ at a time upon being given $N, K$ and the previous combination. The $K$ integers in the vector $J(1) \cdots J(K)$ range in value from 0 to $N-1$, and are always monotonically strictly increasing with respect to themselves in input and output format. If the vector $J$ is set equal to zero, the first combination produced is $N-K, \cdots, N-1$. That initial combination is also produced after $0,1, \cdots, N-1$, the last value in that cycle;

begin integer $B, L$;

$$
\mathrm{B}:=1 \text {; }
$$

mainbody: if $J(B) \geqq B$ then begin $A:=J(B)-B-1$;

$$
\text { for } L:=1 \text { step } 1 \text { until } B \text { do } J(L):=L+A \text {; }
$$

go to exit end;

if $\mathrm{B}=\mathrm{K}$ then go to initiate;

$\mathrm{B}:=\mathrm{B}+1$; go to mainbody;

initiate: for $B:=1$ step 1 until $K$ do $J(B):=N-K-1+B$ exit: end COMBINATION

\section{CERTIFICATION OF ALGORITHM 94}

COMBINATION [J. Kurtzburg, Comm. ACM, June, 1962]

R. E. Grench*

Reactor Eng. Div., Argonne National Laboratory, Argonne, Ill.

* Work supported by U. S. Atomic Energy Commission

Four changes were required in the algorithm.

1. The last sentence in the comment should read: That initial combination is also produced after $0,1, \ldots, K-1$, the last value in that cycle;

2. The integer $A$ was declared,

3. Parentheses were replaced by brackets in the subscript expressions;
4. A semicolon was inserted at the end of the initiate statement. After the above changes were made the body of Algorithm 94 was tested on an LGP-30 computer using the Dartmouth College ALGOL-30 translator. The body tested satisfactorily and the time required to generate one $J$ when $K=5$ and $N=15$ was 30 seconds.

Various tests should be included if this algorithm is to be used as a procedure. These tests might include a statement to check if $K>N$ and if the initial value of $J$ is correct These two possibilities were investigated and it was found that improper $J$ 's are generated.

\section{CERTIFICATION OF ALGORITHM 94}

COMBINATION [J. Kurtzberg, Comm. ACM, June 1962]

RoNald W. MaY

University of Alberta, Calgary, Alberta, Canada

Algorithm 94.was translated into Fortran for the IBM 1620 and run successfully with no corrections. The variable $A$, however, has not been declared.

\section{ALGORITHM 95 \\ GENERATION OF PARTITIONS IN PART-COUNT FORM}

Frank Stockmal

System Development Corp., Santa Monica, Calif.

procedure partgen $(c, N, K, G)$; integer $N, K$; integer array $c$; Boolean G;

comment This procedure operates on a given partition of the positive integer $N$ into parts $\leqq K$, to produce a consequent partition if one exists. Each partition is represented by the integers $c[1]$ thru $c[K]$, where $c[j]$ is the number of parts of the partition equal to the integer $j$. If entry is made with $G=$ false, procedure ignores the input array $c$, sets $G=$ true, and produces the first partition of $N$ ones. Upon each successive entry with $G=$ true, a consequent partition is stored in $c[1]$ thru $c[K]$. For $N=K X$, the final partition is $c[K]=X$. For $N=K X+r$, $1 \leqq r \leqq K-1$, final partition is $c[K]=X, c[r]=1$. When entry is made with array $c=$ final partition, $c$ is left unchanged and $G$ is reset to false;.

begin integer $a, i, j$;

if $\neg \mathrm{G}$ then go to first;

$\mathrm{j}:=2$;

$\mathrm{a}:=\mathrm{C}[1]$

test: if $a<j$ then go to $B$;

$c[j]:=1+c[j]$

$c[1]:=a-j ;$

zero: for $i:=2$ step 1 until $j-1$

do $\mathrm{c}[\mathrm{i}]:=0$;

go to EXIT;

$B: \quad$ if $\mathrm{j}=\mathrm{K}$ then go to last;

$\mathrm{a}:=\mathrm{a}+\mathbf{j} \times \mathbf{c}[\mathbf{j}]$;

$\mathrm{j}:=\mathrm{j}+1$;

go to test;

first: $G:=$ true;

c[1] := N;

$\mathrm{j}:=\mathrm{K}+1$;

go to zero;

last: $\mathrm{G}:=$ false;

EXIT: end partgen 


\section{ALGORITHM 96 \\ ANCESTOR}

ROBERT W. FLOYD

Armour Research Foundation, Chicago, Ill

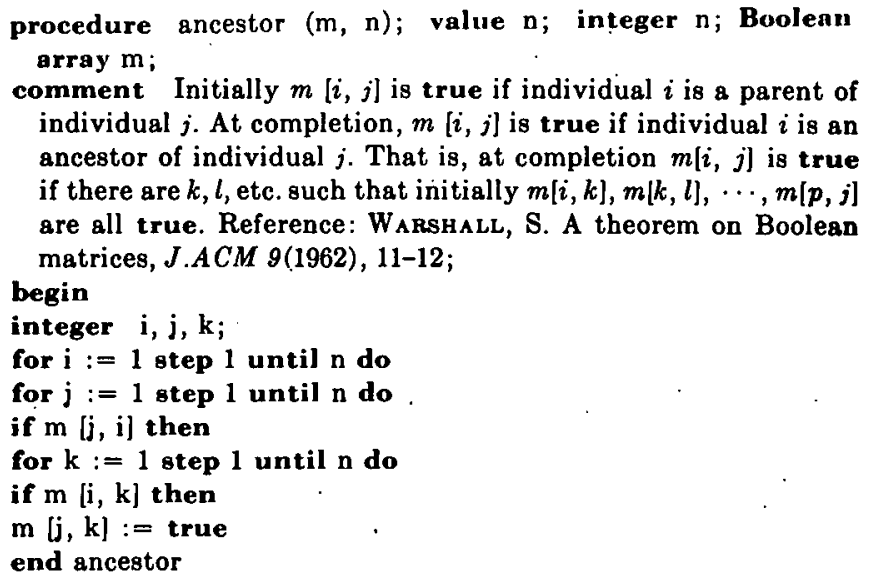

\section{CERTIFICATION OF ALGORITHM 96}

ANCESTOR [Robert W. Floyd, Comm. ACM, June, 1962]

Henry C. Thacher, JR. ${ }^{*}$

Argonne National Laboratory, Argonne, Ill.

* Work supported by the. U.S. Atomic Energy Commission

The body of this procedure was tested on the LGP-30 using the Dartmouth translator. After inclosing conditional statements in begin end brackets (apparently necessary for this translator), the procedure operated satisfactorily for the following matrices:

\begin{tabular}{ll}
\multicolumn{2}{c}{$n=5$, Time: } \\
$8^{\prime} 15^{*}$ \\
FTTFF & FTTTT \\
FFFFT & FFFFT \\
FFFTF $\rightarrow$ & FFFTT \\
FFFFT & FFFFT \\
FFFFF & FFFFF
\end{tabular}

\begin{tabular}{ll}
\multicolumn{1}{c}{$n=6$, Time: } & $13^{\prime} 15^{\prime \prime}$ \\
FTTFFF & FTTTTT \\
FFFTFF & FFFTFT \\
FFFFTF $\rightarrow$ & FFFTFT \\
FFFFFT & FFFFFT \\
FFFFFF & FFFFFF
\end{tabular}

\begin{tabular}{ll}
\multicolumn{2}{c}{$n=9$, Time $31^{\prime} 2^{*}$} \\
FTTFFFFFF & FTTTTTTTT \\
FFFFTFFFF & FFFFTTTTF \\
FFFTTFFFF & FFFTTTTTT \\
FFFFFFFFT & FFFFFTTTT \\
FFFFFTTFF & $\rightarrow$ FFFFFTTTF \\
FFFFFFFTF & FFFFFFFTF \\
FFFFFFFTF & FFFFFFFTF \\
FFFFFFFFF & FFFFFFFFF \\
FFFFFTTFF & FFFFFTTTF
\end{tabular}

The correctness of these results was confirmed by inspection of the network diagrams.

\section{ALGORITHM 97}

SHORTEST PATH

ROBERT W. FLOYD

Armour Research Foundation, Chicago, Ill.

procedure shortest path $(m, n)$; value $n$; integer $n$; array $m$; comment Initially. $m[i, j]$ is the length of a direct link from point $i$ of a network to point $j$. If no direct link exists, $m[i, j]$ is initially 1010 . At completion, $m[i, j]$ is the length of the shortest path from $i$ to $j$. If none exists, $m[i, j]$ is 1010 . Reference: WARSHALL, S. A theorem on Boolean matrices. $J, A C M$ 9(1962), 11-12;

\section{begin}

integer $i, j, k$; real inf, $s$; inf $:=1010$;

for $i:=1$ step 1 until $n$ do

for $j:=1$ step 1 until $n$ do

if $m[j, i]<$ inf then

for $k:=1$ step 1 until $n$ do

if $m[i, k]<$ inf then

begin $s:=m[j, i]+m[i, k]$;

if $s<m[j, k]$ then $m[j, k]:=s$

end

end shortest path

\section{ALGORITHM 98 \\ EVALUATION OF DEFINITE COMPLEX LINE INTEGRALS}

JoHN L. PFaltz

Syracuse University Computing Center, Syracuse, N. Y.

procedure COMPLINEINTGRL(A, B, N, RSSUM);

value $A, B, N$; real $A, B, N$; array $R S S U M$;

comment COMPLINEINTGRL approximates the complex line integral by evaluating the partial Riemann-Stieltjes sum $\sum_{t=1}^{n} f\left(z_{k}\right)\left[z_{t}-z_{t-1}\right]$ where $a \leqq t \leqq b$ and $z_{k} \in\left(z_{t-1}, z_{t}\right)$. The programmer must provide 1$)$ the procedures $\operatorname{GAMMA}(T, Z)$ to calculate $z(t)$ on $\Gamma$, and $\operatorname{FUNCT}(Z, F)$ to calculate function values, and 2 ) the end points $A$ and $B$ of the parametric interval and $N$ the number of subintervals into which $[a, b]$ is to be partitioned;

begin integer $I$; real $T, D E L T$; real array $Z T, Z T L, D E L Z$, ZK, PART $[1: 2] ; \operatorname{RSSUM}[1]:=0.0 ; \operatorname{RSSUM}[2]:=0.0$ DELT $:=(B-A) / N ; T:=A$;

line: $\operatorname{GAMMA}(\mathrm{T}, \mathrm{ZT})$

if $T=A$ then go to next;

for $I:=1$ step 1 until 2 do

begin

$\operatorname{DELZ}[\mathrm{I}]:=\mathrm{ZT}[\mathrm{I}]-\mathrm{ZTL}[\mathrm{I}]$; end;

for $I:=1$ step 1 until 2 do

begin

$\mathrm{ZK}[\mathrm{I}]:=\mathrm{ZTL}[\mathrm{I}]+\mathrm{DELZ}[\mathrm{I}] / 2.0$; end;

FUNCT(ZK, FZ);

PART[1] $:=$ FZ[1] $\times$ DELZ[1] - FZ[2] $\times$ DELZ[2];

PART[2]:= FZ[1] $\times$ DELZ[2] + FZ[2] $\times$ DELZ[1];

for $I:=1$ step 1 until 2 do

begin

RSSUM[I] := RSSUM $[I]+\operatorname{PART}[\mathrm{I}]$; end;

if $\mathrm{T}<\mathrm{B}-(0.25 \times \mathrm{DELT})$ then go to next else go to exit;

next: for $I:=1$ step 1 until 2 do

begin

$\mathrm{ZTL}[\mathrm{I}]:=\mathrm{ZT}[\mathrm{I}] ;$ enć;

$\mathrm{T}:=\mathbf{T}+\mathrm{DELT}$

go to line;

exit: end COMPLINEINTGRL. 


\section{ALGORITHM 99}

\section{EVALUATION OF JACOBI SYMBOL}

Stephen J. Garland and Anthony W. Knapp

Dartmouth College, Hanover, N. H.

procedure Jacobi $(n, m, r)$; value $n, m$;

integer $\mathrm{n}, \mathrm{m}, \mathrm{r}$;

comment Jacobi computes the value of the Jacobi symbol $(n / m)$, where $m$ is odd, by the law of quadratic reciprocity. The parameter $r$ is assigned one of the values $-1,0$, or 1 if $m$ is odd. If $m$ is even, the symbol is undefined and $r$ is assigned the value 2 . For odd $m$ the routine provides a test of whether $m$ and $n$ are relatively prime. The value of $r$ is 0 if and only if $m$ and $n$ have a nontrivial common factor. In the special case where $m$ is prime. $r=-1$ if and only if $n$ is a quadratic nonresidue of $m$;

begin

integer $s$;

Boolean p, q;

Boolean procedure parity $(x)$; value $x$; integer $x$; comment The value of the function parity is true if $x$ is odd, false if $x$ is.even;

begin

parity : $=x \div 2 \times 2 \neq x$

end parity;

if $\neg$ parity $(m)$ then begin $r:=2 ;$ go to exit end;

$\mathrm{p}:=$ true;

loop: $\mathbf{n}:=\mathbf{n}-\mathbf{n} \div \mathbf{m} \times \mathbf{m}$;

$q:=$ false;

if $\mathrm{n} \leqq 1$ then go to done;

even: if $\neg$ parity $(\mathrm{n})$ then

begin

$\mathrm{q}:=\neg \mathrm{q}$

$\mathrm{n}:=\mathrm{n} \div 2$;

go to even

end $n$ now odd;

if $q$ then if parity $((m \uparrow 2-1) \div 8)$ then $p:=\neg p$;

if $\mathrm{n}=1$ then go to done;

if parity $((m-1) \times(n-1) \div 4)$ then $p:=\neg p$;

$\mathrm{s}:=\mathrm{m} ; \mathrm{m}:=\mathrm{n} ; \mathrm{n}:=\mathrm{s}$; go to loop;

done: $r:=$ if $n=0$ then 0 else if $p$ then 1 else -1 ;

exit: end Jacobi

\section{REMARK ON ALGORITHM 99}

EVALUATION OF JACOBI SYMBOL [S. J. Gar-

land and A. W. Knapp, Comm. ACM 6, June 1962]

Ronald W. MaY

University of Alberta, Calgary, Alberta, Canada

One syntactical error was found in this procedure. It occurs in the second if statement following the label even. The statement

if $q$ then if parity $((\mathrm{m} \uparrow 2-1) \div 8)$ then

$$
p:=\neg p
$$

might be changed as follows.

if $q$ then go to $C H E C K$

next 1: if $n=1$ then go to done;

CHECK: if parity $((m \uparrow 2-1) \div 8)$ then

$$
p:=\neg p
$$

go to next 1 ;

The two statements beginning with $C H E C K$ could be inserted before the labcl done and after the statement go to loop;

\section{ALGORITHM 100}

\section{ADD ITEM TO CHAIN-LINKED LIST}

Philip J. Kiviat

United States Steel Corp., Appl. Research Lab., Monroeville, Penn.

procedure inlist (t,info, $m$, list, $n$, first,flag,addr, listfull); integer $n, m$,first,flag, $t$; integer array info,list, addr; comment inlist adds the information pair $\{t$,info $\}$ to the chainlink structured matrix list $(i, j)$, where $t$ is an order key $\geqq 0$, and info $(k)$ an information vector associated with $t$. info $(k)$ has dimension $\mathrm{m}$, list $(i, j)$ has dimensions $(n \times(m+3))$. flag denotes the head and tail of list $(i, j)$, and first contains the address of the first (lowest order) entry in list $(i, j) . \operatorname{addr}(k)$ is a vector containing the addresses of available (empty) rows in list $(i, j)$. Initialization: list $(i, m+2)=$ flag, for some $i \leqq n$. If list $(i, j)$ is filled exit is to listfull;

begin integer $\mathrm{i}, \mathrm{j}$, linkl, link2;

$0:$ if addr $[1]=0$; then go to listfull; $i:=1$;

1: if list $[i, 1] \leqq t$

then begin if list $[i, 2] \neq 0$ then begin link $1:=m+2$; link $2:=m+3$; go to 2 end; else begin if list $[i, m+2]=$ flag then begin $i:=$ flag; linkl := $m+3$; $\operatorname{link} 2:=m+2$; go to 3 end; else begin $\mathrm{i}:=\mathrm{i}+1$; go to 1 end end end;

else begin link $1:=m+3 ; \quad$ link $2:=m+2$ end;

2: if list $\lfloor i$, link2 $\neq \neq$ flag

then begin $k:=i ; \quad i:=$ list $[i$, link2];

if (link2 $=m+2 \wedge$ list $[i, 1] \leqq t) \vee$

(link $2 \neq m+2 \wedge$ list $[i, 1]>t$ ) then go to 4 ;

else go to 1 end;

else begin list [i,link2] := addr [1] end;

3: $\mathrm{j}:=\operatorname{add} \mathbf{r}[1] ;$ list $[\mathrm{j}, \operatorname{link} 1]:=\mathrm{i}$;

list $[j$, link2] $:=$ flag; if link $2=m+2$ then

first := addr $[1]$; go to 5 ;

4: $\mathrm{j}:=\operatorname{addr}[1] ;$ list $[\mathrm{j}, \mathrm{link} 1]:=$ list $[\mathrm{i}$, link 1$]$; list $[i, \operatorname{link} 1]:=$ list $[k$, link2 $]:=\operatorname{add} r[1]$; list $[\mathrm{j}$, link2] $:=\mathrm{i}$;

5: list $[j, 1]:=t$; for $i:=1$ step 1 until $m$ do

list $[j, i+1]:=$ info $[i] ;$ for $i:=1$ step 1 until $n-1$ do addr $[i]:=\operatorname{addr}[i+1] ; \operatorname{addr}[n]:=0$

end inlist

\section{ALGORITHM 101}

REMOVE ITEM FROM CHAIN-LINKED LIST

Philip J. Kiviat

United States Steel Corp., Appl. Res. Lab., Monroeville, Penn.

procedure outlist (vector, $m$, list, $n$, first,flag,addr);

integer $n, m, f i r s t$, flag; integer array vector, list, $a d d r$; comment outlist removes the first entry (information pair with

lowest order key) from list $(i, j)$ and puts it in vector $(k)$; begin integer $i$;

for $i:=1$ step 1 until $m+1$ do vector[i] $:=$ list $\mid$ first,i];

for $i:=n-1$ step -1 until 1 do addr $[i+1]:=$ addr [i];

addr [1] := first;

if list [first, $m+3]=$ flag then

begin list $[1, \mathrm{~m}+2]:=$ flag; first $:=1$;

for $i:=1$ step 1 until $n$ do addr [i] $:=i$ end;

else begin first : $=$ list [first, $m+3$ ];

list [first, $m+2]:=$ flag end;

for $i:=1$ step 1 until $m+3$ do list [addr [1], $i]:=0$

end outlist 


\section{ALGORITHM 102 \\ PERMUTATION IN LEXICOGRAPHICAL ORDER \\ G. F. Schrack and M. Shimrat \\ University of Alberta, Calgary, Alberta, Canada}

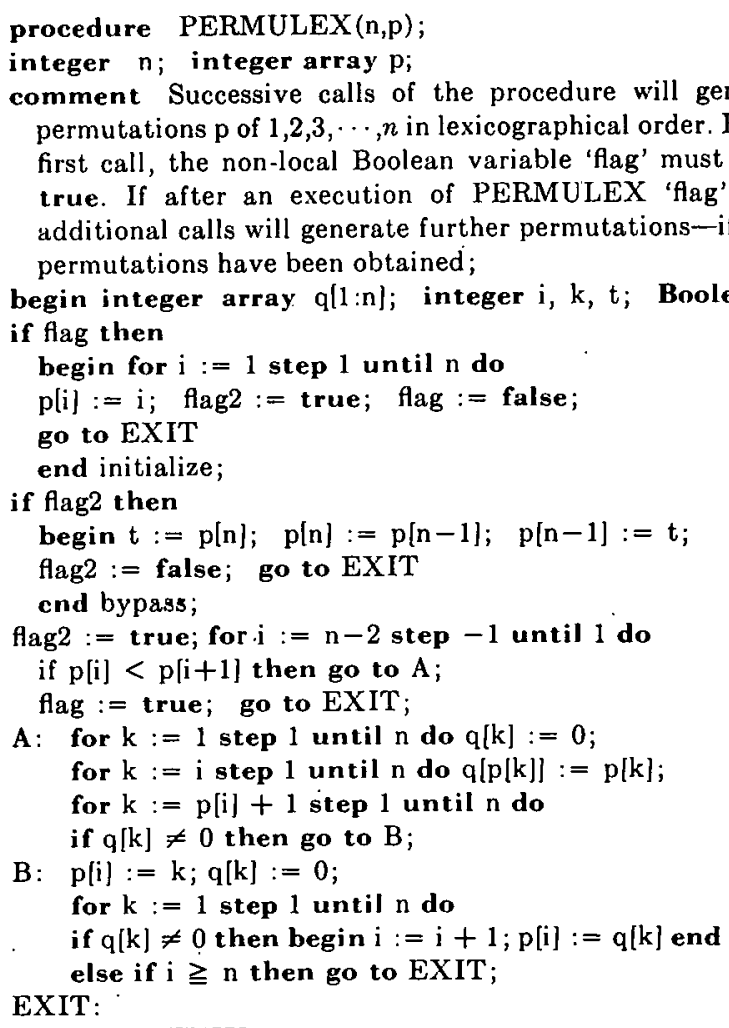

end PERMULEX

\section{ALGORITHM 103}

\section{SIMPSON'S RULE INTEGRATOR}

GuY F. Kuncir

UNIVAC Division, Sperry Rand Corp., San Diego, Calif.

procedure SIMPSON (a, b, f, I, i eps, N);

value $a, b$, eps, $N$; integer $N$;

real $a, b, I, i$, eps; real procedure $f$;

comment This procedure integrates the function $f(x)$ using a modified Simpson's Rule quadrature formula. The quadrature is performed over $j$ subintervals of $[a, b]$ forming the total area $I$. Convergence in each subinterval of length $(b-a) / 2^{n}$ is indicated when the relative difference between successive three-point and five-point area approximations

$$
A_{3, j}=(b-a)\left(g_{0}+4 g_{2}+g_{4}\right) /\left(3 \cdot 2^{n+1}\right)
$$$$
A_{5, j}=(b-a)\left(g_{0}+4 g_{1}+2 g_{2}+4 g_{3}+g_{4}\right) /\left(3 \cdot 2^{n+2}\right) \text {. }
$$

is less than or equal to an appropriate portion of the over-all tolerance eps (i.e., $\left|\left(A_{5, j}-A_{3, j}\right) / A_{5, j}\right| \leqq$ eps $/ 2^{n}$ with $\left.n \leqq N\right)$. SIMPSON will reduce the size of each interval until this condition is satisfied.

Complete integration over $[a, b]$ is indicated by $i=b$. A value $a \leqq i<b$ is indicates that the integration was terminated, leaving $I$ the true area under $f$ in $[a, i]$. Further integration over $[i, b]$ will necessitate either the assignment of a larger $N$, a larger eps, or an integral substitution reducing the slope of the integrand in that interval. It is recommended that this procedure be used between known integrand maxima and minima.; begin integer $m, n$; real $d, h$; array g[0:4], $A[0: 2], S[1: N, 1: 3]$; $\mathrm{I}:=\mathrm{i}:=\mathrm{m}:=\mathrm{n}:=0$;

$\boldsymbol{g}[0]:=f(a)$

$\mathrm{g}|2|:=\mathrm{f}((\mathrm{a}+\mathrm{b}) / 2)$

$\mathrm{g}[4]:=\mathrm{f}(\mathrm{b})$;

$A[0]:=(b)-a) \times(g[0]++\times g[2|+g| 4 \mid) / 2$;

AA: $\quad d:=2\lceil n ; h:=(b-a) / 4 / d$;

$\mathrm{g}[1]:=\mathrm{f}(\mathrm{a}+\mathrm{h} \times(4 \times \mathrm{m}+1))$;

$\mathrm{g}|3|:=\mathrm{f}(\mathrm{a}+\mathrm{h} \times(4 \times \mathrm{m}+3))$;

$\mathrm{A}[1]:=\mathrm{h} \times(\mathrm{g}[0]+4 \times \mathrm{g}[1]+\mathrm{g}[2])$;

$A \mid 2]:=h \times(g[2]+4 \times g[3]+g[4])$;

if al)s $(((A[1]+A[2])-A[0]) /(A[1]+A[2]))>\mathrm{eps} / \mathrm{d}$

then hegin $m:=2 \times m ; n:=n+1$;

if $\mathrm{n}>\mathrm{N}$ then go to $\mathrm{CC}$;

$\mathrm{A}[0]:=\mathrm{A}[1] ; \mathrm{S}[\mathrm{n}, 1]:=\mathrm{A}[2] ;$

$S\{n, 2]:=g[3] ; \quad S\{n, 3]:=g[4] ;$

$\mathrm{g}[4]:=\mathrm{g}[2] ; \quad \mathrm{g}[2]:=\mathrm{g}[1] ;$ go to AA end

else begin $\mathrm{I}:=\mathrm{I}+(\mathrm{A}[1]+\mathrm{A}[2]) / 3$;

$\mathrm{m}:=\mathrm{m}+1 ; \mathrm{i}:=\mathrm{a}+\mathrm{m} \times(\mathrm{b}-\mathrm{a}) / \mathrm{d}$

BB :

if $\mathrm{m}=2 \times(\mathrm{m} \div 2)$ then

begin $\mathrm{m}:=\mathrm{m} \div 2$; $\mathrm{n}:=\mathrm{n}-1$; go to $\mathrm{BB}$ end if ( $11 \neq 1) \vee(n \neq 0)$ then

hegin $A[0]:=S[n, 1] ; \quad g[0]:=g[4] ;$

$\mathrm{g}[2]:=\mathrm{Sin}, 2\} ; \quad \mathrm{g}[4]:=\mathrm{S} \mid \mathrm{n}, 3] ;$ go to AA end

end

CC: end SIMPSON

\section{ALGORITHM 104 \\ REDUCTION TO JACOBI}

H. Rutishauser

Eidg. Technische Hochschule, Zưrich, Switzerland

procedure $m 21$ ( $\mathrm{n}, \mathrm{a}, \mathrm{b}, \mathrm{c}$, inform); value $\mathrm{n}$;

integer $\mathrm{n}$; array $\mathrm{a}, \mathrm{b}, \mathrm{c}$; procedure inform;

comment: $m 21$ transforms symmetric bandmatrix

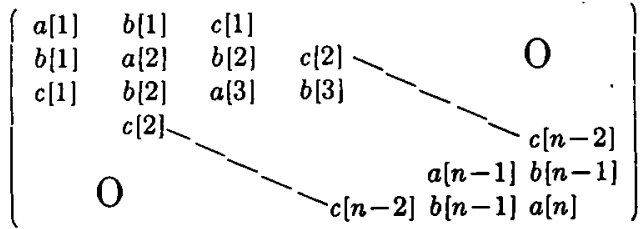

represented by the arrays $a, b, c$ by orthogonal transformation to Jacobi form which is represented by the arrays $a, b$. The method is described in H. Rutishauser, "On Jacobi rotation patterns," to appear in Proc. Symposium in Experimental Arithmetic, Chicago, Apr. 12-14, 1962, Sect. 5. Note that declarations must be given for the arrays $a, b, c$ with subscripts ranging from 1 to $n$. Also procedure inform must be declared. It may serve to use the Jacobi rotations occurring inside $m 21$ also for other purposes;

begin

real $p, g, d, 8$;

integer $\mathrm{k}, \mathrm{j}$;

$\mathrm{b}[\mathrm{n}]:=\mathrm{c}[\mathrm{n}]:=\mathrm{c}[\mathrm{n}-1]:=0$

for $k:=2$ step 1 until $n-1$ do

begin

for $j:=k$ step 2 until $n-1$ do

begin

if $\mathrm{k}=\mathrm{j}$ then 


\section{begin}

$\mathrm{p}:=\operatorname{sqrt}(\mathrm{b}[\mathrm{k}-1) \uparrow 2+\mathrm{c}(\mathrm{k}-1) \uparrow 2)$;

if $p=0$ then go: $: 0 \mathrm{ex}$;

$\mathrm{d}:=\mathrm{b}[\mathrm{k}-1] / \mathrm{p}$;

$\mathrm{s}:=-\mathrm{c}[\mathrm{k}-1] / \mathrm{p}$;

$\mathrm{b}(\mathrm{k}-1]:=\mathrm{p}$;

$c\{k-1\}:=0$

end $k=j$

else

begin

$\mathrm{p}:=\operatorname{sqrt}(\mathrm{c}[\mathrm{j}-2] \uparrow 2+\mathrm{g} \uparrow 2)$

if $\mathrm{p}=0$ then go to $e x$;

$\mathrm{d}:=\mathrm{c}[\mathrm{j}-2] / \mathrm{p}$;

$\mathrm{s}:=-\mathrm{g} / \mathrm{p}$

$\mathrm{c}[\mathrm{j}-2]:=\mathrm{p}$;

$\mathrm{p}:=\mathrm{d} \times \mathrm{b}[\mathrm{j}-1]-\mathrm{s} \times \mathrm{c}[\mathrm{j}-1]$;

$\mathrm{c}[\mathrm{j}-1]:=\mathrm{s} \times \mathrm{b}[\mathrm{j}-1]+\mathrm{d} \times \mathrm{c}[\mathrm{j}-1]$;

$b[j-1]:=p$

end $\mathrm{j} \neq \mathrm{k}$;

common: $\mathrm{g}:=2 \times \mathrm{b}(\mathrm{j}) \times \mathrm{d} \times \mathrm{s}$;

$p:=a[j] \times d \times d-g+a[j+1] \times s \times s ;$

$b[j]:=(a[j]-a[j+1]) \times d \times s+b[j] \times(d \times d-s \times s) ;$

$a[j+1]:=a[j] \times s \times s+g+a[j+1] \times d \times d ;$

$\mathrm{a}[\mathrm{j}]:=\mathrm{p}$;

$\mathrm{p}:=\mathrm{d} \times \mathrm{c}[\mathrm{j}]-\mathrm{s} \times \mathrm{b}[\mathrm{j}+1]$;

$b[j+1]:=s \times c[j]+d \times b[j+1]$;

$\mathbf{c}[\mathrm{j}]:=\mathbf{p}$;

$\mathrm{g}:=-\mathrm{s} \times \mathrm{c}[\mathrm{j}+1]$

$\mathrm{c}[\mathrm{j}+1]:=\mathrm{d} \times \mathrm{c}[\mathrm{j}+1]$;

inform (n, j, d, s);

comment: The Jacobi rotation which has been performed in this turn of the $j$-loop is $A:=U^{T} A U$ with

$$
U=\left(\begin{array}{cccccc}
1 & & & & & \\
& 1 & & & 0 & \\
& & d & s & & \\
& 0 & -s & d & & \\
& & & & 1 & 1
\end{array}\right)
$$

where the $d$ 's and $s$ 's are located at the crosspoints of end $j$;

rows and columns $j$ and $j+1$;

$e x$ : end $k$

end $m 21$

\section{ALGORITHM 105 \\ NEWTON MAEHLY}

F. L. Bauer and J. Stoer

Johannes Gutenberg-Universität, Mainz, Germany

procedure Newton Maehly (a, n, z, eps);

value $\mathrm{n}$, eps;

array a, $\mathrm{z}$;

integer $n$;

real eps;

comment The procedure determines all zeros $z[1: n]$ of the polynomial $p(x):=a \mid 0] \times x \uparrow n+\cdots+a[n]$ of order $n$, if $p(x)$ has only real zeros which have to be all different. The zeros $z[i]$ are ordered according to their magnitude: $z[1]>z \mid 2]>$ $\cdots>z[n]$. The approximations for each zero will be improved by iteration as long as abs $(x 1-x 0)>$ eps $\times$ abs $(x 1)$ holds for two successive approximations $x 0$ and $x 1$; begin real aa, $p p, q q, x 0, x 1$;

integer $i, m, s$;

$\operatorname{array} b, p, q[0: n-1]$

procedure Horner( $p, q, n, x, p p, q q)$;

value $n, x$;

array $p, q$;

real $\mathrm{pp}, \mathrm{x}, \mathrm{qq}$;

integer $n$;

begin real $s, s 1$;

integer $i$;

s : $=\mathbf{s l}:=0$;

for $\mathrm{i}:=0$ step 1 until $\mathrm{n}-1$ do

begin $s:=s \times x+p[i] ; \quad s 1:=s 1 \times x+q[i]$; end ;

$\mathrm{pp}:=\mathrm{s} \times \mathrm{x}+\mathrm{p}[\mathrm{n}] ; \mathrm{qq}:=\mathrm{s} 1$

end;

$\mathrm{p}[0]:=\mathrm{aa}:=\mathrm{a}[0] ; \quad \mathbf{x} 0:=\mathrm{pp}:=0 ; \mathrm{s}:=\operatorname{sign}(\mathrm{s}[0]) ;$

for $\mathrm{i}:=1$ step 1 until $\mathrm{n}$ do

if $s \times a[i]<0$ then

begin if $p p=0$ then $p p:=i$;

end :

if $x 0<\operatorname{abs}(a[i])$ then $x 0:=a b s(a[i])$;

$x 0:=$ if $p p=0$ then 0 else $1+\exp (\ln (\mathrm{abs}(\mathrm{xo} / \mathrm{aa})) / \mathrm{pp}) ;$

comment $x 0$ is a first approximation for the largest zero which may be printed out at this point of the program;

for $i:=0$ step 1 until $n-1$ do b|i] := $(n-1) \times a[i]$;

for $m:=1$ step 1 uhtil $n$ do

hegin

iteration:

Horner $(\mathrm{a}, \mathrm{b}, \mathrm{n}, \mathrm{xu}, \mathrm{pp}, \mathrm{qq}) ; \quad \mathrm{xl}:=\mathrm{x} 0-\mathrm{pp} / \mathrm{qq}$;

if $\operatorname{abs}(x 1-x 0)>\operatorname{eps} \times \operatorname{abs}(x 1)$ then

begin $\times 0:=\times 1$;

comment $x 0$ is the last approximation for the zero being improved, which may be printed out at this point;

go to iteration;

end ;

$\mathrm{z}[\mathrm{m}]:=\mathrm{xl}$

comment $z[m]:=x 1$ is the $m$ th zero of the polynomial;

$\mathrm{pp}:=\mathrm{b}[0]:=\mathrm{b}[0]-\mathrm{aa} ; \mathrm{q}[0]:=\mathrm{pp} ;$

if $m<n$ then

begin for $i:=1$ step 1 until $n-1$ do

begin $p p:=p[i]:=x 1 \times p[i-1]+a(i)$;

$p p:=b[i]:=b(i)-p p$;

end;

$q[i]:=x 1 \times q[i-1]+p p$;

Horner (p, q, n-1, xl, pp, qq);

$\mathrm{x} 0:=\mathrm{x} 1-\mathrm{pp} / \mathrm{qq}$;

comment $x 0$ is a first approximation for the next zero;

end

end Newton Maehly;

CERTIFICATION OF ALGORITHM 105

NEWTON MAEHLY [F. L. Bauer and J. Stoer, Comm. ACM, July 1962]

JoANiE Kondo

Burroughs Corp., Pasadena, Calif.

Algorithm 105 was successfully run on Burroughs 220 computer after the following correction had been made:

for $i:=0$ step 1 until $n-1$ do $b[i]:=(n-1) \times a[i]$ changéd to

for $i:=0$ step 1 until $n-1$ do $b[i]:=(n-i) \times a[i]$.

The following polynomials were tested for real roots using this algorithm :

\begin{tabular}{|c|c|c|}
\hline al & epsilon & 80 \\
\hline 8 & 0.0000001 & $10^{-8}$ \\
\hline$x^{5}-15 x^{4}+85 x^{3}-225 x^{2}+274 x-120$ & 0.000001 & $10^{-5}$ \\
\hline
\end{tabular}


ALGORITHM 106

COMPLEX NUMBER TO A REAL POWER

Margaret L. Johnson and Ward Sangren

Computer Applications, Inc., San Diego, California

procedure POWC $(x, y, w, A, B)$; value $x, y, w$;

real $x, y, w, A, B$;

comment This procedure takes a complex number $(x+i y)$ to a real power $w$. The result is $A+i B=(x+i y)^{\infty}$. This procedure must be used with caution becsuse although it is formally correct, it may not give the desired results. For example, if $w$ is a reciprocal integer it does not follow that the desired power (a root) will be calculated;

begin real THETA, PHI, R;

if $x>0$ then begin THETA :=0.0; go to SOL 1 end;

if $x<0 \wedge y \geqq 0$ then begin THETA $:=3.1415927$ : go to SOL 1 end;

if $\mathrm{x}<0 \wedge \mathrm{y}<0$ then begin THETA := 3.1415927; go to SOL 1 end;

if $\mathrm{x}=0 \wedge \mathrm{y}=0$ then begin $\mathrm{A}:=\mathrm{B}:=0.0$; go to RETURN end; if $x=0 \wedge y<0$ then begin PHI :=1.5707963; go to SOL 2 end;

if $x=0 \wedge y>0$ then begin $P H I:=-1.5707963$; go to SOL 2 end;

SOL 1: PHI $:=\arctan (y / x)+$ THETA;

SOL 2: $\quad R:=8 q r t(x \times x+y \times y)$;

$\mathbf{R}:=\exp (w \times \ln (R))$

$A:=\mathbf{R} \times \cos (w \times P H I)$;

$\mathrm{B}:=\mathrm{R} \times \sin (\mathrm{w} \times \mathrm{PHI})$

RETURN: end POWC

\section{REMARK ON ALGORITHM 106}

COMPLEX NUMBER TO A REAL POWER [Margaret L. Johnson and Ward Sangren, Comm. $A C M$ 5, Jul. 1962]

Grant W. ERwin, Jr.

The Boeing Co., Renton, Wash.

The comment "if $W$ is a reciprocal integer it does not follow that the desired power (a root) will be calculated" might better read "if $W$ is the reciprocal of an integer $N$, the procedure will calculate an $n$th root, but possibly not the particular $n$th root desired. E.g. $w=\frac{1}{3}, x=-1, y=0$ uields $A=\frac{1}{3}, B=\frac{1}{3} \sqrt{3}$ rather than the simpler $A=-1, B=0 . "$

The comment should be made that it is assumed that the arctan function yields a result between $-\pi / 2$ and $\pi / 2$.

The following four corrections should be made:

(1) if $x<0 \wedge y<0$ then begin THETA: $=3.1415927$; should read:

(2)

should read:

$$
\text { ‥ THETA: }=-3.1415927 \text {; }
$$

go to RETURN end:

go to RETURN end;

(3)

should read:

$$
\text { if } x=0 \wedge y<0 \cdots
$$

$$
\text { if } x=0 \wedge y>0 \cdots
$$

(4)

should read:

$$
\text { if } x=0 \wedge y>0
$$

$$
\text { if } x=0 \wedge y<0 \ldots
$$

ALGORITHM 107

GAUSS'S METHOD

JAY W. Counts

University of Missouri, Columbia, Mo.

procedure gauss $(u, a, y)$;

real array a, $y$; real temp; integer $u$;

comment This procedure is for solving a system of linear equations by successive elimination of the unknowns. The augmented matrix is a and $u$ the number of unknowns. The solution vector is $y$. If the system hasn't any solution or many solutions, this is indicated by go to stop;

begin

integer $i, j, k, m, n$;

n $:=0$;

ck0: $\mathbf{n}:=\mathrm{n}+1$;

for $k$ := $n$ step 1 until $u$ do if $a[k, n] \neq 0$ then go to ckl; go to stop;

ck1: if $\mathrm{k}=\mathrm{n}$ then go to $\mathrm{ck2}$; for $m:=n$ step 1 until $u+1$ do

begin temp $:=a[n, m] ; \quad a[n, m]:=a[k, m] ; \quad a[k, m]:=$ temp end;

ck2: for $j:=u+1$ step-1 until n do $a[n, j]:=a[n, j] / a[n, n]$;

for $i:=k+1$ step 1 until $u$ do

for $j:=n+l$ step 1 until $u+1$ do

$a[i, j]:=a[i, j]-a[i, n] \times a[n, j]$;

if $n \neq x$ then go to ck0;

for $i:=u$ step -1 until 1 do

begin

$y[i]:=a[i, u+1] / a[i, i]$

for $k:=i-1$ step -1 until 1 do

$a[k, u+1]:=a[k, u+1]-a[k, i] \times y[i]$

end end;

REMARK ON ALGORITHM 107

GAUSS'S METHOD [J. 'W. Counts, Comm. ACM, July 1962]

P. NAUR

Regnecentralen, Copenhagen, Denmark

Algorithm 107 cannot be recommended since it does not search for pivot and therefore will yield poor accuracy (cf. Remarks on Algorithm 42 above).

\section{ALGORITHM 108 \\ DEFINITE EXPONENTIAL INTEGRALS A}

YURI A. KRUGLYAK

Kharkov State University, Kharkov, U.S.S.R., AND

Donald R. Whitman

Case Institute of Technology, Cleveland, Ohio

real procedure $A s(n, b)$; value $n, b$; integer $n$; real $b$; comment: This procedure computes a value of integral $A_{n-1}(1, b)=\int_{1}^{\infty} x^{n-1} \exp (-b x) d x$ for any given positive integer, $n$, and any positive real parameter, $b$, by the recursion formula $A_{n}(1, b)=A_{0}(1, b)+(n / b) A_{n-1}(1, b)$ with $\dot{A}_{0}(1, b)=\exp (-b) / b$;

begin integer $m$; real $d b$; real array $a[1: n]$; $a[1]:=\exp (-b) / b$;

if $\mathrm{n}=1$ then go to exit; 
comment integral $a[1]=A_{0}(1, b)$ was evaluated; $\mathrm{db}:=1 / \mathrm{b}$; for $\mathrm{m}:=2$ step 1 until $n$ do $a[m]:=$ $\mathrm{a}[1]+\mathrm{db} \times(\mathrm{m}-1) \times \mathrm{a}[\mathrm{m}-1]$

comment integral $a[n]=A_{n-1}(1, b)$ was evaluated; As $:=\mathrm{a}[\mathrm{n}]$ end $\mathrm{As}$;

\section{CERTIFICATION OF ALGORITHM 108}

DEFINITE EXPONENTIAL INTEGRALS A [Yuri

A. Kruglyak and Donald R. Whitman, Comm. ACM 5 (July 1962)]

YuRi A. Kruglyak

Kharkov State University, Kharkov, U.S.S.R. and

Donald R. Whitman

Case Institute of Technology, Cleveland, Ohio

Integrals $A_{n}(1, b)=\int_{1}^{\infty} x^{n} \exp (-b x) d x$ occur in physical problems involving spheroidal coordinates, particularly in quantum chemistry calculations. This algorithm was programmed for the Burrough's 220 computer using Burrough's Algebraic Compiler. The program was used to compute tables of $A_{n}(1, b)$ in the ranges $n=0(1) 15$, and $b=0.01(0.01) 30.14$. For example, for $n=0(1) 15$, and $b=0.25$ and $b=24.0$, the results below were obtained. These are compared with the results (columns 3 and 5) obtained by James Miller, John M. Gerhauser, and F. A. Matsen [Quantum Chemistry Integrals and Tables, University of Texas Press, 1959].

\begin{tabular}{|c|c|c|c|c|}
\hline$n$ & $b=0.25$ & $\begin{array}{c}b=0.25 \text { (Miller } \\
\text { et al.) }\end{array}$ & $b=24.0$ & $\begin{array}{c}b=24.0 \text { (Miller } \\
\text { et al. })\end{array}$ \\
\hline 0 & $.31152031,01$ & $.31152031322856,01$ & $.15729727,-11$ & $.15729727267830,-11$ \\
\hline 1 & $.15576015,02$ & $.15576015661428,02$ & $.16385132,-11$ & $16385132570656,-11$ \\
\hline 2 & $.12772332,03$ & $.12772332842371,03$ & $.17095154,-11$ & $17095154982051,-11$ \\
\hline 3 & $.15357950,04$ & $\overline{15357951442168,04}$ & $.17866621,-11$ & $. \overline{17866621} 640586,-11$ \\
\hline 4 & $.24575835,05$ & $.24575837510601,05$ & $.18707497,-11$ & $. \overline{18707497} 541261,-11$ \\
\hline 5 & $.49151976,06$ & $.49151986541516,06$ & $.19627122,-11$ & $. \overline{9627122} 588926,-11$ \\
\hline 6 & $.11796476,08$ &.$\overline{11796479885167,08}$ & $.20636507,-11$ & $. \overline{20636507} 915061,-11$ \\
\hline 7 & $.33030132,09$ & $. \overline{3303014} 4989988,09$ & $.21748707,-11$ &.$\overline{21748708743056,-11}$ \\
\hline 8 & $.10569642,11$ &.$\overline{10569646079911,11}$ & $.22979295,-11$ & $\overline{2297929} 6848848,-11$ \\
\hline 9 & $.38050711,12$ &.$\overline{38050725887992,12}$ & $.24346962,-11$ & $\overline{24346963586148,-11}$ \\
\hline 10 & $.15220284,14$ & $.1 \overline{5220290355200,14}$ & $.25874294,-11$ & $\overline{2587429} 5428724,-11$ \\
\hline 11 & $.66969248,15$ & $.66969277562880,15$ & $.27588778,-11$ & $.27588779339328,-11$ \\
\hline 12 & $.32145238,17$ & $. \overline{321452} 53230182,17$ & $.29524115,-11$ & $\overline{2952411} 6937494,-11$ \\
\hline 13 & $.16715523,19$ & $. \overline{167155} 31679695, \quad 19$ & $.31721955,-11$ & $. \overline{3172195} 7275639,-11$ \\
\hline 14 & $.93606928,20$ &.$\overline{93606977406291,20}$ & $.34234200,-11$ & $. \overline{3423420} 2345285,-11$ \\
\hline 15 & $.56164156,22$ & $. \overline{561641} 86443775,22$ & $37126102,-11$ & $. \overline{3712610} 3733633,-11$ \\
\hline
\end{tabular}

The accuracy is at least six significant figures over the entire range. This accuracy is completely satisfactory for all quantum chemical calculations.

\section{ALGORITHM 109}

\section{DEFINITE EXPONENTIAL INTEGRALS B}

YURI A. KRUGLYAK

Kharkov State University, Kharkov, U.S.S.R., AND

Donatio R. Whitman

Case Institute of Technology, Cleveland, Ohio

real procedure $B s(n, a)$; value $n, a$; integer $n$; real $a$; comment This procedure computes a value of the integral $B_{n-1}(a)=\int_{-1}^{+1} x^{n-1} \exp (-a x) d x$ for any given positive integer, $n$, and any real parameter, $a$. If $|a|<$ alim an expansion of $\exp (-a x)$ is used, otherwise the recursion formula $B_{n}(a)=$ $\left[(-1)^{n} e^{a}-e^{-a}+n B_{n-1}(a)\right] / a$ with $b_{0}(a)=2 \sinh (a) / a$ is used. The value of alim depends upon the highest $n$ appearing in the calculations and upon the maximum errors in the last significant digits in the library procedures. For example, we have used alim $=8$ for $n_{\max }=16$ with gamma $=1 \times 10^{-8}$. The intrinsic function $\bmod \left(E_{1}, E_{2}\right)$ which requires two integer arguments, is the conventional modulus; begin integer $m$; real alim, delta, gamma, $r$, epsilon, s, $k$, a2, omega, da, jp, jm, q1, q2; real array $b[1: n] ; \quad$ if $a=0$ then

L1: begin if $\bmod (n-1,2)=0$ then

L2: $\quad$ begin $b[n]:=2 / n$; go to exit end L2;

comment integral $b[n]=B_{n-1}(0)$ for odd $n$ was evaluated; $b[n]:=0 ;$ go to exit end $L 1$;

comment integral $b[n]=B_{n-1}(0)$ for even $n$ was evaluated; if abs(a) alim then

L3: begin delta := $\operatorname{gamma} ;$ if $\bmod (n-1,2)=0$ then

IA : begin $r:=2 / n$; epsilon $:=r \times$ delta; $s:=r$;

Even: $\quad k:=k+2$;

$r:=r \times a 2 \times(n+k-2) /(k \times(k-1) \times(n+k))$;

$s:=s+r$; if $r>$ epsilon then go to Even;

$\mathrm{b}[\mathbf{n}]:=\mathbf{s}+\mathbf{r}$

go to exit end L4;

comment integral $b[n]=B_{n-1}(a)$ for odd $n$ and $|a|<$ alim was evaluated;

r $:=2 \times a /(n+1) ;$ omega $:=$ abs $(r \times$ delta $)$;

$\mathrm{s}:=\mathrm{r} ; \mathrm{k}:=1$;

$\mathbf{4 2}:=\mathrm{a} \uparrow 2$

$k:=k+2$;

$r:=r \times a 2 \times(n+k-2) /(k \times(k-1) \times(n+k))$;

$s:=s+r ;$ if abs $(r)>$ omega then go to Odd;

$b[n]:=-(s+r)$; go to exit end L3;

comment integral $b[n]=B_{n-1}(a)$ for even $n$ and $|a|<$ alim was evaluated;

$\mathrm{da}:=1 / \mathrm{a} ; \mathrm{jp}:=\mathrm{da} \times \exp (\mathrm{a}) ; \mathrm{jm}:=(\mathrm{da} \uparrow 2) / \mathrm{jp}$; b[1] := jp-jm;

if $\mathrm{n}=1$ then go to exit;

comment integral $b[1]=B_{0}(a)$ for $|a| \geqq$ alim was evaluated; $\mathrm{q} 1:=-1 ; \mathrm{q} 2:=1 ;$ for $\mathrm{m}:=2$ step 1 until $\mathrm{n}$ do

J.5: begin $b[\mathrm{~m}]:=\mathrm{q} 1 \times \mathrm{jp}-\mathrm{jm}+\mathrm{q} 2 \times \mathrm{da} \times \mathrm{b}[\mathrm{m}-1]$; $\mathrm{q} 1:=-\mathrm{q} 1 ; \quad \mathrm{q} 2:=\mathrm{q} 2+1$ end $L 5$;

comment integral $b[n]=B_{n-1}(a)$ for integer $n \geqq 2$ and $|a| \geqq$ alim was evaluated;

exit: $\quad B s:=b[n]$ end $B s$;

\section{CERTIFICATION OF ALGORITHM 109}

DEFINITE EXPONENTIAL INTEGRALS B [Yuri A.

Kruglyak, D. R. Whitman, Comm. ACM 5 (July 1962)]

YURI A. KRUGLYAK

Kharkov State University, Kharkov, U.S.S.R., and

Donald R. Whitman

Case Institute of Technology, Cleveland, Ohio

Integrals $B_{n}(a)=\int_{-1}^{+1} x^{n} \exp (-a x) d x$ occur in physical problems involving spheroidal coordinates, particularly in quantum chemistry calculations. This algorithm was programmed for the Burroughs-220 computer using a Burroughs Algebraic Compiler. The program was used to compute tables of $B_{n}(a)$ in the ranges $n=0(1) 15$, and $a=0.00(0.01) 32.54$. For example, for $n=0(1) 15$ and $a=0.25$, and $a=24.0$ the results below were obtained. These are compared with the results (columns 3 and 5) obtained by James Miller, John M. Gerhauser, and F. A. Matsen [Quantum Chemistry Integrals and Tables, University of Texas Press, Austin, 1959].

\begin{tabular}{|c|c|c|c|c|}
\hline$n$ & $a=0.25$ & $\begin{array}{c}a=0.25 \text { (Miller } \\
\text { et al.) }\end{array}$ & $a=24.0$ & $\begin{array}{c}a=24.0 \text { (Miller } \\
\text { et al. })\end{array}$ \\
\hline 0 & 84,01 & .20208985344653 , & 34,10 & .1103 \\
\hline 1 & $-.16771064, \quad 00$ &.$\overline{1677106} 6117520$ & $-.10577253,10$ & 772536282,10 \\
\hline 2 & $.67821322,00$ & $. \overline{6792132} 4506375, \quad 00$ & $.10155696,10$ &.$\overline{101556964184,10}$ \\
\hline 3 & $-.10074584,00$ &.$- \overline{10074585827159}$ & $-.97676725,09$ &.$- \overline{976767}$ \\
\hline 4 & 40896479 &.$\overline{40896480211998, \quad 00}$ & $.94091887,09$ & .940918 \\
\hline 5 & $-.72008754,-01$ &.$- \overline{72008756636929,-01}$ & $-.90768866,09$ &.$- \overline{907688}$ \\
\hline 6 & $.29268836, \quad 00$ & $. \overline{29268933} 7517905, \quad 00$ & $.87679129,09$ &.$\overline{8760212}$ \\
\hline 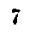 & $-.56030292,-01$ & $-. \overline{5603029} 4023170,-01$ & $-.84798262,09$ & $-.847982638333,09$ \\
\hline 8 & $.22792911, \quad 00$ & $.22792912573392, \quad 00$ & $.82105258,09$ & $.821052542631,09$ \\
\hline 9 & $-.45856272,-01$ & $-. \overline{4525627} 2975462,-01$ & $-.79581870,09$ & $-.795818718590,09$ \\
\hline & $.18664760,00$ &.$\overline{18664761} 544688$ & $.77212229,09$ & $.772122289331,09$ \\
\hline
\end{tabular}




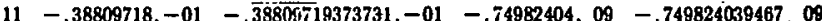

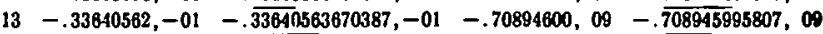
$14.13702696,00 \quad . \overline{13702696892367,00 \quad .69016158,09 \quad .690161591189,00}$ $15-.28686862,-01-. \overline{28686663} 616401,-01-.67236245,09-. \overline{072362427383,00}$

The accuracy is at least six significant figures in the ranges mentioned above. This accuracy is enough for the majority of quantum chemistry calculations.

\section{ALGORITHM 110 \\ QUANTUM MECHANICAL INTEGRALS OF SLATER-TYPE ORBITALS}

YURI A. KRUGLYaK

Kharkov State University, Kharkov, U.S.S.R., AND

Donald R. Whitman

Case Institute of Technology, Cleveland, Ohio

réal procedure INTSOLl (n, r, za, ab, As, Bs) Result:

( $s, i 1, i 2, i 3)$; value $n, r, z a, z b$; integer $n$; real $r, z a, z b$; real array $a[1: 8], b[1: 8], G[1: 2 \times n]$; integer array $b c[1: 2 \times n$, $1: 2 \times \mathrm{n}] ; \quad$ real procedure $\mathrm{As}, \mathrm{Bs}$;

comment Procedure INTSOLI computes the quantum mechanical integrals $s=\left\langle\psi_{n 00}^{a i} \mid \psi_{210}^{b i}\right\rangle \quad$ (overlap integral), il $=\left\langle\psi_{n 00}^{a i}\left|Z_{a}^{*} / r_{a i}\right| \psi_{210}^{b i}\right\rangle \quad$ (exchange integral), $\mathrm{i} 2=\left\langle\psi_{n 00}^{a i}\left|Z_{b}^{*} / \mathrm{r}_{b i}\right| \psi_{n 00}^{a i}\right\rangle$ (coulomb integral), and $\quad \mathrm{i} 3=\left\langle\psi_{210}^{b i}\left|\mathrm{Z}_{a}^{*} / \mathrm{r}_{a i}\right| \psi_{210}^{b i}\right\rangle$ (coulomb integral).

Here $\left|\psi_{n l m}^{a i}\right\rangle$ is a Slater-type orbital of electron $i$ centered on atomic nucleus $a$. The integer $n$ is the effective principal quantum number with values $1,2,3$ and $4, Z_{a}^{*}=z a$ and $Z_{b}^{*}=z b$ are effective nuclear charges. $r_{b i}$ is the distance of electron $i$ from nucleus $b$. The input parameter $r$ is the distance between the two centers $a$ and $b$. All physical quantities are given in atomic units;

begin

integer $q, t, c, m$;

real $\mathrm{g}, \mathrm{zsa}, \mathrm{zsb}, \mathrm{ks}, \mathrm{p}, \mathrm{pt}$, lilya, $\mathrm{s}, \mathrm{k} 1$, exc, il, pppt, k2, sue, i2, pmpt, ptmp, k3, i3;

$\operatorname{bc}[1,1):=b c[2,1]:=b c[2,2]:=1$;

L6: $\quad$ begin bc $[g, 1]:=1$; for $t:=2$ step 1 until $g-1$ do $\mathrm{bc}[\mathrm{q}, \mathrm{t}]:=\mathrm{bc}[\mathrm{q}-1, \mathrm{t}-1]+\mathrm{bc}[\mathrm{q}-1, \mathrm{t}]$; $\mathrm{bc}[\mathrm{q}, \mathrm{q}]:=1$ end $L 6$;

comment binomial coefficients $b c[q, t]=\left(\begin{array}{c}q-1 \\ t-1\end{array}\right)$ were computed using the recursion formula $\left(\begin{array}{l}q \\ t\end{array}\right)=\left(\begin{array}{c}q-1 \\ t-1\end{array}\right)+\left(\begin{array}{c}q-1 \\ t\end{array}\right)$;

procedure $A s(n, b)$ Result: (a[n]); value $n, b$; integer $n$; real $b$;

comment procedure $A s$ computes a value of integral $A_{n-1}(1, b)$ [see Algorithm 108, "Definite Exponential Integrals $A$," by Yuri A. Kruglyak and D. R. Whitman, Comm. ACM (July 1962)]. Any identifier occurring within the $A s$ is specified to be local to the $A s$;

begin integer $m$; real $d b ; a[1]:=\exp (-b) / b$; if $\mathrm{n}=1$ then go to exitAs; $\mathrm{db}:=1 / \mathrm{b}$; for $m:=2$ step 1 until $n$ do $a[m]:=a[1]+$ $\mathrm{db} \times(\mathrm{m}-1) \times \mathrm{a}[\mathrm{m}-1]$

exitAs: end As;

procedure $B s(n, a)$ Result: $(b[n])$; value $n, a$; integer $n$; real $a$;

comment procedure $B s$ computes a value of integral $B_{n-1}(a)$ [see Algorithm 109, "Definite Exponential Integrals $B$ " by Yuri A. Kruglyak and D. R. Whitman, Comm. ACM (July 1962)]. Any identifier occurring within the $B s$ is specified to be local to the $B_{s}$;

begin

Even :

Odd:

exitBs:

S:

I1:

I2:

BA2:

I3:

AB3: 
CERTIFICATION OF ALGORITHM 110

QUANTUM MECHANICAL INTEGRALS OF

SLATER-TYPE ORBITALS [Yuri A. Kruglyak and

Donald R. Whitman, Comm. ACM 5 (July 1962)]

Yuri A. KRUglyak

Kharkov State University, Kharkov, U.S.S.R. and

Donald R. Whitman

Case Institute of Technology, Cleveland, Ohio

This procedure was written and lested in the Burroughs 220 version of the Algol language in the spring of 1961 at Case Institute of Technology. The program was used to compute tables of quatnum mechanical integrals $s, i 1, i 2$, and $i 3$ in the ranges: $r(\AA)=0.64(0.02) 1.40(0.10) 3.10 ; Z_{b}^{*}=0.25(0.50) 3.75,3.90,4.25$, $4.55,4.75,5.20,5.25 ; Z_{\mathrm{a}}^{*}=0.7,1.0$ for $n=1 ; 1.3(1.0) 3.3$ for $n=2$; $0.2,2.2(1.0) 4.2$ for $n=3$; and $0.2,2.2,3.2$ for $n=4$. The table at the right shows typical results 'compared with values from Integrallafeln zur Quantenchemie by H. Preuss (Springer-Verlag, 1957), $\mathrm{Z}$ weiter Band. Accuracy is at least six significant fgures in the ranges mentioned above. This is ample for the overwhelming majority of quantum chemistry calculations.

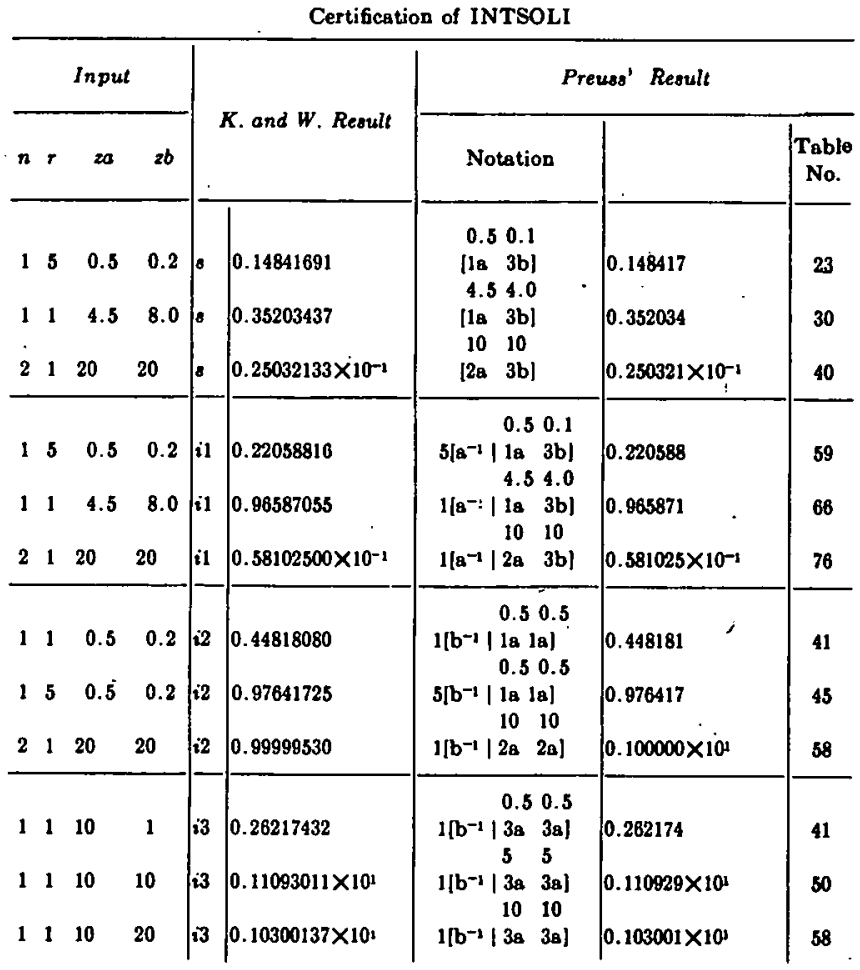

\section{ALGORITHM 111 \\ MOLECULAR-ORBITAL CALCULATION OF MOLECULAR INTERACTIONS}

\section{YuRI A. Kruglyak}

Kharkov State University, Kharkov, U.S.S.R., ANI)

Donald R. Whitman

Case Institute of Technology, Cleveland, Ohio

real procedure SOII $(n, z a, z b, r e m$, coef $2, r 1, E 1, d r 1, d r k 1$, acc, acc1, rk2, rk1, dr2, usy, rk3, dr3, As, Bs, RESULT) Result: (ra, Ep, Em, ca, ca2, ch, ch2, I)Ea, I)Eb, s, i1, i2, i3, haa, hab, hbb); value $n, z a, z b$, rem, coef2, r1, E1, dr1, rk1, drk1, acc, accl, rk2, dr2, asy, rk3, dr3; integer $\mathrm{n}$, rem; real otherwise; real array u[1:8], b[1:8], G[1:8], zsap [1:9], rp[1:8], ans[1:rem]; real procedure $\mathrm{As}, \mathrm{Bs}, \mathrm{RESULT}$;

comment This procedure calculates a one-electron approximation to the energy of interaction of molecular species by the use of the molecular-orbital (MO) method with a linear combination of Slater-type orbitals (LCSTO). The wave function used is $|\phi\rangle=c_{a}\left|\psi_{n 00}^{a}\right\rangle+c_{b} \mid \psi_{210^{\prime}}^{l}$, where $\left|\psi_{n t m}^{a}\right\rangle$ is a ST( ) centered on nucleus $a$. The effective principal quantum number $n$ takes the integral value $1,2,3$ or 4 . The Hamiltonian used is: $\mathfrak{K}_{a b}=$ $-\Delta / 2-Z_{a}^{*} / r_{a}-Z_{b}^{*} / r_{b}+Z_{a}^{*} Z_{b}^{*} / R_{a b}$. Here $Z_{a}^{*}$ and $Z_{b}^{*}$ are effective nuclear charges, $r_{a}$ und $r_{b}$ the distances of the electron from nucleus $a$ and $b$, and $R_{a b}$ is the distance between nuclei $a$ and $b$. The calculations are in atomic units, while the output $r a$ is in Angstroms and $D E a$ and $D E b$ are in kcal/gm-ion. Abbreviations of the following type are used: $Z a=Z_{a}{ }^{*}, \quad r a=$ $R_{a b}(\AA)$, , haa $=\left\langle\psi_{n 00}^{a}\left|\mathcal{H}_{a b}\right| \psi_{n 00}^{a}\right\rangle, D E a=D(a, b+$ electron $), e 1=$ $\left\langle\psi_{n 00}^{a}\left|-\Delta / 2-Z_{a}^{*} / r a\right| \psi_{n 00}^{a}\right\rangle$. The values of coefl and coef2 are 627.71 (kcal/gm.ion) and $0.5291 \AA$, respectively. $r 1, E 1, d r 1, d r 2, d r 3, a c c, a c c 1$, and asy are control parameters. The accuracy of the calculations (acc, acc1) is $1 \times 10^{-5}$. The initial values of $R_{a b}$ and $E_{-}$are conveniently: $r 1=0.4(\AA)$, and $E 1=100$ (a.u.). The steps are: $d r 1=0.1, d r 2=0.4$, and $d r 3=0.01$, all in Angstroms. asy is $-1 \times 10^{-3}$ (a.u.);

begin integer $q, t, c, m, f$; real otherwise;

procedure As(n, b) Result: (a[n]); value $n, b$; integer $n$; real b;

comment any identifier occurring within the $A s$ is specified to be local to the As;

hegin integer $m$; real $d b ; a \mid 1]:=\exp (-b) / b$; if $n=1$ then go to exitAs; db := 1/b;

for $m:=2$ step 1 until $n$ do $a[m]:=a[1]+d b X$ $(m-1) \times a[m-1]$

exitAs: end As;

procedure $B s(n, a)$ Result: $(b[n])$; value $n, a$; integer $n$; real a;

comment any identifier occurring within the $B s$ is specified to be local to the $B s$;

begin integer $m$; real otherwise; if $a=0$ then begin if $\bmod (n-1,2)=0$ then begin $b[n]:=2 / n$; go to exitBs end; $h[n]:=0 ;$ go to exitBs end; if abs(a)<alim then begin delta := gamma; if $\bmod (n-1,2)=0$ then begin $r:=2 / n$; epsilon $:=r \times$ delta; $s:=r ; \quad k:=0 ; a 2:=a \uparrow 2$;

Even: $\quad k:=k+2 ; \quad r:=r \times a 2 \times(n+k-2) /(k(k-1)(n+k))$ $s:=s+r$; if $r>$ epsilon then go to Even; $b[n]:=s+r$; go to exitBs end;

$r:=2 \times a /(n+1) ;$ omega $:=$ abs $(r \times$ delta $)$; $\mathrm{s}:=\mathrm{r} ; \mathrm{k}:=1 ; \mathrm{a} 2:=\mathrm{a} \uparrow 2$

Odd: $\quad k:=k+2 ; r:=r \times a 2 \times(n+k-2) /(k(k-1)(n+k))$; $\mathrm{s}:=\mathrm{s}+\mathrm{r} ;$ if abs(r)>omega then go to Odd; b $[\mathbf{n}]:=-(s+r)$; go to exitBs

end; $d a:=1 / a ; j p:=\operatorname{da} \times \exp (a)$; $\mathrm{jm}:=(\mathrm{du} \uparrow 2) / \mathrm{jp} ; \quad b[1]:=\mathrm{jp}-\mathrm{jm} ;$

if $\mathrm{n}=1$ then go to exitBs; $\mathrm{ql}:=-1 ; \mathrm{q} 2:=1$; for $m:=2$ step 1

until $n$ do begin $b[m]:=q 1 \times j p-j m+$ $\mathrm{q} 2 \times \mathrm{da} \times \mathrm{b}[\mathrm{m}-1]$;

$q 1:=-q 1 ; \quad q 2:=q 2+1$ end

exitBs: end Bs;

procedure Result(coef 1$)$; real coef 1 ;

comment RESULT computes $E p, E m, c a, c a 2, c b, c b 2, D E a$, $D E b, s, i 1, i 2, i 3, n n$, haa, hat, hbb. Important: RESULT and any identifier occurring within the RESULT enter SOLI as nonlocal entities;

hegin $\quad r:=r a \times b r ; \quad r p[1]:=r ;$ for $c:=2$ step 1 until $n+4$ do $r p[c]:=r p[c-1] \times r p[1] ; p:=r \times s u m$; pt $:=$ rXdif; ks $:=r p[n+3] \times z s s$; 
for $c:=1$ step 1 until $n+3$ do begin $A s(c, p)$ Result: (a[c]); Bs(c, pt) Result: (b[c]) end; lilya $:=0$; for $m:=0$ step 1 until $n$ do lilya $:=$ lilya $+b c[n+1, m+1) \times(a[n-m+2] \times$

$(b[m+1]+b[m+3])-b[m+2] \times(a[n-m+1]$ $+a(n-m+3])) ; \quad s:=$ ks $\times$ lilya: $1 \mathrm{il} 2:=2 \times 8$; $\mathrm{kl}:=\mathrm{ks} \times \mathrm{za} / \mathrm{r}$; exc $:=0$; for min $:=0$ step 1 until $\mathrm{n}-1$ do exc $:=\operatorname{exc}+\mathrm{bc}[\mathrm{n}, \mathrm{m}+1] \times(\mathrm{a}[\mathrm{n}-$ $\mathrm{m}+1] \times(\mathrm{b}[\mathrm{m}+1]+\mathrm{b}[\mathrm{m}+3])-\mathrm{b}[\mathrm{m}+2] \times(\mathrm{a}[\mathrm{n}-\mathrm{m}]+$ $\mathrm{a}[\mathrm{n}-\mathrm{m}+2]))$; il $:=\mathrm{k} 1 \times \mathrm{exc}$; pppt := p+pt; $\mathrm{k} 2:=\mathrm{rp}[2 \times \mathrm{n}] \times 2 \mathrm{sbd} ;$ for $\mathrm{c}:=1$ step 1 until $2 \times n$ do begin As(c, pppt) Result: (a[c]); Bs (c, pppt) Result: (b[c]) end; sue := 0; for $m:=0$ step 1 until $2 \times n-1$ do sue := sue $+b c[2 \times n, \quad m+1] \times a[2 \times n-m] \times b[m+1]$; i2 := k2 $\times$ sue; pmpt $:=\mathrm{p}-\mathrm{pt}$; ptmp := -pmpt; k3 := rp[4]×25; for $c:=1$ step 1 until 4 do begin $\mathrm{As}(\mathrm{c}, \mathrm{pmpt})$ Result: (a[c]); Bs(c, ptmp) Result: (b[c]) end; i3 : $=k 3 \times(a[2] \times(b[1]+2 \times b[3])-b[2] \times(a[1]+2 \times$ $a[3])+a[4] \times b[3]-a[3] \times b[4])$;

comment Two-center integrals" $8, i 1-i 2$; and- $i 3$-were computed [see Algorithm 110, "Quantum Mechanical Integrals of SlaterType Orbitals," by Yuri A. Kruglyak and D. R. Whitman, Comm. $A C M$ (July 1962)]; $\mathrm{nn}:=\mathrm{zz} /(2 \times \mathrm{r}) ;$ e2pnn := e2+nn; haa :=e1-e2+nn; hbb := e2pnn-i3; hab :=e2pnn $\times s-i 1$; den $:=2-s \times 1 i 12 ;$ bsr $:=$ haa + hbb-hab $\times 1 i 12$; root := sqrt(bsr $\uparrow 2-2 \times \operatorname{den} \times($ haa $\times$ hbl $-h a b \uparrow 2))$; Ep $:=($ bsr + root $) /$ den; $\mathrm{Em}:=($ bsr - root $) /$ den; ans $[\mathrm{f}]:=\mathrm{Em} ;$ DEa $:=\operatorname{coef} 1 \times(\mathrm{e} 2-\mathrm{Em})$; $\mathrm{DEb}:=$ coef $1 \times(\mathrm{el}-\mathrm{Em}) ;$ Emhaa $:=$ Em-haa; Emhbb := Em-hbb; ES := EmXs; habmES := hab-ES; caDcb1 := habmES/Emhaa; cbl)ca2 := habmES/Emhbb; if $\operatorname{abs}($ Emhaa $)>\operatorname{abs}(E m h b b)$ then begin col := caDcb1 $\uparrow$;

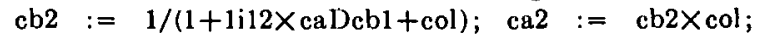
ca $:=\operatorname{sqrt}(\mathrm{ca} 2) ;$ cb $:=\mathrm{ca} / \mathrm{caDcbl}$ go to NATA end; $\operatorname{co} 2:=\operatorname{cbDca} 2 \uparrow 2 ;$ ca2 $:=1 /(1+1 \mathrm{i} 12 \times \operatorname{cbD} \mathrm{ca} 2+\operatorname{co} 2)$; cb2 $:=\operatorname{ca} 2 \times \operatorname{co} 2 ;$ ca $:=\operatorname{sqrt}(\mathrm{ca} 2) ; \quad$ cb $:=$ ca $\times$ cbI)ca2

NATA: end RESULT;

Begin of program: $b c[1,1]:=b c[2,1]:=b c[2,2]:=1$; for $q:=3$ step 1 until 8 do begin $b c[q, 1]:=1$; for $t:=2$ step 1 until $q-1$ do $b c[q, t]:=b c[q-1, t-1]+b c[q-1, t]$ $\mathrm{bc}[\mathrm{q}, \mathrm{q}]:=1$ end;

IZM: $\quad \mathrm{g}:=1$; for $\mathrm{m}:=1$ step 1 until $2 \times \mathrm{n}$ do $\mathrm{g}:=\mathrm{g} / \mathrm{m}$; $\mathrm{G}[2 \times \mathrm{n}]:=\mathrm{g} ; \quad$ zsa $:=\mathrm{za} / \mathrm{n} ; \quad$ zsap $[1]:=\mathrm{zsa} \times 2$; for $c:=2$ step 1

until $2 \times n+1$ do $x$ sap $[c]:=$ zsap $[c-1] \times \operatorname{zsap}[1]$; l) := $\quad \operatorname{ssap}[2 \times \mathrm{n}+1] \times \mathrm{G}[2 \times \mathrm{n}] ;$ DS $:=\operatorname{sqrt}(1))$; el $:=-\operatorname{zsap}[2 \mid \times 0.125 \times(4 \times n-3) /(2 \times n-1)$; $\mathrm{zsb}:=\mathrm{zb} \times 0.5 ;$ sum $:=\mathrm{zsa}+\mathrm{zsb} ;$ dif $:=\mathrm{zsa}-\mathrm{zsb}$; zsb5 := zsh 5 ; zss := JSXsqrt(zsb5); zsbd $:=z s b \times 1) ; \quad z 5:=2 \times z a \times z s b 5 ; \quad z z:=z a \times z b ;$ e2 $:=-(\mathrm{zsh}) \uparrow 2) / 2 ;$ br $:=0.5 / \operatorname{coef} 2 ; \mathrm{f}:=1$; ans $[1]:=\mathrm{E} 1 ; \quad \mathrm{ra}:=\mathrm{rl}$;

KOM: ra $:=$ ra+drl; if $r a>r k l$ then $r a:=$ ra+drkl; $f:=f+1 ; \operatorname{RESULT}(\operatorname{coef} 1) ;$ if ans $[\mathrm{f}]-\operatorname{ans}[\mathrm{f}-1 \mid \leqq$ acc then begin if ra>rk2 then go to $I / M$; go to KOM end; ansf := ans(f]; dl := ra;

CLEV: $\mathrm{ra}:=\mathrm{ra}+\mathrm{dr} 2$; RESULT (coef1); if $\mathrm{e} 1<\mathrm{e} 2$ then begin if $\mathrm{Em}-\mathrm{el} \leqq \mathrm{asy} \wedge \mathrm{ra}<\mathrm{rk} 3$ then go to CLEV; go to KHAR end; if $\mathrm{e} 1 \geqq \mathrm{e} 2$ then begin if $\mathrm{Em}-\mathrm{e} 2 \leqq$ asy $\wedge$ ra< $<$ rk3 then go to CLEV; go to KHAR end;

KHAR: ra $:=\mathrm{d} 1$; ans $[\mathrm{f}-1]:=$ ansf;

CASE: ra $:=r a-d r 3 ; f:=f+1 ;$ RESULT (coef 1 ); if ans $[f]-a n s[f-1] \leqq$ accl then go to $\mathrm{CASE}$; go to IZM end SOI,I;
ALGORITHM 112

POSITION OF POINT RELATIVE TO POLYGON

M. Shimrat

University of Alberta, Calgary, Alberta, Canada

Boolean procedure POINT IN POL YGON $(n, x, y, x 0, y 0)$;

value $n, x 0, y 0 ;$ integer $n ;$ array $x, y$; real $x 0, y 0$;

comment if the points $(x[i], y[i])(i=1,2, \cdots, n)$ are-in this cyclic order-the vertices of a simple closed polygon and $(x 0, y 0)$ is a point not on any side of the polygon, then the procedure determines, by setting "point in polygon" to true, whether $(x 0, y 0)$ lies in the interior of the polygon;

begin integer $i$; Boolean $b$;

$x[n+1]:=x[1] ; \quad y[n+1]:=y[1] ; \quad b:=$ true;

for $i:=1$ step 1 until $n$ do

if $(y<y[i] \equiv y>y[i+1]) \wedge$

$x 0-x[i]-(y 0-y[i]) \times(x[i+1]-x[i]) /(y[i+1]-y[i])<0$

then $b:=\neg b$;

POINT IN POLYGON := $\neg b$;

end POINT IN POLYGON

\section{CERTIFICATION OF ALGORITHM 112}

POSITION OF POINT RELATIVE TO POLYGON

[M. Shimrat, Comm. ACM, Aug. 1962]

RICHARD HaCKER

The Boeing Co., Seattle Wash.

The Boolean procedure POINT IN POL YGON was programmed in FORTRAN for the IBM 7090. The algorithm gave satisfactory results except for a case such as the following:

Let the polygon points be: $(0,0),(1,0),(2,1),(1,2),(0,2)$.

In this case the procedure would not detect that the point $(1,1)$ is in the polygon. However, the correct result was obtained by changing:

$$
\text { if }(y<y[i] \equiv y>y[i+1]) \wedge
$$

to read:

$$
\text { if }(y 0 \leqq y[i] \equiv y 0>y[i+1]) \wedge
$$

\section{ALGORITHM 113 \\ TREESORT}

Robert W. Floyd

Computer Associates, Inc., Woburn, Mass.

procedure TREESORT (UNSORTED, $n, S O R T E D, k$ ); value $n, k$;

integer $n, k ;$ array UNSORTED, SORTED;

comment TREESORT sorts the smallest $k$ elements of the $n$ component array UNSORTED into the $k$-component array SORTED (the two arrays may be the same). The number of operations is on the order of $2 \times n+k \times \log _{2}(n)$. The number of auxiliary storage cells required is on the order of $2 \times n$. It is. assumed that procedures are available for finding the minimum of two quantities, for packing one real number and one integer into a word, and for obtaining the left and right half of a packed word. The value of infinity is assumed to be larger than that of any element of UNSORTED;

begin integer $i, j ;$ array $m[1: 2 \times n-1]$;

for $i:=1$ step 1 until $n$ do $m[n+i-1]:=$ pack (UNSORTED [i], $n+i-1)$; 
for $i:=n-1$ step -1 until 1 do $m[i]:=\operatorname{minimum}(m[2 \times i]$, $m[2 \times i+1])$;

for $j:=1$ step 1 until $k$ do

begin SORTED $[j]:=$ lefl half $(m[1]) ; i:=$ right half $(m[1])$; $m[i]:=$ infinity

for $i:=i \div 2$ while $i>0$ do $m[i]:=\operatorname{minimum}(m[2 \times i], m[2 \times$ $i+1 \mid)$

end

end TIREESORT

\section{ALGORITHM 114}

GENERATION OF PARTITIONS WITH CON STRAINTS

Frank Stocknal

System Development Corp., Santa Monica, Calif.

procedure CP GENERATOR ( $N, K, H, p, F, Z)$; integer $N, K, H$; integer array $p ;$ Boolean $F, Z$;

comment CP GENERATOR generates a partition of $N$ into $K$ parts, no part greater than $H$. Each partition is represented by the array of parts $p[1]$ thru $p[K]$, where $p[1] \geqq p[2] \geqq \cdots \geqq p[K]$ Initial entry is made with $F=$ true and $Z=$ true if parts $=0$ are allowable, or $F=$ true and $Z=$ false if only nonzero parts are desired. Upon initial entry, procedure ignores the input array $p$, sets $F=$ false, and generates the initial partition. Subsequent calls made with $F=$ false will call-c procedure to operate upon the input partition to produce another partition if one exists, so that all possible unpermuted partitions with the specified constraints will be produced if $C P$ GENERATOR is allowed to operate upon its previous output. When this scheme is followed, and initial entry is made with $F=$ true, $Z=$ true, $K=N, H=N$, all possible unpermuted partitions of $N$ will be produced. Upon generating the last partition, procedure resets $F$ to true. The input parameters are restricted as follows: $K \geqq 1, H \geqq 1, \quad p[1] \geqq p[2]$ $\geqq \cdots \geqq p[K]$. For $Z=$ true, $N$ is restricted to the range $0 \leqq N \leqq K H$, and for $Z=$ false, $K \leqq N \leqq K H$. A call should not be made with $p[1]-p[K]<2$ and $F=$ false;

begin integer $a, b, i, j, q, r$;

if $F$ then go to first;

$a:=p[1]-p[2]-2 ; j:=2$;

test: if $p[1]-p[j] \geqq 2$ then go to divide;

$a:=a-1+j \times(p[j]-p[j+1]) ; j:=j+1 ;$ go to test

first: if $Z$ then go to alpha;

$a:=N-K ; p[K]:=0 ;$ go to beta;

alpha: $a:=N ; p[K]:=-1$;

bela: $F:=$ false; $j:=K$;

divide: $\quad b:=H-1-p[j] ; q:=$ entier $(a / b) ; \quad r:=a-b \times q$;

for $i:=1$ step 1 until $q$ do $p[i]:=H$;

if $q=K$ then go to last;

for $i:=q+1$ step 1 until $j$ do $p[i]:=1+p[j]$;

$p[q+1]:=r+p[q+1]$;

if $p[1]-p[K] \geqq 2$ then go to exit;

last: $F:=$ true;

exit: end CP GENERATOR

\section{ALGORITHM 115}

PERM

H. F. TROTTER

Princeton University, Princeton, N. J.

procedure $\operatorname{PERM}(x, n)$; value $n$;

integer $n$; array $x$;

comment This algorithm was inspired by the procedure PERMUTE of Peck and Schrack (Algorithm 86, Comm. ACM

Apr. 1962) and performs the same function. Each call of PERM changes the order of the first $n$ components of $x$, and $n$ ! successive calls will generate all $n$ ! permutations. A nonlocal Boolean variable ' $f$ irst' is assumed, which must be true when PERM is frst called, to cause proper initialization. The first. call of PER.M makes 'first' false, and it remains so (unless changed by the external program) until the exit from the (n!)th call of PERM. At that time $x$ is restored to its original order and ' $f$ r $t$ ' ' is made true.

The excuse for adding PERM to the growing pile of permuta tion generators is that, at the expense of some extra own storage, it cuts the manipulation of $x$ to the theoretical minimum of $n$ ! transpositions, and appears to offer an advantage in speed. It also has the (probably useless) property that the permutations it generates are alternately odd and even;

begin own integer array $p, d[2: n] ;$ integer $k, q$; real $t$;

if $f$ irst then initialize:

begin for $k:=2$ step 1 until $n$ do

begin $p[k]:=0 ; d[k]:=1$ end;

first : = false

end initialize;

$k:=0$;

INDEX: $\quad p[n]:=q:=p[n]+d[n]$;

if $q=n$ then

begin $d[n]:=-1$; go to $L O O P$ end;

if $q \neq 0$ then go to TRANSPOSE;

$d[n]:=1 ; k:=k+1$;

LOOP: if $n>2$ then begin

comment Note that $n$ was called by value;

$n:=n-1$; go to INDEX end $L O O P$;

Final exit: $q:=1$; first $:=$ true;

TRANSPOSE: $q:=q+k ; t:=x[q]$

$x[q]:=x[q+1] ; \quad x[q+1]:=t$

end PERM;

\section{CERTIFICATION OF ALGORITHM 115}

PERM [H. F. Trotter, Comm. ACM, Aug. 1962]

E. S. PhILlips

Michigan State University, East Lansing, Mich.

PERM was translated into ForTran for the CDC 160-A, and it performed correctly. For $n=8$, this method requires 2822 seconds. For comparison, Algorithm 86, PERMUTE, was translated and run on the same machine, requiring 3710 seconds as opposed to 1316 when run on an IBM 1620.

\section{CERTIFICATIUA OF ALGORITHM 11;}

PERM [H. F. Trotter, Comm. ACMI (Aug. 1962)]

G. F. SCHRACK

University of Alberta, Calgary, Alb., Canada

PERM was translated into FORTRAN for the IBM 1620 and it performed satisfactorily. Timing tests were carried out under the same conditions as for PERMLTATION (Algorithm 71) and PERMUTE (Algorithm 86)

PERM is indeed the fastest permutation generator so far encountered. For $n=8$, PERM is $25 \%$ faster than PERMUTE (989 against $1316 \mathrm{sec}$.). The values for $r_{n}$ are (for a definition of $r_{n}$, see Certification of Algorithm 71 , Comm. ACM, Apr. 1962):

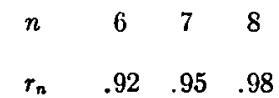




\section{ALGORITHM 116 COMPLEX DIVISION}

ROBERT L. SMITH

Stanford University, Stanford, Calif.

procedure complexdiv $(a, b, c, d)$ results: $(e, f)$;

value $a, b, c, d ;$ real $a, b, c, d$;

comment complexdiv yields the complex quotient of $a+i b$ divided by $c+i d$. The method used here tends to avoid arithmetic overflow or underflow. Such spills could otherwise occur when squaring the component parts of the denominator if the usual method were used;

begin real $r$, den;

if $a b s$ (c) $\geqq a b s$ (d) then

begin $r:=d / c$;

den $:=c+r \times d$

$e:=(a+b \times r) / d e n$

$f:=(b-a \times r) / d e n$;

end

else

begin $r:=c / d$;

den $:=d+r \times c$;

$e:=(a \times r+b) / d e n$

$f:=(b \times r-a) / d e n$

end

end complexdiv

\section{ALGORITHM 117}

\section{MAGIC SQUARE (EVEN ORDER)}

D. M. Collision

Elliott Brothers (London) Limited, Borehamwood, Herts., England

procedure magiceven $(n, x)$; value $n$; integer array $x$; integer $n$;

comment the method of Devedec for even $n$ is described in "Mathematical Recreations" by M. Kraitchik, pp. 150-2. Enter with side of square $n$ to produce a magic square of the integers $1-n \uparrow 2$ in $x$, where $n \geqq 4$;

begin integer $a, b, n 2, n n ;$ Boolean $p, q, r$;

$n 2:=n \div 2 ; n n:=n \times n$

begin

procedure alpha $(p, q, a, h) ;$ value $p, q, a, h$; integer $p, q, a$; Boolean $h$;

Comment pattern $0 / 0 / 0 / \ldots$;

begin integer $r$;

for $r:=p$ step 1 until $q$ do begin

$x[r, a]:=$ if $h$ then $(a \times n-n+r)$ else $(n n-a \times n+$

$1+n-r) ; h:=\neg h$ end;

end alpha;

procedure beta $(p, q, a, h)$; value $p, q, a, h$; integer $p, q, a$; Boolean $h$;

comment pattern $1-1-1-\cdots$;

begin integer $r$;

for $r:=p$ step 1 until $q$ do begin

$x[r, a]:=$ if $h$ then $[n n-a \times n+r)$ else $(a \times n+1-r)$ $h:=\neg h$ end;

end bela;

procedure gamma $(p, q, a, h)$; value $p, q, a, h$; integer $p, q, a$;

Boolean $h$;

comment pattern $/-1-1-\cdots$

begin integer $r$; for $r:=p$ step 1 until $q$ do begin

$x[r, a]:=$ if $h$ then $\left(n n-a \times n+n-r+{ }^{\circ} 1\right)$ else $(a \times n$ $+1-r) ; h:=\neg h$ end;

end gamma;

comment program begins;

$p:=q:=(n-(n \div 4) \times 4=0) ; r:=$ true;

for $a:=1$ step 1 until $(n 2-1)$ do begin

beta $(1, a-1, a, r) ; \quad$ alpha $(a, n 2-1, a$, true);

$x[n 2, a]:=$ if $q$ then $(n n-a \times n+n 2+1)$ else $(n n-a \times$ $n+n 2)$;

alpha $(n 2+1, n, a, \neg q)$;

$q:=\neg q ; r:=\neg r$ end;

alpha $(1, n 2-1, n 2, \neg p) ;$ alpha $(n 2+2, n, n 2$, false);

gamma $(1, n 2-1, n 2+1, p) ; \operatorname{gamma}(n 2+2, n, n 2+1$, true $)$;

$q:=p ; \quad r:=$ true;

for $a:=(n 2+2)$ step 1 until $n$ do begin

beta $(1, n-a, a, q) ; \quad x[n-a+1, a]:=a \times n-a+1 ;$

beta $(n-a+2, n 2-1, a$, true $)$;

if $r$ then for $b:=n 2, n 2+1$ do $x[b, a]:=n n-a \times n+$ $n-b+1$

else begin $x[n 2, a]:=n n-a \times n+n 2$;

$x[n 2+1, a]:=a \times n-n 2+1$ end;

beta $(n 2+2, a-1, a, \neg r) ;$ alpha $(a, n, a$, true $)$;

$\mathbf{q}:=\neg q ; \quad r:=\neg r$ end

for $a:=n 2, n 2+1$ do for $b:=n 2, n 2+1 \mathrm{do}$

$x[b, a]:=$ if $p$ then $(a \times n-n+b)$ else $(n n-a \times n+n-$ $b+1)$

if $\neg p$ then begin

for $a:=n 2, n 2+1$ do $x[n 2-1, a]:=a \times n-n 2+2$

for $b:=n 2, n 2+1$ do $x[b, n 2+2]:=n \times n 2-2 \times n+b$ end;

end end magiceven

CERTIFICATIONS OF ALGORITHMS 1.17 and 118 MAGIC SQUARE (ODD AND EVEN ORDERS)

[D. M. Collison, Comm. ACM, Aug. 1962]

K. M. Bosworth

I.C.T. Ltd., Blyth Road, Hayes, Middlesex, England

The statement within the Booleon procedure beta should be changed from

$x[r, a]:=$ if $h$ then $[n n-a \times n+r)$ else $(a \times n+1-r) ;$

to

$x[r, a]:=$ if $h$ then $(n n-a \times n+r)$ else $(a \times n+1-r) ;$

The procedures were then tested on magic squares of order 3 to 17 inclusive without fault.

CERTIFICATION OF ALGORITHMS 117 AND 118 MAGIC SQLARE (ODD AND, IEVEA ORDERS)

[D. M. Collison, Comm. ACAI, Aug. 196i2]

D. MI. Collisox

Elliott Bros. (London) Ltd., Borchamwood, Herts.,

England

Both algorithms were checked and timed, using al special A t,col. program, with the Elliott. Al,gol translatior on the NationalElliot t. 803. The procedure for odd orders wats the slower:

$\begin{array}{ccc}\text { Procedure } & \text { Sise of Square } & \text { Time } \\ \text { Odd order } & 9 & 10 \mathrm{sec} . \\ & 19 & 45 \mathrm{sec} . \\ \text { Even order } & 10 & 7 \mathrm{sec} . \\ & 20 & 23 \mathrm{sec} .\end{array}$

Because of the different methods used and the lengt h of the even order procedure it wis decided not to combine the two. The smallest square of even order generated is given below:-

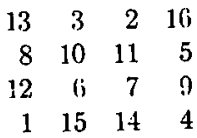


CERTIFICATION OF ALGORITHMS 117 AND 118 MAGIC SQUARES (EVEN AND ODD ORDERS)

[D. M. Collison, [Comm. ACM, Aug. 1962]

P. NAUR

Regnecentralen, Copenhagen, Denmark

MAGICEVEN needed the following correction: Within the body of procedure beta a left square bracket : . . then $[n n$... should be changed to a left parenthesis: ...then ( $n n$...

With this correction it has run successfully in the Gren Aloor system. The squares of even orders from 4 to 20 were generated and checked for magicity in rows and columns, but not in diagonals.

The algorithm contains 11 pairs of superfluous parentheses (10 of which are in conditional expressions) and if the assignments to $n 2$ and $n n$ are moved to the place just following "end gamma;" the inner block becomes unnecessary.

MAGICODD ran without correction in GIER ALgol and produced a few reasonable-looking squares.

Run times are as follows:

$\begin{array}{lcc}\text { Procedure } & \text { Sise of squre } & \text { Time } \\ \text { Magicodd } & 9 & 0.6 \mathrm{sec} \\ & 19 & 2.5 \mathrm{sec} \\ \text { Magiceven } & 10 & 0.9 \mathrm{sec} \\ & 20 & 2.3 \mathrm{sec}\end{array}$

\section{ALGORITHM 118}

\section{MAGIC SQUARE (ODD ORDER)}

D. M. Collison

Elliott Brothers (London) Limited, Borehamwood, Herts., England

procedure magicodd $(n, x)$; value $n$; integer $n$; integer array $x$;

comment for given side $n$ the procedure generates a magic square of the integers $1-n \uparrow 2$. For the method of De la Loubc̀re, see M. Kraitchik, "Mathematical Recreations," p.

149. $n$ must be odd and $n \geqq 3$;

begin integer $i, j, k$;

for $i:=1$ step 1 until $n$ do

for $j:=1$ step 1 until $n$ do $x[i, j]:=0$

$i:=(n+1) \div 2 ; j:=n$;

for $k:=1$ step 1 until $n \times n$ do begin

if $x[i, j] \neq 0$ then begin $i:=i-1 ; \quad j:=j-2$;

if $i<1$ then $i:=i+n$; if $j<1$ then $j:=j+n$ end; $x[i, j]:=k$;

$i:=i+1 ; \quad$ if $i>n$ then $i:=i-n$;

$j:=j+1$; if $j>n$ then $j:=j-n$;

end;

end magicodd

\section{CERTIFICATION OF ALGORITHM 118}

MAGIC SQUARE (ODD ORDER) [D. M. Collison, Comm. ACM, Aug. 1962]

Henry C. Thacher, Jr.*

Reactor Engineering Div., Argonne National Lab., Argonne, Ill.

* Work supported by the U.S. Atomic Energy Commission.

The body of the procedure magicodd was tested on the LGP -30 using the Dartmouth ALGOL 60 translator. No syntactical errors were found. The procedure generated odd-order magic squares satisfactorily. For orders up to 9 , times were as follows (including output on the Flexowriter):

$\begin{array}{cc}\text { Order } & \text { Time }(\mathrm{sec}) \\ 3 & 171 \\ 5 & 422 \\ 7 & 804 \\ 9 & 1285\end{array}$

The $3 \times 3$ square was:

$\begin{array}{rrr}4 & 3 & -8 \\ 9 & 5 & 1 \\ 2 & 7 & 6\end{array}$

SEE AIGORITHM 117

ALGORITHM 119

EVALUATION OF A PERT NETWORK

Burton Eisenman and Martin Shapiro

United Nuclear Corp., White Plains, N. Y.

procedure pert (nmax, $i, j$; $t e, s t$, emax, $l, e s, a l$ );

comment An algorithm describing an iterative procedure for evaluating a PERT network that permits the use of arbitrarily. ordered activities and event identifiers such that an upper triangular matrix type of solution is unnecessary.

It has been observed by investigations of PERT networks, that an $N \times N$ matrix whose rows are designated as predecessor and whose columns are designated as successor events, has an entry in the $(i, j)$-element representing the activity time required in going from event $i$ to event $j$. By elementary transformations, the matrix is transformed generally into an upper triangular matrix. The resultant upper triangular matrix is well ordered (i.e. any activity time appearing in a column is not dependent upon those activity times which appear in columns to the right of $i t$ ).

This precise manipulation generally demands considerable running time. By direct evaluation not requiring a collection of elementary transformations, it is possible to evaluate the net. work with considerable reduction of running time;

integer, nmax, emax;

real $s ! ;$

integer array $i, j, l$;

real array $t e, e s, a t$

comment Given the total number of activities, nmax, the preceding and succeeding event identifiers, $i_{n}$ and $j_{n}$, the corresponding expected time, $t e$, for each activity, and the starting time, $s t$, of the network, this procedure computes the early start and late finish times, $e s_{e}$ and $a l_{e}$, for each event, $l_{e}$, in the network;

begin

procedure $\operatorname{scan}(e, t, l)$;

integer $e, t$;

integer array $l$;

comment Given the mumber of events, $e-1$, contained thus far in vector array, $l$, and an event identifier $i_{n}$ or $j_{n}$, stored in $t$, this procedure scans the existing array, $l$, to determine whether the event should be added to the list or not. If it is to be added, it becomes $l_{e}$ and $e$ replaces the event identifier. If it is not added, $k$ replaces the event identifier.;

begin

integer $k$;

$$
\text { if } e=1 \text { then go to add; }
$$




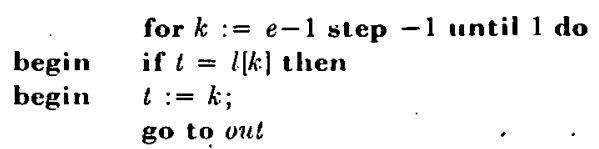

end

end;

add:

out:

$$
\begin{aligned}
& l[\varepsilon]:=\ell \\
& t:=e \\
& e:=e+1
\end{aligned}
$$

end scan;

integer $n, e, s, t, k$

real $a, x$;

$$
e:=1 \text {; }
$$

for $n:=1$ step 1 until nmax,do

begin . $t:=j[n]$;

sian $(e, t, l)$;

$j[n]:=t ;$

$\ell:=i[n]$

$\operatorname{scan}(e, t, l)$;

$i[n]:=\imath$

end;

comment By means of the swltch, $s$, we will either compute the activity times, $a l_{c}$, and transfer the values to the early start vector, $e s_{s}$, or we will compute $a l_{c}$ without any transfer process, in which case the late finish times will be obtained.;

$$
\begin{aligned}
\text { emax }:= & e-1 ; \\
s: & =1 ; \\
a & :=s t ;
\end{aligned}
$$

s1: $\quad k::=$ emax

for $e:=1$ step 1 until emax do

$a \ell[e]:=a$;

s2: $\quad$ for $\mathrm{n}:=1$ step 1 until $n \max$ do

begin if $l[i[n]]>0$ then

begin switch $s:=b 1, b 2$;

b1: $\quad x:=a b s(a t[i[n]])+t e[n]$;

if $x>a b s(a l|j| n] \mid)$ then go io $l 1$;

go to $l 2$;

b2: $\quad x:=\operatorname{abs}(a t[i[n]])-t e[n]$;

if $x<a b s(a l[j[n]])$ then

l1: $\quad a \ell[j[n] \mid:=-x$

12:

end

end;

for $e:=1$ step 1 until emax do

begin if $l[e]<0$ then

begin if at $[e]<0$ then

begin $l[e]:=$ abs $(l[e])$;

$k:=k+1$;

s3: $\quad a t[e]:=a b s(a t[e \mid)$;

go to $l 3$

end;

go to 13

end;

$$
\begin{array}{ll} 
& \text { if } a^{t}[e] \geqq 0 \text { then } \\
\text { begin } & l \mid e]:=-l[e] \\
& k:=k-1 \\
& \text { go to } l 3
\end{array}
$$

end;

go to $s 3$;

13:

end;

if $k \neq 0$ then go to $s 2$;

switch $8:=g 1, g 2$;

gl: $\quad s:=2$; for $n:=1$ step 1 until $n \max$ do

begin $\quad t:=i[n]$;

$i[n]:=j[n]$

end;

$j[n]:=t$

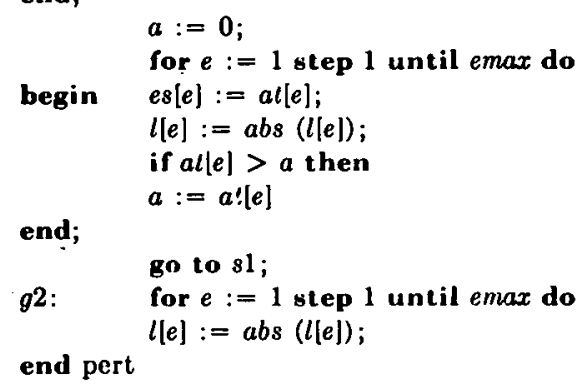

\section{ALGORITHM 120}

\section{MATIRIX INVERSION II}

Richard George*

Particle Accelerator Division Argonne National Laboratory Argonnc, Illinois

* Work supported by the U. S. Atomic Energy Commission.

procedure INVERSION $I I$ ( $n$, a, epsilon, .LL.LRM, della);

comment This is a revision of Algorithm 58. It accomplishes inversion of the matrix $a$, with the result stored in matrix $a$. The order of the matrix is $n$. If in the process of calculating, any pivot element has an alssolute value less than epsilon, there will be a jump to the non-local lahel $A L A R M$. The variable della will contain the value of the determinant of the original matrix on normal exit, zero or a very small number on exit to $A L A R M$.;

value $n$;

array $a$;

real epsilon, delta;

integer $n$;

begin

urray $b, c[1: n] ;$ real $w, y$;

integer array $z[1: n]$; integer $i, j, k, l, p$;

delta $:=1.0$

for $\mathrm{j}:=1$ step 1 until $n$ do

$z[j]:=j$;

for $i:=1$ step 1 until $n$ do

begin

$k:=i ; \quad y:=a[i, i] ; \quad l:=i-1 ; \quad p:=i+1 ;$

for $j:=p$ step 1 until $n$ do

begin

$w:=a[i, j]$

if $a b s(w)>a b s(y)$ then

begin

$k:=j$;

$y:=w$

end;

end;

della $:=$ della $\times y$;

if abs $(y)<$ epsilon then go to $A L A R M$;

$y:=1.0 / y$

for $j:=1$ step 1 until $n$ do

begin

$c[j]:=a[j, k]$

$a[j, k]:=a[j, i]$

$a[j, i]:=-c[j] \times y$

$b[j]:=a[i, j]:=a[i, j] \times y$

end; 


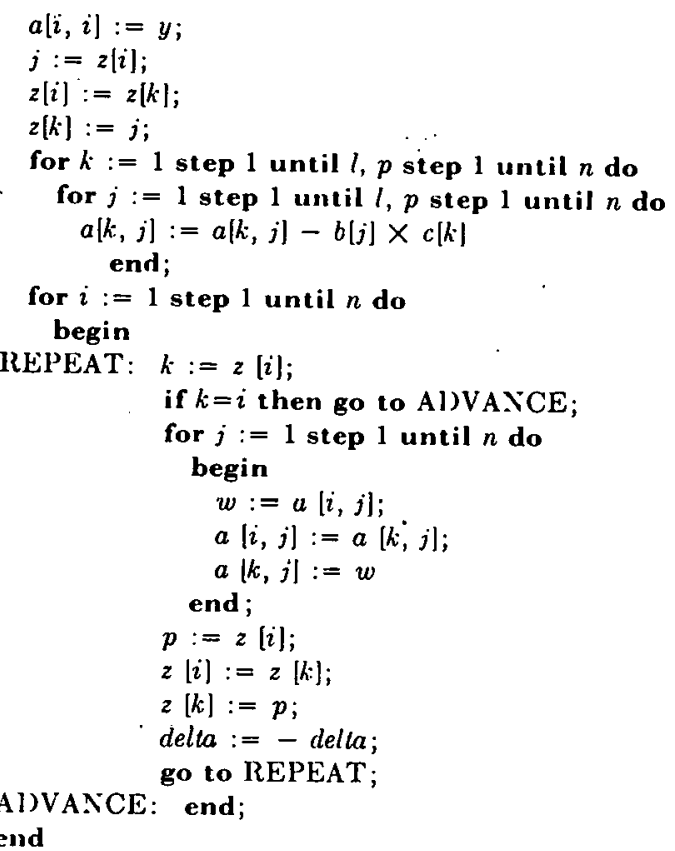

end

\section{CERTIFICATION OF ALGORITHMS 120 ANI) MATRIX INVERSION BY GAUSS-JORDAN}

INVERSION II [R. George, Comm. ACM Aug. 1962] and gjr [by H. Rutishauser, quoted by H. R. Schwarz, Comm. ACM Febr. 1962]

\section{P. NAUR}

Regnecentralen, Copenhagen, Denmark

These two procedures were compared using the Gier Algol system ( 30 bits for the normalized mantissa including sign). The following changes (in part dictated by the requirements of the compiler) were included:

INVERSION II: (1) Epsilon was included in the value part.

(2) The specification label $A L A R M$ was added.

gir: (1) The value part: value $n$, eps was inserted. (2) The second $a$ in the formal parameter part was taken out.

With these changes both procedures ran smoothly through the compiler. In order to obtain a comparison each of them was tested as follows: With a given, rather large value of epsilon the procedure was called to invert a segment of the Hilbert matrix. Upon alarm exit, the value of epsilon was divided by 10 and a fresh call was made. In this way an estimate of the largest permissible epsilon was obtained. When the inverse had been obtained, that element of it which was most in error was found through a comparison with the accurate inverse as calculated by means of INVHILBERT (Algorithm 50, see certification above). A relative error was obtained through division by the largest element of the accurate inverse.

This process was carried out for segments of the Hilbert matrix of orders 2 through 15 . For orders above 9 , the results of the inversion are dominated by errors. Below 9 we obtained the following output:

\begin{tabular}{|c|c|c|c|c|}
\hline eps & Determinant & $\begin{array}{l}\text { Ma } \\
\text { Sutscr. }\end{array}$ & $\begin{array}{c}\text { ximum error } \\
\text { Error }\end{array}$ & $\underset{\text { Relative }}{M a x i m u m ~ e r r o r ~}$ \\
\hline $10-2$ & $8.3333333_{10}-2$ & 2,2 & $2.98_{10}-8$ & $2.48_{10}-9$ \\
\hline $10-3$ & $4.6296284_{10}-4$ & 2,2 & $5.01_{10}-5$ & $2.61_{10}-7$ \\
\hline $10-4$ & $1.653431410-7$ & 3,3 & $3.0610-2$ & $4.7210-6$ \\
\hline $10-5$ & $3.7490001_{10}-12$ & 4,4 & 1.38101 & $7.7210-5$ \\
\hline $10-7$ & $5.3601875_{10}-18$ & 5,5 & 5.78103 & $1.31_{10}-3$ \\
\hline $10-8$ & $4.8485529_{10}-25$ & 5,5 & $3.70_{105}$ & $2.77_{10}-3$ \\
\hline $10-10$ & $1.5221000_{10}-33$ & 6,6 & 3.33109 & $7.8410-1$ \\
\hline
\end{tabular}

Similarly we got for gjr, and the ratio of errors of the two procedures:

\begin{tabular}{|c|c|c|c|c|c|}
\hline \multirow[b]{2}{*}{ Order } & \multirow[b]{2}{*}{ eps } & \multicolumn{3}{|c|}{ Inversion by gjr } & \multirow{2}{*}{$\begin{array}{l}\text { Ratio of errors } \\
\text { INVERSION II } \\
\text { to gjr }\end{array}$} \\
\hline & & $\begin{array}{c}\text { Max } \\
\text { Subscr. }\end{array}$ & $\begin{array}{c}\text { imum error } \\
\text { Error }\end{array}$ & $\underset{\substack{\text { IS nximum } \\
\text { Relative }}}{\text { error }}$ & \\
\hline 2 & $10-2$ & 2,1 & $2.9810-8$ & $2.4810-9$ & 1.0 \\
\hline 3 & $10-3$ & 2,2 & $2.8610-6$ & $1.49_{10-8}$ & 18 \\
\hline 4 & $10-4$ & 4,3 & $1.07_{10}-4$ & $1.65_{10}-8$ & 290 \\
\hline 5 & $10-6$ & 4,4 & 2.48 & $1.39_{10}-5$ & 5.6 \\
\hline 6 & $10-7$ & 5,5 & 4.05103 & $9.18_{10}-4$ & 1.4 \\
\hline 7 & $10-8$ & 5,5 & 4.32106 & $3.2410-2$ & .086 \\
\hline 8 & $10-10$ & 7,7 & 5.55107 & $1.31_{10}-2$ & 60 \\
\hline
\end{tabular}

Although the superiority of gjr, which searches for the pivot in both columns and rows, over INVERSION II, which only searches in the next column, is well brought out in the last culumn of the second table the behavior for $n=7$ is curious and ought to be confirmed elsewhere.

As a further test both procedures were used to invert the matrices produced by Algorithm 52, TESTMATRIX (see certification above). Again, the error of the inverse was found by a comparison with the known inverse. The comparison of the two procedures was made for orders 2 through 23 and revealed a sur. prisingly small difference of accuracy. Typical output was as follows:

$\begin{array}{cccccc}\text { Location and size of max. error } & \text { Ratio of errors } \\ \text { Order } & \begin{array}{c}\text { INVERSION II } \\ \text { Subscr. }\end{array} \text { Error } & \text { Subscr. } & { }_{\text {gjr }} & \text { Error } & \begin{array}{c}\text { INERSION II } \\ \text { bo gir }\end{array} \\ 5 & 5,5 & 8.9410-8 & 5,5 & 8.9410-8 & 1.00 \\ 10 & 10,10 & 3.7610-6 & 10,10 & 3.5210-6 & 1.07 \\ 15 & 15,15 & 2.1210-5 & 15,15 & 1.78_{10}-5 & 1.19 \\ 20 & 20,20 & 6.8110-5 & 20,20 & 6.71_{10}-5 & 1.02\end{array}$

The relative errors of the determinants calculated by INVERSION II increased slowly with $n$, reaching $2.3_{10}-7$ for $n=24$.

Typical execution times were found as follows:

\begin{tabular}{|c|c|c|c|c|}
\hline Order & \multirow{2}{*}{\multicolumn{2}{|c|}{$\begin{array}{c}\text { IN VERSION II } \\
2 \text { seconds }\end{array}$}} & \multicolumn{2}{|c|}{ gjr } \\
\hline 5 & & & & conds \\
\hline 10 & 5 & " & 8 & " \\
\hline 15 & 16 & " & 17 & " \\
\hline 20 & 53 & "“ & 57 & " \\
\hline
\end{tabular}

However, it should be noted that uwing to the automatic segmentation of the program into drum tracks in Gier Algol the execution time may vary somewhat from one program in which a procedure is used to another. The above times do not, in fact, refer to the same program.

SEE ALGORITHM 1.2

\section{ALGORITHM 121 \\ NORMDEV}

DAVID SHAFER

University of Chicago, Chicago, Ill.

procedure NormDev(Random, $A, x)$;

procedure Random; real $A, x$;

comment 'NormDev' uses (1) a procedure 'Random(y)' assumed to produce a random number, $0<y<1$, and (2) the constant $A=\operatorname{sqrt}(2 / p i) \times$ integral $[0: 1] \exp (-x \uparrow 2 / 2) d x$, to produce a positive normal deviate ' $x$ ';

begin real $y$;

$\operatorname{Random}(x) ;$ if $x>A$ then go to large;

$x:=x / A$

1: Random $(y) ;$ if $y<\exp (-x \uparrow 2 / 2)$ then go to EndND; Random $(x)$; go to 1 .

large: $\quad x:=(x-A) /(1-A)$;

2: $x:=\operatorname{sqrt}(1-2 \times \log (x))$;

Random $(y) ;$ if $y<1 / x$ then go to EndND;

$\operatorname{Random}(x)$; go to 2 ; 


\section{ALGORITHM 122}

\section{TRIDIAGONAL MATRIX}

Gerard F. Dietzel

Burroughs Corp., Pasadena, Calif.

procedure TRIDIAG $(n, A, U)$;

integer $n ; \operatorname{array} A, U$;

comment This procedure reduces a real symmetric matrix $A$ of order $n$ to tridiagonal form $(U T) A U$ (UT $=$ transpose of $U$ ) by a sequence of at most $(n-1)(n-2) / 2$ binary orthogonal transformations. Also, the matrix $U$ is calculated. [Cf. W. Givens, "Numerical computation of the characteristic values of a real symmetric matrix," Report ORNL1574 (1954), Oak Ridge Nat. Lab., Tinn., and D. E. Johansen, "A modified Givens method for the eigenvalue evaluation of large matrices," J. ACM \&, 3 (1961)];

begin real fact,c1,c2,loc1,loc $2, t e m p$; integer $i, j, j 1, j 2, j 3, j 4, n 1$; comment Set array $U=$ identity matrix of order $n$;

for $i:=1$ step 1 until $n$ do

begin

$$
\begin{aligned}
& \text { for } j:=i+1 \text { step } 1 \text { until } n \text { do } U[i, j]:=U[j, i]=0 ; \\
& U[i, i]:=1.0
\end{aligned}
$$

end:

comment The reduction of the matrix $A$ begins bere. Only the upper triangular elements of $A$ are used in the computation;

$n 1:=n-2$

for $i:=1$ step 1 until $n 1$ do

begin

$j 1:=i+1 ; \quad j 2:=i+2$

for $j:=j 2$ step 1 until $n$ do

begin

if $A[i, j]=0$ then go to $l a b$;

fact $:=1 / \operatorname{sqrt}(A[i, j 1] \uparrow 2+A[i, j] \uparrow 2)$;

$c 1:=$ fact $\times A[i, j 1] ; \quad c 2:=$ fact $\times A[i, j]$

loc1 :=A[j1,j1]; $\operatorname{loc} 2:=A[j 1, j]$;

$A[j 1, j 1]:=c 1 \uparrow 2 \times \operatorname{loc} 1+2.0 \times c 1 \times c 2 \times \operatorname{loc} 2+c 2 \uparrow 2 \times$ $A[j, j]$

$A[j 1, j]:=-c 1 \times c 2 \times l o c 1+(c 1 \uparrow 2-c 2 \uparrow 2) \times l o c 2+c 1 \times$ $c 2 \times A[j, j]$

$A[j, j]:=c 2 \uparrow 2 \times \operatorname{loc} 1-2.0 \times c 1 \times c 2 \times \operatorname{loc} 2+c 1 \uparrow 2 \times$ $A[j, j]$

$j 3:=j+1$;

for $k:=j 3$ step 1 until $n$ do

begin

temp :=A(j1,k);

$A[j \mathrm{i}, k]:=c 1 \times$ temp $+c 2 \times A[j, k]$

$A[j, k]:=-c 2 \times . t e m p+c 1 \times A[j, k]$

end;

$j 4:=j-1$

for $k:=j 2$ step 1 until $j 4$ do

begin

temp := $A[j 1, k]$;

$A[j 1, k]:=c 1 \times$ temp $+c 2 \times A[k, j]$

$A[k, j]:=-c 2 \times t e m p+c 1 \times A[k, j]$ end;

$A[i, j 1]:=c 1 \times A[i, j 1]+c 2 \times A[i, j]$

$A[i, j]:=0$;

for $k:=1$ step 1 until $n$ do

begin

temp $:=U[k, j 1]$

$U[k, j 1]:=c 1 \times t e m p+c 2 \times U[k, j] ;$

$U[k, j]:=-c 2 \times \operatorname{temp}+c 1 \times U[k, j]$ end;

lab: end

end;

for $i:=1$ step 1 until $n$ do

for $j:=i+1$ step 1 until $n$ do

$A[j, i]:=A[i, j]$

end

CIRTIFICATION ()F AIGGORITHM 122

TRIDIAGONAL MATRIX [Gerard F. Dietzel, Comm. ACM 5 (Sept. 19(i2), 482]

Peter NaUK (Recd 27 Sept. 6:3)

Regnecentralen, Copenhagen, Denmark

TRIDI $A G$ needed the following corrections:

]. Insert $k$ among the local integers to read:

integer $i, j, j 1, j 2, j 3, j 4, n 1, k$;

2. At the end of line 5 of the procedure body, insert the colon to read $U[j, i]:=0$;

3. Change the round parenthesis to a square bracket following for $k:=j 3 \cdots$ to read temp $:=A[j 1, k]$;

With these corrections the algorithm worked satisfactorily with the (iler Al.cor, system. As a test it was tried with the fullowing matrix:

HBH TESTWATRI. $[j, i]=H B H$ TESTMATRI $[i, j]$

$$
=n+1-j
$$

(cf. the Certification of Alg. 85, Comm. ACM 6 (Aug. 1963), 447). As a check the resulting matrix was rotated back again, using the resulting $U$-matrix, and the largest deviation of any element from the original was found.

For comparison the figures obtained by using the algorithms given by Wilkinson in Numerische Mauhematik 4 (1962), 354-376, may be used. Wilkinson's algorithms use Householder's method of obtaining the tridiagonal form. It should be noted that the deviations given in the table below for Householder's method refer to the final result of obtaining the eigenvalues and vectors, and not only the tridiagonal form, and thus include error contributions from a rather longer chain of calculations than the ones given for TRIDIAG. The times, however, only refer to the tridiagonalisation process in both cases.

Largest deviation

TRIDIAG,

householder tridiagonalisation

Time of execution, in Gier

Algol, seconds

TRIDIAG

householder tridiagonalisation

$$
\begin{array}{ccc}
n=5 & n=10 & n=15 \\
1.4_{10}-7 & 7.0_{10}-7 & 2.4_{10}-6 \\
& 1.4_{10}-7 & 1.3_{10}-6
\end{array}
$$

These figures clearly demonstrate the superiority of the Houselolder process. Since, in addition, the Householder method in the form given by Wilkinson uses much less storage for variables. Algorithm 122 cannot be recommended.

\section{ALGORITHM 123}

REAL ERROR FUNCTION, ERF $(x)$

Martin Crawford and Robert Techo

Georgia Institute of Technology, Atlanta, Ga.

real procedure $E r f(x)$; real $x$;

comment $\Phi(x)=\operatorname{Erf}(x)=(2 / \sqrt{\pi}) \int_{0}^{x} e^{-u^{2}} d u$ can be computed by using the recursive relation for derivatives with $\Phi^{l}(x)=$ $(2 / \sqrt{\pi}) e^{-x^{2}}$, where $\Phi^{(n)}(x)=-2 x \Phi^{(n-1)}(x)-2(n-2) \Phi^{(n-2)}(x)$, for $n=2,3, \cdots$. The Taylor's series expansions of $\Phi\left(a_{k}\right)$ are taken about $k+1$ points on the interval $0<a_{k} \leqq x$ and summed to get $\Phi(x)$;

begin real $A, U, V, W, Y, Z, T$; integer $N$;

$Z:=0 ; 1:$ if $x \neq 0$ then

begin if $0.5<a b 8(x)$ then $A:=-\operatorname{sign}(x) \times 0.5$ 


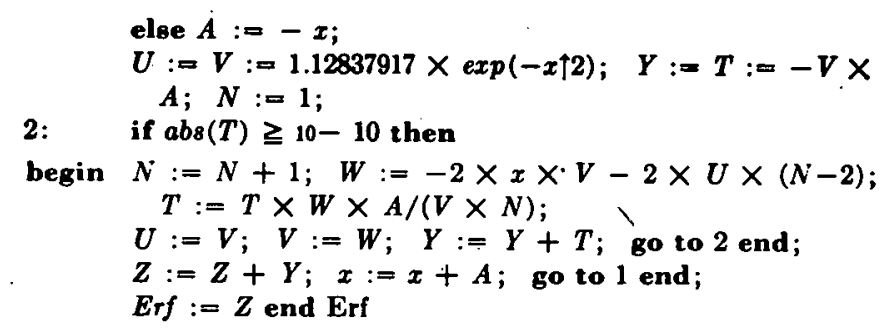

\section{CERTIFICATION OF ALGORITHM 123}

REAL ERROR FUNCTION, ERF (x) [Martin Crawford and Robert Techo, Comm. ACMI, Sept. 196\%]

Henky C. Thacher, JR.*

Argonne National Laboratory, Argonne, Ill.

* Work supported by the U. S. Atomic Energy Commission.

The body of $\operatorname{Er} f(x)$ was tested using the 1)artmouth sCALl compiler for the LGP-30. For $x=0(0.01) 0.3$, the results agreed with tabulated values to 8 in the 7 th decimal place, and for $x=$ $0.4(0.2) 1.6$ the error was less than 1 in the 6 th decimal. These results are compatible with the roundoff error in the arithmetic used. The computing time increased rapidly (by a factor of more than 10) as $x$ increased from 0.01 to 1.6 .

The following comments should be considered by users of the algorithm:

1. The parameter $x$ should he called by value, both to allow the use of expressions, and also to avoid destruction of the actual parameter.

2. The constant ${ }_{10}-10$ in statement 2 determines the accuracy of the computation. Its value should be adjusted to the arithmetic being used, and the accuracy recuired. A machine-independent test could be made by substituting if $\gamma^{*}-\gamma=\xi$ then $\cdots$.

3. For large $x$, the error function is more efficiently calculated from the Laplace continued fraction for $\operatorname{erfc}(x)$. Algorithm 180) is based on this method.

\section{REMARK ON ALGORITHM 123}

$\operatorname{ERF}(x)$ [Martin Crawford and Robert Techo, Comm. $A C M$, Sept. 1962]

D. IBBETSON

Elliott Brothers (London) Ltd.

Elstree Way, Borehamwood, Herts., England

(1) The specification value $x$; was added to allow $x$ to be an expression and to prevent side effects.

(2) The algorithm was then modified to give the Gaussian integral $(1 / \sqrt{2 \pi}) \int_{-\infty}^{x} \exp \left(-\frac{1}{2} u^{2}\right) d u$ by

(a) changing its name to Gauss (x),

(b) inserting $x:=x * 0.70710678$; immediately before $Z:=0$; , and

(c) changing the final statement to Gauss : $=(Z+1) / 2$ end Gauss

(3) The algorithm with the above changes was tested on a National Elliott 803 computer using the Elliott-ALgou translator with ${ }_{10}-8$ substituted for ${ }_{10}-10$. It was found to produce wrong answers when $x= \pm 1$ (corresponding to $\operatorname{Erf}( \pm 1 / \sqrt{ } 2)$ ) giving $0.5 \pm 0.3467899$ instead of $0.5 \pm 0.3413447$.
REMARK ON ALGORITHM 123

$\operatorname{ERF}(x)$ [Martin Crawford and Robert Techo, Comm. ACM 5 (Sept. 1962), 483; 6 (June 1963), 316; 6 (Oct. 1963), 618]

Stephen P. Barton and John F. Wagner (Recd 2 Dec. 63)

General Telephone and Electronics Laboratories, Bayside, New York

This algorithm may err when the Taylor series expands about a root of the $n$ th-order Hermite polynomial; one such error has already been noted [Remark on Algorithm 123, D. Ibbetson, Comm. ACM 6 (Oct. 1963), 618]. The difficulty springs from the Taylor-series truncation criterion, which assumes that the magnitude of successive terms in the Taylor series decreases. This is not always so, as may be seen by relating

$$
\Phi^{(n)}(x) \equiv \frac{2}{\sqrt{ } \pi} \frac{d^{n-1}}{d x^{n-1}}\left(e^{-x^{2}}\right),
$$

$(n \geqq 1)$

to the Hermite polynomial $H_{n}(x)$, which can be defined as

$$
H_{n}(x) \equiv(-1)^{n} e^{x^{2}} \frac{d^{n}}{d x^{n}}\left(e^{-x^{2}}\right)
$$

Therefore

$$
\Phi^{(n)}(x)=\frac{2}{\sqrt{ } \pi}(-1)^{n-1} e^{-x} H_{n-1}(x)
$$

As a result, $\Phi^{(n)}(x)$ vanishes when $x$ is a root of $H_{n-1}(x)$ and the Taylor series may be terminated prematurely.

The algorithm was translated into ForTran II and run on a Scientific Data Systems 910 computer (39-bit mantissa) with the following changes :

(1) The argument was decremented by 0.25 rather than 0.5

(2) The truncation criterion for abs $(T)$ was $10^{-12}$ rather than $10^{-10}$.

Errors, detected for $x=1 / \sqrt{ } 2$ and $x=2.652$, were traced to the above described premature truncation of the relevant Taylor series. These arguments correspond to the roots of $H_{2}(x)$ and $H_{7}(x)$.

The program was therefore modified to sum a fixed number of terms, with special attention to the difficulties that might arise when expanding about roots of $H_{n}(x)$. In particular, in Algorithm 123, line 9 , the coefficient, $A^{n} / n$ !, of the $n$th term in the Taylor expansion, is obtained via the intermediate step of dividing the $(n-1)$-term, $T$, by the $(n-1)$-derivative, $V$. The possibility of dividing by $V=0$ when the Taylor expansion takes place about roots of $H_{n-2}(x)$ was avoided by modifying the program to compute coefficients directly from the recursion relation,

$$
A^{n} / n !=\left[A^{n-1} /(n-1) !\right][A / n]
$$

In selecting the number of terms to be included in each Trylor series, consideration should also be given to the size of the standard decrement (specified as 0.5 in line 3 of Algorithm 123), for it is the combination of these two parameters which largely determines the accuracy and running time. A brief survey suggested that at least 10-digit accuracy could be obtained if a decrement of 0.4 were employed with 16 terms in eăch Taylor series; this resulted in an average running time of about 3.5 seconds per computation for arguments in the range $0 \leqq x \leqq 5.0$.

Reference: H. Margenau and G. M. Murphy, The Mathematics of Physics and Chemistry, pp. 119, 122. D. van Nostrand, 1943. 


\section{ALGORITHM 124 \\ HANKEL FUNCTION}

\section{Luis J. Schaefer}

Purdue University, West Lafayette, Ind.

procedure $H A N K E L(N, X, H)$; value $N, X$; integer $N$; real $X$; array $H$;

comment This procedure evaluates the complex valued hankel function of the first kind for real argument $X$ and integral order $N$ and assigns it to $H$. The individual Bessel- and Neuman-function series are not evaluated separately. Both the real and imaginary parts are generated from the same terms;

begin real $K, P, R, A, S, T, D, L$; integer $Q$; $A:=R:=1 ; H[1]:=H[2]:=S:=0$;

for $Q:=1$ gtep 1 until $N$ do begin $R:=R \times Q ; S:=S+$ $1 / Q$ end; $D:=R / N$

$R:=1 / R ; \quad K:=X \times X / 4 ; \quad P:=(X / 2) \uparrow N ; \quad T:=\ln (K)+$ 1.1544313298631 ;

for $Q:=0, Q+1$ while $Q \leqq N \vee L \neq H[2]$ do

begin $L:=H[2] ; H[1]:=H[1]+A \times K \times R$; $H[2]:=H[2]+A \times(R \times K \times(T-S)-($ if $Q<N$ then $D / P$ else 0));

$A:=A \times K / Q ; . R:=-R /(Q+N) ; \quad S:=S+1 / Q+1 /(Q+N) ;$

if $Q<N$ then $D:=D /(N-Q)$

end; $H[2]:=H[2] \times .31830989$

end

\section{ALGORITHM 125}

\section{WEIGHTCOEFF}

H. RuTishauser

Eidg. Technische Hochschule, Zurich, Switzerland

procedure weightcoeff $(n, q, e, e p s, w, x)$; value' $n$; , real eps;

integer $n$; array $q, e, w, x$;

comment Computes abscissae $x_{i}$ and weight coefficients $w_{i}$ for a Gaussian quadrature method $\int_{0}^{b} w(x) f(x) d x \approx \sum_{i=1}^{n} w_{i} f\left(x_{i}\right)$, where $\int_{0}^{b} w(x) d x=1$ and $w(x) \geqq 0$. The method requires the order $n, \mathbf{a}$ tolerance eps and the $2 n-1$ first coefficients of the continued fraction

$$
\int_{0}^{b} \frac{w(x)}{z-x} d x=\frac{1}{\mid z}-\frac{q_{1} \mid}{\mid 1}-\frac{e_{1} \mid}{\mid z}-\frac{g_{2} \mid}{\mid 1}-\frac{e_{2} \mid}{\mid z}-\cdots
$$

to be given, the latter as two arrays $q[1: n]$ and $e[1: n-1]$ all components of which are automatically positive by virtue of the condition $w(x) \geqq 0$. The method works as well if the upper bound $b$ is actually infinity (note that $b$ does not appear directly as parameter!) or if the density ${ }^{o} w(x) d x$ is replaced by $d \alpha(x)$ with a monotonically increasing $\alpha(x)$ with at least $n$ points of variation. The tolerance eps should be given in accordance to the machine accuracy, e.g. as $10-10$ for a computer with a ten-digit mantissa. The result is delivered as two arrays $w[1: n]$ (the weight coefficients) and $x[1: n]$ (the abscissae). For a description of the method see H. Rutishauser, "On a modification of the QD-algorithm with Graeffe-type convergence" [Proceedings of the IFIPS Congress, Munich, 1962].;

begin

integer $k$;

Boolean test;

real $m, p$

array $g[1: n]$ procedure red $(a, f, n)$; value $n$; integer $n$; array $a, f$; comment subprocedure red reduces a heptadiagonal matrix $a$ to tridiagonal form as described in the paper loc. cit. Since the bulk of the computing time of the whole method is spent in this subprocedure, it would pay to write it in machine code.;

begin

real $c$; integer $j, k$;

for $k:=1$ step 1 until $n-1$ do

begin

for $j:=k$ step 1 until $n-1$ do

begin

$c:=-f[j] \times a[j, 7] / a[j, 2]$

$a[j, 7]:=0$;

$a[j+1,2]:=a[j+1,2]+c \times a[j, 5]$

$a[j, 1]:=a[j, 1]-c \times f[j] \times a[j, 4] ;$

$a[j, 6]:=a[j, 6]-c \times a[j+1,1]$;

$a[j+1,3]:=a[j+1,3]-c \times a[j+1,6]$;

end $j$;

for $j:=k$ step 1 until $n-1$ do

begin

$c:=-f[j] \times a[j, 4] / a[j, 1]$

$a|j, 4|:=0$

$a[j+1,1]:=a[j+1,1]+c \times a[j, 6] ;$

$a[j+1,6]:=a[j+1,6]+c \times a[j+1,3]$

$a[j, 5]:=a[j, 5]-c \times a[j+1,2]$;

$a[j+1,0]:=a[j+1,0]-c \times a[j+1,5]$

end $j$;

for $j:=k+1$ step 1 until $n-1$ do

begin

$c:=-a[j, 3] / a[j-1,6]$;

$a[j, 3]:=0$

$a[j, 6]:=a[j, 6]+c \times a[j, 1]$;

$a[j-1,5]:=a[j-1,5]-c \times f[j] \times f[j] \times a[j, 0] ;$

$a[j, 2]:=a[j, 2]-c \times f[j] \times f[j] \times a[j, 5]$

$a[j, 7]:=a[j, 7]-c \times f[j] \times a[j+1,2]$;

end $j$;

for $j:=k+1$ step 1 until $n-1$ do

begin

$c:=-a[j, 0] / a[j-1,5]$;

$a[j, 0]:=0$;

$a[j+1,2]:=a[j+1,2]+c \times f[j] \times a[j, 7]$

$a[j, 5]:=a[j, 5]+c \times a[j, 2]$

$a[j, 1]:=a[j, 1]-c \times f[j] \times f[j] \times a[j, 6]$

$a[j, 4]:=a[j, 4]-c \times f[j] \times a[j+1,1] ;$

end $j$;

end $k$;

end red;

procedure qdgraefe $(n, h, g, f)$; value $n$;

integer $n$; array $h, g, f$;

comment Subprocedure qdgraeffe computes for a given finite continued fraction

$$
f(z)=\frac{1}{\mid z}-\frac{q_{1} \mid}{\mid 1}-\frac{e_{1} \mid}{\mid z}-\frac{q_{2} \mid}{\mid 1}-\cdots-\frac{q_{n} \mid}{\mid 1}
$$

another one, the poles of which are the squares of the poles of $f(z)$. However qdgraeffe uses not the coefficients $\dot{q}_{1}, \cdots, q_{n}$ and $e_{1}, \cdots, e_{n-1}$ of $f(z)$, but the quotients

$$
\left\{\begin{array}{l}
f_{k}=q_{k+1} / q_{k} \\
g_{k}=e_{k} / q_{k+1}
\end{array}\right\} \quad(k:=1,2, \cdots, n-1)
$$

and the $h_{k}=\ln \left(a b s\left(q_{k}\right)\right) \quad(k:=1,2, \cdots, n)$, and the results are delivered in the same form. Procedure qdgraeffe can be used independently, but requires subprocedure red above; 


\section{begin}

integer $k$; array $a[0: n, 0: 7]$

$g[n]:=f[n]:=0$

for $k:=1$ step 1 until $n$ do

begin

$a[k-1,4]:=a[k-1,5]:=1$

$a[k, 1]:=a[k, 2]:=1+g[k] \times f[k] ;$

$a[k, 6]:=a[k, 7]:=g[k]$;

$a[k, 0]:=a[k, 3]:=0$;

comment The array $a$ represents the heptadiagonal matrix $Q$ of the paper loc. cit., but with the modifications needed to avoid the large numbers and with a peculiar arrangement.;

end $k$;

$a[n, 5]:=0$;

$\operatorname{red}(a, f, n)$

for $k:=1$ step 1 until $n$ do

$h[k]:=2 \times h[k]+\ln (a b s(a[k, 1] \times a[k, 2])) ;$

comment A saving might be achieved by economizing the log-computation in the range $.8 \leqq x \leqq 1.2$;

for $k:=1$ step 1 until $n-1$ do

hegin

$f[k]:=f[k] \times f[k] \times a[k+1,2] \times a[k+1,1] /(a[k, 1] \times a[k, 2]) ;$ $g[k]:=a[k, 5] \times a[k, 6] /(a[k+1,1] \times a[k+1,2])$

end $k$;

end yulyaeffe;

$L 1: \quad x[1]:=q[1]+e[1]$

for $k:=2$ step 1 until $n$ do

begin

$g[k-1]:=e[k-1] \times q[k \mid / x[k-1]$

$x[k]:=q[k]+($ if $k=n$ then 0 else $e[k])-g[k-1]$;.

$g[k-1]:=g[k-1] / x[k]$;

$w[k-1]:=x[k] / x[k-1]$;

$x[k-1]:=\ln (x|k-1|)$;

end $k$;

$x[n]:=\ln (x[n])$;

L2: $p:=1$;

$L 25$ : begin

test $:=$ true

for $k:=1$ step 1 until $n-1$ do

test $:=$ test $\wedge$ abs $(g[k] \times w[k])<$ eps;

if test then go to $L 3$;

qdgraeffe $(n, x, g, w)$;

end;

. $p:=2 \times p$;

go to $L 25$;

comment What follows is a peculiar method to compute the $w_{k}$ from given ratios $g_{k}=w_{k+1} / w_{k}$ such that $\sum_{k-1}^{n} w_{k}=1$, but the straightforward formulae to do this might well produce overflow of exponent.;

$L, 3: \quad w[1]:=m:=0$;

for $k:=1$ step 1 until $n-1$ do

begin

$w[k+1]:=w[k]+\ln (g[k])$

if $w[k]>m$ then $m:=w[k]$;

end $k$;

for $k:=1$ step 1 until $n$ do $w[k]:=\exp (w[k]-m)$;

$m:=0$;

for $k:=1$ step 1 until $n$ do $m:=m+w[k]$;

for $k:=1$ step 1 until $n$ do begin $w[k]:=w[k] / m$; $x[k]:=\exp (x[k] / p)$ end;

end weightcoeff

\section{ALGORITHM 126 \\ GAUSS' METHOD \\ JAY W. Counts \\ University of Missouri, Columbia, Mo.}

procedure gauss $(u, a, y)$;

real array $a, y$; integer $u$;

comment This procedure is for solving a system of linear equations by successive elimination of the unknowns. The augmented matrix is $a$ and $u$ is the number of unknowns. The solution vector is $y$. If the system hasn't any solution or many solutions, this is indicated by the go to error where error is a label outside the procedure.;

begin

integer $i, j, k, m, n$;

$n:=0$;

cho: $n:=n+1$;

for $k:=n$ step 1 until $u$ do if $a[k, n] \neq 0$ then go to $c k 1$; go to error;

ck1: if $k=n$ then go to $c k 2$;

for $m:=n$ step 1 until $u+1$ do

begin end;

$\operatorname{temp}:=a[n, m] ; \quad a[n, m]:=a[k, m] ; \quad a[k, m]:=$ temp

ck2: for $j:=u+1$ step $\because 1$ until $n$ do $a[n, j]:=a[n, j] / a[n, n]$;

for $i:=k+1$ step 1 until $u$ do

for $j:=n+1$ step 1 until $u+1$ do

$a[i, j]:=a[i, j]-a[i, n] \times a[n, j]$;

if $n \neq u$ then go to $c k 0$;

for $i:=u$ step -1 until 1 do

begin

$y[i]:=a[i, u+1] / a[i, i]$

for $k:=i-1$ step -1 until I do

$a[k, u+1]:=a[k, u+1]-a[k, i] \times y[i]$

end end;

\section{ALGORITHM 127 \\ ORTHO}

Philip J. Walsh

National Bureau of Standards, Washington, D. C.

procedure $O R T H O(W, Y, Z, n, f n, m, p, r, a i, a u i, m u i, z e i, X, D E V$, $C O F, S T D, C V, V C V, g m d l, Q, Q 2, E, E P, A, G F, E N F)$;

value $n, m, p, r, a i, a u i, m u i, z e i$;

real $f n, g m d t$;

array $W, Y, Z, X, D E V, C O F, S T D, C V, V C V, Q, Q 2, E, E P, A, G F, E N F$; integer $n, m, p, r, a i, a u i, z e i, m u i$;

switch at $:=a t 1, a t 2 ;$ switch $z e:=z e 1, z e 2$;

switch $a u:=a u 1, a u 2 ;$ switch $m u:=m u 1, m u 2, m u 3$;

comment ORTHO is a general purpose procedure which is capable of solving a wide variety of problems. For a detailed discussion of the applications listed below and other applications, see (1) Philip Davis and Philip Rabinowitz, "A Multiple Purpose Orthonormalizing Code and Its Uses," J. ACM 1 (1954), 183-191, (2) Philip Davis, "Orthonormalizing Codes in Numerical Analysis," in J. Todd (Ed.), A Survey of Numerical Analysis, Ch. 10 (McGraw-Hill, 1962), (3) Philip Davis and Philip Rabinowitz, "Advances in Orthonormalizing Computation," in F. L. Alt (Ed.), Advances in Computers, Vol. 2, pp. 55133 (Academic Press, 1961), (4) Philip J. Walsh and Emilie V. Haynsworth, General Purpose Orthonormalizing Code, SHARE Abstr. $\$ 850$. Applications: (a) orthonormalizing a set. of 
vectors with respect to a general inner product, (b) least squares approximation to given functions by polynomial approximations or any linear combination of powers, rational functions, transcendental functions and special functions, such as those defined numerically by a set of values, (c) curve fitting of empirical data in two or more dimensions, (d) finding the best solution in the 1.s.s. to a system of $m$ linear equations in $n$ unknowns $(n \leqq m)$, (e) matrix inversion and solution of linear systems of equations, (f) expansion of functions in a series of orthogonal functions, such as a series of Legendre or Chebyshev polynomials.

The following information must be supplied to the procedure. (We are considering here the approximation feature of the procedure.)

$n$ the number of components per vector (exsluding augmentation)

$m$ the number of vectors used in the approximation. For a polynomial fit of degree $t$, set $m=t+1$.

$p$ the number of augmented components per vector. A feature of this procedure is that once the approximating vectors have been orthonormalized, they may be used in approximating $r$ functions without repeating the orthonormalization procedure on the original appruximating vecluirs.

r the number of functions to be approximated.

ai a switch control concerning the approximating vectors. With $a i=1$, the procedure selects the first $n$ components of the first row of $[Z]$, supplied by user. The $i$ powers of these values are computed and stored into working location $[X], i=0(1) m-1$. This is the usual set up for a poly. nomial fit. With $a i=2$, the procedure selects the first $n$ components of the first $m$ rows of $[Z]$ supplied by user and stores them into working location $[X]$.

aui a switch control concerning augmentation on the approximating vectors. If $p=0$, this switch is ignored. With $a u i=1$, regular augmentation is applied to the vectors in $[X]$. $p$ zeros are stored after the $n$th component of the first $m$ rows of $[X]$. The $(n+i)$ th component is replaced by $1.0, i=1(1) m$. With aui=2, special augmentation is applied to the vectors in $[X]$. The $p$ components located after the $n$th component of the first $m$ rows of $[Z]$ supplied by the user augment $[X]$.

zei a switch control concerning augmentation on the functions to be approximated. If $r=0$, this switch is ignored. With $z e i=1$, regular augmentation is applied to the functions during the calculation. The $n$ components of the first $r$ rows of $[Y]$ supplied by user will be augmented hy $p$ zeros when moving $[Y]$ to $[X]$. With $z e i=2$, special augmentation is applied. The first $n$ components of the first $r$ rows of $[Y]$ are the functional values supplied by user. The next $p$ components of the first $r$ rows of $[Y]$ are special values also supplied by user.

mui a switch control concerning weights. $[W]$ is an $n \times n$ real, positive definite, symmetric matrix of weights. It is gen: erally diagonal and often the Identity matrix. mui=1 when $[W]=I_{n}$, the matrix $[W]$ need not be supplied. $m u i=2$ when $[W]$ is diagonal, but not $I_{n}$. The procedure is supplied the $n$ diagonal elements of $[W]$, but stored in the first row of matrix $[W] . \quad m u i=3$ when the full weighting matrix is supplied to the procedure.

The following list of matrix arrays is given to aid the user in determining the number of components and vectors in the input and results. $W[1: n, 1: n], \quad Y[1: r, 1: n+p], \quad Z[1: m, 1: n+p]$, $X[1: m+1,1: n+p], \quad D E V[1: r, 1: n], \quad C O F[1: r, 1: p], \quad S T D[1: r]$, $C V[1: p+1,1: p], \quad V C V[1: r, 1: p+1,1: p], \quad Q[1: r, 1: m+1], Q 2, E$, $E P[1: r, 1: m], A[1: m, 1: p], G F[1: m+r], E N F[1: m]$.

The results of the procedure are stored in the following locations. The user must be sufficiently familiar with the theory to know which results are relevant to his application of the procedure. All vectors are stored row-wise in the matrices listed below.
$X \quad$ orthonormal vectors

$D E V$ deviations

COF coefficients

STD standard deviations

$C V$ covariance matrix, stored in upper triangular form.

The $(p+1)$ st row contains the square root of the diagonal elements of the matrix.

$V C V$ variance-covariance matrices, stored in upper triangular form with the $(p+1)$ st rows containing the square root of the diagonal elements. There are $r$ such matrices, the first subscript running over the $r$ values.

gmdl Gram determinant value

$Q \quad$ Fourier coefficients

Q2 squared Fourier coefficients

$E \quad$ sum of the squared residuals

$E P$ residuals

$A$ a lower triangular matrix used to calculate the covariance matrix. $C V=A^{\prime} A$.

GF Gram factors

$E N F$ norms of the approximating vectors;

begin

integer $n p p, n p m, m 1, n 2, m 2, r 1, r b a r, p 2, b e i, r h i, i 18, g a i, s i i, i$. $j$, dei, mui, elz1, elz2, $k$, thi, ali, omi, nii;

array $P K, X P[1: n+p], Q K[1: m+1]$;

real denom,sum, $d k 2, d k, f i, s s, s s q$;

switch $b e:=b e 1$, be2; switch $r h:=r h 1$, rh 2 ; switch $g a:=$ ga1,ga2;

switch $s i:=\operatorname{sil}$,si2; switch $d e:=\operatorname{de1,de2;}$ switch $n u:=$ $n u 1, n u 2$;

switch $t h:=t h 1, t h 2, t h 3 ;$ switch al $:=$ al $1, a l 2$;

switch om $:=$ om 1 ,om 2 ;

$n p p:=n+p ; n p m:=n+m ; m 1:=m-1 ; n 2:=n+1 ; m 2:=m+1$;

$r 1:=0 ; \quad$ rbar $:=r ; \quad p 2:=p+1 ;$ denom $:=$ if $n=m$ then 1.0

else $\operatorname{sqrt}(n-m) ;$ bei $:=\operatorname{rhi}:=i 18:=1$;

if $(p \neq 0)$ then gai $:=s i i:=2$ else $g a i:=s i i:=1$;

boxl : go to at [ai $]$;

at1 : for $j:=1$ step 1 until $n$ do begin

$X(2, j):=Z(1, j) ; X[1, j]:=1.0$ end;

for $i:=2$ step 1 until $m 1$ do begin

for $j:=1$ step 1 until $n$ do

$X[i+1, j]:=X[i, j] \times X[2, j]$ end; go to box 2 ;

at2: for $i:=1$ step 1 until $m$ do begin

for $j:=1$ step 1 until $n$ do

$X[i, j]:=Z[i, j]$ end;

box2: if $p=0$ then go to box 3 else go to $a u[a u i]$;

aul: for $i:=1$ step 1 until $m$ do begin

for $j:=n 2$ step 1 until $n p p$ do

$X[i, j]:=0.0 ; \quad X[i, n+i]:=1.0$ end; go to box 3 ;

au2: for $i:=1$ step 1 until $m$ do begin

for $j:=n 2$ step 1 until $n p p$ do

$X[i, j]:=Z[i, j]$ end;

box3: $\quad$ dei $:=n u i:=e 1 z 1:=e 1 z 2:=k:=1$;

box4: $\quad$ thi $:=1$;

box5: ali $:=$ omi $:=1$; if $p=0$ then go to box 6 else

for $j:=1$ step 1 until $p$ do $P K[n+j]:=0.0$;

box6: go to $m u[m u i]$;

$m u 1$ : for $i:=1$ step 1 until $n$ do $P K[i]:=X[k, i]$; go to $b o x 7$;

$m u 2: \quad$ for $i:=1$ step 1 until $n$ do

$P K[i]:=X[k, i] \times W[1, i]$; go to $b o x 7$;

$m u 3:$ for $i:=1$ step 1 until $n$ do begin sum $:=0.0$;

for $j:=1$ step 1 until $n$ do sum $:=\operatorname{sum}+X[k, j] \times$ $W[i, j] ; \quad P K[i]:=$ sum end;

box 7: go to om[omi];

om 1: for $i:=1$ step 1 until $k$ do begin sum $:=0.0$;

for $j:=1$ stepp 1 until $n p p$ do

sum $:=\operatorname{sum}+P K[j] \times X[i, j] ; \quad Q K[i]:=$ sum end; go to $b o x 8$; 
om2: $\quad d k 2:=0.0 ;$ for $i:=1$ step 1 until npp do

$d k 2:=d k 2+P K[i] \times X[k, i]$;

$d k:=\operatorname{sgrl}(d k 2)$;

$G F[i 18]:=d k ; \quad i 18:=i 18+1$;

for $i:=1$ step 1 until $n p p$ do

$X[k, i]:=X[k, i] / d k$;

omi $:=1$; go to box 6 ;

box8: go to de $\{d e i\}$;

de1: $\quad e 1 z 1:=-e 1 z 1$; if $e 1 z 1<0$ then go to $b o x 8 b$ else go to box $8 a$;

box $8 a$ : for $i:=1$ step 1 until $k-1$ do

$Q K[i]:=-Q K[i] ; \quad Q K[k]:=1.0$;

for $i:=1$ step 1 until $n p p$ do begin

sum $:=0.0 ;$ for $j:=1$ step 1 until $k$ do

sum $:=\operatorname{sum}+X[j, i] \times Q K[j] ;$

$X P[i]:=$ sum end; go to box 9 ;

box8b: ENF $[i 18]:=\operatorname{sqr} t(Q K[k]) ;$ go to box $8 a$;

de2: $\quad e 1 z 2:=-e 1 z 2 ;$ if $e 1 z 2<0$ then go to $b o x 8 c$ else go to box $8 a$;

boxsc: for $i:=1$ step 1 until $m$ do begin

$Q[r 1, i]:=Q K[i] ; \quad Q 2[r 1, i]:=Q K[i] \times Q K[i]$ end;

$Q[r 1, m 2]:=Q K[m 2] ; \quad E[r 1,1]:=Q[r 1, m 2]-Q 2[r 1,1] ;$

for $j:=2$ step 1 until $m$ do

$E[r 1, j]:=E[r 1, j-1]-Q 2[r 1, j]$;

fi $:=1.0$;

for $i:=1$ step 1 until $m$ do begin

if $(f n-f i)>0.0$ then begin if $E[r 1, i]<0.0$ then begin

$E P[r 1, i]:=-\operatorname{sgrt}(a b s(E[r 1, i]) /(f n-f i)) ;$ go to boxsd end

else $E P[r 1, i]:=\operatorname{sqrt}(E[r 1, i] /(f n-f i))$;

go to box $8 d$; end else $E[r 1, i]:=-1.0$

box $8 d: \quad f i:=f i+1.0$; end go to $b o x 8 a$;

box 9 : go to th $[$ thi];

thl : for $i:=1$ step 1 until $n p p$ do

$X[k, i]:=X P[i]$; go to box 10 ;

th2: $\quad$ for $i:=1$ step 1 until $n$ do

$D E V[r 1, i]:=X P[i]$;

for $i:=1$ step 1 until $p$ do

$\operatorname{COF}[r 1, i]:=-X P[n+i] ;$ thi $:=3$; go to th1;

th3: go to box11;

box10: go to allali];

al1: omi $:=$ ali $:=2$; go to boxt;

al2: if $k<m$ then begin $k:=k+1$; go to box4; end else go to boxi2;

box11: go to $n u[n u i]$;

nu1: $n u i:=2$; go to box14;

nu2: $\quad$ ss $:=d k /$ denom; $88 q:=88 \times 88$

$S T D[r 1]:=88$; go to box 14 ;

box12: go to be[bei];

be1 : for $i:=1$ step 1 until $m$ do begin

for $j:=1$ step 1 until $p$ do

$A[i, j]:=X[i, n+j]$ end;

gmd $t:=1.0 ;$ for $i:=1$ step 1 until $m$ do

gmd $:=g m d t \times(G F[i] / E N F[i])$;

gmdt $:=$ gmdt $\times$ gmdt $;$ dei $:=$ bei $:=$ thi $:=2$;

$k:=k+1$; go to box 13 ;

be2: go to box11;

box13: go to galgai];

gal: go to box 11;

ga2: for $i:=1$ step 1 until $p$ do begin

for $j:=i$ step 1 until $p$ do begin

sum $:=0.0$;

for $n i i:=1$ step 1 until $m$ do

sum $:=$ sum $+A[n i i, i] \times A[n i i, j]$;

$C V[i, j]:=$ sum end end;

for $i:=1$ step 1 until $p$ do

$C V[p 2, i]:=\operatorname{sqrt}(C V[i, i]) ; g a i:=1$; go to box 11 ;

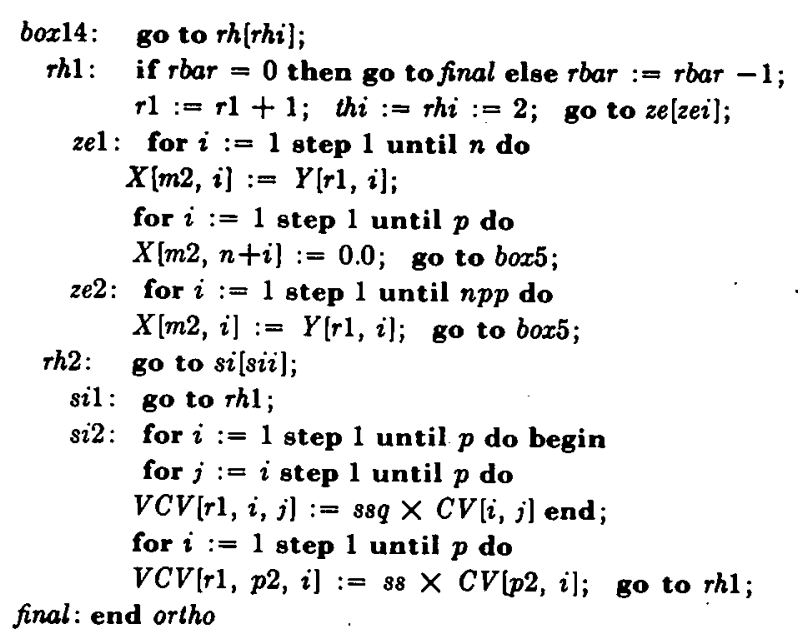

ALGORITHM 128

SUMMATION OF FOURIER SERIES

M. WELLS

University of Leeds, Leeds 2, England*

* Currently with Burroughs Corp:, Pasadena, Calif.

procedure Fourier $(X, r, w, n, A, B)$;

value $n$; real $X, w, A, B$; integer $r, n$;

comment Fourier sums a one-dimensional Fourier series, using a recurrence relation described by Watt [Computer $J .1,4$ (1959) 162]. The parameters are the coefficients $X$, which are selected by $r, w$, the argument and $n$ the total number of terms in the series. On exit $A=\sum_{r=0}^{n-1} X_{r} \cos (r w)$ and $B=\sum_{r=0}^{n-1} X_{r} \sin (r w)$. Fourier is particularly efficient where $X_{r}=0$ for all $r>$ some $r_{1}$ and $X_{r} \neq 0$ for all $r \leqq r_{1}$;; begin real $t, t r, t r 1, \cos w 2$;

trl $:=0 ; \quad \cos w 2:=2 \times \cos (w)$;

for $r:=n-1$ step -1 until 0 do

begin if $X \neq 0$ then go to term end search for nonzero term:

tr $:=0$; go to all zeroes;

term: $t r:=X$; for $r:=r-1$ step -1 until 0 do

begin $t:=\operatorname{tr} \times \cos w 2+X-t r 1 ; \operatorname{tr} 1:=t r ; t r:=t$ end reourrence;

all zeros: $A:=t r-t r 1 \times \cos w 2 / 2 ; B:=t r 1 \times \sin (w)$

end Fourier series

\section{CERTIFICATION OF ALGORITHM 128 [C6]}

SUMMATION OF FOURIER SERIES [M. Wells, Comm. ACM 5 (Oct. 1962), 513]

Henry C. Thacher, Jr.* (Recd. 18 Mar. 1964)

Argonne National Lab., Argonne, Ill.

* Work supported by the U.S. Atomic Energy Commission

The body of Fourier was transcribed for the Dartmouth SCALP translator for the LGP-30 computer. After uniformizing the spelling of zeros (lines 5 and 9 in the procedure body), the program compiled and ran without difficulty.

In the procedure statement for Fourier, the actual parameter corresponding to $X$ should be an expression depending on the actual parameter corresponding to $r$.

The ScAlp program was tested for the finite series: 


$$
\begin{aligned}
& A=\sum_{r=0}^{n-1} \cos r w=\frac{\sin ((n-1) w / 2)}{\sin (w / 2)} \cos (n w / 2)+1 \\
& B=\sum_{r=0}^{n-1} \sin r w=\frac{\sin ((n-1) w / 2)}{\sin (w / 2)} \sin (n w / 2)
\end{aligned}
$$

for $w=0.1,0.2,0.5$ and 1.0 , and for $n=1(1) 51$ : Although the algorithm appears to be numerically correct, the results showed evidence of serious numerical instability, particularly for small values of $w$. For $w=0.1$, and $n=51$, the error in $A$ was .00109 , and in $B,-.00231$. Since the largest $A$ for $n<51$ is 10.5, and the largest $B$ about 20 , the best result obtainable with the $7+$ significomparison, a program summing the same series using a forward recurrence based on the addition formulas for the sine and cosine gave errors of .00012 and -.00018 . It was, however, only about half as fast. cant digit arithmetic of the SCALP system is about .00001 . For

comment start looking for a minimum at midpoint of region; for $j:=1$ step 1 until $n$ do

begin $w n e w[j]:=(l 1[j]+b 1[j]) / 2 ; x t[j]:=w n e w[j]$ $x u b[j]:=t 1[j] ; \quad x 1 b[j]:=b 1[j] ; \operatorname{del} x[j]:=(x u b[j]$ $-x 1 b(j]) / 5$

end;

$$
d 12 x[j]:=\operatorname{de} 1 x[j] \uparrow 2 ; \quad x \min [j]:=x t[j]
$$

$f \min :=\operatorname{GFUN}(x \min )$;

for $j:=1$ step 1 until $n$ do

begin $w:=x t[j]$; for $i:=1$ step 1 until 4 do

begin $x[j, i]:=x 1 b[j]+i \times \operatorname{del} x[j]$; $x t[j]:=x[j, i] ; \quad g[j, i]:=\operatorname{GFUN}(x t)$;

end;

$x t[j]:=w$

$d x \min [j]:=(g[j, 3]-g[j, 2]) / d e 1 x[j]$

$d 2 x m n[j]:=(g[j, 4]-g[j, 3]-g[j, 2]+g[j, 1]) / d 12 x[j]$

end;

comment first and second difference quotients have been computed;

icnt $:=0 ; \quad d \max :=d x \min [1] ; \quad k:=1$;

nustep: for $j:=2$ step 1 until $n$ do

begin if $a b s(d \max )<a b s(d x m n[j])$ then

ALGORITHM 129

MINIFUN

V. W. WHITLEY

Signal Missile Support Agency, White Sands Missile Range, N. Mex.

procedure MINIFUN ( $l 1, b 1$, eps, $n, n c n t, f \min , x \min , k 1$, GFUN);

value $t 1, b 1$, eps, $n, n c n t$; integer $n, n c n t, k 1$; real fmin; real procedure $G F U N$; array $t 1, b 1$, eps, xmin;

comment MINIFUN is a subroutine to find the minimum of a function of $n$ variables, using the method of steepest descent. Input is:

1. $\quad l(i), \quad i=1,2, \cdots, n$, the upper limits of the search region

2. $b 1(i), i=1,2, \cdots, n$, the lower limits of the search region

3. eps $(i), i=1,2, \cdots, n$, the convergence criteris. The function must be a minimun in the region $|x(i)-x \min (i)|$ $\leqq e p_{s}(i)$

4. n, the number of variables (the dimension of the arrays)

5. nent, the maximum number of iterations. The routine searches for a minimum until $|x(i)-x \min (i)| \leqq e p s(i)$ for all $i$, or until $i c n t=n c n t$, whichever happens first.

Output is:

1. $f \min$, the minimum value of the function

2. $x \min (i) ; i=1, \cdots, n$, the point at which the minimum. occurs

3. $k 1$, an error code

If $k 1=1$, a minimum has heen found within the specificl number of iterations ancl the minimum is less than all values of the function at the centers of the planes forming the boundary of the epsilon-cube

If $k 1=2, \Delta x(i) \leqq e p s(i)$ but a new minimum has been found

If $k 1=3, n c n t$ has been exceeded withoit $\Delta x(i) \leqq e p s(i)$. In this case, a test is made to see if the current minimum is a minimum in the epsilon-cube.

MINIFUN has been written as a. ForTran II subroutine and is available from the SMSA Computation Center. It should be noted that the Fortran II deck has been tested only on some relatively simple functions of two variables, such as GFUN $(x, y)=\cos (x y)$. The writer does not claim that the algorithm has been thoroughly tested;

begin integer $j, i$, icnt $k$; real $w, d \max , \operatorname{alamb}, f t$; array wnew $[1: n], x t[1: n], x 1 b[1: n], x u b[1: n]$, $\operatorname{de} 1 x[1: n], d 12 x[1: n], x \min [1: n], x[1: n, 1: 4], g[1: n, 1: 4]$, $d x \min [1: n], d 2 x m n[1: n]$;

\section{begin $d \max :=d x \min [j] ; \quad k:=j$}

end;

end ;

alamb $:=d x \min [k] / d 2 x m n[k] ; \quad w:=x t[k]-a 1 a m b ;$

comment a new coordinatc has been computed for the variable having the largest first partial derivative. It will be checked to see if the new point still lies within the region and search will continue;

if $w<b 1[k]$ then $w:=b 1[k]$ else if $w>t 1[k]$ then $w:=t 1[k]$;

$x t[k]:=w ; f t:=\operatorname{GFUN}(x t)$;

if $f t<f \min$ then go to check else

restart: if $x \ell[k]<w n e w[k]$ then go to $1 b d c h k$

else if $x t[k]=w n e w[k]$ then go to stnubds

else if $t[k]>\cdot x t[k]$ then go to nupbds

else $x t[k]:=1.5 \times$ wnew $|k|$;

nupbds: $x u b \mid k]:=t 1[k] ; \quad x 1 b[k]:=2 \times x t[k]-t 1[k]$; go to newdel ;

stnubls: $x 1 b[k]:=x t[k]-0.5 \times$ wnew $[k] ; x u b[k]:=x t[k]+$ $0.5 \times$ wnew $[k]$;

newde1: $\quad \operatorname{de} 1 x[k]:=0.2 \times(x u b[k]-x 1 b[k]) ; d 12 x[k]:=\operatorname{del} x \mid k] \uparrow 2 ;$

for $i:=1$ step 1 until 4 do

begin $x[k, i]:=x 1 b[k]+i \times d e 1 x[k] ; \quad w:=x t[k]$; $x t[k]:=x[k, i] ; \quad g[k, i]:=\operatorname{GFUN}(x l) ; \quad x t[k]:=w$

end;

$d x \min [k]:=(g[k, 3]-g[k, 2]) / \operatorname{del} x[k] ;$

$d 2 x m n[k]:=(g[k, 4]-g[k, 3]-g \mid k, 2]+g[k, 1]) / d 12 x[k] ;$

icnt $:=i c n t+1$;

if $i c n t>$ ncnt then go to outcd else go to nustep;

$1 b d c h k: \quad$ if $x t[\mathrm{k}] \leqq b 1[k]$ then $x t[k]:=0.5 \times$ wnew $[k]$

else $x 1 b[k]:=b 1[k] ; \quad x u b[k]:=2.0 \times x t[k]-b 1[k] ;$

go to newdel ;

check: $f \min :=f t ; x \min [k]:=x t \mid k\}$;

for $j:=1$ step 1 until $n$ do if $d e l x[j]>$ eps $[j]$ then go to reslart; rechecl: for $j:=1$ step 1 until $n$ do

begin $w:=x \min [j] ; x \min [j]:=w+\operatorname{eps}[j] ; f t:=G F U N$ (xmin);

if $f t<f m i n$ then go to $\operatorname{set} 2 ; \quad x \min [j]:=w-\operatorname{eps}[j]$;

$f t:=G F U N(x \min ) ; \quad$ if $f t<f \min$ then go to $\operatorname{set} 2 ; x \min [j]$ $:=w$

end;

if $k 1<3$ then $k 1:=1$; go to bgend;

8et2: $k 1:=2$; go to bgend;

outcd: $k 1:=3$; go to recheck;

bgend: end MINIFUN; 
REMARK ON ALGORITHM 129 MINIFUN

MINIFUN [V. W. Whitley, Comm. ACM, Nov. 1962]

E. J. WAsscher

Philips Research Laboratories

N. V. Philips' Gloeilampenfabrieken

Eindhoven-Netherlands

Some errors found in Algorithm 129 MINIFUN [Comm. ACM, Nov. 1962] are given below.

In addition, the way "steepest descent" is used to compute the minimum of a function of $n$ variables is not entirely satis. factory. The method for computing first derivatives may be improved in two ways:

1. Instead of computing $\frac{f(x+h)-f(x)}{h}$ it is better to take $\frac{f(x+h)-f(x-h)}{2 h}$. As $f(x-h)$ has been computed by MINIFUN this does not give rise to extra computations.

2. In MINIFUN the choice of $h$ seems rather deliberate. Indeed, $h$ is taken as $.2 \times(x u b-x 1 b)$, where $x u b$ and $x 1 b$ are variable bounds of $x$. In the beginning of the program these bounds are put equal to the fixed bounds $b 1$ and $u b$; afterwards in the iteration process they should tend towards each other, and in the limit they provide the minimum. So especially when a good approximation to the minimum is unknown, $b 1$ and $u b$ have to be taken well apart from each other, which means that $h$ is rather large. At the limit, however, $h$ is very small. It is better to take $h$ in such a way that the nominator $f(x+h)-f(x-h)$ attains an appropriate value.

As the method used by IIINIFUN is the Newton-Raphson method applied to the first derivatives, convergence is not alway's secured-especially since frst and second partial derivatives are estimated with numerical methods.

It should be noted that the test on end of program is not correct. For a further possible decrease of the function one has not to look in the direction of the coordinate axes but in the direction of the steepest descent.

AlGol descriptions of some "steepest descent" programs which were written in the symbolic code of the Philips computer Pascal [cf. H. J. Heijn and J. C. Selman, IRE Trans. EC1O (June 1961), 175-183) are given in Algorithms 203, 204 and 205.

Corrections of MINIFUA:

Printing errors: The line below label nustep should read: begin if abs $(d \max )<a b s(d x \min [j])$ then

The label $1 b d c h k$ should be $l b d c h k$

In comment $M I N I F U N: \quad k 1=2:$ a new minimum has not been found.

The label nustep should be placed before the statement: $d \max :=d x \min [j] ;$ The declaration of $x \min$ should be removed from the blockhead of the procedure body. The 2-dimensional arrays $x[1: n, 1: 4]$ and $g[1: n, 1: 4]$ can be replaced by a real $x$ and a 1 -dimensional array $g[1: 4]$ respectively.

An improvement could be the insertion of the statement

$$
k 1:=1 \text {; }
$$

just before the label nustep.

I am having considerable trouble with the obviously important part played by the array wnew, although it does not change after being set in the first statement of the program. Furthermore it seems to me that wnew plays a double rôle: first the component $w n e w[k]$ is the value of $x t[k]$ before an iteration on $x t[k]$. But then one should insert another statement after label nustep: $w n e w|k|:=x t|k|$; Secondly wnew $[k]$ is to be understood as half the distance between upper and lower bound $t[k]$ and $b 1[k]$, which is only true when $b 1[k]=0$.

Convergence of $d e 1 x[j]$ to 0 is only achieved when $x 1 b[k]$ and $x u b[k]$ are tending towards each other. This indicates that $w n e w[k]$ should go to 0 too. (See statements after label stnubds.)
The following modifications could remove these objections (starting with the line above label restart):

if $f t<f \min$ then go to check else $x t[k]:=$ wnew $[k \mid$

restart: if $x t[k]<w n e w[k]$ then gò to $l b d c h k$;

if $x t \mid k]=$ wnew $[k]$ then go to stnubds;

if $x \iota[k]<\iota 1[k]$ then go to nupbds;

$\operatorname{xt}[k]:=0.5 \times($ wnew $[k]+t 1[k])$;

nupbds: $x u b[k]:=t 1[k] ; \quad x 1 b[k]:=2 \times x \iota[k]-t 1[k] ;$ go to newdel;

slnubds: $x \mathbf{l} b[k]:=x t[k]-0.5 \times(w n e w[k]-x 1 b[k])$ $x u b[k]:=x t[k]+0.5 \times($ wnew $[k]-x 1 b[k]) ;$ (etc.)

lbdchk: if $x t[k]=b 1[k]$ then $x t[k]:=0.5 \times($ wnew $[k]+b 1[k])$; $x 1 b[k]:=b 1[k] ; x u b[k]:=2 \times x t[k]-b 1 \mid k]$; go to newdel; (etc.)

\section{ALGORITHM 130 \\ PERMUTE}

\section{Lt. B. C. Eaves}

U.S.A. Signal Center and School, Fort Monmouth, N. J.

procedure PERMUTE $(A, n, x)$

array $A$; integer $n, x$;

comment Each entry into PERMUTE generates the next permutation of the first $n$ elements of $A$. If $A$ is read as a number $(A[1] A[2] \cdots A[\mathrm{n}])$, each generation is larger than the last: $n:=4, x:=1$
$A[1]$
1
$\begin{array}{lllllll}A[2] & 1 & 8 & 8 & 1 & 1 & 8\end{array}$
$A[3] \quad \begin{array}{llllllll}8 & 1 & 8 & 1 & 8 & 1\end{array}$
$A \mid 4] \quad \begin{array}{rrrrrrrr}8 & 8 & 1 & 8 & 1 & 1 & \text { end }\end{array}$
Permutations $=\frac{4 !}{2 ! 2 !}$

Identical elements in $A$ reduce the number of permutations. The array should be ordered before the first call on PERMUTE. Integer $x$ specifies the first elements whose order should be preserved: $n:=4, x:=3$

$A[1] \quad 1 \quad 1 \quad 1 \quad 1 \quad 4$

$A[2] \quad 2 \quad 2 \quad 2 \quad 4 \quad 1$

$A[3] \quad 3 \quad 4 \quad 2 \quad 2$

$A[4] \quad 4 \quad 3 \quad 3 \quad 3$ end

Before the first call on PERMUTE for a given array, first should be made true. If more is true, then PERMUTE was able to give another permutation;

hegin array $B[1: n]$; integer $f, i, k, m, p ;$ real $r$; own real $t$; if first then $t:=A[x]$; first $:=$ false;

for $i:=1$ step 1 until $n$ do $B[i]:=0$;

for $i:=n$ step -1 until 2 do

begin if $A[i]>\iota \wedge A[i]>A[i-1]$ then go to find; end; more := false; go to exit;

find: for $k:=n$ step -1 until $i$ do begin if $A[k]>\imath \wedge A \mid k]>A[i-1]$ then

begin $B[k]:=A[k] ; m:=k ;$ end; end;

for $k:=n$ step -1 until $i$ do

begin if $B|k|>0 \wedge B|k|<B[m]$ then

begin $B[m]:=B[k] ; f:=k ;$ end; end; $r:=A[i-1] ; \quad A[i-1]:=B[m] ; \quad A[f]:=r$

schell: $p:=i-1 ; m:=n-p$;

for $m:=m / 2-.4$ while $m>0$ do

begin $k:=n-m$;

for $f:=p+1$ step 1 until $k$ do

begin $i:=f$;

comp: $\quad$ if $A[i]>A[i+m]$ then

begin $r:=A[i+m] ; \quad A[i+m]:=A[i]$;

$A[i]:=r ; i:=i-m$;

if $i \geqq p+1$ then go to $c o m p$;

end end end schell;

exil: end PERMUTE 


\section{ALGORITHM 131 \\ COEFFICIENT DETERMINATION*}

\section{H. Smith and M. L. Alle.}

Georgia Institute of Technology, Atlanta 13, Ga.

- This procedure pertains to research work sponsored in part by NSF Grant G-7361.

$\begin{array}{ll}\text { procedure } & D E T(n, G, H) \\ \text { array } & G, H ; \text { integer } n\end{array}$

comment Given the first $n$ coefficients of the power series

$G(z)=g_{1}+g_{2} z+g_{2} z^{2}+\cdots+g_{n} z^{n-1}+\cdots$, and $H(z)=h_{1}+$

$h_{2} z+h_{3} z^{2}+\cdots+h_{n} z^{n-1}+\cdots$, this procedure determines the coefficients $d_{i}, i=1, \cdots, n$, of the power series which is the expansion of the quotient $H(z) / G(z)$. It is assumed that $g_{1} \neq 0$. The arrays $G$ and $H$ initially contain the coefficients of $G(z)$ and contains the coefficient $d_{i}$. The procedure may also be useful in $F(z)=H(z) / G(z)$ is a complex valued function of a complex variable and that $F$ has a pole of order $m$ at $z=b$, where $H(z)=$ $\sum_{k=1}^{\infty} h_{k}(z-b)^{-1}, G(z)=\sum_{k=1}^{\infty} g_{k}(z-b)^{k+m-1}$, and $g_{1} \neq 0$, $h_{1} \neq 0$. 'The required residue at $z=b$ is $d_{n}$ where

$$
\begin{aligned}
D(z) & =\left[\sum_{k=1}^{\infty} h_{L}(z-b)^{k-1}\right] /\left[\sum_{k=1}^{\infty} g_{k}(z-b)^{k-1}\right] \\
& =\sum_{i=1}^{\infty} d_{j}(z-b)^{j-1} .
\end{aligned}
$$

For more on this, one is referred to Einar Hille, "Analytic Function Theory, Vol. I, "Ginn and Co., 1959, pages 242-244;

begin integer $i, j, n$; real alpha, bela alpha $:=1 / G[1]$

for $j:=1$ step 1 until $n$ do

begin beta:=.alpha $\times H[j]$;

for $i:=j+1$ step 1 until $n$ do $H[i]:=H[i]-($ bela $\times G[i-j+1])$ end;

for $j:=1$ step 1 until $n$ do $H[j]:=H[j] \times$ alpha;

end DET $H(z)$, respectively. The integer $n$ is the number of known coefficients in the expansion of $G(z)$ and $H(z)$. At the conclision, $H_{\text {i }}$ calculating residues for certain complex functions. Suppose

$$
\int p_{c}\left(r_{c}^{n} c\right) q_{c} d \tau
$$

which can be expressed in terms of the simple $A_{n}(b)$ and $B_{n}(a)$ functions. The subscript $c$ denotes either of the two nuclei of a diatomic molecule. These integrals include all those one-electron integrals necessary for a conventional energy calculation on a diatomic molecule. In the arguments of allslater $p$ and $q$ are numerical designations for the respective orbitals. $p$ and $q$ are even or odd as they respectively are associated with the "left," $a$, nucleus or "right," $b$, nucleus of a diatomic molecule. Global arrays, fact 1, of 'factorials and binom, of binomial coefficients are assumed. We first define some procedures utilized by allslater. The main program begins at the label set;

begin real norm, r2, alpha, beta, s, clp, clq, bpci;

integer nsum, lsum, peven, qeven, podd, godd, limitp, limitq, $g, h, i, j, n 1 p, n 1 q, \operatorname{lmp}, \operatorname{lm} q$, gama, gamb, aidaa, aidab, gam, aida, num 2 ; real array avalues $[0: 21]$, bualues $[0: 21]$; real procedure $c 1, b p c$, modulus;

real procedure $c 1(l, m, j)$; value $l, m, j$, integer $l, m, j$; begin $c 1:=((-1) \uparrow j) \times$ factl $[2 \times(l-j)] /((2 \uparrow l) \times$ factl $[l-2 \times j-m] \times$ fact $1[l-j] \times$ fact $1[j])$

end $c 1$;

real procedure $b p c(i, j, k)$; value $i, j, k$, integer $i, j, k$;

hegin real $t$; integer $m ; t:=0$;

for $m:=0$ step 1 until $k$ do

begin $t:=t+((-1) \uparrow(k-m)$ $\times$ binom $[i, m] \times$ binom $[j, k-m]$ end

end $b p c$;

real procedure modulus $(i, j)$; value $i, j$; integer $i, j$;

begin modulus $:=1-a b s(i \div j) \times j$

end modulus;

procedure avector $(b, n \max$, avalues); value $b, n \max$; real $b$; integer nmax; real array avalues;

begin integer $m$;

avalues $[0]=\exp (-b) / b$;

if $n \max =0$ then go to exit;

for $m=1$ step 1 until nmax do

begin avalues $[m]=$ avalues $[0]+(m / b) \times$ avalues $[m-1]$ end;

exit: end avector;

procedure bvector(a nmax, bvalues); value $a, n$ max; real $a$; integer nmax; real array bvalues; real procedure modulus; comment This procedure computes a sequence of values for the integral, $B_{n}(a)=\mathcal{S}_{-1} x^{n} e^{-a x} d x$, for $n=0$ to $n=n \max$. If $a \geqq$ alim then $B_{0}(a)$ is computed and upward recursion is used to generate the higher $n$ values. If $a<$ alim then $B_{n \max }(a)$ is computed by series expansion and downward recursion is used to generate the smaller $n$ values. alim is determined within the program by a simplification of a result of Gautschi (J.ACM 8, 21 (1961)). Gautschi has made an analysis of the recursive procedures for the $B_{n}(a)$ which could be taken as a model for workers in'molecular quantum mechanics;

hegin real $f x x, f x y$, numerator, denom, sum, factor 1 , tsum factor2, $t, a a$; integer $m, m n$; hegin if $a b s(a) \geqq((n \max +n \max / 6+3) / 2.3)$ then $u p$ : begin $f x x:=\exp (a)$; $f x y:=1 / f x x$

comment The Slater-type orbitals frequently used in quantum mechanical calculations on atoms and molecules are defined as $p=k(n p, p e) r^{n-1} e^{-(p e) r} Y_{1 p}^{m p}(\theta, \phi)$, where $k(n p, p e)$ is a normalization constant, $Y_{1} m(\theta, \phi)$ is a spherical harmonic with the phase convention $\left[Y_{1}^{m}(\theta, \phi)\right]^{*}=(-1)^{m} Y_{1}^{-m}(\theta, \phi), n p$ is a positive integer, $l p$ is an integer, $l p<n p, m p$ is an integer, $-l p \leqq m p$ $\leqq l p$; and $p e$ is a real positive constant. Algorithm 110, Y. A. Kruglyak and D. R. Whitman (Comm. ACM, July 1962) serves to compute integrals over certain operators of a quite restricted class of Slater-type orbitals, $n p \geqq 4, l p=1, m p=0$. The algorithm given here will compute all integrals of the form

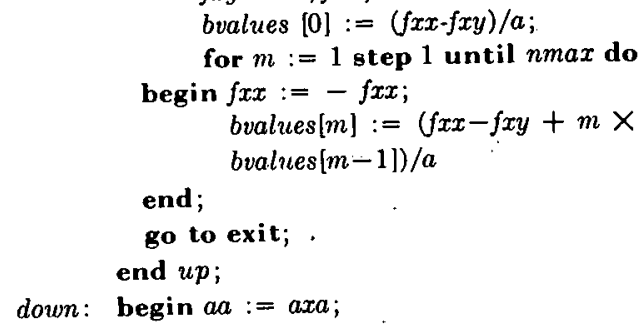
bvalues $[0]:=(f x x-f x y) / a$; 
if modulus $(n \max , 2) \neq 0$ then

setodd: begin numerator $:=n \max +2$;

sum $:=a /$ numerator

factor $1:=-2$

factor2 := 3;

go to compule;

end setodd;

seleven: begin numberalor := nmax +1 ;

sum := 1/numerator;

factorl $:=$ factor2 $:=2$;

end seteven:

compute: begin denom := numerator +2

$\ell:=$ sum

$t:=((((t /$ factor 2$) \times a a)$

/(factor 2-1)) $\times$ numerator)

/denom;

tsum : $=t+8 u m$;

if $($ sum - tsum $)=0$ then

begin bvalues[nmax] := sum $\times$ factor 1 ; go to recur;

end;

begin factor $2:=$ factor $2+2$;

numerator := denom; sum := เsum;

go to compule;

end compule;

recur: begin $f x x:=\exp (a)$;

$f x y:=1 / f x x$

$m n:=n \max -1$;

if $\operatorname{modulus}(n \max , 2) \neq 0$ then

$f x x:=-f x x$;

for $m:=m n$ step -1 until 0 do

begin $f x x=-f x x$;

bvalues $[m]:=(f x x+f x y+a \times$

bvalues $[m+1]) /(m+1)$; end

end down;

end recur;

end;

exit: end buector;

set: begin if $(m p+. m q) \neq 0$ then

begin altslater $:=0.0$; go to exil end;

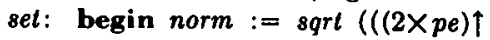

$(2 \times n p+1) \times(2 \times l p+1) \times f a c t 1[l p-m p \mid \times(2 \times q e) \uparrow$

$(2 \times n q+1) \times(2 \times l q+1) \times f a c t 1[l q-m q]) /(f a c t 1[2 \times$

$n p] \times f a c t 1[l p+m p] \times f a c t 1[2 \times n q] \times f a c t 1[l q+m q] \times$ 4));

$n s u m:=n p+n q$

$l s u m:=l p+l q$

$r 2:=r / 2$

norm $:=$ norm $\times(r 2 \uparrow(n s u m+1+n a+n b))$;

alpha $:=r 2 \times(p e+q e)$;

beta $:=r 2 \times(((-1) \uparrow p) \times p e+((-1) \uparrow q) \times q e)$;

num $2:=2$;

avector (alpha, nsum, avalues);

bvector (beta, nsum, bvalues);

peven := modulus $(p+1,2)$;

qeven $:=$ modulus $(q+1,2)$;

podd $:=$ modulus $(p, 2)$;

qodd $:=$ modulus $(q, 2)$

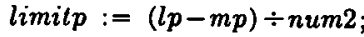

limitq $:=(l q-m q) \div n u m 2$;

$s:=0$

end set;

sum: begin for $g:=0$ step 1 until limitp do

begin $c 1 p:=c 1(l p, m p, g)$;

for $h:=0$ step 1 until limitq do

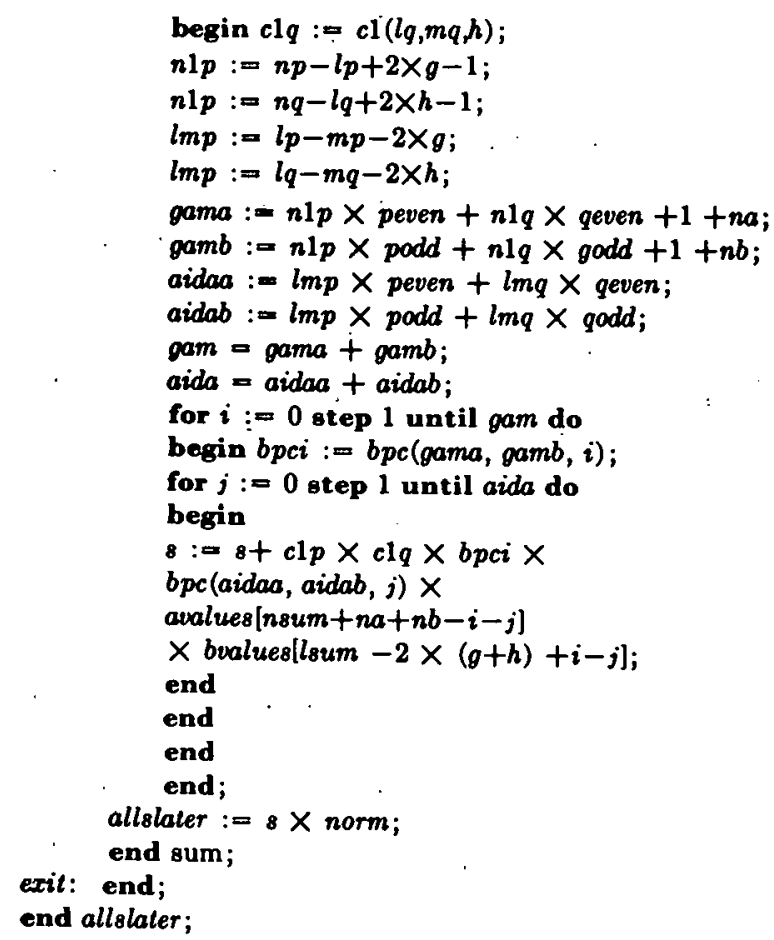

\section{ALGORITHM 133 \\ RANDOM}

Peter G. Behrenz

Mathematikmaskinnämnden, Stockholm, Sweden

real procedure $R A N D O M(A, B, X 0)$;

value $A, B, X 0$ :

real $A, B$;

integer $X 0$;

comment RANDOM generates a rectangular distributed pseudo-random number in the interval $A<B . \quad X 0$ is an integer starting-value. The first time $R A N D O M$ is used in a program $X 0$ should be a positive odd integer with 11 digits, $X 0<2^{35}=$ 34359738368 . The following times $R A N D O M$ is used, $X 0$ should be $X 0=0$. The mathematical method used is $X_{n+1}=5 X_{n}$ $\left(\bmod 2^{25}\right)$. This sequence has period $\left.2^{28}\right) . R A N D O M$ was successfully run on FACIT EDB using FACIT-ALGOL 1, which is a realization of $A L G O L 60$ for FACIT EDB, except for the declarator own, which is not included in FACIT-ALGOL 1. To test $R A N D O M$, we computed $1 / N \sum X_{n}$ and $1 / N \sum X_{n}{ }^{2}$ in the interval 0,1 for $N=500,1000,5000$. The startingvaluc was $X 0=28395423107$. The results were 0.50625 , $0.48632,0.50304$ and $0.34304,0.31681,0.33469$. Theoretically one expects 0.50000 and 0.33333 ;

begin

integer $M 35, M 36, M 37$;

own integer $X$;

if $X 0 \neq 0$ then begin

$X:=X 0 ; \quad M 35:=34359738368 ; \quad M 36:=68719476736$;

$M 37:=137438953472$ end; $X:=5 \times X$;

if $X \geqq M 37$ then $X:=X-M 37$;

if $X \geqq M 36$ then $X:=X-M 36$;

if $X \geqq M 35$ then $X:=X-M 35$;

$R A N D O M:=X / M 35 \times(B-A)+A$ end 
CERTIFICATION OF ALGORITHM 133

RANDOM [Peter G. Behrenz, Comm. ACM, Nov. 196(i2] JENSE H: POORE, $\mathbf{J}_{\mathrm{R}}$.

Iouisiana Polytechnic Institute, Ruston, La.

Algorithm 133 was transliterated into Fortran II for the Il3M 1620 computer. A monitor program performed the test indicated in Algorithm 133 on the generated numbers.

Results of the test are shown in the following chart: The not:1tion used is identical to that used in the algorithm.

\begin{tabular}{|c|c|c|c|}
\hline$x_{11}$ & $\frac{1}{x}=x$ & $\frac{1}{x} z x^{2}$ & \\
\hline 13543288579 & $\begin{array}{l}.4986+80931 \\
.4840396(i+10 \\
.4996829127\end{array}$ & $\begin{array}{l}.3280501242 \\
.31+1520616 \\
.3321160892\end{array}$ & $\begin{array}{l}N=500 \\
N=1000 \\
N=50(0)\end{array}$ \\
\hline $24376589+11$ & $\begin{array}{r}+97141+790 \\
.4997720120 \\
.4986380784\end{array}$ & $\begin{array}{r}.3297990588 \\
.3326801987 \\
.3319949173\end{array}$ & $\begin{array}{l}N=5(0) \\
N=1000 \\
N=50(0)\end{array}$ \\
\hline $3+3597383467$ & $\begin{array}{r}.4062108338 \\
.4974837457 \\
.4929612237\end{array}$ & $\begin{array}{l}.3339214302 \\
.3335720239 \\
.3253+21270\end{array}$ & $\begin{array}{l}N=5(0) \cdots \\
N=1000 \\
N=5(0 x)\end{array}$ \\
\hline $1132+679915$ & $\begin{array}{r}.5313808305 \\
.5167083685 \\
.504381+(i 33 T\end{array}$ & $\begin{array}{l}.31691599122 \\
.3498558251 \\
.3383+29327\end{array}$ & $\begin{array}{l}N=500 \\
N=1000 \\
N=50(0)\end{array}$ \\
\hline
\end{tabular}

REMARK ON ALGORITHM 133

RANDOM (P. G. Behrenz, Comm. ACM, Nov. 1962)

Peter G. Behrenz

Matematikmaskinnämnden, Box 6131, Stockholm 6, Sweden

Replace the declarations in the body of the procedure, by : integer $M 35, M 36, M 37$; own integer $X$;

own integer $X, M 35, M 36, M 37$;

The sequence of $2^{\text {ss }}$ random numbers contains about 15 numbers which are not really random numbers. For details, see R. W. Hamming, Numerical Methods for Scientists and Engineers, p. 384 [McGraw-Hill, 1962]

REMARK ON ALGORITHM 133

RANDOM [Peter G. Behrenz, Comm. ACM 11, Nov. 1962]

Donald L. Laughlin

Missouri School of Mines and Metallurgy, Rolla, Missouri

Algorithm 133 was translated into Fortran II for the IBM 1620 and run successfully. The starting value was changed to 21348759609 and significant results followed.

For $N=500$ and 1000 , the resulting values were: 0.4990157688 , 0.4986269653 and $0.3318717863,0.3290401482$.
ALGORITHM 134

EXPONENTIATION OF SERIES

. Henry E. Fettis

Aeronautical Research Laboratories, Wright-Patterson Air Force Base, Ohio

procedure SERIESPWR $(A, B, P, N)$;

comment This procedure calculates the coefficients $B[i]$ for the series $(f(x))^{P} \equiv g(x) \doteqdot 1+\sum B[i] \times x \uparrow i,(i=1,2, \cdots, N)$ given the coefficients of the series $f(x)=1+\sum A[i] \times x \uparrow i$. $P$ may be any real number;

value $A, P, N$

array $A, B$;

integer $N$;

begin integer $i, k$;

real $p, 8$;

$B[1]:=P \times A[1]$

for $i:=2$ step 1 until $N$ do

begin $s:=0$;

for $k:=1$ step 1 until $i-1$ do

$S:=8+(P \times[i-k]-k) \times B[k] \times A[i-k] ;$

$B[i]:=P \times A[i]+.(s / i)$.

end for $i$;

end SERIESPWR

CERTIFICATION OF ALGORITHMS 134 AND 158

EXPONENTIATION OF SERIES [Henry E. Fettis,

Сомм. ACM, Oct. 1962 and Mar. 1963]

Henry C. Thacher, Jr.

Reactor Engineering Div., Argonne National Laboratory Argonne, Ill.

Work supported by the U.S. Atomic Energy Commission.

The bodies of SERIESPWR were transcribed for the Dartmouth SCALP processor for the LGP-30 computer. In addition to the modifications required by the limitations of this translator, the following corrections were necessary:

1. Add "real $P$;" to the specifications.

2. Delete " $p$," from the declarations in the procedure body.

3. (134 only) Replace " $S$ " by " $s$ " and $[i-k]$ by " $(i-k)$ " in the statement $S:=s+\cdots$.

4. (158 only) Changes last sentence of comment to "Setting $P:=0$ gives the coefficients for $\ln (f(x))$. In this series, the constant term is 0 , instead of 1 as elsewhere;"

5. (158 only) Add the identifier $P 2$ to the declared real variables.

6. (158 only) Make the first statements read:

"if $P=0$ then $P 2:=1$ else $P 2:=P$; $B[1]:=P 2 \times A[1]$;

7. (158 only) Make the statement of the for $k$ loop read

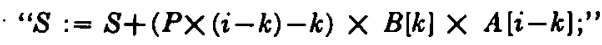

8. Change the last statement to

$$
\text { " } B[i]:=P 2 \times A[i]+S / i \text { end for } i ;
$$

In addition, the following modifications would improve the efficiency of the program:

1. Remove $A$ from the value list.

2. Omit the statement $B[1]:=P \times A[1] ; \quad(P 2 \times A[1]$ in 158 according to correction 6 ) and change the initial value of $i$ in the statement following from 2 to 1.

When these changes were made, both procedures produced the first ten coefficients of the series for $(\exp (x)) \uparrow 2.5$ from the first ten coefficients of the exponential series. The procedures were also used to generate the binomial coefficients by applying them to $(1+x)^{P}$, for $P=2.0$, and 0.5000000 . Algorithm 158 was also tested with $P:=0$ for $1+x$. and for the series expansions for $(\sin x) / x, \cos x$, and $\exp x$. In all cases, the coefficients agreed with known values within roundoff. 
ALGORITHM 135

CROUT WITH EQUILIBRATION AND ITERATION

William Marshall McKeeman*

Stanford University, Stanford, Calif.

* This work was supported in part by the Office of Naval Research under contract Nonr 225(37).

procedure LINE ARS YSTEM $(A)$ order :(n) right-hand sides:(B) number of right-hand sides: $(m)$ answers: $(X)$ determinant:(det, $e x)$ condition of $A:(c n r)$;

integer $n, m, e x$; real $d e t, c n r ; \quad$ real array $A, B, X$;

comment, LINEAR SYSTEM uses Crout's method with row equilibration, row interchanges and iterative improvement for solving the matrix equation $A X=B$ where $A$ is $n \times n$ and $X$ and $B$ are $n \times m$. As special cases one sees that: for $m \leqq 0$, only the determinant of $A$ is evaluated, for $m=1$, the algorithm solves a system of $n$ equations in $n$ unknowns, for $m=n$ and $B=$ the identity matrix, the algorithm inverts $A$.

If the algorithm breaks down for a singular or nearly singular matrix $A$, exit to a non-local label "singular" is provided. Five auxiliary procedures: EQUILIBRATE, CROUT, PRODUCT, $R E S I D U A L S$ and $S O L V E$ are declared with appropriate comments after the end of this procedure. This code is the result of the joint efforts of G. Guthrie, W. McKeeman, Cleve Moler, Margaret Salmon, Alan Shaw and R. Van Wyk. It was written following ideas presented by $\mathrm{J}$. $\mathrm{H}$. Wilkinson as a visiting lecturer in Professor George E. Forsythe's class in Advanced Numerical Analysis at Stanford, 1962;

begin integer array pivot $[1: n]$; integer $i, j, k$; real $m x$; real array $L U[1: n, 1: n], y$, res, $m u l l[1: n] ; \backslash$

comment, remove appropriate factors from the rows of $A \ldots$; EQUILIBRAT'E $(A, n$; mult $)$;

comment $\ldots$ and save the result for the eventual computation of residuals during iteration;

for $i:=1$ step 1 until $n$ do

for $\mathbf{j}:=1$ step 1 until $n$ do $L U[i, j]:=A[i, j]$; comment, decompose the matrix into triangular factors; CROUT (LUU, $n$, pivot, det);

comment, assuming that there was no exit to "singular", evaluate the determinant in the form $\operatorname{det} \times(10.0 \uparrow \mathrm{ex})$;

for $i:=1$ step 1 until $n$ do $y[i]:=L U[i, i] \times \operatorname{mull}[i]$;

$\operatorname{det}:=\operatorname{det} \times$.PRODUCT $(y, 1, n, e x)$;

comment, now begin to process right-hand sides;

for $k:=1$ step 1 until $m$ do

begin integer $i$, count, limit; real normy, $k r$;

$k r:=k$;

comment, scale the right-hand side;

for $i:=1$ step 1 until $n$ do $\operatorname{res}[i]:=B[i, k]:=B[i, k] /$ mull $[i]$; comment, store the first approximation and its $L(1)$ norm; normy :=0;

SOLVE $(L U, n$, res, pivol, $y)$;

for $i:=1$ step 1 until $n$ do

begin

normy $:=$ normy $+a b s(y[i])$;

$X[i, k]:=y^{[}[i]$

end;

comment, enter the iterating loop. The iteration is terminated on the integer "limit" which itself is determined on the basis of the success of the first iteration and a machinedependent real number designated here by "eps". For "eps", the programmer must insert the largest real number such that eps $+1.0=1.0$;

for count $:=1,2$ step 1 until limit do

begin integer $i$; real $t$; comment, compute the residuals of the solution $y$; RESIDUALS $(A, n, B, k, X$, res $)$; comment ... and find the next increment to the solution; SOLVE $(L U, n, r e s, p i v o t, y)$;

comment, set up termination conditions;

if count $=1$ then

begin real normdy;

normdy :=0;

for $i:=1$ step 1 until $n$ do normdy $:=$ normdy $+a b s(y[i])$;

if normdy $=0$ then begin $\mathrm{cnr}:=1.0$; go to enditer end; $\ell:=$ normy/normdy;

comment, The quantity $\|A\| \cdot\left\|A^{-1}\right\|$ (spectral norm) is called the condition number of the matrix $A$. It is a measure of the difficulty in solving the input equation and appears naturally in error bounds for the solution (see Wilkinson [3]). cnr is a direct measure of the error and experimentally approximates the condition number;

$c n r:=((k r-1.0) \times c n r+1.0 /(e p s \times t)) / k r ;$

if $t<2.0$ then go to singular;

limit := $\ln (e p 8) / \ln (1.0 / t)$;

end;

comment, store the new approximation;

for $i:=1$ step 1 until $n$ do $X[i, k]:=X[i, k]:=X[i, k]+y[i]$; end ileration;

enditer:

end right-hand sides

end LINEAR SYSTEM;

procedure EQUILIBRATE (A) order:(n) multipliers:(mult); integer $n$; real array $A$, mull;

comment, scaling the rows of the matrix $A$ to roughly the same maximum magnitude (here, dividing by the largest element) allows the procedure CROUT to select effective pivotal elements for the Gaussian decomposition of the matrix. The iterating procedure will converge to the solution for the equilibrated matrix rather than the input matrix. If the matrix is badly conditioned then the solution is sensitive to perturbations in the input and the scaling division must be done not by the largest element but rather by the power of the machine number base (2 and 10 for binary and decimal machines, respectively) nearest the largest element so as to avoid rounding errors. Equilibration is discussed in reference [3] p. 284;

begin integer $i$; real $m x$;

for $i:=1$ step 1 until $n$ do

begin integer $j$;

$m x:=0.0$; comment, find the largest element;

for $j:=1$ step 1 until $n$ do

if $a b s(A[i, k])>m x$ then $m x:=a b s(A[i, k])$;

if $m x=0.0$ then go to singular;

comment, now store the multiplier and scale the row;

$m u l l[i]:=m x$; comment $:=$ base $\uparrow e x$ for exact scaling;

if $m x \neq 1.0$ then

end

for $j:=1$ step 1 until $n$ do $A[i, j]:=A[i, j] / m x$

end EQUILIBRATE;

procedure CROUT $(A)$ order: $(n)$ pivots:(pivot) interchanges:(sg).

integer $n$; integer array pivot; real array $A$; real $s g$;

comment, this is Crout's method with row interchanges as formulated in reference [1] for transforming the matrix $A$ into the triangular decomposition $L U$ with all the $L[k, k]=1.0$. pivot $k$ ] stores the index of the pivotal row at the $k$-th stage of the elimination for use in the procedure SOLVE;

begin integer $i, j, k$, imax, $p$; real $t$, quot; real procedure $I P 1(A)$ extra term: $(t)$ length: $(f)$;

integer $f$; real $t$; real array $A$; comment non-local $i, j, k$; comment, IP1 forms a row by column inner product of $A$, namely the sum of $A[i, p] \times A[p ; k]$ for $p:=1,2, \ldots, f$, and then adds the extra term $t$. If $f<1$, the value of $I P 1$ is $t$. This procedure is the inner loop of the algorithm. The pro- 
grammer can expect a substantial advantage from substituting a faster and more accurate inner product here;

begin real sum; integer $p$;

sum $:=t$

for $\dot{p}:=1$ step 1 until $f$ do sum $:=\operatorname{sum}+A[i, p] \times A[p, k]$;

$I P 1:=$ sum

end $I P 1$;

$s g:=1.0$

comment, $k$ is the stage of the elimination;

for $k:=1$ step 1 until $n$ do

begin

$t:=0$

for $i:=k$ step 1 until $n$ do

begin comment, computc $L$. Note that the first calls on IP1 are empty;

$A[i, k]:=-I P 1(A,-A[i, k], k-1)$;

if $a b s(A[i, k])>\ell$ then

end;

begin $t:=a b s(A[i, k]) ; \quad i \max :=i$ end

if $t=0$ then go to singular;

comment, $A[$ imax, $k]$ is the largest element in the remainder of column $k$. Interchange rows if necessary and record the change;

$\operatorname{pivot}[k]:=i \max$

if $\operatorname{imax} \neq k$ then

begin

$\mathbf{s g}:=-\mathbf{s g}$

for $j:=1$ step 1 until $n$ do

begin

$t:=A[k, j] ; A[k, j]:=A[i \max , j] ; A[i \max , j]:=t$

end

end;

comment, compute a column of multipliers;

quot $:=1.0 / A[k, k]$

for $i:=k+1$ step 1 until $n$ do $A[i, k]:=A[i, k] \times q u o t$;

comment, and compute a row of $U$;

for $j:=\mathbf{k}+1$ step 1 until $n$ do

end

$A[k, j]:=-I P 1(A,-A[k, j], k-1)$

end $C R O U T$;

real procedure PRODUCT (factors) start:(s) finish:(f) exponent:(ex);

integer $s, f, e x ;$ real array factors;

comment, PRODUCT multiplies the numbers stored from index $s$ through $f$ inclusive in the array "factors", preventing exponent overflow. The answer is normalized so that $1.0>$ abs $(P R O D U C T) \geqq 0.1$. The exponent appears in ex;

begin integer $i$; real $p, p 1$;

ex $:=0 ; p:=1.0$

for $i:=s$ step 1 until $f$ do

begin

$p 1:=$ factors $[i]$;

if $a b s(p 1)<0.1$ then begin $p 1=10.0 \times p 1 ; \quad e x:=e x-1$ end;

$p:=p \times p 1$;

if $p=0$ then begin $e x:=0$; go to fin end;

1: if $a b s(p)<0.1$ then

begin $p:=p \times 10.0 ; \quad e x:=e x-1$; go to 1 end;

$2: \quad$ if $a b s(p) \geqq 1.0$ then

begin $p:=p / 10.0 ; \quad e x:=e x+1 ;$ go to 2 end; end;

fin: $P R O D U C T:=p$

end PRODUCT;

procedure RESIDUALS (A) order:(n) right-hand sides:(B) column of $B:(k)$ approximate solution:(X) residuals:(res); integer $n, k$; real array $A, B ; X$, res;

comment, RESIDUALS computes $b-A y$ where $b$ is the $k$ th column of the right-hand side matrix $B$ and $y$ is the $k$ th column of $X$; real procedure IP2 $(A)$ row: $(i)$ order: $(n)$ approximate solution: $(X)$

column:(k) extra therm: $(t)$;

integer $i, k, n$; real $t$ real array $A, X$;

comment, IP2 forms the inner product of row $i$ of the matrix $A$ and column $k$ of the solution matrix $X$, then adds the single term $t$. It is essential that IP2 be an "accumulating" or double precision inner product as discussed in reference [3] p. 296. The value of $I P 2$ is the rounded single precision result of the double precision arithmetic. The body of the procedure is left undefined;

begin integer $i$;

for $i:=1$ step 1 until $n$ do

res $|i|:=-I P 2(A, i, n, \lambda, k,-B[i, k])$

end KESTLUALS;

procedure $S O L V E(A)$ order:(n) right-hand side:(b) pivots:

(pivol) answer:(y);

integer $n$; integer array pivot; real array $A, b, y$;

comment, SOLVE processes a right-hand side $b$ and then backsolves for the solution $y$ using the $L U$ decomposition provided by CROUT';

begin integer $k, p$; real $t$;

for $k:=1$ step 1 until $n$ do

begin

$l:=b[$ pivol $[k]\rfloor ; \quad b\lfloor$ pivol $[k]]:=b[k] ;$

for $p:=1$ step 1 until $k-1$ do $t:=t-A[k, p] \times b[p]$;

$b|k|:=\ell$

end ...having modified $b$ by $L$ inverse;

comment, now the back solution for $y$;

for $k:=n$ step -1 until 1 do

begin

$\iota:=b[k]$

for $p:=k+1$ step 1 until $n$ do $t:=t-A[k, p] \times y[p]$;

$y[k]:=\imath$

end backsolution

end SOLVE

REFERENCES

1. George E. Forsythe, Crout with Pivoting. Algorithm 16. Comm. ACM S, 2 (Sept. 1960), 507.

2. Derek Johann Roek, Simultaneous System of Equations and Matrix Inversion Routine. Algorithm 92. Comm. ACM 5, 5 (May 1962), 286.

3. J. H. Wilkinson, Error Analysis of Direct Methods of Matrix Inversion, $J . A C M 8,3$ (July 1961), 281-330.

\section{CERTIFICATION OF ALGORITHM 13i}

CROUT WITH EQUILIBRATION AND ITERATION

[William Marshall McKeeman,* Comm. ACM, Nov. 1962]

William Marshall McKeeman,

Stanford University, Stanford, Calif.

* This work was supported in part by the Office of Naval Research under contract Nonr 225(37).

A BALGOL translation of the algorithm was tested for accuracy, proper termination and running time on the Burroughs 220 . The exact inverse of the Hilbert segment of order 6 can be stored in the 8-decimal-digit floating word of the B220 and was used in the accuracy and termination tests. The Hilbert segment $\mathrm{H}_{0}$ is very ill-conditioned (for the spectral norm, $\left\|\mathrm{H}_{6}\right\| \cdot\left\|\mathrm{H}_{\theta}^{-1}\right\|=$ $\left.1.3 \times 10^{7}\right)$. Hence the number of iterations required should not be taken as typical.

The $[n, n]$ element (mathematically ${ }_{i}{ }^{1}=.090909 \cdots$ ) is representative of the behavior of the rest: 
initial solution

first iteration

second iteration

third iteration

fourth iteration

fifth iteration "exact" equilibration
(by powers of 10)

.092587535

.090877240

.090909695

.090909080

.090909091

terminated equilibralion by largest element in row

094091506

.091498265

.091570311

.091568310

.091568365

.091568364

terminated

Conclusions: The iterating procedure terminated correctly, or performed one extra iteration in each case. If the equilibration procedure alters the data, the iteration will converge to the solution for the altered matrix. If the matrix is ill-conditioned, as in the case above, the equilibration may cost a great deal more than it gains. As a practical matter, a machine language substitute for EQUILIBRATE which will not cause rounding of the data is probably the best course of action.

The running time is approximately proportional to $n^{3}$ as expected. If for a given machine, $\mu$ is the floating multiply time in seconds, one can expect that run time will be given by $r t:=1.3 \times$ $\mu \times(n+7) \uparrow 3$ seconds for a call on LINEARSYSTEM with one right-hand side.

The division of run time between the various phases of the algorithm is as follows:

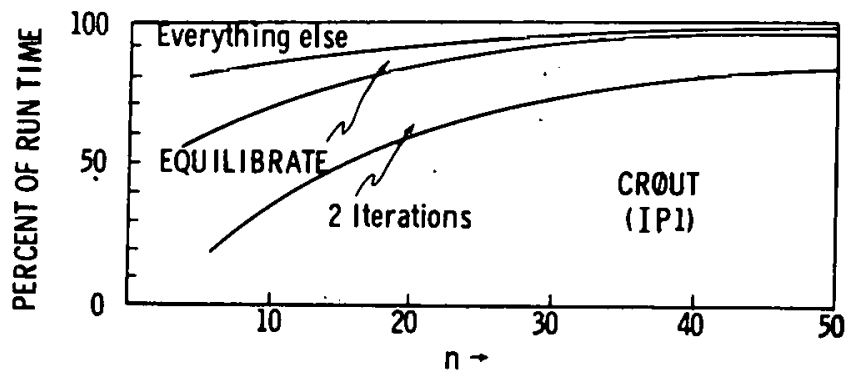

ORDER OF MATRIX

ReFERENCE:

1. SavaGE ANd LuKacs; Tables of inverses of finite segment of the Hilbert matrix. In Olga Taussky (Ed.), Contributions to the Solution of Systems of Linear Equations and the Determination of Eigenvalues, pp. 105-108, Nat. Bur. Standards Appl. Math. Series no. 39, U. S. Government Printing Office, Wash., D.C., 1954.

\section{REMARK ON ALGORITHM 135 [F4]}

CROUT WITH EQUILIBRATION AND ITERATION

[W. M. McKeeman, Comm. ACM 5 (Nov. 1962), 555$557,559]$

William Marshall McKeeman (Recd. 1 Apr. 1964)

Computation Center, Stanford University, Stanford, Calif.

The following corrections to the published algorithm are recom. mended:

1. Two lines above the bottom line of procedure SOLVE one must change

$$
y[k]:=\imath \quad \text { to } \quad y[k]:=\imath / A[k, k]
$$

2. In procedure EQUILIBRATE, all occurrences of the subscript $k$ must be changed to $j$

3 . The statement $c n r:=1.0$ should be added at the start of the body of procedure LINEARSYSTEM, so that $c n r$ will have a value the first time it is used.

4. Line 19 from the end of LINEARSYSTEM should be changed from

if normdy $=0$ then begin $c n r:=1.0 ;$ go to enditer end;

to read

$$
\text { if normdy }=0 \text { then go to enditer; }
$$

This correction makes sure that $\mathrm{cnr}$ retains a reasonable value in case normdy should be 0 for some column.

5. The symbol "." must be removed from the parameter delimiters in the declarations of procedures LINEARSISTEM, RESIDUALS and SOLVE.

6. Four lines above the bottom line of procedure $L I N E A R$ $S Y S T E M$, delete the first occurrence of $X[i, k]:=$

7. In the third line of the heading of procedure $I P 2$, the parameter delimiter

should be changed to

$$
\text { ) extra therm:( }
$$

) extra term:(

\section{AI.GORITHM 136}

\section{ENLARGEMENT OF A GROUP}

\section{WELLS*}

University of Leeds, England

* Currently with Burroughs Corporation, Pasadena, California

procedure Enlarge group $(G, n, g$, Abelian); array $G, g$; integer $n$; Boolean Abelian;

comment This procedure combines the element $g$ with the sul)group $G$, of $n$ elements, to form a new group. The Boolean $A$ belian has the value true if the group to which $G$ and $g$ helong is Abelian. Two procedures, multiply and equal are assumed to be declared: multiply $(G[i])$ by : $(G[j])$ to give : $(G|k|)$ will set the element $G_{k}$ equal to the product of the elements $G_{i}$ and $G_{j}$. equal $(G[i], G[j])$ is a Boolean procedure whuse value is true if, and only if, the elements $G_{i}$ and $G_{j}$ are equal. On leaving the procedure the enlarged group is in $G$, and $n$ is equal to the number of elements in the new sub-group $G$. The procedure will function correctly if $g$ is included in $G$ on entry. It is prob. able that $g$ and the elements of $G$ will be arrays, and the procedure body will, in practice, need to be altered considerably. The procedure has been used successfully in connection with problems of space-group theory;

begin integer $i, j, k$;

for $i:=1$ step 1 until $n$ do

if equal $(G[i], g)$ then go to not new generalor;

$n:=n+1 ; G[n]:=g$;

for $i:=n$ step 1 until $n$ do

begin for $j:=1$ step 1 until $n$ do

begin multiply (G[i], $G[j], G[n+1])$;

for $k:=1$ step 1 until $n$ do

if equal $(G[k], G[n+1])$ then go to not new element 1;

$n:=n+1$

not new element 1 : if Abelian then go to take next element; multiply (G[j], $G[i], G[n+1])$;

for $k:=1$ step 1 until $n$ do

if equal $(G[k], G[n+1])$ then go to not new element 2 ;

$n:=n+1$.

not new element 2: take next element:

end of $\mathrm{j}$-loop;

end of $\mathrm{i}$-loop:

not new generator: end of group enlargement 


\section{ALGORITHM 137}

NESTING OF FOR STATEMENT I

David M. Dahm \& M. Wells*

Burroughs Corp., Pasadena, Calif.

* On leave of absence from the University of Leeds, England.

procedure Fors $1(n, P)$;

value $n$; integer $n$; procedure $P$;

comment Fors 1 generates a nest of $n$ for statements with the procedure $P$ at their center. Two non-local arrays $I$ and $U$, which give the value of the controlled variable and its upper bound for each level are assumed to be declared;

begin integer $j$;

if $\mathbf{n}=0$ then $P$

else for $j:=1$ step 1 until $U[n]$ do

begin $I[n]:=j ;$ Fors $1(n-1, P)$ end end Fors 1

\author{
ALGORITHM 138 \\ NESTING OF FOR STATEMENT II \\ David M. Dahm \& M. Wells* \\ Burroughs Corp., Pasadena, Calif. \\ * On leave of absence from the University of Leeds, Engliand. \\ procedure Fors $2(P)$; \\ procedure $P$; \\ comment Fors 2 performs the same function as Fors 1 , but is \\ more economic of storage.space. It is expected, however, \\ that Fors 1 would be more economic of time. The formal \\ parameter $n$ is now replaced by the non-local integer $n$; \\ begin if $n=0$ then $P$ \\ else for $I[n]:=1$ step 1 until $U[n]$ do \\ begin $n:=n-1 ;$ Fors $2(P)$ end; \\ $n:=n+1$ end Fors 2
}

the number $n$ of divisions for use below

if $d=0$ then go to if $c=0$ then INDETERMINATE

else NO SOLUTION; comment The case $d=0$ occurs when $a^{2}+b^{2}=0$. If $d$ now does not divide $c$ then the equation cannot be solved so;

if $(c \div d) \times d \neq c$ then go to NO SOLUTION;

if $d \neq 1$ then

begin $a:=a / d, \quad b:=b / d ; \quad c:=c / d$ end, which removes the common factor and reduces the equation to the case where $a$ and $b$ are relatively prime;

hegin comment. We shall now find $u_{1}$ and $v_{1}$ in order to express

$1=a u_{1}+b v_{1}$, using the relations $r_{n}=r_{i} v_{i}+r_{i-1} u_{i}$,

$i=n, \dot{n}-1, \cdots, 1, v_{n}=1, \quad u_{n}=0$, and $r_{i+1}=-r_{i} q_{i}+r_{i-1}$, $i=n-1, n-2, \cdots, 1 ;$ integer $u, v$;

if $n=0$ then

begin $v:=0 ; u:=1$ end, which takes care of the case $b=0$

else

begin $v:=1 ; u:=0$;

for $i:=n-1$ step -1 until 1 do

begin integer $\ell$;

$t \cdot:=v ; \quad v:=u-v \times q[i] ; u:=\imath$

end $i$

end the case $n \neq 0$. It remains now to multiply the equality $1=a u_{1}+b v_{1}$ through by $c$;

begin integer $x 0, y 0$;

$x 0:=c \times u \times \operatorname{sign}(a) ; \quad y 0:=c \times v \times \operatorname{sign}(b) ;$ print $(x 0, y 0)$ comment If $x_{0}, y_{0}$ is a particular solution then $x_{0} \pm i b$, $y_{0} \mp i a, i=1,2, \ldots$ gives the general solution. Therefore;

if general solution then

begin $u:=b ; \quad v:=a$;

$A:$ print $(x 0+u, y 0-v)$; print $(x 0-u, y 0+v)$; :

$u:=u+b ; \quad v:=v+a$

if time permits then go to $A$

end general solution and

end solution.

end $u, v$

end 1)iophantus.

\section{ALGORITHM 139}

\section{SOLUTIONS OF THE DIOPHANTINE EQUATION}

\section{J. E. L. PeCK}

University of Alberta, Calgary, Alberta, Canada

procedure Diophantus $(a, b, c)$; integer $a, b, c$;

comment This procedure seeks the integer solutions of the equation $a x+b y=c$, where the integers $a, b, c$ are given. It assumes a non-local integer $M$, which should be as large as storage will allow, two nonlocal labels INDETERMINATE and NO SOLUTION and two non-local Boolean variables 'general solution' and 'time permits' which are self explanatory. It also assumes the procedures abs, sign and print;

begin integer $n, r, s, d, i$; integer array $q[1: M]$

$n:=i:=0 ; \quad d:=8:=\operatorname{abs}(a) ; \quad r:=\operatorname{abs}(b)$

comment $d$ will become the greatest common divisor of $a$ and $b$. If $b=0$ then $d=|a|$. The vector $q$ will retain the successive quotients in the Euclidean algorithm $r_{i-1}=r_{i q i}+r_{i+1}$, $i=1,2, \cdots, n$, where $0 \leqq r_{i+1}<r_{i}, r_{0}=|a|, r_{1}=|b|$, and $r_{n+1}=0$

for $i:=i+1$ while $r \neq 0$ do

begin $n:=i ; d:=r ; \quad q[i]:=s \div d$;

$r:=8-d \times q[i] ; \quad s:=d$ end This records the quotients and

\section{ALGORITHM 140 \\ MATRIX INVERSION}

\section{P. Z. INGERMAN}

University of Pennsylvania, Philadelphia, Penn.

procedure invert (a) of order:(n) with tolerance:(eps) and error exit:(oops);

value $n$, eps; array $a$; integer $n$; real eps; label oops;

comment This procedure inverts a matrix by using elementary row operations. Although the method is not particularly good for ill-conditioned matrices, the simplicity of the algorithm and the fact that the inversion occurs in place make it useful on occasion;

begin integer $i$;

for $i:=1$ step 1 until $n$ do

begin integer $j, k$; real $q$;

$q:=a[i, i]$

if $a b s(q) \leqq a b s(e p s)$ then go to oops;

$a[i, i]:=1$;

if $q \neq 1$ then for $k:=1$ step 1 until $n$ do $a[i, k]:=a[i, k] / q$;

for $j:=1$ step 1 until $n$ do

if $i \neq j$ then

begin $q:=a[j, i] ; \quad a[j, i]:=0$;

for $k:=1$ step 1 until $n$ do $a[j, k]:=a[j, k]-q \times a[i, k]$ end end end 


\section{CERTIFICATION OF ALGORITHM 140}

MATRIX INVERSION [P. Z. Ingerman, Comm. ACM,

Nov. 1962]

Richard George*

Argonne National Laboratory, Argomne, Ill.

- Work supported by the United Stateg Atomic Energy Commission.

Algorithm 140 was tested on the LGP-30, using SCALP, a loadand-go compiler from the Dartmouth College Computation Center, and it was shown to be syntactically correct.

It is indeed a simple procedure. It is so simple because the author has eliminated the very necessary search for largest elements and the row interchanges. As a result, this procedure will fail to invert many non-singular matrices. To be invertable by this procedure, a matrix must be such that all of its leading diagonal submatrices will have non-zero determinants.

One would do well to avoid this algorithm and use one (such as 120 ) which employs the pivoting process.

\section{ALGORITHM 141 \\ PATH MATRIX}

P. Z. INGERMAN

University of Pennsylvania, Philadelphia, Penn.

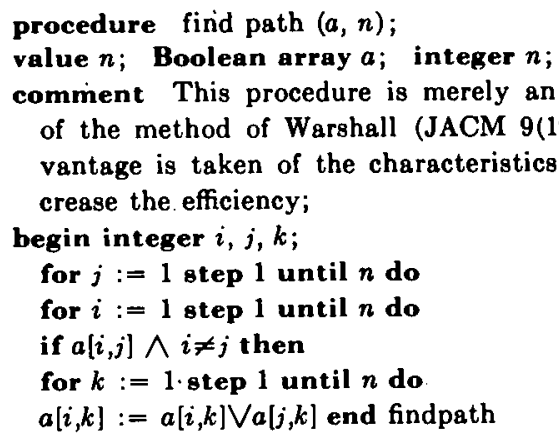

\section{ALGORITHM 142 . \\ TRIANGULAR REGRESSION}

\section{W. L. Hafley and J. S. Lewis}

Aluminum Company of America, Pittsburgh, Penn.

procedure trireg $(n$, nob, dep, pmax);

real pmax; integer $n$, nob, dep;

comment trireg is a multiple regression procedure which develops and inverts only the upper triangular portion of a correlation matrix of order $n$. The $i, j$ th $(i \leqq j)$ matrix element is $r\left(c_{i}+j\right)$ where the $c^{\prime}$ s are cram numbers (ref. Algorithm 67, J. Caffey, Comm. ACM 4, July 1961). dep $<n$ dependent variables are regressed simultaneously. Read $(u)$ is an input procedure for single elements. The input consists of nob obaervations on $n$ variables. The first dep variables are considered dependent and the remaining $n-d e p$ are considered independent variables. Independent variables are dropped when the pivotal element exceeds $p$ max during the inversion. Total variable storage is $14+3 n+n(n+1) / 2$; begin integer $i 1, i 2, i 3, c 1, c 2, c 3, d f$; integer array $c[1: n]$; real $d, p, a$; real array $r[1: n(n+1) / 2], v[1: n], m[1: n]$;

initial : $d f:=0$; for $i l:=1$ step 1 until $n$ do $m[i 1]:=0$; for $i 1:=1$ step 1 until $n(n+1) / 2$ do $r[i 1]:=0$;

input: for $i 1:=1$ atep 1 until nob do begin for $i 2:=1$ step 1 until $n$ do $\operatorname{Read}(v[i 2])$; c1 : = 0; for $i 2:=1$ step 1 until $n$ do begin $d:=v[i 2] ; \quad m[i 2]:=m[i 2]+d$; for $i 3:=i 2$ step 1 until $n$ do begin $c 1:=c 1+1 ; \quad r[c 1]:=r[c 1]+v[i 3] \times d$ end end $i 2$;

end $i 1$;

correlation: $c 1:=1 ; \quad a:=1 /$ nob; for $i 1:=1$ step 1 until $n$ do begin $v[i 1]:=1 / 8 q r t(r[c 1]-(m[i 1] \uparrow 2) \times a)$; $r[c 1]:=1 ; \quad c l:=c 1+n-i 1$ end $i 1$;

$c 1:=1$; for $i 1:=1$ step 1 until $n$ do

begin $d:=a \times m[i 1] ; \quad p:=v[i 1] ; \quad c 1:=c 1+1$; $m[i 1]:=d ;$

for $i 2:=i 1+1$ step 1 until $n$ do

begin $r[c 1]:=(r[c 1]-d \times m[i 2]) \times v[i 2] \times p$; end $i 2$;

end $i 1$;

comment variable $i$ may be dropped from the regression by setting $v_{i}=0$ and $d f$ equal to the number of variables dropped;

eram: $i 1:=-n ; \quad i 2:=n+1$; for $i 3:=1$ step 1 until $n$ do begin $i 1:=i 1+i 2-i 3 ; c[i 3]:=i 1$ end $i 1$;

inversion: for $i 1:=\operatorname{dep}+1$ step 1 until $n$ do begin $c 1:=c[i 1]$; if $v[i 1] \neq 0$ then begin $p:=1 / r[c 1+i 1] ;$ if $p>p \max$ then begin $d f:=d f+1$; go to $Y Y$ end else begin $r[c 1+i 1]:=p$; for $i 2:=1$ step 1 until il -1 do

begin $c 2:=c[i 2] ; \quad a:=p \times r[c 2+i 1]$ for $i 3:=i 2$ step 1 until $n$ while $i 3 \neq i 1$ do begin if $i 3<i 1$ then

begin $c 3:=c[i 3] ; d:=r[c 3+i 1]$ end

alse $d:=-r[c 1+l 3]$;

$r[c 2+i 3]:=r[c 2+i 3]+d \times a$

end $i 3$;

end $i 2$;

for $i 2:=i 1+1$ step 1 until $n$ do

begin $a:=p \times r[c 1+i 2] ; \quad c 2:=c[i 2]$;

for $i 3:=i 2$ step 1 until $n$ do

$r[c 2+i 3]:=r[c 2+i 3]-a \times r[c 1+i 3]$; end $i 2$;

$Z Z:$ for $i 2:=1$ step 1 until $i 1-1$ do begin $c 2:=c[i 2+i 1] ; \quad r[c 2]:=-p \times r[c 2]$ end $i 2$;

for $i 2:=c 1+i 1+1$ step 1 until $n+c 1$ do $r[i 2]:=p \times r[i 2]$

end

end else

$Y Y:$ begin $p:=0 ; \quad r[c 1+i 1]:=0 ;$ go to $Z Z$ end

coeff: $d:=1 /(n o b-n+d e p-l+d f) ;$ for $i l:=1$ step 1 until dep do

if $v[i 1] \neq 0$ then

begin $a:=0 ; p:=1 / v[i 1] ; \quad c 1:=c[i 1] ;$ for $i 2:=\operatorname{dep}$ +1 step 1 until $n$ do

begin if $r[i 2] \neq 0$ then

begin $r[c 1+i 2]:=-r[c 1+i 2] \times v[i 2] \times p ; a:=$ $a+r[c 1+i 2] \times m[i 2]$

end

end $i 2$;

$v[i 1]:=(2-r[c 1+i 1]) \times d /(v[i 1] \uparrow 2)$ 
comment: $v[1: d e p]$ now contains the mean square deviations from regressions for the dependent variables. The coefficients of determination $R^{2}$ may be obtained as $r[c 1+i 1]-1$;

$r[c 1+i 1]:=m[i 1]-a$ else

begin $c 1:=c[i 1]$; for $i 2:=c 1+i 1$ step 1 until $c 1+n$ do $r[i 2]:=0$

$$
\text { end }
$$

end

comment The r-array now contains the constants and coefficients of regression, and the inverse of the correlation matrix of the independent variables that have been kept. The following example will help to locate the information in the $r$ array.

Example: $n=6$ dep $=3$

$$
\begin{aligned}
& r_{1} r_{2} r_{2} \quad r_{4} r_{5} r_{6} \quad b_{01} \quad j b_{11} b_{21} b_{21} \\
& r_{7} r_{8} r_{0} r_{10} r_{11} \quad b_{02} \quad b_{12} \cdot b_{22} b_{12} \\
& r_{12} r_{12} r_{14} r_{16} \quad b_{02} \quad b_{13} b_{12} b_{32} \\
& r_{18} r_{20} \text {. } r^{22} r^{28} \\
& r_{31} \quad r^{32}
\end{aligned}
$$

The variances and covariances of the regression coefficients for the $j$ th dependent variable can be determined by-

$\operatorname{Var}\left(b_{i i}\right)=r^{i i} \times v_{i} \times v_{i}^{2}$

Covar $\left(b_{i j} b_{h i}\right)=r^{i k} \times v_{j} \times v_{i} \times v_{k}$;

end trireg

\section{ALGORITHM 144 \\ TREESORT 2}

Arthur F. KaUpe, JR.

Westinghouse Electric Corp., Pittsburgh, Penn.

procedure TREESORT 2 (UNSORTED, $n$, SORTED, $k$, ordered); value $n, k$;

integer $n, k$; array UNSORTED, SORTED; Boolean procedure ordered;

comment TREESORT 2 is a generalized version of TREESORT

1. The Boolean procedure ordered is to have two real arguments. The array SORTED will have the property that ordered (SORTED [i], SORTED[j]) is true when $j>i$ if ordered is a linear order relation;

begin integer $i, j ;$ array $m 1[1: 2 \times n-1]$; integer array $m 2$ [1:2×n-1];

procedure minimum; if ordered ( $m 1[2 \times i], m 1[2 \times i+1])$ then begin $m 1[i]:=m 1[2 \times i] ; \quad m 2[i]:=m 2[2 \times i]$ end else

begin $m 1[i]:=m 1[2 \times i+1] ; \quad m 2[i]:=m 2[2 \times i+1]$ end $m i n i-$ mum;

for $i:=n$ step 1 until $2 \times n-1$ do begin $m 1[i]:=U N S O R T E D$ $[i-n+1] ; \quad m 2[i]:=i$ end

for $i:=n-1$ step -1 until 1 do minimum;

for $j:=1$ step 1 until $k$ do

begin $S O R T E D[j]:=m 1[1] ; \quad i:=m 2[1] ; \quad m 1[i]:=$ infinily;

for $i:=i \div 2$ while $i>0$ do minimum end

end TREESORT 2

\section{ALGORITHM 143}

TREESORT 1

Arthur F. Kaupe, Jr.

Westinghouse Electric Corp., Pittsburgh, Penn.

procedure TREESORT 1 (UNSORTED, $n$, SORTED, $k$ ); value $n, k$;

integer $n, k$; array UNSORTED, SORTED;

comment TREESORT 1 is a revision of TREESORT (ALGORITHM 113) which requires neither the "packed" array $m$ nor the machine procedures pack, left half, right half, and minimum. The identifier infinity is used as nonlocal real variable with value greater than any element of UNSORTED;

begin integer $i, j$; array $m 1[1: 2 \times n-1]$; integer array $m 2[1: 2 \times n-1]$

procedure minimum; if $m 1[2 \times i] \leqq m 1[2 \times i+1]$ then begin $m 1[i]:=m 1[2 \times i] ; \quad m 2[i]:=m 2[2 \times i]$ end else begin $m 1[i]:=m 1[2 \times i+1] ; \quad m 2[i]:=m 2[2 \times i+1]$ end minimum;

for $i:=n$ step 1 until $2 \times n-1$ do begin $m 1[i]:=$ UNSORTED $[i-n+1] ; \quad m 2[i]:=i$ end

for $i:=n-1$ step -1 until 1 do minimum;

for $j:=1$ step 1 until $k$ do

begin SORTED $[j]:=m 1[1] ; \quad i:=m 2[1] ; \quad m 1[i]:=$ infinity;

for $i:=i \div 2$ while $i>0$ do minimum end

end TREESORT 1

\section{ALGORITHM 145}

ADAPTIVE NUMERICAL INTEGRATION BY SIMPSON'S RULE

William Marshall McKeeman*

Stanford University, Stanford, Calif.

* This work was supported in part by the Office of Naval Research under contract Non4 225(37).

real procedure Integral $(F)$ limits: $(a, b)$ tolerance: $(e p s)$;

real procedure $F$; real $a, b, e p s$;

begin comment Integral will numerically approximate the integral of the function $F$ between the limits $a$ and $b$ by the application of a modified Simpson's rule. Although eps is a measure of the relative error of the result, the actual error may be very much larger (e.g. whenever the answer is amall because a positive area cancelled a negative area). The procedure attempts to minimize the number of function evaluations by using small subdivisions of the interval only where required for the given tolerance;

integer level;

real procedure Simpson $(F, a, d a, F a, F m, F b, a b s a r e a, e s t, e p s)$; real procedure $F$; real $a, d a, F a, F m, F b$, absarea, est, eps;

begin comment Recursive Simpson's rule; real $d x, x 1, x 2$, est 1 , est2, est $3, F 1, F 2, F 3, F 4$, sum; $d x:=d a / 3.0 ; \quad x 1:=a+d x ; \quad x 2:=x 1+d x ;$ $F 1:=4.0 \times F(a+d x / 2.0) ; \quad F 2:=F(x 1) ;$ $F 3:=F(x 2) ; \quad F 4:=4.0 \times F(a+2.5 \times d x) ;$ est1 : $=(F a+F 1+F 2) \times d x / 6.0$; $e s t 2:=(F 2+F m+F 3) \times d x / 6.0$; $e s t 3:=(F 3+F 4+F b) \times d x / 6.0$; absarea $:=$ absarea-abs $(e s t)+a b s(e s t 1)+a b s(e s t 2)+a b s(e s t 3)$; sum $:=e s t 1+e s t 2+e s t 3$;

. level $:=$ level +1 ; 
Simpson : = if $(a b 8(e s t-8 u m) \leqq e p 8 \times a b s a r e a \wedge e s t \neq 1.0) \vee$ level $\geqq 7$ then sum

else Simpson (F, a, dx, Fa, F1, F2, absarea, est1, ep8/3.0)

+ Simpson $(F, x 1, d x, F 2, F m, F 3$, absarea, est2, eps/3.0)

$+\operatorname{Simpson}(F, x 2, d x, F 3, F 4, F b, a b s a r e a, e s t 3, e p 8 / 3.0)$;

level : = level -1 ;

end Simpson; '

level : = 1;

Integral := Simpson $(F, b-a, F(a), 4.0 \times F((a+b) / 2.0), F(b)$,

end Inlegral 13 $1.0,1.0$, eps)

\section{CFRTIFICATION OI: AJGORITHM 145}

\section{ADAl'TIVE NUMFRICAL INTEGRATION BY}

SIMPSON'S RULE [W. Iclieeman, Comm. ACM,

Dec. 1962]

Wm. M. Mcheeman

Stanford, University, Stanford, Calif.

Suggested chunges in the code:

1. Replace all occurrences of eps/3.0 by eps/1.7.

2. Replace level $\geqq 7$ by level $\geqq 20$.

3. The second parameter, $a$ in the final call of Simpson wais omitted; insert it.

With the above changes, a BALGos, translation of Integral has been tested successfully on a large number of functions: An example of its behavior is given below:

Machine: Burroughs 220,8 decimal digit floating-point mantiss:t. $f(x)=1.0 /$ sgrt $(a b s(x))$; which has a pole at the origin.

$n=-9.0 ; b=1000.0 ;$ correct answer $=206.0$;

\begin{tabular}{l|l|l}
\hline \multicolumn{1}{c|}{ eps } & computer answer & relative error \\
\hline 0.1 & 200.22251 & 0.028 \\
0.01 & 206.00221 & 0.0000107 \\
0.001 & 206.00092 & 0.0000045 \\
0.0001 & 205.99985 & 0.0000007 \\
\hline
\end{tabular}

If the recursion was allowed to go thirty levels deep we found:

\section{$\begin{array}{lll}0.0001 & 206.00005 & 0.0000002\end{array}$}

The graph below shows the aduptive clustering of the points of evaluation around the pole of the function (taken from the first example above).

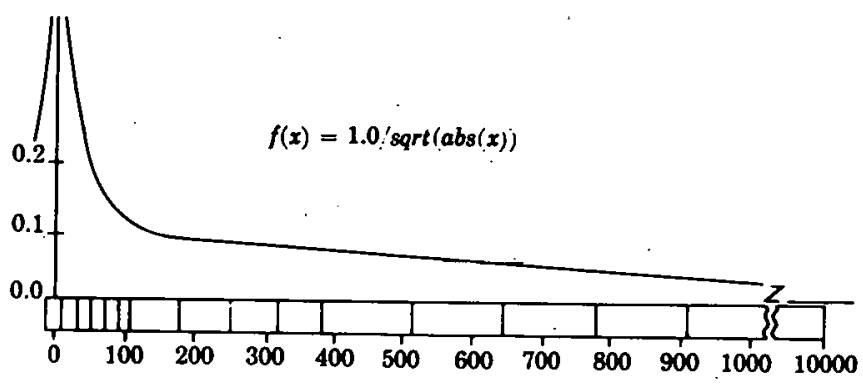

Each verticul line represents a point of evaluation for the function during the execution of the call:

integral $(f,-0.0,10000.0,0.1)$;
ALGORITHM 146

MULTIPLE INTEGRATION

Wrilam Marshall McKegman*

Stanford University, Stanford, Calif.

- This work was supported in part by the Office of Naval Research under contract Non4 225(37).

real procedure MultipleIntegral $(F)$ limits: $(a, b)$ order: $(n)$ tolersnce: $(e p s)$;

real procedure $F$; real array $a, b$; real eps; integer $n$;

begin comment $F$ is a function of $n$ variables which are stored in an internal array $x$. MultipleInlegral approximates the multiple integral of $F$ between the $n$ pairs of limits stored in the parameter arrays $a$ and $b$. For a mesh of $k$ steps on each axis, the number of function evaluations required for an integral of $n$th order is approximately $k \uparrow n$. One consequence is that the practical limit on $n$ is quite small. Another is that any inefficiency in the (undefined) procedure Inlegral will reflect itself to the $n$th power in MultipleIntegral. The adaptive procedure Integral is recommended;

real array $x[1: n+1]$; integer axis;

real procedure Integral $(F)$ limits: $(a, b)$ tolerance: $(e p s)$;

real procedure $F$; real $a, b$, eps;

begin comment The body of procedure Integral is left undefined. For it one may substitute any procedure of the same name that evaluates the integral of a function of a single variable between the real limits $a$ and $b$;

end Integral;

real procedure $M I(y)$; real $y$;

begin comment Recursive multiple integration;

$x[a x i s]:=y$;

axis $:=$ axis -1 ;

$M I:=$ if axis $=0$ then $F(x)$ else

Integral (MI, a[axis], b[axis], eps/n);

axis : = axis +1 ;

end $M I$;

axis $:=n+1$;

MultipleIntegral : = $M I(0)$

end MultipleIntégral

\section{CERTIFICATION OF ALGORITHM 146}

MULTIPLE INTEGRATION [W. M. McKeeman, Comm. ACM 5 (Dec. 1962), 604]

Niklaus Wirth (Recd. 6 Jan. 1964)

Computer Science Div., Stanford U., Stanford, Calif. .

Algorithm 146 was translated into a generalized ALgoL [cf. N. Wirth, A generalization of ALGOL, Comm. ACM 6 (Sept. 1963), 547-554] and successfully run on the Stanford IBM 7090 computer. Algorithm 60, Romberg Integration [Comm. ACM \& (June 1961), 255; 6 (Mar. 1962), 168; 6 (May 1962), 281] was used for the real procedure Integral.

The main disadvantage of Algorithm 146 is that the bounds of the domain of integration must be constant, i.e. the domain must always have the form of a rectangular hyperbox. 
comment Computes the logarithmic derivative of the factorial function defined by:

$$
\Psi(x)=\frac{(x !)^{\prime}}{x !}=\frac{\Gamma^{\prime}(x+1)}{\Gamma(x+1)}
$$

We make use of the expansion: (1) $\Psi(x)=\ln x+1 / 2 x-$ $1 / 12 x^{2}+1 / 120 x^{4}-1 / 252 x^{5}+\epsilon$, (2) $\epsilon<1 / 240 x^{2}$ and of the recursion relation, (3) $\Psi(x)=\Psi(x+n)-(1 /(x+1)+\ldots+1 /(x+n))$. For $x<-1$ we use: $(4) \cdot \Psi(-x)=\pi \tan \pi(x+0.5)+\Psi(x-1)$. The value of $x$ is increased up to $a$. Then $\Psi$ is calculated by and (1). The error is then less than $1 / 240 a^{8}$;

begin real $p s i, p e i ; \quad p s i:=0$;

if $x>-1 \wedge x \neq 0$ then go to pos;

if $x=0$ then begin $p s i:=-0.5772156649$; go to exit end;

begin integer $x 1 ; x 1:=x$;

if $x=x 1$ then go to errexit end

comment $p s i$ is infinite;

pei $:=3.141592654 ; x:=-x-1$;

$p 8 i:=p e i \times \tan (p e i \times(x+0.5))$;

pos: if $x \geqq a$ then go to large;

$x:=x+1 ; p s i:=p s i-1 / x$; go to pos;

large: begin real $y ; y:=1 / x$;

$p s i:=p s i+\ln (x)+y / 2-y \uparrow 2 / 12+y \uparrow 4 / 120-y \uparrow 6 / 252$

exit: $p$ sif $:=p s i$

end $p$ sif

\section{CERTIFICATION OI ALGORITHM 147}

ISSIF [D. Amit, Comm. AC'M., Dec. 62]

Hexry C. Thacher, JR.*

Reactor Eng. Div., Argonne National Lab., Argonne, Ill.

- Work supported by the U.S. Atomic Energy Commission.

The following minor errors were noted in this algorithm:

a. (3) in the comment should read $\varepsilon<1 / 240 x^{8}$.

b. The function tan is not a standard ALGOL function. It should be declared, or replaced by $\sin () / \cos ()$.

c. The block labelled large should be closed by inserting end inmediately after 252 .

The efficiency of the progran would be improved by the following modifications:

a. Let the statement

$$
\text { if } x=0 \text { then begin ... end; }
$$

be the first statement of the procedure body.

b. Delete the condition $x \neq 0$ from the if clause,

$$
\text { if } x>-1 \wedge x \neq 0 \text { then. . }
$$

c. Delete the declaration of $p e i$, and the assignment of the value of 3.141592654 to $p e i$ in the statement

psi : = pei $\times \sin ($ pei $\times(x+0.5)) / \cos (p e i \times(x+0.5))$;

replace $p e i$ by the value 3.141592654 .

d. Replace the block labelled large by:

large: begin real $y ; x:=1 / x ; y:=x \times x$;

$p s i:=p 8 i-\ln (x)+x / 2-((y / 252-0.008333333333) \times y+$ $0.08333333333) \times y$ end;

With these changes, the body of the procedure was translated and run on the L,GP-30 conputer using the Dartmouth Scalp processor. The program was used to tabulate $p s i f(x)$ for $x=-1$ $(0.5) 0(0.005) 1.250$. With $a=3.0$ the results agreed with tabulated values to within 3 in the 6 th decimal place. This is considered satisfactory, since one decimal place is lost in applying the recurrence. Running time, including output on the Flexowriter and computation of new values of the independent variable, averaged about 30 seconds per value.

It should be observed that $p \operatorname{sif}(x)$ is $\Psi(x+1)$ as tabulated, for example, by Jahnke-Emde-Losch.
ALGORITHM 148

\section{TERM OF MAGIC SQUARE}

D. M. Collison

Elliott Brothers (London) Ltd., Borehamwood, Herts.

integer procedure magiclerm $(x, y, n)$; value $x, y, n ;$ integer $x, y, n$

comment for the magic square $s[1: n, 1: n]$, magicterm generates the element $s[x, y]$, where $n>2$ and $n$ is odd. De la Loubere's method is used;

begin integer $b, c$; $b:=y-x+(n-1) \div 2 ; c:=y+y-x$

if $b \geqq n$ then $b:=b-n$ else if $b<0$ then $b:=b+n$;

if $c>n$ then $c:=c-n$ else if $c \leqq 0$ then $c:=c+n$;

magicterm $:=b \times n+c$

end magicterm

CERTIFICATION OI: ALGORITHM 148

TERMI OF MAGIC SQLARE [D. M. Collison, Comm. $A C M$, Dec. 62]

Dмiткі тноко

San Jose State College, San Jose, Calif.

This algorithm was translated into Fortran and Forgo for the IBM 1620. No changes in the program were necessary. The elements of magic squares of odd orders up to 15 were generated satisfactorily:

\section{CERTIFICATION OF ALGORITHM 148}

TERM OF MAGIC SQLARE [D. II. Collinson, Comm. $A C M$, Dec. 1962]

J. N. R. BAKNECUT

University of Alberta, Calgary; Calgary, Alberta, Canada

MAGICTERM was translated into Fortran for the IBM 1620 The procedure was tested for terms of squares up to order 13 . Correct results were obtained. For determination of complete squares operuting time was not significantly different from $\mathrm{Al}$ gorithm 118 .

\section{ALGORITHM 149}

\section{COMPLETE ELLIPTIC INTEGRAL}

\section{J. N. Merner}

Burroughs Corp., Pasadena, Calif.

comment The following two procedures, along with a test program were compiled and run by Peter Naur on the DISADEC computer. Compilation time for the .9 pass compiler was less than 10 seconds. The elliptic integral of the form

$$
\int_{0}^{\pi / 2} \frac{d t}{\sqrt{a^{2} \cos ^{2} t+b^{2} \sin ^{2} t}}
$$

is evaluated by replacing $a$ and $b$ by their arithmetic and geometric means, respectively. ELIP $\&$ is a nonrecursive procedure to accomplish the same thing;

reál procedure $\operatorname{ELIP} 1(a, b)$; value $a, b$; real $a, b$;

$E L I P 1:=$ if $a b s(a-b)<10-8 \times a$

then $3.14159265 / 2 / a$

else $E L I P 1((a+b) / 2$, sqrl $(a \times b))$;

real procedure $\operatorname{ELIP} \&(a, b)$; value $a, b ;$ real $a, b$; begin real $C$;

$L: \quad C:=(a+b) / 2 ; \quad b:=\operatorname{sqri}(a \times b) ; a:=c ;$

if $a b s(a-b)<_{10}-8 \times a$ then $E L I P 2:=3.14159265 / 2 \% a /$

else go to $L$ end

SEE ALGORITHM 55 


\section{ALGORITHM 150 \\ SYMINV2}

\section{H. Rutishauser}

Eidg. Technische Hochschule, Zurich, Switzerland

procedure syminv $2(a, n)$ result: (a) exit: $(f a i l)$; value $n$; integer $n$; array $a$; label fail;

comment syminv 2 obtains inverse of a symmetric matrix $a$ of order $n$ by a method which is similar to that given by Busing and Levy [Comm. ACM 5 (1962), 446] but requires no interchanges of rows and columns nor storage space for an additional matrix $Q$, yet is numerically equivalent. The procedure requires the upper triangular part of $a$ to be given and overwrites it by the upper triangular part of the inverse which is again denoted by $a$. All pivots are chosen on the diagonal, and if all further diagonal elements which are eligible as pivots vanish (this is impossible for a positive definite matrix a) then exit through fail occurs;

\section{begin}

real bigajj;

integer $i, j, k$;

real array $p, q[1: n]$

Bò̀lean array $r[1: n]$;

for $i:=1$ step 1 until $n$ do $r[i]:=$ true;

grand loop:

for $i:=1$ step 1 until $n$ do

begin.

search for pivot:

bigajj.:=0;

for $j:=1$ step 1 until $n$ do

begin

if $r[j] \wedge a b s(a[j, j])>b i g a j j$ then

begin

$b_{i g a j j}:=a b s(a[j, j]) ;$

$k:=j$

end:

end;

if $b i g a j j=0$ then go to fail;

preparation of elimination step $i$ :

$r[k]:=$ false;

$q[k]:=1 / a[k, k]$

$p[k]:=1$

$a[k, k]:=0$;

for $j:=1$ step $1^{i}$ until $k-1$ do

begin

$p[j]:=a[j, k]$

$q[j]:=($ if $r[j]$ then $-a[j, k]$ else $a[j, k]) \times q[k]$

$a[j, k]:=0$

end;

for $j:=k+1$ step 1 until $n$ do

begin

$p[j]:=$ if $r[j]$ then $a \mid k, j]$ else $-a|k, j|$;

$q[j]:=-a[k, j] \times q[k]$;

$a[k, j]:=0$

end;

elimination proper:

for $j:=1$ step 1 until $n$ do

for $k:=j$ step 1 until $n$ do

end grand loop

$$
a[j, k]:=a[j, k]+p[j] \times q[k]
$$

end syminv2
CERTIFICATION OF ALGORITHM 150

SYMINV2 [H. Rutishauser, Comm. ACM 6 (Feb. 1963), 67]

Peter Naur (Recd 27 Sept. 63)

Regnecentralen, Copenhagen, Denmark

Since the translator refuses to run programs with more than one occurrence of the same identifier in a formal parameter list, the second $a$ was taken out when this procedure was run with the Gier Algol system [cf. also the discussion in Comm. ACM 6 (July 1963), 390]. Otherwise it ran smoothly. For testing the accuracy, segments of the Hilbert matrix were inverted and the results multiplied by the original segment and compared with the unit matrix. The largest deviation in any element was found to be:

$\begin{array}{cccc}\text { Order } & \begin{array}{c}\text { Max. deviation from elements } \\ \text { of the unit matrix }\end{array} & \text { Order } & \begin{array}{c}\text { Max. deriotion from elements } \\ \text { of the unit malrix }\end{array} \\ 2 & -1.4910-8 & 6 & -7.3210-3 \\ 3 & -2.3810-7 & 7 & -3.5910-1 \\ 4 & -1.5310-5 & 8 & -2.95101 \\ 5 & -3.3610-4 & 9 & -1.25101\end{array}$

These figures may be sompared direstly with the ones related to Algorithms 120, INVERSION II, and gjr [Comm. ACM 6 (Aug. 1963), 445]. A comparison shows that all three algorithms yield about the same accuracy, with syminv 2 being the best in most cases, however. This is not too surprising since the knowledge that the matrix is symmetric ought to simplify the calculation considerably.

The lengths of the three procedures after translation are as follows:

$\begin{array}{lc} & \text { Number of } \\ \text { GIER words } \\ \text { syminv2 } & 216 \\ \text { INVERSION II } & 279 \\ \text { gjr } & 302\end{array}$

Execution times for syminv2 in Gier Algol are:

$\begin{array}{cc}\text { Order } & \text { Time (sec) } \\ 5 & 1 \\ 10 & 3.5 \\ 15 & 10.5 \\ 20 & 23\end{array}$

This is about half the time of execution of INVERSION II or gjr.

REMARK ON ALGORITHM 150

SYMINV2 [H. Rutishauser, Comm. ACM, Feb. 1963]

H. Rutishauser

Eidg. Technische Hochschule, Zurich, Switzerland

procedure syminv $2(a, n)$ result : $(a)$ exit : (fail); $\cdots$ indicates that the value of parameter " $a$ " is changed by the computing process (the matrix $a$ is changed into its inverse, whereby the given matrix is destroyed). In any procedure call, the two actual parameters corresponding to the two $a$ 's must be identical, otherwise the action of the procedure will be undefined (by virtue of the substilulion rule). The user may also change the procedure heading into syminv $2(a, n)$ exit : (fail); $\cdots$ without changing the effect of the procedure.

Editor's Note: The ALCOR group has adopted the rule that if the value of a parameter is changed by the execution of the procedure, then the parameter should be listed twice. Although the Algol 60 Report does not forbid listing a formal parameter twice, it would appear that a compiler which thus restricts the language could not accept some of the examples given in the Algol 60 Report: 
REMARK ON ALGORITHM 150

SYMINV2 [H. Rutishauser, Comm. ACM, Feb. 1963]

Arthur Evans, Jr.

Carnegie Institute of Technology, Pittsburgh, Pennsylvania

The identifier " $a$ " appears twice in the procedure heading as a formal parameter. It is not clear that this situation has any meaning in Algol. Indeed, it is not at all obvious how one might translate the procedure. If the actual parameters corresponding to the two formal parameters with the same identifier are different there is no way for the translator (or for the reader) to distinguish which ' $a$ ' is to be used. Further, it would take a detailed examination of the published algorithm to determine how this situation might be corrected. It is certainly not clear that it would be safe merely to delete one occurrence of the formal parameter ' $a$ ', since the operation of the algorithm might require that two separate matrices be available.

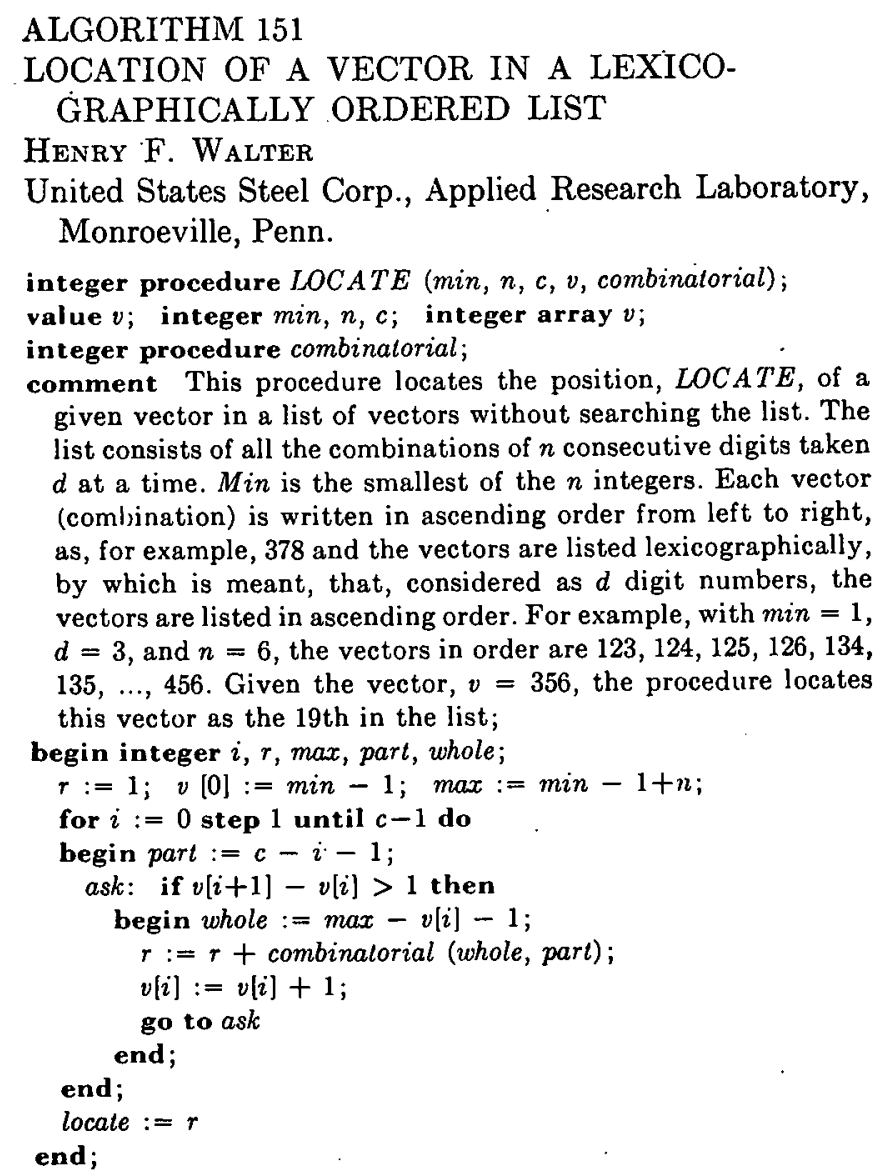

ALGORITHM 152

\section{NEXCOM}

JoHN Hopley

Peat, Marwick, Mitchell \& Co., London, England

procedure nexcom (char, $N$, setcomplete, nullvector);

array char; integer $N$;

label setcomplete, nullvector;

comment char is a column vector containing $N$ elements each of which is either 1 or 0 . Nexcom transforms char into another vector containing the same number of 1 's and 0 's, but in a different sequence. Startin ; with char in the state of havin $r 1$ in each of the element positions $1, \ldots, r$ and zeros elsewhere then repeated application of nexcom generates all " $\mathrm{Cr}$ patterns of char. The procedure terminates if the presented vector char has 1 in each of the positions $N, N-1, \ldots N-r+1$ and zeros elsewhere. Termination is indicated by exit through the formal label 'selcomplele'. If char is the null vector then procedure exists through the formal label 'nullvector';

begin integer $n, p, m$;

comment find the first 1 in char;

for $n:=1$ atop 1 until $N$ do if

char $[n]=1$ then go to $A$;

go to nullvector;

comment how many adjacent 1 's;

$A: \quad p:=0$;

for $m:=n+1$ step 1 until $N$ do

if char $[m]=1$ then $p:=p+1$ else go to $B$

comment Have all combinations been generated;

$B:$ if $p+n=N$ then go to setcomplete;

comment Set up next combination; $\operatorname{char}[n+p+1]:=1$;

for $m:=n+p$ step -1 until $n$ do $\operatorname{char}[m]:=0$;

for $m:=1$ step 1 until $p$ do $\operatorname{char}[m\}:=1$;

end nexcom;

\section{ALGORITHM 153 \\ GOMORY}

F. L. BAUER

Johannes Gutenberg-Universität, Mainz, Germany

procedure Gomory $(a, m, n)$ result: (a) exit: (no solution);

value $m, n$;

integer $m, n$;

integer array $a$;

label no solution;

comment Gomory algorithm for all-integer programming. The objective of this procedure is to determine the integer solution of a linear programming problem with integer coefficients only. The tableau-matrix $a$ consists of $m+1$ rows and $n$ columns. The top row of $a$ is the objective row, the last column represents the right-hand sides. The tableau-columns, with the exception of the last column, have to be lexicographically positive. The algorithm is finished if all entries in the last column, except the top most entry, are nonnegative. Then the top most entry of the last column represents the value of the objective function. The other entries of the last column define the coordinates of the optimal solution. There are always the same variables connected with the same rows. The exit no solution is used if a row is found which has a negative entry in the last column, but otherwise only nonnegative entries;

begin integer $i, k, j, l, r$;

real lambda;

integer array $\ell[1: n-1], c[1: n]$; 


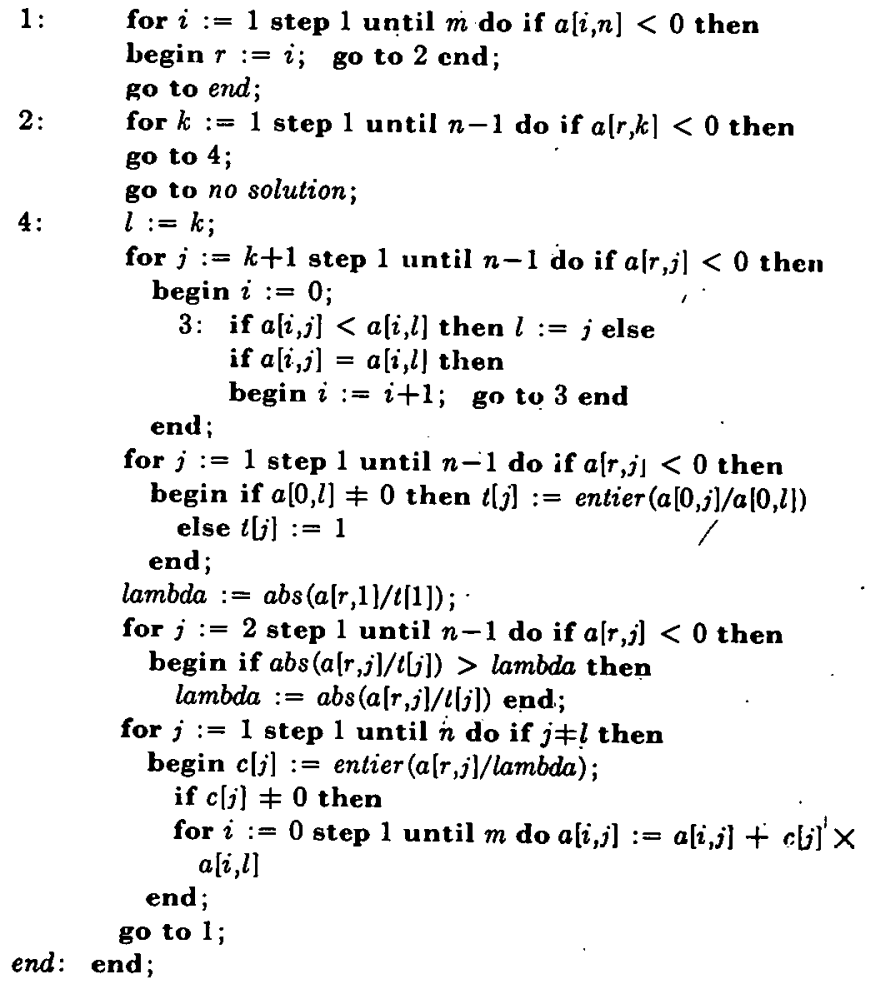

\section{CERTIFICATION OF ALGORITHM 153}

GOMORY [F. L. Bauer, Comm. ACM 6, Feb. 1963]

B. Lefkowitz AND D. A. D'Esopo*

Stanford Research Institute, Menlo Park, California

- Work supported by Office of Naval Research.

GOMORY was hand-coded in BALGOL for the Burroughs 220 and in FORTRAN for the CDC 1604. The following corrections should be made:

The statement

lambda: $=\operatorname{als}(a[r, 1] / t[1])$;

should read

lambda $:=a b s(a[r, l] / t[l])$;

The statement

for $j:=2$ step 1 until $n-1$ do if $a[r, j]<0$ then

should read

for $j:=1$ step 1 until $n-1$ do if $a[r, j]<0$ then

The following changes to Bauer's program were made to in. crease its efficiency and reduce storage requirements.

Change the statement

begin integer $i, k, j, 1, r$;

to read

begin integer $i, k, j, 1, r, c, t$;

Change the statement

real lambda;

to read

real lambda, lambd;

Delete the statement

integer array $t[1: n-1], c[1: n]$

Before the statement

for $j:=1$ step 1 until $n-1$ do if $a[r, j]<0$ then

insert the statement

lambda: $=1.0$;

Change the statement

hegin if $a[0, l] \neq 0$ then $\iota[j]:=\operatorname{entier}(a[0, j] / a[0, l])$

to read

begin if $a[0, l] \neq 0$ then $t:=\operatorname{entier}(a[0, j] / a[0, l])$
Change the statement

else $t[j]:=1$

to read

else $t:=1$

After the statement

else $t[j]:=1$

insert the statements

lambd $:=-\dot{a}[r, j] / t$

lambda := if lambda < lambd then lambd else lambda:

Delete the statements starting with

lambda $:=a b s(a[r, 1] /([1])$;

$u p$ to and including

lambda $:=a b s(a[r, j] / t[j])$ end;

Change the statement

begin $c \mid j]:=$ entier $(a[r, j] / l a m b d a)$;

to read

begin $c:=$ entier $(a[r, j] /$ lambda $)$;

Change the statement.

if $c[j] \neq 0$ then

to read

if $c \neq 0$ then

Change the statement

for $i:=0$ step 1 until $m$ do $a[i, j]:=a[i, j]+c[j] \times$ to read

for $i:=0$ step 1 until $m$ do $a[i, j]:=a|i, j|+c \times$

'The "tie-breaking" procedure embodied in the three statements beginning at

$$
3: \text { if } a[i, j]<a \mid i, l] \text { then } l:=j \text { else }
$$

will fail if the two columns being compared are identical. Although this cannot happen on the first iteration, it may occur later. To test for this condition change the two statements beginning with

$$
\text { begin } i:=i+1 \text {; go to } 3 \text { end }
$$

to read

begin $i:=i+1$; if $i>m$ then go to 31 else go to 3 end; 31 : end:

The revised algorithm yielded satisfactory answers on a ten equation-seven variable problem in 159 iterations and a 35 .equation 14-variable problem in 447 iterations.

The following comments may be helpful for preparing a problem for GOMORY. The problem constraints must be stated in the form:

$$
\sum_{j} \dot{a}_{i} x_{j}+s_{i}=b_{i}
$$

where the $s_{i}$ are slack variables. The columns representing these slack variables need not appear in the initial tableau-matrix $a$.

Since the only variables in the solution that will necessarily be non-negative are the $s_{i}$, any non-negativity constraints on the other variables must be among the above equations (e.g. the constraint $x_{i} \geqq 0$ is represented by $-x_{i}+s_{k}=0$ ).

The size of the integers in the $b$ vector substantially affects the number of iterations.

The requirement that all but the last tableat-columns be lexicographically positive means that the first nonzero element in these columns must be positive.

Editor's Note: Prof. Bauer wishes to indicate that for the Algorithm 153, GOMORY, credit is due to $\mathrm{Ch}$. Witzgall, who wrote the draft. 


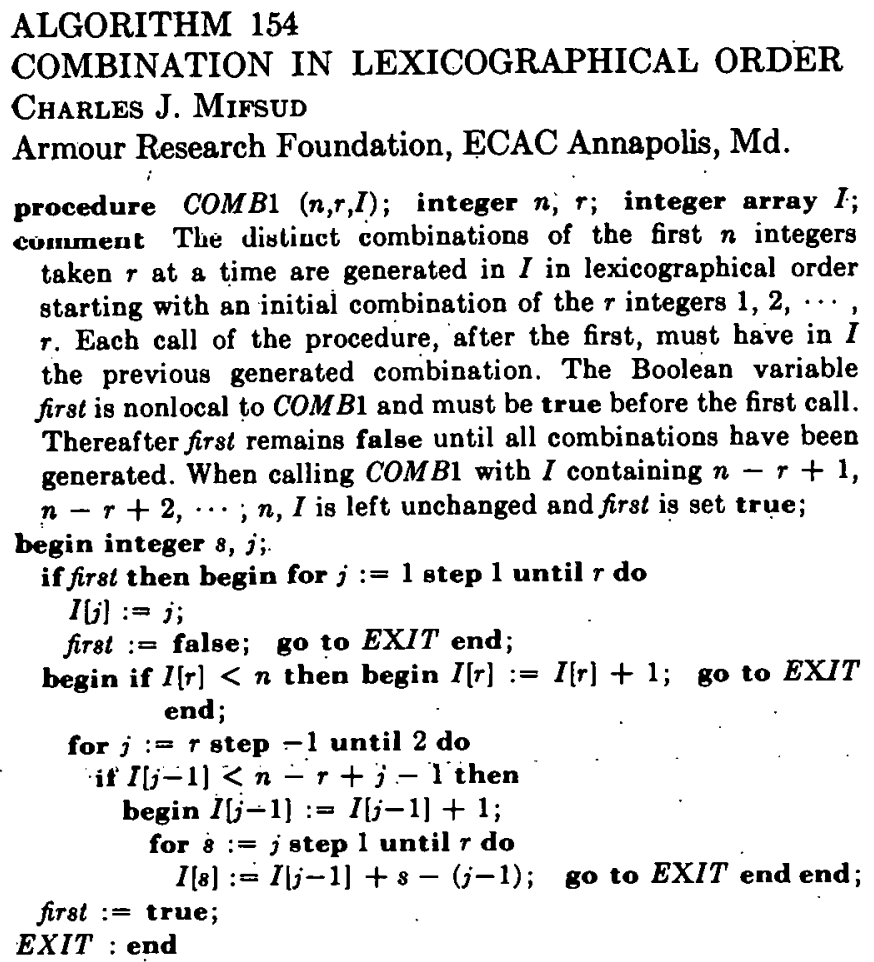

CERTIFICATION OF ALGORITHM 154 COMBINATION IN LEXICOGRAPHICAL ORDER [Charles J. Mifsund, Comm. ACM, Mar. 1963]

K. M. Bosworth

I.C.T. Ltd., Hayes, Middlesex, England

This procedure was tested

$$
\text { for } r:=1 \text { step } 1 \text { until } n \text { with } n=6
$$

with correct results.

\section{ALGORITHM 155 \\ COMBINATION IN ANY ORDER}

Charles J. Mifsud

Armour Research Foundation, ECAC Annapolis, Md.

procedure $\operatorname{COMB2}(m, M, n, r, s, S, T O T A L)$; integer array $m$, $M, S ;$ integer $n, r, 8, T O T A L$;

comment Each call of COMB2 generates a distinct combination $S$, (if possible) of the $n$ integer values of $J$ taken $r(r>1)$ at a time if $J$ consists of $m[1]$ integers each equal to $M[1]$, and $m[2]$ integers each equal to $M[2]$, and so on, there being 8 integers available. TOTAL must be set to zero before the first call of COMB2 and thereafter TOT $A L$ is increased by one after each new combination is generated. To speed up the machine operation arrange the $s$ integers in $M$ such that $m[1] \geqq m[2] \geqq \cdots \geqq$ $m[8]$;

begin integer $i, j, t, p$; own integer array $J[1: n], I[1: r]$; own Boolean first; if TOT $A L=0$ then begin $t:=1 ; p:=0$; for $j:=1$ step 1 until $s$ do

begin $p:=p+m[j]$;

for $i:=t$ step 1 until $p$ do

begin $J[i]:=M[j]$;

$t:=t+1$ end end;

first : = true end;

1: $\operatorname{COMB1}(n, r, I)$;

if first then go to $E X I T$;

if $I[1]=1$ then go to 2 else go to 3 ;

2: for $j:=2$ step 1 until $r$ do

if $(J[I[j]]=J[I[j]-1]) \wedge(I[j]>I[j-1]+1)$ then go to 1 ; go to 4;

3: if $J[I[1]]=J[I[1]-1]$ then go to 1 else go to 2 ;

4: for $j:=1$ step 1 until $r$ do $S[j]:=J[I[j]]$

: TOT $A L:=$ TOT $A L+1$;

EXIT: end

\section{CERTIFICATION OF ALGORITHM 155}

COMBINATION IN ANY ORDER [Charles J. Mifsud, Comm. ACM, Mar. 1963]

K. M. Büswók'tr

I.C.T. Ltd., Hayes, Middlesex, England

This procedure was tested using

$$
\begin{array}{llll}
m[1]=4 & m[2]=3 & m[3]=2 & m[4]=2 \\
M[1]=4 & M[2]=7 & M[3]=9 & M[4]=16
\end{array}
$$

and for $r:=1$ step 1 until $s$

It is correctly generated for $r=1$ the four combinations $4,7,9$, 16 , as well as the ten combinations for $r=2$, the eighteen combinations for $r=3$, and the twenty -six combinations for $r=4$.

Changes made due to compiler limitations were (i) systematic changes of upper case letters where there was conflict due to having only one case of letters, (ii) transfer of own declared variables tc non-local variables, and (iii) integer labels to identifiers.

\section{ALGORITHM 156}

ALGEBRA OF SETS

Charles J. Mifsud.

Armour Research Foundation, ECAC Annapolis, Md.

procedure INOUT $(A, n, S U M)$; real array $A$; integer $n$;

real $S U M ;$
comment $S U M=\sum_{1} A_{i}-\sum_{2} A_{i} A_{j}+\sum_{i} A_{i} A_{j} A_{k}-\cdots \pm$ $A_{1} A_{2} \cdots A_{n}$ is formed where the symbols $\sum_{1}, \sum_{2}, \sum_{8}, \cdots$, $\sum_{n-1}$ stand for summation of the possible combinations of the numbers $A_{1}, A_{2}, \cdots, A_{n}$ taken one, two, three, $\cdots,(n-1)$ at a time;

begin real $j$, part, $T$; integer $i, r$; integer array $I[1: n]$;

Boolean first; $r:=S U M:=0 ; j:=-1$

$B$ : first $:=$ true; $r:=r+1$; part $:=0$;

$A$ : $\operatorname{COMB1}(n, r, I)$;

if first then begin $j:=-1 \times j ;$ part $:=j \times$ part; $S U M:=S U M+$ part;

if $r<n$ then go to $B$ else go to EXIT end;

$T:=1$;

for $i:=1$ step 1 until $r$ do

$T:=A[I[i]] \times T$

parl $:=$ part $+T ;$ go to $A$;

EXIT: end 


\section{CERTIFICATION OF ALGORITHM 156}

ALGEBRA OF SETS [Charles J. Mifsud, Comm. ACM, Mar. 196.3]

K. M. Bosworth

I.C.T. Ltd., Hayes, Middlesex, England

One correction required in this procedure is the systematic change of label $A$ to avoid conflict with the formal parameter array $A$.

The procedure was then tested for $n=9$ and $A i=i, \quad i=1$, $\cdots, n$, producing the correct answer $S U M=1$.

Two other tests with arbitrary values of $A i$ and $n=4$ were also correct.

\section{ALGORITHM 157}

\section{FOURIER SERIES APPROXIMATION}

Charles J. Mifsud

Armour Research Foundation, ECAC Annapolis, Md.

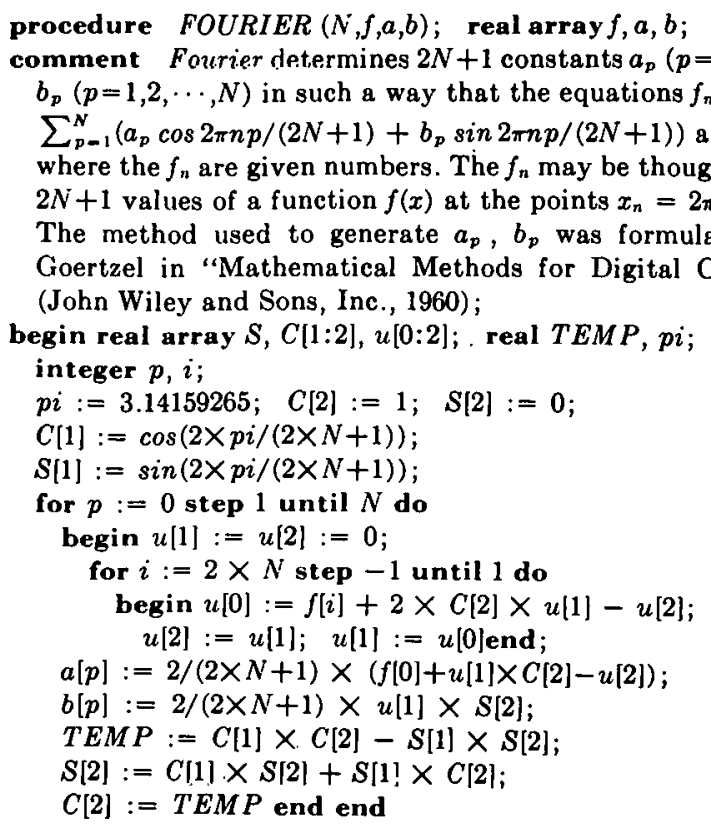

\section{REMARK ON ALGORITHM 157}

FOURIER SERIES APPROXIMATION [C. J. Mifsud, Comm ACM, Mar. 1963]

RICHARD GeORGE*

Argonne National Laboratory, Argonne, Ill.

This algorithm was written in FAP language for the 32-K IBM 704. It was tested on a sawtooth curve, and the sawtooth was recreated by summing the expansion up through the $2 N+1$ constants, with excellent results.

The arrays $S, C$ and $u$ are never referenced with a variable subscript. For a saving of time, I suggest that simple variables be used instead.

By declaring one additional real variable, one can bring the phrase

$$
2 /(2 \times N+1)
$$

outside of the for loops, because $N$ does not change through the procedure. This results in a saving of $4 N+2$ mult-ops.
REMARK ON ALGORITHM 157

FOURIER SERIES APPROXIMATION [Charles J.

Mifsud, Comm. ACM, Mar. 1963]

George R. Schubert*

University of Dayton, Dayton, Ohio

- Undergraduate research prcject, Computer Science Program, Univ. of Dayton.

Algorithm 157 has been modified to fit $2 N$ data points and has run successfully on the Burroughs 220 using BALGOL. With the modifications, $2 N$ constants $a_{p}(p=0,1, \cdots, N)$ and $b_{p}$ $(p=1,2, \cdots, N-1)$ are determined such that the equation $f_{n}=a_{0} / 2+\sum_{p-1}^{N-1}\left(a_{p} \cos \pi n p / N+b_{p} \sin \pi n p / N\right)+a_{N} / 2 \cos \pi n$ is satisfied.

In the modified procedure, the second and third lines after the integer declaration should read:

$C[1]:=\cos (p i / N)$;

$S[1]:=\sin (p i / N)$;

The second for statement should read:

for $i:=2 \times N-1$ step -1 until 1 do

The lines containing the $a$ and $b$ coefficients should read:

$a[p]:=(f[0]+u[1] \times C[2]-u[2]) / N$;

$b[p]:=(u[1] \times S[2]) / N$;

Reference: R. W. Hamming, Numerical Methods for Scientists and Enginecrs, pp. 68-73 (McGraw-Hill, 1962).

\section{ALGORITHM 158 (ALGORITHM 134, REVISED) EXPONENTIATION OF SERIES}

HENRY E. FETTIS

Aeronautical Research Laboratories, Wright-Patterson Air Force Base, Ohio

procedure $S E R I E S P W R(A, B, P, N)$; value $A, P, N$; array $A, B$; integer $N$;

comment This procedure calculates the first $N$ coefficients $B[i]$ of the series $g(x)=f(x) \uparrow P$ given the first $N$ coefficients of the series

$$
f(x)=1+\sum A[i] \times x \uparrow i \quad(i=1,2,-,-, N) .
$$

$P$ may be any real number. Setting $P:=0$ gives the coefficients for $L N(g(x))$;

begin integer $i, k$;

real $P, S$;

if $P=0$ then $B[1]=A[1]$;

else $B[1]:=P \times A[1]$;

for $i:=2$ step 1 until $N$ do

begin $S:=0$;

for $k:=1$ step 1 until $i-1$ do

$S:=S+(P \times(N-k)-k) \times B[k] \times A[N-k]$;

$B[i]:=P \times A[i]+(S / i)$

end for $i$;

end SERIESPWR

CERTIFICATION OF ALGORITHM 158

EXPONENTIATION OF SERIES [H. E. Fettis, Comm.

$A C M$, Mar. 1963]

J. Dennis Lawrence

Lawrence Radiation Laboratory, Livermore, Calif.

This procedure was translated into Fontran and run on the Remington-Rand Larc Computer. Three changes are necessary.

(1) The last line of the comment should read for the natural logarithm of $f(x)$; 
(2) The third line from the end should read

$$
S^{\prime}:=S+(P \times(i-k)-k) \times B[k] \times A[i-k] ;
$$

(This line was given correctly in algorithm 134.)

(3) The second line from the end apparently should read

$$
B[i]:=A[i]:=(S / i) \text {; }
$$

for the case $P=0$ only. Probably the best way to incorporate this is by making two changes:

(a) Change the if clause to read

if $P=0$ then $R:=1$ else $R:=P ; \quad B[1]:=R \times A[1]$;

(b) Change the second line from the end to read

$$
B[i]:=R \times A[i]+(S / i) ;
$$

A large number of examples were run quite successfully; the following give representative samples.

(1) $\left(1+2 x+3 x^{2}+0.5 x^{8}\right)^{2}=1+4 x+10 x^{2}+13 x^{8}+11 x^{4}+3 x^{5}+0.25 x^{6}$ (using $A[4]:=A[5]:=A[6]:=0$ ).

(2) Setting $P:=1$ gives $B[i]:=A[i]$.

(3) Let $f(x)=e^{x}=1+\sum_{i=1}^{n} \frac{1}{i !} x^{i}$ and let $P=\ln 2=.693147181$.

Then $g(x)=2^{x}=1+\sum_{i=1}^{B} \frac{(\ln 2)^{i}}{i !} x^{i}$. (See Table 1.)

(4) Let $f(x)=e^{x}$ and $P=-1$. Then $g(x)=e^{-x}$. For $P=0$, apparently the constant term of $g(x)$ should be zero instead of one.

\section{TABLE 1}

\begin{tabular}{r|c|c}
\hline & $A[i]$ & $B[i]$ \\
\cline { 2 - 3 } 1 & 1.000000000 & 0.693147181 \\
2 & 0.500000000 & 0.240226507 \\
3 & 0.166666667 & 0.055504109 \\
4 & 0.041666667 & 0.009618129 \\
5 & 0.00833333 & 0.001333356 \\
6 & 0.001388889 & 0.000154035 \\
7 & 0.000198413 & 0.000015253 \\
8 & 0.000024802 & 0.000001322 \\
9 & 0.000002756 & 0.000000102 \\
10 & 0.000000276 & 0.000000007 \\
\hline
\end{tabular}

(5) Let $f(x)=e^{x}$ and $P=0$. Then $g(x)=x$.

(6) Let $f(x)=\sum_{i=0}^{n} x^{n}$ and $P=0$. Then $g(x)=\ln \left(1-x^{n}\right)-\ln (1-x)=$ $\sum_{i=1}^{n} \frac{1}{i} x^{i}$. (See Table 2.)

TABLE 2

\begin{tabular}{r|c|c}
\hline & $A[i]$ & $B[i]$ \\
\cline { 2 - 3 } 1 & 1.0 & 1.000000000 \\
2 & 1.0 & 0.500000000 \\
3 & 1.0 & 0.333333340 \\
4 & 1.0 & 0.250000000 \\
5 & 1.0 & 0.200000000 \\
6 & 1.0 & 0.166666670 \\
7 & 1.0 & 0.142857140 \\
8 & 1.0 & 0.125000000 \\
9 & 1.0 & 0.111111110 \\
10 & 1.0 & 0.100000000 \\
11 & 1.0 & 0.090909100 \\
12 & 1.0 & 0.083333330 \\
13 & 1.0 & 0.076923080 \\
14 & 1.0 & 0.071428580 \\
15 & 1.0 & 0.066666660 \\
\hline
\end{tabular}

ALGORITHM 159

DETERMINANT

David W. DigBY

Oregon State University, Corvallis, Ore.

real procedure Determinant $(X, n)$;

value $n$; integer $n$; $\operatorname{array} X$;

comment Determinant calculates the determinant of the $n$-by$n$ square matrix $X$, using the combinatorial definition of the determinant. This algorithm is intended as an example of a recursive procedure which is somewhat less trivial than Factorial (Algorithm 33);

begin real $D$; integer $i$; Boolean array $B[1: n]$;

procedure Thread $(P, e, i)$;

value $P, e, i$; real $P$; integer $e, i$;

if $i>n$ then $D:=D+P \times(-1) \uparrow e$ else if $P \neq 0$ then

begin integer $j, f$;

$f:=0$;

for $j:=n$ step -1 until 1 do

if $B[j]$ then $f:=f+1$ else

begin

$B[j]:=$ true

Threat $(P \times X[i, j], e+f, i+1)$

$B[j]:=$ false;

end of loop;

end of Thread;

for $i:=1$ step 1 until $n$ do

$B[i]:=$ false;

$D:=0$

Thread $(1,0,1)$

Determinant $:=D$

end Determinant;

\section{CERTIFICATION OF ALGORITHM 159}

DETERMINANT [David W. Digby, Comm. $A C M$, March 1963]

ArNold Lapidus

Courant Institute of Mathematical Sciences, New York

University, New York, N. Y.

Algorithm 159 was translated into Fortran II for the IBM 7090 as part of a test of Fortran subroutines designed to facilitate the implementation of recursive procedures. As expected, the numerical results were poor. For the Hilbert matrices $H_{n}=\left(a_{i j}\right)$, $a_{i j}=1 /(i+j-1)$, results were as follows:

$\begin{array}{lcc}n & \text { Det } \Pi_{n} \text { (true) } & \begin{array}{c}\text { Det } H_{n} \text { (computed by } \\ \text { Algorithm 159) }\end{array} \\ 2 & 8.3333333(-2) & 8.3333332(-2) \\ 3 & 4.6296296(-4) & 4.6296231(-4) \\ 4 & 1.6534392(-7) & 1.6519334(-7) \\ 5 & 3.7492951(-12) & -2.9103830(-11)\end{array}$

Determinants of order 4 and 6 with integer elements were also evaluated. The algorithm gave full accuracy for these.

\section{ALGORITHM 160 \\ COMBINATORIAL OF M THINGS}

TAKEN N AT A TIME

M. L. Wolfson aNd H. V. Whight

United States Steel Corp., Momroeville, Penn.

integer procedure combination $(m, n)$;

value $n$; integer $m, n$; 
comment culculates the number of combinations of $m$ things taken $n$ at a time. If $n$ is less than half of $m$, then the progran calculates the combinations of $m$ things taken $m-n$ at a time which is the exact equivalent of $m$ things taken $n$ at a time;

begin integer $p, r, i$;

$p:=m-n$

if $n<p$ then begin $p:=n ; n:=m-p$ end;

if $p=0$ then begin $r:=1$; go to exit end;

$r:=n+1$;

for $i:=2$ step 1 until $p$ do $r:=(r \times(n+i)) / i$;

exit: combination $:=r$

end combination

\section{CERTIFICATION OF ALGORITHM 160}

COMBINATORIAL OF $M$ THINGS TAKEN $N$ AT

A TIME [M. L. Wolfson and H. V. Wright, Comm. $A C M$,

Apr. 1963]

DMitri Thoro

San Jose State College, San Jose, Calif.

Algorithm 160 was translated into Fortran II and Fongo for the IBM 1620. Correct results were obtained for values of $m$ up to 20

\section{CERTIFICATION OF ALGORITHM 160} COMBINATORIAL OF M THINGS TAKEN N AT

A TIME [M. L. Wolfson and H. V. Wright, Comm. $A C M$; April 1963]

ROBERT F. BLAKELY

Indiana Geological Survey, Bloomington, Ind.

Algorithm 160 was translated into Atco, a compiler for the Control Data Corp. G-15 computer (formerly the Bendix G-15).

With the restriction that $m \geqq \dot{n} \geqq 0$, correct results were obtained for all integer values of $m$ and $n$, where $0 \leqq m \leqq 10$. Several other values were tested and all results were correct.

\footnotetext{
AI.(i)IRITHM 161

COMBINATORIAJ, ()F: MI 'THINGS

PAKLA ONE AT A TIML, TWO AT A TIME, UP TO N AT A TIME

H. V. Wriciht aNd M. J. Wolrson

United States Steel Corp., Monrocville, Penn.

procedure combination vector $(m, n, v)$;

integer $m, n$; integer array $v$;

comment calculates all combinations of $m$ things taken from 1 to $n$ at a time. The result is a vector, $v$, within which the first clement is the combination of $m$ things taken 1 at a time, the second element is the combinations of $m$ things taken 2 at a time, the third element taken 3 at a time, $\cdots$, and the $n$th element taken $n$ at a time.

begin integer $i$;

$v[1]:=m$;

for $i:=2$ step 1 until $n$ do

$v[i]:=(v[i-1] \times(m-i+1)) / i$;

end combination vector
}

\section{CERTIFICATION OF ALGORITHM 161}

COMBINATORIAL OF $M$ THINGS TAKEN ONE AT

A TIME, TWO AT A TIME, UP TO $N$ AT A TIME

[H. V. Wright and M. L. Wolfson, Comm. ACM, Apr. 1963]

Dmitri Thoro

San Jose State College, San Jose, Calif.

Algorithm 161 was translated into ForTran II and Forgo for the IBM 1620. Correct. results were obtained for values of $m$ up to 20 .

\section{CERTIFICATION OF ALGORITHM 161}

COMBINATORIAL OF M THINGS TAKEN ONE AT

A TIME, TWO AT A TIME, UP TO N AT A TIME

[H. V. Wright and M. L. Wolfson, Comm. ACM, Apr. 1963]

David H. Coluins

Indiana Geological Survey; Bloomington, Ind.

Algorithm 161 was translated into Algo, a compiler for the Control Data Corp. G-15 computer (formerly the Bendix G-15).

With the restriction that $m \geqq n \geqq 1$, correct results were obtained for all integer values of $m$ and $n$, where $1 \leqq m=n \leqq 15$. Several other values were tested (including cases where $m \neq n$ ) and all results were correct.

\section{ALGORITHM 162}

\section{XYMOVE PLOTTING}

l'Rei) G. STоck'o.

Shell Development Co., Imeryville, Calif.

procedure $x y$ move $(X Z, Y Z, X N, Y N)$; value $X Z, Y Z, X N, Y N$; integer $X Z, Y Z, X N, Y N$;

comment xymove computes the code string required to move the pen of a digital incremental $X, Y$-plotter from an initial point $(X Z, Y Z)$ to a terminal point $(X N, Y N)$ by the "best" approximation to the straight line between the points. The permitted elemental pen movement is to an adjacent point in a plane Cartesian point lattice, diagonal moves permitted. The eight permitted pen movements are coded

$$
\begin{aligned}
& 1=+Y, \quad 2=+X+Y, \quad 3=+X, \quad 4=+X-Y, \\
& 5=-Y, \quad 6=-X-Y, \quad 7=-X, \quad 8=-X+Y .
\end{aligned}
$$

The approximation is "best" in the sense that each point traversed is at least as near the true line as the other candidate point for the same move.

xymove does not use multiplication or division.;

begin integer $A, B, D, E, F, T, I$, move;

:omment code $(J)$ is a procedure which returns a value of code according to the following table:

$\begin{array}{rrrrrrrrr}J & 1 & 2 & 3 & 4 & 5 & 6 & 7 & 8 \\ \text { code } & 1 & 2 & 3 & 2 & 3 & 4 & 5 & 4 \\ J & 9 & 10 & 11 & 12 & 13 & 14 & 15 & 16 \\ \text { code } & 5 & 6 & 7 & 6 & 7 & 8 & 1 & 8\end{array}$

plot (mote) is a procedure which sends move to the plotter as a plot ter command.;

if $X Z=X N \wedge Y Z=Y N$ then go to return; 


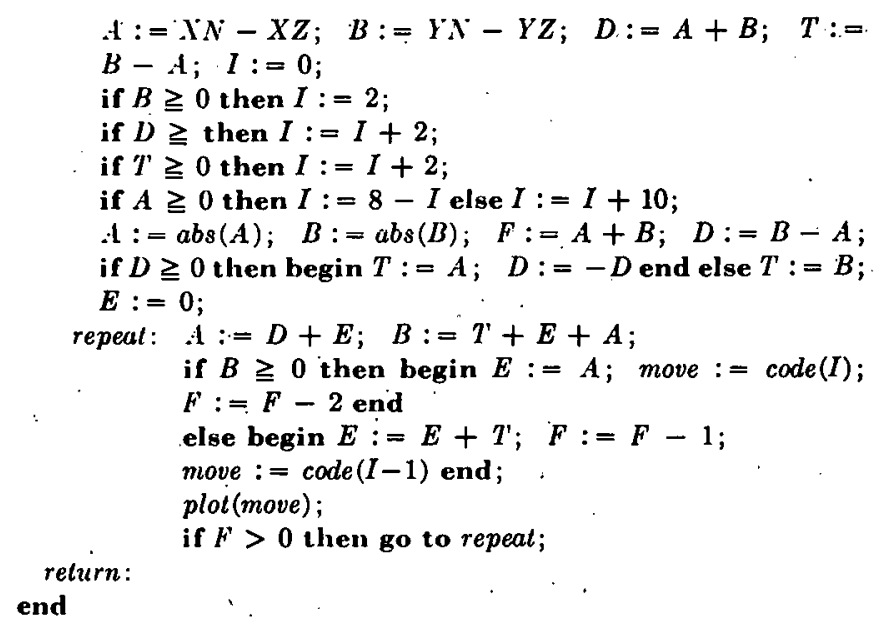

\section{CERTIFICATION OF ALGORITHM 162}

XYMOVE PLOTTING [Fred G. Stockton, Comm. ACM, Apr. 1863]

William E. Fletcher

Bolt, Beranek and Newman Inc., Los Angeles, Calif.

The line in the body of the procedure which read:

$$
\text { if } D \geqq \text { then } I:=I+2 \text {; }
$$

was corrected to read:

$$
\text { if } D \geqq 0 \text { then } I:=I+2 \text {; }
$$

With this one change the body of the procedure was transliterated into DECAI,-BBN and successfully, run on a PDP-1 computer utilizing the cathode ray tube output to display the path of a simulated digital incremental plotter.

\section{REMARK ON ALGORITHM 162 [J6]}

XYMOVE PLOTTING [F. G. Stockton, Comm. ACM 6

(Apr. 1963), 161;6 (Aug. 1963), 450]

D. K. Cavin (Recd. 10 Feb. 1964)

Oak Ridge National Laboratory, Oak Ridge, Tenn.

The following modifications were made to Algorithm 162 to decrease the average execution time. The last nine lines of Algorithm 162 are replaced by the following: move $:=\operatorname{code}(I-1) ; \quad I:=\operatorname{code}(I)$;

repeat: $A:=D+E ; B:=T+E+A$ if $B \geqq 0$ then begin $E:=A ; F:=F-2 ; p \operatorname{lot}(I)$ end else begin $E:=E+T ; F:=F-1 ; p l o t$ (move) end; if $F>0$ then go to repeat; return:

end

It is obvious that on any movement containing more than two elemental pen movements the use of the code procedure in the loop is redundant, since no more than two of the eight permitted pen movements are necessary for the approximation of any line. Therefore moving the call of the code procedure outside of the basic loop reduces the execution time whenever the $\mathrm{X}, Y$ movement requires more than two elemental pen movements. The procedures were coded in CODAP, the assembly language for the CDC 1604-A, and this modified version was 'approximately 40 percent faster in the loop than the original version. The timing comparisons used numbers in the range -2000 to 2000 with heavy emphasis on the subrange -150 to 150 . The typographical error noted in the certification (Comm. $A C M$, August 1963) was corrected in both codes.

(A referes verifies that Algorithm 162 does indeed run; as changed.-G.E.F.].

\section{ALGIORITHM 163}

\section{MODIFIED HANKEL FUNCTION}

\section{Henry E. Fetris}

Aeronautical Research Laboratories, Wright-Patterson Air Force Base, Ohio

procedure $E X P K(P, X, E)$; real $P, X ; E$;

comment this procedure calculates the modified Hankel Function $e^{x} K_{p}(x)$ to within a given accuracy $E$ from the integral representation:

$$
e^{x} K_{p}(x)=\int_{0}^{\infty} e^{x(1-\dot{c o s h})} \cosh (p t) d t
$$

begin real $F ; G, H, R, S, T, U, Y, Z, Z P$;

$R:=0.0$

$H:=1.0$;

iteration: begin

$G:=R$

$T:=.5 \times H$

$S:=0$;

$Z:=\exp (T)$

$\dot{U}:=Z \times Z$

integration: begin

$Y:=X \times(1-.5 \times(Z+1 / Z))$

if $P=0$ then $Z P:=1$

else $Z P:=Z \uparrow P$;

$F:=.5 \times \exp (Y) \times(Z P+1 / Z P)$;

$S:=S+F$

$Z:=Z \times U$

end;

if $F \geqq E$ then go to integration

else $R:=H \times S$;

$H:=.5 \times H$;

end;

if $a b 8(\mathrm{R}-\mathrm{G}) \geqq \mathrm{E}$ then go to iteration

else $E X P K:=R$

end $E X P K$

\section{CERTIFICATION OF ALGORITHM 163}

MODIFIED HANKEL FUNCTION [Henry E. Fettis, Comm. ACM, Apr. 1963]

Henry C. ThaCher, Jr.*

Argonne National Laboratory, Argonne, Ill.

Since this algorithm is a function declaration, the procedure declaration should be:

$$
\text { real procedure } E X P K(D, X, E) ; \cdots
$$

Otherwise, no syntactical errors were noticed.

The body of the procedure was translated and run on the LGP-30 computer, using the Dartmouth ScaLP system. Results for $E=0.0001, X=0.1(0.1) 1.0, P=0,0.3333333,0.6666667$ and 1.000000 agreed with values tabulated in Jahnke-Emde-Losch to the 3-4D given in the tables, except for errors discovered in the table of $2 / \pi K_{2 / 3}(x)$.

With $X=0$, the program ended in floating-point overflow. The algorithm itself, or the call of the procedure, should include a test to insure that the variable is greater than eps, where eps is chosen to prevent exceeding machine capacity.

The algorithm was found to be excessively slow. Times on the LGP-30 were of the order of 6 minutes. A considerable saving in time could be realized by improving the quadrature formula, currently the simple midpoint formula, repeated completely for each iteration. A more effective method would be a modified Romberg algorithm. A procedure based on the latter approach is being developed in this division. 


\section{ALGORITHM 164}

\section{ORTHOGONAL POLYNOMIAL LEAST SQLARES} SURFACE FIT

\section{R. F. Clark, R. N. Kubik, L. P. Phillips}

The Babcock \& Wilcox Co., Atomic Energy Div., Lynchburg, Va.

procedure surfacefit $(x, u, y, w, z, n \max , \operatorname{mmax}, i \max , j \max )$ result: (beta, phi, zcomp, minsgd, minsqdcomp, sumdifcomp, maxilifcomp);

real array $x, u, y, w, z, p h i$, beta, zcomp;

integer $n \max , \operatorname{mmax}, i \max , j \max$;

real minsgd, minsqdcomp, sumdifcomp, maxdifcomp;

comment this is a transliteration of an operating program written in Burroughs A LGoL for the B-220. It fits, in the least squares sense, a polynomial function of two independent variables to values of a dependent variable specified at points on a rectangular grid in the plane of the independent variables. The use of orthogonal polynomials leads to a particularly simple system of linear equations rather than the ill-conditioned system which arises from the usual normal equations. It also provides a measure of the improvements resulting from each new term included which further leads, in this algorithm, to an automatic selection of a "best". degree polynomial function as determined by Gauss' criterion. The initial normalization of the variables results in significant reduction of round off errors in many cases. This algorithm is developed more fully in BAW-182. For a very similar approach to this and related problems see Cadwell, J. H., "Least Squares Surface Fitting Program", The Compuler J. $\$$ (1961), 266 and.Cadwell, J. H., and Williams, D. E., "Some Orthogonal Methods of Curve and Surface Fitting," The Computer J. 4 (1961), 260. A further reference is Gauss, C. F., "Theoria Combinationis Observationum Erroribus Minimis Obnoxial," Gauss Werke 4 (Gottingen 1873), 3-93. $x[i]$ and $y[j]$ are the independent variables, $z[i, j]$ is the dependent variable. $u[i]$ and $w[j]$ represent the weights corresponding to $x[i]$ and $y[j]$, respectively. nmax is one more than the maximum degree of $x$ to be considered. mmax is one more than the maximum degree of $y$ to be considered. imax is the number of $x$ 's, and $j$ max is the number of $y$ 's. beta $[n, m]$ is a measure of the improvement resulting from the inclusion of the $x^{n} y^{m}$ th term. phi $[n, m]$ is the polynomial coefficient for the $x^{r} y^{m}$ th term. Note the degree of the resulting polynomial may be less than the maximum degree specified as a result of the application of Gauss' criterion. zcomp is the computed dependent variable.

$$
\begin{aligned}
& \text { minsqd }=\left(\frac{\sum_{i, j} u[i] \cdot w[j] \cdot z[i, j]^{2}-\sum_{n, m} \text { beta }[n, m]}{i \max \cdot j \max }\right)^{1 / 2} \\
& \text { minsqdcomp }=\left(\frac{\sum_{i, j} u[i] \cdot w[j](z[i, j]-z \operatorname{comp}[i, j])^{2}}{i \max \cdot j \max }\right)^{1 / 2} \\
& \text { sumdifcomp }=\frac{\sum_{i, j}|z[i, j]-z \operatorname{com} p[i, j]|}{i \max \cdot j \max }
\end{aligned}
$$

$\operatorname{maxdifcomp}=\max \mid z(i, j)-2 \operatorname{comp}((i, j) \mid$

minsqd and minsqdcomp are equal if computation is exact. In practice they will not be equal due to the imprecise nature of calculation. A wide discrepancy indicates excessive errors in calculation;

\section{begin}

real array $a, b$, denpa $[1: n \max ], c, d$, denga $[1: \operatorname{mmax}]$, alpha[1:nmax, 1:mmax], $p[1: n \max , 1: i \max ], q[1: \operatorname{mmax}, 1: j \max ]$, $p c[1: n \max , 1: n \max ], q c[1: m \max , 1: m \max ]$;

integer $n, m, i, j, 8, t, r$;

real sumx, sumy, sumz, meanx, meany, meanz, numa, dena, denb, numc, denc, dend, alph, sumz8q, gausscrit, trialgausscrit, betasum, rescomp, poly; comment normalization of variables;

sumx $:=$ sumy $:=$ sumz $:=0.0$;

for $i:=1$ step 1 until imax do

$\operatorname{sum} x:=\operatorname{sum} x+x[i]$;

meanx := sumx/imax;

for $\mathrm{i}:=1$ step 1 until imax do

$x[i]:=x[i]-\operatorname{mean} x$

for $\mathbf{j}:=1$ step 1 until jmax do

sumy : = sumy $+y[j]$;

meany $:=$ sumy/jmax;

for $j:=1$ step 1 until jmax do

$y[j]:=y[j]-$ meany

for $i:=1$ step 1 until imax do begin

for $j:=1$ step 1 until jmax do

sumz $:=\operatorname{sumz}+z[i, j]$ end

meanz $:=\operatorname{sumz} /(i \max \times j \max )$;

for $i:=1$ step 1 until imax do begin

for $j:=1$ step 1 until $j \max$ do

$z[i, j]:=z[i, j]-$ meanz end;

comment evaluate orthogonal polynomials;

numa : = dena $:=0.0$;

for $i:=1$ step 1 until imax do begin

$p[1, i]:=1.0$;

numa $:=$ numa $+u[i] \times x[i]$;

dena $:=$ dena $+u[i]$ end;

$a[2]:=$ numa/dena;

for $i:=1$ step 1 until imax do

$p[2, i]:=x[i]-a[2]$;

for $n:=3$ step 1 until nmax do begin

numa $:=\operatorname{den} a:=\operatorname{den} b:=0.0$;

for $\mathrm{i}:=1$ step 1 until imax do begin

numa := numa $+u[i] \times x[i] \times p[n-1] \uparrow 2 ;$

dena $:=\operatorname{dena}+u[i] \times p[n-1, i] \uparrow 2$;

denb $:=\operatorname{den} b+u[i] \times p[n-2, i] \uparrow 2$ end;

$a[n]:=$ numa/dena; $b[n]:=\operatorname{den} a / \operatorname{den} b$;

for $i:=1$ step 1 until imax do

$p[n, i]:=(x[i]-a[n]) \times p[n-1, i]-b[n] \times p[n-2, i]$ end;

numc $:=$ denc $:=0.0$;

for $j:=1$ step 1 until $j$ max do begin

$q[1, j]:=1.0$;

numc $:=n u m c+w[j] \times y[j]$;

denc $:=\operatorname{denc}+w[j]$ end;

$c[2]:=$ numc/denc;

for $j:=1$ step 1 until jmax do

$q[2, j]:=y[j]-c[2]$

for $m:=3$ step 1 until mmax do begin

numc $:=$ denc $:=$ dend $:=0.0$;

for $j:=1$ step 1 until jmax do begin

numc $:=n u m c+w[j] \times y[j] \times q[m-1, j] \uparrow 2$;

denc $:=$ denc $+w[j] \times q[m-1, j] \uparrow 2$;

dend $:=$ dend $+w[j] \times q[m-2, j] \uparrow 2$ end;

$c[m]:=$ numc/denc; $d[m]:=$ denc/dend;

for $j:=1$ step 1 until $j \max$ do

$q[m, j]:=(y[j]-c[m]) \times q[m-1, j]-d[m] \times q[m-2, j]$ end ;

comment evaluate contribution of each orthogonal polynomial

to the minimization of the residuals;

for $n:=1$ step 1 until $n \max$ do begin

$\operatorname{denpa}[n]:=0.0$;

for $i:=1$ step 1 until imax do

$\operatorname{denpa}[n]:=\operatorname{den} p a[n]+u[i] \times p[n, i] \uparrow 2$ end;

for $m:=1$ step 1 until mmax do begin

denqa $[m]:=0.0$

for $j:=1$ step 1 until jmax do

$\operatorname{den} q a[m]:=\operatorname{den} q a[m]+w[j] \times q[m, j] \uparrow 2$ end;

for $n:=1$ step 1 until nmax do begin

for $m:=1$ step 1 until mmax do begin

alph : $=0.0$ 
for $i:=1$ step 1 until $i m a x$ do begin

for $j:=1$ step 1 until jmax do

alph $:=$ alph $+u[i] \times w[j] \times z[i, j] \times p[n, i] \times q[m, j]$ end;

alpha $[n, m]:=a l p h /($ denpa $[n] \times$ denqa $[m])$;

beta $[n, m]:=$ alpha $[n, m] \times$ alph; end end;

comment application of Ciuuss' criterion to determine the degree polynomial which yields the closest fit to the given data. Guuss' criterion is, strictly speaking, applicable only to cases where the weights $u[i]$ and $w[j]$ are unity;

sumzzsq : $=0.0$;

for $i$ : = 1 step 1 until $i$ max do begin

for $j:=1$ step 1 until $j \max$ do

sumzsq : $=8 u m z s q+u[i] \times w[j] \times z[i, j] \uparrow 2$ end;

$8:=t:=1$;

for $n:=1$ step 1 until nmax do begin

betasum: : 0.0 ;

for $m:=1$ step 1 until mmax do begin

for $r:=1$ step 1 until $n$ do

betasum : = belasum + bela $[r, m]$;

if betasum $>$ sumz8q then trialgausscrit $:=0.0$

else

Irialgausserit-: $=-(8 u m z 8 q-b e l a s u m) /(i \max \times j \max -n \times(m) ;$

if $n=1 \wedge m=1$ then gausscrit : = trialgausscrit;

if gausscril = trialgausscrit then begin

if $n \times m<8 \times t$ then begin

$8:=n$

$\ell::=m$ end end;

if gausscrit $>$ trialgausscril then begin

gausscril : =,trialgaus8crit;

$8:=n$

$t:=m$ end end end;

$n \max :=8$;

$\operatorname{mmax}:=\ell$;

minsqd $:=($ gausscrit $\times(i \max \times j \max -n \max \times \operatorname{mmax}) /(i \max \times j \max ))$

$\uparrow 1 / 2 ;$

comment evaluation of orthogonal polynomial coefficients;

for $n:=1$ step 1 until $n$ max do begin

$p c[n, m]:=1.0$;

for $8:=1$ step 1 until $n-1$ do begin

$p c[n, 8]:=-a[n] \times p c[n-1,8]$;

if $8 \neq 1$ then $p c[n, 8]:=p c[n, 8]+p c[n-1,8-1]$;

if $8 \not \ldots-1$ then $p c[n, 8]:=p c[n, s]-b[n] \times p c[n-2, s]$ end end;

for $m:=1$ step 1 until mmax do begin

$q c[m, m]:=1.0$;

for $t:=1$ step 1 until $m-1$ do.begin

$q c[m, t]:=-c[m] \times q c \mid n \iota-1, t]$

if $t \neq$ then $q c[m, t]:=q c[m, t]+q c[m-1, t-1]$;

if $t \neq m-1$ then $q c[m, t]:=q c[m, t]-d[m] \times q c[m-2, t]$

end end;

comment evaluation of approximating polynomial coefficients;

for $s:=1$ step. 1 until $n$ max do begin

for $t:=1$ step 1 until $\operatorname{mmax}$ do begin

$\operatorname{phi}[8, t]:=0.0$;

for $n:=8$ step 1 until $n$ max do begin

for $m:=t$ step 1 until mmax do

phi $[8, t]:=\operatorname{phi}[8, t]+\operatorname{alpha}[n, m] \times p c[n, 8] \times q c[m, t]$ end end end;

comment evaluation of dependent zariables using the approximating. polynomial;

minsqdcomp $:=$ sumdifcomp $:=$ maxidifcomp $:=0.0$;

for $i$ : 1 step 1 until imax do begin

for $j:=1$-step 1 until $j \max$ do begin

$z \operatorname{comp}[i, j]:=0.0$

for $8:=$ nmax gtep -1 until 1 do begin

poly : $=$ phi $[8, \operatorname{mmax}]$ for $t:=\operatorname{mmax}-1$ step 1 until 1 do

poly $:=$ poly $\times y[j]+$ phi $\mid s, t\}$

$z \operatorname{comp}[i, j]:=z \operatorname{comp}[i, j] \times x[i]+$ poly end;

rescomp : $=z[i, j]-z \operatorname{comp}[i, j]$

zcomp $[i, j]:=\operatorname{zcomp}[i, j]+\operatorname{meanz}$;

minsqdcomp $:=\operatorname{minsqdcomp}+u[i] \times w[j] \times$ rescomp $\uparrow 2$

sumdifcomp $:=$ sumdifcomp + abs (rescomp);

if $a b s$ (rescomp) $>$ maxdifcomp then

$\operatorname{maxdifcomp}:=a b s($ rescomp $)$ end end;

$\operatorname{minsqdcomp} .:=.(\operatorname{minsqdcomp} /(i \max \times j \max )) \uparrow 1 / 2$

sumdifcomp : = sumdifcomp $/($ imax $\times$ jmax $)$;

end surfacefit

CERTIFICATION OF ALGORITHM 164

ORTHOGONAL POLYNOMIAL LEAST SQUARES

SURFACE FIT [R. E. Clark, R. N. Kubik, L. P. Phillips, Comm. ACM, April 1963]

C. V. Bitterli

Johns Hopkins Univ. Applied Physics Lab., Silver Spring, Md.

The SURF-ACEFIT algorithm-was translated into-ForTRAN. and successfully run on an IBM 7094. It was necessary to make the following corrections:

(a) 12th line after

comment evaluate orthogonal polynomials;

should read

numa $:=n u m a+u[i] \times x[i] \times p[n-1, i] \uparrow 2 ;$

(b) 2nd line after

comment evaluation of orthogonal polynomial coefficients; should read

$$
p c[n, n]:=1.0
$$

(c) 12 th line after

comment evaluation of orthogonal polynomial coefficients;

should read

$$
\text { if } t \neq 1 \text { then } q c[m, \ell]:=q c[m, t]+q c[m-1, t-1]
$$

(d) 8th line after

comment evaluation of dependent variables using the approxi mating polynominal

should read

$$
\text { for } t:=\operatorname{mmax}-1 \text { step }-1 \text { until } 1 \text { do }
$$

The following function was used to generate data for checking this algorithm:

$$
\begin{aligned}
& z=1-x+y-x y+x^{2}-y^{2} \\
& \text { for } \quad x=0,1,2,3,4 \\
& \text { and } \quad y=0,1,2,3,4
\end{aligned}
$$

The resulting polynomial was:

$$
z=x-5 y-x y+x^{2}-y^{2}
$$

which is correct for the normalized variables.

It should be pointed out in the comment for this procedure that the resulting polynomial is in the normalized variables and not the original variables. 
ALGORITHM 165

\section{COMPIETE ELLIPTIC INTEGRALS}

Henky C. Thacher, Jir.*

Reactor Eng. Div., Argonne National I,ab., Argonne, III.

* Work supported by the U.S. Atonic Energy Commission.

procedure $K A N D E(m l, K, E, t o l$, alarm);

value $m 1$, $t o l$;

real $m l, K, E$, tol;

label alarm;

comment this procedure computes the complete elliptic integrals $K(m 1)=\int_{0}^{\pi / 2}\left(1-(1-m 1) \sin ^{2} v\right)^{-1 / 2} \mathrm{~d} \nu$ and $E(m 1)=$ $\int_{0}^{\pi / 2}\left(1-(1-m 1) \sin ^{2} \nu\right)^{1 / 2} \mathrm{~d} \nu$ by the arithmetic-geometricmean process. The accuracy is limited only by the accuracy of the arithmetic.

Except for the provision of tests for pathological values of the parameter, the calculation of $K$ is only a slight modification of the second procedure of Algorithm No. 149 (Comm. ACM. 6 (Dec. 1962), 605). These integrals may also be approximated to limited (6I)) accuracy by Algorithms 55 and 56 (Comm. ACM. 4 (Apr. 1961), 180). Unless the square-root is exceptionally fast, the latter algorithms are probably more efficient for $6 \mathrm{D}$-accuracy.

The complementary parameter, $m 1$, is chosen as the independent variable, rather than the parameter, $m$, the modulus, $k$ or the modular angle $\alpha$, because of the possibility of serious loss of significance in generating $m 1$ from the other possible independent variables when $m 1$ is small and $d K / d m l$ is very large. These variables are related liy $m 1=1-m=1-k^{2}=$ $\cos ^{2} \alpha$.

The formal parameter, tol, determines the relative accuracy of the result. To prevent entering a nonterminating loop, tol should not be less than twice the relative error in the square root routine. If $m 1 \leqq 0$ or if $m 1>1$, the procedure exits to alarm. $K(0)=\infty$ while $E(0)=1.0000000$.

The body of this procedure has been tested using the Dartmouth ScalP processor for the LGP-30. With $t o l=5_{10}-7$, results agreed with tabulated values to within 3 in the seventh significant digit;

begin real $a, b, c$, sum, temp;

integer fact;

if $m 1>1 \vee m 1 \leqq 0$ then go to alarm;

$a,:=$ fact $:=1$;

$b:=\operatorname{sqrl}(m 1)$

temp $:=1-m 1$;

sum.: = $\mathbf{0}$

iter: sum : = sum + temp;

$c:=(a-b) / 2$

fact $:=$ fact + fact;

temp : $=(a+b) / 2$

$b:=\operatorname{sqrt}(a \times b)$;

$a:=$ temp

temp : = fact $\times c \times c$

if abs $(c) \geqq$ tol $\times a \vee$ temp $>$ tol $\times$ sum then go to iter

sum $:=$ sum + temp;

$K:=3.141592654 /(a+b)$;

comment $p i$ must be given to the full accuracy desired;

$E:=K \times(1-$ sum $/ 2)$

end
ALGORITHM 166

MONTECARLO

R. D. RoDmaN

Burroughs Corp., Pasadena, Calif.

procedure montecarlo ( $n, a, r o w, t o l, m x m$, inv, test, count);

value $n$, row, tol, mxm; integer $n$, row, mxm, count;

real tol; real array $a$, inv, test;

comment this procedure will compute a single row of the inverse of a given matrix using a monte carlo technique. $n$ is the size of the matrix, array $a$ is the matrix, row indicates which inverse row is to be computed, $t o l$ is a tolerance factor and thus a criterion for terminating the process, $m x m$ is 1000 times the maximum number of random walks to be taken, after which the process is terminated, array inv contains the inverse row, array test contains the innerproduct of $i n v$ with the rowth column of $a$, count is the number of random walks executed upon termination. real procedure $R A \dot{N} D O M$ must be declared in the blockhead of procedure MONTE CARLO and generates a single random number between 0 and 1 . If $a$ is the matrix to be inverted, the absolute value of the largest eigenvalue of the matrix $I-a$ ( $I$ is the unit matrix) must be less than one to assure convergence. This procedure is easily adapted to finding a single unknown from a set of simultaneous linear equations;

begin integer $i, j, k, n w k$, lastwalk, walk; real res, $p, g$;

real array $\operatorname{sum}[1: n], v[1: n, 1: n]$;

start: $\quad p:=(n-1) / n \times n$;

for $i:=1$ step 1 until $n$ do for $j:=1$ step 1 until $n$ do $v[i, j]:=$ if $i \neq j$ then $-a[i, j] / p$ else $(1-a[i, j]) / p$ $n w k:=1000$;

count $:=$ res $:=0$

for $k:=1$ step 1 until $n$ do test $[k]:=\operatorname{sum}[k]:=0$

start1: lastwalk $:=$ row $; g:=1$;

start2: walk: $=(R A N D O M / p)+1$.

if walk $>n$ then go to $s t o p$;

$g:=v[$ lastwalk,walk] $\times g$; lastwalk $:=$ walk;

go to start2;

stop: count := count $+1 ;$ sum[lastwalk $]:=$ sum[lastwalk $]+g$; if count < $n w k$ then go to start1;

for $k:=1$ step 1 until $n$ do $i n v[k]:=n \times \operatorname{sum}[k] /$ count; for $i:=1$ step 1 until $n$ do for $k:=1$ step 1 until $n$ do $\operatorname{test}[i]:=i n v[k] \times a[k, i]+\operatorname{test}[i]$;

for $i:=1$ step 1 until row-1, row +1 step 1 until $n$ do res $:=a b s($ test $[i])+$ res; res, : = abs (test [row $]-1)+r e s ;$ if res < tol then go to exit;

if count $\geqq 1000 \times m x m$ then go to exit;

$n w k:=n w k+1000 ;$ res $:=0$;

for $k:=1$ step 1 until $n$ do test $[k]:=0$;

go to start1;

exit: end of monte carlo inversion procedure

\section{REMARK ON ALGORITHM 166}

MONTECARLO INVERSE [R. D. Rodman, Comm. ACM, Apr. 1963]

R. D. RODMAN .

Burroughs Corp., Pasadena, Calif.

The algorithm contained two errors:

(1) The line which reads

start: $p:=(n-1) / n \times n$;

should read

start: $p:=(n-1) / n \uparrow 2$; 
(2) The line which reads start2: walk: $=($ random $/ p)+1$;

should read

start2: walk: $=$ entier $(($ random $/ p)+1)$;

After making the preceding corrections, procedure montecarlo was transliterated into EXTENDED ALGOL and run successfully on the Burroughs B-5000. Convergence occurred in all cases where the matrix satisfied the conditions set down in the comment statement of the algorithm. It was found that convergence was quickest and the routine most practical for matrices with eigenvalues small relative to one.

\section{ALGORITHM 167}

\section{CALCULATION OF CONFLUENT DIVIDED DIFFERENCES}

W. Kahan and I. Farkas

-Institute of Computer Science, University of Toronto, Canada

real procedure $D V D F C(n, X, V, B, W) ; \operatorname{integer} n$; real array $X, V, B, W$;

comment $D V D F C$ ca.culates the forward divided difference $\Delta f\left(X_{1}, X_{2}, \cdots X_{n}\right) . \quad n$ is an integer which takes the values $n=1,2,3, \cdots$ in turn. $X$ is a real array of dimension at least $n$ in which $X[i]=X_{i}$ for $i=1,2, \cdots, n$. The values $X_{i}$ need not be distinct nor in any special order, but once the array $X$ is chosen it will fix the interpretation of the arrays $B$ and $V$. If $X[1], X[2], \cdots, X[n]$ are in monotonic order, then the effect of roundoff upon any $n$th divided difference is no more than would be caused by perturbing each $f(X[i])$ by $n$ units at most in its last significant place. But if the $X$ 's are not. in monotonic order, the error can be catastrophic if some of the divided differences are relatively large. $V$ is a real array of dimension at least $n$ containing the values of the function $f(X)$ and perhaps its derivatives at the point $X_{i} . \quad V[i]=f^{m}\left(X_{i}\right) / m$ ! and $m=m_{i}$ for $i=1,2,3, \cdots, n$. $m_{i}$ is the number of times that. the value of $X_{i}$ has previously appeared in the array $X . B$ is a real array of dimension at least $n$ containing backward divided differences. Before a reference to $D V D F C$ is executed one should have $B[i]=\Delta f\left(X_{i}, X_{i+1}, \cdots, X_{n-1}\right)$ for $i=1,2, \cdots, n-1$. After that reference to $D V D F C$ is executed one will find $B[i]=$ $\Delta f\left(X_{i}, X_{i+1}, \cdots, X_{n-1}, X_{n}\right)$ for $i=1,2, \cdots, n-1, n$. When $n=1$ the initial state of $B$ is irrelevant. $W$ is a real array of dimension $(2+\bar{m})$ at least, where $\bar{m}$ is the maximum value of $m_{i}$ for $i=1,2, \cdots, n$. W is used for work space;

begin real $D E N O M$; integer $i, j, N K, N I N$;

if $n=1$ then go to $L 1$;

$N K:=1$;

for $i:=1$ step 1 until $n$ do

begin

if $X[i]=X[n]$ then begin $N K:=N K+1$;

end $i$

$W[N K]:=V[i]$ end

for $i:=n$ step -1 until 2 do

begin $W[1]:=B[i-1] ; B[i]:=W[2]$

$N I N:=$ if $n-i+2<N K$ then $n-i+2$ else $N K$;

for $j:=N I N$ step -1 until 2 do

begin

$D E N O M:=X[n]-X[i+j-3]$;

if $D E N O M \neq 0$ then go to $L 2$;

$W[j]:=W[j+1]$;

if $N K-j-1 \neq 0$ then go Cont;

$N K:=N K-1$;

go to Cont;
$L 2: \quad W[j]:=(W[j]-W[j-1]) / D E N O M$;

Cont: end $j$

end $i$;

$B[1]:=W[2]$;

go to $L 3$;

$L 1: \quad B[1]:=V[1]$

L3: $D V D F C:=B[1]$

end $D V D F C$

The following program segment is an example of how $D V D F C$ can

be used to construct a table of forward or backward differences.

for $n:=1$ step 1 until $N$ do

begin

$X[n]:=\cdots ; V[n]:=\cdots ; F[n]:=\operatorname{DVDFC}(n, X, V, B, W)$ end;

The array $F$ can be used in $F \backslash E W T(z, N, X, F, R, D, E)$ or the array $B$ in $B N E W T(z, N, X, B, P, D, E)$. See algorithms "New'ton interpolation with forward (backward) divided differences."

$D V D F C$ has been written as a ForTran II function and is available from I.C.S., University of Toronto;

SEE ALGORITHM 169

\section{ALGORITHM 168}

NEWTON INTERPOLATION WITH

BACKWARD DIVIDED DIFFERENCES

W. Kahan and I. Farkas

Institute of Computer Science, University of Toronto, Canada

procedure $B N E W T(z, N, x, B, P, D, E)$; value $z, N$; real $z, P, D, E$; integer $N$; real array $X, B$;

comment $X$ is a real array of dimension at least $N$ in which $X[i]=X_{i}$ for $i=1,2,3, \cdots, N$. The values $X_{i}$ need not be distinct nor in any special order, but once the array $X$ is chosen it will fix the interpretation of the array $B .^{\circ} B$ is a real array of dimension at least $N$ and contains the backward divided differences $B[i]=\Delta f\left(X_{i}, X_{i+1}, \cdots, X_{N}\right) \quad i=1,2, \cdots, N$. If two or more of the values $X_{i}$ are equal then some of the $B$ 's must be confluent divided differences, see algorithm: "Calculation of confluent divided differences." $P$ is the value of the following polynomial in $z$ of degree $\mathrm{N}-1$ at most, $B(N)+\left(z-\mathrm{N}_{N}\right)$. $\left\{B(N-1)+\left(z-X_{N-1}\right)\left\{B(N-2)+\cdots+\left(z-X_{2}\right) B(1)\right\} \cdots \|\right\}$ This polynomial is an interpolation polynomial which would, but for rounding errors, match values of the function $f(x)$ and any of its derivatives that $D V D F C$ might have been given. $D$ is the value of the derivative of $P . E$ is the maximum error in $P$ caused by roundoff during the execution of $B N E W T$. The error estimate is based upon the assumption that the result of each floating point arithmetic operation is truncated to $27 \mathrm{sig}$ nificant binary digits as is the case in Fortran programs on the 7090. BNEWT has been written as a Fortran II subroutine and is available from I.C.S., University of Toronto;

begin real $z 1$; integer $i$;

$P:=D:=E:=0$;

for $i:=1$ step 1 until $N$ do

begin

zl : $=z:-X[i]$;

$D:=P+z 1 \times D$;

$P:=B[i]+z 1 \times P$

$E:=a b s(P)+E \times a b s(z 1)$

end;

$E:=(1.5 \times E-a b s(P)) \times 3_{10}-8$

end $B N E W T$ 
AIGORITHM 169

\section{NEWTOA INTERPOLATION WITH}

FORWARD DIVIDED DIFTERENCES

\section{W. KaHaI aNd I. Farkas}

Institute of Computer Science, Eniversity of Toronto, Canada

procedure $F . V E W T(z, x, X, F, R, D, E)$; value $z, \lambda$; real $z, R, D, E$; integer $N$; real array $X, F$;

comment $X$ is a real array of dimension at least $\lambda$ in which $X[i]=X_{i}$ for $i=1,2, \cdots, X$. The values $X_{i}$ need not be distinct nor in any special order, but once the array $X$ is chosen it will fix the interpretation of the array $F$. $F$ is a real array of dimension at least $N$ and contains the forward divided differences $F[i]=\Delta f\left(X_{1}, x_{2}, \cdots, X_{i}\right) \quad i=1,2, \cdots, X$. If two or more of the values $X_{i}$ are equal then some of the $F$ 's must be confluent divided differences, see algorithm: "Calculation of confluent divided differences." $R$ is the value of the following polynomial in $z$ of degree $N-1$ at most, $F(1)+\left(z-X_{1}\right) \cdot$ $\left.\left\{F(2)+\left(z-X_{2}\right)\left\{F(3)+\cdots+\left(z-X_{N-1}\right) F(N)\right\} \cdots\right\}\right\}$. This polynomial is an interpolation polynomial which would, but for rounding errors, match values of the function $f(x)$ and any of its derivatives that $D V D F C$ might have been given. $D$ is the value of the derivative of $R$. $E$ is the maximum error in $R$ caused by roundoff during the execution of F.YEW'. 'The error estimate is based upon the assumption that the result of each floating-point arithmetic operation is truncated to 27 significant binary digits as is the cuse in Fortran programs on the 7090. FNEWT has been written as a Fortran II subroutine and is available from I.C.S., Inniversity of Toronto;

begin real $z 1$; integer $i$;

$R:=D:=E:=0$;

for $i:=N$ step -1 until 1 do

begin

$21:=z-X[i]$

$D:=R+z 1 \times D$

$R:=F[i]+z 1 \times R$;

$E:=a b s(R)+a b s(z 1) \times E$

end;

$E:=(1.5 \times E-a b s(R)) \times 3_{10}-8$

end $F . Y E W T$
CERTIFICATION OF ALGORITHM 167

CALCULATION OF CONFLUENT DIVIDED DIFFERENCES [W. Kahan and I. Farkas, Comm. $A C M$, Apr. 1963]

CERTIFICATION OF ALGORITHM 168 NEWTON INTERPOLATION WITH BACKWARD DIVIDED DIFFERENCES [W. Kahan and $I$. Farkas, Comm. ACM, Apr. 1963]

CERTIFICATION OF ALGORITHM 169

NEWTON INTERPOLATION WITH FORWARD DIVIDED DIFFERENCES [W. Kahan and $I$.

Farkas, Comm. ACM, Apr. 1963.]

Henry C. Thacher, Jr.*

Argonne National Laboratory, Argonne, Ill.

The bodies of these procedures were tested on the LGP-30 computer using the Dartmouth SCAlP compiler. Compilation and execution revealed no syntactical or mathematical errors.

It is to be noted that, although with Algorithm 169, reducing the value of $N$ from that used to generate $F$ leads to an interpolation polynomial based on fewer points, this is not true for Algorithm 168 . This flexibility could be supplied by adding an additional formal parameter, deg, say, to the procedure, and by making the for statement read:

$$
\text { "for } i:=N-\operatorname{deg} \text { step } 1 \text { until } N \text { do } . . . "
$$

The logic of the error estimate in Algorithms 168 and 169 is not entirely clear. However, it appears that the estimate can be adjusted for different precision. of arithmetic by adjusting the constant $310-8$ appropriately. For the Scalp arithmetic, this constant was changed to $1_{10}-7$.

The algorithms were tested on the examples given by MilneThomson [The Calculus of Finite Differences, p. 4, Macmillan, 1951] and by Milne [Numerical Calculus, p. 204, Princeton, 1949]. In both examples, Algorithm 167 reproduced the divided difference table, and both Algorithms 168 and 169 reproduced the input values. As a check of the calculation of confluent divided differences, values of the exponential function of its first two derivatives at $x=5.0$ and 6.0 were used. The difference table shown in Table A was obtained.

TABLE A

\begin{tabular}{|c|c|c|c|c|c|c|c|c|}
\hline$n$ & $X(n)$ & $V[n]$ & $B[n]$ & $B[n-1]$ & $B[n-2]$ & $B[n-3]$ & $B \mid n-4]$ & $B[n-5]$ \\
\hline 1 & 5.0 & 148.4132 & 148.4132 & & & & & \\
\hline 2 & 5.0 & 148.4132 & 148.4132 & $148 .+132$ & & & & \\
\hline 4 & 6.0 & 403.4288 & 403.4287 & 403.4287 & 148.4132 & 41.81091 & & \\
\hline 5 & 5.0 & 74.20658 & 148.4132 & 255.0155 & 148.4132 & 41.81091 & 9.415191 & \\
\hline 6 & 6.0 & 201.7144 & 403.1287 & 255.0155 & 148.4132 & 53.30115 & 11.49023 & 2.075043 \\
\hline
\end{tabular}

The forward differences lie along the top diagonal.

Use of these results with $B N E W T$ and with FNEWT gave the following results, for $N=6$.

\begin{tabular}{|c|c|c|c|c|c|c|}
\hline \multirow{2}{*}{$z$} & \multicolumn{3}{|c|}{ BNE $\mathrm{ET}$} & \multicolumn{3}{|c|}{$F_{N} B W T$} \\
\hline & $P$ & $D$ & $E$ & $R$ & $D$ & $\boldsymbol{E}$ \\
\hline
\end{tabular}




\section{ALGORITHM 170}

REDUCTION OF A MATRIX CONTAINING POLYNOMIAL ELEMENTS

\section{Patl E. Hennion}

Giannini Controls Corp., Astromechanics Res. Div., Berwyı, Pèini.

real procedure POLYMATRIA (A, NCOL, $N, C O E, N P 1)$; vulue $A, N C O L, N$; real array $A$; integer $N C O L, N$;

comment this procedure will expand a general determinant, where each of the elenients are polynomials in the Laplace complex variable. This program is useful for the investigation of dynamic stability problems when using the transfer function approach. The process is one of triangularization of a polynomial matrix with real coefficients whereupon multiplication of the diagonal elements the determinant polynomial is formed. The polynomial matrix as defined herein is a matrix whose elements are polynomials of the form $\sum_{i=0}^{N} a_{i} x^{i}$. When such a matrix is triangularized, all elements below the main diagonal are nulled. Then upon expanding, the nonvanishing terms are those formed by the product of these diagonal elements. Hence stability criteria may be checked by evaluating the roots of the characteristic equation thus formed using some suitable root extracting routine.

Consider the polynomial matrix with quadratic elements $(N=2)$. In this case the three-dimensional input matrix $A$ is size $A[1: N C O L, 1: N C O L, 1: M]$, where $N C O L$ is the order of the matrix and $M=N \times N C O L+1$. Here the first subscript of $A$ refers to the row, the second to the column, and the third to the polynomial coefficient. Therefore, prior to entry the constant term of a general polynomial element is contained in $A[i, j, 1]$, the linear term is contained in $A[i, j, 2]$, and the quadratic term in $A[i, j, 3]$. Upon completion of the routine, the coefficients of the determinant polynomial are contained in COE $[1: M]$. The constant coefficient being in $C O E$ [1], the linear coefficient in $C O E[2]$, the quadratic coefficient in $C O E[3]$, etc. The variable $\Lambda^{\prime} P 1$ will specify the number of coefficients of the determinant polynomial. In general $I P I \neq M$ since some terms may vanish during the expansion.

If the polynomials comprising the matrix elements are not all of equal degree, set $N$ prior to entry equal to the degree of the highest ordered polynomial;

begin real $8 a, 8 b$; integer $i, j, k, j 1, j 2, j 3, j 4, j 5, j 6, j 7, j 8, j 9, j 10$, $j 11, N P 1, M$; urray $C: 1[1: M], C 2[1: M], C O E[1: M]$; integer array $M A T[1: N C O L, 1: N C O L]$;

start: $M:=N \times N(O L-1$, for $i:=1$ step unti 1 NCOOL, do begin for $j:=1$ step 1 until $N^{\prime} C O L$ do begin $M A T[i, j]:=0$; for $k:=1$ step 1 until $M$ do begin

if $A[i, j, k] \neq 0$ then $M A T\{i, j]:=k$; end end end; $j 1:=1$ :

I0: $\quad j 9:=0 ;$ for $i:=j l$ step 1 until NCOL do begin

if $M A T\{i, j 1\}<0$ then go to exit;

else if $M A T\{i, j 1\}=0$ then go to $L 1$

elve $j 9:=j n+1 ; j 3:=i$;

$L 1$ : end; if $(j 9-1)<0$ then ko to exit

else if $(j y-1)>0$ then ko to $l .2$

else if $(j 3-j 1)<0$ then go to exit

else if $(j 3-j 1)=0$ then go to $L 12$

else for $j:=j 1$ step 1 until $N^{\circ}(O O L$ do

begin $j 2:=M A X(M A T[j 3, j], M A T[j 1, j]) ; j 4:=M A T[j 3, j]$;

$M A T[j 3, j]:=M A T[j 1, j] ; M A T[j 1, j]:=j 4 ;$

for $k:=1$ step 1 until $j 2$ do

begin $8 a:=A[j 3, j, k] ; \quad A[j 3, j, k]:=A[j 1, j, k]$;

$A[j 1, j, k]:=-8 a$; end end; go to $L 12$;

L2: $\quad j 3:=j 1+1 ;$ for $i:=j 3$ step 1 until $N C O L$, do begin

$L 3$ : if $(M A T[i, j 1])<0$ then go to exit

else if $\left[M A^{\prime}[i, j 1]\right)=0$ then go to $L 11$

else if $\left(M A T^{\prime}[j 1, j 1]\right)<0$ then go to exit

else if $(M A T[j 1, j 1])=0$ then go to $L A$

else if $(M A T[i, j 1]-M A T[j 1, j 1]) \geqq 0$ then go to $L 5$ else
I4: for $j: \doteq j 1$ step 1 until $N C O L$ do begin

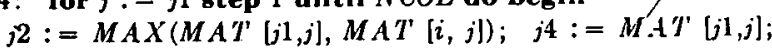
$\operatorname{MAT}(j 1, j]:=M A T^{\prime}[i, j] ; \quad M A T[i, j]:=j 4 ;$ for $k:=1$ step 1 until. $j 2$ do begin sa: $=A[i, j, k]$; $A[i, j, k]:=A[j 1, j, k] ; \quad A[j 1, j, k]:=-s a$; end end; go to $L 3$; comment Interchange row $i$ with $j$;

L5: $\quad j 7:=\operatorname{MAT}[i, j 1] ; \quad j 5:=\operatorname{MA} A^{\prime}[j 1, j 1] ; j 6:=j 7-j 5$ $s b:=A[i, j 1, j 7] / A[j 1, j 1, j 5]$;

if $(a b s(8 b)-4)<0$ then go to $J A$

else if $(j 6)<0$ then go to exil

else if $(j 6)=0$ then go to $L 4$ else

$I f:$ for $j:=j 1$ step 1 until $N C O L$ do begin $j 5:=M A T[j 1, j]$; for $k:=1$ step 1 until $j 5$ do begin $j 7:=k+j 6$

if $(j 7-M)>0$ then go to $L 10$ else

L7: if $\left.(a b 8(A[i, j, j]]-s b \times A[j 1, j, k])-2_{10}-8\right) \leqq 0$ then go to $L 8$ else $A[i, j, j]:=A[i, j, j]\}-s b \times A[j 1, j, k]$; go to $L 9$;

L8: $A[i, j, j]]:=0$;

$L 9$ : end end;

L10: for $j:=j 1$ step 1 until $N C O L$ do begin

$j 7:=\operatorname{MAX}(M A T[i, j], M A T[j 1, j]+j 6) ; \operatorname{MAT}[i, j]:=0 ;$ for $k:=1$ step 1 until $M$ do begin if $(A[i, j, k]) \neq 0$ then $M A T[i, j]:=k$ end end;

L11: end; go to $l 0$;

$L 12: \quad j 1:=j 1+1 ; \quad$ if $(j 1-. Y(O L)<0$ then go to $L 0$ else

for $j:=1$ step 1 until . IC:OL do begin

$j 2:=M . A T[j, j]$;

for $k:=1$ step 1 until $j 2$ do ( $1[k]:=A[j, j, k]$;

J.13: if $(j-1)<0$ then go to exit

else if $(j-1)=0$ then go to $L 14$

else for $k:=1$ step 1 until $X P 1$ do $C 2[k]:=\operatorname{COE}[k]$;

for $k:=1$ step 1 until $M$ do ('OE $[k]:=0$;

if $(j 2)<0$ then go to exil

else if $(j 2)=0$ then go to $L 15$

else for $k:=1$ step 1 until $j 2$ do begin

for $j 10:=1$ step 1 until.$P P 1$ do hegin

$j 11:=k+j 10-1$;

$C O E[j 11]:=C O E[j 11]+(: 1[k] \times C 2[j 10]$;

end end; $N P 1:=j 11$; go to $L 15$;

I.14: for $k:=1$ step 1 ulitil $j 2$ do $\operatorname{COE}[k]:=C 1[k]$;

$X P 1:=j 2$;

L15: end;

eril: end POLYMATRIX

\section{CERTIFICATION OF ALGORITHM 170 [F3]}

REDUCTION OF A MATRIX CONTAINING POLY-

NOMIAL ELEMENTS [P. E. Hennion, Comm. ACM

6 (April 1963), 165; 6 (Aug. 1963), 450]

Karen B. Priebe (Recd. 18 Dec. 1963 and 18 Feb. 1964)

Woodward Governor Co., Rockford, Ill.

Algorithm 170 was translated into FAST for the NCR 315 and gave satisfactory results with the following corrections:

1. real procedure ... integer $N C O L, N$; should be replaced by

procedure POLYMATRIX (A, NCOL, N, COE, NP1); value $N C O L, N$; real array $A, C O E$;

integer $N C O L, N, N P 1$;

2. At the end of the first comment add:

The global integer procedure $M A X$ is assumed and furnishes the maximum of two integers.

3. integer $i, j, k, \ldots \operatorname{COE}[1: M]$;

should be replaced by

integer $i, j, k, j 1, j 2, j 3, j 4, j 5, j 6, j 7, j 8, j 9, j 10, j 11, M$;

array $C 1, C 2[1: N \times N C O L+1]$; 
4. Immediately after start: the statement

$$
N P_{1}:=N+1 \text {; }
$$

should be added, and the third line after start: i.e.,

for $k:=1$ step 1 until $M$ do begin

should be replaced by

for $k:=1$ step 1 until $N P 1$ do begin

5. The third line after $L 10$ : i.e.,

$$
\text { for } k:=1 \text { step } 1 \text { until } M \text { do ... }
$$

should be replaced by

$$
\text { for } k:=1 \text { step } 1 \text { until } \overrightarrow{j 7} \text { do ... }
$$

The last two changes simply shorten both of the indicated ros statements.

[EDITOR's Note. In addition to the above corrections, we have two comments on the Remark on Algorithm 170 by Hennion, loc. cit., p. 450 :

First, the semicolon at the end of the first line after $L O$ MUST be removed.

Second, correction (4) is irrelevant.

The referee confirms that a transcription into Burroughs Extended Alool of the program as corrected by Mrs. Priebe runs on the B5000.-G.E.F.]

\section{REMARK ON ALGORITHM 170}

REDUCTION OF A MATRIX CONTAINING POLYNOMIAL ELEMENTS [P. E. Hennion, Comm. ACM, Apr. 1963]

P. E. Hennion

Giannini Controls Corp., Berwyn, Penn.

Four typographical errors were found upon reviewing the procedure. The following corrections should be made:

(1) The increment for the for statement of line start:, should be 1 .

(2) The colon at the end of the third line after line start:, should be replaced by a semicolon.

(3) The semicolon at the end of the first line after line $L O$ :, may be removed.

(4) The last statement of the first column should read:

$$
M A T[i, j]:=\downarrow k ; \text { end end; }
$$

\footnotetext{
ALGORITHM 173

ASSIGN

Otomar Hájek

Research Institute of Mathematical Machines, Prague, Czechoslovakia

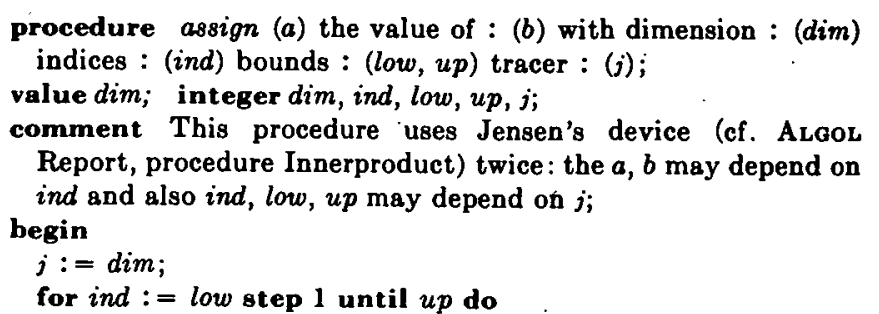


recursive procedures are prescribed. Luckily, the only parameter which varies during the recursive call in the body of Assign is called by value (it is the parameter dim which determines depth of recursion). The body of Assign was replaced by (i) a procedure declaration Ass(dim), whose body is that of the original $A$ ssign, but with the recursive call of Assign replaced by that of $A s s$, and (ii) a single statement, the activation of Ass(dim).

The resulting procedure was tested (on the National-Elliott 803 in the Computing Center), on a rather large set of examples, including those described in the text following Algorithm 173. It was found that in the last example, matrix multiplication, indices $i_{1}$ and $i_{8}$ should be interchanged throughout.

No changes of the algorithm itself were necessary. It seems that the modification described above, motivated by limitations of Elliott-Alciol, also improve efficiency, at least for large dimensions of the arrays concerned.

\section{ALGORITHM 174}

\section{A POSTERIORI BOUNDS ON A ZERO OF A POLYNOMIAL* \\ Allan GibB}

University of Alberta, Calgary, Alberta, Canada

comment The procedures below make use of Algorithm 61, Procedures for Range Arithmetic [Comm. ACM 4 (1961)]. It is assumed that the procedures below and the range arithmetic procedures are contained in an outer block and, therefore, that the procedures are available as required. Together the procedures make possible an attempt to determine absolute bounds on a zero of a polynomial given an initial estimate of the zero. The procedures below are given for the complex case but may readily be adapted for the real case;

procedure $R n g P l y C(N, A, Z, P)$;

comment RngPlyC finds bounds $[P 1, P 2]+\mathrm{i}[P 3, P 4]$ on the value of an $n$th degree polynomial $\Sigma_{k=0}^{n}\left\{\left[a_{4 k+1}, a_{4 k+2}\right]\right.$ $\left.+\mathrm{i}\left[a_{4 k+3}, a_{4 k+4}\right]\right\} z^{k}$ with complex range coefficients for a complex range argument $z=[Z 1, Z 2]+\mathrm{i}[Z 3, Z 4]$;

integer $N$; array $A, Z, P$;

begin integer $K, J$; array $X, Y[1: 4]$;

$P[1]:=P[2]:=P[3]:=P[4]:=0$;

for $K:=4 \times N$ step -4 until 0 do

begin for $J:=1$ step 1 until 4 do $X[J]:=A[K+J]$;

RNGMPYC (P[1], $P[2], P[3], P[4], Z[1], Z[2], Z[3], Z[4], Y[1]$, $Y[2], Y[3], Y[4])$;

RNGSUMC (Y[1], $Y[2], Y[3], Y[4], X[1], X[2], X[3], X[4]$, $P[1], P[2], P[3], P[4])$

end

end;

procedure $R n g A b s C(A, C)$;

comment $R n g A b s C$ produces the range absolute value $[C 1, C 2]$

of the complex range number $[A 1, A 2]+\mathrm{i}[A 3, A 4]$;

array $A, C$;

begin array $B[1: 4]$;

RANGESQR $\quad(A[1], A[2], B[1], B[2])$;

RANGESQR (A[3], $A[4], B[3], B[4 \mid)$;

RANGESUM (B[1], $B[2], B[3], B[4], C[1], C[2])$;

$C[1]:=\operatorname{sgrt}(C[1])$;

$C[2]:=\operatorname{sgrl}(C[2])$;

comment It is assumed that the accuracy of the sqrt routine used is known and that the maximum error in $\operatorname{sqr}(C)$ is $\pm K$ $\times$ CORRECTION $(C) . K$ is to be replaced below by its appropriate numerical value;
$C[1]:=C[1]-K \times$ CORRECTION $(C[1])$;

$C[2]:=C[2]+K \times$ CORRECTION $(C[2])$

end;

procedure $B n d Z r P l y C(N, Z O R, Z O J, A, W)$;

integer $N$; real $Z O R, Z O J ; \quad \operatorname{array} A, W$;

comment BndZrPlyC attempts to determine bounds $[W 1, W 2]$ $+\mathrm{i}[W 3, W 4]$ on a zero of an $N$-th degree polynomial in $z$ with complex range coefficients. It is assumed that an estimate $Z O=Z O R+\mathrm{i} Z O J$ of the zero is available. The following theorem is used. Assume $f$ is regular at $z_{0}$ with $f^{\prime}\left(z_{0}\right) \neq 0$. Let $h_{0}=-f\left(z_{0}\right) / f^{\prime}\left(z_{0}\right)$, let $\Delta$ be the region $\left|z-z_{0}\right| \leqq r\left|h_{0}\right|$, and assume that $f$ is regular in $\Delta$. If, for some $r>0,\left|f^{\prime}(z)\right| \geqq(1 / r)$. $\left|f^{\prime}\left(z_{0}\right)\right|$ for all $z \in \Delta$ then $\Delta$ contains a zero of $f$ (see [1], pp. 29-31); begin integer $I, J$; array $B[1: 4 \times N !, E, F, F P, D[1: 4], A F$, $A F P, G[1: 2]$

real $R H, R H S, N L, N R, R, R N L, R N R$;

for $I:=1$ step 1 until $N$ do

begin $J:=4 \times I$

RANGEMPY (I, $I, A[J+1], A[J+2], B[J-3], B[J-2])$ :

RANGEMPY $(I, I, A[J+3], A[J+4], B[J-1], B[J])$

end;

$E[1]:=E[2]:=Z O R ; \quad E[3]:=E[4]:=Z O J ;$

$R n g P l y C(N, A, E, F)$;

RngAbsC $(F, A F)$;

$R n g P l y C(N-1, B, E, F P)$;

$R n g A b s C(F P, A F P)$;

$R A N G E D V D(A F[1], A F[2], A F P[1], A F P(2], N L, N R)$;

$R:=2$;

1: R.ANGEMPY $(R, R, N R, N R, R N L, R N R)$;

RANGESUM (ZOR, ZOR, -RNR, RNR,W[1],W[2]);

RANGESUM(ZOJ, ZOJ, -RNR, RNR, W[3], W[4]);

comment We have replaced the disk of the theorem by a square; $\operatorname{RngRlyC}(N-1, B, W, D)$;

$\operatorname{Rng} A b s C(D, G)$;

if $G[1]=0$ then go to failure 1 ;

comment failure 1 and failure 2 are non-local labels;

$R A N G E D V D(A F P[2], A F P[2], R, R, R H, R H S)$;

if $G[1]<R H S$ then

begin $R:=2 \times R$;

if $R>1024$ then go to failure 2 ;

go to 1

end

end

comment The following procedure may replace BndZrPlyC above;

procedure $B n d Z r P l y C 2(N, Z O R, Z O J, A, W)$;

integer $N$; array $A, W$; real $Z O R, Z O J$;

comment BndZrPlyC2 is similar to $B n d Z_{r} P l y C$ above. The theorem used here follows. If, in the disk $\left|z-z_{0}\right| \leqq 2\left|h_{0}\right|$ we have $\left|f^{\prime \prime}(z)\right| \leqq\left|f^{\prime}\left(z_{0}\right)\right| /\left(2\left|h_{0}\right|\right)$, then there is a unique zero in the disk (see [2, pp. 43-50];

begin integer $I, J$; array $B[1: 4 \times N], C[1: 4 \times N-4], F, D, P$, $S[1: 4], X, T, Q, Y[1: 2]$; real $V, V P, R, R L$;

for $I:=1$ step 1 until $N$ do

begin $J:=4 \times 1$;

RANGEMPY $(I, I, A[J+1], A[J+2], B[J-3], B[J-2])$;

RANGEMPY $(I, I, A[J+3], A[J+4], B[J-1], B[J])$

end;

for $I:=1$ step 1 until $N-1$ do

begin $J:=4 \times I$;

RANGEMPY $(I, I, B[J+1], B[J+2], C[J-3], C[J-2])$;

RANGEMPY $(I, I, B[J+3], B[J+4], C[. J-1], C[J])$

end;

$D[1]:=D[2]:=Z O R$;

$D[3 \mid:=D[4]:=Z O J$

$\operatorname{Rng} P \operatorname{Ply} C(N, A, D, F)$; 
$\operatorname{Rng} \dot{P} l \bar{C} \bar{C}(\bar{N}-1, \bar{B}, \bar{D}, \bar{P})$

$R n g A b s C(F, T)$;

$R n g A b s C(P, X)$;

if $X[1]=0$ then go to failure1;

comment failure1.and failure 2 are non-local labels;

$R A N G E D V D(T[1], T[2], X[1], X[2], Q[1], Q[2])$;

$R A N G E M P Y(2,2, Q[2], Q[2], R L, R)$;

$R N G S U M C(-R, R,-R, R, Z O R, Z O R, Z O J, Z O J, W[1], W[2]$, $W[3], W[4])$;

$\operatorname{Rng} P \operatorname{ly} C(N-2, C, W, S)$;

$\operatorname{Rng} A b s C(S, Y)$;

$R A N G E D V D(X[1], X[1], R, R, V, V P)$;

if $Y[2]>V$ then go to failure 2

end

References:

1. Gibb, Allan. ALGOL procedures for range arithmetic. Tech. Report No. 15, Appl. Math. and Statistics Laboratories, Stanford University (1961).

2. OstrowsKI, A. M. Solution of equations and systems of equations. Academic Press, New York, 1960.

* These procedures were developed under Office of Naval Research Contract Nonr-225(37) at Stanford University. The author wishes to thank Professor George E. Forsythe for assistance with this work.

\section{ALGORITHM 175}

SHUTTLE SORT

C. J. Shaw. and T. N. Trimble

System Development Corporation, Santa Monica, Calif.

procedure shuttle sort ( $m$, Temporary, $N$ );

value $m$; integer $m$; array $N[1: m]$;

comment This procedure sorts the list of numbers $N[1]$ through $N^{\prime}[\mathrm{m}]$ into numeric order, by exchanging out-of-order number pairs. The procedure is simple, requires only Temporary as extra storage, and is quite fast for short lists (say 25 numbers) and fairly fast for slightly longer lists (say 100 numbers). For still longer lists, though, other methods are much swifter. The actual parameters for Temporary and $N$ should, of course, be similar in type;

begin integer $i, j$;

for $i:=1$ step 1 until $m-1$ do

begin

for $j:=i$ step -1 until 1 do

begin

if $N[j] \leq N[j+1]$ then go to Test;

Exchange: Temporary $:=N[j] ; \quad N[j]:=, N[j+1]$;

Test: end of $i$ loop

$N[j+1]:=$ Temporary; end of $j$ loop;

end shuttle sort

\section{CERTIFICATION OF ALGORITHM 175}

SHUTTLE SORT [C. J. Shaw and T. N. Trimble, Comm. $A C M$, June 1963]

GeORGe R. Schubert**

University of Dayton, Dayton, Ohio

- Undergraduate research project, Computer Science Program, Univ. of Dayton.

Algorithm 175 was translated into BALGOL and ran successfully

on the Burroughs 220 . The following actual sorting times were observed:

Number of llems
25
50
100
250
500

The algorithm can le extended so that the sort is made on one array, while retaining a one-to-one correspondence to a second array. This is done by inserting immediately before end of the $j$ loop the following:

Temporary $:=Y[j] ; \quad Y[j]:=Y[j+1] ; \quad Y[j+1]:=T e m p o-$ rary; where $Y[k]$ is the element to be associated with $N[k]$. Other variations are obviously possible.

\section{REMARK ON ALGORITHM 175}

SHUTTLE SORT [C. J. Shaw and T. N. Trimble, Comm. $A C M \quad 6$ (June 1963), 312; G. R. Schubert, Comm. $A C M 6$ (Oct. 1963), 619; O. C. Juelich, Comm. ACM 6 (Dec. 1963), 739]

Otтo C. Juelich (Recd. 18 Dec. 1963)

North American Aviation, 4300 E. Fifth Ave., Columbus, Ohio

The appearance of Schubert's certification has caused me to restudy the algorithm. What I supposed were crrors amount to a rearrangement of the order in which the comparisons are carried out. The efficiency of the algorithm is not much affected by the rearrangement, since the number of executions of the statements labeled Exchange remains the same.

\section{REMARK ON ALGORITHM $17 \bar{j}$}

SHUTTLE SORT [C. J. Shaw and T. N. Trimble, Comm. $A C M$ 6, June 1963]

O. C. JUELICH

North American Aviation, Inc., Columbus, Ohio

The authors of this algorithm do well to remind the reader that "Shuttle Sort" is not an efficient procedure, except for lists of items so short that they do not justify the housekeeping apparatus needed by the usual sorting routines.

The algorithm as published is not free from errors. The statement

for $j:=i$ step - 1 until 1 do

should be replaced by eilher:

for $j:=m-1$ step -1 until $i$ do

or

for $j:=1$ step 1 until $m-i$ do

In the former case the process can be visualized as placing the $i$ th smallest element in place on the $i$ th pass; in the latter the $i$ th largest element is put in place on the $i$ th pass.

The label "Test" should precede the delimiter "end of $j$ loop" rather than the "end of $i$ loop". The algorithm can be slightly accelerated by rewriting the body of the procedure:

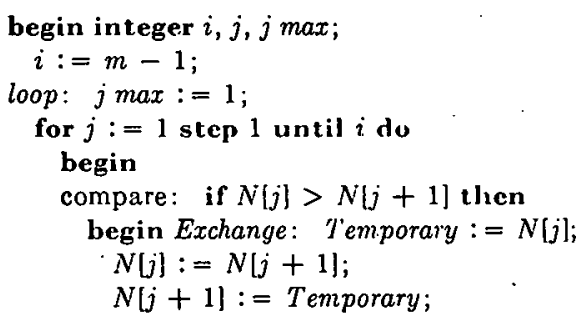




\author{
$j \max :=j$ \\ end Exchange; \\ end of $j$ loop; \\ $i:=j \max$ \\ if $i>1$ then go to loop; \\ end shutlle sort
}

The revised procedure body will eliminate redundant iterations when some of the data is already ordered.

It was studied in this form by R. L. Boyell and the writer on the Ordvac at Ballistics Research Laboratories, Aberdeen Proving Ground, in 1955. For randomly ordered data the $i$-loop may be expected to be executed about $m-\sqrt{ } m$ times.

\section{ALGORITHM 176 \\ LEAST SQUARES SURFACE FIT}

T. D. ARthurs

The Boeing Company, Transpört Division, Renton, Wash.

procedure $\operatorname{SURFIT}(F, z, W, m, n)$ answers: $(a, e, r m s)$;

integer $m, n$; real $r m s ; \operatorname{array} F, z, W, e$;

procedure Invert, sqrt;

comment Given a set of $m$ ordinates and the corresponding values of $n$ prescribed general functions, $\left(f_{i}\right)$, of one or more linearly independent variables, this procedure fits the points, in the least squares sense, with a function of the form $a_{1} f_{1}+a_{2} f_{2}$ $+\ldots+a_{n} f_{n}$ where $a_{i}$ are the unknown coefficients. Also computed are the vectors of residuals $\left(e_{j}\right)$ and their lengths ( $r m s$ ). Provision.is made for weighting the data points. Essentially, the matrix equation $F^{T} W F a=F^{T} W z$ is solved, where $a$ is the vector of unknowns, $W$ is an $m \times m$ diagonal matrix of data point weights, $z$ is the vector of ordinate values and $F$ is the $m \times n$ matrix of corresponding function values. The availability of a procedure Invert, which replaces a real matrix with its inverse, is assumed;

begin integer $i, j, k$; real sqsum, $g$; array $G[1: n, 1: n]$; comment $G$ is working space for the inversion procedure; sqsum :=0;

for $i:=1$ step 1 until $n$ do

for $j:=1$ step 1 until $n$ do

hegin $G[i, j]:=0$;

for $k:=1$ step 1 until $m$ do

$G[i, j]:=G[i, j]+F[k, i] \times F[k, j] \times W[k]$

end $j$;

Invert $(G, n)$;

for $i:=1$ step 1 until $n$ do

begin $a[i]:=0$;

for $j:=1$ step 1 until $m$ do

begin $g:=0$;

for $k:=1$ step 1 until $n$ do

$g:=g+G[i, k] \times F[j, k]$

$a[i]:=a[i]+g \times z[j] \times W[j]$

end $j$

end $i$;

for $i:=1$ step 1 until $m$ do

begin $e[i]=y[i]$;

for $j:=1$ step 1 until $n$ do

$e[i]:=e[i]-a[j] \times F[i, j]$;

$s q s u m:=s q s u m+e[i] \uparrow 2$

end $i$;

$r m s:=s q r t(s q s u m / m)$

end SURFIT
ALGORITHM 177

LEAST SQUARES SOLUTION WITH CONSTRAINTS

M. J. SYNGE

The Boeing Company, Transport Division, Renton, Wash.

procedure $\operatorname{CONLSQ}(A, y, w, n, m, r)$ results: $(x)$ residuals: $(e, r i n s)$;

real $r m s ;$ integer $n, m, r$; array $A, y, w, x, e$; procedure $a b s, S U R F I T$;

comment This procedure solves an overdetermined set of $n$ simultaneous linear equations in $m$ unknowns, $A x=y$. The first $r$ equations $(r \leq m)$ are satisfied exactly and the remaining $n-r$ are satisfied as well as possible by the method of least squares. Each equation is assigned a weight from the vector $w$, although the first $r$ weights have no relevance. This procedure may be used for curve or surface fitting when the approximating function or its derivatives are required to have fixed values at a number of points;

begin integer $i, j, k, i i$, ick; integer array $i c[1: m]$; array $B[1: n-r, 1: m-r]$; real $A$ max;

for $i:=1$ step 1 until $r$ do

begin $k:=1$; for $j:=2$ step 1 until $m$ do

begin if abs $(A[i, j])>a b s(A[i, k])$ then $k:=j$; “ eud; $i c[i]:=k ; \quad A \max :=A[i, k] ;$ for $j:=1$ step 1 until $m$ do $A[i, j]:=A[i, j] / A \max ; \quad y[i]:=y[i] / A \max$;

for $i i:=1$ step 1 until $r$ do

begin if $i i=i$ then go to skip; $A \max :=A[i i, k]$;

for $j:=1$ step 1 until $m$ do

$A[i i, j]:=A[i i, j]-A[i, j] \times A \max$;

$y[i i]:=y[i i]-y[i] \times A \max$;

skip: end $i i$

end $i$;

$i c k:=r+1 ;$ for $j:=1$ step 1 until $m$ do

begin $k:=1$;

repeat: if $j=i c[k]$ then go to next;

$k:=k+1 ;$ if $r \geqq k$ then go to repeat;

$i c[i c k]:=j ; \quad i c k:=i c k+1$;

next: end $k$;

for $i:=r+1$ step 1 until $n$ do

begin for $k:=1$ step 1 until $r$ do

$y[i]:=y[i]-y[k] \times A[i, i c[k]]$

for $j:=r+1$ step 1 until $m$ do

begin $B[i, j]:=A[i, i c[j]]$;

for $k:=1$ step 1 until $r$ do

$B[i, j]:=B[i, j]-A[i, i c[k]] \times A[k, i c[j]]$ end $j$

end $i$;

$\operatorname{SURFIT}(B, y[r+1: n], w[r+1: n], n-r, m-r, x[r+1: m]$, $e[r+1: n], r m s)$;

comment The procedure SURFIT is called to solve the reduced set of $n-r$ simultaneous linear equations in $m-r$ unknowns, $B x_{2}=y_{2}^{\prime}$, which have no constraints;

for $j:=r+1$ step 1 until $m$ do $x[i c[j]]:=x[j]$;

for $j:=1$ step 1 until $r$ do

begin $x[i c[j]]:=y[j]$

for $i:=r+1$ step 1 until $m$ do

$x[i c[j]]:=x[i c[j]]-A[j, i c[i]] \times x[i c[i]]$

end $j$

end CONLSQ

REMARK ON ALGORITHM 177

LEAST SQUARES SOLUTION WITH CONSTRAINTS

[Michael J. Synge, Comm. ACM, June 63]

Michael J. Synge

The Boeing Co., Transport Division, Renton, Wash.

In row-reducing the constraint equations, CONLSQ does not 
use full pivoting nor does it detect redundancy or inconsistency of the constraints; it was felt that the constraints were likely to be few in number and well-conditioned. However, these omis sions may be made good by replacing the statement

$$
i c k:=i c k+1 \text {; }
$$

by

$$
\text { done: } i c k:=i c k+1 \text {; }
$$

and substituting the lines below for the first seven lines of the first compound statement of CONLSQ. If inconsistency is found, the procedure exits to the nonlocal label inconsistent. A roundoff tolerance, eps, is used in checking consistency, and some numerical value (e.g. $10^{-0}$ ) should be substituted for it.

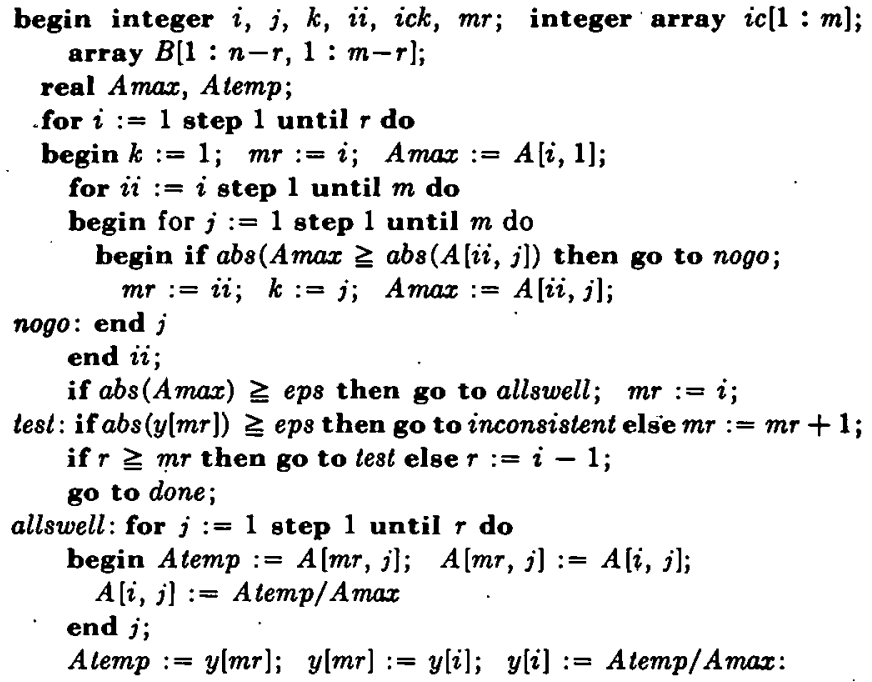

The Algorithm then continues with the line: for $i i:=1$ step 1 until $r$ do

\section{ALGORITHM 178 \\ DIRECT SEARCH}

Arthur F. Kaupe, JR.

Westinghouse Electric Corp., Pittsburgh, Penn.

procedure direct search ( $p s i, X, D E L T A$, rho, delta, $S$ );

value $K, D E L T A$, rho, della; integer $K$; array $p s i$; real $D E L T A$, rho, delta; real procedure $S$;

comment This procedure may be used to locate the minimum of the function $S$ of $K$ variables. A discussion of the use of this procedure may be found in: Robert Hooke and T. A. Jeeves, 'Direct Search' Solution of Numerical and Statistical Problems [J. ACM 8, 2 (1961), 212-229]. The notation is essentially that used in Appendix $B$ of the cited paper. The exceptions being the spelling of the Greek letters and the introduction of notation to distinguish between the process of calculating a value of $S$ and the value itself-thus $S$ (phi) and $S p h i$. A modified version of this procedure acceptable to the $\mathrm{BAC}$ compiler for the Burroughs 205 and 220 computers has been prepared and run successfully; begin real $S S, S p s i$, Sphi, theta; array $p h i[i: K] ; \cdots$ integer $K, k$; procedure $E$; for $k:=1$ step 1 until $K$ do $\cdots$

begin phi $[k]:=$ phi $[k]+D E I . T A ; \quad S p h i:=S($ phi $) ;$

if $S p h i<S S$ then $S S:=S p h i$ else

begin phi $[k]:=$ phi $[k]-2 \times D E L T A ; \quad S p h i:=S(p h i)$ :

if $S p h i<S S$ then $S S:=S p h i$ else phi $[k]:=$ phi $[k]+D E L I ' A$ end $E$;
Start: $S p s i:=S(p s i)$

1: $S S:=S p s i$

for $k:=1$ step 1 until $K$ do phi $[k]:=p s i[k] ; E$;

if $S S<S p s i$ then begin

2: for $k:=1$ step 1 until $K$ do begin

theta $:=p s i[k]$;

psi $[k]:=p h i[k \mid ;$

phi $[k]:=2 \times$ phi $[k]-$ theta end;

Spsi : =SS; SS :=Sphi $:=S(p h i) ; E$;

if $S S<S p s i$ then go to 2 else go to 1 end;

3: if DEIT'A $\geq$ delta then begin DELTA : - rho $\times$ DEL'TA; go to 1 end end

\section{ALGORITHM 179 \\ INCOMPLETE BETA RATIO*}

Oliver G. Ludwig

Mathematical Jaboratory and Department of Theoretical Chemistry, University of Cambridge, Fngland

* Based in part on work done at Carnegie Institute of Technology, Pittsburgh, Pennsylvania and supported by the Petroleum Research Fund of the American Chemical Society and by the National Science Foundation.

real procedure incompletebeta $(x, p, q$, epsilon);

value $x, p, q$; real $x, p, q$, epsilon;

begin real finsum, infsum, temp, temp 1, term, term 1, qrecur, index; Boolean alter

comment This procedure evaluates the ratio $B_{x}(p, q) / B_{1}(p, q)$, where $B_{x}(p, q)=\int_{0}^{x} t^{p-1}(1-t)^{q-1} d t$, with $0 \leqq x \leqq 1$ and $p, q>0$, but not necessarily integers. It assumes the existence of a nonlocal label, alarm, to which control is transferred upon entry to the procedure with invalid arguments. Also assumed is a procedure to evaluate $\int_{0}^{\infty} t^{p} e^{-t} d t$ which is called factorial (p), (cf. e.g. Algorithm 80, March, 1962);

if $x>1 \vee x<0 \vee p \leqq 0 \vee q \leqq 0$ then go to alarm;

if $x=0 \vee x=1$ then hegin incompletebeta: $=x$; go to End end;

comment This part interchanges arguments if necessary to ob. tain better convergence in the power series below;

if $x \leqq 0.5$ then alter $:=$ false else

begin alter $:=$ true; $\operatorname{tem} p:=p ; \quad p:=q ; q:=\operatorname{temp} ; \quad x:=$ $1-x$ end;

comment This part recurs on the (effective) $q$ until the power series bclow does not allernate;

finsum $:=0 ;$ term $:=1 ;$ lemp $:=1-x ;$ qrecur $:=$ index $:=q$;

for index $:=$ index -1 while index $>0$ do

begin qrecur:= index;

lerm : = term $\times($ grecur +1$) /($ temp $\times(p+q r e c u r))$;

finsum : $=$ finsum + term

end;

comment This part sums a power series for non-integral efiective $q$ and yields unity for integer $q$;

infsum $:=\operatorname{term}:=1$; index $:=0$;

comment In the following statement the convergence criterion might well be altered to term $>$ epsilon, since infsum $>1$ always, thus saving one divide per cycle at the cost, perhaps, of a -few more cycles;

for index $:=$ index +1 while $($ term/infsum $)>$ epsilon do

begin lerm $:=$ term $\times x \times$ (index-qrecur $) \times(p+$ index-1)/ (index $\times(x+$ index $)) ;$ infsum $:=$ infsum + term end;

comment This part evaluates most of the necessary factorial functions, minimizing the number of entries into the factorial procedure; 
temp $:=\operatorname{temp} 1:=$ factorial $($ qrecur -1$)$

term $:=$ term $1:=$ factorial $($ qrecur $+p-1)$;

for index : = qrecur step 1 until $(q-0.5)$ do

begin temp $1::=$ temp $1 \times$ index;

term $1:=$ term $1 \times($ index $+p)$

end;

comment This part combines the partial results into the final une;

lemp $:=x \cdot \uparrow p \times($ infsum $\times$ term $/(p \times$ temp $)+$ finsum $\times$ term $1 \times$ $(1-x) \uparrow q /(q \times$ lemp 1$)) /$ factorial $(p-1)$;

incompletebeta $:=$ if alter then $1-$ temp else temp;

end: end incompletebeta

\section{AlGORITHM 180}

ERROR FUNCTION-LARGE X

HeNRY C. THACHER, JR.*

Argonne National Laboratory, Argonne, Ill.

* Work supported by the U.S. Atomic Energy Commission.

real procedure $\operatorname{erf} L(x)$; value $x$; real $x$;

comment This procedure evaluates the error function of real argument, $\operatorname{erf}(x)=(2 / \sqrt{ } \bar{\pi}) \int_{0}^{x} e^{-u^{2}} d u$ by the Laplace continued

fraction for the complementary error function: $\operatorname{erf}(x)=1-$ $(1 /(1+v /(1+2 v /(1+3 v /(1+\cdots))))) /\left(\sqrt{\pi} x e^{x^{2}}\right)$ where $v=1 /$ $\left(2 x^{2}\right)$. Successive even convergents of the continued fraction are evaluated, using an algorithm suggested by Maehly, until the full accuracy of the arithmetic being used is attained.

The continued fraction converges for all $x>0$. For small $x$, however, convergence may be excessively, slow, and overflow may occur. In this region, the Taylor series converges satisfactorily, and algorithms such as No. 123 are suitable.

For $x \leqq 0$, the procedure calls the global procedure alarm.

The body of this procedure has been checked on the LGP-30 computer, using the Dartmouth Self Contained Algol Processor. The program was used to tabulate $\operatorname{erf}(x)$ from $0.9(.1) 5.0$. The maximum error was $2 \times 10^{-6}$, which is explainable by roundoff errors. The number of convergents calculated ranged from 36 for $x=0.9$ to 2 for $x \geqq 3.3$. Overflow occurred for $x=0.87$;

begin integer $m$; real $B \min 2, B \min 3, P, R, T, v, v 2$;

if $x \leq 0$ then alarm;

$v:=x \times x$

$T:=-0.56418958 / x / \exp (v)$;

comment The constant $0.56418958 \ldots \subseteq \pi^{-1 / 2}$, and should

be given to the full accuracy required of the procedure;

$v:=0.5 / v$

$P=v \times T$

$v 2:=v \times v$

$T:=T+1$

$m:=0$;

$R:=B \min 3:=B \min 2:=1$;

for $m:=m+1$ while $T \neq R$ do

begin $R:=T$;

$B \min 3:=v \times(m-1) \times B \min 3+B \min 2$

$T:=B \min 2$;

$B \min 2:=v \times m \times B \min 2+B \min 3$;

$T:=R-P / B \min 2 / T$

$P:=m \times(m+1) \times v 2 \times P$

end while;

$\operatorname{erf} L:=T$

end

\section{ALGORITHM 181 \\ COMPLEMENTARY ERROR FUNCTION- LARGE $\mathbf{X}$}

Henry C. Thacher, Jr.*

Argonne National Laboratory, Argonne, Ill.

- Work supported by the U. S. Atomic Energy Commission.

real procedure $\operatorname{erfcL}(x)$; value $x$; real $x$;

comment This procedure evaluates the complementary error function, $\operatorname{erfc}(x)=1-\operatorname{erf}(x)=(2 / \sqrt{\pi}) \int_{x}^{\infty} \exp \left(-u^{2}\right) d u$ by

the Laplace continued fraction:

$\operatorname{erfc}(x)=(1 /(1+v /(1+2 v /(1+3 v /(1+\cdots))))) /\left(\sqrt{x} x e^{x^{2}}\right)$

where $v=1 /\left(2 x^{2}\right)$. Successive even convergents of the continued fraction are evaluated, using an algorithm suggested by Maehly, until the full accuracy of the arithmetic being used is attained.

The continued fraction converges for all $x>0$. For small $x$, however, convergence may be excessively slow, and overflow and round-off accumulation may occur. In this region, the Taylor series converges satisfactorily.

For $x \leqq 0$, the procedure calls the global procedure alarm.

The body of this procedure has been checked on the LGP-30 Computer, using the Dartmouth Self Contained Algol Processor, for $x=1.2(0.1) 5.0$. Results were generally correct to 1 in the 6th significant digit, although a few errors were as large as 6 in that digit. The errors are believed to be due to round-off only. The number of convergents calculated ranged from 46 for $x=1.2$ to 10 for $x=5.0$.

Overflow occurred for $x=1.183$;

begin integer $m$; real $B \min 2, B \min 3, P, R, T, v, v 2$;

if $x \leq 0$ then alarm;

$v:=x \times x$

$T:=0.56418958 / x / \exp (v)$;

comment The constant $0.56418958 \cdots=\pi^{-1 / 2}$, and should be given to the full accuracy required of the procedure;

$v:=0.5 / v$

$v 2:=v \times v$

$P:=v \times T$

$m:=R:=0$

$B \min 3:=B \min 2:=1$;

for $m:=m+2$ while $K \neq T$ do

begin $R:=T$;

$B \min 3:=v \times(m-1) \times B \min 3+B \min 2$;

$T:=B \min 2$

$B \min 2:=v \times m \times B \min 2+B \min 3$;

$T:=R-P / B \min 2 / T$;

$P:=m \times(m+1) \times v 2 \times P$

end while;

erfc $L:=T$

end

\section{CERTLFICATION OF ALGORITHM 181 [S15]}

COMPLEMENTARY ERROR FUNCTION-LARGE

$\mathrm{X}$ [Henry C. Thacher, Jr., Comm. ACM 6 (June 1963), $315]$

I. Clauusen and L. Hansson (Recd. 20 Aug. 1964)

DAEC, Ris $\varnothing$, Denmark.

The procedure erfcL was tested in Gren-Algol with 29 significant bits and the number-range $a b s(x)<2 \uparrow 512$ (approx. 1.310154). The statement $m:=R:=0$; was corrected to $m:=0 ; R:=$ 0 ; (Because $m$ and $R$ are of different type; of. Sec. 4.2.4 of the Algol Report, Comm. ACM 6 (Jan. 1963), 1-17.-Ed.] After this the tests were successful. The procedure was checked a.o. for $x=1.19(-0.01) 0.72$. The differences from table values increased from $10-8$ at $x=1.1$ to $710-8$ at $x=0.75$. Overflow occurred at $x=0.71$. 


\section{ALGORITHM 182}

\section{NONRECURSIVE ADAPTIVE INTEGRATION}

W. M. McKeeman and Larry Tesler

Stanford University, Stanford, Calif.

real procedure Simpson $(F)$ limits : $(a, b)$ tolerance : $(e p s)$;

real procedure $F$; real $a, b$, eps; value $a, b, e p s$;

begin comment A nonrecursive translation of Algorithm 145.

Note that the device used here can be used to simulate recursion

for a wide class of algorithms;

integer $l v l$;

switch return $:=r 1, r 2, r 3$;

real array $d x, e p s p, x 2, x 3, F 2, F 3, F 4, F m p, F b p$,

est2, est3 [1:30], pual[1:30, 1:3];

integer array $\operatorname{rtrn}[1: 30]$;

real absarea, est, $F a, F m, F b, d a, s x, e s t 1, s u m, F 1$;

comment the parameter setup for the initial call;

$l v l:=a b s a r e a:=e s t:=0 ; d a:=b-a$;

$F a:=F(a) ; F m:=4.0 \times F((a+b) / 2.0) ; F b:=F(b) ;$

recur:

$l v l:=l v l+1 ; \quad d x[l v l]:=d a / 3.0$

$s x:=d x[l v l] / 6.0 ; \quad F l:=4.0 \times F(\mathrm{a}+d x[l v l] / 2.0)$;

$x 2[l v l]:=a+d x[l v l] ; \quad F 2[l v l]:=F(x 2[l v l]) ;$

$x 3[l v l]:=x 2[l v l]+d x[l v l] ; \quad F 3[l v l]:=F(x 3 \mid l v l]) ;$

epsp[lvl] := eps; $F 4[l v l]:=4.0 \times F(x 3[l v l]+d x[l v l]) ;$

$F m p[l v l]:=F m ; \quad$ est $1:=(F a+F 1+F 2[l v l]) \times s x$

$F b p[l v l]:=F b ; \quad$ est $2[l v l]:=(F 2[l v l]+F 3[l v l]+F m) \times s x ;$ $e s t 3[l v l]:=(F 3[l v l]+F 4[l v l]+F \mathrm{~b}) \times s x$

sum $:=e s t]:+e s t 2[l v l]+e s t 3[l v l] ;$

absarea $:=a b s a r e a-a b s(e s t)+a b s(e s t 1)+a b s(e s t 2[l v l])+$ abs (est3[lvl]);

if $(a b s(e s t-s u m) \leq e p s p[l v l] \times$ absarea $) \vee(l v l \geq 30)$ then

begin comment done on this level;

up $: l v l:=l v l-1$

pval $[l v l, r t r n[l v l]]:=$ sum;

go to return [rirn $[l v l]]$

end;

$r$ trn $[l v l]:=1 ; \quad d a:=d x[l v l] ; \quad F m:=F 1$

$F \mathrm{~b}:=F 2[l v l] ;$ eps $:=\operatorname{epsp}[l v l] / 1.7 ;$ est $:=$ est1;

go to recur; $r 1$ :

$\operatorname{rtrn}[l v l]:=2 ; d a:=d x[l v l] ; \quad F a:=F 2[l v l] ;$

$F m:=F m p[l v l] ; \quad F b:=F 3[l v l] ; \quad$ eps $:=e p s p[l v l] / 1.7$;

est $:=e s t 2[l v l] ; \quad a:=x 2[l v l] ;$ go to recur; $r 2$

rtrn $[l v l]:=3 ; \quad d a:=d x[l v l] ; F a:=F 3[l v l]$;

$F m:=F 4[l v l] ; \quad F b:=F b p[l v l] ; \quad$ eps $:=e p s p[l v l] / 1.7 ;$

est $:=$ est $3[l v l] ; \quad a:=x 3[l v l] ;$ go to recur; $r 3$ :

sum $:=\operatorname{pval}[l v l, 1]+\operatorname{pval}[l v l, 2]+\operatorname{pval}[l v l, 3]$;

if $l v l>1$ then go to $u p$;

Simpson := sum

end Simpson

\section{CERTIFICATION OF ALGORITHM 182}

NONRECURSIVE ADAPTIVE INTEGRATION [W.

M. McKeeman and Larry Tesler, Comm. ACM 6 (June 1963), 315]

Harold S. Butler (Recd 8 Nov. 1963; rev. 6 Dec. 1963)

Stanford Linear Accelerator Center, Stanford, Calif.

A BaLgol transliteration of Simpson has been prepared at Stanford by its authors and it has been used in a number of problems involving numerical integration. Its value was most strikingly displayed when it was utiliżed in a triple integral in which the final integration was over a strongly peaked function that spanned seven orders of magnitude. Simpson effectively minimized the number of evaluations and completed the integration five times faster than alternate schemes to subdivide the region of interest. The values of the integral agreed with independent calculations well within the required tolerance.

The following changes should be made to the published algorithm:

Line 13 should be changed to:

lvl $:=0 ;$ absarea $:=e s t:=1.0 ; d a:=b-a ;$

Line 17 should read:

$s x:=d x[l v l] / 6.0 ; \quad F 1:=4.0 \times F(a+d x[l v l] / 2.0) ;$

Line 20 should read:

epsp $[l v l]:=e p s ; \quad F 4[l v l]:=4.0 \times F(x 3[l v l]+d x[l v l] / 2.0)$

The condition of line 27 should be changed to:

if $((a b s(e s t-s u m) \leqq e p s p[l v l] \times$ absarea $) \wedge(e s t \neq 1.0)) \vee$ $(l v l \geqq 30)$ then

ALGORITHM 183

REDUCTION OF A SYMMETRIC BANDMATRIX TO TRIPLE DIAGONAL FORM

H. R. SchWARZ

Swiss Federal Institute of Technology, Zürich, Switzerland

procedure bandred $(a, n, m)$; value $n, m$; integer $n, m$; array $a$;

comment bandred reduces a real and symmetric matrix of band type (order $n, a[i, k]=0$ for $|i-k|>m$ ) by a sequence of orthogonal similarity transformations to triple diagonal form. The procedure represents a generalization of the algorithm $m 21$ by H. Rutishauser. Due to symmetry only the upper part of the band matrix must be given and these elements are denoted for convenience in the following way: $a[i, 0](i=1,2, \cdots, \mathrm{n})$ represents the diagonal element in the $i$ th row, and $a[i, k](i=1,2$, $\cdots, n-b$ and $k=1,2, \cdots, m$ ) represents the generally nonzero element in the $i$ th row and the $k$ th position to the right of the diagonal. After completion of the reduction, the elements of the symmetric triple diagonal matrix are given by $a[i, 0](i=1,2$, $\cdots, n)$ and $a[i, 1](i=1,2, \cdots, n-1)$;

begin integer $r, k, i, j, p, r r$; real $b, g, c, s, c 2, s 2, c s, u, v$;

for $r:=m$ step -1 until 2 do

begin

for $k:=1$ step 1 until $n-r$ do

begin

for $j:=k$ step $r$ until $n-r$ do

begin

comment This compound statement describes the rotation involving the $i$ th and $(i+1)$ st rows and columns in order to reduce either $a[j, r]$ or the off-band element $g$ to zero, respectively. This rotation produces a new off-band element $g$ (in general different from zero) provided $i+r<n$

if $j=k$ then

begin if $a[j, r\}=0$ then go to endk; $b:=-a[j, r-1] / a[j, r]$

end

else

begin if $g=0$ then go to endk; $b:=-a[j-1, r] / g$

end;

$s:=1 / \operatorname{sgrt}(1+b \times b) ; \quad c:=b \times s$

$c 2:=c \times c ; s 2:=s \times s ; c s:=c \times s$; $i:=j+r-1$;

cross elements:

$u:=c 2 \times a[i, 0]-2 \times c s \times a[i, 1]+s 2 \times a[i+1,0] ;$ 
$v:=s 2 \times a[i, 0]+2 \times c s \times a[i, 1]+c 2 \times a[i+1,0]$

$a[i, 1]:=c s \times(a[i, 0]-a[i+1,0])+(c 2-s 2) \times a ́[i, 1]$;

$a[i, 0]:=u ; \quad a[i+1,0]:=v$;

column rotation:

for $p:=j$ step 1 until $i-1$ do

begin

$u:=c \times a[p, i-p]-s \times a[\dot{p}, i-p+1] ;$

$a[p, i-p+1]:=s \times a[p, i-p]+c \times a[p, i-x+1]$

$a[p, i-p]:=u$

end $p$;

if $j \neq k$ then

row rotation:

$a[j-1, r]:=c \times a[j-1, r]-s \times g$

$r r:=$ if $r \leq n-i$ then $r$ else $n-i$;

for $p:=2$ step 1 until $r r$ do

begin

$u:=c \times a[i, p]-s \times a[i+1, p-1]$

$a[i+1, p-1]:=s \times a[i, p]+c \times a[i+1, p-1]$

$a[i, p]:=u$

end $p$

if $i+r<n$ then

new g: begin $g:=-s \times a[i+1, r]$

$a[i+1, r]:=c \times a[i+1, r]$

end

end $j$;

endk: end $k$

end $r$

end bandred

\section{ALGORITHM 184}

\section{ERLANG PROBABILITY FOR CURVE FITTING}

\section{A. Colker}

U. S. Steel Applied Research Laboratory

Monroeville, Penn.

procedure ERLANG (X,XO, M, VARS, C, FACTORIAL, P); value $X O, M, V A R S, C$; integer $C$; real array $X, P$;

integer procedure $F A C T O R I A L$;

comment Computes the Erlang probability for the $i$ th interval by $\int_{0}^{x_{i}} f(x) d x-\int_{0}^{x_{i}-1} f(x) d x$ where $f(x)=+\left[\left(K_{\mu}\right)^{K} /(K-1) !\right]$ . $\left(x-x_{0}\right)^{K-1} e^{-K \mu\left(x-x_{0}\right)}$ where $\mu=1 / M, K=\left(M-X_{0}\right)^{2} V A R S$ is the upper boundary for the class intervals, $X_{0}$ is the lower boundary of the first class interval, $M$ is the mean of the Erlang, VARS is the variance corrected by Sheppard's correction, $C$ is the number of class intervals and $P_{i}$ is the calculated probability;

\section{begin}

integer $I, J, K, F$; real array $X E[0: C]$;

for $I:=1$ step 1 until $C$ do

$X E[I]:=X[I]-X O$

$X E[0]:=0$;

$M E:=M-X O$

$K:=0.5+(M E \uparrow 2) / V A R S$

$U:=K / M E$;

$S P:=0$;

for $I:=1$ step 1 until $C$ do

begin

$S U M 1:=0$

$S U M 2 \cdot:=0$

for $J:=0$ step 1 until $K-1$ do

begin

$F:=F A C T O R I A L(J)$

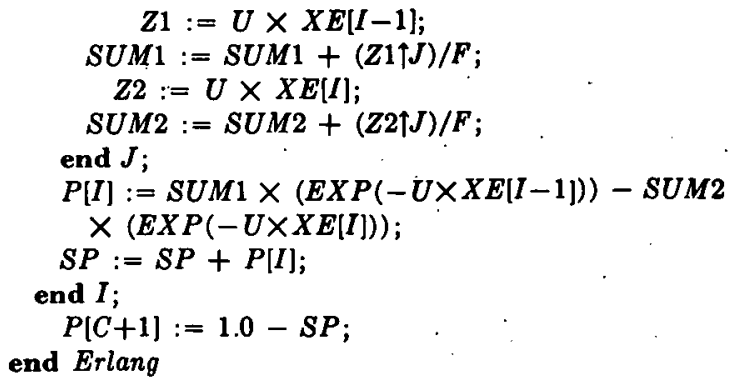

ALGORITHM 185

NORMAL PROBABILITY FOR CURVE FITTING

A. Colker

U. S. Steel Applied Research Laboratory

Monroeville, Penn.

procedure NORMAL $(X, M, V A R S, C, H A S T I N G S, P)$

value $M, V A R S, C$; integer $C$; real array $X, P$;

real procedure HASTINGS;

comment Computes the normal probabilities for the $i$ th interva] by $\int_{0}^{x_{i}} f(x) d x-\int_{0}^{x_{i}-1} f(x) d x$.where $f(x)$ is Hastings' approxi. mation to the normal interval. Hastings' formula is

$$
\phi\left(X_{n i}\right)=\frac{1}{2}\left[1-\left(1+a_{1} X_{n i}+a_{2} X_{n i}^{2}+a_{3} X_{n i}^{3}+a_{4} X_{n i}^{4}+a_{5} X_{n i}^{5}\right)^{-8}\right]
$$

where $a_{1}=0.09979268, a_{2}=0.04432014, a_{3}=0.00969920$, $a_{4}=-0.00009862$, and $a_{5}=0.00058155$. The $X_{n i}$ are normalized boundary values of $X_{i}$ where $X_{n i}=\left(X_{i}-M\right) / \sqrt{ } V A R S$, where $M$ is the mean and $V A R S$ is the variance corrected by Sheppard's correction, $C$ is the number of class intervals and $P_{i}$ the calculated probability;

begin

integer $I$; real array $X N[1: C]$;

for $I:=1$ step 1 until $C$ do $X N[I]:=(X[I]-M) / S Q R T(V A R S)$;

$P[1]:=0.5-H A S T I N G S(A B S(X N[1]))$;

for $I:=2$ step 1 until $C$ do

begin

if

$X N[I]<0$ then

$P[I]:=$ HASTINGS $(A B S(X N[I-1]))-H A S T I N G S$

$(A B S(X N[I])) ;$ else

begin

if $(X N[I]>0) \wedge(X N[I-1]<0)$

then $P[I]:=H A S T I N G S(X N[I])+H A S T I N G S$

$(A B S(X N[1-1])) ;$ else

$P[I]:=$ HASTINGS $(X N[I])-H A S T I N G S(X N \mid I-1])$; end;

end $I$;

$P[C+1]:=0.5-H A S T I N G S(X N[C]) ;$

end NORMAL 


\section{ALGORITHM 186}

\section{COMPLEX ARITHMETIC}

R. P. VAN DE RIET

Mathematical Centre, Amsterdam, Holland

procedure Complex arithmetic $(a, b, R, r)$; value $a, b$; array $a, b, R, r$;

comment This procedure assigns the value $a^{2}+b^{2}$ to $R$ and the value $(a+i b) /(a-i b)$ to $r$, where $a, b, R$ and $r$ are complex numbers. These two arithmetic expressions are of course fully arbitrary. They serve only to demonstrate the use of the procedures $P, Q, S, T, J$ and $U$. With them one can build up any arithmetic expression with complex variables, as easily as one can form them with real variables in ALgol 60 (As one sees immediately these procedures can-easily be extended for use in quaternion arithmetic or general vector and tensor calculus). We focus attention to the value call of the procedure-parameters, which is essential. Furthermore, we notice that the depth or height of the accumulator $H$ is the number of right-handed brackets placed one after another not counting the brackets which occur in parameter-delimeters. It is perhaps superfluous to mention that this procedure was tested on the $\mathrm{X} 1$ computer of the Mathematical Centre.;

begin integer $i, k$; array $H[1: 4,1: 2]$;

integer procedure $P(i, j)$; value $i, j$; integer $i, j$;

comment $P$ forms the product of the $i$ th and $j$ th element of $H$; begin real $a ; k:=k-1 ; a:=H[i, 1] \times H[j, 1]-H[i, 2]$ $\times H(j, 2] ; \quad H[k, 2]:=H[i, 1] \times H[i, 2]+H[i, 2] \times$

end; $H[j, 1] ; \quad H[k, 1]:=a ; \quad P:=k$

integer procedure $Q(i, j)$; value $i, j$; integer $i, j$; comment $Q$ forms the quotient of the $i$ th and $j$ th element of $H$ : begin real $a, b ; k:=k-1 ; b:=H[j, 1] \uparrow 2+H[j, 2] \uparrow 2$; $a:=(H[i, 1] \times H[j, 1]+H[i, 2] \times H[j, 2]) / b$

$H[k, 2]:=(H[i, 2] \times H[j, 1]-H[i, 1] \times H[j, 2]) / b$; $H[k, 1]:=a ; Q:=k$

end;

integer procedure $S(i, j)$; value $i, j$; integer $i, j$; comment $S$ forms the sum of the $i$ th and $j$ th element of $H$;

begin $k:=k-1 ; H[k, 1]:=H[i, 1]+H \mid j, 1]$;

end;

$$
H[k, 2]:=H[i, 2]+H[j, 2] ; S:=k
$$

integer procedure $T(a)$; array $a$;

comment $T$ assigns to the $k+1$ th element of $H$ the complex variable $a$;

begin $k:=k+1 ; \quad H[k, 1]:=a[1] ; \quad H[k, 2]:=a[2] ; \quad T:=k$ end;

integer procedure $J(i$, expi); integer $i$; real expi; comment $J$ assigns to the $(k+1)$ th element of $H$ a complex variable which is decomposed in real and imaginary part;

begin $k:=k+1 ; i:=1 ; \quad H[k, 1]:=\exp i ; \quad i:=2$;

$$
H[k, 2]:=\text { expi; }
$$

$$
J:=\boldsymbol{k}
$$

end;

procedure $U(i, R)$; value $i$; integer $i$; array $R$;

comment $U$ a.ssigna to $R$ the $i$ th clcment of $H$;

begin $R[1]:=H[i, 1] ; \quad R[2]:=H[i, 2] ; \quad k:=0$ end;

$k:=0 ; \quad U(S(P(T(a))$ times: $(T(a)))$ plus: $(P(T(b))$ times: $(T(b))), R)$;

comment $(a \times a)+(b \times b)=: R ; U(Q(S(T(a))$ plus:

$(P(J(i, i-1))$ times: $(T(b))))$ divided by: $(S(T(a))$

plus: $(P(J(i, 1-i))$ times: $(T(b)))), r)$;

comment $(a+(i \times b)) /(a+(-i \times b))=: r$

end Complex Arithmetic;

The contents of this Algorithm are published in the Technical Note TN 27, Mathematical Centre, Nov. 1962.

\section{ALGORITHM 187}

\section{DIFFERENCES AND DERIVATIVES}

\section{R. P. vaN DE RIET}

Mathematical Centre, Amsterdam, Holland

begin real $h$; integer $i, k$; array $A[1: 50]$;

comment This program calculates, only to demonstrate the procedures $D E L T A$ and $D E R$, the third derivative of the exponential function with a sixth order difference scheme. We do not propose to use these procedures in actual calculations, for as we observed with the $\mathrm{X} 1$ computer of the Math. Centre, they work, but very slowly as a consequence of the strong recursiveness of the procedures. In actual programming one has to take the trouble to write out the well-known formula of Gregory, or for higher derivatives to multiply this formula a number of times by itself, ther one has to collect the same function-values. All this trouble is taken over by the computer if one uses the procedures described below. My purpose, however, in publishing these procedures lies not in the numerical use but in a demonstration of the flexibility of Algol 60, if one uses the recursiveness property of procedures.;

real procedure $\operatorname{SUM}(i, h, k, t i)$; value $k$; integer $i, k, h$; real $t i$

begin real $s ; \quad s:=0 ; \quad$ for $i:=h$ step 1 until $k$ do $s:=s+t i$ $S U M:=s$

end;

real procedure $D E L T A(N, k, k 0, f k)$; value $N, k 0$; real $f k$; integer $N, k, k 0$;

comment $N$ is the order of the forward difference which is calculated from a set of function-values with equidistant parameter-values;

begin integer $i$;

DELTA $:=$ if $N=1$

then $S U M(k, k 0, k 0+1,(-1) \uparrow(k+1-k 0) \times f k)$

else $D E L T A(1, i, k 0, D E L T A(N-1, k, i, f k))$

end;

real procedure $D E R(O R, N, h, k, k 0, f k)$; value $O R, N, h, k 0$ real $f k, h_{i}$

integer $O R, N, k, k 0$;

comment $O R$ is the order of the derivative, calculated from a given set of function-values $f(k)$, with equidistant parametervalues, the error is of the order $h \uparrow(N+1-O R)$, where $h$ is the steplength. $k 0$ is the point where the derivative is calculated;

begin integer $i$;

$D E R:=$ if $O R=1$

then $S U M(i, 1, N, D E L T A(i, k, k 0, f k)$ $\times(-1) \uparrow(i+1) / i) / h$

else $D E R(1, N+1-O R, h, i, k 0, D E R(O R-1, N-1, h$

end; $k, i, f k))$

for $i:=1$ step 1 until 50 do $A[i]:=\exp (i / 50)$;

for $i:=1$ step 1 until 25 do $A[i]:=\operatorname{DER}(3,6, .02, k, i, A[k])$ end

The contents of this Algorithm are published in the Technical Note TN 27, Mathematical Centre, Nov. 1962. 


\section{ALGORITHM 188 \\ SMOOTHING 1.}

F. RODRIGUEz-GiL

Central University, Caracas, Venezuela

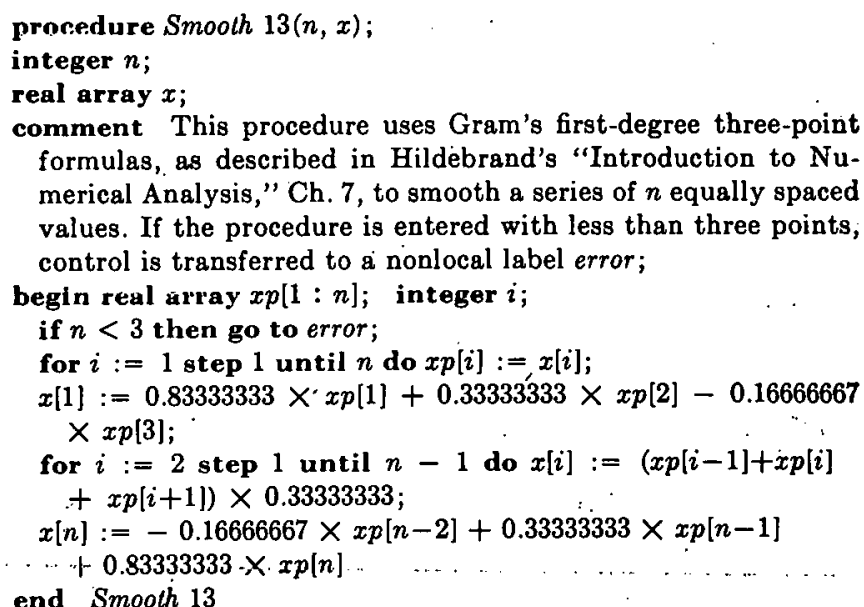

\section{ALGORITHM 189}

SMOOTHING 2

F. Rodriguez GiL

Central University, Caracas, Venezuela

\section{ALGORITHM 190 COMPLEX POWER}

\section{A. P. Relph}

The English Electric Co. Ltd., Whetstone, England

procedure Complex power $(a, b, c, d, n, x, y)$; value $a, b, c, d, n$; real $a, b, c, d, x, y$; integer $n$;

comment This procedure calculates $(x+i y)=(a+i b) \uparrow(c+i d)$ where $i$ is the root of -1 . In the complex plane, with a cut along the real axis from 0 to $-\infty, p$ is the sum of the principal value of the argument of $(a+i b)$ and $2 n \pi$ ( $n$ is positive, negative or zero depending on the solution required). arclan is assumed to be in the range $-\pi / 2$ to $\pi / 2$. The case $n=0, d=0$ is given by Algorithm 106;

begin real $p, r, v, w$; if $a=0$ then begin if $b=0$ then begin $x:=y:=0$; go to $L$ end else $p:=1.57079633 \times$ $(\operatorname{sign}(b)+4 \times n)$

end

else begin $p:=6.28318532 \times n+\arctan (b / a)$ if $a<0$, then begin if $b \geqq 0$ then

if $a<0$ then begin if $b \geqq 0$ then
$\begin{gathered}p \\ \text { else } \\ \text { end }\end{gathered}:=p-3.14159265$
end;
$r:=.5 \times \ln (a \uparrow 2+b \uparrow 2) ; \quad v:=c \times p+d \times r ;$
$w:=\exp (c \times r-d \times p) ;$
$x:=w \times \cos (v) ; \quad y:=w \times \sin (v) ;$

\section{ALGORITHM 191 HYPERGEOMETRIC}

A. P. RELPH

The English Electric Co. Ltd., Whetstone, England

procedure Hypergeometric ( $a 1, a 2, b 1, b 2, c 1, c 2, z 1, z 2)$ Results: $(s 1, s 2)$; value $a 1, a 2 ; b 1, b 2 . c 1, c 2, z 1, z 2$; real $a 1, a 2, b 1, b 2$, $c 1, c 2, z 1, z 2, s 1, s 2$;

begin comment calculates the hypergeometric function $1 F 2(a, b, c, z)$ with complex parameters $(a=a 1+i a 2$, etc);

real $d, y 1, y 2$; integer $n$;

procedure comp mult ( $a 1, a 2, b 1, b 2, c 1, c 2)$; value $b 1$, $a 2, b 1, b 2$; real $a 1, a 2, b 1, b 2, c 1, c 2$;

begin comment calculates the product of the two complex numbers $(a 1+i a 2)$ and $(b 1+i b 2)$ where $i$ is the root of -1 ;

$c 1:=a 1 \times b 1-a 2 \times b 2 ; c 2:=a 2 \times b 1+$ $a 1 \times b 2$

end; $\cdots \cdots \cdots+\cdots$

$s 1:=y 1:=1 ; \quad s 2:=y 2:=0$;

for $n:=1$ step 1 until 100 do

begin $d:=n \times((c 1+n-1) \uparrow 2+c 2 \uparrow 2)$;

comp mult $(a 1+n-1, a 2, y 1 / d, y 2 / d, y 1, y 2)$;

comp mult ( $y 1, y 2, b 1+n-1, b 2, y 1, y 2)$;

comp mult $(y 1, y 2, c 1+n-1,-c 2, y 1, y 2)$;

comp mull ( $(y 1, y 2, z 1, z 2, y 1, y 2)$;

if $s 1=s 1+y 1 \wedge s 2=s 2+y 2$ then go to $L$ $s 1:=s 1+y 1 ; s 2:=s 2+y 2$

end;

$L:$ end 
CERTIFICATION OF ALGORITHMS 191 AND 192 HYPERGEOMETRIC AND CONFLUENT HYPERGEOMETRIC [A. P. Relph, Comm. ACMI 6 (July 1963), 388]

Henry C. Thacher, JR.* (Recd 2 Dec. 1963)

Argonne National Laboratory, Argonne, Ill.

- Work supported by the C.S. Atomic Energy Commission.

The bodies of these two procedures were transcribed for the Dartmouth Scalp processor for the LGP-30 computer. No syntactical errois were found, and the programs gave results agreeing within roundoff (7D) with tabulated values for the following special cases: ${ }_{2} F_{1}\left(0.5,0.5 ; 1 ; k^{2}\right)=(2 / \pi) K(k) ;{ }_{2} F_{1}\left(0.5,-0.5 ; 1 ; k^{2}\right)=$ $(2 / \pi) E(k)$ where $K$ and $E$ are complete elliptic integrals of the first and second kinds; ${ }_{1} F_{1}(.5 ; 1 ; i y)=J_{0}(x)$, and with ${ }_{1} F_{1}(-1 ; 0.1 ; x)$; ${ }_{1} F_{1}(-0.5 ; 0.1 ; x)$, and ${ }_{1} F_{1}(-0.5 ; 0.5 ; x)$.

It should be observed that the function calculated by 191 is ${ }_{2} F_{1}(a, b ; c ; z)$, not $\backslash F_{2}(a, b ; c ; z)$ as stated in the comment. These programs evaluate the functions by direct summation of the hypergeometric series. They are, therefore, relatively general, but inefficient. Precautions must also be taken against attempting to compute outside the range of effective convergence of the series.

\section{ALGORITHM 192}

\section{CONFLUENT HYPERGEOMETRIC}

\section{A. P. RELPH}

The English Electric Co. Inc., Whetstone, England

procedure Confluent hypergeometric $(a 1, a 2, c 1, c 2, z 1, z 2)$

Result : $(s 1, s 2)$; value $a 1, a 2, c 1, c 2, z 1, z 2$;

real $a 1, a 2, c 1, c 2, z 1, z 2, s 1, s 2$;

begin comment calculates the confluent hypergeometric function $1 F 1(a, c, z)$ with complex parameters $(a=a 1+i a 2$, etc);

\section{real $d, y 1, y 2$; integer $n$;}

procedure comp mult ( $a 1, a 2, b 1, b 2, c 1, c 2)$; value $a 1, a 2, b 1, b 2$; real $a 1, a 2, b 1, b 2, c 1, c 2$;

begin comment calculates the product of the two complex numbers $(a 1+i a 2)$ and $(b 1+i b 2)$ where $i$ is the root of -1 ; $c 1:=a 1 \times b 1-a 2 \times b 2$

end; $c 2:=a 2 \times b 1+a 1 \times b 2$

$s 1:=y 1:=1 ; \quad s 2:=y 2:=0$;

for $n:=$ step 1 until 100 do

begin $d:=n \times((c 1+n-1) \uparrow 2+c 2 \uparrow 2)$;

comp mult (a1+n-1, a2, y1/d, y2/d, y1,y2);

comp mult ( $y 1, y 2, c 1+n-1,-c 2, y 1, y 2)$;

comp mult ( $y 1, y 2, z 1, z 2, y 1, y 2)$;

if $s 1=s 1+y 1 \wedge s 2=s 2+y 2$ then go to $L$;

ol $:=s 1+y 1 ; s 2:=s 2+y 2$

$L:$ end

end:

SEE AIGORITHM 191

\section{ALGORITHM 193 \\ REVERSION OF SERIES}

Henry E. Fettis

Aeronautical Research Laboratories, Wright-Patterson Air Force Base, Ohio

procedure SERIESRVRT $(A, B, N)$;

value $A, N$; array $A, B$; integer $N$;

comment This procedure gives the coefficients $B[i]$ for the series $x=y+\Sigma B[i] \times y \uparrow i(i=2,3, \cdots, n)$ when the coefficients $A[i]$ of the series $y=x+\Sigma A[i] \times x \uparrow i$ are given. The procedure uses successive approximations after writing $y_{L_{+1}}=x-\Sigma B[i] \times$ $y_{L} \uparrow i(i=2,3, \cdots, L+2$ and $L=0,1, \cdots, N-2)$ starting with $y_{0}=x$;

begin integer $i, j, k, m$;

array $Q, R[0: N]$;

real $s$;

$A[1]:=B[0]:=0$;

$B[1]:=1$;

for $k:=1$ step 1 until $N-1$ do

begin $B[k+1]:=0$;

for $i:=0$ step 1 until $k+1$ do

$R[i]:=0$

for $j:=k+1$ step -1 until 1 do

begin $Q[0]:=R[0]-A[j]$;

for $i:=1 \cdot \operatorname{stcp} 1$ until $k+1$ do

$Q[i]:=R[i]$;

for $i:=0$ step 1 until $k+1$ do

begin $s:=0$;

for $m:=0$ step 1 until $i$ do

$\mathbf{s}:=s+B[m] \times Q[i-m]$;

$R[i]:=s$

end for $i$;

end for $j$;

for $i:=2$ step 1 until $k+1$ do $B[i]:=R[i]$

end for $k$;

end SERIESRVRT

\section{CERTIFICATION OF ALGORITHM 193}

REVERSION OF SERIES [Henry E. Fettis, Comm. $A C M$ 5, 1962]

HenRY C. Thacher, JR.*

Argonne National Laboratory, Argonne, Ill.

Work supported by the U. S. Atomic Energy Commission

The body of Algorithm 193 was tested on the LGP-30 using the Algol 60 translator developed by the Dartmouth College Computer Center. No syntactical errors were found. The program successfully found the first four coefficients for the series for $\ln (1+y)$ from the first four coefficients of the series for $y=e^{x}-1$.

\section{ALGORITHM 194}

ZERSOL

Carlos Domingo

Ùniversidad Central, Caracas, Venezuela

procedure $Z E R S O L(h, Y I, m$, epsi, $F, f, Z)$; real $h$, epsi, $f$; array $Y I, Z$; integer $m$; procedure $F$;

comment $Z E R S O L$ finds the simple zeros of the solution $Y 1(Y 0)$ of the set of $m$ first order differential equations $Y_{j}=F j(Y 0$, $Y 1, \cdots, Y m) . h$ is the step of integration, epsi the error with 
which the zeros are to be determined (assuming no error in the process of integration). $F(Y S, j, v)$ is a procedure which calcu-. lates the functions $F j$, taking the arguments from the array $Y S$ and leaving the results in $v$. The search for zeros stops when $Y 0>f$. The zeros are stored as elements of the array $Z$. $M R$ is a $4 \times 4$ matrix with the coefficients of a Runge-Kutta method. For example $M R$ may be row-wise $0.5,1,0.5,0,1-a$, $1-a, 1-a, 0.5,1+a, 1+a, 1+a, 0, \frac{1}{6}, \frac{1}{3}, 0.5,0.5$, where $a=\operatorname{sgrt}(2)$;

begin real $v, r, d$; integer $j, s, n, k$; array $Q[1: m], Y S[0: m]$, $Y A L[0: m], Y T[1: m], M R[1: 4,1: 4] ;$ switch $S:=N O Z, Z E R$; $n:=1$;

for $d:=h$ while $Y I[0] \leqq f$ ilo

begin $s:=1$

$R 1:$ for $j:=1$ step 1 until $m$ do

begin $Q[j]:=0.0 ; Y S[j]:=Y I[j] ; Y T[j]:=Y I[j]$ end; $Y S[0]:=Y I[0]$

$R 2:$ for $k:=1$ step 1 until 4 do

$\operatorname{begin} Y S[0]:=Y S[0]+M R[k, 4] \times d$;

for $j:=1$ step 1 until $m$ do

begin $F(Y S, j, v) ; \quad v:=v \times d$

$r:=M R[k, 1] \times v-M R[k, 2] \times Q \mid j] ;$

$Y T[j]:=Y T[j]+r$;

$Q[j]:=Q[j]+3.0 \times r-M R[k, 3] \times v$

end;

for $j:=1$ step 1 until $m$ do $Y S[j]:=Y T[j]$

end;

go to $S(s)$;

NOZ: if $\operatorname{sign}(Y I[1]) \neq \operatorname{sign}(Y S[1])$ then go to $I T$;

$T R$ : for $j:=0$ step 1 until $m$ do $Y I[j]:=Y S[j]$; go to $R 2$;

$I T: \quad s:=2$;

for $j:=0$ step 1 until $m$ do $Y A L[j]:=Y S[j]$;

$Z E R: \quad d:=d / 2$;

if $d \leqq$ epsi then go to $S T Z$;

if $\operatorname{sign}(Y I[1])=\operatorname{sign}(Y S[1])$ then go to $T R$ else go to $R 1$;

STZ: $Z[n]:=Y I[0]:=Y I[0]+d ; n:=n+1$

for $j:=\mathrm{C}$ step 1 until $m$ do $Y I[j]:=Y A L[j]$

end;

end

\section{ALGORITHM 195 \\ BANDSOLVE}

Donald H. Thurnau

Marathon Oil Co., Littleton, Colo.

procedure $B A N D S O L V E(C, N, M, \dot{V})$; value $N, M$; integer $N, M$; real array $C, V$;

comment $B A N D S O L V E$ is effective in solving the matrix equation $A X=B$ when the matrix $A$ is of large order and sparse such that a narrow band centered on the main diagonal includes all the non zero elements. Parameter $N$ is the order of $A$, and $M$ is the width of the band, necessarily an odd number of elements. $B A N D S O L V E$ is very efficient because it operates oniy on the band portion of the matrix $A$, given in the $N$ by $M$ array $C$. The band elements of a given row of $A$ appear in the same row of $C$ but shifted such that element $A[i, j]$ becomes $C[i, j-i+(M+1) / 2]$. All band elements whether zero or non -zero must be given. The values of undefined elements of $C$, such as $C[1,1]$ or $C[N, M]$, are irrelevant. The array $V$ initially contains the vector $B$. After solution, the array $V$ contains the answer vector $X$. The contents of array $C$ are destroyed during solution which is done by Gauss elimination with row interchanges, followed by back substitution; begin integer $J M, L R, I, P I V, R, J$; real $T$;

$L R:=(M+1) \div 2$

for $R:=1$ step 1 until $L R-1$ do

for $I:=1$ step 1 until $L R-R$ do

begin for $J:=2$ step 1 until $M$ do

$C[R, J-1]:=C[R, J]$

$C[R, M]:=C[N+1-R, M+1-I]:=0$

end of row shifting and zero placement;

for $I:=1$ step 1 until $N-1$ do

begin $P I V:=I$;

for $R:=I+1$ step 1 until $L R$ do

if $a b s(C[R, 1])>a b s(C[P I V, 1])$ then

$P I V:=R$;

if $P I V \not x I$ then

begin $T:=V[I]$;

$V[I]:=V[P I V]$

$V[P I V]:=T$

for $J:=1$ step 1 until $M$ do

begin $T:=C\{I, J]$

$C[I, J]:=C[P I V, J]$

$C[P I V, J]:=T$

end $J$

end of row inlerchango;

$V[I]:=V[I] / C[I, 1]$;

for $J:=2$ step 1 until $M$ do

$C[I, J]:=C[I, J] / C[I, 1]$;

for $R:=I+1$ step 1 until $L R$ do

begin $T:=C[R, 1]$

$V[R]:=V[R]-T \times V[I]$

for $J:=2$ step 1 until $M$ do

$C[R, J-1]:=C[R, J]-T \times C[I, J]$

$C[R, M]:=0$

end $R$;

if $L R \neq N$ then $L R:=L R+1$

end of triangularization;

$V[N]:=V[N] / C[N, 1]$

$J M:=2$

for $R:=N-1$ step -1 until 1 do

begin for $J:=2$ step 1 until $J M$ do $V[R]:=V[R]-C[R, J] \times V[R-1+J]$

if $J M \neq M$ then $J M:=J M+1$

end of back solution

end $B A N D S O L V E$

ALGORITHM 196

MULLER'S METHOD FOR FINDING ROOTS OF AN ARBITRARY FUNCTION

ROBERT D. RODMAN

Burroughs Corp., Pasadena, Calif.

procedure $M U L L E R$ ( $p 1, p 2, p 3, m x m$, nrts, ep1, ep2, sw1, sw2, $s w 3, s w r, r r t, i r t)$

value $p 1, p 2, p 3, m x m, n r t s, e p 1, e p 2, s w 1, s w 2, s w 3, s w r$;

integer $m x m$, nrts; boolean $s w 1, s w 2, s w 3, s w r$;

real $p 1, p 2, p 3, e p 1, e p 2$; array $r r t, i r t$;

begin comment procedure $M U L L E R$ finds real and complex roots of an arbitrary function. $p 1, p 2$, and $p 3$ are starting values. Roots nearest these points are found first. $m x m$ is the maximum number of iterations to be made in finding any one root. ep1 and $e p 2$ are specified as tolerance parameters. If $A B S\left(\left(X_{i+1}-X_{i}\right) /\right.$ $\left.X_{i+1}\right)<e p l$ or if the function value and modified function value are both less than ep2, a root has been found. If $s w 1$ is true, 
then each iterant of each root is printed. If $s w 2$ is true, the value of each root found is printed. If $s w 3$ is true, then, when applicable, the complex conjugate of each root found is admitted as a root. If swr is true, only real roots are found. $r r t$ and irt contain the real, and imaginary parts of each root found. Procedure function is the function generator and procedure complex performs necessary complex operations;

boolean bool; integer $c 1, r t c, i$, itc; real $r x 1, r x 2, r x 3, i x 1$, $i x 2$, ix3, rroot, iroot, rdnr, idnr, $t 1$, itl, frroot, firoot, $r f x 1, r f x 2$, $r f x 3$, if $x 1$, if $x 2$, if $x 3$, th, ih, rlam, ilam, rdel, idel, $t 2, i t 2, t 3, i t 3, t 4$, it4, rg, ig, rden, iden, rfunc, ifunc;

switch $j:=m 2, m 3, m 4, m 7, m 11$;

procedure function (reale, imag, reval, ieval);

value reale, imag; real reale, imag, reval, ieval;

begin comment Coding for this procedure must be inserted at compile time. reale and imag are the real and imaginary parts of the dependent variable. reval and ieval, the real and imaginary parts of the function;

end function;

procedure complex $(a, i a, b, i b, k, c, i c)$;

value $a, i a, b, i b, k$; integer $k$;

real $a, i a, b, i b, c, i c$;

begin real $t e m p ;$ switch $j:=m p y, d v d, 8 q t$; go to $j[k]$;

mpy: $\quad c:=a \times b-i a \times i b ; \quad i c:=a \times i b+i a \times b$; go to exil;

dvd: if $(i b=0) \wedge(b=0)$ then begin ic $:=0 ; c:=1$; go to exit end; lemp $:=b \uparrow 2+i b \uparrow 2$; $c:=(a \times b+i a \times i b) / t e m p ; i c:=(i a \times b-a \times i b) / t e m p$; go to exit;

sqt: if $(i a=0) \wedge(a<0)$ then

begin $c:=0 ; \quad i c:=s q r t(-a)$ end

else if $i a=0$ then

begin $c:=\operatorname{sqrl}(a) ; \quad i c:=0$ end

else begin temp := sqrt $(a \uparrow 2+i a \uparrow 2)$

$c:=\operatorname{sqrt}((\operatorname{temp}+a) / 2)$

ic $:=$ if $(\operatorname{lemp}-a)<0$ then 0

else sqrt $((t e m p-a) / 2)$ end

if $((b+c) \uparrow 2+(i b+i c) \uparrow 2)<((b-c) \uparrow 2+(i b-i c) \uparrow 2)$

then begin $c:=b-c ; \quad i c:=i b-i c$ end

else begin $c:=b+c ; \quad i c:=i b+i c$ end;

exit: end of complex;

slart : for $i:=1$ step 1 until $n r t s$ do $r r t[i]:=i r t[i]:=0 ; r l c:=0$;

$m 0: \quad i x 1:=i x 2:=i x 3:=c 1,:=$ iroot $:=i t c:=0$;

rroot $:=p 1 ;$ bool $:=$ false;

$m 1: \quad c 1 ;:=c 1+1 ; \quad r d n r:=1 ; i d n r:=0$;

for $i:=1$ step 1 until $r t c$ do

begin

complex $(r d n r, i d n r$, rroot-rrt $[i]$, iroot-irt $[i], 1, t 1, i t 1)$;

$r d n r:=t 1 ; i d n r:=i t 1$

end;

function (rroot, iroot, $t 1, i t 1$ );

complex ( $t 1, i t 1, r d n r, i d n r, 2$, frroot, firoot);

go to $j[c 1]$;

$m 2: \quad r f x 1:=$ frrool; $\quad$ if $x 1:=$ firoot; rroot $:=p 2$; go to $m 1$;

$m 3: \quad r f x 2:=$ frroot; if $x 2:=$ froot; rrost $:=p 3$;

go to $m 1$;

$m 4: \quad r f x 3:=$ frroot; if $x 3:=$ firoot; $\quad r x 1:=p 1$;

$r x 2:=p 2 ; \quad r x 3:=p 3 ; \quad r h:=r x 3-r x 2$;

$i h:=i x 3-i x 2$;

complex (rh, ih, $r x 2-r x 1, i x 2-i x 1,2$, rlam, ilam);

rdel $:=$ rlam +1 ; idel $:=$ ilam;

$m 9: \quad$ if $(r f x 1=r f x 2) \wedge(r f x 2=r f x 3) \wedge(i f x 1=i j x 2) \wedge(i f x 2=i f x 3)$

then begin rlam $:=1$; ilam $:=0$; go to $m 8$ end;

complex $(r f x 1, i f x 1$, rlam, ilam, $1, t 1, i t 1)$;

complex $(r f x 2$, if $x 2$, rdel, idel, $1, t 2, i t 2)$;

$t 1:=\imath 1-t 2+r f x 3 ; \quad i \ell 1:=i \ell 1-i l 2+i f x 3$ complex (rdel, idel, rlam, ilam, 1, t2, it2);

complex ( $t 1, i t 1$ t2, it2, $1, t 3, i t 3)$;

complex $(r f x 3, i f x 3, t 3, i t 3,1, t 1, i t 1)$;

$\iota 1:=-4 \times t 1 ; \quad i t 1:=-4 \times i t 1$;

complex $(r f x 3, i f x 3$, rlam + rdel, ilam+idel, 1, t2, it2);

complex (rdel $\uparrow 2-i d e l \uparrow 2,2 \times r d e l \times i d e l, r f x 2, i f x 2,1, t 3, i t 3)$;

complex (rlam $\uparrow 2-i l a m \uparrow 2,2 \times$ rlam $\times$ ilam, $r f x 1, i f x 1,1$,

$t 4, i t 4)$;

$r g:=t 4-t 3+t 2 ; \quad i g:=i t 4-i t 3+i t 2 ;$

if $s w r \wedge((r g \uparrow 2+t 1)<0)$ then

begin $r$ den $:=r g$; iden $:=i g:=0$ end

else complex $(r g \uparrow 2-i g \uparrow 2+i 1,2 \times r g \times i g+i t 1, r g, i g, 3$, rden, iden);

complex $(-2 \times r f x 3,-2 \times i f x 3, r d e l, i d e l, 1, t 1, i t 1)$;

complex ( $11, i t 1$, rden, iden, 2, rlam, ilam);

$m 8: \quad i t c:=i i c+1$;

$r x 1:=r x 2 ; \quad r x 2:=r x 3 ; \quad r f x 1:=r f x 2 ; \quad r f x 2:=r f x 3$;

$i x 1:=i x 2 ; \quad i x 2:=i x 3 ; \quad$ if $x 1:=i f x 2 ; \quad$ if $x 2:=i f x 3$

complex (rlam, ilam, rh, ih, $1, i \mathrm{1}, i t 1)$;

$r h:=\imath 1 ;$ ih $:=i t 1$;

$m 6: \quad r d e l:=$ rlam $+1 ;$ idel $:=$ ilam; $r x 3:=r x 2+r h$;

$i x 3:=i x 2+i h ; \quad c 1:=3$; rroot $:=r x 3$;

iroot $:=i x 3$; go to $m 1$;

$m 7: \quad r f x 3:=$ frroot; if $x 3:=$ firoot;

function ( $r x 3, i x 3$, rfunc, ifunc);

complex $(r f x 3, i f x 3, r f x 2, i f x 2,2, t 1, i t 1)$;

if $(t 1 \uparrow 2+i t 1 \uparrow 2)>100$ then

begin $\operatorname{rlam}:=\operatorname{rlam} / 2 ; \quad r h:=r h / 2 ;$ ilam $:=$ ilam $/ 2$;

ih $:=i h / 2$; go to $m 6$ end;

if $s w 1$ then...

comment option to output iterant and associated function values;

$\iota 1:=r x 3-r x 2 ; \quad i t 1:=i x 3-i x 2$

complex $(t 1, i t 1, r x 2, i x 2,2, t 2, i t 2)$

if sqrt $(t 2 \uparrow 2+i t 2 \uparrow 2) \leqq e p 1$ then go to $f n 1$;

if $^{\prime}(\operatorname{sqr\ell }(r f x 3 \uparrow 2+i f x 3 \uparrow 2) \leqq e p 2) \wedge$

(sqrt (rfunc $\uparrow 2+i f u n c \uparrow 2) \leqq e p 2)$ then go to fin 2 ;

go to if $i t c \geqq m x m$ then $f i n 3$ else $m 9$;

fin 1 : if $s w 2$ then...

comment option to output root; go to $\mathrm{ml2}$;

fin2: if $s w 2$ then ...

comment option to output root; go to $m 12$;

fin3: if sw2 then ...

comment no convergence, option to output last iterant;

bool $:=$ true;

$m 12: \quad r t c:=r l c+1 ; \quad r r t[r t c]:=r x 3 ; \quad i r t[r t c]:=i x 3$;

if $r \ell c$ Z n rts then go to exit;

if $(A B S(i x 3)>e p 1) \wedge s w 3 \wedge \neg$ bool then

begin $i x 3:=-i x 3$; function $(r x 3, i x 3$, rfunc, ifunc); rroot $:=r x 3 ;$ iroot $:=i x 3 ; \quad$ cl $:=4$; go to $m 1$;

$m 11$ : if $s w 2$ then ...

comment the complex conjugate of the last root found is accept-

able. Option to output this root;

$r t c:=r t c+1 ; \quad \operatorname{rrt}[r t \bar{c}]:=r x 3 ; \quad \operatorname{irt}[r t c]:=i x 3$

end else go to $m 0$;

if $r i c<n r t s$ then go to $m 0$

exit: end of procedure $M U L L E R$ 


\section{ALGORITHM 197}

\section{MATRIX DIVISION}

M. WELLS

University of Leeds, Leeds, England

procedure Pos Div $(b, c, m, n$, solve);

value $m, n$, solve; array $b, c$; integer $m, n$; Boolean solve;

comment The matrix $c$, with $m$ rows and $n$ columns, is divided

by the positive definite matrix $b$, of order $m$, 'by the square root method (see Fadeeva, V. N., Computational Methods of Linear Algebra, Chap 2, \$10). The upper triangle of $b$ is replaced by an upper triangular matrix $N$ such that $N^{t} N=b$. The other elements of $b$ are undisturbed. The matrix $c$ is replaced by $b^{-1} c$. The Boolean solve is used as a switch. If its value is true, then it is assumed that an earlier entry to Pos Div has left the matrix $N$ in place, and a further division of $c$ by $b$ takes place;

begin integer $i, j, k$;

real procedure $\operatorname{dot}(a, b, p, q)$;

value $q$; real $a, b$; integer $p, q$;

comment This is innerproduct, modified to define a function designator;

begin real $s ; s:=0$;

for $p:=1$ otop 1 until $q$ do $s:=s+a \times b$;

$d o t:=s$ end $d o t$;

Start of program: if solve then go to back substitution;

for $i:=$ step 1 until $m$ do

begin $b[i, i]:=\operatorname{sqrt}(b[i, i]-\operatorname{dot}(b[j, i] \uparrow 2,1, j, i-1))$;

for $j:=i+1$ step 1 until $m$ do

$b[i, j]:=(b[i, j]-\operatorname{dot}(b[k, i], b[k, j], k, i-1)) / b[i, i]$

end formation of upper triangular matrix;

back substiluton: for $i:=1$ step 1 until $n$ do

begin for $j:=1$ step 1 until $m$ do

$c[i, j]:=(c[i, j]-\operatorname{dot}(b[k, j], c[i, k], k, j-1)) / b[j, j]$

for $j:=m \cdot$ step -1 until 1 do

$c[i, j]:=(c[i, j]-\operatorname{dot}(b[j, m+1-k], c[i, m+1-k], k, m-j)) /$ $b[j, j]$

end of double back substitution

end of Pos Div

\section{CERTIFICATION OF ALGORITHM 197}

MATRIX DIVISION [M. Wells, Comm. ACM 6 (Aug. 1963), 443]

M. Wells (Recd 18 Nov. 63)

University of Leeds, Leeds, England

The procedure was tested on a Ferranti Pegasus, using the As.gol compiler developed by the de Havilland Aircraft Company at Hatfield. The line after the one labelled 'start of program' should read

$$
\text { for } i:=1 \text { step } 1 \text { until } m \text { do }
$$

(the first 1 was omitted). read

The statement labelled back substituton is incorrect, and should

back substitution: for $j:=1$ step 1 until $n$ do

begin for $i:=1$ step 1 until $m$ do

$c[i, j]:=(c[i, j]-\operatorname{dot}(b[k, i], c[k, j], k, i-1)) / b[i, i]$

for $i:=m$ step -1 until 1 do

$c[i, j]:=(c[i, j]-\operatorname{dot}(b[i, m+1-k], \quad c[n+1-k, j], k, m-i)) / b[i, i]$ end of double back substitution.

With these changes the program was operated successfully on a number of small test problems. The procedure is only applicable to symmetric positive definite matrices, and no systematic attempt has yet been made to assess the accuracy of the results.

The word 'symmetric' should be inserted before 'positive definite' in the comment.

It is interesting to note that the original; incorrect version of the procedure will divide one symmetric matrix by another, and so can be used for matrix inversion.
ALGORITHM 198

ADAPTIVE INTEGRATION AND MULTIPLE INTEGRATION

William Marshall McKeeman

Stanford University, Stanford, Calif.

begin comment This program illustrates the declaration and call of a procedure used to numerically approximate definite in tegrals and multiple integrals. The in tegrand is an expression substituted for the first formal parameter and must be a function of the simple variable replacing the second formal parameter. Multiple integration is accomplished by substituting a complete call of Integral for the first formal parameter. Note that in this case that the limits of integration on the inside calls may be functions of the variable of integration on the outer call. The parameter rule selects a Newton-Cotes formula which matches a polynomial of degree $=$ rule to the function in the interval of integration. (See Hamming, Numerical Methods for Scientists and Engineers, Sec. 12.2). In any case, the procedure integral adapts its step size to the function in seeking to minimize the number of function evaluations. The program has been tested and run on a variety of functions using the ALgol compiler on the Burroughs $B-5000$;

real procedure Inlegral $(F)$ a function of the real variables: $(x)$ between limits: $(a, b)$ polynomial degree: (rule) tolerance: (eps);

value $a, b$, rule, eps; integer rule;

real $F, x, a, b$, eps;

begin comment set up the parameters for the recursion before calling the procedure $\mathrm{NC}$;

switch $n c t:=R 1, R 2, R 3, R 4, R 5, R 6, R 7$;

real array $c f$, fn $[1:$ rule +1$]$;

integer $k$; real $d a, a b$;

real procedure $N C(F, x, a, d a, f n, k, c f, r u l e, e p s, e s, a b, 1 v 1)$;

value $a, d a$, rule, eps, es, $1 v 1$; real array $c f$;

integer $k, r u l e, 1 v 1$; real $F, x, a, d a, f n, e p s, e s, a b$;

begin comment $\mathrm{NC}$ is the adaptive heart of Integral;

real array $f c[1$ :rule $+1,1$ :rule +1$]$, est, $x x[1:$ rule +1$]$;

integer $i, j$; real $d x$, int, $e p$;

real procedure $S U M(t e r m$, index, upperlimil);

real term; integer index, upperlimit;

begin real $t ; t:=0$;

for index := 1 step 1 until upperlimit do

$\ell:=t+t e r m$

$S U M:=t$

end of $S U M$;

comment begin the integration by evaluating $F$ on the mesh points;

for $k:=1$ step 1 until rule +1 do $f c[k ; k]:=f n$;

$d x:=d a /($ rule $\times($ rule +1$))$;

$x:=a$;

for $i:=1$ step 1 until rule +1 do

for $j:=1$ step 1 until rule do

begin

if $j=1$.then $x x[i]:=x$;

if $i \neq j$ then $f c[i, j]:=c f[j] \times F$

$x:=x+d x$;

end having done all necessary function evaluations;

for $i:=1$ step 1 until rule do

$f c[i$, rule +1$]:=f c[i+1,1]$

ep $:=$ eps $/$ sqri $($ rule +1$)$;

comment eps/(rule +1$)$ is the value to give an absolute error bound of eps in the final answer. It proves too strict in practice;

$d x:=d x \times$ rule;

comment compute the integrals of the subintervals;

for $i:=1$ step 1 until rule +1 do

est $[i]:=\operatorname{SUM}(f c[i, j], j$, rule +1$) \times d x$

$a b:=a b-a b s(e s)+S U M(a b s(e s t[i]), i$, rule +1$)$; 
comment $a b$ is the area under $a b s(F)$. It is used in computing the relative error upon which to terminate;

int := SUM (est[i],i,rule+1);

if $1 v 1 \geqq 100 /($ rule +1$)$ then go to error;

$N C:=$ if $a b s(e s-i n t) \leqq e p s \times a b \wedge e s \neq 1.0$ then int

else $S U M(N C(F, x, x x[i], d x, f c[i, j], j, c f, r u l e, e p, e s t \mid i], a b, 1 v 1+1)$, i,rule+1);

go to réturn;

error; $N C:=$ int;

comment abs $(e s-i n t)$ is the approximate error caused by terminating the recursion. In most cases, termination at this level will not adversely affect the accuracy of the result; return:

end of $N C$;

comment now initialize the Newton-Cotes coefficients;

go to nct [rule];

$R 1: \quad c f[1]:=c f[2]:=1.0 / 2.0 ;$ go to compute;

$R 2: c f[1]:=c f[3]:=1.0 / 6.0 ; \quad c f[2]:=4.0 / 6.0$;

comment $R 1$ is trapezoidal rule, $R 2$ is Simpson's rule;

go to compute;

R3: $\quad c f[1]:=c f[4]:=1.0 / 8.0$

$c f[2]:=c f[3]:=3.0 / 8.0 ;$ go to compute;

$R 4: \quad c f[1]:=c f[5]:=7.0 / 90.0$;

$c f[2]:=c f[4]:=32.0 / 90.0$;

$c f[3]:=12.0 / 90.0 ;$ go to compute;

$R 5: \quad c f[1]:=c f[6]:=19.0 / 288.0$;

$c f[2]:=c f[5]:=75.0 / 288.0$;

$c f[3]:=c f[4]:=50.0 / 288.0 ;$ go to compute;

$R 6: \quad c f[1]:=c f[7]:=41.0 / 840.0$;

$c f[2]:=c f[6]:=216.0 / 840.0$

$c f[3]:=c f[5]:=27.0 / 840.0$;

$c f[4]:=272.0 / 840.0 ;$ go to compute;

$R 7: \quad c f[1]:=c f[8]:=75.1 / 1728.0$;

$c f[2]:=c f[7]:=357.7 / 1728.0$;

$c f[3]=c f[6]:=134.3 / 1728.0$;

$c f[4]:=c f[5]:=298.9 / 1728.0$;

compute: $d a:=b-a$;

for $k:=0$ step 1 until rule do

begin

$x:=a+k \times d a / r u l e ;$

$f n[k+1]:=F \times c f[k+1]$;

end;

$a b:=1.0$;

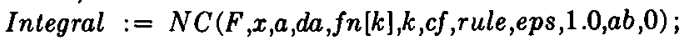

end of Integral;

comment Now evaluate the integral of $1.0 / \operatorname{sqri}(a b s(x+y))$

on the unit disk in the $x, y$-plane;

real $x, y$, answer;

answer := Inlegral(Integral (1.0/sqrt $(a b s(x+y)), x$,

$-\operatorname{sqrt}(1.0-y \uparrow 2), \operatorname{sqrt}(1.0-y \uparrow 2), 7,0.001), y,-1.0,1.0,3,0.001)$;

end of program;

\section{ALGORITHM 199}

CONVERSIONS BETWEEN CALENDAR DATE AND JULIAN DAY NUMBER

Robert G. Tantzen

Air Force Missile Development Center, Holloman AFB, New Mex.

procedure $J D A Y(d, m, y, j)$;

integer $d, m, y, j$; comment $J D A Y$ converts a calendar date, Gregorian calendar, to the corresponding Julian day number $j$. From the given day $d$, month $m$, and year $y$, the Julian day number $j$ is computed without using tables. The procedure is valid for any valid Gregorian calendar date. When transcribing $J D A Y$ for other compilers, be sure that integers of size $3 \times 10^{6}$ can be handled;

begin integer $c, y a$;

if $m>2$ then $m:=m-3$

else begin $m:=m+9 ; \quad y:=y-1$ end;

$c:=y \div 100 ; y a:=y-100 \times c$;

$j:=(146097 \times c) \div 4+(1461 \times y a) \div 4+(153 \times m+2) \div 5+d+1721119$ end $J D A Y$

procedure $J D A T E(j, d, m, y)$;

in teger $j, d, m, y$;

comment $J D A T E$ converts a Julian day number $j$ to the corresponding calendar date, Gregorian calendar. Since $j$ is an integer for this procedure, it is correct astronomically for noon of the day. $J D A T E$ computes the day $d$, month $m$, and year $y$, without using tables. The procedure is valid for any valid Gregorian calendar date. When transcribing $J D A T E$ for other compilers, be sure that integers of size $3 \times 10^{6}$ can be handled;

begin $j:=j-1721119$;

$y:=(4 \times j-1) \div 146097 ; j:=4 \times j-1-146097 \times y$

$d:=j \div 4$;

$j:=(4 \times d+3) \div 1461 ; \quad d:=4 \times d+3-1461 \times j ;$

$d:=(d+4) \div 4$

$m:=(5 \times d-3) \div 153 ; \quad d:=5 \times d-3-153 \times m$;

$d:=(d+5) \div 5$;

$y:=100 \times y+j ; \quad$ if $m<10$ then $m:=m+3$

else begin $m:=m-9 ; \quad y:=y+1$ end;

end $J D A T E$

procedure $K D A Y(d, \dot{m}, y a, k)$;

integer $d, m, y a, k$;

comment $K D A Y$ converts a calendar date, Gregorian calendar, to the corresponding serial day number $k$. From the given day $d$, month $m$, and the last two decimals of the year, ya, the serial day number $k$ is computed without using tables. The procedure is valid from 1 March $1900 \quad(k=1)$ to 31 December 1999 $(k=36465)$. To obtain the Julian day number $j$ (valid at noon) use $j=k+2415079$;

begin if $m>2$ then $m:=m-3$

else begin $m:=m+9 ; y a:=y a-1$ end;

$k:=(1461 \times y a) \div 4+(153 \times m+2) \div 5+d$

end

procedure $K D A T E(k, d, m, y a)$; .

integer $k, d, m, y a$;

comment $K D A T E$ converts a serial day number $k$ to the corresponding calendar date, Gregorian calendar. It computes day $d$, month $m$, and the last two decimals of the year, ya, without using tables. The procedure is valid from $k=1$ (1 March 00) to $k=36465$ (31 December 99) for any one century. For the 20th Century the relation between $k$ and theulian day number $j$ (at noon) is $j=k+2415079$;

begin ya $:=(4 \times k-1) \div 1461 ; d:=4 \times k-1-1461 \times y a$; $d:=(d+4) \div 4 ; m:=(5 \times d-3) \div 153$;

$d:=5 \times d-3-153 \times m$

$d:=(d+5) \div 5$

if $m<10$ then $m:=m+3$

else begin $m:=m-9 ; y a:=y a+1$ end; end $K D A T E$ 


\section{CERTIFICATION OF ALGORITHM 199 [Z] CONVERSIONS BETWEEN CALENDAR DATE AND} JULIAN DAY NUMBER [Robert G. Tartzen, Comm. $A C M 8$ (Aug. 1963), 444].

DA vid K. OpPenhem (Recd. $10 \mathrm{Jul} .64$ and 27 Jul. 64) System Development Córp., Santa Monica, Calif.

Algorithm 199 was translated into JovisL J3 and tested on the $P h i l c o$ 2000. Input was generated with a random number generator that produced uniformly distributed dates between the years 1583 and 2583 . The results were checked for 50 different dates in that range.

The procedures as written place unnecessary restrictions on some of the parameters. Expressions cannot always be used as inputs to the procedures. Also, the original input to $J D A Y$, $J D A T E$ and $K D A Y$ will be modified during the operation of the respective procedures. It should also be noted that in many implementations of ALGOL the use of parameters called by name may be more expensive than those called by value. The call by name is a far more powerful tool than is necessary for most of the parameters of these procedures. For these reasons the following changes are suggested:

1. In procedure $J D A Y$

change: integer $d, m, y, j$;

to: value $d, m, y$; integer $d, m, y, j$;

2. In procedure $J D A T E$

change: integer $j, d, m, y$; to: value $j$; integer $j, d, m, y$;

3. In procedure $K D A Y$

change: integer $d, m, y a, k$;

to: value $d, m, y a$; integer $d, m, y a, k$;

4. In procedure $K D A T E$

change: integer $k, d, m, y a$;

to: value $k$; integer $k, d, m, y a$;

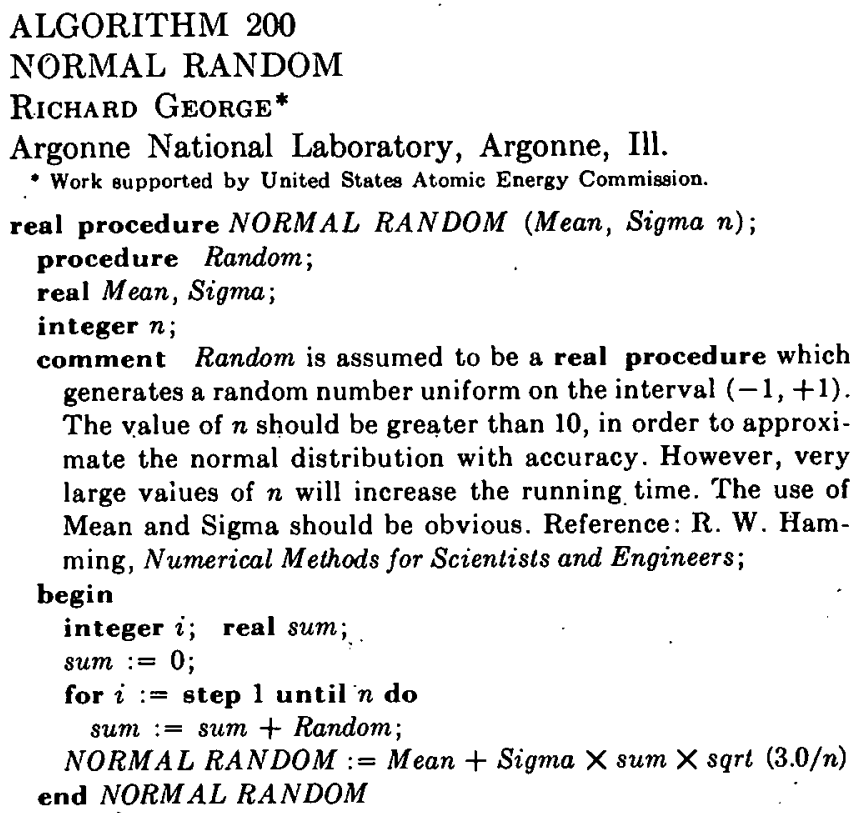

ALGORITHM 201

SHELLSORT

J. Boothroyd

English Electric-Leo Computers, Kidsgrove, Staffs, England

procedure Shellsort $(a, n)$; value $n$; real array $a$; integer $n$; comment $a[1]$ through $a[n]$ of $a[1: n]$ are rearranged in ascending order. The method is that of D.A. Shell, (A high-speed sorting procedure, Comm. ACM 2 (1959), 30-32) with subsequences chosen as suggested by $\mathrm{T}$. $\mathrm{N}$. Hibberd (An empirical study of minimal storage sorting, SDC Report SP-982): Subsequences depend on $m_{1}$ the first operative value of $m$. Here $m_{1}=2^{k}-1$ for $2^{k} \leq n<2^{k+1}$. To implement Shell's original choice of $m_{1}=$ $[n / 2]$ change the first statement to $m:=n$;

hegin integer $i, j, k, m$; real $w$;

for $i:=1$ step $i$ until $n$ do $m:=2 \times i-1$;

for $m:=m \div 2$ while $m \neq 0$ do

begin $k:=n-m$;

for $j:=1$ step 1 until $k$ do

begin for $i:=j$ step $-m$ until 1 do

begin if $a[i+m] \geqq a[i]$ then go to 1 ;

$w:=a[i] ; \quad a[i]:=a[i+m] ; \cdot a[i+m]:=w ;$ end $i$

$1:$ end $j$

end $m$

end Shellsort;

CERTIFICATION OF ALGORITHM 201

SHELLSORT [J. BOOTHROYD, Comm. ACM 6 (Aug. 1963), 445]

M. A. BatTY (Recd 27 Jan. 1964)

English Electric Co., Whetstone, Nr. Leicester, England

This algorithm has been tested successfully using the DEUCE Algol Compiler. When the first statement of the algorithm was replaced by the statement

$$
m:=n
$$

to implement Shell's original choice of $m_{1}:=n / 2$, a slight increase in sorting time was observed with most of the cases tested.

\footnotetext{
ALGORITHM 202

GENERATION OF PERMUTATIONS IN LEXICOGRAPHICAL ORDER

MoK-Kong Shen

Postfach 74, München 34, Germany

procedure $P E R L E(S, N, I, E)$;

integer array $S$; integer $N$; Boolean $I$; label $E$;

comment If the array $S$ contains a certain permutation of the $N$ digits $1,2, \cdots, N$ before call, the procedure will replace this with the lexicographically next permutation. If initialization is required set the Boolean variable $I$ equal true, which will be changed automatically to false through the first call, otherwise set $I$ equal false. If no.further permutation can be generated, exit will be made to $E$. For reference see $B I T \&$ (1962), 228-231;

begin integer $j, u, w$

if $I$ then begin for $j=1$ step 1 until $N$ do $S[j]:=j$; $I:=$ false; go to Rose end;
} 
$w:=N$;

Lilie: if $S[w]<S[w-1]$ then

begin if $w=2$ then go to $E$;

$w:=w-1$; go to Lilie

end;

$u:=S[w-1]$;

for $j:=N$ step -1 until $w$ do

begin if $S[j]>u$ then

begin $S[w-1]:=S[j]$;

$-S[j]:=u$; go to Tulpe

end

end;

Tulpe: for $j:=0$ step 1 until $(N-w-1) / 2+0.1$ do

begin $u:=S[N-j]$

$S[N-j]:=S[w+j] ; \quad S[w+j]:=u$ end;

Rose:

end PERLE

\section{ALGORITHM 203 \\ STEEP1}

\section{E. J. WASSChER}

Philips Research Laboratories

N. V. Philips' Gloeilampenfabrieken

Eindhoven-Netherlands

procedure $S T E E P 1(l b, x s, u b, d x, x \min , f \min , n$, eps, relax, dxmax, ela, psi, pmax, zela, FUNK);

value $d x, n, e p s$, relax, dxmax, eta, psi, pmax, zeta;

integer $n$;

real $f \min$, eps, relax, dxmax, eta, psi, pmax, zela;

array $l b, x s, u b, d x, x \min$; real procedure $F U N K$;

comment $S T E E P 1$ is a subroutine to find the minimum of a differentiable function of $n$ variables, using the method of steepest descent. It mainly consists of three parts: (1) a subroutine $A T I V E$, for computing the partial derivatives, (2) a subroutine $S T E P$, for computing the components of an array $x$ step $[1: n]$, which is a new approximation of $x \min [1: n],(3)$ the compound tail of the procedure body. Both subroutines are only called for once, but by writing the program in this way it is quite easy to change the flow of the program.

Significance of the parameters: $l b(i), u b(i)$ are lower and upper bounds for the independent variables. $x 8(i)$ is the starting value for $x \min (i) . x \min (i)$ is the computed $i$ th component of the minimum, $f \min$ the value of the function in $x$ min. $n$ is the number of variables. eps is a small number which is a measure of the desired accuracy-rather of $f \min$ than of $x \min (i)$. FUNK $(x)$ is the function to be minimized. The other parameters are described in the comments on the three parts mentioned;

begin integer $j$; real alpha, $p$; array $x 8 t e p, d f d x, d f p r[1: n]$; procedure $\triangle T I V E$;

begin real beta, gamma, lambda; Boolean $A, B$;

comment 1. A useful estimate for the derivative is $\frac{f(x+d x)-f(x-d x)}{2 d x}$, where $d x$ should be small, but not so small that roundoff noise dominates. This may be achieved by taking $d x$ such that eta $<\left|\frac{f(x+d x)-f(x-d x)}{f(x)}\right|<100$ eta, where eta is a measure for the relative roundoff error. When $|f(x)|<1$ it is better to replace the denominator by a constant. In the programthe parameter $p 8 i$ is used for this purpose. The components $d x(i)$ are used as a frst guess. When the derivative is 0 , the program enlarges $d x$ until $d x>d x \max$.
$A T I V E$ computes $d f d x[1: n]$ in $x$ min. The previously computed partial derivatives $d f p r[1: n]$ as well as relax are used for relaxation purposes. See comment 3 . The Boolean $A$ is used when $x+d x$ or $x-d x$ crosses the boundary $u b$ or $l b$. In that case $f$ min has to be recomputed afterwards. The Boolean $B$ is of a somewhat complicated nature. It may be seen that $d x$ has the character of an own array for ATIVE. In the neighborhood of the minimum this may have the following effect: A step in one variable is taken such that $f(x+d x)$ becomes equal to $f(x-d x)$. Then in the next call for $A T I V E d x$ has to be doubled, etc. By using the Boolean $B$ it is possible to keep $d x$ constant near the minimum.

A similar effect may occur in the large. When $f(x)$ tends to a constant for $x$ tending to $+\infty$ and $-\infty$, then for $|x|$ large $d x$ has to be taken large. It is only possible to make $d x$ smaller in the neighborhood of the minimum by reducing $d x$ after each call of $A T I V E$.

From the last two remarks one may deduce that the first guess for $d x(i)$ should be made with considerable care. Tabulating the function near the starting point may be very helpful; begin ATIVE: lambda := 0;

for $j:=1$ step 1 until $n$ do

begin

large: $A:=B:=$ false; if $x \min [j]+d x[j]>u b[j]$

then begin $x \min [j]:=u b[j]-d x[j] ; \quad A:=$ true end else if $x \min [j]-d x[j]<l b[j]$

then begin $x \min [j]:=l b[j]+d x[j] ; A:=$ true end;

small: $x \min [j]:=x \min [j]+d x[j] ;$ alpha $:=F U N K(x \min )$; $x \min (j]:=x \min [j]-2 \times d x[j] ;$ beta $:=F U N K(x \min )$; $x \min [j]:=x \min [j]+d x[j] ;$ if $A$ then $f \min :=F U N K$ (xmin);

$A:=$ false;

if alpha $-f \min >0 \wedge$ beta $-f \min >0$

then begin $B:=$ true; go to comp end;

gamma $:=a b s(($ alpha-bela $) /($ if $a b s(f m i n)<p s i$ then psi else $f \min )$ );

if gamma $>100 \times$ eta then

begin $d x[j]:=.2 \times d x[j]$; go to small end;

if gamma $<$ eta then

begin $d x[j]:=2 \times d x[j] ;$ if $d x[j]<d x \max$ then

' go to large else $d x[j]:=d x \max$ end

comp: $d f d x[j]:=(a l p h a-b e t a) /(2 \times d x[j])$;

lambda := lambda $+d f d x[j] \uparrow 2$;

if $\neg B$ then $d x[j]:=.5 \times d x[j]$

end for; lambda:= sqrt (lambda);

for $j:=1$ step 1 until $n$ do

$d f d x[j]:=d f d x[j] / l a m b d a$

end procedure $A T I V E$;

procedure STEP;

comment 2. A step is taken in all variables at the same time. The order of magnitude of the step in one variable should be of the order of magnitude of this variable. To accomplish this three weighting factors are given to the partial derivatives: 1) $\lambda=\left(\sum_{i=1}^{n}\left(\frac{\partial f}{\partial x_{i}}\right)^{2}\right)^{-1}$ (see subroutine $A T I V E$ ),

2) $\left|x_{i}\right|$, or when small, zeta,

3) a number $p$, which is put equal to 1 at the beginning of the program and which tends to 0 at the minimum.

After a decrease of the function the step is accepted and $p$ is multiplied by 1.5. After an increase $p$ is divided by 2. pmax replaces $p$ when $p$ becomes greater than $p$ max;

begin for $j:=1$ step 1 until $n$ do

begin alpha $:=(1-$ relax $) \times d f d x[j]+$ relax $\times d f p r[j]$

$x \operatorname{siep}[j]:=x \min [j]-p \times$ alpha $\times$

(if $a b s(x \min [j])<$ zeta then zeta else abs $(x \min [j]))$;

$\operatorname{dfpr}[j]:=$ alpha;

if $x s t e p[j]>u b[j]$ then $x s t e p[j]:=u b[j]$ 
else if $x$ step $[j]<l b[j]$ then $x s t e p[j]:=l b[j]$

end for

end $S T E P$;

comment 3 . In the next part-the compound tail-the calls for $A T I V E$ and $S T E P$ are organized. The values 1.5 and .5 of the factors of $p$ are not very important. During the iteration $p$ gets an optimal value, which slowly varies. Only at the end $p$ rapidly tends to 0 . The programme was tested on the functions $\frac{y^{2}+1}{x^{2}+1}$ and $\frac{(x-y)^{2}-2}{(x+y)^{2}+2}$, the latter being the first one except for a rotation of the $x y$-plane over $\pi / 4$ radians. In the first case a "gutter" coincides with the $x$-axis, while for $x>0$ and $|y| \gtrless 1 \frac{\partial f}{\partial x} \lessgtr 0$.

In the second case, where the gutter is along the line $x=y$, the relaxation is especially interesting, because with relax $=0$ (and pmax $=100$ ) the iteration follows the gutter in an unstable way. With starting values $x=-14$ and $y=21$ from $x=y=26$ about 300 steps were taken along the gutter with $p$ about .01 . With relax $=.35$ and $p$ max $=.5$ we had about 150 steps from $x=y=23$. In the gutter itself relax $=.85$ gave the best results, but in that case the gutter was reached ai $x=y-63$.

Other parameter values were: $z e t a=p s i=1, d x \max =100$, eta $=10^{-9}$ with eps $=10^{-8}$ gave $f$ min in 10 figures correctly and $x \min [i]$ in 4 to 6 figures for various starting values of $x 8[i]$;

$p:=1$;

for $j:=1$ step 1 until $n$ do

begin $x \min [j]:=x s[j] ; \operatorname{dfpr}[j]:=0$ end; fmin $:=F U N K$ $(x \min )$;

deriv: $A T I V E$;

next: $S T E P$;

alpha :=FUNK $\cdot($ sstep $)$

if alpha $<f \min$ then

begin $f \min :=$ alpha; $p:=1.5 \times p$

if $p>p \max$ then $p:=p \max$;

for $j:=1$ step 1 until $n$ do $x \min [j]:=x s t e p[j]$;

go to deriv end;

$p:=.5 \times p$;

if $p>$ eps then go to nexl;

comment As $p$ has become smaller than eps this is the end of

STEEP1. The program ATIVE takes up rather a lot of computer time by the way it chooses a value for $d x(i)$. A thorough simplification is obtained by taking $d x(i)$ as $10 \uparrow-3 \times a b s(x \min [i])$, where again $x \min [i]$ may be replaced by zeta. Further, at the cost of some loss of accuracy, computing time is saved by taking $\frac{f(x+h)-f(x)}{h}$ as an estimate for the derivative. This program, as far as it differs from $S T E E P 1$, is described in algorithm 204, $S T E E P 2$. An interesting compromise between the two methods is obtained by interchanging the computation of $d x$ and $d f d x$ in $A T I V E$ of STEEP1 ald omitting the iteration on $d x$. This routine $A T I V E$, which has to be used in $S T E E P 1$, is given by J. G. A. Haubrich in algorithm 205; end $S T E E P 1$

\section{CERTIFICATION OF ALGORITHM 203 [EA]}

STEEP1 [E. J. Wasscher, Comm. ACM 9 (Sept. 1963), 517]

Phillp Wallack (Recd. 25 May 1964)

Republic Aviation Corp., Farmingdale, L. I., N. Y.

STEEP1 was tranglated into ForTraN IV and run on the IBM 7094. The program was tested on the function. $x^{4}+y^{4}-1$, with starting values $x=y=1.5$. Other parameter values were those suggested in the body of the algorithm. After 17 steps the values of the variables were $x=.0180, y=.0191$, and the function value $f \min =-.9999999$.

I feel that good programming practice requires that a count be kept of the number of steps taken in $S T E E P 1$ and the number of iterations in $A T I V E$, with.running checks on both these quantities to control looping. Counters were set up for this purpose in the version of the program $I$ ran.

\section{ALGORITHM 204}

\section{STEEP2}

E. J: WAs8ChER

Philips Research Laboratories

N. V. Philips' Gloeilampenfabrieken

Eindhoven-Netherlands

procedure STEEP2 $(l b, x 8, u b, d x, x \min , f \min , n, e p 8$, relax dxmax, pmax, zeta, FUNK);

value $d x, n$, eps, relax, dxmax, pmax, zeta;

integer $n$;

real $d x, f m i n$, eps, relax, dxmax, pmax, zela;

array $l b, x s, u b, x \min$; real procedure $F U N K$;

comment $d x$ should now be taken about $10 \uparrow-3$, drmax could be taken equal to 1 . As the program is equal to STEEP1 after the declaration of the procedure ATIVE, the ALGoL description is cut off there;

begin integer $j$; real alpha, $p$;

array xstep, dfdx, dfpr [1:n];

procedure ATIVE;

begin real beta, lambda; lambda :=0; for $j:=1$ atep I until $n$ do

begin alpha $:=d x \times($ if $a b s(x \min [j])<d x \max$ then $d x \max$ else $a b s(x \min [j]))$; if $x \min [j]+$ alpha $>u b[j]$ then alpha :=-alpha; $x \min [j]:=x \min [j]+$ alpha; beta $:=F U N K(x \min )$; $x \min [j]:=x \min [j]-$ alpha; $d f d x[j]:=($ beta $-f \min ) /$ alpha; lambda := lambda $+d f d x[j] \uparrow 2$

end for; lambda : = sqrt (lambda);

for $j:=1$ step 1 until $n$ do $d f d x[j]:=d f d x[j] /$ lambda; end procedure $A T I V E$

\section{ALGORITHM 205 ATIVE}

J. G. A. HAUBRICB

Philips Research Laboratories

N. V. Philips' Gloeilampenfabrieken

Eindhoven-Netherlands

procedure ATIVE;

begin real beta, lambda; Boolean $A$;

comment This routine may replace $A T I V E$ in STEEP1. The significance of eta has alightly changed;

lambda := 0;

for $j:=1$ step 1 until $n$ do

begin $A:=$ false; alpha $:=d x[j]$;

if $x \min [j]+$ alpha $>u b[j]$ then

begin $x \min [j]:=u b[j]-$ alpha; $A:=$ true end

else if $x \min [j]$ - alpha $<l b[j]$ then

begin $x \min [j]:=l b[j]+$ alpha; $A:=$ true end; $x \min [j]:=x \min [j]+d x[j] ;$ alpha $:=\operatorname{FUNK}(x \min )$; $x \min [j]:=x \min [j]-2 \times d x[j] ;$ beta $:=F U N K(x \min )$; $x \min [j]:=x \min [j]+d x[j] ;$ if $A$ then $f \min :=F U N K$ (xmin);

$d f d x[j]:=($ alpha - beta $) /(2 \times d x[j])$; lambda:= lambda+dfdx[j] $\uparrow 2$;

if alpha $-f \min >0 \wedge$ beta $-f \min >0$ then go to end;

beta $:=a b s((a l p h a-b e t a) /($ if $a b s(f \min )<$ psi then psi else $f$ min $))$; if beta $>$ eta then $d x[j]:=.3 \times d x[j]$ else

begin $d x[j]:=\times d 3 x[j] ; \quad$ if $d x[j]>d x \max$ then $d x[j]:=d x \max$ end; end: end for;

lambda := sqrt (lambda);

for $j:=1$ step 1 until $n$ do $d f d x[j]:=d f d x[j] /$ lambda

end procedure $A T I V E$ 


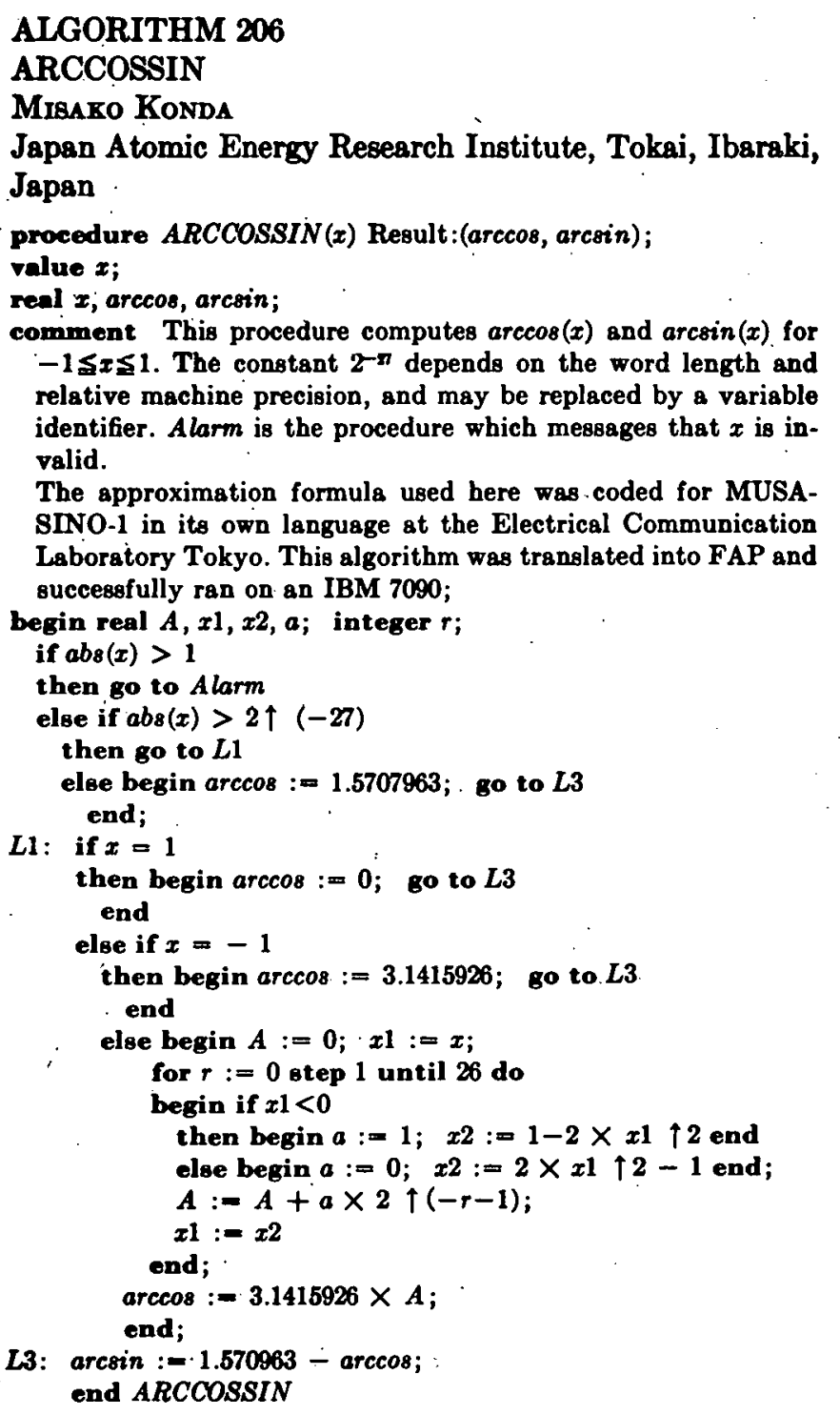

\section{ALGORITHM 207}

STRINGSORT

\section{J. BoothroYD}

English Electric-Leo Computers, Ltd.

Staffordshire, England

procedure stringsort $(a, n)$; comment elements $a[1] \cdots a[n]$ of $a[1: 2 n]$ are sorted into ascending sequence using $a[n+1] \cdots$ a[2n] as auxiliary storage. Von Neumann extended string logic is employed to merge input strings from both ends of a sending area into output strings which are sent alternitely to either end of a receiving area. The procedure takes advantage of naturally occurring ascending or descending order in the original data;

value $n$; integer $n$; array $a$;

begin integer $d, i, j, m, u, v, z$; integer array $c[-1: 1]$; switch $p:=j z 1$, str $i$; switch $q:=$ merge, $j z 2$

oddpass: $i:=1 ; j:=n ; c[-1]:=n+1 ; c[1]:=2 \times n$; allpass: $d:=1$; go to firststring

merge: if $a[i] \geqq a[z]$

then begin go to $p[v]$,

$j z 1: \quad$ if $a[j] \geqq a[z]$

then $i j$ : begin if $a[i] \geqq a[j]$

$$
\begin{aligned}
& \text { then str } j: \text { begin } a[m]:=a[j] ; \\
& j:=j-1 \text { end } \\
& \text { else str } i: \text { begin } a[m]:=a[i] ;
\end{aligned}
$$

end

end

else begin $v:=2 ;$ go to str $i$ end

else begin $u:=2$;

jz2: if $a[j] \geqq a[z]$

then go to str

else begin $d:=-d ; \quad c[d]:=m$;

firstatring: $m:=c[-d]$;

$v:=u:=1$;

go to $i j$

end

end;

$z:=m ; m:=m+d ;$ if $j \geqq i$ then go to $q[u]$

if $m>n+1$ then begin comment evenpass; $i:=n+1$ $j:=2 \times n ; c[-1]:=1 ; c[1]:=n ;$ go to allpass end

end stringsort;

else if $m<n+1$ then go to oddpass

CERTIFICATION OF ALGORITH̀M 207 [M1]

STRINGSORT [J. Boothroyd, Comm:ACM 6 (Oct. .1963), 615]

Charles R. Buair (Recd. 31 Jul. 1964)

Department of Defense, Washington 25, D. C.

STRINGSORT compiled and ran successfully without correction on the ALDAP translator for the CDC 1604A. The following sorting times were observed.

$\begin{array}{cc}\text { Number of llems } & \text { Time in Seconds } \\ 10 & 0.03 \\ 20 & 0.05 \\ 50 & 0.20 \\ 100 & 0.38 \\ 200 & 1.03 \\ 500 & 3.22 \\ 1000 & 6.43 \\ 2000 & 12.85 \\ 5000 & 38.72 \\ 10000 & 90.72\end{array}$

\section{ALGORITHM 208}

\section{DISCRETE CONVOLUTION}

William T. Foreman, JR.

Collins Radio Co.

Newport Beach, Calif.

procedure Discrete Convolution ( $m, n$, prs) result: (Conv); integer $m, n$; real procedure prs; real array conv;

comment This procedure finds the probability distribution of the sum of $m$ independent variables, each with a known distribution over the nonnegative integers. $\dot{A}$ real procedure prs with results $p r[k]$ is assumed to find each probability distribution in 
succession. The maximum sum for which probabilities are computed must be fixed by the user. The number of iterations is roughly $m^{2} n / 2$. The procedure prs will in general depend on additional parameters and should include the read-in of the parameters for that distribution. It may include the selection of one function from a set;

begin integer $i, j, k, i x 1, i x 2$;

real array prob $[1: 2,0: m], \operatorname{pr}[0: m]$;

$i:=1 ; \quad i x 1:=1 ; \quad i x 2:=2 ;$ prs $(m)$ result: $(p r)$;

for $j:=0$ step 1 until $m$ do $\operatorname{prob}[i x 1, j]:=\operatorname{pr}[j]$;

for $i:=2$ step 1 until $n$ do

begin

if $i x 1=1$ then begin $i x 2:=1 ; \quad i x 1:=2$ end

else begin $i x 2:=2 ; \quad i x 1:=1$ end

prs $(m)$ result: $(p r)$;

for $j:=0$ step 1 until $m$ do

begin

$\operatorname{prob}[i x 1, j]:=0$;

for $k:=0$ step 1 until $j$ do end $j$

$\operatorname{prob}[i x 1, j]:=\operatorname{prob}[i x 1, j]+\operatorname{pr}[k] \times \operatorname{prob}[i x 2, j-k]$

end $i$

for $j:=0$ step 1 until $m$ do $\operatorname{conv}[j]:=\operatorname{prob}[i x 1, j]$

end Discrete Convolution

comment The convolution of discrete probability series is

- isomurphic to the multiplication of polynomials. A useful vari-

ation is to omit the parameters $i, n$ and have prs recognize the end of input. A Fortran program using this procedure has been run on the IBM 7090 to find the sum of queue lengths in a teletype switching center, where messages arrived according to the Poisson distribution and message lengths were distributed negative-exponentially. The following was used as the probability procedure;

procedure $p r s(m)$ result: $(p r)$

value $m$; procedure read;

real array $p r$; integer $m$;

begin real trafficrate, linespeed, rho; integer $j$;

read (trafficrate, linespeed);

rho := trafficrate/linespeed;

$\operatorname{pr}[0]: 1$ - rho;

for $j:=1$ step until $m$ do $\operatorname{pr}[j]:=\operatorname{rho} \times \operatorname{pr}[j-1]$

end $p r s$

\section{ALGORITHM 209 \\ GAUSS}

D. IвBETSON,

Elliott Brothers (London) Ltd.,

Elstree Way, Borehamwood, Herts., England

real procedure Gauss $(x)$; value $x$; real $x$;

comment Gauss calculates $(1 / \sqrt{2 \pi}) \mathcal{S}_{-\infty}^{x} \exp \left(-\frac{1}{2} u^{2}\right) d u$ by means of polynomial approximations due to A. M. Murray of Aberdeen University;

begin real $y, z, w$;

if $x=0$ then $z:=0$

else

begin $y:=a b s(x) / 2$;

if $y \geqq 3$ then $z:=1$

else if $y<1$ then

begin $w:=y \times y$

$z:=((()((((0.000124818987 \times w$

$-0.001075204047) \times w+0.005198775019) \times w$

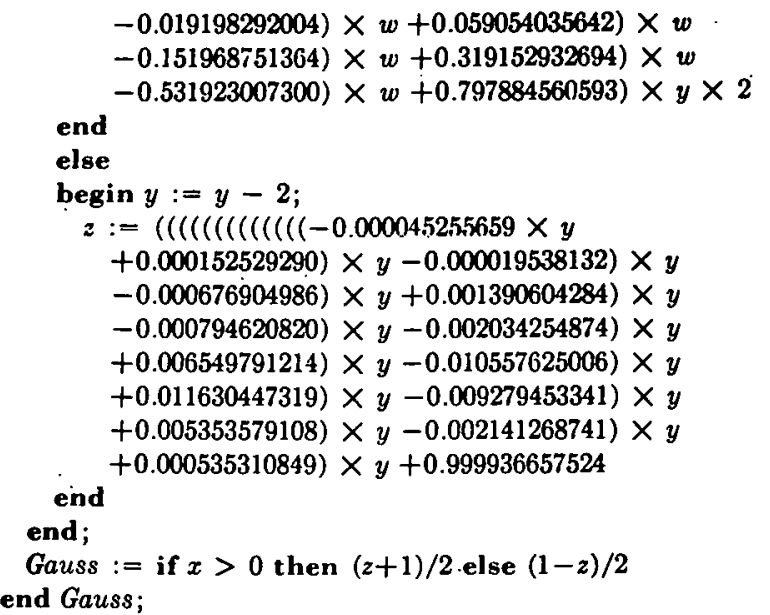

CERTIFICATION OF ALGORITHM 209

GAUSS [D. Ibbetson, Comm. ACM 6 (Oct. 1963), 616]

(Pvt.) G. W. Gladfelter (Rend 4 Nov. 63)

RA17667701, 1st Inf. Battle Group U.S. Military Academy (9822), West Point, N.Y.

The algorithm was translated into ForTRAN for the GE 225 and used to publish a table of the error function. No errors were found in the algorithm and the table produced agreed with the published tables at hand ( 6 significant figures).

\section{CERTIFICATION OF ALGORITHM 209 [S15]}

GAUSS [D. Ibbetson, Comm. ACM 6, Oct. 1963, 616] M. C. Pike

Statistical Research Unit of the Medical Research Council,

University. College Hospital Medical School, London, England

This procedure was tested on an Elliott 803 computer using the standard Elliott Aloor compiler. The expression

$$
2 \times \operatorname{Gauss}(x)-1
$$

was evaluated for $x=0(.01) 6$ and the answers checked with those given in Tables of Probability Functions, vol. II, U.S. National Bureau of Standards, Washington, D.C., 1942, where they are given to 15 decimal places. There was a maximum error of 1 in the 8th decimal place.

ALGORITHM 210

\section{LAGRANGIAN INTERPOLATION}

GEORGE R. SchubERT*

University of Dayton, Dayton, Ohio

- Undergraduate research project, Computer Science Program, Univ. of Dayton.

procedure $L A G R A N G E(N, u, X, Y, A N S)$; real array $X, Y$; integer $N$; real $u, A N S$;

comment This procedure evaluates an $N$ th degree Lagrange polynomial, given $N+1$ data coordinates, and $u$ the value where interpolation is desired. $X$ is the abscissa array and $Y$ the ordinate array. $A N S$ is the resultant value of the function 
at $u$. The notation is that used in R. W. Hamming, Numerical Methods for Scientists and Engineers, pp. 94-95 (McGraw-Hill Book Company, Inc., 1962);

begin integer $i, j$; real $L$;

$A N S:=0.0$

for $j:=$ step 1 until $N+1$ do

begin $L:=1.0$;

for $i:=$ step 1 until $N+1$ do

begin if $i \neq j$ then $L:=L \times(u-X[i]) /(X[j]-X[i])$

end;

$A N S:=A N S+L \times Y[j]$

end end

\section{CERTIFICATION OF ALGORITHM 210}

HERMITE INTERPOLATION [George R. Șchubert,

Comm. ACM, Oct. 1963]

Thomas A. Dwyer

Argonne National Laboratory, Argonne, Ill.

The body of HERMITE was transcribed for the Dartmouth SCaLP processor for the LGP-30 computer and ran successfully without corrections. It was tested using the error function and its derivatives. Roundoff error in the LGP-30 began to appear for values of $n$ greater.than 3 . For $n$ equal to 2 (third degree polynomial) the interpolated value agreed with the function within machine limitations (six significant figures) for steps in the argument data of 0.005 .

\section{ALGORITHM 211}

\section{HERMITE INTERPOLATION}

George R. Schubert*

University of Dayton, Dayton, Ohio

- Undergraduate research project, Computer Science Program, Univ. of Dayton.

procedure $H E R M I T E(n, u, X, Y, Y 1, A N S) ; \quad$ real array $X, Y$, $Y 1$;

integer $n$; real $u, A N S$;

comment This procedure evaluates $a(2 n+1)$ th degree Hermite polynomial, given the value of the function and its first derivative at each of $n+1$ points. $X$ is the abscissa array, $Y$ the ordinate array, and $Y 1$ the derivative array. $A N S$ is the interpolated value of the function at $u$. REFERENCE: R. W. Hamming, Numerical Methods for Scientists and Engineers, pp. 96-97 (McGraw-Hill Book Company, Inc., 1962);

begin integer $i, j$; real $h, a$; $A N S:=0.0$;

for $j:=1$ step 1 until $n+1$ do

begin $h:=1.0 ; a:=0.0$;

for $i:=1$ step 1 until $n+1$ do

begin if $i=j$ then go to out;

$h:=h \times(u-X[i]) \uparrow 2 /(X[j]-X[i]) \uparrow 2$;

$a:=a+1.0 /(X[j]-X[i])$

out: end;

$A N S:=A N S+h \times((X[j]-u) \times(2 \times a \times Y[j]-Y 1[j])+Y[j])$

end end

\section{ALGORITHM 212 \\ FREQUENCY DISTRIBUTION}

Malcolm D. Gray

The Boeing Co., Seattle, Wash.

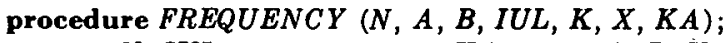
integer $N, I U L ;$ integer array $K A$; real $A, B, K$; real array $X$;

comment Given a set $X$ of variables in some interval $I=[a, b]$ such that $a \leqq \min x, \max x \leqq b, F R E Q U E N C Y$ determines the frequency distribution of $X$ over $k$ equal, half open subintervals of $I$. The interval $I$ is transformed to the interval $J=[0, k]$ with unit subintervals by $x^{\prime}=\left(x_{i}-a\right) /[(b-a) / k], \quad i=1,2, \cdots$, $n$, and considering $x^{\prime}=L \times M, L$ and $M$ integers. The value $L$ then immediately determines the subinterval and $M$ is used for boundary points. If $I U L=0$, the subintervals are open on the upper end, except the $k$ th. On entry, the array $K A$ is assumed identically zero; on return, $K A[i]$ contains the frequency of $X$ in the ith subinterval;

begin integer $i, L$; real $B A K, X P$;

$B A K:=(B-A) / K$;

for $i:=1$ step 1 until $N$ do begin

$X P:=(X[i]-A) / B A K$;

$L:=$ entier $(X P)$;

if $X P=L$ then go to $p 2$ else $L:=L+1$; go to $p 5$;

$p 2:$ if $I U L=0$ then go to $p 3$ else if $L=0$ then $L:=L+1$; go to $x 5$;

p3: if $X P \neq K$ then $L:=L+1$;

$p 5: K A[L]:=K A[L]+1$;

end;

end FREQUENCY

\section{ALGORITHM 213}

\section{FRESNEL INTEGRALS}

Malcolm D. Gray

The Boeing Co., Seattle, Wash.

real procedure $F R E S N E L(w, S, C)$; value $w$; real $S, C$; comment FRESNEL computes the Fresnel sine and cosine integrals $S(w)=\int_{0}^{w} \sin \left[(\pi / 2) t^{2}\right] d t$ and $C(w)=\int_{0}^{w} \cos \left[(\pi / 2) \iota^{2} \mid d l\right.$ using the series expansions

$$
\begin{aligned}
& S(w)=w \sum_{i=1}^{\infty} \frac{(-1)^{i+1} x^{2 i-1}}{(4 i-1)(2 i-1) !} \text { and } \\
& \qquad C(w)=w \sum_{i=1}^{\infty} \frac{(-1)^{i+1} x^{2 i-2}}{(4 i-3)(2 i-2) !}
\end{aligned}
$$

for $|w|<\sqrt{22 / \pi}$ and $x \equiv \pi w^{2} / 2$, and using the asymptotic series

$$
\begin{aligned}
& S(w)=\alpha-\frac{1}{\pi w}[P(x) \sin (x)+Q(x) \cos (x)], \\
& C(w)=\alpha-\frac{1}{\pi w}[P(x) \cos (x)-Q(x) \sin (x)]
\end{aligned}
$$

where $|w| \geqq \sqrt{22 / \pi}, \quad x \equiv \pi w^{2} / 2$,

$$
Q(x)=1-\sum_{i=2}^{\infty} \frac{(-1)^{i}(4 i-5) ! !}{(2 x)^{2 i-2}}, \quad P(x)=\sum_{i=1}^{\infty} \frac{(-i)^{i+1}(4 i-3) ! !}{(2 x)^{2 i-1}},
$$

and $n ! ! \equiv n(n-2)(n-4) \cdots 1$. If $w \geqq 0$, then $\alpha=\frac{1}{2}$, or if $w<0$, then $\alpha=-\frac{1}{2}$. 
This algorithm is a translation of a FAP coded subroutine currently in use on the IBM 7094 at the Boeing Company. The FAP program yields the following errors when tested at 0.05 increments of $x$ :

\begin{tabular}{|c|c|c|c|}
\hline \multicolumn{2}{|c|}{$x$. } & $\Delta S$ & $\Delta C$ \\
\hline 0.00 , & 1.00 & $<1 \times 10^{-7}$ & $<1 \times 10^{-7}$ \\
\hline 1.05, & 8.65 & $<1 \times 10^{-8}$ & $<1 \times 10^{\circ}$ \\
\hline 8.70 , & 10.30 & $3 \times 10^{-6}$ & $2 \times 10^{-}$ \\
\hline 10.35, & 11.00 & $5 \times 10^{-6}$ & $4 \times 10$ \\
\hline 11.05, & 12.15 & $<1 \times 10^{-6}$ & $3 \times 10^{-6}$ \\
\hline 12.20 & 15.00 & $<1 \times 10^{-8}$ & $<1 \times 10^{-6}$ \\
\hline
\end{tabular}

where $\Delta S$ and $\Delta C$ are the approximate average absolute deviations (over the range) from the reference. The user must supply $S(w)=C(w)= \pm \frac{1}{2}$ if $w \rightarrow \pm \infty$. REFERENCES: ALGORITHMS 88-90, J. L. Cundiff, Comm. ACM, May 1962; Born, M. and Wolf, E., Principles of Optics, Pergamon Press (1958), pp. $369-431$;

begin real $x, x 2$, eps, term; integer $n ;$ eps $:=0.000001$; $x:=w \times w / 0.6366198$

$x 2:=-x \times x ;$ if $x \geqq 11.0$ then go to asympt;

begin real frs, frsi;

frs $:=x / 3 ; n:=5 ;$ lerm $:=x \times x 2 / 6$

frsi $:=$ frs + term $/ 7$

loops: if abs(frs-frsi) $\leqq$ eps then go to send; frs := frsi; term $:=$ term $\times x 2 / n /(n-1) ;$ frsi $:=$ frs + term $/(n+n+1)$ $n:=n+2$; go to loops;

send: $\quad S:=$ frsi $\times w$; end;

begin real $f r c$, frci; frc $:=1 ; n:=4 ;$ term $:=x 2 / 2 ;$ frci $:=1+$ term $/ 5$

loopc: if abs(frc-frci) $\leqq$ eps then go to cend; frc := frci; term $:=\operatorname{term} \times x 2 / n /(n-1) ;$ frci $:=f r c+$ tern $/(n+n+1)$; $n:=n+2 ;$ go to loopc;

cend: $\quad C .:=f r c i \times w$; end; go to aend;

asympt: begin real $S 1, S 2$, half, temp; integer $i$; $x 2:=4 \times x 2 ;$ term $:=3 / x 2 ; \quad S 1:=1+$ term; $n:=8$ for $i:=1$ step 1 until 5 do begin $n:=n+4$; term $:=$ term $\times(n-7) \times(n-5) / x 2 ; \quad S 1:=S 1+$ term if abs(term) $\leqq$ eps then go to next; end $i$;

next: for $i:=1$ step 1 until 5 do begin $n:=n+4$; term $:=$ term $\times(n-5) \times(n-3) / x 2 ; \quad S 2:=S 2+$ term; if abs(term) $\leqq$ eps then go to final; end $i$;

final: if $w<0$ then half $:=-0.5$ else half $:=0.5 ;$ term $:=\cos (x)$; temp $:=\sin (x) ; \quad x 2:=3.1415927 \times w$;

$C:=$ half $+($ temp $\times S 1-$ term $\times S 2) / x 2$

$S:=$ half $-($ term $\times S 1+$ temp $\times S 2) / x 2$; end;

aend: end FRESNEL

\section{CERTIFICATION OF ALGORITHM 213 [S20]}

FRESNEL INTEGRALS [M.D. Gray, Comm. ACM 6

(Oct. 1963), 617]

Malcolm Gray (Recd. 29 May 1964 and, revised, 11 June 1964)

Computer Science Div., Stanford U., Stanford, Calif. (now at The Boeing Company, Seattle, Wash.)

Necessary changes to the algorithm are:

(1) in the first line, replace real $S, C$; with real $w, S, C$;

(2) in the formula for $P(x)$, replace $(-i)^{i+1}$ with $(-1)^{i+1}$

(3) - the statement beginning

loopc: if $a b s(f r c-f r c i)$

should read

loopc: if $a b s(f r c-f r c i)$
(4) in the body, replace the line next: for $i:=1$ step 1 until 5 do begin $n:=n+4$; with the lines

next: term $:=\$ 2:=0.5 \% ; n:=4$

for $i:=1$ step 1 until 5 do begin $n:=n+4$

The procedure (with the above changes) was executed on the Burroughs B5000 at Stanford University and gave results as indicated in the slgorithm.

Communications from Helmut Lotsch of the W. W. Hansen Laboratories, Stanford University, and from Harold Butler of the Los Alamos Scientific Laboratory, Los Alamos, New Mexico, state that they found these same errors, and after the corrections were made, similar results were obtained. Mr. Lotsch's work was done on the B5000 and Dr. Butler's work was done on the IBM 7090.

\section{ALGORMITH 214}

q-BESSEL FUNCTIONS $I_{n}(t)$

\section{J. M. S. Simõ̀es Pereirs}

Gulbenkian Scientific Computing Center, Lisbon, Portugal

procedure $q$ Bessel $(t, q, n, j, 8)$; integer $n, j$; real $t, q, 8$; array 8;

comment This procedure computes values of any $q$-Bessel function $I_{n}(t)$ for $n$ integer (positive, negative or zero) by the use of the well-known expansion

$$
I_{n}(t)=\sum_{k=0}^{\infty} \frac{q^{i k(t-1)+\left(n(n+k)(n+k-1) t^{n+2 k}\right.}}{(q)_{k}(q)_{n+k}}
$$

where $|q|<1, \quad(q)_{n}=(1-q)\left(1-q^{q}\right) \cdots\left(1-q^{n}\right), \quad(q)_{0}^{\dot{0}}=1$ and $1 /(q)_{-n}=0 \quad(n=1,2,3, \cdots)$. (See L. Carlitz, The product of $q$-Bessel functions, Port. Math. 21 (1962), 5-9.) Moreover, $j$ denotes the number of terms (at lesst 2) retained in the summation, and $8[i]$ stands for the sum of the first $i+1$ terms of the expansion. This procedure bas been translated into Fortan for the IBM 1620 and run successfully;

begin integer $k, m, p$; real $c, u ; m:=a b s(n) ; c:=1$; if $n=0$ then go to $A$;

for $p:=1$ step 1 until $m$ do $c:=c \times(1-q \uparrow p) ;$ if $n<0$ then go to $B$;

$A: u:=q \uparrow(n \times(n-1) / 2) \times(t \uparrow n) / c ; s[0]:=u$;

for $k=1$ step 1 until $j$ do

begin $u:=u \times q \uparrow(n+2 \times k-2) \times(t \uparrow 2) /((1-q \uparrow k) \times(1-q \uparrow(n+k)))$; $s[k]:=s[k-1]+u$ end;

$B: u:=q \uparrow((m-1) \times m / 2) \times t \uparrow(n+2 \times m) / c ; 8[m]:=u$;

for $k:=m+1$ step 1 until $j$ do

begin $u:=u \times q \uparrow(n+2 \times k-2) \times(t \uparrow 2) /((1-q \uparrow k)(1-q \uparrow(n+k)))$; $s[k]:=s[k-1]+u$ end

end

\section{REMARK ON ALGORITHM 214}

$q$-BESSEL FUNCTIONS $I_{n}(l)$ [J. M. S. Simões Pereira, Comm. ACM 6 (Nov. 1963), 662]

J. M. S. Simões Pereira (Recd 6 Jan 1964)

Gulbenkian Scientific Computing Center, Lisbon, Portugal

Corrections :

1. Insert a dummy statement labeled $C$ just before the final end.

2. Add a statement go to $C$ just before the label $B$.

3. Add a colon in the clause for $k:=1$ step 1 until $j$ do ... 


\section{ALGORITHM 215}

\section{SHANKS}

Henry C. Thacher, Jr.*

Argonne National Laboratory, Argonne, Ill.

- Work supported by the U. S. Atomic Energy Commission

procedure Shanks (nmin, nmax, kmax, S);

value $n$ min, $n \max , k \max$;

integer nmin, nmax, $k$ max

array $S$;

comment This procedure replaces the elements $S[n$ min] through

$S[n \max -2 \times k \max ]$ of the array $S$ by the $e[k \max ]$ transform of

the sequence $S$. The elements $S[n \max -2 \times k \max +1]$ through

$S[n \max -1]$ are destroyed. The $e[k]$ transforms were discovered by $D$. Shanks ( $J$. Math. Phys. 94 (1955), 1-42). e[1] is equivalent to the (della) $\uparrow 2$ transformation. The $e[k]$ transforms are particularly valuable in estimating $B$ in sequences which may be written in the form $S[n]=B+\sum a[i] \times q[i] \uparrow n \quad(i=1,2, \cdots$, k).

The transformation is carried out by the epsilon algorithm (Wynn, P., M.T.A.C 10 (1956), 91-96). ALool procedures for applying the algorithm to series of complex terms are given by Wynn (BIT \& (1962), 232-255).

The body of this procedure has been tested using the Dartmouth -Self-Contained ALgol. Processor for the LGP-30 computer. It gave the following results on the sequence for the amaller zero of the Laguerre polynomial, $L[2](x)$ :

\begin{tabular}{|c|c|c|c|c|}
\hline 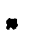 & $S[m]$ & $e[1](S[m])$ & $e[2](S \mid m])$ & $e[J P(S[\omega])$ \\
\hline 0 & 0.0000000 & 0.5714285 & 0.5857432 & 0.5857616 \\
\hline 1 & 0.5000000 & 0.5851059 & 0.5857854 & 0.5857859 \\
\hline 2 & 0.5625000 & 0.5857318 & 0.5857861 & 0.5857861 \\
\hline 3 & 0.5791016 & 0.5857816 & & \\
\hline 4 & 0.5838396 & 0.5857859 & & . \\
\hline 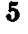 & 0.5852172 & & & \\
\hline & 0.5856198 & Tru & lue 0.58 & 1375 \\
\hline
\end{tabular}

These results are in satisfactory agreement with those given by by Wynn (1956):

begin integer $j, k, \lim j, \operatorname{limk}$, two kmax;

real $T 0, T 1$;

two $k \max :=k \max +k \max$

$\lim j:=n \max$

for $j:=$ nmin step 1 until lim $j$ do

begin $T 0:=0$;

$\lim :=j-n$ min;

if limk $>$ two kmax then limk $:=$ two kmax $\rangle$ limk $:=\operatorname{limk}-1$ :

for $k:=0$ atep 1 until limk do

begin $T 1:=S[j-k]-S[j-k-1]$

if $T 1 \neq 0$ then $T 1:=T 0+1 / T 1$ else

if $S[j-k]=1099$ then $T 1:=T 0$ else

T1 : $=1099$;

comment 1099 may be replaced by the largest number representable in the computer;

$T 0:=S[j-k-1]$

$S[j-k-1]:=T 1$

end for $k$

end for $j$

end Shanks

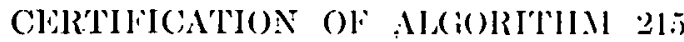

SHANKS |H. C. Thacher, Ji., Comm. AC:ll is (Nov. $1963), 662$

LarRy Schumaker (Recd. 16 Dec. 633)

Computation Ctr., Stanford I:, Stanford, Calif.

Algorithm 215 was coded in lextended Aldiol, for the Burroughs B-500) and was tested on a large number of secuences. ()ne ap- parent typographical error was noted. The statement $\lim :=$ $j-$ nmin should have read limk $:=j-$ nmin. 'The following tahles were reproduced exactly: (a) tables on p. 5 and p. 33 of [1]; (b) Table I on p. 95 of [2]; (c) Tables III and IV on p. 28 of [3].

REFERENCES:

1. Shanks, D. Non-linear transformations of divergent and slowly convergent sequences. J. Malh. Phys. 34 (1955), 1-42.

2. Wrnn, $P$. On a device for computing the $e_{m}\left(S_{n}\right)$ transformation. M'T'AC 10 (1956), 91-96

3. Wrnn, $P$. On repeated applic:ation of the $\epsilon$-algurithm. ('hiffers 4 (1961), 19-22.

\section{ALGORITHM 216 \\ SMOOTH}

Richard George*

Argonne National Laboratory, Argonne, Ill.

- Work supported by the U. S. Atomic Engergy Commission.

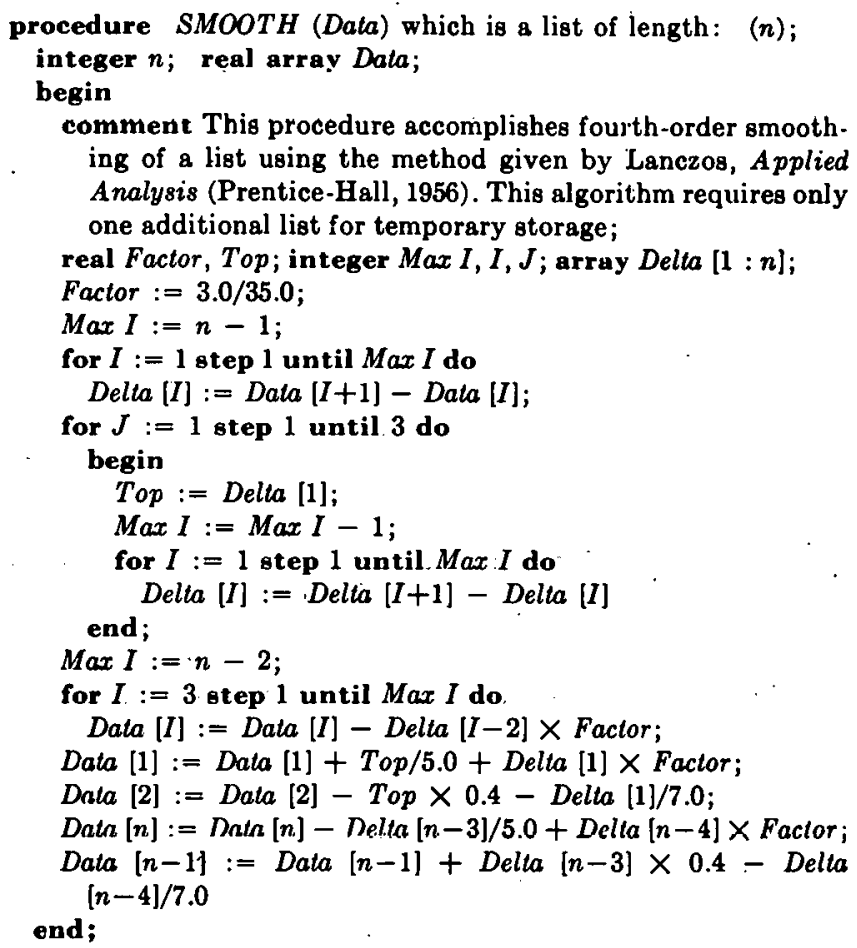

\section{ALGORITHMI 217 \\ MININIUM EXCESS COST CURVE}

Wililiam A. Briggs

Marathon Oil Co., Findlay, Ohio

procedure MINIMUM EXCESS COST CURVE (nodes, links, source, sink, $I, J$, crash, normal, slope, node, lij, ERROR);

value nodes, links, source, sink;

integer nodes, links, source, sink;

integer array $, I, J$, crash, normal, slope, node, lij;

comment This procedure utilizes a network-type description of 
a project to compute the minimum cost involved in expedition of the project completion date. Project tasks are identified and completion order specified by the vector pair $I, J$, which contain node numbers of the events starting and ending each task. The tasks are parameterized within the vectors crash, normal, and slope-which contain the crash or minimum task completion times, the normal task completion times, and the increased cost per unit decrease in task duration (the slope of the time-costcurve), which must be a nonzero integer. The procedure initially determines the normal-duration critical path, then successively reduces the durations of the tasks with the flattest cost slope, adjusting the critical path, until minimum durations are reached. The FORD-FULKERSON labeling technique is utilized. Each task must proceed from a lower-numbered node to a higher-numbered one-if not, exit to the nonlocal label $E R R O R$ is made. Nodes should be numbered sequentially, starting at the initial event (source) and continuing to the final event (sink). The maximum node number is equivalent to the value nodes, while the value links denotes the total number of tasks. The arrays are of dimensions $I, J$, crash, normal, slope, lij $[1:$ links $]$ and node $[1:$ nodes $]$;

begin integer $m, n, \iota b, n j i, n i j, l e x, k f, n j, n i, n t v$, ord, infinity, temp;

integer array labl[1:nodes, $1: 3], f[1:$ links, $1: 2]$;

comment infinity is herein used to represent the largest available integer;

for $m:=1$ step 1 until links-1 do

if $I[m] \geqq J[m] \vee I[m]>I[m+1] \vee J[m]>J[m+1]$ then go to ERROR;

if I links] $\geqq J[$ links] then go to ERROR;

for $n:=1$ step 1 until nodes do $\operatorname{labl}[n, 1]:=\operatorname{labl}[n, 2]:=$ $\operatorname{labl}[n, 3]:=\operatorname{node}[n]:=0$

for $m:=1$ step 1 until links do

begin $f[m, 1]:=f[m, 2]:=0$ temp $:=\operatorname{node}[I[m]]+$ normal $[m]$

if node $[J[m]]<$ temp then node $[J[m]]:=t e m p$

end;

$n t v:=$ ord $:=0 ; \quad t b:=\operatorname{node}[\operatorname{sink}]$

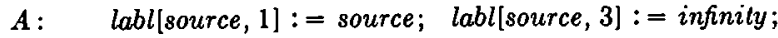

for $m:=1$ step 1 until links do

begin if $l a b l[I[m], 1]=0$ then go to $B$;

if labl[J[m],1] $\neq 0$ then go to $C$;

$n j i:=\operatorname{node}[J[m]]-\operatorname{node}[I[m]]$;

if $n j i \neq$ normal $[m]$ then go to $A 1$;

lex $:=\operatorname{slope}[m]-f[m, 1]$

if lex $\leqq 0$ then go to $A 1$;

$k f:=1$; go to $A 2$;

$A 1: \quad$ if $n j i \neq \operatorname{crash}[m]$ then go to $C$;

lex $:=$ infinity; $k f:=2$;

A2: $\quad \operatorname{labl}[J[m], 1]:=I[m] ; \quad \operatorname{labl}[J[m], 2]:=k f ;$

if $\operatorname{labl}[I[m], 3]>$ lex then go to $A 3$;

labl $[J[m], 3]:=\operatorname{labl}[I[m], 3] ;$ go to $A 4$;

A3: $\quad \operatorname{labl}[J[m], 2]:=$ lex;

A4: if $J[m]=\operatorname{sink}$ then go to $D$ else go to $C$;

$B: \quad$ if $l a b l[J[m], 1]=0$ then go to $C$;

$n i j:=\operatorname{node}[I[m]]-\operatorname{node}[J[m]]$;

if $n i j \neq$ normal $[m] \vee f[m, 1]=0$ then go to $B 1$;

lex $:=f[m, 1] ; \quad k f:=-1$; go to $B 2$;

$B 1: \quad$ if $n i j \neq$ normal $[m] \vee[m, 2]=0$ then go to $C$;

lex $:=f[m, 2] ; \quad$ kf $:=-2$;

$B 2: \quad l a b l[I[m], 1]:=J[m] ; \quad l a b l[I[m], 2]:=k f ;$

if $\mid l a b l[J[m], 3 \mid>$ lex then go to $B 3$;

labl $[I[m], 3]:=\operatorname{labl}[J[\mathrm{~m}], 3]$; go to $B 4$;

B3: $\quad l a b l[I[m], 3]:=$ lex;

$B 4: \quad$ if $I[m]=\operatorname{sink}$ then go to $D$;

$C$ : end;

for $n:=1$ step 1 until nodes do if $\operatorname{labl}[n, 1]=0$ then node $[n]:=$ node $[n]-1$;

$F: \quad$ for $n:=1$ step 1 until nodes do $l a b l[n, 1]:=\operatorname{labl}[n, 2]:=$ $\operatorname{labl}[n, 3]:=0$

go to $A$;

$D: \quad$ if labl[sink, 3] = infinity then go to OUT;

$n t v:=n t v+l a b l[\operatorname{sink}, 3] ; \quad n j:=\operatorname{sink}$;

$G: \quad n i:=l a b l[n j, 1]$

if $l a b l[n j, 2]>0$ then go to $G 1$;

for $m:=1$ step 1 until links do if $I[m]=n j \wedge J[m]=$ $n i$ then $f[m,-\operatorname{labl}[n j, 2]]:=f[m,-l a b l[n j, 2]]+$ labllsink, 3];

G1: for $m:=1$ step 1 until links do if $I[m]=n i \wedge J[m]=$ $n j$ then $f[m, l a b l[n j, 2]]:=f[m, l a b l[n j, 2]]+\operatorname{labl}[\operatorname{sink}, 3]$; if $n i=$ source then go to $O U T$;

$n j:=n i$; go to $G$;

OUT: for $m:=1$ step 1 until links do

begin $l i j[m]:=\operatorname{node}[J[m]]-\operatorname{node}[I[m]]$

if $l i j[m]>$ normal $[m]$ then $l i j[m]:=$ normal $[m]$ end;

ord $:=(t b$-node[sink $]) \times n t v ; \quad l b:=$ node $[\operatorname{sink}] ;$

if labl $\{\operatorname{sink}, 3]=$ infinily then $n t v:=$ infinity;

ANS: comment as control passes through here-

ord is the ordinate of the minimum project excess cost curve at a total project duration of node[sink],

successive values of ord plotted versus node[sink] gener-

ate the minimum project excess cost curve.

node $[1:$ nodes $]$ contains the event times at each node

lij $[1$ : links $]$ contains the durations of each task

$n t v$ is the slope of the cost curve back in time from total duration node[sink].

these values should be printed in some readable form; if $n t v<$ infinity then go to $F$;

end MINIMUM EXCESS COST CURVE;

\section{ALGORITHM 218}

KUTTA MERSON

Phyllis M. Lukehart*

Argonne National Laboratory, Argonne, Ill.

procedure KuttaMerson ( $n, t, y, e p s, h, f c t, f i r s t)$;

value $n$, eps:

integer $n$;

real $t$, eps, $h$;

real array $y$;

Boolean first;

procedure $f c t$;

comment This procedure integrates the system of ordinary firstorder differential equations $y[i]=f[i](t, y[1], y[2], \cdots y[n])$ from $t=t$ to $t=t+h$ by the Kutta-Merson method (L. Fox, Numerical Solution of Ordinary and Partial Differential Equations, p. 24, Pergamon Press, 1962). The working interval of calculation is adjusted by the procedure so that the maximum absolute error of the dependent variables is less than eps. For optimum error control, the equations should be scaled so that all dependent variables have approximately the same magnitude. Input variables for the procedure are $n$, the number of equations, $t$, initial value of the independent variable, $y$, array of initial values of dependent variables, eps, allowable error, $h$, the total interval, $f c t$, a procedure evaluating the derivatives, and first, a Boolean variable which indicates whether the working interval has been adjusted to secure the desired accuracy. On the initial call of the procedure for a given system, first 
should be true. It will be set false by the procedure, and the proper working interval determined. The procedure $f c t$ has as formal parameters the simple real variable $t$, and the real arrays $y$ and $f$. For $i=1,2,3, \cdots, n$ it must assign to $f[i]$ the value of the first derivative of $y[i]$ appropriate to the values of $t$ and $y$. The body of this procedure has been tested using the Dartmouth Scalp compiler for the LGP-30 computer. For the equation $d y / d t=-2 t y^{2}$ and input data $t=1, y=.5, h=1$, $e p s=.0001$, the average error was .000003 and the time was 30 min. For the linear boundary value problem $d^{2} y / d l^{2}=-1-$ $\left(l^{2}+1\right) y, y( \pm 1)=0$, the maximum error was .0000024 (L. Collatz, The Numerical Trentment of Differential Equations, pp. 145, 225, Springer-Verlag, Berlin, 1960) and the time, $90 \mathrm{~min}$. More accuracy may be achieved by using a smaller value of eps;

begin integer $i$, loc;

real error;

array $y 0, y 1, y 2, f 0, f 1, f 2[1: n]$;

own integer ploc;

own real $h c$;

Boolean increase;

if first then begin $h c:=h ;$ ploc $:=1$; first $:=$ false end; loc $:=0$;

nexl: $f c t(l, y 0, f 0)$;

for $i:=1$ step 1 until $n$ do

$y 1[i]:=y 0[i]+h c / 3 \times f 0[i]$

$f c t(t+h c / 3, y 1, f 1)$;

for $i:=1$ step 1 until $n$ do

$y 1[i]:=y 0[i]+h c / 6 \times j 0[i]+h c / 6 \times f 1[i]$

$f c t(t+h c / 3, y 1, f 1)$;

for $i:=1$ step 1 until $n$ do

$y 1[i]:=y 0[i]+h c / 8 \times f 0[i]+3 \times h c / 8 \times f 1[i]$;

$f c t(t+h c / 2, y 1, f 2)$;

for $i:=1$ step 1 until $n$ do

$y 1[i]:=y 0[i]+h c / 2 \times f 0[i]-3 \times h c / 2 \times f 1[i]+2 \times h c \times$ f2[i];

$f c t(t+h c, y 1, f 1)$

for $i:=1$ step 1 until $n$ do

$y 2[i]:=y 0[i]+h c / 6 \times \mathcal{N}[i]+2 \times h c / 3 \times f 2[i]+h c / 6 \times f 1[i] ;$

increase : = true;

for $i:=1$ step 1 until $n$ do

begin error : $=a b s(.2 \times(y 1[i]-y 2[i]))$;

comment To test on relative error change this expression to $a b s(.2-.2 \times y 2[i] / y 1[i])$

if error $>$ eps then

begin $h c:=h c / 2$;

ploc $:=2 \times$ ploc;

loc $:=2 \times l o c$;

go to next

end;

if error $\times 64>$ eps then increase $:=$ false

end $i$;

$t:=t+h c$

for $i:=1$ step 1 until $n$ do

$y 0[i]:=y 2[i]$

loc $:=l o c+1$

if loc $<$ ploc $\wedge$ increase $\wedge$ loc $=l o c \div 2 \times 2 \wedge$ pioc $>1$ then

begin $h c:=2 \times h c$;

loc $:=l o c \div 2$

ploc $:=$ ploc $\div 2$

end;

go to next

end KuttaMerson
CERTIFICATION OF ALGORITHM 218 [D2]

KUTTA MERSON [Phyllis M. Lukehart, Comm. $A C M$

6 (Dec. 1963), 737]

Karen Borman Priebe (Recd. 10 Feb. 1964)

Woodward Governor.Company, Rockford, Illinois

Algorithm 218 was translated into FAST for the NCR 315 and gave satisfactory results with the following corrections, if the equations were scaled as recommended in the comment of the original algorithm. Ignoring this scaling can lead to results that do not satisfy the intended error criterion.

1. procedure KuttaMerson ( $n, t, y, e p s, h, f c t, f i r s t, x)$; instead of

procedure KuttaMerson ( $n, t, y, e p s, h, f c t, f(r 8 t)$;

2. real array $y, x$;

instead of

real array $y$;

3. if first then begin for $i:=1$ step 1 until $n$ do $y 0[i]:=y[i]$ $h c:=h$.

instead of

if first then begin $h c:=h ; \quad \cdots$

4. if loc $<$ ploc then

begin

if increase $\wedge l o c=(l o c \div 2) \times 2 \wedge p l o c>1$ then

begin

$h c:=2 \times h c$;

loc $:=\ln s \div 2$;

ploc $:=$ ploc $\div 2$

end;

go to next

end;

for $i:=1$ step 1 until $n$ do $x[i]:=y 0[i]$

end KuttaMerson

instead of

if loc $<$ ploc $\wedge$ increase..

end KuttaMerson

5. The following sentences should be added to the initial comment of the procedure:

The values of the dependent variables at $t+h$ are placed in the array $x$. Note that the values of $t$ and first are changed as sideeffects of the procedure. (As originally written, KuttaMerson seemed unable to obtain the values of the solution at $t$ or to trans. mit the values of the solution at $t+h$ to the outside program! Ed.)

6. Change array to array in the body of the procedure.

7. Insert after own integer ploc;

$$
\text { own array } y 0[1: n] \text {; }
$$

Delete yo from the existing array declaration.

\section{ALGORITHM 219 \\ TOPOLOGICAL ORDERING FOR PERT NET- WORKS \\ RoBert H. KaSE \\ Atlantic Refining Co., Philadelphia, Penn:}

procedure Topological Ordering $(i, j, t r i, n, n e)$;

integer $n, n e$; integer array $i, j, t r i$;

comment Nodal points $i$ and $j$ represent activities in a PERT

network. $n$ is the number of activities. $t r i$ is a tape record index 
vector locating where additional data for each activity is stored.;

begin integer $a, b$; integer array $n i, n j$, event $[1: n]$;

comment An event vector is set up containing ne events.

New nodal numbers $n i$ and $n j$ are assigned for all activities.; ne $:=n i[1]:=1 ;$ event $[1]:=i[1]$;

begin for $a:=2$ step 1 until $n$ do

begin for $b:=1$ step 1 until $n e$ do

if $i[a]=e v e n t[b]$ then begin $n i[a]:=b$;

go to repeal 1 end;

end;

$n i[a]:=n e:=\dot{n} e+1 ;$ event $[n e]:=i[a]$

repeal 1: end;

begin for $a:=1$ step 1 until $n$ do

begin for $b:=1$ step 1 until ne do

if $j[a]=$ event $[b]$ then begin $n j[a]:=b$;

go to repeat 2 end;

end;

$n j:=n e:=n e+1 ;$, event $[n e]:=j[a]$

repeal 2: end;

begin integer $t$, bigtal; integer array rank, con $[1: n e]$;

comment Event ranking (topological ordering);

for $a:=1$ step 1 until $n e$ do

begin $\operatorname{rank}[a]:=1 ; \operatorname{con}[a]:=0$ end;

bigtal $:=1$

pass: $t:=0$;

for $a:=1$ step 1 until $n$ do

begin if $\operatorname{rank}[n j[a]] \leqq \operatorname{rank}[n i[a]\}$ then

$\operatorname{rank}[n j[a]]:=\operatorname{rank}[n i[a]]+1$ else go to fill;

if $\operatorname{rank}[n j[a]]>$ bigtal then

bigtal $:=\operatorname{rank}[n j[a]] ; \quad t:=1$

fill: $\operatorname{con}[\operatorname{rank}[n i[a]]]:=\operatorname{con}[\operatorname{rank}[n j[a]]]:=1$

end;

if $t \leqq 0$ then go to new;

for $a:=1$ step 1 until bigtal do

begin if $\operatorname{con}[a]=0$ then go to Loop end;

comment Loop should be a label of a procedure statement which calls a subroutine to detect those events which may be in a loop in the PERT network or the label of a print out indicating that loop(s) exist in the network. In any case

a loop exists and further problem processing is impossible.;

for $a:=1$ step 1 until bigtal do con $[a]:=0$;

go to pass;

comment Reassignment of a new nodal number, $n i$, to all activities;

new: $t:=1$;

for $a:=1$ step 1 until bigtal do

begin for $b:=1$ step 1 until ne do

if $\operatorname{rank}[b]=a$ then begin event $[b]:=t ; \quad t:=t+1$ end end;

for $a:=1$ step 1 until $n$ do $n i[a]:=$ event $[n i[a]]$

end;

comment Using the new nodal number, $n i$, activities ( $i$ and $j$ ) and their corresponding $t r i$ may now be arranged in topological sequence with conventional sort routines. Sorting should be done on $n i$;

end
ALGORITHM 220

GAUSS-SEIDEL

Peter W. Shantz

University of Waterloo, Waterloo, Ontario, Canada

procedure GAUSS-SEIDEL $(n, A, B, t o l)$;

value $n$, tol; array $A, B$; real tol; integer $n$;

comment GAUSS-SEIDEL solves a system, $A x=B$, of $n$ simultaneous linear equations in $n$ unknowns. $A$ is the matrix of coefficients, $B$ an inhomogeneous vector. The standard GaussSeidel iterative technique is employed until $\left|x_{R}^{(i)}-x_{K}^{(i-1)}\right|<t o l$ for all $K$, where $x_{K}^{(i)}$ denotes the $i$ th iterant of the unknown $x_{K}$. (Cf. Ralph G. Stanton, Numerical Methods for Science and Engineering, Ch. 8);

begin array $X, Y[1: n]$; integer $i, j, K$;

for $i:=1$ step 1 until $n$ do $X[i]:=Y[i]:=0$;

START: for $i:=1$ step 1 until $n$ do begin $Y[i]:=B[i]$

for $j:=1$ step 1 until $n$ do

$Y[i]:=$ if $i=j$ then $y[i]$ else

$Y[i]-A[i, j] \times Y[j]$

$Y[i]:=Y[i] / A[i, i]$

end $i$

comment Now test for convergence;

for $K:=1$ step 1 until $n$ do

if $a b s(Y[K]-X[K]) \geq t o l$ then

begin for $i:=1$ step 1 until $n$ do

$X[i]:=Y[i] ;$ go to $S T A R T$

end convergence test;

end GAUSS-SEIDEL

\section{CERTIFICATION OF AND REMARK ON}

ALGORITHM 220

GAUSS-SEIDEL [P. W. Shantz, Comm. $A C M \quad 6$ (Dec. 1963), 739]

A. P. Batson (Recd 6 Jan. 1964)

University of Virginia, Charlottesville, Va.

NikLa Us Wirth (Recd 6 Jan. 1964)

Computer Science Div., Stanford U., Stanford, Calif.

[Editor's Note. Two substantially equivalent contributions were received on the same day, and so the editor has merged them.-G.E.F.]

The following errors were detected.

1. The procedure cannot communicate the solution to the outside block unless $X$ (or $Y$ ) is made a parameter of the procedure.

2. The identifier GAUSS-SEIDEL may not contain a hyphen.

3. In the fourth line after the label START change $y[i]$ to $Y[i]$.

With the above errors corrected, GAUSS SEIDEL was successfully run on the Stanford 7090 computer in Wirth's Extended Algol, and on the Virginia Algol compiler for the Burroughs 205.

The following improvements would be desirable.

1. Avoid repeated reference to the subscripted variable $Y[i]$ inside the $j$ loop.

2. Permit the user to initialize the array $X$ to an appropriate value at the start of the iteration.

3. Modify tol to be a relative error, rather than an absolute error.

4. Incorporate a guard against nonconvergence. 


\section{SUBJECT INDEX}

List of Modified SHARE Classification

Al Real Arithmetic, Number Theory

A2 Complex Arithmetic

$B 1$ Trig and Inverse Trig Functions

B2 Hyperbolic Functions

B3 Exponential and Logarithmic Functions

B4 Roots and Powers

$\mathrm{Cl}$ Operations on Polynomials and Power Series

$\mathrm{C} 2$ Zeros of Polynomials

C5 Zeros of One or More Transcendental Equations

C6 Summation of Series, Convergence Acceleration

Dl Quadraturc

D2 Ordinary Differential Equations

D3 Partial Differential Equations

D4 Differentiation

El Interpolation

E2 Curve and Surface Fitting

E3 Smoothing

E4 Minimizing or Maximizing a Function

Fl Matrix Operations, Including Inversion

F2 Eigenvalues and Eigenvectors of Matrices

Al--Real Arithmetic, Number Theory

7 Euclidean

35 Sieve of Eratosthenes

61 Range Arithmetic

68 Augmentation

72 Compositions

93 Generalized Arithmetic

95 Partitions

99 Jacobi Symbol

114. Partitions

139. Diophantine Equation

A2--Complex Arithmetic

116 Complex Divide

186 Complex Arithmetic:

$\mathrm{Bl--Trig}$ and Inverse Trig Functions

206 Arccossin

M. Konda

B2--Hyperbolic Functions

B3--Exponential and Logarithmic Functions
$46 \operatorname{Exp}(Z), z$ Complex
J. R. Herndon
$48 \log (z), z$ Complex
J. R. Herndon

B4--Roots and Power

53 Roots of Complex Numbers J. R. Herndon

106 Powers of Complex Numbers M. L. Johnson,

W. Sangren

190 Powers of Complex Numbers A. P. Relph

Cl--Operations on Polynomials and Power Series

29 Polynomial Shifter
131 Divide Power Serie

J. G. Mackinney

134 Exponentiate Power Series

V. H. Smith,

M. L. Allen

158 Exponentiate Power Series

H. E. Fettis

H. E. Fettis

' 193 Revert Power Series
F3 Determinants

F4 Simultaneous Linear Equations

F5 Or thogonalization

Gl Simple Calculations on Statistical Data

G2 Correlation and Regression Analysis

G5 Random Number Generators

G6 Permutations and Combinations

G7 Subset Generators and Classifications

H Operations Research, Graph Structures

I5 Input--Composite

J6 Plotting

K2 Relocation

Ml Sorting

M2 Data Conversion and Scaling

O2 Simulation of Computing Structure

$S$ Approximation of Special Functions. Functions are Classified SO1 to S22, Following FletcherMiller-Rosenhead, Index of Math. Tables

Z All Others

C2--Zeros of Polynomials

3 Bairstow

30 Bairstow-Newton

59 Resultant Method

75 Rational Roots-Integer Coeff.

78 Rational Roots-Integer Coeff.

105 Newton-Maehly

A. A. Grau

K. W. Ellenberger

E. H. Bareiss,

M. A. Fisherkeller

J. E. L. Peck

C. Perry

F. L. Bauer,

J. Stoer

A. Gibb

174 Bounds of Zeros

C5--Zeros of One or More

Transcendental Equations

2 Regula Falsi

J. Wegstein

4 Bisection

S. Gorn.

15 Regula Falsi.

25 Real Zeros:

26 Regula Falsi

196 Mullers Method

H. C. Thacher

B. Leavenworth

J. G. Herriot

R. D. Rodman

C6--Summation of Series, Convergence Acceleration

8 Euler Sum

128 Fourier Series Summation

157 Fourier Series Summation

215 Epsilon

P. Naur

M. Wells

C. J. Mifsud

H. C. Thacher

D1--Quadrature

1 Quadrature

32 Multiple Integral

60 Romberg Method

84 Simpsons Rule

98 Complex Line Integral

103 Simpsons Rule

125 Gaussian Coefficients

145 Adaptive Simpson

146 Multiple Integral

182 Adaptive Simpson

198 Adaptive, Multiple Integral
R. J. Herbold

R. D. Freeman

F. L. Bauer

P. E. Hennion

J. L. Pfaltz

G. F. Kuncir

H. Rutishauser

W. M. Mckeeman

W. M. Mckeeman

W. M. McKeeman,

L. Tesler

W. M. McKeeman 
D2 - - Ordinary Differential Equations

9 Runge-Kutta P. Naur

1194 Zeros of O.D.E. System C. Domingo

218 Kutta-Merson

P. M. Lukehart

D3 - Partial Differential Equations

D4--Differentiation

79 Difference Expression Coeff. T. P. Giammo

El--Interpolation

18 Rational Interp.--

Cont. Fract. R. W. Floyd

70 Aitken Interpolation $\quad$ C. J. Mifsud

77 Interpolation, Diffn., Integrn. P. E. Hennion

167 Confluent Divided Differençes

W. Kahan, I. Farkas

W. Kahan, I. Farkas Differences

169 Interpolation-Divided Differences

W. Kahan, I. Farkas

187 Diffces. and Derivs. Recursive

210 Lagrange Interpolation

$R$. P. van de Riet

G, R, Schubert

G. R. Schubert

211 Hermite Interpolation

E2--Curve and Surface Fitting

28 Least Squares by Orthog. Polyn.

37 Economization

38 Economization

74 Least Squares with Constraints

91 Chebyshev Fit

164 Surface Fit

176 Surface Fit

177 Least Squares with Constraints

J. G. Mackinney

K. A. Brons

K. A. Brons

J. E. L. Peck

A. Newhouse

R. E. Clark,

R. N. Kubik,

L. P. Phillips

T. D. Arthurs

M. J. Synge

E3--Smoothing

188 Smoothing

189 Smoothing

216 Smoothing

F. Rodriguez-Gil

F. Rodriguez-Gil

R. George

E4--Minimizing or Maximizing a Function

129 Minimize Function of N Variables $\quad$ V. W. Whitley

178 Minimize Function of N Variables

A. F. Kaupe

203 Minimize. Function of $\mathrm{N}$ Variables

E. J. Wasscher

204 Minimize Function of N Variables

E. J. Wasscher

205 Minimize Function of N Variables

J. G. A. Haubrich

Fl--Matrix Operations, Including Inversion

42 Inversion

T. C. Wood

50 Inverse of Hilbert Matrix

J. R. Herndon

51 Inverse of Perturbed Matrix

52 Inverse of Test Matrix

58 Inversion-Gaussian Elimination

66 Inversion-SQRT Method

67 Cram Matrix

J. R. Herndon

J. R. Herndon

D. Cohen

J. Caffrey

J. Caffrey

120 Inversion-Gaussian Elimination

140 Inversion
150 Inverse of Symmetric Matrix

166 Monte Carlo Inverse

197 Matrix Division

H. Rutishauser

R. D. Rodman

F2--Eigenvalues and Eigenvectors of Matrices

85 Jacobi Method

T. G. Evans

104 Reduction-Band to Tridiagonal H. Rutishauser

122 Givens Tridiagonal Reduction G. F. Dietzel

183 Reduction-Band to Tridiagonal H. R. Schwarz

F3--Determinants

41 Determinant Evaluation

159 Determinant Evaluation

170 Determinant-Polynomial

Elements

J. G. Solomon

D. W. Digby

P. E. Hennion

F4--Simultaneous Linear Equations

16 Crout with Pivoting

17 Solve Tridiagonal Matrix

24 Solve Tridiagonal Matrix

G. E. Forsythe

C. F. Sprague

43 Crout with Pivoting

92 Simultaneous Equations-Iterative Solution

107 Gaussian Elimination

12.6 Gaussian Elimination

135 Crout with Equilibration

B. Leavenworth

H. C. Thacher

195 Band Solve

220 Gauss-Seidel

D. J. Roek

J. W. Counts

J. W. Counts

W. M. Mckeeman

D. H. Thurnau

P. W. Shantz

F5--Orthogonalization

127 Orthonormalization $\quad$ P. J. Walsh

Gl--Simple Calculations on Statistical Data

208 Discrete Convolution $\quad$ W. T. Foreman

212 Determine Distrib. Fcn. from Data

M. D. Gray

G2--Correlation and Regression Analysis

39 Correlation Coefficients P. Sassouni

142 Triangular Regression W. L. Hafley,

J. S. Lewis

G5--Random Number Generators

121 Random Normal D. Shafer

133 Random Flat P. G. Behrenz

200 Random Normal R. George

G6--Permutations and Combinations

71 Permutations R. R. Coveyou,

J. G. Sullivan

86 Permutations

J. E. L. Peck,

87 Permutations

94 Combinations

102 Permutations

115 Permutations

130 Permutations

152 Combinations

154 Combinations

155 Combinations

156 Combinations

160 Combinations

161 Combinations

G. F. Schrack

J. R. Howell

J. Kurtzberg

G. F. Schrack,

M. Shimrat

H. F. Trotter

B. C. Eaves

J. Hopley

C. J. Mifsud

C. J. Mifsud

C. J. Mifsud

M. L. Wolfson,

H. V. Wright

M. L. Wolfson,

H. V. Wright

202 Permutations

Mok-Kong Shen

G7--Subset Generators and Classifications

81 Subsequences

82 Subsequences

83 Classifications
B. H. Mayoh

B. H. Mayoh

B. H. Mayoh 


$\begin{aligned} 27 & \text { H--Operations Research, Graph Structures } \\ 40 \text { Critical Path Scheduling } & \text { R. Silver } \\ 69 \text { Chain Tracing } & \text { B. Leavenworth } \\ 96 \text { Ancestor } & \text { B. H. Mayoh } \\ 97 \text { Shortest Path } & \text { R. W. Floyd } \\ 119 \text { PERT Network } & \text { R. W. Floyd } \\ & \text { B. Eisenman, } \\ 141 \text { Find Path } & \text { M. Shapiro } \\ 153 \text { Integer Programming } & \text { P. Z. Ingerman } \\ 217 \text { Min. Access Cost Curve } & \text { F. L. Bauer }\end{aligned}$

I5--Input--Composite

$\begin{aligned} 162 & \frac{\text { J6--Plotting }}{\text { XY Plotter }} \\ 173 & \frac{\text { K2--Relocation }}{\text { Transfer Array Values }} \\ & \text { M1--Sorting } \\ 23 & \text { Sort } \\ 63 & \text { Sort } \\ 64 & \text { Sort } \\ 65 & \text { Sort } \\ 76 & \text { Sort } \\ 113 & \text { Treesort } \\ 143 & \text { Treesort } \\ 144 & \text { Treesort } \\ 151 & \text { Locate in a List } \\ 175 & \text { Shuttle Sort } \\ 201 & \text { Shell Sort } \\ 207 & \text { String Sort }\end{aligned}$

F. G. Stockton

O. Hajek

W. Feurzeig

C. A. R. Hoare

C. A. R. Hoare

C. A. R. Hoare

I. Flores

R. W. Floyd

A. F. Kaupe

A. F. Kaupe

H. F. Walter

C. J. Shaw,

T. N. Trimble

J. Boothroyd

J. Boothroyd

M2--Data Conversion and Scaling

O2--Simulation of Computing Structure

100 Processing of Chain-Linked List P. J. Kiviat

101 Processing of Chain-Linked List

137 Nested for Statement

P. J. Kiviat

D. M. Dahm,

M. Wells

138 Nested for Statement

D. M. Dahm,

M. Wells

S--Approximation of Special Functions

S03 19 Binomial Coefficients R. R. Kenyon 33 Factorial $\mathrm{N}$

M. F. Lipp

S13 14 Complex Exponential Integral

A. Beam

20 Real Exponential Integral S. Peavy

108 Exponential Integral

Y. A. Kruglyak,

D. R. Whitman

109 Exponential Integral

Y. A. Kruglyak,

D. R. Whitman

R. M. Collinge

M. F. Lipp

J. R. Herndon

W. Holsten

34 Gamma Function

54 Gamma Function

80 Gamma Function

147 Derivative of Gamma Function

179 Beta Ratio

Sl5 11 Hermite Polynomial

123 Error Function

180 Error Function

D. Amit

O. G. Ludwig

G. M. Galler

M. Crawford,

R. Techo

H. C. Thacher
181 Error Function

185 Error Function

209 Error Function

S16 13 Legendre Polynomial

47 Associated Legendre Function

62 Associated Legendre Function

S17 21 Bessel Function

22 Riccati-Bessel Function

44 Bessel Function

49 Spherical Neumann Function

124 Hankel Function

.163 Hankel Function

S18 5 Bessel Function

6 Bessel Function

214 Bessel Function

S19 57 Berbei Function

S20 88 Fresnel Integrals

89 Fresnel Sine Integral

90 Fresnel Cosine Integral

213 Fresnel Integrals

S2l 55 Elliptic Integral--First Kind

56 Elliptic Integral--Second Kind

73 Incomplete Elliptic Integral

149 Elliptic Integral

165 Elliptic Integral

S22 10 Chebyshev Polynomial

12 Laguerre Polynomial

36 Chebyshev Polynomial

110 Physics Integrals

111 Physics Integrals

132 Physics Integrals

184 Erlang Probability Function

191 Hypergeometric Fcn. (Complex)

192 Confluent Hypergeometric Fcn. (Complex)

\section{Z--All Others}

45 Interest Refinement

112 Point Inside Polygon

117 Magic Square

118 Magic Square

136 Enlarge A Group

148 Magic Square

199 Calendar Conversion

219 Topological Ordering
H. C. Thacher

A. Colker

D. Ibbetson

G. M. Galler

J. R. Herndon

J. R. Herndon

W. Börsch-Supan

H. Oser

M. E. Wojcicki

J. R. Herndon

L. J. Schaefer

H. E. Fettis

D. S. Clarke

D. S. Clarke

J. M. S. Simoes

Pereira

J. R. Herndon

J. L. Cundiff

J. L. Cundiff

J. L. Cundiff

M. D. Gray

J. R. Herndon

J. K. Herndon

D. K. Jefferson

J. N. Merner

H. C. Thacher

G. M. Galler

G. M. Galler

A. J. Gianni

Y. A. Kruglyak,

D. R. Whitman

Y. A. Kruglyak,

D. R. Whitman

J. C. Browne

A. Colker

A. P. Relph

A. P. Relph

P. Z. Ingerman

M. Shimrat

D. M. Collis on

D. M. Collison

M. Wells

D. M. Collison

R. G. Tantzen

R. H. Kase 


\section{AUTHOR INDEX}

Listed below are the names of contributors and the numbers of their Algorithm, Certification, or Remark.

Alexander, N. P., 40

Alexander, W. J., 20, 30

Allen, M. L., 131

Amit, D., 147

Arthurs, T. D., 176

Bareiss, E. H., 59

Barnecut, J. N. R., 148

Barton, S. P., 123

Batson, A. P., 220

Batty, M. A., 201

Bauer, F. L., 60, 105, 153

Beam, A., 14

Behrenz, P. G., $31,58,84,133$.

Bitterli, C. V., 164

Blair, C. R., 207

Blakely, R. F., 160

Boothroyd, J., 201, 207

Borsch-Supan, W., 21

Bosworth, K. M., 117, 118, 154, 155, 156

Breed, L. M., 68

Bridges, J. F., 37, 38

Briggs, W. A., 217

Brons, K. A., 37, 38

Brown, P. J., 35, 71

Browne, J. C., 132

Broyden, C. G., 66

Buchner, K. H., 60

Butler, H. S., 182

Caffrey, J., 66, 67

Cavin, D. K., 162

Clark, E. S., 79

Clark, R. E., 164

Clarke, D. S., 5, 6

Clausen, I., 181

Claussen, R., 7

Cohen, D., 58

Cohen, K. J., 30

Colker, A., 184, 185

Collens, D. S., 48

Collinge, R. M., 25, 31

Collins, D. H., 161

Collison, D. M., 72, 78, 86, 87, 117, 118,148

Conger, R. A., 58

Counts, J. W., 107, 126

Coveyou, R. R., 71

Crawford, M., 123

Cundiff, J. L., 88, 89, 90

Dahm, D. M., 137, 138

D'Esopo, D. A., 153

Dietzel, G. F., 122

Digby, D. W., 159

Domingo, C., 43, 194

Dubay, G. H., 52

Dwyer, T. A., 210

Eaves, B. C., 130

Eisenman, B., 119

Ellenberger, K. W., 30

Erwin, G. W., 106

Evans, A., 150

Evans, T. G., 85

Farkas, I., 167, 168, 169

Fettis, H. E., 134, 158, 163, 193

Feurzeig, W., 23
Filsak, Z., 173

Fisherkeller, M. A., 59

Fletcher, W. E., 162

Flores, I., 76

Floyd, R. W., 18, 96, 97, 113

Forsythe, G. E., 15, 16

Foreman, W. T., 208

Freed, B. H., 41

Freeman, R. D., 32

Galler, G. M., 10, 11, 12, 13

Garland, S. J., 99

George, R., 19, 47, 51, 58, 84, 93, 120, 140, 157, 200,216

Giammo, T. P., 79

Gianni, A. J., 36

Gibbs, A., 61, 174

Gilbert, H. E., 52

Gladfelter, G. W., 209

Gorn, S., 4

Grau, A. A., 3

Gray, M. D., 88, 89, 90, 212, 213

Grench, R. E., 94

Hacker, R., 112

Hafley, W. L., 142

Hajek, O., 173

Hale, R. P.; 91

Halstead, M. H., 78

Hansson, L., 181

Haubrich, J. G. A., 205

Hellberg, L., 40

Hellerman, L., 72

Hennion, P. E., 77, 84, 170

Herbold, R. J., I

Herndon, J. R., 3, 10, 13, 46, 47, 48, 49, 50, 51, 52, $53,54,55,56,57,62$

Herriot, J. G., 15, 26

Hillmore, J. S., 35, 52, 63, 64, 65, 71, 75, 85

Hoare, C. A. R., 63, 64, 65

Hoffman, I. A., 40

Holsten, W., 80

Hopley, J., 152

Howell, J. R., 87

Ibbetson, D., 123, 209

Ingerman, P. Z., 45, 140, 141

Is oda, K., 74

Jeffer son, D. K., 73

Johnson, M. L., 34, 48, 106

Juelich, O. C., 175

Kahan, W., 167, 168, 169

Kase, R. H., 219

Kaupe, A. F., 1 43, 144, 178

Kenyon, R. R., 19

Kiviat, P. J., 100, 101

Knapp, A. W., 42, 99

Konda, M., 206

Kondo, J., 105

Kriebel, D. C., 7.3

Kruglyak, Y. A., 108, 109, 110,111

Kubik, R. N., 164

Kuncir, G. F., 103

Kurtzberg, J., 94 
Lapidus, A., 159

Laughlin, D. L., 133

Lawrence, J. D., 158

Leavenworth, B., 24, 25, 40

Lefkowitz, B., 153

Lewis, J. S., 142

Lipp, M. F., 33, 34

Ludwig, O. G., 179

Lukehart, P. M., 218

Mackinney, J. G., 28, 29

MacMillan, D. B., 28

May, R. W., 94, 99

Mayoh, R. H., 69, 81, 82, 83

McKeeman, W. M., 135, 145, 146, 182, 198

Merner, J. N., 149

Meyer, N. A., 73

Mifsud, C. J., 70, 154, 155, 156, 157

Naur, P., 8, 9, 42, 50, 52, 85, 91, 107, 117, 118 , $120,122,150$

Nestor, C. W., 53

Newhouse, A., 27, 91

Ogden S., 72

Oppenheim, D. K., 199

Oser, H., 22

Peavy, S., 20

Pcck, J. E. L., 71, 74, 75, 86, 139

Perry, C.. 78

Perstein, M. H., 93

'Pfaltz, J. L., 98

Phillips, E. S., 115

Phillips, L. P., 164

Pike, M. C., 209

Poore, Jr., J. H., 133

Priebe, K. B., 170,218

Rader, P. J., 4, 14

Randell, B., 50, 63, 64, 65, 66, 76

Ranshaw, R. W., 23

Relph, A. P. 46, 48, 57, 67, 70,75, 84, 190, 191, 192

Rice, H. G., 68

Rodman, R. D., 166, 196

Rodriguez-Gil, F., 43, 188, 189

Rotenberg, L. J., 41

Roek, D. J., 92

Russell, L. J., 63, 64, 65

Rutishauser, H., 104, 125,150

Sangren, W., 34, 48, 106

Sassouni, P., 39

Schaefer, L. J., 124

Schrack, G. F., 71, 86, 87, 102, 115

Schubert, G. R., 157, 175, 210,211

Schumaker, L., 215

Schwarz, H. R., 183

Scowen, R. S., 173

Shafer, D., 121

Shamen, P., 42

Shant $z$, P. W., 220

Shapiro, M., 119

Shaw, C. J., 175

Shen, Mok-Kong, 202

Shimrat, M., 87, 102, 112

Silver, R., 27

Simoes Pereira, J. M. S., 214

Smith, R. L., 116

Smith, V. H., 131

Solomon, J. G., 41

Sprague, C. F., 17
Steck, B., 19

Stockmal, F., 95, 114

Stockton, F. G., 162

Stoer, J., 105

Struble, G., 58

Sullivan, J. G., 71

Synge, M. J., 177

Tantzen, R. G., 199

Techo, R., 123

Tesler, L., 182

Thacher, Jr., H. C., 2, 3, 8, 14, 15, 16, 18, 32, 37, 43,

$55,57,60,96,118,123,128,134,147,149,158,163$, $165,167,168,169,180,181,191,192,193,215$

Thoro, D., 148, 160, 161

Thurnau, D. H., 195

Trimble, T. N., 175

Trotter, H. F., 115

Vandergraft, J. S., 3

van de Riet, R. P., 73, 186, 187

Vrchovecka, L., 173

Wagner, J. F., 123

Wallack, P., 203

Walsh, P. J., 127

Walter, H. F., 151

Wasscher, E. J., 129, 203, 204

Wegstcin, J., 2

Wells, M., 128, 136, 137, 138, 197

Whitley, V. W., 129

Whitman, D. R., 108, 109, 110, 111

Whittier, V. E., 77

Wilkenson, J. H., 2, 3, 15, 25, 26

Wirth, N., 146, 220

Witty, R. D., 27

Wolfson, M. L., 160,161

Wojcicki, M. E., 44

Wood, T. C., 35, 42

Wright, C. B., 45

Wright, H. V., 160, 161 
